Friederike Faust

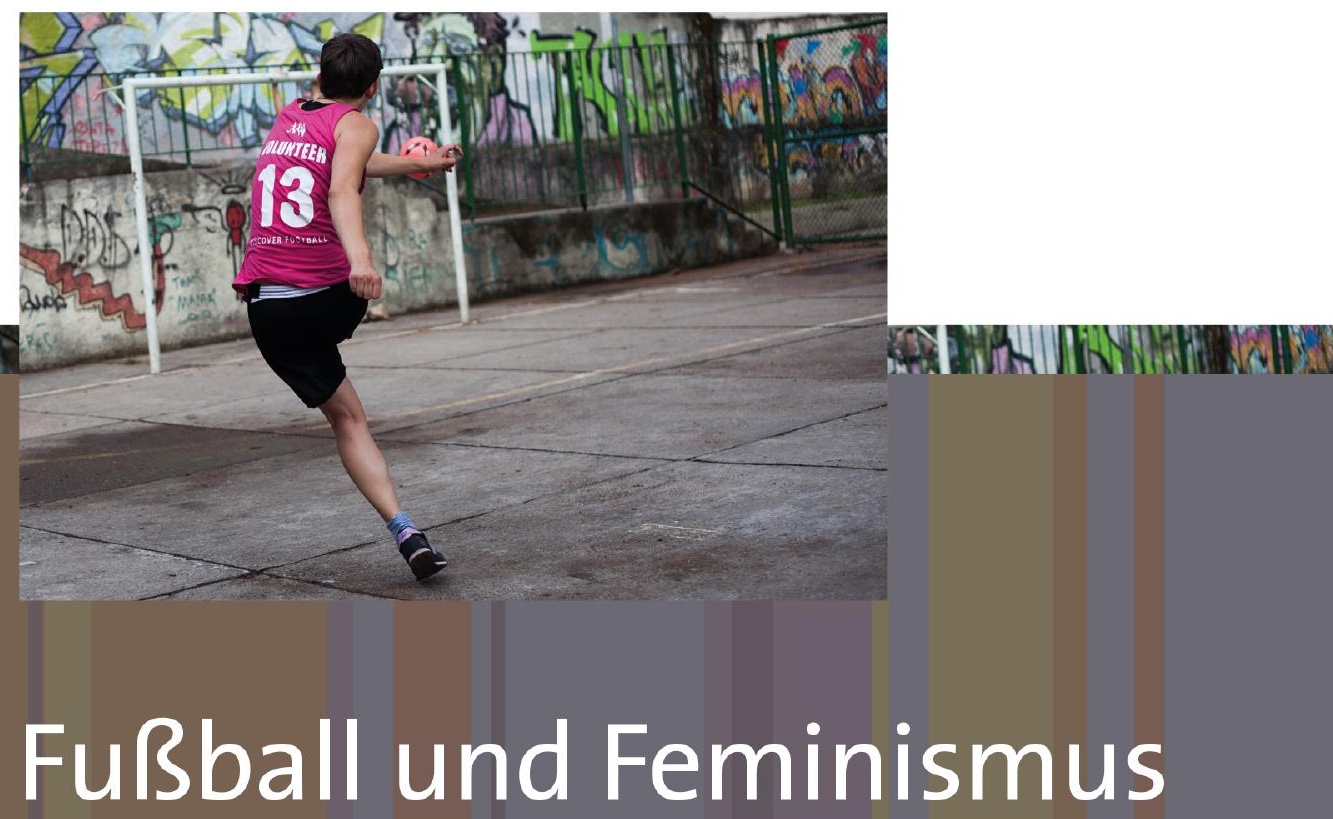

Eine Ethnografie geschlechterpolitischer Interventionen 
Friederike Faust

Fußball und Feminismus 
Friederike Faust

\section{Fußball und Feminismus}

Eine Ethnografie geschlechterpolitischer Interventionen

Budrich UniPress Ltd.

Opladen • Berlin • Toronto 2019 
Bibliografische Information der Deutschen Nationalbibliothek

Die Deutsche Nationalbibliothek verzeichnet diese Publikation in der Deutschen

Nationalbibliografie; detaillierte bibliografische Daten sind im Internet über

http://dnb.d-nb.de abrufbar.

(C) 2019 Dieses Werk ist beim Verlag Budrich UniPress erschienen und steht unter der Creative Commons Lizenz Attribution-ShareAlike 4.0 International (CC BY-SA 4.0): https://creativecommons.org/licenses/by-sa/4.0/

Diese Lizenz erlaubt die Verbreitung, Speicherung, Vervielfältigung und Bearbeitung bei Verwendung der gleichen CC-BY-SA 4.0-Lizenz und unter Angabe der UrheberInnen, Rechte, Änderungen und verwendeten Lizenz.

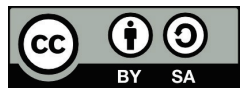

Dieses Buch steht im Open-Access-Bereich der Verlagsseite zum kostenlosen Download bereit (https://doi.org/10.3224/86388819).

Eine kostenpflichtige Druckversion kann über den Verlag bezogen werden. Die Seitenzahlen in der Druck- und Onlineversion sind identisch.

$\begin{array}{ll}\text { ISBN } & 978-3-86388-819-0 \text { (Paperback) } \\ \text { eISBN } & 978-3-86388-453-6 \text { (eBook) } \\ \text { DOI } & 10.3224 / 86388819\end{array}$

Umschlaggestaltung: Bettina Lehfeldt, Kleinmachnow - www.lehfeldtgraphic.de Titelbildnachweis: Foto: Dana Rösinger

Typografisches Lektorat: Anja Borkam, Jena - kontakt@lektorat-borkam.de 


\section{Inhalt}

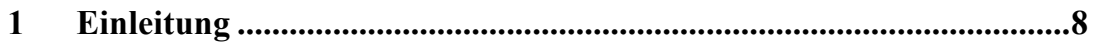

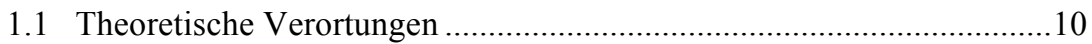

1.2 Fußball und Feminismus als Forschungsfeld ........................................15

1.3 Leitende Frage und Aufbau der Arbeit................................................17

2 Forschungsdesign und methodologische Aussichtspunkte ................20

2.1 Forschungsprozess, Methoden und multiple Positionen .......................21

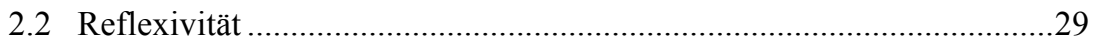

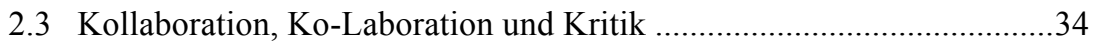

Teil I: Die Entstehung eines günstigen Moments .......................................49

3 Theoretische Ausgangspunkte: Friction und Schnittstellen..............50

4 Verbandsfußball: Historische, geschlechtliche und gesellschaftliche Dimensionen .................................................................52

4.1 Fußball als Kultur: Vereinswesen ....................................................53

4.2 Aus Geschlechterperspektive: Fußball und Frauen .................................58

4.3 Verwobenheiten: Sport, Staat und Ökonomie ......................................70

5 Diskursive Formationen des Fußballs ................................................76

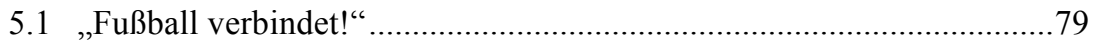

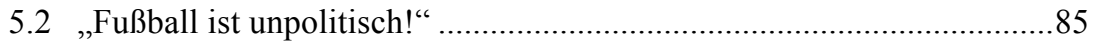

5.3 „Fußball empowert Frauen!“‘..........................................................92

6 Zwischenstand: ein Momentum für Fußball und Feminismus ......103

Teil II: Die Formierung einer Organisation ...........................................106

7 Theoretische Ausgangspunkte: Organisationen und ihre multiplen Gestalten ......................................................................107

8 Rechtskräftiger Verein und verlässliche Partnerorganisation - Verwaltungen und Projektförderung ................................................111

8.1 Ein Verein werden: „Es ist ja nicht nur Glaubwürdigkeit““.................112

8.2 Arbeit institutionalisieren: „Ihr macht das unglaublich

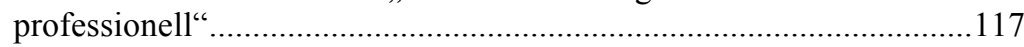

8.3 Sich engagieren: „Selbstständige Köpfe und kräftige Hände““.............123 
9 Städtische Sportveranstaltung und feministische Initiative -

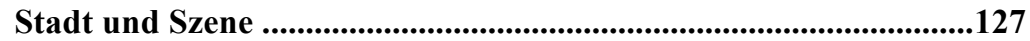

9.1 Sich stadträumlich verorten: „In the heart of Kreuzberg“.....................127

9.2 Stadträumlich navigieren: „DF ist doch eine Institution hier“ .............130

10 Fußballverein und Amateurin - Verbandsfußball..........................132

10.1 Eine Alternative bieten: ,Selbstorganisiert und transparent“"..............133

10.2 Sich eingliedern: „Den Wettkampf ernst nehmen“ “.............................136

11 Expertin und Betroffene - Social Movement Market ........................142

11.1 Diskursiv manövrieren: „So ein roter Faden“.....................................143

11.2 Strategisch essentialisieren: „Das ist nicht die politische Realität“ .....155

11.3 Netzwerk verwalten: „Beziehungsarbeit“ .........................................160

11.4 Expertise schaffen: „Mit differenziertem Wissen auftrumpfen“..........163

11.5 Erfahrung privilegieren: „Wir sind viel sympathischer“.....................165

12 Aktivistin und Fußballerin - die Mitgliederschaft ............................169

12.2 Subjektivität affirmieren: „Female football activist“ ...........................173

12.3 Identitäten verhandeln: „Frauen und Lesben“.....................................176

13 Mitstreitende und Lernende - weltweite Frauenfußballinitiativen ...........................................................181

13.1 Erwartungen auswählen: „Why do you consider football important as a woman*?".

13.2 Machtbeziehungen verkomplizieren: „Western women are not more emancipated than Muslim women!"

13.3 Ähnlichkeiten herstellen: „Wir hatten ganz ähnliche

Erfahrungen“"

14 Zwischenstand: Durch multiple Anforderungen navigieren...........200

Teil III: Geschlechterpolitische Interventionen..........................................207

15 Theoretische Ausgangspunkte: politisches Handeln und Topografien der Macht ....................................................................209

16 Forderungen stellen............................................................................217

16.1 Emotional Stellung beziehen: ,Figo, we will kill you““........................219

16.2 Erfahrungen mobilisieren: „What discouraged you?“.........................223

16.3 Privates politisieren: „That is discrimination“ ....................................226 
16.4 Plausible Lösungen vorschlagen: „IST-Beschreibung und SOLLForderung".....

16.5 Stimme formatieren: „We, representatives of girls’ and women's football" 241

16.6 Gehört werden: „Es ist einfach unser Recht“ .....................................244

17 Alternativen präfigurieren ..................................................................251

17.1 Raumzeitliche Gegenwelt aufbauen: „So ein Inselgefühl“ “.................254

17.2 Leistung modifizieren: „Es geht nicht nur um Fußball“ „.....................259

17.3 Fußball politisieren: „Es lässt sich mit Fußball gut Politik

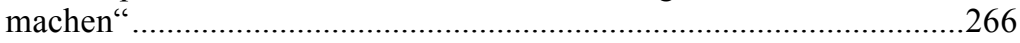

17.4 Geschlechterverhältnisse umkehren: „Dein Chef hat mir das erlaubt" 270

17.5 Multiple Räume erleben: „Überhaupt keinen Zugang finden“ “...........278

17.6 Gesehen werden und mobilisieren: „It is a thin line”. 285

18 Zwischenstand: Sich zwischen multiple Strategien und Positionen bewegen......................................................................................293

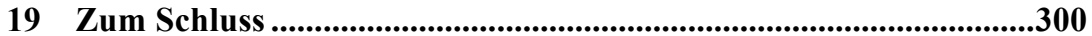

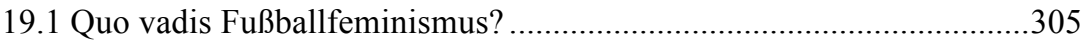

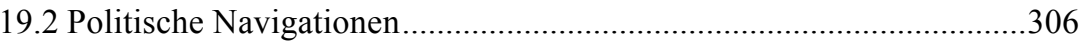

19.3 Kritik und die Lücken im paraethnografischen Wissen .......................309

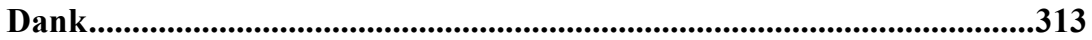

Literatur- und Quellenverzeichnis...........................................................315 


\section{Einleitung}

Im Mai 2013 lädt das Bundesministerium des Innern (BMI) zur fünften Weltsportministerkonferenz nach Berlin. Die angestrebte „Bestandsaufnahme der weltweiten Entwicklungen im Sport und die Formulierung strategischer Ziele zu aktuellen Themen der internationalen Sportpolitik“ (MINEPS V 2013) fokussiert unter anderem den Zugang zum Sport als grundlegendes Recht für alle und adressiert die geschlechtergerechte Teilhabe. Neben den sportpolitischen Länderdelegationen sind auch Vertreter_innen der Sportverbände, Wissenschaftler_innen und Nicht-Regierungsorganisationen (NGOs) anwesend. Eine dieser NGOs ist die in Berlin ansässige Frauenrechts- und Frauenfußballorganisation Discover Football, die ich zu diesem Zeitpunkt bereits seit einem Jahr begleite. Das BMI hatte die kleine NGO eingeladen, im Vorfeld an einem Erfahrungsaustausch zur Teilhabe von Mädchen und Frauen am Sport teilzunehmen und sich auf der Konferenz in Form eines Posters zu präsentieren. Dieses Poster reiht sich nun im Vorraum des Plenarsaals ein in ein Meer an Stellwänden und informiert über die Vision des gemeinnützigen Vereins: einen diskriminierungsfreien Zugang zum Sport als fundamentales Recht für alle. Darunter stellen pinke, wie handgeschrieben wirkende Großbuchstaben die dafür eingesetzten Mittel vor:

\section{„We empower girls and women through football we challenge gender roles in society we enable exchange and networking}

we are creating a powerful international network for women's football“"

Mit mir sind bereits vier weitere Mitglieder von Discover Football eingetroffen und haben Broschüren und Flyer ansehnlich um das Poster drapiert, als ein fünftes Mitglied deutlich übermüdet dazueilt und eine Schachtel voller DVDs bringt. Bis tief in die Nacht hinein habe sie an der Fertigstellung der digitalen Version des Handbuchs „Frauen Macht Fußball“ gearbeitet, damit es heute verteilt werden könne. Nach den Eröffnungsreden und Ansprachen der Bundeskanzlerin, des Innenministers und weiterer hochrangiger Vertreter_innen aus Politik und Sport ist Mittagspause. Wir Frauen von Discover Football stecken die Köpfe zusammen und beratschlagen, wie wir die sich darbietenden Kontaktmöglichkeiten nutzen könnten. Birgit schlägt vor: „Wir sollten uns allen Delegationen aus den Ländern vorstellen“, und erhofft sich dadurch möglichst viele Kontakte zu Frauenfußballteams weltweit, die sie dann zu Projekten einladen könnte. Saskia und Nadine möchten gerne noch einmal mit einer Wissenschaftlerin und Frauensport-Aktivistin sprechen. Sie wollen sich bei ihr nach Möglichkeiten der Einflussnahme auf das zu verabschiedende Communiqué erkundigen, denn in dem Entwurf vermissen sie die Benennung von Sexismus und Homophobie als zu bekämpfende Diskriminierungsformen. Celia hat bereits gute Kontakte zum Frauenfußball in Benin 
und Togo und möchte daher mit den sportpolitischen Delegationen dieser Länder sprechen. Ich schlage vor, den bereits bestehenden Kontakt zur Gesellschaft für Internationale Zusammenarbeit und zum Bundesministerium für wirtschaftliche Zusammenarbeit und Entwicklung $\mathrm{zu}$ intensivieren, schließlich habe ein Vertreter mir gegenüber kürzlich Interesse an einer $\mathrm{Zu}$ sammenarbeit mit Discover Football signalisiert. Nach dieser kurzen Absprache verstreuen wir uns in den Konferenzräumen und verfolgen unsere Missionen. Am Ende der Konferenz haben wir zahlreiche Visitenkarten gesammelt, Informationsmaterial verteilt, bestehende Kontakte vertieft, und die Sportminister innen haben die „Berliner Erklärung“ verabschiedet. In ihr heben die Regierungsbeauftragten die „Bedeutung von Gender Mainstreaming unter den Leitbegriffen Vielfalt, Wahlmöglichkeit, Befähigung und Ermutigung bei Maßnahmen zur Stärkung der Teilhabe von Mädchen und Frauen am und durch Sport" hervor, weisen darauf hin, dass dies ,auch bedeutet, Frauen in Sportorganisationen und in Entscheidungspositionen einzubinden", und verpflichten sich unter anderem, Hindernisse für die sportliche Partizipation von Frauen zu verringern, Diskriminierungen und sexuelle Belästigung zu verfolgen sowie die Präsenz von Frauen in Entscheidungsgremien zu fördern (MINEPS V 2013a).

Die Sportminister_innen wollen damit verändern, was zahlreiche sozialwissenschaftliche Studien, historische Untersuchungen und publizistische Beiträge für unterschiedliche Sportarten - aber besonders für den Fußball herausarbeiten und analysieren: die Dominanz des Männlichen und die Marginalisierung des Weiblichen. Wie für viele andere Länder diagnostizieren sie auch für Deutschland: Fußball ist ein Männersport. Mit diesem Resümee machen sie auf die Dringlichkeit geschlechterpolitischer Interventionen in jene Sportarten aufmerksam, die als besonders männlich gelten. Fußball, ein Männersport - so wurde das Spiel mit dem Ball auf dem grünen Rasen lange Zeit auch von den Vereinen und Verbänden verstanden und gelebt. Aber nun einigen sich die Sportminister_innen und Vertreter_innen der Sportverbände auf explizite Maßnahmen zur Förderung des Frauensports und des Abbaus geschlechterbasierter Benachteiligungen.

Ist Fußball immer noch ein Männersport? Der „Schutzraum für moderne Vorstellungen der vormodernen männlichen Natur“" (Sülzle 2011, 252) scheint ins Wanken geraten zu sein. Nicht nur kicken immer mehr Mädchen und Frauen selbst und mischen die fußballerische „Arena der Männlichkeit“ (Kreisky/Spitaler 2006) auf, auch kritisieren Aktivist_innen, Journalist_innen, Funktionär_innen und Politiker_innen öffentlich die Benachteiligung von Frauen und können sich dabei auf die 1979 verabschiedete Frauenrechtskonvention und ihre Forderung nach diskriminierungsfreier Teilhabe an Sport und Leibesübungen berufen. Eine Stimme in diesem Chor ist der Verein Discover Football, der für Geschlechtergerechtigkeit im Fußball eintritt. 
In dieser Arbeit erkunde ich ausgehend von meiner Feldforschung bei Discover Football, wie die scheinbar selbstverständliche Zuweisung von Geschlechterrollen und ihre Hierarchisierung im Fußball plötzlich öffentlich und effektiv in Zweifel gezogen werden. Ich untersuche, wie die über viele Jahrzehnte stabilisierten Geschlechterverhältnisse, die normal, ja fast schon natürlich erscheinen, in Frage gestellt und als problematisch erachtet werden und wie sie schließlich von einer zivilgesellschaftlichen Organisation adressiert und verändert werden.

\subsection{Theoretische Verortungen}

Mein Forschungsinteresse gilt den fußballerischen Geschlechterverhältnissen und ihrer Infragestellung und fokussiert insbesondere, wie im Fußball vergeschlechtlichte Subjekte zueinander ins Verhältnis gesetzt werden, wie dieses Verhältnis kritisiert und letztlich aktiv bearbeitet wird. Im Zentrum steht dabei die Frage, wie zivilgesellschaftliche Akteur_innen in den Fußball als ein Feld intervenieren, das sich in organisatorischer, struktureller und kultureller Hinsicht deutlichen von staatlichen und internationalen Regierungsinstitutionen unterscheidet. Ich greife dafür auf theoretische Überlegungen zum Verhältnis von Geschlecht und Sport zurück und ergänze diese mit dem Konzept der Problematisierung.

Die Theoretisierungen der Geschlechterverhältnisse im Sport, wie sie sich vor allem in der Sportsoziologie und vereinzelt auch in den Ethno-, Kulturund Politikwissenschaften finden, sind eng mit den Denkbewegungen der internationalen Gender und Queer Studies verknüpft (Caudwell 2011). In den 1970er und 1980er Jahren entstehen entlang der analytischen Kategorie Frau und auf Basis der Unterscheidung zwischen biologischem und sozialem Geschlecht (sex und gender) Forschungen über intrakategorielle Gemeinsamkeiten und geteilte Unterdrückungserfahrungen (ebd., 331). Liberalfeministische Ansätze analysieren Geschlecht als gesellschaftliche Machtbeziehung und gehen von einem bipolaren, aber ungleichen Geschlechterverhältnis aus, das sich über den Sport naturalisiert und Frauen als ,die Anderen' positioniert (ebd.). Sport erscheint aufgrund des kapitalistisch inspirierten Leistungsstrebens und Wettkampfgedankens sowie der Gewaltaspekte als ,training ground for maintaining the domination/submission relationships characteristic for patriarchy“ (Bennett et al. 1987, 378). Iris Marion Young (1980) argumentiert, dass die Objektifizierung von Frauen in industrialisierten Ländern bei diesen unterbrochene, zögerliche und fragmentierte Bewegungen produziert. Sportliches Ungeschick und Unvermögen - gerade in Sportarten, die raumgreifenden, entschlossenen und kontaktfreudigen Körpereinsatz erfordern sind damit nicht an das biologische Geschlecht gebunden, sondern erst durch 
eine geschlechtsspezifische Sozialisation erlernt (ebd.; Bennett et al. 1987). Carol Hagemann-White bezeichnet den Fußball als „maßgebliche Sozialisationsinstanz" $(1993,77)$, in der Männlichkeit konstruiert und von Jungen und Männern erlernt wird. Sport wird so als Feld konzipiert, in dem über Leistung und Wettkampf ein soziales Geschlecht erlernt und patriarchale Kontrolle über Frauen, ihre Körper und ihre Selbstwahrnehmung ausgeübt wird.

Intersektionale bzw. interdependente Ansätze tragen zu einer Dezentrierung des Patriarchats als eines Hauptmechanismus der Macht bei, indem sie auf die Vielzahl interagierender, sich kreuzender oder miteinander verwobener Machtbeziehungen hinweisen und Geschlecht zu anderen sozialen Identitätskategorien ins Verhältnis setzen (Walgenbach et al. 2007; Binder/Hess 2011, 16). Sie zeigen, dass soziale Kategorisierungen wie Geschlecht, Sexualität, race, Alter oder soziale Herkunft auch im Sport miteinander verwoben wirken und Machtverhältnisse multiskalar und sich gegenseitig durchdringend verlaufen (Hargreaves 2000, 6; Elling/Knoppers 2005; Pelak 2005; Scraton et al. 2005; Degele/Janz 2011; Lau 2011; Dietze 2012; Ochsner 2012; Degele 2013). Den universalisierenden und essentialisierenden Tendenzen der eindimensionalen Analyse der Kategorie Frau wirken sie durch den Verweis auf intrakategorielle Diversität von Frauen entgegen, was schließlich auch zu einer Revision der Geschlechteropposition führt: Anstatt allein Jungen und Männer als Akteure der Unterdrückung zu denken, wird auch die Beteiligung privilegierter Frauen bei der sportlichen Exklusion weniger privilegierter Frauen thematisiert (Caudwell 2011, 334). Gleichzeitig warnt Judith Butler davor, die Ordnungskategorien als analytische Ausgangspunkte und horizontale Aneinanderreihungen von möglichen Identitäten der Untersuchung voranzustellen. Subjektivierung ist vielmehr ein fortdauernder Prozess, der immer wieder neu situierte Subjekte hervorbringt (2006, 143). Eine interdependente Betrachtung fußballerischer Geschlechterverhältnisse geht also davon aus, dass Identitäten und Machtverhältnisse immer situativ aufgerufen, aktualisiert oder auch temporär vergessen oder irrelevant werden (vgl. Hirschauer 2001). Vergeschlechtlichende Subjektivierung im Fußball ist daher das Produkt eines nur bedingt vorhersehbaren $\mathrm{Zu}$ sammenspiels vieler verschiedener Ungleichheitsverhältnisse und Identitätskategorien. Eine ethnografische Herangehensweise begegnet diesem Zusammenspiel mit größtmöglicher „Offenheit gegenüber der Komplexität und Kontingenz gesellschaftlicher Problemlagen“ und ist darauf ausgerichtet, „möglichst sensibel möglichst viele Kategorien im Spiel“ ${ }^{\text {zu halten }}$ (Binder/Hess 2011, 52).

Mit der poststrukturalistischen Wende rückt die Frage nach der Herstellung von Geschlecht im Sport in den Fokus. Die zentrale Analysekategorie verschiebt sich weg von Frau/en hin zu Gender: An die Stelle der Geschlechterdifferenz als analytischem Ausgangspunkt tritt nun das Interesse an der Herstellung von Körpern, biologischen und sozialen Geschlechtern 
(Caudwell 2011, 334), und die situative Interaktion gewinnt neben der Sozialisation an Bedeutung. Anknüpfend an die Praxistheorien und die Ethnomethodologie wird Sport als vergeschlechtlichende Praxis untersucht. Männlichkeit und Weiblichkeit erscheinen als Produkte des ständigen interaktiven „Zeigens gekonnter körperlicher Bewegungen und entsprechender Verhaltens- und Redeweisen" (Schmidt 2012, 46; vgl. West/Zimmerman 2009, 114). Im Sinne der praxistheoretischen Perspektive auf die Herstellung sozialer Ordnung in verkörperten Praktiken erscheint die ,wechselseitige Konstitution von Subjekt, Körper und sozialer Welt [...] im Sport so gut fassbar wie an kaum einer anderen Praxis“ (Alkemeyer 2006, 290). Die Körper- und Geschlechterverhältnisse im Fußball gelten als historisch-kulturell geformte „Effekte einer sozialen Praxis“ (Sobiech/Ochsner 2012, 10), und sie reproduzieren sich nicht nur durch Körper, Verhaltens- und Bewegungsweisen, sondern auch in Zuordnungen zu und Selbstpositionierungen in verschiedenen Handlungsspielräumen (ebd.; Pfister 2003). Dabei werden Geschlechter nicht nur hergestellt, sondern auch hierarchisch angeordnet: Jungen und Männer werden als rational, aktiv, stark und aggressiv gedacht und daher Frauen und Mädchen, die als emotional, schwach und unterwürfig konstituiert werden, übergeordnet (Braunmüller et al. 2016, 85).

Die feministische Sportforschung im anglo-amerikanischen Raum widmet sich vor allem der kulturell-diskursiven Subjektivierung. Was in der Ethnomethodologie von Candance West und Don Zimmerman (2009) noch als biologisches Geschlecht (engl. sex) angelegt ist, wird bei Butler selbst als kulturelle Norm gedacht. Mit dem Begriff der Performativität beschreibt sie „die ständig wiederholende und zitierende Praxis, durch die der Diskurs die Wirkung erzeugt, die er benennt" $(1997,22)$. Die Norm, das als biologisch imaginierte Geschlecht, wird durch ihre Aufführung erst hervorgebracht und dadurch gleichzeitig stabilisiert (ebd., 32). Normalisierung und Stabilität des zweigeschlechtlichen Systems sind damit Effekte der Performativität, und das Sporttreiben vor allem in männlich konnotierten Sportarten wird zu einer „masculinizing practice“ (Whitson 2002, 229). Maskulinität und Femininität sind demnach weder Körpermerkmale noch Charaktereigenschaften, sondern ,a product of discourse, practices, and social relations that construct the situation of women in patriarchal societies in ways that typically disable women in relation to men" (ebd.). Geschlechterdifferenz ist damit Effekt und nicht Grundlage von Machtformationen. Donna Haraway (1995) setzt diesem sozialkonstruktivistischen Interesse an Diskursen und Sprachhandlungen einen Fokus auf Materialität und Stofflichkeit entgegen und warnt vor einer vorschnellen Preisgabe des Körperlichen. Ohne zu re-biologisieren, verweist sie darauf, dass der Körper ,,mehr ist als ein leeres Blatt für soziale Einschreibungen“ und „Rohmaterial von Kultur“ (ebd., 92f.). Statt als passive Wissensobjekte untersucht sie Körper als materiell-semiotische Akteure, die aktiv an der Produktion von Bedeutungen über Geschlecht beteiligt sind. Die 
Grenzen werden situativ und interaktiv gezogen, sie sind nicht präexistent, sondern vielmehr verschiebbar. Gender ist damit die „situierte Differenz und Verkörperung" (ebd., 94).

Die Interventionen der Queer Studies in die Geschlechtertheorie ergänzen die Entnaturalisierung der Geschlechterverhältnisse um Sexualität als weitere Differenzierungskategorie sowie um die Dekonstruktionen der Zweigeschlechtlichkeit. Michael Warner (1993, xxi) macht mit dem Begriff der Heteronormativität jenen Diskurs beschreibbar, der aufbauend auf der zweigeschlechtlichen Ordnung Heterosexualität als Norm konstituiert, die Gegensätze ,männlich ‘ und, weiblich' durch gegenseitiges Begehren miteinander verbindet und als elementare Form sozialer Beziehungen und ihres Fortbestandes naturalisiert. Damit wird Sport als heteronormativierendes Feld und Praxis untersuchbar (vgl. Scraton/Flintoff 2002; Caudwell 2006; Eng 2008). In den Fokus geraten so die uneindeutigen Zwischenräume zwischen und die Ausdifferenzierungen innerhalb der Kategorien ,männlich ${ }^{\text {‘ }}$ und ,weiblich', sodass diese in den Plural gesetzt und um viele weitere Konzepte von geschlechtlichen und sexuellen Identitäten und Praktiken sowie deren Instabilitäten und Unbestimmbarkeiten ergänzt werden können. Gefragt wird nicht allein danach, wie Weiblichkeit im Fußball hergestellt wird, sondern welche Weiblichkeiten situativ zum Einsatz kommen, wie sich Spielerinnen mithilfe von Körperpraktiken und Geschlechtsmarkern welche Geschlechtlichkeiten aneignen, die Spielräume der Heteronormativität und des Patriarchats kreativ für sich nutzten und mit nicht-normativen Geschlechterperformanzen wie z.B. der „female masculinity“ (Halberstam 2012) oder der „femme-inity“ (Caudwell 1999) die Zwischenräume der Zweigeschlechtlichkeit bespielen (vgl. Palzkill 1995; Cox/Thompson 2000; Eng 2006). Sportpraxen junger Frauen in männlich dominierten Sportarten werden darauf hin untersucht, wie sie Geschlechterrollen modellieren, indem sie kompetitives, schmerzhaftes und risikobereites Verhalten mit Weiblichkeitsdarstellungen kombinieren und selbstbestimmte Geschlechtsidentitäten entwerfen (vgl. Young 1997; Pfister/Fasting 2004).

Die vorliegende Arbeit versteht die fußballerischen Geschlechterverhältnisse als historisch gewachsene und durch soziale Praktiken hergestellte, hierarchische Anordnung von Körpern und Subjekten entlang zweigeschlechtlicher, heteronormativer Prinzipen. Als Produkt sich wiederholender, Norm zitierender Praktiken im Sport - sowohl das aktive Sporttreiben, das Organisieren und Verwalten als auch das Rezipieren - ist diese relationale Anordnung kontingent und damit auch anfällig für Abweichungen und Veränderungen.

Die Arbeit nimmt diese Überlegungen als Ausgangspunkt und geht mit Blick auf die jüngsten Entwicklungen in Fußball, Sport- und Geschlechterpolitik einen Schritt weiter: Nachdem ausführliche Erkenntnisse zu Beschaffenheit, Konstruktion und Reproduktion der fußballerischen Geschlechterver- 
hältnisse sowie erste Überlegungen zu deren Brüchigkeit und Widersprüchlichkeit vorliegen, kontextualisiert diese Arbeit sowohl die Reproduktion wie auch Veränderbarkeit der Geschlechterverhältnisse in einem breiten gesellschaftspolitischen Kontext. Konkret frage ich, wie historische Entwicklungen und gegenwärtige diskursive Formationen, Sport- und Geschlechterpolitiken auf internationaler, staatlicher und zivilgesellschaftlicher Ebene sowie übergeordnete Paradigmen es möglich machen, dass die fußballerischen Geschlechterverhältnisse ihre Selbstverständlichkeit verlieren und kritisierbar werden, wie andere Verhältnisse imaginiert werden und wie an konkreten Veränderungen und utopischen Alternativen gearbeitet wird.

Um zu untersuchen, wie die Geschlechterverhältnisse im Fußball in Zweifel gezogen werden, greife ich auf Michel Foucaults Begriff der Problematisierung zurück, mit dem er die Geschichte des Denkens charakterisiert (2005, 731f.). Mit diesem lässt sich untersuchen, wie und unter welchen Umständen Ordnungen, Wahrheiten und Situationen ihren Status des Selbstverständlichen verlieren und in den Bereich des kritischen - hier: feministischen Denkens und Handelns eintreten. Foucault führte diesen Begriff erst spät und rückblickend auf seine historischen Arbeiten ein:

„Die Geschichte des Denkens ist die Analyse der Art und Weise, wie ein unproblematisches Erfahrungsfeld oder eine Reihe von Praktiken, die als selbstverständlich akzeptiert wurden, die vertraut und, unausgesprochen' sind, also außer Frage stehen, zum Problem werden, Diskussionen und Debatten hervorruft, neue Reaktionen anregt und eine Krise der bisherigen stillschweigenden Verhaltensweisen, Gewohnheiten, Praktiken und Institutionen bewirkt." (Foucault 1996, 78)

Mit dem Begriff der Problematisierung verweist Foucault auf den gesellschaftlichen Konstruktionsprozess von Problemen (Klöppel 2010, 255). Er beschreibt damit, wie „ein Handlungsbereich und ein Verhalten“ verunsichert, ihrer Vertrautheit beraubt werden oder wie in ihrem Umfeld Schwierigkeiten entstehen, sodass diese ,ins Feld des Denkens“ eintreten (Foucault 2005, 732). Problematisierungen stellen daher Ausgangspunkte für Veränderungen und Transformationen dar (Klöppel 2010, 260). Dabei geben die Art und Weise, wie Probleme benannt und beleuchtet werden, bereits bestimmte Lösungswege vor und schließen andere durch die Problembestimmung im Vorfeld aus (Foucault 2005, 733). Problematisieren impliziert damit die Transformation bisheriger Selbstverständlichkeiten bei gleichzeitiger Stabilisierung anderer Gewohnheiten, die sich in den Lösungsmöglichkeiten fortschreiben (Klöppel 2010, 260).

Gesellschaftliche Probleme als Prozesse zu erfassen lässt diese nicht nur als kontingent erscheinen, sondern auch als aktives Tun. Poblematisierungen sind damit Produkte konkreter Situationen und historischer Entwicklungen, sie sind verkörpert und an Akteur_innen gebunden und erschließen sich somit dem ethnografischen Blick auf Alltagspraktiken. Um Problematisierungen als Bündel von Praktiken zu fassen und beschreibbar zu machen, umkreise ich 
Foucaults Konzept in drei Schritten: Erstens frage ich nach den Umständen und Bedingungen ihrer Möglichkeit, zweitens nach den Akteur_innen und dem entsprechenden verkörperten Wissen und drittens nach den konkreten Praktiken der Problembearbeitung. Ich gehe also explorativ und nicht repräsentativ vor und erkunde die Materialisierung von Problematisierungen in sozialen Praktiken anhand eines lokalisierbaren Forschungsfeldes.

\subsection{Fußball und Feminismus als Forschungsfeld}

„DISCOVER FOOTBALL advocates for a world in which girls and women can do sports without being discriminated against for any reason." (Discover Football o.J.)

Die Problematisierung der fußballerischen Geschlechterverhältnisse ist in jenen gesellschaftlichen Feldern und Momenten greifbar, in denen sich Fußball und Geschlechterpolitik überschneiden. Diese Schnittstelle bildet mein Forschungsfeld. Sie institutionalisiert und materialisiert sich unter anderem in der Frauenfußball- und Frauenrechtsorganisation Discover Football, die ich als Ort meiner ethnografischen Feldforschung gewählt habe.

Discover Football (DF) ist ein in Berlin ansässiger eingetragener Verein, der sich mit internationalen Projekten für Frauenrechte, Geschlechtergerechtigkeit und gegen Diskriminierungen im Fußball einsetzt. Zur Zeit meiner Feldforschung trafen sich die zwei bis drei hauptamtlichen Projektkoordinatorinnen und die vielen ehrenamtlichen Mitarbeiter_innen einmal in der Woche zum sogenannten Plenum, um die Aktivitäten des Vereins zu planen. $\mathrm{Zu}$ diesen gehörte seit ihrer Gründung 2009 das internationale Frauen-FußballKultur-Festival, das die Organisation alle zwei Jahre in Berlin-Kreuzberg veranstaltete und wofür sie sozial und politisch engagierte Frauenfußballteams aus der ganzen Welt einlud. 2012 organisierte DF zum ersten Mal ein Expertinnenforum mit internationalen Trainerinnen, Spielerinnen und Funktionärinnen und wiederholte dieses Projektformat in den kommenden Jahren mit wechselnden thematischen und regionalen Schwerpunkten. Aus diesen Foren entstanden unter anderem Interviewfilme mit den Teilnehmerinnen, ein Handbuch mit Erfahrungsberichten und Handlungsstrategien sowie eine Deklaration mit Forderungen an die Fußballverbände. 2012 reiste DF mit zwei Kleinbussen zu den Austragungsorten der Fußball-Europameisterschaft der Männer nach Polen und in die Ukraine, zeigte vor Ort eine mobile Ausstellung und organisierte Fußballturniere und Podiumsdiskussionen. 2014 fand eine ähnliche Reise zur WM der Männer in Brasilien statt. Zudem ergänzte DF mit der Gründung eines eigenen Frauen-Fußballvereins die lokale sportliche Infrastruktur und engagierte sich fortan vermehrt in der Berliner Sportpolitik und im lokalen Fußballverband. Hinzu traten viele weitere klei- 
nere Projekte: DF organisierte Filmvorführungen, leitete Workshops auf Kongressen, organisierte Podiumsdiskussionen und lokale Fußballturniere, reiste zu Turnieren und präsentierte dort die eigene Arbeit, arbeitete mit anderen Fußballvereinen und -initiativen an einer verbesserten lokalen Infrastruktur für den Frauenfußball und setzte sich zusammen mit anderen Menschenrechtsorganisationen für den verbesserten Schutz von Frauen und LGBTI-Personen ein. Stets prangerte DF die Benachteiligung von Frauen im Fußball, den Sexismus und die Herabwürdigung von Spielerinnen an.

Meine Forschung führte ich von Juli 2012 bis Dezember 2015 durch. Sie fiel damit in einen Zeitraum der langsamen, aber kontinuierlichen Veränderung und des institutionellen Wachsens von DF. So konnte ich den Prozess der Formierung und Stabilisierung als Organisation erfassen: Zwar gab es zwischenzeitlich immer wieder Sorgen um das finanzielle und organisatorische Überleben, doch gelang es DF, sich als glaubwürdige Frauenrechts- und Frauenfußballorganisation lokal, national und auch international zu etablieren. In diesem Sinne ist die Darstellung dieser Organisationsformierung auch die Geschichte eines kleinen Erfolgs - sowie der damit zusammenhängenden Kompromisse, Abstriche, Kontroversen und Zwickmühlen. DFs Erfolg wurde durch Veränderungen in der geschlechterpolitischen Landschaft des Fußballs begünstigt: Mit einem Mal fand die Kritik an den fußballinternen Benachteiligungen und Diskriminierungen Gehör und Unterstützung, und etwa zeitgleich entdeckten regierende Institutionen Mädchen und Frauen als Zielgruppen und Sport als Medium ihrer Politiken. Diese günstigen Umstände nutzte DF mit Motivation und Geschick, fand eine Nische und füllte sie nach und nach aus.

Mir ist keine andere international agierende feministische Organisation bekannt, die aus dem Fußball selbst käme und diesen ins Zentrum ihrer geschlechterpolitischen Mittel und Ziele gerückt hätte. Viele gemeinnützige Organisationen integrieren Sport in ihre entwicklungs- und integrationspolitische Arbeit, jedoch dient er meist als Mittel für sportferne Zwecke. Natürlich bekämpfen zahlreiche Initiativen und Projekte Diskriminierungen im Fußball, üblicherweise konzentrieren sie sich aber auf den Männerfußball und adressieren Geschlecht und Sexualität lediglich nebenbei. Dieses Alleinstellungsmerkmal von DF begründete die Wahl des Feldausschnitts, stellte mich aber zugleich vor das Problem, dass eine umfassende Anonymisierung nicht möglich sein würde. Ich habe mich daher unter Absprache mit der Organisation gegen eine Anonymisierung entschieden. Alle Protagonist_innen dieser Arbeit tragen jedoch Pseudonyme.

Ich beschreibe Argumente, Vorgehensweisen und Politiken von DF explizit als feministisch, auch wenn diese Bezeichnung von DF selbst kaum verwendet wird. Damit kennzeichne ich, was mich an ihrem Vorgehen interessiert: wie es dominante Geschlechterverhältnisse herausfordert und umgestaltet (vgl. Thayer 2010, 180). Dabei denke ich im Plural, sodass die Kategorie 
,Feminismen' ganz unterschiedliche Positionen, Argumente, Methoden, Ästhetiken und Ziele - radikal-, differenz-, gleichheits-, queer-feministische und andere - beinhalten und die unterschiedlichen Ansichten innerhalb der Gruppe sowie ihrer Kooperationspartner_innen und Teilnehmer_innen umfassen kann. Feminismen stellen demnach eine von mehreren möglichen Ausgestaltungen von und Herangehensweisen an Geschlechterpolitiken dar. Mit Geschlechterpolitik bezeichne ich sämtliche Politiken sowohl von zivilgesellschaftlichen, verbandsfußballerischen als auch staatlich-regierenden und -verwaltenden Akteur_innen, die sich mit den Geschlechterverhältnissen beschäftigen, aber nicht notwendigerweise auf eine Umgestaltung der dominanten Verhältnisse abzielen.

\subsection{Leitende Frage und Aufbau der Arbeit}

Von meinen Beobachtungen bei DF ausgehend diskutiere ich die Frage nach der geschlechterpolitischen Anzweiflung und Umarbeitung bestehender Verhältnisse im Fußball in einem Dreischritt. Im Sinne einer ,anthropology of the contemporary" (Rabinow et al. 2008) wende ich mich den Schnittstellen von Fußball und Feminismus sowie den Möglichkeitsbedingungen der Problematisierung und den dabei wirkenden Rationalitäten zu. Diese verfolge ich über das lokalisierte Forschungsfeld hinaus in historische Tiefen und gegenwärtige gesellschaftliche Breiten hinein, und löse mich damit von früheren holistisch ausgerichteten, orts- und kulturgebundenen Ansätzen. Jeder der drei Schritte hat zum Ziel, von der Empirie ausgehend aktuelle theoretische Diskussionen zu kommentieren und zugleich in Form von Zwischenergebnissen der Antwort auf die forschungsleitende Frage näherzukommen. Dieser Dreischritt gliedert sich also entlang folgender Fragen:

- Unter welchen Umständen werden die fußballerischen Geschlechterverhältnisse öffentlich problematisierbar?

- Wie und entsprechend welchen Anforderungen formiert sich eine Organisation, um als Bearbeiterin der Geschlechterverhältnisse anerkannt zu werden?

- Wie wird in die Geschlechterverhältnisse problematisierend interveniert?

Dementsprechend widmet sich der erste Teil den geschichtlichen, politischen, sportkulturellen und gesellschaftlichen Umständen aus historischer und diskursanalytischer Perspektive. Ich folge dabei Foucaults Auffassung, dass die Aufgabe wissenschaftlicher Kritik darin liegt, die Wurzeln der Problematisierungen sichtbar zu machen, die verschiedenen sie einschränkenden sowie ermöglichenden Bedingungen und die vielzähligen zur Verfügung stehenden 
Antwortmöglichkeiten aufzuzeigen (Rabinow 2004, 41). Ich erkunde dafür die historische Entwicklung des Verbandsfußballs, um einerseits herauszuarbeiten, wie sich eine spezifische Geschlechterordnung und ein verbandsfußballerischer Habitus zu Selbstverständlichkeiten festigten, und um andererseits zu zeigen, dass feministische Infragestellungen dieser Verhältnisse historische Konjunkturen durchliefen. Aus diskursanalytischer Perspektive untersuche ich dominante diskursive Formationen des Fußballs und zeige daran, wie einerseits diskursive Schnittmengen zwischen Fußball, Sportpolitik und Feminismus entstehen, andererseits auch zunächst kaum gehörte Zweifel an der fußballerischen Geschlechterordnung plötzlich plausibel werden. Ich frage daher zunächst nach den „Ereignissen“ (Foucault 2005), die den fußballerischen Geschlechterverhältnissen den Status des Selbstverständlichen nehmen und sie zum Gegenstand des Denkens und Zweifelns machen. Die historischen und diskursanalytischen Betrachtungen werden analytisch von Anna Tsings Konzept der Friction (2005) gerahmt. Dieses erlaubt, an der Schnittstelle von Fußball, Geschlecht und feministischer Politik gegenwärtig ein Momentum zu identifizieren, das die Problematisierung fußballerischer Geschlechterverhältnisse plausibel und möglich macht und eine Vielzahl von Institutionen, Erwartungen, Argumenten und Interessen versammelt.

Im zweiten und dritten Schritt löse ich mich von der historischen und diskursiven Ebene und richte den Fokus auf Discover Football als gegenwärtige Akteurin geschlechterpolitischer Problematisierungen. Der zweite Teil der Arbeit begreift jene Versammlung heterogener Institutionen, Erwartungen, Argumente und Interessen als eine komplexe Landschaft, in der sich die von mir untersuchte Organisation erfolgreich formieren muss, will sie als Bearbeiterin der Geschlechterverhältnisse anerkannt werden. $\mathrm{Zu}$ Beginn dieses zweiten Teils entwickle ich anknüpfend an aktuelle Diskussionen der Organisations- und Politikethnologie ein prozessuales Verständnis von Organisationen und untersuche diese folglich nicht als starre Entitäten, sondern als kontinuierlichen Prozess des Sich-Organisierens. Dementsprechend gliedert sich der zweite Teil entlang der verschiedenen Kompetenzregime und der jeweiligen Formationen als Organisation. Er schließt mit einem Resümee über das Navigieren zwischen verschiedenen Formationen und durch heterogene Anerkennungsregime als zentrale Praktik des Sich-Organisierens auf zivilgesellschaftlicher Ebene in spätkapitalistischen Demokratien.

Im dritten Teil gehe ich von jenen komplexen gesellschaftspolitischen Landschaften aus und verstehe sie als typisch für spätkapitalistische Regierungsverhältnisse, die sich nicht vertikal in ein Oben und Unten gliedern lassen. Dabei verfolge ich die Frage, wie die fußballerischen Geschlechterverhältnisse bearbeitet werden, und untersuche entsprechende feministische Interventionen zivilgesellschaftlicher Organisationen als politisches Handeln. So knüpfe ich an politikethnologische und feministische Perspektiven auf Protest, soziale Bewegungen und NGOs an und entwickle davon ausgehend 
ein Verständnis zivilgesellschaftlichen politischen Handelns. Geschlechterpolitisches Problematisieren erscheint in diesem Licht als nuanciertes Handeln jenseits eines Dualismus von Widerstand einerseits und Kooption/Regierung andererseits, das multiple, teils widersprüchliche Effekte hat. Anhand zweier verschiedener Vorgehensweisen - dem Stellen von Forderungen und dem Präfigurieren fußballerischer Alternativen - zeige ich, welche Selbstverständlichkeiten sich in die politischen Strategien eingeschrieben haben, welchen Möglichkeitsbedingungen sie unterliegen und welche Wirkungen sie entfalten. Ausgehend von der Gleichzeitigkeit beider Vorgehensweisen schließe ich diesen Teil mit Überlegungen zur Beweglichkeit zwischen Strategien, Positionen und Zukünften als wesentlichem Modus geschlechterpolitischen Handelns.

Abschließend führe ich die Beobachtungen zusammen und schlage ein Verständnis geschlechterpolitischer Interventionen seitens zivilgesellschaftlicher Organisationen vor, das Beweglichkeit als zentralen Modus der Anzweiflung, Bearbeitung und Veränderung bestehender Verhältnisse im Feld des Fußballs und im Kontext spätkapitalistischer demokratischer Gesellschaften versteht. Trotz der prinzipiellen Unabgeschlossenheit ethnografischer Erkenntnisse und Produkte (Marcus 2009, 26) ermöglicht die Bearbeitung dieser Fragestellung weitergehende Diskussionen über die Umstände, Möglichkeiten und Effekte der Problematisierung gegenwärtiger Verhältnisse in komplexen Gesellschaftsgefügen. Jede diskutierte Frage wirft dabei neue auf: Ich schließe mit Überlegungen zum Zusammenspiel wissenschaftlicher Erkenntnis und ethnografischem Feld und eruiere, welchen Beitrag zukünftige Forschungen zu zivilgesellschaftlichem politischen Handeln sowohl für reflexive Aktivist_innen als auch für ein besseres Verstehen aktueller politischer Bewegungen leisten können. 


\section{Forschungsdesign und methodologische Aussichtspunkte}

Im Frühjahr 2013 reise ich zusammen mit Mitgliedern von DF auf Einladung einer Partnerorganisation in eine serbische Kleinstadt. Wir sind in einem kleinen Sportinternat am Stadtrand untergebracht. Am Morgen nach der Anreise trudeln die ersten zum Frühstück im Aufenthaltsraum ein. Nachdem ich mir einen Teller mit Frühstück und ein Glas Saft geholt habe, versuche ich mich mit vollen Händen auf den Boden zu setzen, plumpse aber ungeschickt hin und verschütte meinen Saft. Alle lachen. Mit gespielter Empörung verteidige ich mich: Es sei schließlich sehr schwer, sich ohne Hilfe der Hände so hinzusetzen. Martina probiert es aus, für sie ist es kein Problem. Alle anderen sind so ungeschickt wie ich. Wir kichern viel, gleichzeitig notiere ich die Erlebnisse des gestrigen Abends in meinem Tagebuch. Kerstin vermutet, dass ich all das Rumgealbere aufschreibe. Ein wenig später schreibe ich weiter an meinen Feldnotizen, während sich Linda, Nadine und Simone Frühstück machen. Saskia lässt sich auf einen Sessel fallen, ein Bein von sich gestreckt. Linda neckt Saskia: „Saskia übt ihre Topmodell-Posen“. Saskia ist gespielt empört. Simone, mit einem Blick zu mir, sagt scherzhaft: „Fritzi notiert: ,auch keine reflektierteren Gespräche als anderswo“.“ Ich lache, sie lacht. (Feldnotiz, Mai 2013)

Diese kurze Sequenz beschreibt einen typischen Moment meiner mehrjährigen ethnografischen Feldforschung bei DF. Sie verdeutlicht nicht nur die Besonderheit der ethnografischen Methode, sich als Forscherin selbst inmitten des Forschungsfeldes aufzuhalten, sondern auch die Spezifik der Feldforschung in sozialen Gruppen, deren Mitglieder in vielerlei Hinsicht der Forscherin ähneln, ihren kulturanalytischen Blick teilen und reflexiv auf sich selbst richten.

Im Folgenden stelle ich zunächst meine Methodologie vor (2.1). In der Feldnotiz deuten die Verwendung meines Spitznamens sowie das gemeinsame Lachen und Scherzen auf eine vertrauensvolle und freundschaftliche Beziehung hin, sodass ich auch auf die Herausforderung multipler Rollen im Feld eingehe. Unter dem Stichwort „Reflexivität“ (2.2) zeige ich, wie ich die oben beschriebene Ähnlichkeit und die tiefe Immersion in das Feld erstens methodisch nutzte und wie ich ihr zweitens mithilfe der Feldforschungssupervision reflexiv begegnete. Schließlich zeichne ich meinen Versuch nach, den dialogischen Charakter von Feldforschung in eine kollaborative Methodologie zu übertragen (2.3). Ich diskutiere das Vorgehen sowohl unter forschungsethischen wie auch epistemologischen Aspekten und erkunde Möglichkeiten und Schwierigkeiten wissenschaftlicher Kritik in politisch aktiven und reflexiven Feldern. 


\subsection{Forschungsprozess, Methoden und multiple Positionen}

Mein Forschungsinteresse führte mich zu einer zivilgesellschaftlichen Organisation, die ich an der Schnittstelle verschiedener historischer Entwicklungen und aktueller gesellschaftlicher Diskurse um Fußball und Geschlechterpolitik vermutete. Anknüpfend an eine praxistheoretische Perspektivierung sozialer Phänomene gehe ich davon aus, dass das Soziale immer im konkreten alltäglichen Vollzug durch unterschiedliche Akteur_innen hervorgebracht wird (Reckwitz 2003; Schmidt 2012). Ins Zentrum ethnografischer Forschung rücken soziale Alltagspraktiken, ihre Akteur_innen und das sie anleitende praktische Wissen. Ethnografieren meint dann zunächst, wie Sherry Ortner zusammenfasst, „looking at and listening to real people doing real things in a given historical moment, past or present, and trying to figure out how what they are doing or have done will or will not reconfigure the world they live in“ $(1996,2)$. Als zentrale Methode der gegenwartsbezogenen Ethnografie bietet sich die Feldforschung an, da sie das Beobachten und Zuhören in den alltäglichen Interaktionen ermöglicht und damit soziale Praktiken in ihrem situativen Vollzug erfassen kann, statt sie lediglich zu erfragen oder als einzelne Phänomene experimentell $\mathrm{zu}$ isolieren. Ihr Ziel ist die tiefgehende Analyse und dichte Beschreibung im Sinne eines ,understanding through richness, texture and detail" (ebd. 2006, 43), ermöglicht durch Kontextualisierung und ein „Denken in Relationen“ (Lindner 2003, 178). Ethnografie will soziale und kulturelle Phänomene in ihrer Komplexität und Kontextualität verstehen - und nicht als vermeintliche Kausalitäten erklären. Sie strebt nicht danach, Wahrheiten im Sinne eines positivistischen Wissenschaftsverständnisses aufzudecken, sondern danach, ein Verstehen der „multiple truth apparent in others' lives“ (Emerson et al. 1995, 3) zu ermöglichen.

\subsubsection{Teilnehmen und beobachten}

Seit Bronislaw Malinowskis stationärer Langzeitfeldforschung auf den Trobriand Inseln ist die teilnehmende Beobachtung zum ,methodischen wie wissenschaftskulturellen Königsweg" in den Ethnologien ${ }^{1}$ erkoren worden (Kaschuba 1999, 197; vgl. Illius 2003). Teilnehmende Beobachtung fokussiert einen recht kleinen Ausschnitt sozialer Realität (in meinem Falle eine einzige Organisation), um dort die Herstellung, Reproduktion, Umarbeitung und Aushandlung des Sozialen in konkreten Praktiken zu untersuchen.

1 Hierzu zähle ich die ethnografisch arbeitenden Disziplinen der Social (UK) und Cultural Anthropology (USA) und ihrer deutschen Schwesterdisziplin der Völkerkunde/Ethnologie/Kultur- und Sozialanthropologie sowie die Zweige des Vielnamensfachs, also die Volkskunde, Europäische Ethnologie, Kulturanthropologie und die empirische Kulturwissenschaft. 
Meine Feldforschung führte ich vom Sommer 2012 bis zum Frühjahr 2014 durch, kehrte aber bis Dezember 2015 immer wieder punktuell ins Feld zurück. Als ehrenamtliche Unterstützerin von DF blieb ich auch darüber hinaus aktiv. Während der gut eineinhalb Jahre intensiver Feldforschung nahm ich an den regelmäßigen, ein- bis zweiwöchig stattfindenden Gruppentreffen („Plena“) im Büro der Organisation sowie an Treffen einzelner Arbeitsgruppen („Gruppentreffen“) teil, besuchte das zweimal wöchentlich stattfindende Fußballtraining und spielte gelegentlich bei den Ligaspielen am Wochenende mit. Während einer gegenseitigen Kennenlernzeit vor Forschungsbeginn fuhr ich mit der Gruppe auf eine dreiwöchige Projektreise durch Polen und die Ukraine. Während meiner Forschung arbeitete ich als Organisatorin und Beobachterin mit an der Herausgabe eines Handbuchs und half bei der Organisation und Durchführung zweier Expertinnenforen sowie des dritten und vierten internationalen Frauen-Fußball-Kultur-Festivals (2013 und 2015). $\mathrm{Zu}$ den selbstorganisierten Veranstaltungen und den Arbeitstreffen gesellten sich weitere Termine in der Berliner Fußballlandschaft. So nahm ich an Treffen des Berliner Fußball-Verbands (BFV) für den Mädchenund Frauensport teil, vertrat Discover Football auf dem sogenannten Präventionstag des BFV und besuchte Veranstaltungen befreundeter Vereine. Auf meinem Programm standen auch Besuche bei Politiker_innen, Verwaltungsangestellten und Spender innen, um DFs Anliegen vorzubringen und/oder um Preise und Spenden in Empfang zu nehmen. Hinzu kamen viele private Treffen: Ich wurde zu Geburtstagen eingeladen, in Kleingruppen trafen wir uns zum public viewing oder verabredeten uns zum gemeinsamen Besuch von Demonstrationen oder für andere Freizeitaktivitäten. So gab es Wochen, in denen jeder Tag andere Aktivitäten für mich bereithielt, die sich weit über die Büroräume der Organisation in die ganze Stadt und auch darüber hinaus erstreckten. In der Zeit zwischen diesen Aktivitäten übernahm ich organisatorische Aufgaben, die ich meist von zu Hause aus erledigte: Ich half beim Verfassen von Anträgen und Bewerbungen, organisierte Workshop-Module mit, recherchierte Finanzierungsmöglichkeiten, übernahm Verwaltungsaufgaben im Fußballverein und brachte mich auch sonst an vielen Stellen in die (E-Mail-)Diskussionen ein. So wurden auch jene Momente zu Forschungssituationen, in denen ich alleine am Computer arbeitete und ich mich selbst beim Erledigen der Aufgaben beobachtete.

Meine Rolle als aktives Mitglied und gleichzeitige Beobachterin gestaltete ich aus, indem ich die Aktivitäten häufig protokollierte. Diese formale Auflage für eingetragene Vereine eröffnete mir eine gute Gelegenheit der Datenerhebung. Auf diese Weise erfasste ich den Alltag der Vereinsarbeit, die zum strukturierenden Element meines eigenen Alltags geworden war. 


\subsubsection{Multiple Positionen und partielle Perspektive}

Durch die Übernahme von Aufgaben und Verantwortungen erhielt ich Einblicke in das für mich bis dahin unbekannte Feld von Feminismus und Sport und konnte den Alltag einer translokal agierenden Organisation auf eine Art erleben, wie es für Außenstehende sonst wegen der professionellen Öffentlichkeitsarbeit nicht möglich ist. Eine nahe Teilnahme und das Aktiv-Werden als Gruppenmitglied ermöglichten mir mitzuerleben und festzuhalten, „how people grapple with uncertainty and confusion, how meanings emerge through talk and collective action, how understandings and interpretations change over time" (Emerson et al. 1995, 4). Da ich es mit einer öffentlich auftretenden Gruppe zu tun hatte, die in ihrer Selbstdarstellung geübt war, war die intensive Teilnahme besonders erkenntnisbringend. Von einer externen Person geführte Interviews, so meine Vermutung, hätten vor allem ein eingeschränktes und spezifisch vorstrukturiertes Repertoire an Erzählungen aktualisiert. Als Externe hätte ich zwar etwas über DFs Sicherheiten und Überzeugungen erfahren und daraus auf blinde Flecken und Selbstverständlichkeiten rückschließen können. Die internen und alltäglichen Aushandlungsprozesse hinter den geschlechterpolitischen Praktiken, Zweifel und Unsicherheiten, moralische Abwägungen und das selbstkritische Befragen des eigenen Tuns wären mir allerdings verborgen geblieben.

Dennoch war die Wahl meiner Forschungsposition nicht allein eine strategische Entscheidung meinerseits. Vielmehr weist das Feld der Forscherin eine Position zu, die nur eingeschränkt verhandelbar ist. Gerade in horizontal strukturierten und durch aktives Mitmachen und kollektive Entscheidungsprozesse gekennzeichneten Forschungsfelder ist für eine passive Begleiterin kein Platz vorgesehen (Hamm 2013, 63). In meinem Fall wünschten auch die Mitglieder von DF meine aktive Teilnahme, damit sie mich, meine Absichten, Denkweisen und politischen Haltungen einschätzen und besser abwägen konnten, was sie fortan mit mir teilen wollten.

Grundlage dafür, dass ich die Rolle als aktiv mitarbeitende Forscherin bzw. forschendes Mitglied einnehmen und mich in die Gruppe einpassen konnte, war eine gewisse Ähnlichkeit zu den Mitgliedern. Zwar besaß ich keinerlei Fußballerfahrung, doch hatte ich wie die meisten Mitglieder studiert, entstammte einem bürgerlichen, weißen Elternhaus, war Ende 20, sportlich und hielt soziales und politisches Engagement für außerordentlich wichtig. Wir teilten trotz kleinerer Unterschiede Welten und übergeordnete Ziele von Geschlechtergerechtigkeit und Emanzipation, sodass mich die anderen bald einschätzen konnten und auch ich schnell ihre Sprech- und Diskussionsweisen, Witze und Andeutungen, Intentionen und Abwägungen deuten und kontextualisieren konnte.

Die Feldnotiz zu Kapitelbeginn veranschaulicht die durch die aktive Teilnahme und die unübersehbare Ähnlichkeit entstandene Doppelrolle: Durch 
das Scherzen und das Eingebunden-Sein in die Kommunikation wurde mein Status als Gruppenmitglied, Mitstreiterin und Freundin deutlich. Das gleichzeitige Notizenschreiben markierte mich als beobachtende Forscherin. Insofern das Notieren nicht zu stören schien, oder gar freundschaftliche Scherze darüber möglich waren, wird ersichtlich, dass meine Doppelrolle von den Anwesenden nach einer längeren Phase des gegenseitigen Kennenlernens akzeptiert wurde und sie sich ihres Status als Beobachtete meist bewusst waren. Doch dieses Rollengleichgewicht war fragil:

Eines Abends traf ich mich mit Annike auf ein Bier. Nachdem wir über persönliche Dinge geredet hatten, kam die Sprache irgendwann auf Fußball und ich schaltete für sie unbemerkt in den Forscherinnenmodus und fragte immer konkreter nach. Ich wusste, was sie sagen wollte, doch versuchte ich durch mein Nachfragen geteiltes Alltagswissen explizit und damit für die Forschung greif- und verwertbar zu machen. Mein Gegenüber war sichtlich verwundert ob meiner plötzlichen Begriffsstutzigkeit. Als ich ihren irritierten Blick bemerkte, musste ich lachen und erklärte mich, sodass auch sie darüber lachen konnte. (Feldnotiz, 20.8.2014)

Im Kontext der gemeinsamen Vereinsarbeit trat ich sichtbar als Forscherin und Gruppenmitglied zugleich in Erscheinung. Interviewsituationen und Aufnahmegeräte erinnerten immer wieder an meinen Beobachterinnenstatus, und es gab viele Situationen, in denen ich von meiner wissenschaftlichen Arbeit erzählte. Diese Eindeutigkeit meiner Doppelrolle verschwamm, sobald sich der Arbeitskontext in einen privaten wandelte und der Rahmen der Begegnung als Freundschaft und Freizeit markiert war. Für mich bedeuteten die Doppelrolle und ihre verschwimmenden Grenzen auch, mit einem inneren Konflikt umzugehen, der sich intensivierte, je enger die persönlichen Beziehungen zu den Mitgliedern wurden. Dem schlechten Gewissen, aus Freundschaften eigene Vorteile für eine Qualifizierungsarbeit zu ziehen und die gegenwärtige Situation für weitere Zwecke als die persönliche Beziehungsarbeit zu nutzen, begegnete ich, indem ich eben jenen Konflikt thematisierte, sodass auch die anderen damit einen Umgang finden konnten. ${ }^{2}$

Diese Rollenbesetzungen im Feld eröffneten nicht nur Zugänge, sondern verschlossen zugleich andere. Aufgrund der aktiven Mitarbeit wurde ich von den internationalen Projektteilnehmenden als Gruppenmitglied und Organisatorin identifiziert. Wenn ich ihre Meinungen und Eindrücke erfragen wollte, antworteten sie also nicht einer unabhängigen Forscherin, sondern einer Repräsentantin jener Organisation, die sie eingeladen hatte. Damit ist keineswegs gesagt, dass eine Antwort wahrer wäre als eine andere und eine andere Situierung im Feld ,objektivere' Daten ermöglicht hätte. Vielmehr ist dies ein

$2 \mathrm{Zu}$ diesem inneren Konflikt und der Vermischung privater und forschungsöffentlicher Beziehungen siehe das zweite Kapitel bei Emerson et al. (1995).

$\mathrm{Zu}$ dieser typischen Angst der Forscherin siehe Lindner (1981). 
Hinweis, eben jene Situiertheit bei der Analyse dieser Daten unbedingt methodisch zu berücksichtigen (hierzu Kap. 2.2).

Mein Hauptaugenmerk war damit auf die Berliner Organisationgruppe und ihre internen Prozesse gerichtet und verfolgte weniger die Effekte dieser Praktiken über den lokalen Kontext hinaus. Aber auch diese Perspektive ist nur partiell. Sie wurde maßgeblich dadurch selektiert, dass ich nie alle parallel verlaufenden Arbeitsprozesse erfassen konnte, mit manchen Mitgliedern engeren Kontakt hatte als mit anderen und mich selbst entlang von Identitätskategorien spezifisch verortete und verortet wurde. Die Perspektive beschränkt sich zudem auf einen konkreten zeitlichen Ausschnitt und kann darüber hinausreichende Entwicklungen der Gesamtgruppe, einzelner Personen und Meinungen nicht erfassen. Die vorliegende Arbeit bietet daher lediglich eine Lesart innerhalb einer ,multiplicity of truth composed from different points of view“; sie ist damit notgedrungen partiell und kein ,singular statement about the project as it is" (Mosse 2006, 942).

\subsubsection{Interviews}

Zur teilnehmenden Beobachtung gehört eine unüberschaubare Zahl an informellen Gesprächen, die in den jeweiligen Situationen nicht als Interviews ausgewiesen werden (Breidenstein et al. 2015, 80f.). So nutzte ich viele Gespräche, um mich über konkrete Arbeitsabläufe, Entscheidungsprozesse oder Ereignisse $\mathrm{zu}$ informieren und um sicherzugehen, dass ich etwas richtig verstanden hatte. In anderen Momenten war die Interaktion durch die Benennung und die Aufzeichnung durch ein Tonbandgerät für alle Beteiligten klar als Interview gekennzeichnet. Zusätzlich zu semi-strukturierten Kleingruppeninterviews führte ich, teilweise in kollaborativer Zusammenarbeit mit einem Mitglied der Gruppe (dazu Kap. 2.3.1), narrative biografische Interviews mit anderen Frauen von DF in Anlehnung an Fritz Schütze (1983). So forderten wir unsere Interviewpartnerinnen auf, ihre Lebensgeschichte mit besonderem Fokus auf den Fußball zu erzählen. Die Interviews dauerten zwischen einer und drei Stunden und wurden, wie auch die anderen Interviews, anschließend vollständig transkribiert. Bei der Auswertung war zu berücksichtigen, dass biografische Erzählungen immer als identitätsgenerierende Arbeit an der eigenen Biografie zu verstehen sind, die nach bestimmten Narrativen organisiert sind. ${ }^{3}$ Dies ermöglicht zu verstehen, welche Erklärungsmodelle für die eigene Erfahrung herangezogen werden, welche historischen, kulturellen und sozialen Prozesse diese Erfahrungen bedingten und wie sie zu dem gegenwärtigen geschlechterpolitischen Engagement sinnhaft in Beziehung gesetzt werden. Mit anderen Worten: Ich wollte nicht wissen,

$3 \mathrm{Zu}$ der Anwendbarkeit und den Schwierigkeiten von Schützes Interviewmethode siehe Pfister (1999, 25), Schmidt-Lauber (2007, 176f.). 
was tatsächlich geschah, sondern wie die Akteur_innen ihren biografischen Erfahrungen mithilfe ihres geteilten Deutungsrahmens Sinn verleihen.

Feldforschungsethiken erinnern an die Verantwortung der Forscherin, Privatsphären zu respektieren. In diesem Sinne erfüllte das biografische Interview einen weiteren Zweck. Meine starke Involviertheit ins Feld und die persönlichen Beziehungen ließen die Grenzen zwischen forschender und privater Interaktion verschwimmen, sodass wir auch viele private und persönliche Geschichten austauschten. Utz Jeggle (2014) betont den Unterschied zwischen beiden Gesprächssituationen: Das Interview plant immer über den Moment hinaus und dient einem weiteren Zweck. Wie schnell dieser weitere Zweck bei meinen Gesprächspartner_innen in Vergessenheit geriet, macht nicht nur die Situation mit Annike deutlich, sondern auch folgendes Gespräch, das im Anschluss an ein Gruppentreffen stattfand:

Bevor ich gehe, drehte sich das Gespräch um unterschiedliche Verhaltensweisen auf dem Fußballplatz. Saskia grinst mich an: Ob ich das nicht aufschreiben wolle, das sei doch wichtig für mich. [...] Kerstin bemerkt: „Ach ja, ich vergess' immer, dass du das ja machst. Gefährlich." Ich lächle, die Ironie in ihrer Stimme ist nicht zu überhören. (Feldnotiz, 6.6.2013)

Bemerkungen wie die von Kerstin veranlassten mich, die Verantwortung nicht allein den Forschungspartner_innen zu überlassen, zwischen den Gesprächssituationen zu unterscheiden und ihre Aussagen dementsprechend zu kontrollieren. Um freundschaftliche Vertrauensverhältnisse nicht auszunutzen, verwendete ich nur jene privat-biografischen Informationen, die ich in den eindeutig als Interview markierten Situationen erhielt. Die Möglichkeit des Verschweigens konnte so von den Interviewten genutzt werden. Dieses Vorgehen ermöglichte, dass ich etwas als Geheimnis erkennen und respektieren konnte, was ich vielleicht auf anderem Wege oder in anderen Situationen erfuhr, mir aber in den Interviews vorenthalten wurde. ${ }^{4}$

\subsubsection{Artefakte}

Artefakte sind „Kristallisationen unserer kulturellen Praxis, weil sich in ihnen Denkweisen, Wertehorizonte und Nutzungsformen ,verewigen“", sich in ihnen Beziehungen materialisieren und symbolisieren (Kaschuba 1999, 224). Da ich unter diesen Begriff auch digitale Produkte wie die Online-Präsenz von DF in sozialen Medien und auf der eigenen Homepage sowie die tägliche

4 Jeggle (2014) diskutiert beispielreich die Frage, was in einer Forschungssituation als Lüge gelten und wie die Forscherin mit Geheimnissen und Verschwiegenem umgehen kann. Emerson et al. beschreiben Momente des bewussten Weghörens und Nicht-Aufschreibens als kurze Auszeit von der Forscherinnenrolle und als Möglichkeit, das Vertrauen zwischen Forscherin und Feld nicht auszunutzen $(1995,36)$. Selbstverständlich formten diese Informationen mein Bild von einzelnen Personen und der Gesamtsituation mit. 
E-Mail-Kommunikation zähle, ist zu erahnen, mit welcher Fülle an materieller Kultur ich mich konfrontiert sah. Ich sammelte ohne Anspruch auf (aber mit dem Bemühen um) Vollständigkeit Artefakte, die sich wie folgt kategorisieren lassen:

- Materialien, die von der Gruppe für eine breite Öffentlichkeit hergestellt wurden. Darunter fallen sowohl Flyer, ein Handbuch, Plakate, Broschüren und Merchandise-Produkte wie Taschen, Pullover, T-Shirts, Paninihefte, Aufkleber und Buttons, als auch digitale Produkte wie Pressemitteilungen, bearbeitete und online veröffentlichte Fotos, Videos und die gesamte Online-Repräsentanz auf Website und Facebook.

- Interne oder nur für eine eingegrenzte Gruppe zugängliche Produkte wie Anträge auf finanzielle Förderung und Abschlussberichte, E-Mails, Bewerbungen um Preise und Entwürfe für später veröffentlichte Produkte.

- Artefakte, die nicht von DF hergestellt wurden, sich aber auf DF beziehen. Darunter fallen Zeitungs- und Zeitschriftenartikel sowie Radio- und Fernsehberichte über die Projektarbeit, publizierte Interviews mit Gruppenmitgliedern sowie Einladungen zur Teilnahme an Tagungen, Netzwerktreffen, Aktionstagen, Podiumsdiskussionen und Ehrungen, aber auch Bewerbungen von Teams und einzelnen Personen zur Teilnahme an DF-Veranstaltungen.

In den Artefakten und ihrer Einbettung in Arbeitsprozesse wurde die textuelle Ebene der geschlechterpolitischen Interventionen greifbar. Ortner betont, dass ein vollständiges anthropologisches Projekt neben dem Beobachten und Erfragen von Praktiken immer auch die kulturellen, ideologischen und diskursiven Formationen berücksichtigen und beide zusammenführen muss. Nur so lassen sich die kulturellen Bedeutungen und strukturellen Arrangements greifen, die Praktiken hervorbringen, ermöglichen oder beschränken (1996, 2). Der methodische Mix aus diskursiver Bild- und Textanalyse, beobachtender Teilnahme und thematisch fokussierter Interviewführung ermöglichte mir, mich explorativ der Konstellation von Sport, Politik und Geschlecht aus verschiedenen Perspektiven zu nähern und ihre Relationen zu erfassen.

\subsubsection{Softwaregestützte qualitative Datenanalyse}

Meine Daten verwaltete und analysierte ich, abgesehen von gewissen Artefakten wie Kleidung, digital mithilfe des Datenbearbeitungsprogramms MAXQDA. Ich nutzte die Software nicht als eigenständige Methode, sondern als Hilfsmittel zur Organisation und Strukturierung der Daten (vgl. Sattler 2014, 477). Dabei folgte ich dem von Georg Breidenstein et al. (2015, 133138) diskutierten Kodierungsvorgehen, um an analytischen Kategorien zu 
arbeiten. Dementsprechend kodierte ich einen ersten Datensatz induktiv und offen, indem ich die Kodes aus meinem empirischen Material herleitete und auch viele Aspekte kodierte, die mir zunächst für mein Forschungsinteresse weniger relevant erschienen. Danach ordnete ich die Kodes nach entsprechenden Kategorien mit mehreren Hierarchieebenen. So entstand z.B. die Kode-Kategorie $<$ Politik $>$ mit (unter anderem) dem Unterkode $<$ Strategien, um politische Ziele zu erreichen>, der sich wiederum in die Unterkodes $<$ Stimme geben $>$ und $<$ Räume schaffen $>$ gliederte. Die aus dieser ersten Kodierungsphase gewonnenen Kodes leiteten das Kodieren des zweiten Datensatzes an, sodass dieser hauptsächlich (aber nicht ausschließlich) fokussiert bzw. axial kodiert wurde (ebd., 136). Durch den Prozess des Kodierens und Re-Kodierens kristallisierten sich Kategorien heraus. So handelt es sich bei den zentralen Begriffen des dritten Teils - Stimme, Raum, Sichtbarkeit, Erfahrung - um emische Begriffe, die durch die Kodierung zu analytischen Kategorien abstrahiert wurden.

Ich möchte hier nicht ausführlich die Vor- und Nachteile computergestützter Datenanalyse diskutieren; diese können in neueren Methodenhandbüchern nachgelesen werden (bspw. Gibbs et al. 2002; Flick 2016). Einen Aspekt möchte ich jedoch aufgreifen. Kodieren heißt Benennen, und jede Benennung ist ein Akt der Festschreibung und Vereindeutigung (Breidenstein et al. 2015, 137). Ethnografie zielt jedoch darauf ab, Widersprüche, Facettenreichtum und Uneindeutigkeiten des Feldes zu erfassen und in ihrer Gleichzeitigkeit verstehbar zu machen. Die Vorgehensweise des Kodierens, ReKodierens und Ordnens der Kodes ist ein Angebot, das Datenmaterial zu organisieren und zu strukturieren. Es ist ein erster Schritt zur Textproduktion, zur Erschaffung einer kohärenten Lesart sozialer Unübersichtlichkeit. Die Kritik der Writing-Culture-Debatte am ethnografischen Schreiben gilt meines Erachtens bereits für die computergestützte Datenanalyse: Die entsprechende Funktionslogik des Programms - die Vergabe von Kodes (alles ist benennbar!) und deren Hierarchisierung (alles lässt sich zueinander anordnen!) suggeriert, dass es eine Ordnung gibt, die nur gefunden werden muss. Dieses Verständnis von Kultur - sei es eine strukturalistische Grammatik oder ein symbolisches Bedeutungsnetz - wird längst angezweifelt (vgl. Rabinow et al. 2008). Auf der Suche nach sozialen Praktiken kann das Kodieren helfen, doch sollten die gebildeten Kategorien stets als einer von vielen Konstruktionsschritten einer Lesart des Feldes, keineswegs als Freilegungen einer einzigen Wirklichkeit verstanden werden. Um zu verhindern, dass Vielstimmigkeit, Widersprüche und Nuancen verloren gehen, gestaltete ich die Kodes möglichst breit. Sie dienten mir vor allem zur besseren Handhabung und zum thematischen Querlesen eines sehr großen, chronologisch und sequentiell geordneten Datenkorpus (vgl. Breidenstein et al. 2015, 137f.). 


\subsection{Reflexivität}

Die bereits thematisierte tiefe Immersion in das Forschungsfeld durch aktive Teilnahme bringt konkrete Herausforderungen für die ethnografische Feldforschung mit sich. Reflexivität hat sich seit den 1980er Jahren als zentraler Imperativ im Umgang mit Positionalität und Subjektivität im Forschungsund Schreibprozess in die einschlägigen Einführungswerke der Europäischen Ethnologie eingeschrieben (bspw. Kaschuba 1999; Brednich 2011). Diese Forderung reagiert auf die Kritik der Writing-Culture-Debatte innerhalb der Social und Cultural Anthropology an der Unsichtbarkeit der Forscherin im ethnografischen Text sowie der Verschleierung ethnografischer Produktionsund Konstruktionsprozesse. Feministische Ansätze haben dieses Versteckspiel des Forscherinnensubjekts weiterführend kritisiert und auf die Standortgebundenheit jeder Wissensproduktion hingewiesen. Diese Diskussionen mündeten in der Einsicht, dass die Perspektivität der Wissenschaft nicht aufgehoben, sondern nur permanent reflektiert werden kann und Ethnografie daher als ein Prozess der Gegenstandskonstruktion zu verstehen ist (vgl. u.a. Fabian 1993; Berg/Fuchs 1993). Lila Abu-Lughod (1991) wie auch Donna Haraway (1995) plädieren daher für ein Verständnis situierten und partiellen Wissens, das auf der gesellschaftlichen, kulturellen und historischen Positionierung des Forscherinnensubjekts aufbaut. Reflexivität möchte ein Korrektiv dieser erkenntnistheoretischen Herausforderung darstellen und einen anderen Modus der Wissensproduktion dadurch begründen, dass beständig die eigene Positionalität kenntlich gemacht und mit anderen Positionen in Beziehung gesetzt wird (Robertson 2002, 785; Langenohl 2009).

Ich begegnete meiner Positionalität, die entscheidend durch meine Involvierung in das Forschungsfeld sowie meine Ähnlichkeit zu den Mitgliedern von DF geprägt war, auf zweierlei Weise. Erstens nutzte ich die eigene Position und die damit verbundene Körperlichkeit meiner Perspektive gezielt als Erkenntnisquelle und Methode, und zweitens fand ich in der Feldforschungssupervision ein geeignetes Instrument für den Umgang mit der daraus entspringenden Forscherin-Feld-Beziehung. Beide Vorgehensweisen, die ich gleich ausführlicher skizziere, können auf die Gefahren einer vorschnellen und verkürzten Befolgung des Reflexivitätsimperativs reagieren. So kritisiert einerseits Pierre Bourdieu jene „narzisstische Reflexivität“ (1993), wenn der ethnografische Text um die Forscherin kreist, anstatt sich dem Untersuchungsgegenstand zu widmen. Auf der anderen Seite läuft Reflexivität Gefahr, bloßes Lippenbekenntnis zu bleiben, wenn der Positioniertheit, Verkörperung und Situiertheit von Wissensproduktion mit einem ritualisierten, checklistenartigen Bekenntnis entlang von Identitätskategorien wie Geschlecht, sexueller Orientierung, Ethnizität, Religion und sozioökonomischem Status begegnet wird. Jennifer Robertson (2002) kritisiert, dass sich 
Ethnografinnen entlang der gängigen Kategorien entweder bereits im Vorfeld der Forschung festschreiben oder diese erst im Schreibprozess dem empirischen Material voranstellen. Dadurch werden die Kategorisierungen als kontextungebunden und selbstevident behandelt und wirken essentialisierend auf die Forscherin wie auch , die Anderen' des Feldes. Ich folgte während der Forschung, der Auswertung und des Schreibens Robertsons Vorschlag, stets davon auszugehen, dass sich Forscherin und Feld kontinuierlich und situativ als Subjekte hervorbringen. Daher verzichte ich in dieser Einleitung auf eine Selbstpositionierung. Stattdessen werde ich als forschende und mitwirkende Person im zitierten empirischen Material immer wieder in Erscheinung treten und meine Positionalität an den relevanten Stellen transparent machen. Dies setzt voraus, die einzelnen Situationen immer wieder mithilfe des eigenen Körpereinsatzes und der Feldforschungssupervision danach zu befragen, wie meine persönliche Geschichte und Position hineinwirkten, welche Facetten eines multiplen Selbst in ihr aktualisiert wurden und wie diese mit den anderen, sich ebenfalls verändernden Positionen in Beziehung standen.

\subsubsection{Der Forscherinnenkörper als Erkenntnissubjekt}

Ethnografie ist nicht nur ein intellektueller und moralischer Konstruktionsund Interpretationsakt, sondern immer auch ein körperlicher Prozess in Raum und Zeit (Ortner 2006, 42). Anstatt die eigene Körperlichkeit und die emotionalen Reaktionen auf Ereignisse im Feld als unliebsame Störfaktoren möglichst objektiver Beobachtungen zu disqualifizieren, wollte ich sie methodisch nutzbar machen. Unterschiedliche Ansätze in den Ethnologien haben den Forscherinnenkörper als Mittel der Erkenntnisgewinnung theoretisiert. Dabei beziehen sich manche auf die Leibesphänomenologie Maurice Merleau-Pontys und auf Thomas Csordas Embodiment-Begriff (bspw. Coffey 1999; Okely 2007) oder orientieren sich vornehmlich an Bourdieus HabitusKonzept (bspw. Wacquant 2003; 2011; Müller 2013); andere knüpfen an George Devereux und ethnopsychoanalytische Ansätze an und machen mit dem Phänomen der Gegenübertragung Feldforschungserfahrungen für die Analyse fruchtbar (bspw. Davies/Spencer 2010; Bonz 2016). Auch innerhalb der Anthropologie der Sinne gibt es Bestrebungen, die sinnliche Wahrnehmung der teilnehmenden Forscherin methodisch zu nutzen (bspw. Bendix 2006; Arantes 2014). Dass der Forscherinnenkörper als Erkenntnissubjekt und -quelle effektiv eingesetzt werden kann, ist dabei unumstritten, das Wie bleibt hingegen wenig ausbuchstabiert (Linska 2012, 118f.). ${ }^{5}$

5 Für das Vielnamenfach bieten bspw. Bendix (2006), Mohr/Vetter (2014) Ausnahmen und stellen einzelne Schritte zur Analyse der sinnlichen, respektive körperlichen Wahrnehmungen vor. Das im Folgenden skizzierte Vorgehen geschieht in Auseinandersetzung mit und in Anlehnung an diese Ansätze. 
Stefan Heissenberger und ich haben in Anlehnung an Pierre Bourdieus Konzept des Praxissinns (1987, 129f.) und Loïc Wacquants Carnal Sociology (2003; 2011) einen Antwortvorschlag unterbreitet, dem ich im Verlauf meiner Forschung folgte (vgl. Faust/Heissenberger 2016). Der eigene Körper wird dabei als Quelle und Instrument der Erfahrung verstanden, von dessen Reaktionen und Lernprozessen aus auf Dynamiken und Eigenschaften des Feldes geschlossen werden kann. Um solche analytischen Schritte zu ermöglichen, werden die eigenen körperlichen und emotionalen Veränderungen ebenfalls schriftlich festgehalten, immer wieder in diachroner Perspektive verglichen und mit den aus Interviews und Feldbeobachtungen gewonnenen Daten angereichert. Reflexive Methoden und Momente, wie sie die Feldforschungssupervision, aber auch die Präsentation von Daten in Kolloquien bereitstellen, verhindern vereinfachte und verkürzte Rückschlüsse. Denn, wie Jochen Bonz zurecht bemerkt, sollte es immer Standard sein, „von der Verschiedenheit des Wahrnehmens auszugehen" (2016, 21).

Dieses methodische Vorgehen ermöglichte mir nachzuvollziehen, wie sich nach und nach der praktische Sinn des Feldes in meinen eigenen Körper einschrieb. Gerade das Fußballspiel als körperlich-sportliche Aktivität, aber auch die Vereinsarbeit beinhalteten verkörperte und habitualisierte Denk- und Handlungsroutinen, nicht-verbalisierte Erfahrungen, Positionierungen und Selbstbezüge, welche durch die Teilnahme als „Minisozialisation“ (Scheer 2011, 71) fassbar wurden. ${ }^{6}$ Dabei gehe ich wie Bonz keinesfalls davon aus, dass die eigenen Erfahrungen mit denen der Forschungsprotagonist innen identisch sind, noch verstehe ich vermeintlich geteilte Erfahrungen als vordiskursive und damit , authentischere' Erkenntnisquellen. Vielmehr dient die Beobachtung der eigenen körperlichen Reaktionen und Wahrnehmungen als Ausgangspunkt einer intersubjektiven Annäherung an relevante Erfahrungen, die dann von der Forscherin verbalisiert und in Interviews, informellen Gesprächen oder Gruppendiskussionen mit den Protagonist_innen besprochen werden können. In diesen Momenten des Redens über Erfahrungen werden

6 Allgemein zur teilnehmenden Beobachtung als sportethnologischer Methode siehe Sands (2002). Für eine ausführliche Abwägung von externer und interner Beobachterinnenposition im Sport siehe Archetti/Dyck (2003). Sie stellen fest, dass die meisten intern Forschenden und damit auch aktiv am Sport Partizipierenden selbst aus den jeweiligen Sportarten oder Fangemeinden stammen. Dies trifft auf mich nicht zu. Ich wagte mich mit dem Fußball auf ein für mich in vielerlei Hinsicht fremdes Terrain. Bourdieu verteidigt den eigenen Körpereinsatz in der Sportforschung mit Blick auf die besondere Relevanz verkörperten, nicht-versprachlichten Wissens: ,[D]ie Sportler gehören eben zu jenen Praktikern, bei denen das sie betreffende Verstehen über den Körper vermittelt wird. Häufig kann man nichts anderes sagen als: ,Schau her und mach es wie ich.““(1992, 205) Mit Blick auf die sportlichen Emotionspraktiken (Kap. 10.2.2) gebe ich Bourdieu Recht, schließe mich bezüglich des sportlich-technischen und -taktischen Könnens jedoch Müller an, dass das Erlernen einer Sportart „eine Kombination aus sinnlich-mimetischen und reflexiven Prozessen“ (2013, 159) im Training erfordert, sodass Sport paradoxerweise gerade „,keine angemessene Analogie für den Praxissinn“ ist (ebd., 174). 
Unterschiede, Gemeinsamkeiten, Nuancen und Ambivalenzen sowie das diskursive Gerüst zur Versprachlichung von Erfahrungen sichtbar. Mithilfe der einzelnen methodischen Schritte - wahrnehmen, aufschreiben, diachron nachverfolgen, mit anderen Daten abgleichen, kollaborative Rückkoppelungen und reflexive Überprüfung - werden verkörperte und emotionale Dimensionen der sportlichen und organisatorischen Praktiken mithilfe des eigenen Körpereinsatzes greifbar (Faust/Heissenberger 2016).

Dieses Vorgehen unterscheidet sich dabei grundlegend von der Autoethnografie. Zwar teilen beide Ansätze die Auffassung, dass körperliche Präsenz und emotionale Reaktionen der Forscherin wichtige Einblicke in das Feld eröffnen. Jedoch meint Autoethnografie eine Methode des Forschens und eine Form des wissenschaftlichen Schreibens, die primär aus der eigenen Erfahrung heraus soziale Phänomene verstehen möchte und diese Erfahrung auch im Text zentral setzt (vgl. Ploder/Stadlbauer 2013). Das von mir verfolgte Vorgehen hingegen betrachtet den eigenen Körpereinsatz als gleichberechtigten ,, integralen Bestandteil unseres methodologischen Instrumentariums“" (Bendix 2006, 72). Indem die eigenen körperlichen Erfahrungen systematisch und methodisch kontrolliert in die Analyse eingehen, können sie dann im schriftlichen Endprodukt in den Hintergrund treten.

\subsubsection{Feldforschungssupervision als reflexive Methode}

Meine Forschung stellte mich bei der Auswertung der erhobenen Daten vor zwei zentrale Probleme: Durch die Miteinbeziehung der eigenen körperlichen Erfahrungen stand ich erstens vor der Herausforderung, von diesen adäquate Rückschlüsse auf das Feld zu ziehen, dabei die verschiedenen Rollen und Erwartungen auseinanderzuhalten und keine voreiligen Verallgemeinerungen vorzunehmen. Es galt, die Wirkungen, die das Feld auf mich hatte, und jene, die meine Kopräsenz auf das Feld ausübte, in ihren Verwobenheiten zu verstehen und dabei eigene blinde Flecken und implizite Vorannahmen aufzudecken. ${ }^{7}$ Durch die große Ähnlichkeit, die mich mit den Akteur_innen des Feldes verband, und dadurch, dass die Feldforschung quasi vor meiner eigenen Haustür stattfand und sich keine geografische Distanz in eine analytische übersetzen ließ, suchte ich zweitens nach Wegen, die vertrauten Aspekte des

7 An anderer Stelle zeige ich (Faust 2017), wie in der gruppenbasierten Deutungswerkstatt die persönlichen Dispositionen der Forscherin von den reaktiven Effekten des Feldes auf die Forscherin unterschieden werden können. Konkret meint das in diesem Fall: Die Supervisionsgruppe zeigte mir anhand meiner Feldnotizen von einem Fußballturnier, dass zwar ein gewisser Leistungsanspruch durchaus in meinem Feld vorhanden, dieser jedoch nicht so groß war, wie ich ihn selbst aufgrund meiner eigenen Erwartungen an mich erlebte - und bewahrte mich so vor möglichen Fehlübertragungen und -schlüssen. 
Feldes zu befremden. ${ }^{8}$ Gerade die Wahrung bzw. Wiedergewinnung von Distanz ermöglicht Kritikfähigkeit gegenüber den Politiken und Moralvorstellungen des Feldes (Bourdieu/Wacquant 1996). Reflexivität kann diese Distanzierungen ermöglichen und somit Hindernisse zu analytischen Vorteilen wenden:

„Gerade engagierte Forschende in sozialen Bewegungen, die sowohl die aktivistische Rolle als auch die der Wissenschaftlerin/des Wissenschaftlers einnehmen, bietet das ethnographische Oszillieren zwischen Teilnahme und Beobachtung einen methodischen Rahmen, in dem ihre hybride Positionierung von einem Hindernis zu einem Vorteil wird.“"(Hamm 2013, 64)

Neben den etablierten Techniken der Reflexion, wie dem Führen eines Tagebuchs, kollegialer Gespräche, regelmäßiger Präsentation von Daten und Deutungsversuchen, erwies sich die ethnografische Feldforschungssupervision als besonders hilfreiches und ausgearbeitetes Instrument zur Entflechtung der Verwobenheiten und zum Oszillieren zwischen Teilnahme und Beobachtung. Daher möchte ich sie im Folgenden darstellen und ihr Erkenntnispotential für die Ethnologien verdeutlichen, in dessen Methodenspektrum sie bislang noch keinen zentralen Platz einnimmt.

Seit 2014 gibt es in Berlin eine Feldforschungssupervisionsgruppe unter Leitung der Kulturanthropologin und Supervisorin Almut Sülzle, die sich an ethnografisch zu Sport forschende Kultur- und Sozialwissenschaftler innen richtet. Durch regelmäßige Teilnahme wurde meine Datenauswertung von diesem außeruniversitären Reflexionsrahmen begleitet und bot mir einen zusätzlichen Raum, mein Material unter besonderer Berücksichtigung meiner eigenen Positionalität und meinen Reaktionen auf Handlungen und Äußerungen der Feldakteur innen auszuwerten.

Die ethnografische Feldforschungssupervision wurde von Barbara WittelFischer auf Anregung von Jeggle 1998 in Tübingen für die Arbeit am Datenmaterial entwickelt und von Brigitte Becker et al. in der Zeitschrift für Volkskunde vorgestellt (2013). Zu den regelmäßigen Treffen bringen die Teilnehmenden Ausschnitte aus Feldnotizen, Erinnerungsprotokollen oder Interview-Transkripten mit, in denen sie selbst sowie Teile des Feldes sichtbar werden. Das Textmaterial wird in der Gruppe diskutiert, nicht aber mit einem methodenkritischen Blick gelesen und bewertet. Im Gegenteil gilt es eben jene Texte auszuwählen, in denen Spannungen zu spüren sind, unterschwellig andere Themen mitverhandelt oder methodische Patzer sichtbar werden, und die im Nachgang Irritationen hinterlassen - eben jene Situationen, deren Analyse durch thematische und emotionale Verwobenheiten er-

8 Siehe Hirschauer/Amann zur Diskussionen der Notwendigkeit befremdender Strategien in der Feldforschung in der eigenen Kultur (1997). Wichtig hierbei ist stete Aufmerksamkeit dafür, dass augenscheinlich Vertrautes womöglich doch nicht so selbstverständlich ist, wie zuerst angenommen. Vielmehr können gleiche Symbole im Feld andere und vor allem verschiedene Bedeutungen tragen. 
schwert wird. Während der von der Supervisorin moderierten Gruppendiskussion ist die Textgeberin nur als passive Zuhörerin anwesend. Die Diskussion zielt darauf, dass ,die unterschiedlichen Stimmen, die sich im Forschungsmaterial finden, zum Sprechen gebracht werden - quasi durch die Münder der Teilnehmer_innen“, denn in „den Übertragungen und Gegenübertragungen, die in der Gruppe stattgefunden haben, spiegeln sich häufig zentrale Strukturen, Wirkungsweisen und Emotionen aus dem Feld“" (Sülzle 2016, 32). Dieses Vorgehen will so die weniger sichtbaren Dimensionen der ethnografischen Sequenz wie Selbstverständlichkeiten, Tabuisiertes, unausgesprochene Machtverhältnisse oder Unerwünschtes greifbar machen (Becker et al. 2013, 195f.). ${ }^{9}$

Für die Forschung über Sport und feministischen Aktivismus ist die Supervision vor allem deshalb hilfreich, da es sich hier um besonders körperliche und emotional diskutierte Themen und Felder handelt, die allerdings aufgrund ihrer Allgegenwärtigkeit und Alltäglichkeit in der eigenen Kultur der Forscherin viele Selbstverständlichkeiten und damit ethnografisch blinde Flecken bereithalten (Sülzle 2016, 34). Für mich bot sich so die Möglichkeit, sowohl Vertrautes zu befremden, ohne dieses essentialisierend zu verfremden, als auch den Prozess des langsamen Vertraut-Werdens nachzuvollziehen und aus ihm zu lernen. Gleichzeitig diente die Supervision mir als reflexives Instrument, um mir meiner eigenen Verstrickungen mit dem Feld bewusst zu werden und sie bei der Auswertung zu berücksichtigen. Dabei wurden sowohl die Positionalität der Forscherin in den jeweiligen Situationen immer wieder thematisiert als auch emotionale und körperliche Reaktionen in Beziehung zu Positionen und Prozessen im Feld gesetzt. Der Vorteil dieser Form der Reflexivität liegt in einem Zugewinn an Kontextualität und Differenzierung der Deutungen; sie unterstützte mich während des Forschungsprozesses in der beständigen Modifizierung, Anpassung und Verortung meiner Hypothesen.

\subsection{Kollaboration, Ko-Laboration und Kritik}

In der ethnografischen Sequenz unserer Serbien-Reise (Kap. 2) wird das Interesse der Vereinsmitglieder am Forschungsprozess und dem entstehenden Wissen deutlich. Dies motivierte mich, mein Vorgehen möglichst transparent und partizipativ zu gestalten und mich an kollaborativen Ansätzen zu orien-

9 Beispiele finden sich bei Becker et al. (2013), Sülzle (2016) und in den Beiträgen in Bonz et al. (2017). Inzwischen gibt es in mehreren Städten Feldforschungssupervisionsgruppen, die unterschiedlich eng an die jeweiligen Institute des Vielnamenfachs gekoppelt sind. 
tieren. Kollaboration ${ }^{10}$ meint die - in den verschiedenen Ansätzen unterschiedlich weitreichende - Miteinbeziehung der untersuchten Personen in den Prozess der Wissensproduktion. Kollaborative Ansätze reagieren auf die Einsicht, dass Feldforschung kein ,individual, case-bounded project" (Marcus 2009, 26), sondern ein fortdauernder dialogischer und kooperativer Prozesses ist. Die Idee der konsequenten und transparenten Zusammenarbeit adressierte zunächst marginalisierte und benachteiligte Gruppen und wurde als eine ethische Antwort auf die Kritik an einer hierarchischen und ausbeuterischen Beziehung zwischen forschendem und beforschtem Subjekt vorgeschlagen. In dieser forschungsethischen Dimension soll die Dialoghaftigkeit auf allen Stufen der Feldforschung umgesetzt und transparent gemacht und damit die Autorität der Forschenden dezentriert werden (Lassiter 2004). Manche kollaborativen Ansätze gehen noch einen Schritt weiter in Richtung engagierter Wissenschaft und stellen die Zusammenarbeit in den Dienst der politischen Kämpfe ihrer Kollaborateur_innen, mit denen sie explizit sympathisieren und die sie mit wissenschaftlichen Mitteln unmittelbar zu unterstützen versuchen (vgl. Fluehr-Lobban 2008).

Mit der Zunahme ethnografischer Forschungen im Sinne eines studying up in technokratischen, politischen und ökonomischen Expertinnenmilieus begannen Ethnolog_innen das epistemologische Potential der Zusammenarbeit $\mathrm{zu}$ eruieren. Die Kollaborateur_innen und Protagonist innen werden dabei als Forschungspartner_innen gedacht und die Möglichkeiten gemeinsamer Wissensproduktion als epistemisches statt ethisches Projekt verhandelt (vgl. Holmes/Marcus 2005; Bister/Niewöhner 2014; Islam 2015). Jörg Niewöhner (2016) schlägt daher vor, zwischen Kollaboration als primär politischem und ethischem Vorhaben und Ko-Laboration als vorrangig epistemologischem Forschungsmodus $\mathrm{zu}$ unterscheiden. Ko-Laboration meint das temporäre gemeinsame Arbeiten, um anthropologisches Wissen zu generieren. Sie verbündet sie sich nicht mit den Protagonist innen zum Zwecke eines unmittelbaren außerdisziplinären politischen Ziels.

$\mathrm{Zu}$ Beginn meiner Forschung motivierten mich vor allem ethische Überlegungen zur engen Zusammenarbeit mit den Aktivist innen. Zwar wollte ich meine Forschung nicht in den Dienst der aktivistischen Kämpfe stellen, doch wollte ich in emanzipativen Feldern auf autoritative Methoden verzichten. Schnell musste ich jedoch zweierlei feststellen: Erstens lässt sich ein auf Zusammenarbeit basierendes Vorgehen keinesfalls im Vorhinein exakt planen, sondern muss pragmatisch mit den Wünschen und Handlungszwängen aller Beteiligten umgehen. Zweitens waren die Wünsche und Interessen der Fußball-Aktivist_innen keineswegs einheitlich: Während die einen die $\mathrm{Zu}$ -

10 ,Kollaboration“ ist die Übersetzung des englischen Begriffs ,collaboration“, die die unglückliche Konnotation der Komplizenschaft und des Verrats z.B. im Nationalsozialismus trägt (Binder/Hess 2013, 24). Ich verwende diesen Begriff aus Mangel an Alternativen, um einen politisch oder ethisch motivierten partizipativen Forschungsmodus zu beschreiben. 
sammenarbeit mit mir nutzten, um Sorgen und mögliche Schwierigkeiten in Bezug auf meine Forschung zu diskutieren, zeigten sich andere interessiert daran, mit ihrem eigenen akademischen Wissen meinen Blick zu ergänzen; andere wiederum nutzten die Gelegenheiten, um abseits des schnelllebigen Aktivismus-Alltags ihr Tun zu reflektieren. Dementsprechend vielschichtig gestaltete sich unsere Zusammenarbeit, und es etablierten sich nach und nach verschiedene Formate nebeneinander: Während z.B. gemeinsame Treffen es ermöglichten, über DFs Vorgehen zu diskutieren und anthropologisches Wissen zu generieren, wurde das Gegenlesen meiner Texte dafür genutzt, der hierarchischen Beziehung zwischen Forscherin und Feld zu begegnen.

Mit Blick auf mein eigenes Vorgehen erscheint es mir daher hilfreich, Ko-Laboration und Kollaboration als unterschiedliche Dimensionen der $\mathrm{Zu}$ sammenarbeit $\mathrm{zu}$ behandeln, die auf die multiplen Bedingungen und Interessen in jenem Beziehungsgeflecht reagieren, „das bisher als Forschende und Feld klar bezeichnet war" (Bister/Niewöhner 2014, 7). Im Folgenden zeichne ich meine Versuche der Zusammenarbeit entlang ihrer forschungsethischen (kollaborativen) und epistemologischen (ko-laborativen) Dimensionen nach. Abschließend diskutiere ich, welchen Beitrag wissenschaftliche Kritik in reflexiven politischen Feldern leisten kann, wenn sie deren Kämpfe nicht unmittelbar unterstützen kann und will.

\subsubsection{Kollaboration als ethisches Projekt}

Kollaborative Methodologien werden häufig unter forschungsethischen Fragen diskutiert und verschiedene Vorgehen zur Teilung der Repräsentationsmacht zwischen Forscherin und Beforschten vorgeschlagen (vgl. Low/Merry 2010; Binder/Hess 2013). Feministische Ansätze unterstreichen zudem den emanzipativen Aspekt der geteilten Arbeit an der akademischen Repräsentation (Enslin 1994). Ich schließe mich Elizabeth Enslin (1994) an, dass Forschen in emanzipatorischen Kontexten auf die autoritative und intransparente Entwicklung von Interpretationen und Argumenten verzichten und sich stattdessen um größtmögliche Partizipation der Forschungssubjekte bemühen sollte. Mein Vorhaben wurde von DF begrüßt - jedoch weniger aus dem abstrakten Wunsch nach geteilter Repräsentationsmacht, sondern erstens aufgrund praktischer Notwendigkeiten, Handlungszwängen und Ängsten und zweitens aufgrund des eigenen wissenschaftlichen Interesses.

Als in der oben wiedergegebenen Sequenz einer gemeinsamen Reise nach Serbien zwei Protagonistinnen meiner Forschung scherzhaft darüber mutmaßten, was ich wohl in mein Feldtagebuch notiert hätte, drückten sie ein reges Interesse an dem über sie entstehenden Wissen aus - schließlich kommentierten sie nicht nur, dass ich etwas schrieb, sondern auch was ich schrieb. Dieses Interesse gründete dabei nicht nur in ihrer eigenen Vertraut- 
heit mit dem kultur- und sozialwissenschaftlichen Genre, das sie zu potentiellen Leser_innen machte. Es reagierte auch auf die sich überschneidenden gesellschaftlichen Beziehungen und Bereiche, in die sie als Organisation und ich als Wissenschaftlerin eingebunden waren. Wie ich in dieser Arbeit noch zeigen werde, hängen das Überleben einer Organisation und ihre Handlungsfähigkeit maßgeblich von der Anerkennung durch viele Stakeholder ab, sodass politisches Handeln immer auch multiple Selbstdarstellungen beinhaltet. Ethnografie kann zu einer Bedrohung für die Beforschten werden, wenn die Forscherin durch Publikationen ebenfalls Zugang zu jener wissenschaftlichaktivistischen Öffentlichkeit hat, in der auch die Beforschten agieren und sich legitimieren (Thayer 2010, 172). ${ }^{11}$ Wenn nicht nur Ethnolog_innen selbst, sondern auch ihre Texte zu ,active agents in the worlds they describe“ (Mosse 2006, 952) werden, stellen sich neue forschungsethische Fragen.

Im Gegensatz zu anderen ethnografische Studien konnte ich auf diese Gefahr nicht mit konsequenter Anonymisierung reagieren. Die Gruppenmitglieder wünschten daher, mich über mehrere Monate, in denen ich als Ehrenamtliche mitarbeiten würde, kennenzulernen, bevor sie über die Forschung entscheiden wollten. Da ihnen aufgrund ihrer eigenen wissenschaftlichen Bildungswege klar war, dass eine Forschung offen und kritisch verlaufen müsse und daher immer die Möglichkeit bestand, dass die ethnografische Beschreibung stark von der Selbstrepräsentation abweichen würde, diente diese Probezeit auch dazu, sich zu vergewissern, dass ich nicht aus einer ideologischen oder wissenschaftlichen Perspektive heraus schreiben würde, die der ihren diametral entgegenstünde und aus ihrer Sicht zu einer Fehlinterpretation ihrer Praktiken und Argumente führen könnte. ${ }^{12}$ Bei mir weckte dies die bekannten

11 Ich musste einsehen, dass ich zwar übergeordnete Ziele mit einem Feld teilte, meine gut gemeinten Intentionen, mit meiner Forschung zu einem besseren Verstehen emanzipativer Kämpfe beizutragen, jedoch negative, gar konträre Effekte haben könnten. Die Parallele zwischen Feld und Forschung ist unübersehbar, jedoch wurden die negativen Effekte gut gemeinter NGO-Interventionen in der Ethnologie viel breiter diskutiert als die negativen Effekte ihrer eigenen wohlmeinenden Forschungen. Später gehe ich mit der Frage nach Kritik genauer auf jene Angst des Feldes vor der ethnografischen Repräsentation ein. Auch Mosse (2006) wurde mit den Bedenken seiner Forschungspartner_innen konfrontiert, dass seine Forschung in entwicklungspolitischen Organisationen diese diffamieren und ihnen schaden könnte. Er thematisiert diese Erfahrung mit Bezug auf die Beziehung zwischen Ethnografin und Feld: Gerade insider ethnography bedeutet, bestimmte Grenzen zum Feld zu überschreiten, sodass auch der Schreibprozess keinen Rückzug bietet: „Those interlocutors - neighbours, friends, colleagues, or co-professionals - who directly experience ethnographic objectifications now surround the anthropologist at her or his desk; they raise objections, make new demands to negotiate public and published interpretations. When desk collapses into field, something important has changed in the structure of ethnographic practice. We are starkly confronted with the essentially relational nature of anthropological knowledge, no longer an object in our possession.“ (Ebd., 937) Seine Analysen des daraus entstandenen Konflikts sind nicht nur aufschlussreich, sondern auch spannend.

12 Solche Erfahrungen hatten sie immer wieder mit Journalist innen gemacht, von deren Berichterstattung sie sich fehldargestellt fühlten, wenn diese mit exotisierenden oder stereo- 
Ängste der Forscherin vor dem Feld: Werden sie mich mögen? Werden sie mich als Wissenschaftlerin anerkennen? (Lindner 1981; Warneken/Wittel 1997) In ihrem Wunsch kristallisierte sich aber auch eine weitere Sorge: die Angst des Forschungsfeldes vor der ethnografischen Repräsentation.

Im Sinne des forschungsethischen Grundprinzips do no harm, d.h. allen an der Forschung Beteiligten keinen Schaden zuzufügen (American Anthropological Association 2012), erschien mir als Umgang mit diesen Sorgen ein kollaboratives Vorgehen passend. So konnte ich die ,Beforschten“ in die Wissensproduktion einbeziehen, anstatt ihnen ganz zum Schluss einen fertigen Text vorzulegen. Wir vereinbarten, dass ich Entwürfe für Artikel, Vorträge und Kapitel den Gruppenmitgliedern zur Lektüre schicken und jedes Zitat autorisieren lassen würde. So konnten sie kommentieren und mich auf potentielle Schwierigkeiten hinweisen, die durch das fahrlässige Ausplaudern von Interna entstehen könnten. Nicht alle fanden die Zeit oder hatten die Muße, meine Kapitel- und Artikelentwürfe zu lesen und zu kommentieren. Daher veranstaltete ich sogenannte kollaborative Treffen: Ich lud die Mitglieder der Organisation in Kleingruppen von drei bis sieben Personen zu mir nach Hause ein und stellte in einem informellen Rahmen mein Vorhaben vor und zur Diskussion, wobei ich die Diskussionen wiederum protokollierte. Ich ergänzte diese größeren Treffen mit vielen kollaborativen Gesprächen: Bei Verabredungen mit einzelnen Mitgliedern berichtete ich ihnen vom aktuellen Stand meiner Arbeit und schilderte meine Gedanken und Unsicherheiten darüber, wie die ein oder andere Situation zu interpretieren sei. Meist stiegen sie interessiert ein, stellten eigene Vermutungen an, erzählten mir von ähnlichen Situationen und wogen meine Argumente ab, stimmten zu oder widerlegten sie. ${ }^{13}$ Diese kollaborativen Treffen begegnen der Angst vor der ethnografischen Repräsentation, indem sie die Protagonist_innen in kleinen Schritten nachvollziehen lassen, was sich über Jahre hinweg über sie im Ent-

typisierenden Worten und Bildern über ihre Projekte berichteten oder mit biologischen oder essentialisierenden Argumenten die Frage nach der fußballerischen Geschlechtergerechtigkeit diskutierten. Viele Forscher innen basieren ihre organisationsinternen Forschungen auf ihrer aktiven Mitarbeit (u.a. Riles 2007; Thayer 2010; Lau 2011). Unausgesprochen bleibt in den mir bekannten Arbeiten dabei häufig, dass dem auch eine Prüfung der Forscherin, ihres Wissens, ihrer Einstellungen und Vertrauenswürdigkeit vorausgeht und es gewisser Gemeinsamkeiten und geteilter politischer, weltanschaulicher oder wissenschaftlicher Positionen bedarf.

13 Interessant ist, dass sie kaum inhaltlich Einspruch erhoben. An manchen Stellen wiesen sie mich auf Gegenbeispiele und Differenzierungen hin, wenn sie meine Deutungen zu einseitig oder zu pauschal fanden, siehe bspw. Saskias Einwand in Kap. 11.4. Dies waren in den meisten Fällen wertvolle Ergänzungen und bewogen mich zu differenzierterer Darstellung. Die meisten Kommentare zu Artikelentwürfen und Zitaten drückten die Sorge aus, dass durch die Widergabe wörtlicher Rede oder flüchtig geschriebener E-Mails ,,jedes gesprochene Wort ein bisschen dumm und unakademisch" wirkt (E-Mail, 16.9.2016). Dies ist eine durchaus ernstzunehmende Sorge, da sie als junge Frauen stets ihre Professionalität unter Beweis stellen mussten. Ich reagierte darauf, indem ich an jenen Stellen, an denen es um den Inhalt, und nicht die Form geht, Zitate von Grammatik- und Tippfehlern bereinigte. 
stehen befindet. Zudem ermöglichen sie, eigene Interessen, Fragen und Situationsdeutungen einzubringen, auf Aspekte hinzuweisen, die durch ein Öffentlichwerden Schaden anrichten könnten, und Sorgen zu äußern, die dann in weiteren Analysen, in Richtungsentscheidungen und beim Schreiben berücksichtigt werden können.

Neben der Möglichkeit, Sorgen zu artikulieren, bot die Dezentrierung der ethnografischen Autorität mittels kollaborativer Formate den Protagonist_innen auch die Möglichkeit, eigenen wissenschaftlichen Interessen nachzugehen. Zusammen mit einem Mitglied der Gruppe experimentierte ich mit Formen des gemeinsamen Schreibens: Wir identifizierten eine Teilfrage, die in engem Bezug zu meinem Gesamtvorhaben stand und uns beide interessierte, führten biografische Interviews mit unseren Mitstreiterinnen, präsentierten eine erste Auswertung auf einer Tagung und verfassten schließlich einen Zeitschriftenbeitrag (vgl. Faust/Kösters 2016).

An der geteilten Autorinnenschaft wurden jedoch auch Schwierigkeiten deutlich, die weniger sichtbar bereits in den kollaborativen Treffen und Gesprächen angelegt waren. Neben dem Eingeständnis, dass aus raumzeitlichen Gründen nie eine gesamte Gruppe, sondern immer nur einzelne Personen oder Kleingruppen in einen solchen Wissensproduktionsprozess eingebunden werden können, traten auch weitere Aspekte zu Tage, die Fragen an die Umsetzbarkeit der ethischen Grundidee kollaborativen Vorgehens richten. Meine Kollegin und ich fanden uns schnell in der Situation, dass die meiste Arbeit von mir übernommen wurde. Diese Arbeitsteilung schien vor dem finanziellen Hintergrund gerechtfertigt - wurde ich durch ein Stipendium für die wissenschaftliche Tätigkeit entlohnt, musste sie dies in ihrer Freizeit neben der Lohnarbeit machen. Dies führte jedoch dazu, dass ich den größten Textanteil produzierte und die meiste Theoriearbeit leistete, meine Kollegin stets kommentierte und überarbeitete. Erstens geriet so der feministisch-emanzipative Aspekt in Zweifel, da meine Kollegin zusätzliche Arbeitet schulterte und zugleich von dem Schuldgefühl plagte wurde, nicht genügend beizutragen. Zweitens wirft dies die skeptische Frage nach der tatsächlichen Dezentrierung der ethnografischen Autorität auf (vgl. Islam 2015, 238), war hier doch ein deutliches Ungleichgewicht spürbar. Meiner Kollegin, die selbst sozialwissenschaftlich ausgebildet war, fiel es schwer, aus Zeitmangel die Theoriebildung abzugeben. Das kollaborative Vorgehen kollidierte also in der Praxis mit den raumzeitlichen Bedingungen und wurde so einerseits meinem emanzipativen Grundgedanken und andererseits den wissenschaftlichen Ambitionen und Ansprüche meiner Kollegin nicht gerecht. Wir stellten im Laufe der Zusammenarbeit fest, dass eine traditionelle Forscherin-BeforschtenBeziehung eher verfestigt, denn zugunsten partnerschaftlicher Zusammenarbeit aufgelöst wurde. Dieses Ungleichgewicht ist auch in den kollaborativen Treffen angelegt, wenn ich Einsprüchen, Kritikpunkten und Änderungswünschen zwar Raum gab, anschließend aber behutsam abwog, ob und wie ich 
sie in meine Analyse einfließen lassen könnte. So löste sich der initiierte Dialog zugunsten der Autorität der Forscherin auf. Die forschungsethische Dimension der Zusammenarbeit verliert ihr emanzipatives Potential, wenn sie den Partizipant_innen letztlich mehr Last auferlegt als ihnen nützt, und sie verfehlt ihre ethische Intention und wird zu einer „besonders raffinierte[n] Form des Abschöpfens von Wissen" (Hamm 2013, 60), wenn sie sich nicht an den Notwendigkeiten und Möglichkeiten des Feldes ausrichtet.

Als ich meine Ko-Autorin um eine Einschätzung dieser Zeilen bat, erhielt ich folgenden Kommentar:

„Ich glaube, ich würde es insgesamt nicht ganz so negativ bewerten. Obwohl ich unzufrieden damit war, dass ich nicht das Gefühl hatte, wirklich auf Augenhöhe mit Dir den Text zu erarbeiten und mir die Theorie anzueignen und auch immer das schlechte Gewissen hatte, dass Du den Löwenanteil machst und ich mich nur dranhänge (also auch so Ownership-Fragen an dem Produkt) würde ich das wieder machen. Natürlich gerne unter anderen Bedingungen aber auch unter denselben. Es war ja trotzdem bereichernd für mich. Und sich ein bisschen theoretisch mit den Thema auseinandersetzen ist ja immer noch besser, als sich gar nicht damit auseinanderzusetzen." (Autorisierung, JK, 16.3.2016)

Meine Kollegin und ich resümierten, dass geteilte Autorinnenschaft vor allem dann sinnvoll ist, wenn Aufwand und Nutzen für alle Seiten geklärt und absehbar sind. Ich schließe mich daher dezidiert nicht jenen Ansätzen an, die die Miteinbeziehung von „participants/collaborators“ von der Entwicklung des Forschungsdesigns bis hin zur Publikation mit dem gemeinsamen Ziel, die Lebensverhältnisse zu verbessern, als ,ethically conscious“ und „morally preferable" gegenüber hierarchischeren Forschungsmodellen verteidigen (Fluehr-Lobban 2008, 175). Partizipative Ansätze sollten nicht aufgrund aktueller Trends engagierter Forschung heraus durchgeführt werden, sondern sich allein an den Interessen, Möglichkeiten und Bedingungen der Beteiligten orientieren sollten.

Auch David Graeber zweifelt an der Verallgemeinerbarkeit eines solchen Vorgehens und fragt mit Blick auf Forschungen in progressiven politischen Bewegungen, die die Aktivist innen über die ethnografischen Beschreibungen mitbestimmen lassen, skeptisch: „Does this apply to all social movements, and if not, what are our criteria for selection?" (2016, 7) Er führt uns damit vor Augen, dass der Imperativ nach partnerschaftlicher Wissensproduktion nicht für alle politischen Felder gültig gemacht wird, sondern eine spezifische politische Positionierung des Forschungsvorhabens und des Feldes impliziert. Oder wer würde gerne rechtspopulistische Protestgruppen mitentscheiden lassen, ob sie als fremdenfeindlich und rassistisch beschrieben werden sollten? Graeber stellt fest:

„So at the very least, all academic radicals feel they have a right to decide what is a social movement that should have the right to decide on its own designation, and what is not." (ebd.) 
Aber auf welcher Basis wird diese Entscheidung getroffen? Diese Frage lässt sich von sozialen Bewegungen auf sämtliche soziale Gruppen übertragen und führt letztlich zu den von den Forschungsperspektiven selbst verfolgten Politiken. ${ }^{14}$ Ich möchte daher Kollaboration explizit nicht als ethisch generalisierbare Antwort auf die Kritik an der machtvollen ethnografischen Repräsentation befürworten, noch glaube ich, ,that power inequalities between the interpreter and the interpreted can be dialogued away" (Mosse 2006, 937). Sie bietet sich vielmehr als Möglichkeiten an, eine konkrete Forscherin-FeldBeziehung vor dem Hintergrund einer genau bestimmten Forschungsperspektive situativ entlang der jeweils relevanten Moralvorstellungen, Interessen und Handlungszwänge auszuhandeln und dabei Bedenken, Sorgen und Interessen der Forschungspartner_innen zu begegnen.

\subsubsection{Ko-Laboration als epistemologisches Projekt}

In den kollaborativen Treffen ging es jedoch nicht nur um mögliche Sorgen und ethische Fragen. Sie wurden von manchen auch genutzt, um über das eigene Tun selbstkritisch oder mit analytischem Interesse zu diskutieren. Gerade in Feldern, die ihr eigenes Tun immer wieder thematisieren und reflektieren, und in Hinblick auf mein Forschungsinteresse an den Aushandlungen geschlechterpolitischer Praktiken bietet eine enge Zusammenarbeit epistemologisches Potential. Dabei finden sich aber auch Fallstricke, auf die ich ziemlich bald stieß. Ich skizziere in Folgenden meinen Umgang damit, um sodann zu zeigen, wie ko-laborative Momente ethnologische Einsichten ermöglichen können.

In der Feldnotiz über unsere Gruppenreise nach Serbien zu Beginn des Kapitels kommentierte Linda ironisch-kritisch Saskias Weiblichkeitsdarstellung, Simone wiederum scherzte selbstironisch über diese für feministische Kontexte „unreflektierte“ Albernheit. Hierin zeichnet sich bereits ab, dass die Protagonist_innen meiner Forschung mithilfe wissenschaftlicher Theorien

14 Weder Graeber noch mir fällt hierzu eine Antwort ein. Hier kann nur gemutmaßt werden; und diese Mutmaßungen führen zu Debatten innerhalb der Ethnologien, wie sie auch in die Politikethnologie hineinwirken. Ich gehe darauf zu Beginn des dritten Teils ein. Hier möchte ich lediglich die Vermutung formulieren, dass fachinterne Auseinandersetzungen mit der eigenen Beteiligung an den Verbrechen des 20. Jahrhunderts, sei es am Nationalsozialsozialismus (Volkskunde) oder am Kolonialismus (Völkerkunde), zu einer links-intellektuellen politischen Positionierung der Fächer auf Seiten der Machtlosen und Marginalisierten (Schuller/Lewis 2017, 637) und einem analytischen „focus on the low rather than on the high, on the ordinary rather than on the event" beitrugen (Rabinow et al. 2008, 73). Für das Vielnamenfach identifiziert Warneken das „Leitmotiv Widerständigkeit“ (2006, 207ff., zit. n. Binder/Hess 2013, 32), das zur Fokussierung von Widerstand, Protesten und gegenkulturellen Phänomenen führte. Es gilt daher immer wieder zu fragen, wie sich Selbstverständlichkeiten, Politiken und Moralvorstellungen des Fachs in seine Methodologien einschreiben (vgl. Niewöhner 2016). 
und Konzepte Bezug auf ihr eigenes Tun nahmen. Auch in anderen Situationen zeigten sich Wissenspraktiken moderner und universitärer Institutionen in ihrem Alltag: Sie diskutierten mithilfe soziologischer und psychologischer Kategorien ihre eigenen organisatorischen Tätigkeiten oder politischen Argumentationen, sammelten Daten, schrieben Anträge, organisierten Arbeitstreffen und Seminare, gestalteten offizielle Dokumente und produzierten Informationen sowie ihre eigenen Repräsentationen (vgl. Schuller/Lewis 2017, 639f.; Riles 2007, 4). DF beschrieb und begründete die eigene Arbeit mit ähnlichen Worten, wie ich sie zur Analyse vorgesehen hatte. In meinem Dissertationsexposé konzipierte ich meinen Forschungsgegenstand z.B. mit folgenden Worten:

„Anknüpfend an Bourdieu ist Fußball als soziales Feld zu konzeptualisieren, welches durch die Verteilung von und den Kampf um verschiedene Kapitalsorten und damit verbundenen Kräfteverhältnissen zwischen den sozialen Akteur_innen strukturiert ist. Die dort vorherrschende androzentrische Ordnung kann als männliche Herrschaft beschrieben werden, die gerade durch ihre sanfte, symbolische Gewalt wirkmächtig ist."

Daran anschließend wollte ich mein Forschungsfeld fragen:

„In Anlehnung an das intersektionale Forschungsparadigma stellen sich die forschungsleitenden Fragen, wie - erstens - die Ausgrenzungsmechanismen Homophobie und Sexismus verschränkt wirken und welche weiteren Ausschlusskategorien noch auftreten. Zweitens, wie werden Geschlecht und Geschlechterrollen im sozialen Feld des Fußballs konstruiert und welche Subjektpositionen werden Fußballerinnen dabei zugewiesen?"

Kurz nachdem ich mit der Feldforschung begonnen hatte, entdeckte ich im DF-Büro eine Ausgabe der Zeitschrift feministische studien, die sich 2012 dem „Sport als Kult der Geschlechter“ widmete. Ein Artikel darin stammte von DF. Unter dem Titel „DISCOVER FOOTBALL - mit Fußball für Gleichberechtigung und Solidarität" beschreiben die Autorinnen die Arbeit des Vereins und erklären diese vor dem kulturellen und historischen Kontext des Fußballs:

„Die historisch bedingten Unterschiede [in der gegenwärtigen Behandlung des Frauen- und Männerfußballes; FF] werden zudem durch eine androzentrische Wahrnehmung des Fußballs überlagert. Fußball ist - gerade in Ländern wie Deutschland, wo er sehr populär ist - eindeutig männlich konnotiert [...]. Die androzentrische Wahrnehmung führt dazu, dass das Engagement von Frauen im Fußball systematisch ausgeblendet und unterschätzt wird." (Sperschneider et al. 2012, 113)

Weitere Großkonzepte wie Sexismus, Heteronormativität und Patriarchat fächerten in Flyern, Broschüren und Interviews das Theoriespektrum auf. Die Akteur_innen meines Feldes verwendeten also die gleichen Wissenspraktiken - theoretische Konzepte und Argumentationen sowie Publikationen - wie ich und hatten auf die von mir gestellten Forschungsfragen die Antworten sofort 
parat. Wenn die ethnografischen Fragen jenen Fragen, die die Akteur_innen im Feld selbst stellen, zu sehr ähneln, muss nach dem Grund gesucht und ein Umweg eingeschlagen werden (vgl. Hodžić 2017, 11).

Scott Lash (1994) bezeichnet den Fall, wenn die Subjekte der Forschung die soziologische Arbeitsweise in ihren Alltag integriert haben, als Doppelungen reflexiver Moderne und macht damit auf die Herausforderung aufmerksam, die entsteht, wenn die Protagonist innen selbst ihre Organisationskultur analysieren und die ethnografisch zu beschreibenden Praktiken bereits theoriegetränkt sind (vgl. Rabinow et al. 2008; Islam 2015): Wie ist mit diesen Repräsentationen umzugehen (Schuller/Lewis 2017, 640)? Was, wenn das Begriffsinstrumentarium zur Analyse des Feldes vom Feld selbst für eigene Zwecke eingesetzt wird? Gerade für engagierte Forschungen, die mit den Zielen der untersuchten politischen Bewegungen sympathisieren und dort auf Ähnlichkeiten treffen, ergeben sich aus diesen reflexiven Thematisierungen mit ähnlichen Wissenspraktiken zwei epistemologische Herausforderungen. Erstens ist die Gefahr, dass sich die Sozialwissenschaften die Probleme des Feldes zu eigen machen (Bourdieu/Wacquant 1996, 271), in der Zusammenarbeit mit Expertinnenkulturen besonders groß. Douglas Holmes und George Marcus warnen daher davor, das para-ethnografische Wissen des Feldes als ethnografische Beschreibung zu übernehmen und die Rolle einer Ethikberaterin oder Hausanthropologin einzunehmen, deren Aufgabe in der Verbesserung oder Schärfung des feldspezifischen para-ethnografischen Wissens liegt. Eine solche Forschung verfehlt ihr primäres Ziel, wissenschaftliches Wissen zu produzieren, wenn sie lediglich eloquentere Selbstdarstellungen des Feldes liefert (Homes/Marcus 2005, 250).

Zweitens und daran anknüpfend stand ich vor der Frage, was meine Forschung dem Wissen des Feldes noch hinzufügen könnte. Martina Klausner et al. (2015) schlagen in solchen Fällen eine die Selbstbeschreibungen und -kritiken des Feldes ergänzende Lesart vor und bauen dafür auf Foucaults Überlegung auf: „Die Leute wissen was sie tun; häufig wissen sie, warum sie das tun, was sie tun; was sie aber nicht wissen, ist, was ihr Tun tut." (Foucault, zit. n. Dreyfus et al. 1994, 219) Ich begann daher die Verwendung wissenschaftlicher Konzepte ebenfalls als Praxis zu verstehen (Islam 2015, 236) und ihr konkretes Wirken im Feld selbst zum Untersuchungsgegenstand $\mathrm{zu}$ machen. Ich adaptierte die vom Feld vorgegebenen Probleme nicht automatisch als anthropologische Probleme, sondern als soziale Phänomene der Problematisierung. Dies ließ mich meine forschungsleitenden Fragen erweitern und verändern: Statt nur nach der spezifischen Konstituierung der Geschlechterverhältnisse zu fragen, untersuchte ich, wie feministische Theorien von Geschlecht und Sport von DF selbst argumentativ eingesetzt wurden; statt ihr Vorgehen mithilfe intersektionaler Ansätze zu befragen, beobachtete ich, wie Intersektionalität selbst als Paradigma auf Praktiken im Feld wirkte; und statt DF anhand von soziologischen Organisa- 
tionstypen zu klassifizieren, analysierte ich, wie unterschiedliche Organisationstypen im Feld mit Bedeutung versehen und als ständige Referenzpunkte für die eigene Positionierung genutzt wurden.

Gerade die kollaborativen Treffen und Gespräche boten hier wertvolle methodische Ergänzungen in mehrfacher Hinsicht. Die Situationsdeutungen der Protagonist_innen dienten mir erstens als wichtige Informationsquellen: Eingebrachte Zweifel, Ergänzungen und Abwägungen bereicherten meine Beobachtungen, sodass ich die gruppeninterne Polyphonie fassen, Ungenauigkeiten oder gar fehlerhafte Darstellungen vermeiden konnte und auf „occasional insightful leaps of imagination, but also their self-serving biases and their moments of ingenuousness" aufmerksam gemacht wurde (Islam 2015, 238). Zweitens wurden diese Einwürfe zu Untersuchungsgegenständen, denn in ihnen schlugen sich Interessensschwerpunkte, Deutungsweisen und kulturelle Wissensbestände der Protagonist_innen nieder, welche mir wiederum neue Denkimpulse gaben. Wenn z.B. die Diskussion immer wieder um das eigene Weiß-Sein der Gruppe kreiste, fragte ich mich, warum genau dies so zentral war, und konnte davon ausgehend einen moralisch-ideologischen Diskurs im städtischen Umfeld als wichtige Referenzgröße identifizieren.

Ko-laborative Formate bieten sich als Instrument an, die reflexiven Fähigkeiten und Interessen der Forschungspartner_innen epistemologisch zu nutzen (Holmes/Marcus 2005). In dieser ko-laborativen Dimension schufen die Treffen und Gespräche temporäre Räume des gemeinsamen Nachdenkens in Form einer ,conversation situated within a very particular relationship and undertaken in a very particular time and place - a dialogue about rather than a final statement on any particular ethnographic topic" (Lassiter 2005, 95). Dabei kann Wissen produziert werden, das sich an den Relevanzen und Logiken des Feldes orientiert, anstatt diese anhand von mehr oder weniger expliziten normativen Meta-Narrativen zu dekonstruieren (Niewöhner 2016). Zurückkommend auf die Angst des Feldes vor der ethnografischen Repräsentation frage ich im Folgenden, was ein solches Wissen in politischen Feldern tut bzw. tun kann.

\subsubsection{Gutes tun, oder: Was macht Kritik?}

Meine Arbeit baut wie jede andere auf einem politisch-ideologischen Fundament auf, sodass mich mit meinem Feld ein übergeordnetes Streben nach Emanzipation und Geschlechtergerechtigkeit verband. Der Kritik, politisch engagierte Wissenschaft würde mit sozialwissenschaftlichen Ansprüchen und Erkenntnisinteressen kollidieren, kontern Beate Binder und Sabine Hess an Bourdieu (2004) und Butler (2001) anknüpfend:

„Insofern ist es auch kurzsichtig, in allen Formen wissenschaftlichen Engagements ein Aufgeben wissenschaftlichen Ethos zu vermuten - schon allein deshalb, weil das 
Nichts-Tun ebenso Ausdruck einer spezifischen politischen Grundhaltung und ebenso Teil einer ,Politik der Wahrheit' ist, die versucht, das, was denkbar, sagbar und wissbar ist, vorzugeben (vgl. Butler 2001, 8).“ (Binder/Hess 2013, 27)

Diese Bemerkung, zusammen mit der Mahnung, dass ,the anthropology anthropological" bleiben müsse und Feldarbeit stets Vorrang vor Ethischem und Politischem eingeräumt werden solle (Rabinow et al. 2008, 73), stellte mich vor die Frage nach dem Ort meiner eigenen politisch-ideologischen Haltung. Während ich als Mitglied die Arbeit und Ziele der untersuchten Organisation unmittelbar unterstützte und unweigerlich mit formte, musste der Ort des Politischen innerhalb meiner Forschung erst noch gefunden werden. ${ }^{15}$ Weder zielte meine Arbeit darauf, im Sinne einer public ethnography (Lassiter 2005) die Anliegen, Sichtweisen und Rechte meines Feldes einer breiten Öffentlichkeit nahezubringen, noch wollte ich im Sinne einer advocacy anthropology (Low/Merry 2010, 210f.) als Anwältin und FürSprecherin des Feldes fungieren. Erstens benötigte DF solche Unterstützung gar nicht, schließlich verfügte die Gruppe selbst über eine äußerst eloquente und breit rezipierte Stimme. Und zweitens lag mein primäres Interesse darin, zu einem besseren wissenschaftlichen Verstehen gegenwärtiger politischer Prozesse beizutragen. ${ }^{16}$ Das so produzierte Wissen wollte ich, wie z.B. von Craig Calhoun $(2008, \mathrm{xx})$ vorgeschlagen, in die politischen Kämpfe einspeisen und sie wissenschaftlich kritisch begleiten, anstatt ihre Anwaltschaft zu übernehmen. Genau wie mein Feld war also auch ich besten Willens, mit den mir zur Verfügung stehenden Mitteln Gutes zu tun.

Umso mehr wunderte ich mich über Bedenken aus dem Feld: Zwar begrüßten DF-Mitglieder die neu geschaffenen Räume zum gemeinsamen Nachdenken und zeigten großes Interesse an meiner Perspektive, doch bemerkten sie mit sorgenvollem Blick auf die Publikation, dass ich ,ja auch kritisch sein müsse“ und ob ihnen diese Kritik nicht auch schaden könnte. In diesem Fall kreiste die Angst vor der ethnografischen Repräsentation weniger darum, dass Vertrauliches öffentlich gemacht werden könnte, als vielmehr um die Ergebnisse wissenschaftlicher Analyse. Das kollaborative Vorgehen

15 Engagiertes Forschen fordert die Forschende ständig dazu auf, sich moralisch in Beziehung zum Feld zu setzen und stets die Beziehung zwischen Forscherin und Partizipant_innen zu befragen. Dissens und Missmut, aber auch plötzliche Übereinstimmung und Begeisterung verstehe ich dann nicht als unwillkommene Störenfriede einer angestrebten Objektivität, sondern als Hinweise auf die eigene Haltung sowie auf das Feld. So bietet engagiertes Forschen eine Chance, sich im Sinne Haraways (1995) der eigenen Situiertheit bewusst zu werden, sie transparent zu machen und damit die Verantwortung für die stets immer nur partielle und nie objektive eigene Perspektive zu übernehmen.

16 Ansätze wie advocacy anthropology, action anthropology oder participatory action research wurden für die Forschung mit sozial benachteiligten Gruppen, nicht jedoch mit sozialen Bewegungen entwickelt. Eine Übertragung konfrontiert die Forscherin damit, dass sie von der untersuchten Gruppe gar nicht wirklich zur Vertretung ihrer Bedürfnisse und Anliegen gebraucht wird, sodass die Partizipation an einem Forschungsprojekt nur eingeschränkt attraktiv ist (Hamm 2013, 63f.). 
ermöglichte zwar, Zweifel an Deutungen zu äußern und zu diskutieren, aber letztlich nicht, meine Analyse ohne plausible Gegenargumente zu verhindern.

Um zu verstehen, woher die Angst des Feldes vor der wissenschaftlichen Kritik rührte, aber auch um den politischen Beitrag zu eruieren, den wissenschaftliches Wissen und eine kritische Haltung leisten können, bedarf es einer Auseinandersetzung mit dem sozialwissenschaftlichen Verständnis von Kritik. Foucault definiert einen Kritikbegriff, auf dem bis heute viele sozialund kulturwissenschaftliche Untersuchungen aufbauen. Kritik meint demnach zunächst ,die Bewegung, in welcher sich das Subjekt das Recht herausnimmt, die Wahrheit auf ihre Machteffekte hin zu befragen und die Macht auf ihre Wahrheitsdiskurse hin“ (Foucault 1992, 15).

Die Angst meines Feldes vor dem ethnografischen Text schien zu steigen, wenn dieser darauf zielte, Machteffekte sichtbar zu machen. Grundlage dieser Befürchtung war eine Vorstellung davon, was jene Wissenschaft macht, die sich selbst als kritisch bezeichnet, bzw. wie diese Kritik in einer für sie relevanten Öffentlichkeit rezipiert werden würde. Die Aktivist_innen verstanden darunter auf Basis eines Kritik-Begriffs, der von der Wissenschaft in aktivistische Felder gewandert war und dort mit moralischen Diskussionen verknüpft wurde, das investigative Aufdecken von reproduzierten Ausschlüssen und Herrschaftsverhältnissen, welches ein unmittelbares Verantwortlichmachen der handelnden Akteur_innen nach sich ziehen würde. Sie selbst verorteten sich in einer städtischen Bewegungsszene, die eng mit meinem akademischen Kontext verknüpft war und welche ich in Kapitel 9 näher beschreibe. In diesem ,politischen beziehungsweise politisierten Feld [eines] zivilgesellschaftlichen Aktivismus und beruflicher Tätigkeit" (Binder 2013, 8) schien ein liberales Verständnis des Politischen die Bewertung zivilgesellschaftlicher Initiativen zu leiten. Chantal Mouffe (2014) hat die moralische Dimension liberaler Theorien des Politischen dahingehend kritisiert, dass sie der Illusion eines harmonischen gesellschaftlichen Konsens ohne Exklusionen nachhingen und damit das zentrale Element des Politischen - den antagonistischen Widerstreit - moralisch bewerteten. Diese liberale moralische Haltung wurde im lokalen Kontext von DF mit Vorstellungen von ,richtigem' und ,falschem' politischen Handeln verknüpft und bildete den Rahmen, um wissenschaftliche Kritik zu rezipieren, zu deuten und in lokalisierte politische Praktiken zu übersetzen. Wissenschaftliche Kritik will zeigen, warum und wie Menschen tun, was sie tun, und macht auf Selbstverständlichkeiten, Machtverhältnisse und Subjektivierungen aufmerksam (Foucault 1992; Fassin 2008). Die Beobachtung, dass und wie die Akteur_innen doch einigermaßen regiert würden und ihr Handeln damit dominante Formen und Normen stabilisiere, würde innerhalb des Wissenschaft-Aktivismus-Nexus entlang der Vektoren Differenz und Inklusion bewertet. Ausschließungen durch die Reproduktion dominanter Herrschaftsformen und -normen würden entsprechend eines liberal-pluralistischen Ideals von gesellschaftlichem Kon- 
sens als moralisch falsch evaluiert werden. Was DF fürchtete, war also ein Kritikverständnis im Sinne des Aufdeckens von Fehlern entlang moralischer (,Das ist schlecht!“) Überzeugungen und mit dem Autoritätsanspruch zu beurteilen, was besser oder richtiger sei (vgl. Fassin 2008, 339; 2017, 10). ${ }^{17}$ Eine solche Kritik kann nicht wissenschaftlich-analytisch wirken, denn sie bleibt dabei in der Moral des Feldes verstrickt und begründet lediglich nach der gleichen Argumentationslogik immer neue Machtformen.

Im Unterschied zu ihrem aktivistischen Pendant will sozialwissenschaftliche Critique, wie Butler (2001) im Anschluss an Foucault (1992) betont, nicht über ihren gesellschaftlichen Gegenstand, seine gegenwärtige Zurichtung und möglichen Ausschließungen von einem festen normativen Referenzpunkt aus richten. Im Gegenteil, sozialwissenschaftliche Critique will die Bedingungen der Möglichkeiten verstehen und aufzeigen, warum gegenwärtige Zustände im Zusammenspiel mit ihren ökonomischen, sozialen, kulturellen und politischen Relationen so und nicht anders gewachsen sind, als richtig oder falsch bewertet werden und wie aktuelle Wissensbestände ihre Reproduktion begünstigen, anstatt sie zu unterbrechen (Calhoun 2008, xxv; Butler 2001). ${ }^{18}$ Geschehnis und Zustand, Rationalität und Selbstverständlichkeit werden so „vom Indikativ zum Konjunktiv, vom Singular zum Plural, vom Notwendigen zum Kontingenten“ (Rabinow 2004, 41). Die kritische anthropologische Haltung meint daher explizit nicht das Verantwortlichmachen einzelner Personen und auch nicht die verwüstende Dekonstruktion entlang eines normativen Richtwertes, sondern das Verstehen der genealogischen Pfade und der Möglichkeitsbedingungen, die die spezifischen Weisen der Problematisierung und das Tun der Akteur_innen hervorbringen (vgl. Niewöhner 2016).

In dieser kritischen wissenschaftlichen Haltung liegt meines Erachtens auch das politische Potential eines auf Zusammenarbeit basierenden Vorgehens. Calhoun formuliert den Beitrag, den akademisches Wissens für soziale Bewegungen jenseits einer Anwaltschaft leisten kann:

„The kind of expertise that academics offer will seldom be simply accumulated facts, and especially not about domains in which activists work. But researchers may be able to analyze data in ways that reveal previously unseen or at least inadequately demonstrated patterns in the facts. They may be able to clarify understanding of some of the broader contexts that influence the specific domains in which activists work. They may be able to help activists reflect on their own movements and struggles,

17 Während die deutsche Sprache keinen Unterschied kennt, findet sich im Englischen für diese Form des Kritisierens der Begriff criticism in Abgrenzung zur critique; siehe Butler (2001); Brown (2009); Kehr/Caduff (2015); Fassin (2017).

18 Das Postulieren von besseren Alternativen, die den Anspruch auf Wahrheit oder moralische Überlegenheit erheben, schließt Foucault (1992) aus seinem Kritikverständnis sogar aus. Die kritische Haltung zeichnet sich gerade dadurch aus, dass sie alle Wahrheiten kontinuierlich auf ihre Regierungseffekte hin befragt. 
partly through knowledge of how other struggles have played out. They may bring knowledge to tactics to expand the repertoires of activist." (Calhoun 2008, xx)

Akademisches Wissen vermag Grautöne, Widersprüche und Komplexitäten herauszuarbeiten und nebeneinander bestehen zu lassen; im Gegensatz zum Bewegungswissen muss es nicht sofort zu Diagnosen, Lösungen und Forderungen verdünnt und zu Skandalisierung und Mobilisierung zugespitzt werden (Binder/Hess 2013, 9f.). Es macht jene für die Aktivist innen kaum sichtbaren, da zu Selbstverständlichkeiten geronnenen und in Alltagsroutinen des Denkens und Handelns eingeschriebenen Bedingungen und vorstrukturierte Möglichkeiten ihres Tuns sichtbar, und es eruiert weniger greifbare und mittelbarere Effekte.

Damit eine solche wissenschaftlich-kritische Perspektive nicht nur zu einem besseren wissenschaftlichen Verstehen zivilgesellschaftlicher Geschlechterpolitik beitragen, sondern auch dem reflexiven Interesse der Protagonist innen begegnen und einen politischen Ort besetzen kann, muss sie, wie oben beschrieben, vom Feld und den dortigen Relevanzen, Anliegen und Widersprüchen ausgehen (Islam 2015, 244; Niewöhner 2016, 13f.). Die Aufgabe der Wissenschaftlerin ist es, diese Anliegen zu mobilisieren und sie im Kontext von anderen Feldern, durch andere Denkweisen, Fragestellungen und theoretische Brillen zu beleuchten (Niewöhner 2016, 11), um sie dann wieder in neuem Licht mit den Forschungspartner_innen zu diskutieren:

„So there's a movement away from and back, and that's the movement of critique, in which your purpose in continuing discussions with your first, orienting subjects is to bring the reality of something that's in their calculus back to their discussions." (Rabinow et al. 2008, 74)

Im Aktivismus-Alltag können solche Diskussionsangebote „reflexive Mikrosituationen schaffen, in denen ein nicht an unmittelbare Notwendigkeiten geknüpftes Nachdenken möglich ist" (Hamm 2013, 68). Treffen zur gemeinsamen Arbeit an der ethnografischen Deutung unter Einbezug von in der Zwischenzeit durch viele verschiedene Felder, Theorien und Denkweisen gewanderten Problematisierungen vermögen es, ,Möglichkeitsräume freizusetzen" (Rabinow 2004, 41), die im besten Falle zur Imagination von und Arbeit an Alternativen, anderen Möglichkeiten und neuen Zukünften führen (Calhoun 2008, xxv). Sie können neue Perspektiven eröffnen, die dann jedoch nicht in eine gemeinsame Strategie münden, sondern von allen Partizipierenden für ihre Zwecke - aktivistische wie akademische - genutzt werden können (vgl. Niewöhner 2016). 


\section{Teil I: Die Entstehung eines günstigen Moments}

„Die Zukunft des Fußballs ist weiblich“, kündigte Joseph „Sepp“ Blatter, zunächst Generalsekretär, dann Präsident des Weltfußballverbandes FIFA, seit 1995 wiederholt an und verwies damit auf das zunehmende Interesse von Mädchen und Frauen an jenem Sport, der lange als reine Männerdomäne galt (Krull 2007; Weinreich 2011). Nicht nur die Sportminister_innen vieler Länder haben sich die Geschlechterverhältnisse des Sports zum Thema gemacht, auch viele Verbände bekunden öffentlich ihre Ambitionen, den Frauenfußball zu fördern. So trug der Deutsche Fußball-Bund 2011 die Weltmeisterinnenschaft im eigenen Land mit großem Einsatz aus, lockte Millionen Zuschauer_innen vor die Fernseher und intensivierte zugleich die Förderung des Frauenbreitenfußballs. 2004 gründete sich das Frauen-Fußballnetzwerk F_in zur Vernetzung und Stärkung von Frauen in Sport und Fanszene, LSBTISportveranstaltungen diversifizieren die Freizeitangebote (Heissenberger 2018) und die Initiative Fußballfans gegen Homophobie prangert den Heterosexismus in der Fußballwelt an. Zugleich entstehen in Stadtteilen niedrigschwellige Mädchen-Fußballligen zur Förderungen von Integration und Selbstbehauptung, und Medien berichteten am Beispiel von Bibiana Steinhaus breit über die Schwierigkeiten, als weibliche Schiedsrichterin in die Männer-Bundesliga aufzusteigen (bspw. Wagner 2017). Sabine Behn und Viktoria Schwenzer diagnostizieren daher, dass ,in jüngster Zeit einiges in Bewegung geraten ist" (2006, 45). Die Geschlechterverhältnisse des Fußballs, konkret: die Benachteiligung von Mädchen und Frauen in Form von Unterrepräsentanz, mangelnder Anerkennung und Förderung sowie erschwerten Entwicklungschancen und offener Abwertung sind $\mathrm{zu}$ einer öffentlich thematisierten Angelegenheit geworden. Zugleich zeigen die skizzenhaften Beispiele sowie die Feldnotiz von der Weltsportministerkonferenz in der Einleitung dieser Arbeit, dass die Auseinandersetzung über Teilhabe und Rechte von Frauen weit über die Institutionen des Verbandsfußballs hinaus in andere gesellschaftliche Bereiche führt.

In diesem ersten Teil zeichne ich nach, wie die fußballerischen Geschlechterverhältnisse öffentlich und effektiv problematisierbar wurden. Ich frage, welche historischen Entwicklungen, feldübergreifenden Verflechtungen sowie gegenwärtigen Diskurse und Politiken es ermöglichen, dass die fußballerischen Geschlechterverhältnisse ,unsicher“ werden, ihre „Vertrautheit" verlieren und „,ins Feld des Denkens“ eintreten (Foucault 2005, 732). Dafür skizziere ich den historischen und diskursiven Kontext des Fußballs und die sich in diesem konstituierende Geschlechterordnung sowie die sich erschließenden Möglichkeiten geschlechterpolitischer Problematisierungen. Damit bietet dieser erste Teil auch eine historische und diskursive Einbettung der Verwobenheiten von Fußball und Feminismus, die das Forschungsfeld 
dieser Ethnografie darstellt. Es kommt damit dem unter anderem von Rolf Lindner formulierten europäisch-ethnologischen Forschungsprogramm nach, das verlangt, ,nicht nur das Phänomen zu kontextualisieren, das heißt, in Abhängigkeit von systemischen Prozessen zu sehen, sondern es auch als Teil eines komplexen Wirkfeldes mit Antipoden, mit Figurationen, mit Sedimentbildungen zu begreifen" $(2001,15)$. Feldforschung braucht daher auch immer eine Erforschung des Feldes, in dem das zu untersuchende Phänomen angesiedelt ist (ebd.).

\section{Theoretische Ausgangspunkte: Friction und Schnittstellen}

Doch wie lassen sich jene sich überschneidenden gesellschaftlichen Bereiche, historischen Sedimente und diskursiven Formationen samt heterogener Akteur_innen, Rationalitäten und Politiken denken, die die Geschlechterverhältnisse plötzlich problematisierbar machen?

Ich fasse diesen Moment des Aufeinandertreffens und der Verdichtung verschiedener Politiken, historischer Entwicklungen und Diskurse mithilfe von Anna Tsings globalisierungstheoretischem Konzept der Friction. Ihr ethnologisches Konzept ist darauf ausgelegt, sowohl kleinere Kontroversen als auch sämtliche kulturelle, diskursive, politische, soziale und andere Kontexte in den Blick zu nehmen und dabei den Fokus auf durch Spannung erzeugte produktive Effekte zu richten (2005). Als Reibungen versteht Tsing dabei die unbequemen, instabilen, ungleichen und kreativen Dimensionen der Interaktionen und Verbindungen über Differenzen hinweg (ebd., 4). Sie fasst damit die Dynamiken in jenem „Moment, in dem Akteure, institutionelle Strukturen, Dinge, Technologien und Diskurse aufeinandertreffen [...], in dem der normale Lauf der Dinge unterbrochen wird oder eine neue Richtung einschlägt" (Adam/Vonderau 2014, 22). Reibungen können auch in unauffälligen Alltagssituationen auftreten, in denen unterschiedliche Vorstellungen aufeinandertreffen (ebd.). Tsing betont dabei den produktiven Effekt dieser Spannungsmomente, die zugleich immer auch Restriktionen beinhalten:

,[A] study of global connections shows the grip of encounter: friction. A wheel turns because of its encounter with the surface of the road; spinning in the air it goes nowhere. Rubbing two sticks together produces heat and light; one stick alone is just a stick. As a metaphorical image, friction reminds us that heterogeneous and unequal encounters can lead to new arrangements of culture and power." (Tsing 2005, 5)

Das Bild der aneinander geriebenen Stöcke weist auch darauf hin, dass Friction nicht zwangsläufig konflikthaft verlaufen muss. Vielmehr scheint Fric- 
tion hier ein Berührungsmoment zu beschreiben, das sowohl konflikthaft und spannungsreich durch Reibung Neues produziert, als auch konfliktlos, aber dynamisch Unterschiedliches zu unvorhersehbaren Konstellationen zusammenfügt. Der Ausgang eines solchen Zusammentreffens ist unvorhersehbar, doch nicht beliebig. Er ist durch Sedimentierungen historischer Entwicklungen wie gegenwärtiger Verhältnisse vorstrukturiert und eingegrenzt:

„Roads are a good image for conceptualizing how friction works: Roads create pathways that make motion easier and more efficient, but in doing so they limit where we go. The ease of travel they facilitate is also a structure of confinement. Friction inflects historical trajectories, enabling, excluding, and particularizing." (ebd., 6)

Neue Arrangements und Wege werden hier nicht als Ergebnisse der Anstrengungen einzelner Akteur_innen, sondern als Resultate verdichteter Interaktionen und Situationen verstanden, was der diesen Teil leitenden Frage in die Hände spielt, die nach den Umständen und Kontexten fragt, unter denen zum gegenwärtigen Zeitpunkt die Geschlechterverhältnisse des Fußballs problematisiert werden können.

Um solch ein „feldübergreifendes Verständnis von [Forschungs-]Feld“ (Lindner 2003, 182) zu erarbeiten, folge ich den Problematisierungen ausgehend von meiner Feldforschung bei DF in andere gesellschaftliche Bereiche und historische Tiefen. Ich beginne zunächst mit einem historischen Rückblick auf den Verbandsfußball (4.1) und die sich darin entwickelnden Geschlechterverhältnisse (4.2). Ich zeige, wie sich vereins- und verbandskulturelle Rationalitäten im heutigen Fußball sedimentierten und sich eine spezifische Vereinskultur und Geschlechterordnung herausbildete. Gleichzeitig wird ersichtlich, wie diese Geschlechterverhältnisse Konjunkturen der Stabilität und Instabilität durchliefen und gegenwärtig Durchlässigkeit bieten, um in Zweifel gezogen zu werden. Dies ist für den weiteren Verlauf der Arbeit relevant, da sich in diesen historischen Sedimenten Bedingungen, Möglichkeiten und Hindernisse feministischer Interventionen in den Fußball einlagern. Anschließend beleuchte ich das Verhältnis zwischen Verbandssport, Staat und Marktwirtschaft, um Wege, aber auch Grenzen der Problematisierung durch Institutionen und Akteure außerhalb des Verbandsfußballs zu eruieren (4.3). Im fünften Kapitel folge ich den diskursiven Formierungen des Fußballs in verschiedene gesellschaftliche Bereiche hinein. Entlang der Aussagen „Fußball verbindet“ (5.1), „Fußball ist unpolitisch“ (5.2) und „Fußball empowert“ (5.3) zeige ich, wie ein Nexus aus Macht und Wissen akzeptierte Wahrheiten über Fußball produziert, diese miteinander verflechtet und dabei heterogene Personengruppen und Institutionen wie den Verbandsfußball, staatliche Verwaltungen der Innen-, Außen- und Entwicklungspolitik, zivilgesellschaftliche Initiativen, feministische Bewegungen sowie Sozial-, Kultur- und Sportwissenschaft zu machtvollen Konstellationen verbindet. Während dabei kontingente Wahrheiten über Fußball stabilisiert werden, ist zugleich zu beobachten, wie andere destabilisiert und als Argumentations- 
grundlage zur Kritik an den Geschlechterverhältnissen genutzt werden. Ich schließe diesen Teil mit dem Argument, dass diese spannungsreiche, doch produktive Fricton gegenwärtig ein Momentum für die plausible und effektive Anzweiflung und Umarbeitung der männlichen Dominanz im Fußball erzeugt. Dieser erste Teil der Arbeit umreißt damit den Kontext sowie die Bedingungen, unter denen sich schließlich eine Organisation als kompetente Problembearbeiterin formieren (Teil II) und geschlechterpolitisch in den Fußball eingreifen kann (Teil III).

\section{Verbandsfußball: Historische, geschlechtliche und gesellschaftliche Dimensionen}

Fußball wird in Deutschland sowohl auf Sportplätzen, in Turnhallen und großen Stadien als auch auf öffentlichen Wiesen und Garagenhöfen, in Wendekreisen und privaten Gärten, auf Bolzplätzen, Schulhöfen und in Bunten Ligen, Stadtteilturnieren oder Betriebssportgruppen gespielt. Fußball umfasst also neben den allgegenwärtigen Vereinen und kommunalen Sportstätten eine Vielfalt an sportlichen (Selbst-)Organisationen, Orten, Ambitionen und Motivationen. Im Zentrum dieser Arbeit steht die Frage nach den Praktiken der geschlechterpolitischen Problematisierungen des Fußballs, die immer wieder territoriale, vor allem nationale Grenzen physisch wie argumentativ überschreiten und sich translokal $\mathrm{zu}$ verankern und $\mathrm{zu}$ vernetzen suchen. Mit einem ethnografischen Ansatz möchte ich komplexe soziale Phänomene anhand ihrer Materialisierung an spezifischen Lokalitäten und in konkreten Situationen verstehen. Daher fokussiere ich die Schnittmenge von Fußball, Geschlecht und Politik anhand einer in Berlin ansässigen Frauenfußball- und Frauenrechtsorganisation. Diese ist, wie im Folgenden noch gezeigt wird, als eingetragener, gemeinnütziger Verein auch ein Produkt des spezifisch deutschen Vereinswesens und -rechts, und ihre Aktivitäten richten sich vor allem an jene Geschlechterverhältnisse, wie sie ihnen im deutschen Verbandsfußball begegnen. Aus diesem Grund grenze ich den historischen Rückblick auf die Entwicklungen des Vereins- und Verbandsfußballs in Deutschland ein und meine damit jene eingetragenen Vereine und entsprechenden Dachverbände, die am regulären Spielbetrieb der Fußballligen teilnehmen, für diese Zwecke die kommunalen Infrastrukturen nutzen und mit der staatlichen Verwaltung interagieren, sowie ihre sportlichen und organisatorischen Praktiken. 


\subsection{Fußball als Kultur: Vereinswesen}

Historische Arbeiten untersuchen die Entstehung des Fußballs in Deutschland meist entlang zentraler gesellschaftlicher Konfliktlinien: Fußball wurde so einerseits als moderner Sport verstanden, der eine gewisse disziplinlos wirkende Ausgelassenheit sowie das Prinzip Leistung dem traditionsreichen, disziplinierenden Turnen und den gemeinschaftlich und ohne Konkurrenzgedanken betriebenen Leibesertüchtigungen entgegensetzte. Andererseits wurde die Entwicklung des Fußballs entlang der Spannung zwischen bürgerlichem Sportverständnis und sozialdemokratischem Arbeitersport untersucht. Wenn ich im Folgenden meinen Fokus vorrangig auf die Entwicklung des Vereinsund Verbandsfußballs richte, greife ich diese Diskussionen immer wieder auf, um zu zeigen, wie sich aus diesen gesellschaftlichen Kämpfen im und um Fußball eine spezifische fußballerische Vereinskultur herausbildete, durch die feministische Interventionen navigieren müssen.

\subsubsection{Die Entstehung des deutschen Vereinswesens}

Die Kultur der Sportvereine wurzelt im deutschen Vereinswesen, das sich aus der städtisch-bürgerlichen Gesellschaft zu Beginn des 18. Jahrhunderts entwickelte und sich seither durch die Freiwilligkeit des Beitritts und die Offenheit seiner Zugehörigkeit auszeichnet (Martischnig 1982, 10f.; Bausinger 2006, 44; Nathaus 2009, 11). Seit jeher verfügen Vereine über eine eigenständige Verwaltung und formale Mitgliedschaften und zeichnen sich durch die Festlegung und Verfolgung eines bestimmten Zwecks und den Verzicht auf Ausschüttung finanzieller Gewinne aus (Nathaus 2009, 11). Von ihren ständisch-bündischen Vorläufern übernahmen sie Abzeichen, Fahnen und Stammtischzeichen und bildeten spezifische Normen, Traditionen und Zeichensetzungen heraus (Martischnig 1982, 10f.). Neben dem hohen Sozialkapital und den Freizeitgestaltungsmöglichkeiten, die Vereine boten, erhob die Vereinsmitgliedschaft den Einzelnen in den Stand eines ehrbaren Bürgers und verlieh ihm öffentliches Ansehen, welches mit der Dauer der Mitgliedschaft wuchs (Nathaus 2009, 105ff.). Während sich zunächst die meisten Vereine aus dem Bürgertum heraus gründeten, setzte zur Mitte des 19. Jahrhunderts und im Zuge der massenhaften Arbeitswanderung, der Expansion der Städte und Ausdifferenzierung sozialer Schichten eine große Welle der Vereinsbildung ein (Nathaus 2009, 105). Emotionale Beziehungen und gemeinschaftliche Zwecke, die vorher ihren Platz in den Nachbarschaften und Dörfern hatten, verlagerten sich nun in die Vereine (Bausinger 2006).

Im Bereich der Leibesübungen entstanden früh zahlreiche Turnvereine, die sich auszeichneten durch Geselligkeit jenseits der Standesgrenzen, eine feste lokale Verankerung in Stadt oder Stadtteil sowie ein demonstratives, 
öffentliches Auftreten mit Stiftungsfesten und Vorführungen (Nathaus 2009, 111).19 Turnen wurde dabei von den Gründungsvätern als rein männliche Tätigkeit verstanden, die den Frauen untersagt war und sie aus den Turnvereinen ausschloss (Pfister 2006, 28f.).

Auch in den meisten anderen Vereinssparten waren weibliche Mitglieder nicht zugelassen. Carola Lipp zeigt anhand des Königreichs Württemberg, dass es nicht nur offizielle Beitritts- oder Gründungsverbote waren, die Frauen die Partizipation am Vereinswesen erschwerten. Sowohl die bürgerlichen Kleidungs- als auch Schicklichkeitsregeln behinderten eine kontinuierliche Vereinigung: Bürgerliche Frauenkleidung war ungeeignet für längere Aufenthalte im Freien und Kühlen, sodass regelmäßige Treffen in den nasskalten Jahreszeiten auf ein Minimum reduziert wurden. Zudem galt es für Frauen als unschicklich, - insbesondere am Abend - unbegleitet auf Veranstaltungen zu gehen, sodass sich die Frauenkreise meist tagsüber trafen und zu gemischtgeschlechtlichen Treffen eine begleitende Anstandsperson gefunden werden musste (1986, 289f.). Im Vormärz und in der Revolution 1848/49 veränderten sich die Öffentlichkeitsstrukturen und Frauen drangen sukzessive und abhängig von den jeweiligen Gesetzen der Länder in die bürgerliche Öffentlichkeit ein. Einerseits wurde ihnen der Zutritt - freilich vorwiegend als passive Mitglieder - zu Vereinssparten wie Museums-, Geselligkeits- und Gesangsvereinen gewährt, andererseits gründeten sich dort, wo dies rechtlich gestattet war, eigenständige, solidarisch orientierte Fraueninitiativen (ebd.; Kuby 1986). Wie in Kapitel 4.2 gezeigt wird, dauerte es dennoch weitere 100 Jahre, bis sich Frauen sukzessive die volle Mitgliedschaft in Sportvereinen eröffnete.

\subsubsection{Der Aufstieg des Fußballs als Wettkampfsport}

$\mathrm{Zu}$ den traditions- und heimatverbundenen Lokalvereinen bürgerlichen Ursprungs gesellten sich zu Beginn des 20. Jahrhunderts vermehrt die alternativen Freizeitangebote der Arbeiterbewegung. ${ }^{20}$ Die zunehmende staatliche Repression kommerziellen Freizeitvergnügens im Kaiserreich sowie die Begünstigung des Vereinswesens bewirkten einen steigenden Zulauf zu den Vereinen und deren langsame Öffnung hin zur populärkulturellen Unterhaltungsformen der Massen. Der Fußball als neuartige, ausländische Bewegungsform stieß zunächst auf heftigen Widerstand aus der Deutschen Turnerschaft, dem größten Dachverband der Turnbewegung. Doch zugleich bot das

19 Zwar entwickelten sich Turnen und Sport als zwei eigenständige Phänomene, doch aufgrund ihrer ständigen gegenseitigen Bezugnahme und Abgrenzungen ist ihre Entwicklung eng verzahnt. Eisenberg (1999, 250-260) deutet den Konflikt zwischen Turnen und Sport als „Kulturkampf“ zwischen traditioneller und moderner Bürgerlichkeit. Daher streife ich an den relevanten Stellen immer wieder die Turnbewegung.

Ausführlich zur Arbeitersportbewegung siehe Schönberger (1995). 
populärkulturelle Vergnügen am Wettstreit einen Anknüpfungspunkt, um moderne Sportarten wie Fußball, Tennis und Boxen, die im ausgehenden 19. Jahrhundert von Großbritannien durch Geschäftsreisende und Touristen nach Kontinentaleuropa gekommen waren, in die Vereinskultur zu integrieren. So entstanden zum Ende des 19. Jahrhunderts die ersten Fußballvereine, und der Deutsche Fußball-Bund (DFB) begann kurz nach seiner Gründung 1900 mit der Ausrichtung von Wettbewerben (Brändle/Koller 2002, 39f.; Nathaus 2009, 122ff.; Eisenberg 2014, 10). So institutionalisierte sich das Leistungsprimat im Vereins- und Verbandsfußball. Wettbewerbe als ,ritualisierte, standardisierte spielerische Performanzen" (Tauschek 2013, 16) verbinden das Spielerische mit dem Wettstreit. Es ist der Wettkampf, der Leistung im Sinne des ständigen Übertrumpfens anderer und der eigenen Leistung begünstigt, indem er den direkten Vergleich durch das Gegeneinander-Antreten oder mithilfe von Messinstrumenten ermöglicht (Bausinger 2006, 130f.). Bis heute sind es die Verbände, die die nötigen Infrastrukturen, wie Regelwerke, Personal, Orte und Bewertungskriterien, bereitstellen und den Leistungsvergleich formalisieren.

Zugleich war die Entstehung der Fußballvereine durch die Spannung zwischen bürgerlichen und arbeiterbewegten Vereinen geprägt, wie Rolf Lindner und Heinrich Breuer unter anderem anhand des Ruhrgebietsvereins Schalke 04 zeigen. Vor dem Ersten Weltkrieg wurde der Fußball weitestgehend von (angehenden) Akademikern beherrscht und Arbeitervereine wie Schalke 04 konnten sich zunächst nur schwer und unter Anpassungszwang im bürgerlichen Spielbetrieb des DFB behaupten (1978, 51). Obwohl sich bereits zu Beginn des 20. Jahrhunderts zahlreiche Arbeiterfußballvereine gründeten, streifte der Fußball sein bürgerliches Image erst nach dem Ersten Weltkrieg ab. Deutsche Soldaten kamen im Krieg mit dem Spiel in Berührung und brachten es mit nach Hause, woraufhin das Fußballinteresse sprunghaft anstieg - was sich auch auf die Mitgliederzahlen auswirkte: 1930 spielten etwa 140000 Männer im Arbeitersport und der im bürgerlichen Lager gegründete DFB zählte zur gleichen Zeit 900000 Mitglieder, die zum großen Teil proletarischer Herkunft waren (Lindner 1983, 31). In dieser Zeit nach dem Ersten Weltkrieg erhielt der Fußball sein Image als Proletariersport, das bis heute in der Konstruktion einer Fußballmännlichkeit und anderen habituellen Formen in den Verbänden zum Ausdruck kommt. ${ }^{21}$

21 In der historischen Fußballforschung gehen die Meinungen auseinander, ob Fußball tatsächlich als Arbeiter- und Proletariersport bezeichnet werden kann. Fürtjes (2012) überprüft sowohl die These vom Fußball als Arbeitersport und schlussfolgert, dass es sich beim Fußball um ein kontinuierlich schichtenübergreifendes Massenphänomen handelt. In dieser Arbeit ist jedoch weniger die tatsächliche Sozialstruktur der Vereinsmitglieder, Spieler_innen und Zuschauer innen von Interesse, sondern vielmehr die Beobachtung, dass der „Mythos vom Proletariersport" (ebd., 317) sich behaupten konnte und damit auch Stoff für spezifische Männlichkeitsentwürfe bot. 


\subsubsection{Vereinskultur und die Krise des Verbandsfußballs}

Trotz der Expansion der Organisationsform Verein unter anderem durch die wachsende Popularität des Fußballs nahmen Ansehen und Attraktivität des Vereinswesens in der zweiten Hälfte des 20. Jahrhunderts ab und das Klischee der spießbürgerlichen Vereinsmeierei breitete sich aus (Nathaus 2009, 269ff.). Kurt Tucholsky karikiert diesen Habitus in seinem Gedicht „Das Mitglied“ (1961) und Bausinger fasst Tucholskys Skizze zusammen: „das falsche Pathos der Funktionäre und Festredner, der bemühte Formalismus der kleinen Funktionärselite, der Jahrmarkt der Eitelkeiten mit Wiederwahl und Anstecknadel und Ehrenmitgliedschaft" - und räumt ein, dass dies gewiss ein karikierendes Zerrbild, aber „sicher kein reines Phantasieprodukt“ sei (2006, 43). ${ }^{22}$ So benennt er neben den positiven Effekten der Vereinskultur wie dem Erlernen demokratischer Diskussions- und Arbeitskultur und der Gemeinschaftsstiftung auch problematische Folgen: etwa die bürokratische Überformung durch Statuten und Mitgliederverwaltung, die Übernahme alter Einflussstrukturen und innerer Rangordnungen sowie die Vernetzung unter den Vereinen, die zu Vetternwirtschaft und verdeckten, lokalen Machtgefügen führen können. In der auf einen Zweck hin ausgerichteten Struktur liegt auch eine Falltür in den Traditionalismus: Zwar manifestieren sich neue Trends und Veränderungen häufig in Vereinsgründungen, ,[a]ber das Neue wird ja sehr schnell alt, und die Vereine, festgelegt auf diesen einen Gegenstand, verteidigen dann das Alte mit Klauen und Zähnen“" (ebd., 48).

Während das Vereinswesen seine Attraktivität einbüßt und sich viele sportlich aktive Menschen vom Vereinssport ab- und dem individuellen oder dem kommerziell organisierten Sport zuwenden, leidet der Vereinssport deutlich geringer unter Mitgliederschwund als andere Vereinssparten. Dies ist zum einen der Beliebtheit des Sports geschuldet, zum anderen der kontinuierlich wachsenden Zahl an weiblichen Neumitgliedern (Nathaus 2009, 275).

Im beliebtesten Vereinssport, dem Fußball, zeichnet sich jedoch gegenwärtig eine andere Krise ab. „Der Fußball ist tot“ - mit diesem Jahresmotto erklärt die Deutsche Akademie für Fußball-Kultur 2019 zum Trauerjahr und listet darunter eine Auswahl an Büchern, die das Motto verständlich machen: „FIFA Mafia“ (Kistner 2011), „Das wunde Leder“" (Gmünder/Zeyringer 2017), „Football Leaks“ (Buschmann/Wulzinger 2016) oder auch „Die dunkle Seite des Balls“ (Josuttis 2017) (Deutsche Akademie für Fußball-Kultur 2019). Gegenwärtig ist zu beobachten, wie der professionelle Fußball und die ihn organisierenden Verbände zunehmend und von verschiedenen Seiten in die Kritik geraten - sei es aufgrund von Korruption wie bei den Vergaben der $\mathrm{WM}$, aufgrund der, die sich unter anderem in nicht mehr zu rechtfertigenden

22 Für eine kritische, humorvolle und gelungene Skizze der Vereinskultur mit ihren historischpolitischen Verstrickungen siehe Eva Menasses Beschreibung des Tennisclubs Schneuzl im Roman „Vienna“ (2005). 
Transfersummen, reinen Sitzplatztribünen oder sehr hohen Eintrittspreisen ausdrückt, oder aufgrund der Verletzungen von Umweltstandards und Arbeitsrechten auf den weltweiten Stadionbaustellen. Mit Blick auf die Kritik am Weltverband FIFA diagnostizieren Jürgen Mittag und Jörg-Uwe Nieland daher eine „Zeitenwende“, also einen „Strukturbruch im Machtgefüge des organisierten internationalen Fußballs“, der zu einem ,grundlegenden Paradigmenwechsel“ führen könnte $(2016,198)$. Diese Krise der Verbände findet ihren Ausdruck in Deutschland am deutlichsten und am besten organisiert in den Fanprotesten gegen Kommerzialisierung, wie sie sich z.B. gegenüber dem Verein RB Leipzig entladen (Bresemann/Duttler 2017, 137).

Für den Fortlauf der Arbeit ist relevant, dass die vor allem ökonomischen Entwicklungen innerhalb des Verbandsfußballs in Deutschland, die mit der Einführung des Berufsfußballs in den 60er Jahren begannen, aber auch international zu vermehrten Protesten führten, gegenwärtig die Organisation des Fußballspiels sowie die internen Strukturen der Verbände grundlegend in Zweifel ziehen und den Machtanspruch der Verbände problematisieren. Die diesen Teil leitende These, dass gegenwärtig ein besonders günstiger Moment zur Problematisierung der fußballerischen Geschlechterverhältnisse besteht, erfährt hier notwendige Kontextualisierung, denn ich vermute, dass die vielfältige Kritik am verbandlichen Machtgefüge auch feministischen Probematisierungen den Weg ebnet. Zugleich gilt es Mittags Analyse zu berücksichtigen, die zu dem Schluss kommt, dass die Proteste trotz ihrer Vielzahl ,zumeist situativ, punktuell und auch nur begrenzt nachhaltig“ sind (2011). $\mathrm{Zu}$ fragen bleibt daher, inwiefern Proteste gegen Kommerzialisierung, Korruption und für Arbeits- und Menschenrechtsstandards im Profifußball der Männer auch für eine konkrete feministische Umarbeitung im Sinne breiter Bündnisse dienlich sind.

Festzuhalten ist, dass Fußball als populärkulturelles Massenphänomen innerhalb eines Spannungsverhältnisses entstand: Bürgerlich-konservative Kräfte behaupteten ein politisch neutrales Sportkonzept gegenüber sozialdemokratischen und sozialistischen Bestrebungen; gleichzeitig konkurrierte die Sinnfreiheit des Freizeitvergnügens mit den nationalistisch-disziplinierenden Tendenzen der Turnerschaft; Arbeiterfußballvereine konnten sich nur schwer gegenüber den bürgerlichen Fußballvereinen behaupten. Trotz dieser vielen Spannungen traf der Fußball mit dem Vereinswesen auf eine relativ stabile Organisationsform, die einen bestimmten Habitus bereits hervorgebracht hatte. Im Verein verbanden sich interne Demokratie und soziale Aufstiegsmöglichkeiten durch Engagement und Leistung mit Repräsentation, Hierarchie und Traditionalismus. Die Ausübung des Fußballs als Wettkampfsport sowie die Hoheit der Verbände über die Austragung des Wettkampfs - seit vielen Jahrzehnten die maßgebliche Organisationsform des Fußballs - ermöglichten die Etablierung des Leistungsprimats im Spitzen- und Breiten- 
fußball. Die zunehmende Verquickung von Fußball und Markt führt dabei gegenwärtig zu einer grundlegenden Infragestellung der Verbände, ihrer internen Strukturen und der Art, wie sie den sportlichen Wettkampf organisieren. Die Vergeschlechtlichung dieser Vereinskultur sowie die Kritik an ihr ist bereits in Form allgemeiner Partizipationshindernisse für Frauen angedeutet worden - im Folgenden wird sie anhand des Verbandsfußballs genauer beleuchtet.

\subsection{Aus Geschlechterperspektive: Fußball und Frauen}

Viele historische und sozialwissenschaftliche Studien befassen sich mit der Geschichte von Frauen im Fußball und begründen aus ihr heraus die aktuellen Geschlechterverhältnisse. ${ }^{23}$ Sie beschreiben diese Geschichte als „Entwicklung des Fußballs zum Männersport“ (Müller 2007, 115), in der ,women have always played a marginal role and that is still true today" (Pfister 2003a, 128). Das Augenmerk liegt zumeist auf den vielen „Schwierigkeiten und Hindernissen" (Selmer 2013, 50), mit denen Frauen im Fußball konfrontiert und letztlich marginalisiert oder gar aus ihm exkludiert wurden. Diese haben sich zwar über die Jahrzehnte immer wieder verändert, doch werden sie stets als Fortführung der männlichen Herrschaft mit anderen Mitteln verstanden. Dabei konzentrieren sich die meisten sportfeministischen Geschichtsnarrationen darauf zu zeigen, wie das weibliche Subjekt in Abgrenzung zum männlichen als untergeordnet produziert wurde. Es erscheint mir durchaus plausibel, die Geschichte des Frauenfußballs auf diese Weise zu erzählen. In diesem Sinne beleuchte ich im Folgenden, wie im Verbandsfußball Männlichkeiten und Weiblichkeiten als Gegensätze historisch konstituiert wurden und wie diverse heteronormative Ordnungsprinzipien die männliche Herrschaft bis heute aufrechterhalten. Zugleich gilt es dabei - wie bei allen anderen Geschichtsnarrationen auch - zu berücksichtigen: „It extracts coherence from confusion, reduces multiplicity to singularity and [...] imposes sequential order on otherwise chaotic and contingent occurrences." (Scott 2012, 50f.). Ich ergänze daher eine zweite Narration, die den Blick vermehrt auf die Konjunkturen in den Geschlechterverhältnissen richtet und so die Durchlässig-

23 Sie beziehen sich dabei meist auf ausführliche Aufarbeitungen der Geschichte des Frauenfußballs. In der Sportsoziologie schließen Pfisters historische Arbeiten über den Frauensport auch den Fußball ein $(1991 ; 1999 ; 2006 ; 2011)$, die Historiker Hoffmann und Nendza (2011) sowie die Sportjournalisten Hennies und Meuren (2009) haben die Geschichte des Frauenfußballs in Deutschland ausführlich aufgearbeitet. Für den Frauenfußball in der DDR legte Linne (2011) eine umfassende historische Untersuchung vor. 
keit, Bruchstellen und Spielräume sichtbar werden lässt. ${ }^{24}$ So werden die gegenwärtigen Problematisierungen der fußballerischen Geschlechterverhältnisse als historisch gewachsen und geformt erkennbar und erscheinen nicht als plötzliche, geschichtslose Phänomene.

\subsubsection{Die Anfänge}

Seitdem die Sportart Fußball zum Ende des 19. Jahrhunderts ihren Weg von Großbritannien ins wilhelminische Deutschland gefunden hatte, galten sowohl die Ränge der Stadien als auch die Fußballplätze und Vereinsheime als Männerdomänen. Eingeführt als wichtiges Instrument der Erziehung und männlichen Sozialisation an den elitären englischen Internatsschulen im späten 18. und frühen 19. Jahrhundert, war der moderne Fußball von vornherein darauf ausgelegt, sportliche Eigenschaften zu fördern, die damals wie heute als männlich gelten: Mut, Stärke, Taktik, Risikobereitschaft, aggressiver Körperkontakt, Ausdauer etc. (Dunning 1994; Brändle/Koller 2002). ${ }^{25}$ Zwar stieß er, wie bereits dargestellt, auf große Abwehr, doch fand er gerade aufgrund seiner disziplinierenden Funktion auch Befürworter. Schon 1874 führte Konrad Koch, Braunschweiger Gymnasiallehrer, die Sportart an seiner Schule ein. Das fußballerische „Stahlbad des Charakters“ galt ihm als Ort, um ,harte" Männlichkeit anzueignen und zu demonstrieren (Koch 1900, zit. n. Pfister 2012, 43). Bereits die Anfänge des Fußballspiels zeichnen dieses also als vergeschlechtlichte und vergeschlechtlichende Praktiken, durch die Männlichkeit erlernt und dargestellt wird. Das Leistungsprimat, das die Verbände durch die Institutionalisierung des Wettbewerbs festigten, ist also keineswegs neutral. Vielmehr basiert die Deutung von schlechter, guter oder gar Höchstleistung auf ganz bestimmten sozialen und biologischen Körpernor-

24 Mit diesen Konjunkturen gingen jeweils Krisen der Maskulinität einher. Da mein Fokus auf den Auswirkungen auf den Frauenfußball liegt, werde ich diese nicht näher betrachten. Nachzulesen sind sie bei Messner (1994). Schultz (2014) blickt auf die Geschichte des Frauensports in den USA anhand von ,points of change“ und identifiziert sieben verschiedene Ereignisse, die sich auf die sportlichen Geschlechterverhältnisse auswirkten. Ähnlich wie Schultz Wendepunkte soll auch hier durch die Betonung von Konjunkturen der Problematisierung eine andere Periodisierung der Sportgeschichte vorgeschlagen werden, die nicht allein chronologisch entlang zentraler politisch-ökonomischer Ereignisse verläuft (ebd., 4).

25 Um 1880 wandelte sich in Großbritannien der Fußball vom Gentlemen-Sport zum Arbeitersport. Die große Beliebtheit zog immer größere Schichten der Bevölkerung auf den Rasen und die Verkürzung der Arbeitszeiten ermöglichte auch den Arbeitern die aktive Teilhabe. Fußball, zum Populärsport gewandelt, konnte so nicht länger als Distinktion des guten Geschmacks dienen, sodass sich die britischen Gentlemen exklusiveren - teureren und komplizierteren - Sportarten zuwandten. Als der Fußball Deutschland erreichte, galt er jedoch noch als gut-bürgerlicher Sport (Brändle/Koller 2002, 33). 
men. Der junge, fitte, weiße, cis-männliche Sportkörper wurde als Maßstab dem Leistungsprinzip eingeschrieben.

Zum Ende des 19. Jahrhunderts öffnete sich die bis dahin den Männern vorbehaltene und am weitesten verbreitete körperliche Ertüchtigungsform, das Turnen, verhalten und gegen Widerstände auch für Mädchen und Frauen. Das Mädchenturnen - und kurze Zeit später auch ihre Beteiligung an den im Freien veranstalteten Sportspielen der Spielbewegung - sollte gesundheitlichen Problemen des weiblichen Geschlechts wie Nervenschwäche, Bleichsucht, nervösen Leiden und Rückgratverkrümmungen begegnen. Um die Jahrhundertwende wagten sich vereinzelt auch Frauen aus der Mittel- und Oberschicht in andere Sportarten wie Schwimmen, Radfahren, Skilaufen, Rodeln, Tennis und Golf vor und beteiligten sich an der Teamsportart Feldhockey. ${ }^{26}$ Als sie sich am Fußball versuchten, stießen sie jedoch auf Widerstände (Pfister 2012, 44f.). Doch es gab auch Fürsprecher_innen. Gertrud Pfister $(1980 ; 2012)$ beschreibt, wie Ärztinnen durch ihre eigenen unkonventionellen beruflichen Biografien sowie ihre Sporterfahrungen zu Anwältinnen des Frauenfußballs wurden. Z.B. stellte Hope Bridges Adams-Lehmann die biologische Begründung für die Schwäche der Frauen in Frage und beschrieb sie stattdessen als Konsequenz der Umstände:

„Nicht die körperliche Beschaffenheit der Mädchen verbietet ihnen, an den Arbeiten und Spielen der Knaben teilzunehmen, sondern ihr Ausschluss von diesen Arbeiten und Spielen ist die Ursache für den verkümmerten Körper und den verkümmerten Geist.“ (Adams-Lehmann 1899, 131, zit. n. Pfister 2012, 49)

Sie kritisierte, dass die Frau, ,gefesselt am Gängelbad des Vorurtheils und der Verhältnisse“ zu „einem Wesen zweiten Ranges“ gemacht wurde (AdamsLehmann 1899, 2, zit. n. ebd.). Sporttreiben sollte diese Schwäche überwinden. Sie forderte koedukativen Sportunterricht und die Beteiligung von Mädchen und Frauen an vielen Sportarten wie dem Fußball.

Auch die in Berlin praktizierende Kinder- und Sportmedizinerin Alice Profé forderte die uneingeschränkte Teilhabe von Mädchen und Frauen am Sport. Wie Adams-Lehmann identifizierte sie die Hindernisse für den Frauensport in den Vorurteilen und der gesellschaftlichen Benachteiligung von Frauen. Auch sie plädierte für die weibliche Sportpartizipation mit gesundheitlichen Argumenten. Ihr Einsatz für Gleichberechtigung wurde vermutlich vor allem durch ihr Engagement im radikalen Flügel der bürgerlichen Frauenbewegung motiviert (Profé 1980; 1980a).

Pfister resümiert über diese frühen frauensportpolitischen Forderungen, dass es ihnen trotz großen Einsatzes nicht gelang, ,Mädchen und Frauen den Weg zu als ,unweiblich' geltenden Leibesübungen oder gar zum Fußballspiel zu ebnen“ (2012, 54). Die im Kaiserreich gegründeten FrauenFußballinitiativen mussten aufgrund von Anfeindungen und Widerständen 
aufgeben. Dennoch betrachte ich diese als erste Versuche der Problematisierung der fußballerischen Geschlechterverhältnisse, denn sie zeigen, dass es in den Anfangsjahren weder vollkommen ausgeschlossen war, als Frau Fußball zu spielen, noch undenkbar, die ,Frauenfrage' auch anhand der fußballerischen Partizipation zu diskutieren. Auffallend ist dabei der Einfluss frauenbewegter Ideen auf die Argumentation, die hier vor allem auf Partizipation und Gleichbehandlung abzielte.

\subsubsection{Die Pathologisierung der Fußballerin}

Dieses Möglichkeitsfenster schoss sich in den 1920er und 30er Jahren sukzessive. Marion Müller zeichnet hierfür die sich verfestigenden und naturalisierenden Geschlechterdifferenz verantwortlich. So wurden Frauen im Zuge der funktionalen Polarisierung der Geschlechter mit Verweis auf biologische Differenzen und entsprechende Charakterunterschiede aus dem Ballsport ausgeschlossen (2007).27

In der Weimarer Republik wuchs Sport zu einem Massenphänomen heran. Körperliche Ertüchtigung wurde zur Mode, die auch Frauen einschloss, ohne im Widerspruch zu Weiblichkeitsnormen zu stehen - jedoch nur, solange sich die ,weibliche" Sportphilosophie in Abgrenzung zum ,männlichen" konkurrenz- und leistungsorientierten Wettkampfgedanken verstand (Messner 1994, 68). Sportliche Leistungen wurden so weiterhin unter dem Differenzparadigma betrachtet. Frauen galten als das schwache Geschlecht, als kleiner, breithüftiger, kurzatmiger und damit als für den Fußball ungeeignet (Pfister 2006, 34; vgl. Pfister 1991). Sie sollten vor allem jene Sportarten ausüben, die sich durch Anmut und die Sorge um Körper und Hygiene auszeichneten (Bromberger 2006, 41f.). ${ }^{28}$ Die Zweigeschlechtlichkeit als binäres System wurde so über den sportlichen Wettkampf festgeschrieben:

„Sport ist Kampf. Die Funktionen des männlichen Körpers entsprechen dem männlichen Charakter und stimmen mit der Forderung auf Kampf und Höchstleistung überein. Der Mann kann im Kampf heldische Größe erreichen, das echte Weib nie, denn die weibliche Eigenart entbehrt des Kampfmomentes. Dadurch wird das Weib das

27 Zur deutlich geringeren Geschlechterdifferenzierung im vormodernen Ballsport siehe Müller (2007): Als ,unvollkommener Mann' hatten Frauen damals an Ballspielen teil, erst die moderne funktionale Geschlechtertrennung machte sie zu völlig , anderen Wesen'. Pfister $(2012,44)$ merkt an, dass im Kaiserreich die Teilnahme von Frauen an ausgewählten Turnübungen und Spielen mit Verweis auf die reproduktive Gesundheit ausdrücklich empfohlen wurde, da nur starke Frauen wehrhaften Nachwuchs gebären könnten.

28 Lönnecker $(2013,212)$ zeigt, dass Studentinnen an deutschen Universitäten der Zwischenkriegsjahre bereits vor 1927 in vereinsähnlichen Zusammenschlüssen Fußball spielten. Aufgrund der sozialen Ächtung des Frauenfußballs galt dieser unter den bürgerlichen Studentinnen jedoch „kaum als sozialädaquate Sportart für künftige Akademikerinnen und Führungspersönlichkeiten“. 
ergänzende Wesen. Das Weib darf nicht zum sportlichen Wettkampf antreten.“ (Dawin-Herne 1926, 34f., zit. n. Hoffmann/Nendza 2011, 24).

Die Ausgrenzung von Frauen aus bzw. Schlechterstellung im Sport geschah über die Verbindung von Leistung und Körper. Sei es die Annahme, dass Frauenkörper und -charakter nicht zu gleicher Leistung fähig wären, oder sei es die Warnung, dass ihrem Körper durch übermäßige sportliche Anstrengung Schaden drohte: Leistungsprimat und Geschlechterdifferenz verflochten sich hier zur Selbstverständlichkeit und maßen dem Männlichen Höherwertigkeit bei. Untermauert wurde diese neue Selbstverständlichkeit durch medizinische Argumente, in denen sich die Furcht vor physischen Veränderungen des Frauenkörpers in Form von Muskelzuwachs, einer tieferen Stimmlage, zunehmender Gesichtsbehaarung und dem Verlust der Gebärfähigkeit ausdrückte (Pfister 2006, 43). Zugleich weisen sie hin auf die Angst vor der Verunsicherung der Geschlechterdifferenz durch symbolische Vermännlichung der Frau.

Die medizinische Warnung gliedert sich in die Argumentationslogik der damaligen Zeit ein, die auch durch psychiatrische und sexualwissenschaftliche Thesen beeinflusst war. Esther Newton zeigt, wie unter anderen der Psychiater Richard von Krafft-Ebing durch die Verknüpfung von Maskulinität, lesbischem Begehren und feministischen Forderungen ,any gender-crossing or aspiration to male privilege as a symptom of lesbianism" pathologisiert hatte (Newton 1989, 287). In dieses Kategorisierungsschema hatte der Sexualforscher Havelock Ellis auch enge Freundschaften unter Frauen einbezogen, was sich bald als Verdacht gegenüber Frauen-Sportteams äußerte und diese mit dem damaligen Stigma der Homosexualität belegte (ebd. 1989, 287f.). Spielerinnen wurden durch die neue medizinische wie psychiatrische Kategorie der ,männischen Lesbe', die männliche Kleidung und Verhaltensformen präferiere und ihre Rolle als Ehefrau und Mutter vernachlässige, ins Abseits gedrängt und als emanzipierte Frauen bloß- und ihre Anständigkeit in Frage gestellt (Griffin 2002; Pfister 2006, 33). ${ }^{29}$ In dieser Zeit entstand der Diskurs um das fußballerische ,Mannweib', welcher bis heute Frauen im Fußball einschüchtert und kontrolliert und ein Dilemma schafft, indem er Weiblichkeit, spezifische Sportarten und entsprechende Körperformen als inkompatibel erklärt (Griffin 2002). ${ }^{30}$

29 Zur Institutionalisierung des Heterosexismus und der Entwicklung des Stereotyps der mannish lesbian am Bespiel des US-amerikanischen Schulsports siehe Cahn (1994).

30 ,The female in sport is described as mannish, muscle bound, unpretty, unhappy, having hormone problems, having menstrual problems, hating men, loving women. She's called a jockette, a butch, a dyke, a lezzie“, so Bennett et al. (1987, 373) in den 1980er Jahren. Durch den kulturellen Wandel zu einem Lifestyle der Sportivität (vgl. Kaschuba 1997) haben sich Weiblichkeit und Sportlichkeit im Kontext von Schönheitsidealen angenähert. Doch noch immer werden bestimmte körperliche Folgen des Sports, bspw. Muskelzuwachs an den ,falschen' Stellen, als unweiblich diffamiert. 
Die Ablehnung des Frauenfußballs bekam auch Lotte Specht zu spüren, die 1930 den ersten Damenfußball-Club in Frankfurt gründete:

\begin{abstract}
„Meine Idee, die kam nicht nur aus der Liebe zum Fußballsport, sondern vor allen Dingen frauenrechtlerisch. Ich habe gesagt, was die Männer können, können wir auch. [...] Die Männer, die haben sogar Steine nach uns geworfen. Und die Zeitungen haben uns durch den Kakao gezogen und geschimpft. Also, es wehte schon der braune Wind 1930. Die deutsche Frau raucht nicht, die deutsche Frau spielt keinen Fußball usw. Wir haben nur ein Jahr existiert, auch weil die Zeitungen so übel geschrieben haben und manche Eltern den Mädchen das verboten haben." (Lotte Specht 1999, zit. n. Hoffmann/Nendza 2011, 28)
\end{abstract}

Specht stellte, wie zuvor Adams-Lehmann und Profé, den Frauenfußball in einen politisch-rechtlichen Kontext und machte ihn damit zu einer frauenrechtlichen und nicht rein sportlichen Angelegenheit. Doch mit dem aufziehenden „braunen Wind“ schloss sich endgültig das Möglichkeitsfenster sowohl für das aktive Fußballspiel als auch für frauenbewegte Kritik. ${ }^{31}$

Nach der „Gleichschaltung“ des DFB 1933 untersagte der Nationalsozialistische Reichsbund für Leibesübungen den Frauenfußball mithilfe einer einfachen Mitteilung:

„Es gibt Sportarten, in denen wir die Frau nicht als Sportausübende treffen, weil ihre Eigenarten nicht dem Wesen der Frau entsprechen. Zu diesen Sportarten gehört auch der Fussball. [...] Zu hart, bei allem fairen Einsatz der Kräfte wird in diesen Sportarten um den leistungskrönenden Sieg gerungen, oder zu gross sind die Anstrengungen, die in diesen Sportkämpfen an den Körper gestellt werden müssen, als dass die Frau sie als Durchschnittsleistung je erreichen könnte. Oft aber widerspricht der männliche Kampfcharakter der einzelnen Sportart dem Wesen der Frau, die wir von Sportarten bewusst ausgeschaltet sehen wollen, die ihr die Würde des Weibes im Wettkampf nehmen müssten." (Nationalsozialistischer Reichsbund für Leibesübungen: NSRLFussball-Pressedienst vom 5. März 1936, zit. n. Hoffmann/Nendza 2011, 33)

Zwar hatten bereits 1932 empirische medizinische Studien, durchgeführt von frauenbewegten Ärztinnen, belegt, dass das Fußballspiel in keiner Weise die Reproduktionsfähigkeit der Spielerinnen beeinträchtige, dennoch hielt sich diese Annahme hartnäckig noch über Jahrzehnte (Pfister 1980, 36). Gerade im Nationalsozialismus, der den Sport ideologisch zur Schaffung eines neuen Menschen einsetzte, kam der Frau und ihrer Gebärfähigkeit eine entscheidende Rolle zu. Ihre Gesundheit galt es auch mit Leibesübungen zu erhalten, Fußball jedoch schien dafür gänzlich ungeeignet (Pfister 2006). ${ }^{32}$

31 Dennoch waren die Reaktionen auf den Frauenfußball nicht durchweg negativ: Die Frankenthaler Zeitung berichtete am 17. Juli und am 3. August 1930 voller Stolz über das erste Frauenfußballspiel in der Region. Die zweite Tageszeitung des Ortes, das Frankenthaler Tageblatt, hingegen ignorierte das Spiel (Thoma 2013, 66f.).

32 Interessant ist, dass im Nationalsozialismus der weibliche Leistungssport generell deutlich zunahm und Frauen den sportlichen Wettkampfbetrieb während des Krieges sogar dominierten. Eisenberg $(1999,401)$ sieht die Ursache hierfür in der propagandistischen Verwertung des Leistungssports als Demonstration von Leistungswillen und Wehrhaftigkeit. 


\subsubsection{Marginalisierung und Verbot des Frauenfußballs nach dem Zweiten Weltkrieg}

Ab den 1950er Jahren hielten Frauen sukzessive Einzug in die meisten Sportarten - nur in besonders maskulin konnotierten Sportarten dauerte es länger. In der DDR wurde der Frauenfußball zwar gestattet und offizielle Wettbewerbe ausgetragen, jedoch war er nur im Freizeit- und Erholungssport angesiedelt, da er sich als damals nicht-olympische Disziplin nicht als nationales Prestigeprojekt anbot. Der Männerfußball im Leistungssportsektor hingegen genoss die entsprechende Förderung und Wertschätzung (Linne 2011). ${ }^{33}$

In Westdeutschland nahm die Marginalisierung des Frauenfußballs einen drastischeren Weg. Auf dem Bundestag des Deutschen Fußball-Bundes (DFB) 1955 beklagten die Funktionäre:

„Im Kampf um den Ball verschwindet die weibliche Anmut, Körper und Seele erleiden unweigerlich Schaden und das Zurschaustellen des Körpers verletzt Schicklichkeit und Anstand.“ (DFB Jahrbuch 1955, zit. n. Hoffmann/Nendza 2011, 47)

Schließlich wurde aus ,ästhetischen Gründen und grundsätzlichen Erwägungen" (ebd.) den Vereinen unter Androhung von Strafe verboten, FrauenAbteilungen zu unterhalten und die eigenen Plätze für Frauen freizugeben. Schieds- und Linienrichtern wurde das Leiten von Frauen-Spielen untersagt ( Hoffmann/Nendza 2011, 47). ${ }^{34}$ Der Frauenfußball wurde just zu jener Zeit im Organisationsbereich des DFB verboten, als Frauen-Partien im Ruhrgebiet bis zu 10000 Zuschauende anlockten und die Männer-Nationalmannschaft 1954 dank eines „Wunders von Bern“ die WM gewann. Dies war kein Zufall, so argumentiert Nina Degele auf Basis von Aussagen der heutigen DFBVize-Präsidentin und Fußballpionierin Hannelore Ratzeburg:

„This was a time when men returning home at the end of the war were facing a collapse of their supposed natural male dominance in the light of the emergence of active, energetic women who had begun rebuilding Germany without them: further adding to their humiliation.“ (Degele 2012)

33 Der Frauenfußball in der DDR findet hier nur spärlich Erwähnung, da für die Arbeit vor allem die Entwicklung der Geschlechterverhältnisse innerhalb des westdeutschen Verbandsfußballs entscheidend ist, da die hier gewachsenen Strukturen nach der Wiedervereinigung auch auf die neuen Bundesländer ausgeweitet wurden. Nur wenige der damals an Betrieben angegliederten Teams konnten sich bis heute erfolgreich halten. Prominentes Beispiel ist der Verein Turbine Potsdam.

34 Mit welchen Mitteln der DFB das Verbot auch außerhalb seines eignen Reglements durchzusetzen versuchte, wird im Geschäftsbericht der Stadion GmbH für das Geschäftsjahr 1957/58 deutlich, der auf ein Frauenfußballspiel Bezug nimmt, welches kurz vorher in einem Frankfurter Stadion ausgetragen wurde: ,[D]a der DFB gegen Damenfußball ist und in Zukunft Stadien, die Schauplatz derartiger Damenfußballspiele sind, nicht mehr mit Länderspielen und dergleichen bedenken wird, ist der Damenfußball nicht über das Anfangsstadion hinausgekommen und befindet sich bereits in Auflösung." (Zit. n. Thoma 2013, 70). 
Der Titelgewinn läutete ein Ende der gefühlten Demütigung ein. Rosa Diketmüller weist zudem darauf hin, dass die „Normalisierung des Lebens“ nach dem Weltkrieg offensichtlich auch die Rückkehr zu vermeintlich traditionellen Geschlechterverhältnissen bedeutete $(2002,206)$.

\subsection{4 „Segregierte Re-Inklusion“ der Frauen in den Fußball}

Trotz offiziellen Verbots seitens des Dachverbandes gründeten sich in der zweiten Hälfte der 1950er Jahre eigene Frauenvereine und -verbände (Hoffmann und Nendza 2011). 1957 organisierten DFB-unabhängige Frauenfußballverbände sogar eine Europameisterinnenschaft in Berlin. Nach und nach kam es zu öffentlichen Diskussionen um und Zweifeln am Frauenfußball-Verbot des DFB. Z.B. beschloss die Stadt Oberhausen 1958 nach langen und hitzigen Diskussionen, ihre Spielstätten dem Trainings- und Wettkampfbetrieb der Frauen (samt Eintrittsgebühren) zur Verfügung zu stellen. Die Gegner_innen des Frauenfußballs lenkten letztlich ein: Sie hätten sich überzeugen lassen, dass die Frauen den Sport durchaus ernst nehmen und keinen Rummel daraus machen würden. Ein Mitglied des kommunalen Sportausschusses stellte fest: „Aus Gründen der Gleichberechtigung müssen wir unseren Sportlerinnen die gleichen Chancen bieten wie den Männern." (NRZ vom 21.11.1958, zit. n. Hoffmann/Nendza 2011, 76). Diese Diskussion spiegelte auch den internationalen gesellschaftspolitischen Kontext der 1960er Jahre und die Entstehung sozialer Bewegungen:

„The student rebellion of 1968 triggered off sweeping social changes, ranging from ,sexual liberation' (Oswald Kolle) to the forming of citizens' lobbies and initiatives, and the development of a new women's movement. In this period of upheaval and paradigm shift, women's football, also encouraged by initiatives abroad, succeeded in establishing itself in West Germany.“ (Pfister 2003a, 134f.)

Als dann auch noch einige Fußballvereine drohten, sich vom DFB abzuwenden und einen eigenen Dachverband zu gründen, lenkte der DFB auch auf Raten des europäischen Dachverbandes UEFA ein und erlaubte ab 1970 Mädchen und Frauen offiziell das Fußballspielen. Markwart Herzog zitiert aus einem Pressebericht des 1. FC Kaiserslautern von 1970. Dieser rechtfertigt seine Entscheidung, eine Abteilung für „Damen-Fußball“ zu gründen, damit, „die Fußballbegeisterung der Mädchen und Frauen - auch so manche Ehefrau will die Fußballstiefel anziehen - in geordnete Bahnen gelenkt" zu haben (zit. n. Herzog 2013, 88). ${ }^{35}$ Degeles Deutung (2012) ist daher sicherlich richtig, dass mit der Aufhebung des Verbots und der Unterstellung unter das Reglement des DFB die Kontrolle über die Sport treibenden Frauen be-

35 Bei der Originalquelle handelt es sich um einen anonymen, undatierten Artikel ohne Überschrift und Verfassername, der dem Privatarchiv von Petra Ziller entstammt, die diesen auf den 5.6.70 datierte. 
halten bzw. zurückgewonnen werden sollte. Dennoch darf nicht übersehen werden, dass sich der Ausschluss von Frauen aus dem offiziellen Spielbetrieb durch das konstante und hartnäckige Spielen vieler Frauen, das Gewinnen von Unterstützer innen sowie die gestiegene gesellschaftliche Aufmerksamkeit für Geschlechterfragen zu einem öffentlichen Problem entwickelte, welches (auch) unter allgemeinen Gleichstellungsfragen verhandelt wurde.

Hier fällt auf, wie für einen kurzen Zeitraum der Ausgang in der Schwebe gehalten wird, doch schon bald nur eine Lösung plausibel erscheint. Die Etablierung separater Frauenfußballstrukturen im Sinne eines radikalen $\mathrm{Fe}-$ minismus stand kurz als Drohung im Raum, doch die damals wie heute dominante liberalfeministische Definition des Problems als ein Mangel an gleichen Chancen und Zugängen machte eine weitere Separation in Form eigener Frauenverbände und -vereine unmöglich und die gleichberechtigte Eingliederung in bestehende verbandsfußballerische Strukturen als (einzig) plausible Lösung unumgänglich. ${ }^{36}$

$\mathrm{Ab}$ diesem Zeitpunkt sollten Frauen unter veränderten Regeln und Bedingungen kicken. Sie sollten fortan, so wollte es der Dachverband, ohne Stollenschuhe und mit Brustpanzer geschützt sowie mit einem kleineren Ball nur zweimal 30 Minuten (anstatt zweimal 45 Minuten) spielen. Müller interpretiert die Aufnahme des Frauenfußballs in den offizielle Spielbetrieb des Dachverbandes daher als „,segregierte Inklusion der Frauen“, die statt einer Aufweichung der fußballerischen Geschlechterdifferenz deren Fortschreibung bewirkte $(2007,132)$. Diese Deutung ist sicherlich richtig, doch muss auch die Tatsache berücksichtigt werden, dass die meisten Regeln (bis auf die verkürzte Spielzeit) bald wieder abgeschafft wurden, da sie sich in der Praxis nicht bewährten, nicht umgesetzt werden konnten oder schlicht nicht eingehalten wurden und sich auch niemand besonders für sie interessierte. Dies zeigt zum einen, dass die fußballerischen Geschlechterverhältnisse durchaus Durchlässigkeiten boten, und zum anderen, dass diese Spielräume von den Akteur_innen genutzt wurden, um sich den vergeschlechtlichenden und unterordnenden Praktiken wirkungsvoll zu widersetzen.

Inzwischen wuchs der Männerfußball zur populärsten Sportart an und stieg zur „hegemonialen Sportkultur“ (Markovits/Hellerman 2004, 10) ${ }^{37}$ in Deutschland auf. Und auch der Frauenfußball erhielt kontinuierlich wachsenden Zulauf. Jedoch dauerte es auch nach Einführung von offiziellen Wettbewerben wie Ligen und Pokalturnieren noch einige Jahrzehnte, bis sich der

36 Bischoff beobachtet solch „geschickte Schachzüge“, mit denen sportlichen Alleingängen von Frauen „der Wind aus den Segeln genommen“" wurde, auch zu anderen Zeiten und in anderen Sportarten $(1986,133)$. Zu den verschiedenen feministischen Strömungen und ihren Sportpolitiken siehe Costas/Guthrie (1994), Hargreaves (1994).

37 Als hegemoniale Sportkultur bezeichnen Markovits/Hellerman die sozialen und kulturellen Dimensionen der Sport-Rezeption. Der hegemoniale Status einer Sportkultur hängt von dem Sprechen über, Wahrnehmen von und Fühlen einer Sportart ab, nicht von der zahlenmäßigen Betätigung $(2004,10 \mathrm{f}$.). 
DFB zu einer expliziten Förderung des Mädchen- und Frauenfußballs durchringen konnte und schließlich 2011 die FIFA-Weltmeisterschaft der Frauen im eigenen Land mit großem Einsatz austrug.

Abseits des organisierten Sports begannen in den 1980er Jahren vermehrt politisch aktive Frauen nach Sportangeboten zu suchen, die nicht nach männlichen und heteronormativen Prinzipien organisiert waren. Im Zuge der feministischen Gesundheitsbewegung und der politischen Kämpfe um den weiblichen Körper begann die Suche nach Sport- und Bewegungsformen jenseits männlich definiertem Erfolgs- und Dominanzstreben einerseits und Schlankheitsgymnastik andererseits (Kugelmann 1993, 141). So entstanden zahlreiche alternative Angebote von Paartanz über Selbstverteidigung und Baumklettern bis hin zu Volleyball und Fußball, die den verbandlichen Strukturen mit Skepsis und Angst vor Vereinnahmung gegenüberstanden und Leistungsstreben, Kräftemessen und körperliche Manipulationen als genuin männliche Prinzipien ablehnten (ebd.; Bischoff 1993; 2008).

An dieser Schnittstelle zwischen organisiertem Fußball und frauen- und lesbenbewegten Sport- und Bewegungsangeboten besteht ein wissenschaftliches Desiderat. Die Frage muss offen bleiben, wie diese alternativen Sportangebote auf den organisierten Sport wirkten, ob und wie feministische Ideen in die Vereine und Verbände Einzug hielten und dort auf Veränderungen in den Geschlechterverhältnissen, den Sportverständnissen und den Bewegungsformen hinwirkten.

\subsubsection{Ein Paradigmenwechsel?“ Der Frauenfußball-Boom}

Während das erste Jahrhundert des Fußballs in Deutschland durch Konjunkturen der Marginalisierung weiblicher Fußballpraxis sowie mehr oder weniger deutliche Problematisierungen dieser Geschlechterverhältnisse charakterisiert war, zeichnete sich um die Jahrtausendwende ein Boom des Frauenfußballs - nicht nur in Deutschland - ab, sodass Diketmüller (2006) skeptisch nach einem Paradigmenwechsel fragt.

Der DFB verzeichnete weiterhin einen konstanten Zuwachs an weiblichen Mitgliedern. Im Jahr 2000 zählte er rund 820000 Mädchen und Frauen in seinen Vereinen, 2015 waren es schon über eine Million (Deutscher FußballBund 2000, 2; 2015, 2). ${ }^{38}$ Auch die Einschaltquoten beim Finale der Weltmeisterinnenschaften 2003 und 2007 gingen weit in die Millionen. Treibende Kraft hinter der Übertragung der Frauenspiele im Fernsehen und der Förde-

38 Zum Vergleich: 2000 waren knapp 5500000 Jungen, Männer und Senioren als Mitglieder beim DFB registriert. Auch ihre Zahl stieg bis 2015 um rund 300000 neue männliche Mitglieder an. Hennies/Meuren zufolge zeichneten die enormen Erfolge der deutschen FrauenNationalmannschaft für die steigende Beliebtheit verantwortlich, bemerken jedoch, dass das öffentliche Interesse weniger der Bundesliga als allein der Nationalmannschaft mit ihren Stars gelte (2009, 127ff.). 
rung des Frauenfußballs auf Profi- wie Amateurebene war der damalige DFB-Präsident Theo Zwanziger. Der DFB zielte mit der Austragung der FIFA-Weltmeisterschaft der Frauen 2011 in Deutschland auch auf einen Entwicklungsschub des Frauenfußballs. Nicht nur sollte eine gesteigerte Akzeptanz des Sports zu hohen Zuschauer_innenzahlen führen, es ging auch darum, Fußball als Frauen- und Mädchensport auf Vereinsebene zu etablieren und voranzutreiben. Durch die Markteinführung einer Fußball-Barbiepuppe sowie diverse Werbeauftritte der Nationalspielerinnen, die Medienpartnerschaft mit dem Frauenmagazin Brigitte und figurbetonte Trikots versuchte der DFB die Attraktivität, sprich die Weiblichkeit und Heterosexualität der Spielerinnen, zu betonen - zusammengefasst im offiziellen WM-Slogan „2011 von seiner schönsten Seite“. ${ }^{39}$ Die explizite Frauenfußballförderung fiel in eine Zeit, in der ,[j]ene traditionelle Männlichkeit, die sich durch Dominanz, Führungsansprüche und Potenzgehabe auszeichnet, [...] überflüssig, ja störend" wurde (Alkemeyer 2011, 6). An die Stelle der traditionellen Männlichkeit traten nun die systemkompatiblen Spieler des modernen Konzeptfußballs (ebd.). Mit Blick auf diese Entwicklungen - erodierende Fußballmännlichkeit und Frauenfußball-Boom - gehe ich von einer weiteren konjunkturellen Schwäche männlicher Herrschaft im Fußball in den ersten zehn Jahres des 21. Jahrhunderts aus.

Zugleich deute ich die in der Barbiepuppe symbolisierten Feminisierungsbestrebungen als Reaktion auf eben diese temporäre Brüchigkeit und als Indiz für einen Strategiewechsel in den Herrschaftsmechanismen. Hennies und Meuren verweisen auf eine Studie der Sportsponsoring-Agentur Experience von 2009, wonach Frauen- und Männerfußball gesellschaftlich differenziert betrachtet werden und Frauenfußball vor allem mit normativ aufgeladenen Attributen wie fair, ehrlich und sympathisch bewertet wird $(2009,127)$. Damit deuten sie eine Entwicklung an, die auch Müller diagnostiziert: Die gestiegene Anerkennung und Aufmerksamkeit basiere auf einer Abspaltung des Frauenfußballs als eigenständiger Sportart vom ,richtigen', sprich Männerfußball $(2007,136)$. Dieser bleibe durch die unterschiedlichen Attributierungen - Frauenfußball $=$ technisch gut, schön anzusehen und fair; Fußball $=$ hart, aggressiv, herausfordernd - weiterhin eine Männerdomäne. Jayne Caudwell sieht in der Gleichzeitigkeit von Frauenfußballförderung und separation eine Strategie, die Geschlechterbinarität zu festigen:

„And yet, the same governing bodies are keen to be seen promoting girls' and women's competitive football at local, national and international levels, that is, as long as women and girls' participation is distinct from men's competitive and men's elite

39 Zur medialen Heterosexualisierung siehe Schaaf (2012), Schaaf/Nieland (2011). Die „Feminisierung des Frauenfußballs“ (Schaaf 2012, 139) kann als weiterer Versuch gedeutet werden, den Spielerinnenkörper seines transgressiven Potentials zu entledigen und in die heteronormative Geschlechterordnung einzubinden. 
football performances. In many ways, these governing bodies aim to differentiate not only women from men, but also femininity from masculinity.“ (Caudwell 2011, 335)

Diese Differenzierung vollzieht sich eher auf diskursiver denn auf struktureller Ebene, denn es gelten weiterhin die gleichen Spielregeln und es kommt zu keiner signifikanten Aufspaltung in Männer- und Frauenvereine. In Vereinen und Verbänden entstehen zwar separate Abteilungen, jedoch nutzen sie weiterhin dieselbe Infrastruktur. Zu vermuten ist, dass die symbolische Differenz dazu dient, den Frauenfußball zu einem populären und damit vermarktbaren Phänomen zu machen, ohne dabei die Geschlechterhierarchie des klassischen (=männlichen) Fußballs in Gefahr zu bringen, da diese aufgrund der abnehmenden männlichen Konnotation bereits Brüche bekommen hat. Vor diesem Hintergrund möchte ich Diketmüllers Frage deutlich verneinen: Im deutschen Verbandsfußball ist eine Veränderung hin zu einer geschlechtlich neutralen Konnotation oder einer Gleichbehandlung und -bewertung des Männer- und Frauenfußballs nicht abzusehen. Gerade seit dem Rücktritt Zwanzigers und einem Abebben des öffentlichen und medialen Interesses nach 2011 ist von einem Paradigmenwechsel wenig zu spüren.

Rückblickend auf die Geschichte des Verbandsfußballs schlussfolgere ich, dass sich die zentralen Prinzipien wie Leistungsprimat und Vereinskultur nicht nur klassenspezifisch entsprechend der proletarischen Konnotation ausdifferenzierten, sondern immer auch vergeschlechtlicht sind. Fußball konnte sich so zunächst als männliche Sportart entwickeln. Mit Blick auf die Gegenwart ist jedoch festzuhalten, dass die Entwicklung der 1990er und 2000er Jahre Brüche und Risse in der männlichen Hegemonie hinterlassen hat. Schon 1986 bemerken Cheryl Cole und Susan Birrell, dass ,[t]he very presence of women in the male preserve of sport is evidence of a ,leaky hegemony “", und deuten die Unmöglichkeit an, untergeordnete Gruppen vollkommen zu kontrollieren (1986, 24, zit. n. Birrell/Theberge 1994, 366). Diese Unmöglichkeit hat in der Vergangenheit immer wieder zu Konjunkturen der Problematisierung der Geschlechterverhältnisse geführt, welche wiederum unterschiedliche Reaktionen provozierten. Seien es die homophoben und pathologisierenden Stigmata und Appelle an die weibliche Sittlichkeit, offizielle Verbote, Verniedlichungen und Trivialisierungen oder die heterosexualisierenden und feminisierenden Bestrebungen - sie alle sind zugleich Reaktionen auf die in Zweifel geratenen Geschlechterverhältnisse als auch Auslöser für erneute Kritik. Die steigende Zahl der Spielerinnen, ein zunehmendes öffentliches Bewusstsein sowie mittlerweile drohende Strafen durch verbandsfußballerische Gerichtsbarkeiten für offenen Sexismus und Homophobie im Fußball, Frauenfußball-Förderprogramme und lokale Initiativen weisen darauf hin, dass sich diese undichten Stellen in der männlichen Herrschaft vergrößern und sich leichte Verschiebungen abzeichnen. 
Zugleich wird ersichtlich, dass die Geschlechterverhältnisse mit Rückgriff auf Argumente, wie Gleichstellung und Gesundheit, problematisiert wurden, die aus anderen sozialen Feldern, wie Medizin, Politik und Frauenbewegung, in den Fußball importiert wurden. Bevor ich diese Argumentationen näher untersuche, beleuchte ich im Folgenden zunächst das Verhältnis zwischen Verbandsfußball und staatlicher Verwaltung. So wird ersichtlich, wie staatliche Politiken und marktwirtschaftliche Kräfte auf die fußballerischen Geschlechterverhältnisse einwirken können, und es zeichnen sich Wege ab, Einfluss auf den Fußball jenseits verbandsinterner Gremien zu nehmen.

\subsection{Verwobenheiten: Sport, Staat und Ökonomie}

Wie in der Einleitung zu diesem Teil bereits angeklungen ist, reichen die Wege, Rationalitäten und Akteur_innen gegenwärtiger Problematisierungen weit über den Bereich des Verbandsfußballs hinaus in andere gesellschaftliche Domänen. Um Möglichkeiten, aber auch vielleicht überraschende Grenzen der geschlechterpolitischen Intervention in den Fußball zu eruieren, beleuchte ich hier zunächst das Verhältnis zwischen Sport, Staat und Ökonomie. Das Verhältnis zwischen Verbandssport und staatlichen Regierungsund Verwaltungsinstitutionen wandelte sich in seiner Geschichte häufig und ist gegenwärtig de jure durch Autonomie und Partnerschaft gekennzeichnet, während sich de facto über die staatliche Sportförderung Möglichkeiten der (geschlechter-)politischen Einflussnahme eröffnen. Auch marktwirtschaftliche Entwicklungen wirkten sich mittel- und unmittelbar auf die Partizipation von Frauen aus, scheinen gegenwärtig jedoch überraschend wenig Einfluss auf die Geschlechterverhältnisse des Breitenfußballs zu haben.

\subsubsection{Sport und Staat}

Fußballvereine in Deutschland sind in der Regel entsprechend der pyramidenförmigen Struktur des Verbandswesens Mitglieder in Bezirks- oder Kreisverbänden, die ihrerseits wiederum Mitglieder in den Landesverbänden sind, die sich in fünf Regionalverbände einordnen, denen wiederum der DFB übergeordnet ist. Gleichzeitig sind die einzelnen Sportfachverbände in den sportartenübergreifenden Verbänden, also den Kreis-/Bezirks-/Stadt- und Landessportbünden, organisiert, an deren Spitze der Deutsche Olympische Sportbund (DOSB) firmiert. Die Regelung der internen Angelegenheiten obliegt den Fachverbänden, die über eigene Regelwerke und Statuten sowie Gerichtsbarkeiten verfügen. Die Verbände organisieren die Wettkämpfe, bilden sportliches Personal wie Schiedsrichter_innen und Trainer_innen aus 
und vertreten die Interessen ihrer Sportart in sportartenübergreifenden Verbänden, in internationalen Fachverbänden sowie gegenüber dem Staat. Letzteres beweist nicht nur ein Blick auf die Liste der registrierten Lobbyist innen des deutschen Bundestages, sondern zeigen auch zahlreiche Veranstaltungen auf lokaler Ebene, wo Verbands- und Vereinsvertreter_innen bei Parteien oder Stadträt_innen vorsprechen.

Das Verhältnis zwischen Staat und Sport wird gegenwärtig von drei allgemein anerkannten Prinzipien gestaltet: Das Prinzip der Autonomie sichert dem Sport eigenständige Organisation und Regelungen seiner Angelegenheiten $\mathrm{zu}$, was unterem anderen bedeutet, dass der Sport über eine eigene Gerichtsbarkeit verfügt. Das Subsidiaritätsprinzip schreibt vor, dass öffentliche Gelder stets nur die Eigenmittel ergänzend vergeben und beansprucht werden dürfen, sodass eine vollständige Abhängigkeit vom Staat verhindert wird. Das dritte Prinzip der partnerschaftlichen Zusammenarbeit schreibt die gleichrangige Zusammenarbeit auf allen Ebenen der zahlreichen Verflechtungen zwischen Staat und Sport vor. So sollen diese Prinzipien die Unabhängigkeit des Sports bewahren und gleichzeitig zur engen Abstimmung verpflichten (Haring 2010, 35f.; Deutscher Bundestag 2014, 20).

Das Bestreben, staatliche Inanspruchnahme und Abhängigkeit zu verhindern, speist sich dabei vorrangig aus einer kritischen Auseinandersetzung mit Verquickungen von Sport und Staat in der jüngeren Vergangenheit, wie sie besonders in der politischen Instrumentalisierung im Nationalsozialismus zum Ausdruck kam (vgl. Eisenberg 1999, 397ff.; Nathaus 2009, 202; Haring 2010, 37). Während in der DDR Vereine zwar erlaubt, Initiativen jenseits der staatlich eingesetzten Vereinigungen jedoch als Ausdruck von Protest gegen Staat und Sozialismus kritisch beobachtet wurden (Agricola 1997, 45), wurde in der BRD die staatliche Verbands- und Vereinsförderung der Vorkriegszeit wieder aufgenommen. Dank der drei oben genannten Prinzipien treten Verbände und Vereine dem Staat nun selbstbewusst als förderungswürdige Experten gegenüber (Nathaus 2009, 271; vgl. Haring 2010, 41).

Trotz der zugesicherten Autonomie bieten sich dennoch Wege der politischen Einflussnahme an. Zwar sind juristische Mittel aufgrund der eigenen Gerichtsbarkeit des Fußballs eingeschränkt, doch öffnen sich auch hier Möglichkeiten punktuell in den Sport hineinzuregieren, etwa durch Ermittlungen in Sachen Steuerhinterziehung, wie sie jüngst die US-Justizbehörden gegen die FIFA einleiteten. Eine deutlich konstantere und institutionalisierte Einflussmöglichkeit bietet hingegen die staatliche Sportpolitik auf Bundes-, Landes- und Kommunalebene (Haring 2010). Die umfangreichste Förderung des Breiten- und Leistungssports auf Bundesebene übernimmt das Bundesministerium des Inneren; das Bundesverteidigungsministerium finanziert über die Bundeswehr den Leistungssport; das Bundesministerium für Familie, Senioren, Frauen und Jugend unterstützt Programme des Mädchen-, Frauen- und Jugendsports sowie zur Bewegung im Alter; das Bundesministe- 
rium für wirtschaftliche Zusammenarbeit und Entwicklung (BMZ) und sein ausführendes Organ, die Gesellschaft für internationale Zusammenarbeit (GIZ), fördern Sport-for-Development-Programme im Ausland, und das Auswärtige Amt (AA) unterstützt im Rahmen der auswärtigen Kulturpolitik internationale Sportprojekte. Gerade die Förderpolitiken des AA wie des BMZ korrespondieren mit inter- und transnationalen Sportpolitiken, wie sie etwa von der EU im Rahmen des Erasmus-Plus-Programms zum Zwecke der europäischen Integration durch Sport oder den von den Vereinten Nationen (UN) befürworteten Sport-for-Development-and-Peace-Programmen vorgesehen sind, die in der letzten Dekade deutlich zugenommen haben. ${ }^{40}$

Auf Länderebene wird der Sport hingegen in einem Ressort - meinst Inneres und Kultus - gebündelt. Die Länder fördern den Vereinssport entweder durch den Bau von Sportstätten oder durch Pauschalüberweisungen an die Landessportbünde. Dabei adressieren sie sowohl Breiten-, als auch Spitzensport, während die Kommunen vorrangig den Breitensport unterstützen, indem sie Sportstätten zur Verfügung stellen und verwalten, bei der Anschaffung von Trainingsmaterialien und der Ausbildung von Übungsleiter_innen helfen, Programme für die Arbeit mit bestimmten Gruppen bezuschussen und ehrenamtliche Leistungen auszeichnen. Die Finanzierungshilfen durch Bund, Länder oder Kommunen sind stets an konkrete Förderrichtlinien geknüpft.

An der Sportförderung fällt auf, dass der Sport auf Bundesebene keine klare Ressortzugehörigkeit genießt sowie auf den verschiedenen Ebenen auf unterschiedliche Bedarfe reagiert. Diese Aufteilung zeigt zum einen, dass Sportförderung ein politisches Querschnittsthema ist. Zum anderen deutet sie eine gewisse Flexibilität oder Vielfalt an, da sich der Sport scheinbar problemlos mit den unterschiedlichen Zuständigkeiten und Zielen der Ressorts und Verwaltungsebenen verbinden lässt. Für den Fortlauf der Arbeit ist der Hinweis entscheidend, dass die entsprechenden Sportförderinstitutionen keinem einheitlichen Programm für die Sportentwicklung zu folgen scheinen, sondern entsprechend ihrer Wirkfelder, Themen und aktuellen Schwerpunktsetzungen divergieren. So mag z.B. die Breitensportförderung des sogenannten Frauenministeriums dem Prinzip der Gleichstellung folgen, während das BMI auf größtmögliche ehrenamtliche Partizipation achtet oder das kommunale Sportamt Integration und soziales Miteinander anvisiert.

Die Einschätzungen zum Ausmaß der staatlichen Steuerung des Sports gehen allerdings stark auseinander. Der Historiker Klaus Nathaus räumt ein, dass Vereine und Verbände dem Staat gegenüber zwar grundsätzlich verpflichtet seien, jedoch könne „bei der Freigebigkeit der öffentlichen Hand

40 Siehe hierzu den aktuellen Sportbericht der Bundesregierung. Diese Informationen basieren auf dem 13. Sportbericht (Deutscher Bundestag 2014). Zum Anstieg der außenpolitischen Sportförderung siehe Deutscher Bundestag $(2010,1)$, Bundesministerium für wirtschaftliche Zusammenarbeit und Entwicklung (2014). Ausführlich zur auswärtigen Sportpolitik siehe Jacobsen (2015, 79ff.). 
von einer Steuerung des Vereinswesens kaum die Rede sein“ $(2009,271)$. Dem hält der Sportsoziologie Klaus Heinemann entgegen, dass die staatliche Förderung die sportliche Autonomie zur Erosion bringe und den Sport an einem ,goldenen Zügel‘ führe (1996; vgl. Haring 2010, 36).

An dieser Stelle ist die Frage nach dem Ausmaß des Einflusses staatlicher Politiken auf den Sport nicht weiter relevant. Doch gilt es mit Blick auf den weiteren Verlauf der Arbeit erstens festzuhalten, dass der Staat mit der Sportförderung ein effektives Mittel zur Hand hat, um Einfluss auf Belange des Sports auszuüben. So wird der Fußball immer auch von unterschiedlichen Sportpolitiken sowie gesellschaftspolitischen Veränderungen mitgestaltet (vgl. Güldenpfennig 2000, 320). Hier deuten sich Wege der geschlechterpolitischen Problematisierungen an, die auch über staatliche Verwaltungen führen können. Aufgrund der verschiedenen Ressortzuständigkeiten, aber auch parteipolitischer Ausrichtungen und aktueller thematischer Konjunkturen sind diese keineswegs einheitlich, sondern folgen verschiedenen Politiken und mobilisieren dabei unterschiedliche Schlagwörter. Zweitens erweckt die Beobachtung, dass die Autonomie des Sports gegenüber der staatlichen Verwaltung dennoch behauptet und in Prinzipien festgeschrieben wird, den Eindruck, als handle es sich hier um einen kulturellen Topos, der eine spezifische Funktion erfüllt und der daher in Kapitel 5.2 unter dem Schlagwort der politischen Neutralität weiter ausgeleuchtet wird.

\subsubsection{Sport und Ökonomie}

Ökonomische und marktwirtschaftliche Entwicklungen wirken bis heute auf unterschiedlichen Wegen stark in den Sport hinein, sei es, dass Sponsoring die (finanziellen) Spielräume des Fußballs vergrößert und zugleich den Stadionraum zunehmend kommerzialisiert, dass der Nexus aus Mode und Sportivität die Beliebtheit einzelner Sportarten fördert, Spieler_innen über Werbeverträge ihre Sportkarrieren finanzieren können oder aber Kommerzialisierung Proteste weckt und den Verbandsfußball problematisierbar macht. Da die Verstrickungen von Sport und Marktwirtschaft vielzählige sind, konzentriere ich mich hier auf die ökonomischen Effekte auf den Frauenfußball.

Jaime Schultz (2014) hat anhand des US-amerikanischen Frauensports gezeigt, wie marktwirtschaftliche Neuerungen auch die Entwicklungen des Frauensports beeinflusst haben: Zum einen waren es sportunabhängige Produktentwicklungen, wie die Markteinführung des Tampons, zum anderen sportabhängige, wie die Erfindung des Sport-BHs, die Frauen das Sporttreiben erleichterten und in der Folge rein quantitativ die Geschlechterverhältnisse im Sport des 20. Jahrhunderts veränderten. Ab den 1980er Jahren, so beobachten z.B. Shari Dworkin und Michael Messner (2002), begünstigte die Entdeckung des Frauensports als Absatzmarkt und Zielscheibe von Marke- 
tingbemühungen und des fitten, disziplinierten Frauenkörpers als konsumförderndes Bild einen Frauensportboom. Mit Sicherheit hat die allgemein zunehmende Akzeptanz von und Begeisterung für Frauenbreitensport auch auf den Fußball ausgestrahlt und an der steigenden Zahl von Spielerinnen mitgewirkt, obwohl das damalige vermarktbare Schönheitsideal als auch die entsprechenden Sportarten nicht den Frauenfußball und den entsprechenden Spielerinnenkörper einschlossen. Eine vorsichtige Ökonomisierung des Frauenfußballs in Deutschland scheint sich erst im Zuge der Weltmeisterinnenschaft 2011 und der sogenannten Charme-Offensive des DFB abzuzeichnen (vgl. RPO 2010). Die Kooperationen mit dem Hersteller von Barbie-Puppen oder einem beliebten Frauenmagazin lockten sicherlich einige Mädchen und Frauen auf die Fußballplätze und verhalfen dem Frauenfußball zu einem besseren Ansehen - allerdings war diese Unterstützung an die Bedingung eines heteronormativen Erscheinungsbilds gebunden (vgl. Nieland 2013; Schaaf 2013). Gleichzeitig nahm der 2019 zur Frauen-WM produzierte Werbespot der Commerzbank Vorurteile und gängige Schmähungen provokant aufs Korn und präsentierte selbstbewusste, schlagfertige und leistungsstarke Nationalspielerinnen. Er zeigt zum einen, dass marktwirtschaftliche Einflüsse nicht unweigerlich gefällige, passive Weiblichkeitsbilder produzieren, zum anderen verdeutlicht er, dass die Problematisierung der fußballerischen Geschlechterverhältnisse inzwischen selbst vermarktbar geworden ist - zumindest im Bereich des professionellen Hochleistungsfußballs.

Während meiner ethnografischen Forschung auf Amateurebene waren marktwirtschaftliche Effekte jedoch nicht zu verzeichnen. So war die Auswahl an Frauenfußballbekleidung gering und Sponsor_innen ließen sich für Frauenteams nur schwer finden. Sportartikelhersteller hatten zwar das Konzept des Frauen-Empowerments in Verbindung mit GuerillaMarketingstrategien entdeckt, doch konzentrierten sich ihre Veranstaltungen vor allem auf Lauf-, Fitness- und Extremsportarten. Fußball, von ihnen vermarktet als wilder Straßenfußball, richtete sich vorrangig an Männer (vgl. Grimm 2016). Im Gegensatz zur Aerobic der 1980er, dem Tennis der 90er und den aktuellen Individualsportarten scheint der Frauenfußball auf Amateurebene dennoch weiterhin durch das immer enger werdende Netz gezielter marktwirtschaftlicher Kräfte zu rutschen. Daher bezweifle ich gegenwärtig noch eine signifikante Breitenwirkung dieser Bestrebungen auf die fußballerischen Geschlechterverhältnisse, zugleich aber vermute ich, dass sich dies in naher Zukunft auch verändern wird. Für diese Arbeit gilt es festzuhalten, dass gerade auf der Breitensport- und Amateurebene des Frauenfußballs marktwirtschaftliche - anders als staatlich-verwaltende - Einflusswege kaum zur Verfügung stehen, um die fußballerischen Geschlechterverhältnisse zu problematisieren.

Festzuhalten bleibt, dass der organisierte Fußball in Deutschland von einer tradierten Vereinskultur gekennzeichnet ist, die sich im Zuge von 200 Jahren 
Vereinswesen entwickelte und sich durch demokratische Partizipation und Gemeinschaft Gleicher einerseits, Traditionalismus, ämterbezogene Prestigevorstellungen sowie Repräsentation andererseits auszeichnet. Es hat sich gezeigt, dass diese Kultur stets vergeschlechtlicht ist und das Leistungsprinzip nicht nur institutionalisierte, sondern fest mit den Geschlechterverhältnissen und ihrer Legitimation verband. Durch soziale Stigmatisierungen, strukturelle Ausschlüsse und Trivialisierung, Verniedlichung oder Heterosexualisierung von Spielerinnen wurde Fußball als Raum und Praxis heteronormativer Männlichkeit konstituiert und behauptet, und der Verweis auf unterschiedliche Leistungen oder Leistungsfähigkeit begründet bis heute die Benachteiligung von Frauen im Fußball. Zugleich wurden jene Geschlechterverhältnisse immer wieder angezweifelt und mehr oder weniger öffentlich und erfolgreich problematisiert. Der Rückblick auf die Geschichte von Frauen im Fußball hat zudem gezeigt, dass sich etwa seit der Jahrtausendwende eine konjunkturelle Instabilität in der männlichen Dominanz im Fußball abzeichnet, die sich nicht allein aus dem Fußball selbst heraus erklären lässt, sondern auch durch externen Einflüsse, wie frauenbewegte Kritik oder geschlechterpolitische gesellschaftlichen Veränderungen, koproduziert wurde. Ein kurzer Einblick in die Verwobenheiten von Staat, Ökonomie und Sport hat Wege der Einflussnahme skizziert, auf denen geschlechterpolitische Problematisierungen in den Verbandsfußball hineinwirken können. Ich habe vorgeschlagen, diese Schnittfläche von verbandsfußballerischen, feministischen, sportpolitischen und zu Teilen auch ökonomischen Rationalitäten, historischen Sedimenten, Politiken und Akteur innen mit Tsings Konzept der Friction (2005) als spannungsreiches, produktives und zugleich im weiteren Verlauf unvorhersehbares Aufeinandertreffen zu denken, um zu verstehen, wie die fußballerischen Geschlechterverhältnisse ihre Selbstverständlichkeit, Wahrheit und Natürlichkeit verlieren.

Nachdem in diesem Teil sowohl historische Konjunkturen der Instabilität und potentielle Wege der Problematisierung ausgeleuchtet wurden, die die männliche Dominanz im Fußball anzweifelbar machen, sowie historische Sedimentierungen - sei es in Form von gewachsener Vereinskultur, sportfördernder Verwaltungsstrukturen als auch etablierter frauenbewegter Argumentationen -, die diese Problematisierungen vorstrukturieren und begrenzen, beleuchte ich im nächsten Teil die diskursiven Dimensionen dieses Aufeinandertreffens. Mich interessiert, welche Wahrheiten über den Fußball hinterfragbar werden - während sich andere stabilisieren - und damit die fußballerische Geschlechterordnung auf eine bestimmte Art kritisierbar machen. 


\section{Diskursive Formationen des Fußballs}

In diesem Kapitel widme ich mich den diskursiven Dimensionen des Fußballs und untersuche, wie (Un-)Sagbarkeiten verhandelt werden und dabei Fußball auf spezifische Art mit hervorgebracht wird. Für die Untersuchung geschlechterpolitischen Handelns ist dies deshalb relevant, da sich politische Konflikte immer auch über und mittels Sprache vollziehen (Hilhorst 2003, 8). Zugleich geben Diskurse Möglichkeiten und Begrenzungen vor, wie politische Themen und Ereignisse gedacht und gedeutet werden. Ich zeige, wie verschiedene Argumente und Deutungsweisen zusammenarbeiten und Wahrheiten über den Fußball produzieren, an anderer Stelle jedoch miteinander im Widerstreit stehen. Gleichzeitig stelle ich die Genealogien und Kontingenzen der verschiedenen Wahrheiten durch historische Einordnungen dar. Zur Erfassung der diskursiven Dimensionen des günstigen Moments bediene ich mich Foucaults diskursanalytischer Konzepte. Diese ermöglichen es, hinter einzelnen diskursiven Praktiken Aussagesysteme zu rekonstruieren, in die Machtverhältnisse eingeflochten sind. Damit werden nicht nur Aussagen darüber möglich, wie der performative Akt des Sprechens Fußball auf spezifische Art konstituiert, sondern auch darüber, wie Machtverhältnisse und Sprecherpositionen über Sag- und Unsagbares entscheiden.

Mit Foucault und im Anschluss an die Sprechakttheorie fasse ich konkrete, sprachliche und kommunikative Aussageereignisse als Praktiken, die einerseits die Dinge, von denen sie sprechen, hervorbringen (Foucault 1994, 74) und andererseits Produkte und Produzenten einer diskursiven Formation sind. Aussageereignisse (synonym: Äußerungen oder diskursive Ereignisse) gehen auf typisierte, abstraktere Aussagen zurück und stellen deren materiale Erscheinungsform dar. Eine Menge an Aussagen (wenngleich die einzelnen Äußerungen zeitlich weit auseinanderliegen und in unterschiedlichen Formen auftreten mögen) bildet dann eine diskursive Formation, wenn ihre thematische und begriffliche Anordnung eine bestimmte Regelhaftigkeit besitzt (ebd., $41 \mathrm{ff}.) .{ }^{41}$ Da es im Folgenden jedoch nicht nur um die Frage geht, wie und nach welchen Regeln Fußball konstituiert wird, sondern auch, wie sich welche Formierungen durchsetzen und andere in den Bereich des Unsagbaren geraten, ziehe ich Foucaults spätere Überlegungen über die prozessualen Dimensionen diskursiver Formationen und Praktiken ergänzend heran. Mithilfe seines genealogischen Vorgehens lassen sich die Bedeutung von

41 Nur kurz möchte ich hinweisen auf die Diskussion zwischen Diskurs- und Praxistheoretiker_innen über den zentrale Analysegegenstand - Diskurs oder Praktik - und die Frage, welcher dem anderen vorgängig ist. $\mathrm{Zu}$ einer ausführlichen Diskussion sowie ihrer nur vermeintlichen Opposition siehe Reckwitz (2008), Füssel/Neu (2010). Auch der Diskursbegriff selbst wird bereits bei Foucault unterschiedlich verwendet. Hier folge ich seinem Diskursbegriff aus „Archäologie des Wissens“ (1994), mit dem er jedes Aussagesystem, das die Aussageereignisse durch Regeln zusammenhält, bezeichnet (vgl. Keller 2008, 131ff.). 
Macht/Wissen-Regimen herausarbeiteten und strategisch-taktische Kämpfe und Auseinandersetzungen sowie die Fragen nach legitimen und nichtlegitimen Sprecher_innen und nach Beherrschungs-, Ausweich- und Ausschlusskriterien fokussieren (bspw. Foucault 2003). Mit der Einführung des Dispositiv-Begriffs, verstanden als Netz, das „Diskurse, Institutionen, architekturale Einrichtungen, reglementierende Entscheidungen, Gesetze, administrative Maßnahmen, wissenschaftliche Aussagen, philosophische, moralische oder philanthropische Lehrsätze, kurz: Gesagtes ebenso wohl Ungesagtes“ verknüpft (Foucault 1978, 119f.), verliert der Diskurs seinen exklusiven Status in Foucaults Theorie. ${ }^{42}$ Das Dispositiv verbindet so Vergegenständlichungen und diskursive wie nicht-diskursive Praktiken. Es erlaubt zu untersuchen, wie z.B. ein Diskurs zu einer politischen Leitlinie, zur Rechtfertigung von Praktiken oder gar zur Gründung einer Organisation wird. Eine der Hauptfunktionen des Dispositivs besteht darin, ,zu einem gegebenen historischen Zeitpunkt [...] auf einen Notstand (urgence) zu antworten" (ebd., 120). Sebastian Friedrich (2015) verdeutlicht, wie der Dispositivbegriff dem hier vorliegenden Forschungsinteresse an geschlechterpolitischen Problematisierungen in die Hände spielt: Notstand und Krise, bzw. die entsprechenden Reaktionen und Umgänge damit, ähneln dem, was Foucault in späteren Schriften als Problematisierung fasste. Friedrichs Schlussfolgerungen über das Verhältnis zwischen Problematisierung und Dispositiv erscheinen mir für die Analyse des Verhältnisses zwischen verschiedenen Aussagen über Fußball hilfreich:

„Ein Dispositiv kann eher als Folge einer entsprechenden Problematisierungsweise gefasst werden. In einem Dispositiv verdichten sich verfestigte Rede- und Handlungsweisen sowie Vergegenständlichungen. Das Feld, auf dem Problematisierungen sich bewegen, ist ein dynamischeres. Es sind sich durchgesetzte [sic] Problematisierungen, die sich in einem Dispositiv verdichten." (Friedrich 2015, 41)

Dies gilt, so meine ich, auch umgekehrt: Während Problematisierungen sich erst noch zu Dispositiven verfestigen können, können sie gleichzeitig auch Folge von oder Indizien für erodierende Dispositive sein. Sie weisen dann auf Momente hin, in denen die Wahrheiten spezifischer Macht/Wissen-Regime in Zweifel gezogen werden, in denen ein Feld ,den Punkt seines Aufbrechens erreicht“, und deuten ,die Momente seiner Diskontinuität, die Stellen, an denen es an der Konstitution jener Intelligibilität scheitert, für die es steht", an (Butler 2001, 10). Problematisierungen weisen damit auf Instabilitäten in Diskursen hin und markieren Zwischenräume zwischen Nicht-mehr- und Noch-nicht-Dispositiven.

42 Foucault drückt dies so aus: ,Jetzt dagegen will ich versuchen zu zeigen, daß das, was ich Dispositiv nenne, ein sehr viel allgemeinerer Fall der Episteme ist. Oder eher, daß die Episteme, im Unterschied zum Dispositiv im Allgemeinen, das seinerseits diskursiv und nichtdiskursiv ist, und dessen Elemente sehr viel heterogener sind, ein spezifisches diskursives Dispositiv ist.“ (1978, 123, Herv. i. Orig.). 
Im Folgenden zeige ich, wie Aussagen einer diskursiven Formation eingebettet in Macht/Wissen-Regime den Fußball auf spezifische Weise hervorbringen. Dabei nehme ich weder eine erschöpfende Diskurs- oder Dispositivanalyse vor, noch überprüfe ich einzelne Äußerungen über Fußball auf ihren Wahrheitsgehalt. Der moderne Verbandsfußball in seiner Singularität soll dabei nicht als monokausales Produkt einzelner Elemente, sondern in seiner Kontingenz als ein möglicher Effekt vieler und verschiedenartiger Beziehungen, Verkettungen, Interaktionen und überlagernder Prozesse erkennbar werden (vgl. Foucault 1992, 37). Gleichzeitig wird das Augenmerk auf die instabilen Momente dadurch gerichtet, dass Aussagen immer auf potentielle Widersprüche und Gegen-Aussagen hin überprüft werden. Für den Verlauf der Arbeit können so die Bedingungen des Sag- und Akzeptierbaren abgesteckt werden, um dann zu fragen, zu welchen Graden Problematisierungen möglich sind, um bestehende Geschlechterarrangements des Fußballs anzugreifen.

Dabei rekonstruiere ich nicht sämtliche Aussagen zum Fußball erschöpfend und en detail, dazu wären es zu viele. Vielmehr spiegelt die Analyse den mein Vorgehen ausgehend von meiner Feldforschung bei DF wider und konzentriert sich dementsprechend auf jene Aussageereignisse, die mir dort begegneten und den diskursiven Kontext meines Feldes bildeten. Die Analyse ist daher partiell und entsprechend Zeit und Ort meiner Forschung auch situiert; sie ist nicht ohne genaue Überprüfung auf andere Kontexte zu übertragen. Aus dieser Perspektivierung heraus verfolgte ich jene regelhaft auftretenden Äußerungen aus der Lokalität meiner Feldforschung hinaus und in internationale, nationale und lokale politische und verbandssportliche Bereiche sowie in wissenschaftliche und zivilgesellschaftliche Felder hinein. Dafür konsultierte ich die Statuten der Sportverbände sowie die Förderrichtlinien und Rechenschaftsberichte staatlicher Verwaltungsorgane, die die Arbeit von DF (potentiell) unterstützten, warf einen Blick auf die Selbstverständnisse kooperierender Partnerorganisationen sowie auf Stellungnahmen, Rechenschaftsberichte und Pressemitteilungen von parteipolitischen Unterstützer_innen und Institutionen der öffentlichen Sportförderung. Literatur- und Internetrecherchen ermöglichten mir dann, die einzelnen Aussageereignisse auch historisch zu kontextualisieren und mit anderen Äußerungen in anderen Situationen in Verbindung zu setzen, sodass ich schließlich drei Aussagen über Fußball abstrahieren konnte: Fußball als verbindende, sozial integrative Kraft (4.1), als politisch, respektive unpolitisch (4.2) sowie als emanzipativ und charakterbildend (4.3). Dabei gilt es jeweils zu fragen, wie diese Aussagen akzeptabel wurden bzw. wie ihre Akzeptabilität angezweifelt wurde (vgl. Foucault 1992, 34). Schließlich ist noch anzumerken, dass die meisten Äußerungen sich auf Sport beziehen und Fußball einschließen. Dies gilt jedoch nicht im Umkehrschluss: Ist von Fußball die Rede, kann meist aufgrund seiner herausragenden Popularität nicht unmittelbar auf andere Sportarten rückgeschlossen werden. 


\section{1 „Fußball verbindet!“}

Im Frühjahr 2014 organisierte die Frauenfußballorganisation eine Podiumsdiskussion. Als Podiumsgast hielt der Grünen-Politiker und damalige Bundestagsabgeordnete Christian Ströbele der provokanten Frage, ob Sportgroßveranstaltungen angesichts der Menschenrechtsverletzungen gänzlich abgeschafft gehörten, die positiven Effekte entgegen:

„Trotzdem haben Olympische Spiele oder auch solche Fußballturniere einen Sinn, wenn die Sportler zusammenkommen. Ich erinnere mich, so im Laufe der Zeit haben ja solche Spiele auch dazu beigetragen, bestimmte Diskriminierungen abzubauen. Also wenn zum Beispiel eine Fußballweltmeisterschaft ist, ich hab dann immer mit der nigerianischen Fußballmannschaft gebangt, dass sie möglichst hoch kommt, dass sie Solidarität bekommt. Und das ging, glaube ich, sehr vielen so. Das wertet natürlich auch sehr viel mehr die Bevölkerung auf, als wenn die Öl exportieren oder ähnliches machen. Und deshalb finde ich, man kann das nicht so schwarz oder weiß sehen." (Feldnotiz, März 2014)

Ströbele konkretisiert hier am Beispiel Nigerias eine Aussage, die zehn Jahre zuvor der damalige UN-Generalsekretär, Kofi Annan, formelhaft prägte, als er 2005 zum internationalen Jahr des Sports erklärte mit den Worten: „Sport is a universal language. At its best it can bring people together, no matter what their origin, background, religious beliefs or economic status" (Generalsekretär der Vereinten Nationen 2004). Ich verfolge hier die Spuren einzelner Äußerungen aufgrund ihrer Ähnlichkeit in Bezug auf diese spezifische Konstitution von Sport und rekonstruiere eine Aussage der diskursiven Formation, die ich unter dem Credo „Fußball verbindet “ zusammenfasse. Diese Spurensuche entlang von Politik-Dokumenten, medialen Äußerungen und Selbstdarstellungen führt mich sowohl zum internationalen und nationalen Verbandssport als auch zu nationalen und trans-/internationalen politischen Institutionen und Akteur_innen. Die Aussage weist dabei starke Überschneidungen zur im nächsten Abschnitt skizzierten Aussage „Fußball empowert“ auf. Zu Analysezwecken und weil ich getrennte historische Entwicklungen vermute, werden beide hier separat dargestellt.

\subsection{1 „Fußball verbindet“ im Verbandssport}

Die Statuten vieler Sportverbände führen internationale Begegnungen und daraus resultierende Völkerverständigung als einen ihrer Verbandszwecke auf und beziehen sich dabei auf die vergemeinschaftenden Effekte des Sports. So sieht die Olympische Charta den Höhepunkt der olympischen Bewegung „im Zusammenbringen der Athleten der Welt zu dem großen Fest des Sports“ erreicht und bestimmt das Ziel des Olympismus darin, „den Sport in den Dienst der harmonischen Entwicklung der Menschheit zu stellen, um eine 
friedliche Gesellschaft zu fördern, die der Wahrnehmung der Menschenwürde verpflichtet ist" (Deutsche Olympische Akademie 2013, 7). Die verbindende und friedensfördernde Kraft finden sich im zentralen Emblem der fünf ineinandergreifenden Ringe - stellvertretend für die fünf Kontinente - symbolisiert. Die FIFA rechtfertigt in einer offiziellen Stellungnahme ihre Entscheidung, die Weltmeisterschaft 2018 trotz der kriegerischen Gewalt in der östlichen Ukraine und der Annexion der Krim in Russland stattfinden zu lassen, mit der friedensstiftenden Wirkung des Fußballs:

„Die Ausrichtung der FIFA Fussball-Weltmeisterschaft ${ }^{\mathrm{TM}}$ mit der damit verbundenen globalen Aufmerksamkeit kann [...] ein wirksamer Katalysator für einen konstruktiven Dialog zwischen Menschen und Regierungen sein und zu positiven gesellschaftlichen Entwicklungen beitragen. Die FIFA Fussball-Weltmeisterschaft ${ }^{\mathrm{TM}}$ bringt vom Beginn der Qualifikation bis zur Endrunde des Turniers Mannschaften und Nationen aus aller Welt im Geiste des Fairplays und Respekts zusammen.“ (FIFA 2014)

Auch die von Ex-FIFA Präsident Blatter stets herangezogene Metapher der großen FIFA-Familie des weltweiten Verbandsfußballs reiht sich ein in die Konstitution und Legitimation von Megaevents des Hochleistungssports. Eine solche diskursive Hervorbringung des Sports scheint nicht zwischen Leistungs- und Breiten-, Profi- und Amateur- sowie Team- und Individualsport zu differenzieren. Besonders deutlich wird die Aussage im Programm Integration durch Sport des Deutschen Olympischen Sportbundes (DOSB). Ein Programmflyer stellt dar, was der Sport zu leisten vermag, indem er in einem leuchtenden Rot die "soziologische Definition“" von Integration als „Verbindung einer Vielheit von einzelnen Personen oder Gruppen zu einer gesellschaftlichen und kulturellen Einheit" hervorhebt (Deutscher Olympischer Sportbund 2012a). Die sportliche Integrationsleistung wird von einem Foto von Kindern in einer Turnhalle belegt: Im Vordergrund trägt ein Mädchen mit Kopftuch eine Gymnastikmatte und grinst in die Kamera, angelächelt von dem blonden, ebenfalls mattentragenden Mädchen neben ihr. Das Programm Integration durch Sport erklärt die vergemeinschaftende Wirkung durch die körperlichemotionale Erfahrung von Zusammengehörigkeit im Sport sowie das bewusste Erlernen von Akzeptanz, Regelkonformität und Respekt auch gegenüber Gegner_innen (Göttlich 2008, 216f.). ${ }^{43}$

Aus der zugeschriebenen sportinternen Fähigkeit, Menschen zusammenzubringen und den Austausch unter ihnen anzuregen, leitet sich die

43 Bemerkenswert erscheint mir, dass viele der Integrationsprojekte von sportwissenschaftlichen Instituten begleitet und evaluiert werden. Diese wenden sich zwar gegen eine naive Vorstellung eines Integrationsautomatismus, stellen jedoch die zugrunde liegende Annahme nicht in Frage (für einen Überblick vgl. Bröskamp 2011). Mit Titeln wie „Integrationsmotor Sportverein“ (Braun/Finke 2010) verstärken sie jene Aussage. Sozialwissenschaften stützen die Integrationsfunktion oft mit Bezug zum Konzept des Sozialkapitals, bspw. Sherry (2010), Spaaij (2011), Vermeulen/Verweel (2011). 
Wirkung als gesamtgesellschaftlich Frieden und Zusammenhalt fördernd ab. Andreas Göttlich identifizierte in seiner Analyse des Integration-durchSport-Programms eine ansteigende Skala:

„Nach Aussage des Datenmaterials kann der Sport zunächst einmal ganz einfach ,Kontakte fördern'. Darüber hinaus kann er ,helfen, Vorurteile abzubauen', oder auch ,Gelegenheit bieten, sich kennen zu lernen und näher zu kommen'. Offensiver wirkt die Behauptung, der Sport überwinde ,problemlos soziale und kulturelle Unterschiede', und schließlich wird ihm gar das Vermögen zugeschrieben, , die Beteiligten zu einem Ganzen`zu integrieren.“ (ebd., 216)

Diese Übersteigerung klingt bereits bei Pierre de Coubertin, dem Begründer des Olympismus, an: Die olympische Idee ermögliche weit mehr als bloße gegenseitige Toleranz durch internationale Begegnung. Olympismus stelle eine geistige Haltung dar, basierend auf gesellschaftlicher Verantwortlichkeit, Menschenwürde und der Achtung universell gültiger moralischer Prinzipien, die über die sportliche Höchstleistung vermittelt werden sollten (Deutsche Olympische Akademie 2013, 7).

\subsection{2 „Fußball verbindet“ in der Politik}

Doch nicht nur Sportverbände rahmen so ihre sportlichen Aktivitäten und stellen den Sport damit als (global-)gesellschaftliche, integrative Kraft dar. Diese Aussage findet sich ähnlich unter staatlichen und inter-/transnationalen Institutionen, wie die Zitate von Ströbele und Kofi Annan bereits andeuteten. Darin attestieren sie dem Sport die Fähigkeit, jene Differenzen zu überwinden - kultureller Hintergrund, nationale Herkunft, ökonomischer Status und Religion -, die als Ursachen von kriegerischen Konflikten gelten.

Zum Vermögen, Brücken zwischen Menschen zu bauen, tritt die Fähigkeit, nationale wie kulturelle Stereotype abzubauen. So wog Ströbele die negativen Seiten der Sportgroßveranstaltungen wie Korruption und Menschenrechtsverletzungen gegen den positiven Effekt der Ausdifferenzierung nationaler und kultureller Stereotype ab. Zur Interpretation des Wettkampfs zwischen Athlet_innen als vergemeinschaftende Begegnung gesellt sich in seiner Rede der Aspekt der medialen Verbreitung der sportlichen Leistung. Diese setze nationalen und kulturellen Stereotypen, die von ökonomischen und politischen Aspekten dominiert werden, differenziertere Bilder entgegen. Da Athlet_innen als Repräsentant_innen der Nation auftreten, strahlen die revidierten nationalen oder kulturellen Bilder auf die gesamte Bevölkerung aus. Die mediale Übertragung multipliziere diese mit dem Fernsehpublikum, was letztlich zu einem Abbau von Diskriminierungen in breitem Maße führe und Solidarität unter geografisch weit entfernt lebenden Menschen fördere.

Unter den politischen Institutionen sind es vor allem Reden, Pressemitteilungen, Leitlinien und Programme der Vereinten Nationen (UN), in denen 
sich diese Aussage materialisiert, wie Simon Darnell (2012) mit Blick auf das Sport-for-Development-and-Peace-Programm der UN veranschaulicht. Darnell datiert die Entdeckung der friedensfördernden Wirkung des Sports durch die transnationalen Organe der UN auf das erste Jahrzehnt des 21. Jahrhunderts. Aus diesen Jahren stammen Annans Worte, die den Sport zu einer universellen Sprache erhoben und bald Einzug in die Auswärtige Kultur- und Bildungspolitik der Bundesrepublik hielten:

„Es ist unbestritten: Sport ist wichtig für das Gemeinwesen und hat herausragende gesellschaftspolitische Bedeutung. Sport bietet jungen Menschen Perspektiven und sinnvolle Freizeitgestaltung, Sport bildet durch Wertevermittlung, Sport trägt zur Gesunderhaltung der Menschen bei, Sport hat große wirtschaftliche Bedeutung und ist ,international': kein anderer gesellschaftlicher Bereich ist so global, so grenzübergreifend wie der Sport. Die ,Sprache“ des Sports wird weltweit gesprochen und verstanden." (Auswärtiges Amt 2012)

Konkret kann die Sprachmetapher so übersetzt werden: Wer die Sprache, also eine Sportart erlernt, trägt damit zu Völkerverständigung und der Überwindung von Grenzen bei. Mit der Alphabetisierung in einer Sportart erschließen sich den Sporttreibenden weitreichendere Werte, sodass die verbindende Wirkkraft des Sports durch seine erzieherischen Effekte unterstützt wird. Die friedensstiftende Wirkung des Sports entfaltet sich jedoch nur durch aktives Eingreifen. Im Report der UN von 2005 wird dies explizit betont:

„The world of sport presents a natural partnership for the United Nations' system. By its very nature sport is about participation. It is about inclusion and citizenship. Sport brings individuals and communities together, highlighting commonalities and bridging cultural or ethnic divides. [...] When these positive aspects of sport are emphasized, sport becomes a powerful vehicle through which the United Nations can work towards achieving its goals." (UN Inter-Agency Task Force on Sport For Development and Peace 2005, i)

Der Report naturalisiert die verbindende und damit friedensfördernde Wirkung des Sports. Jedoch entfaltet sich diese Natur nicht selbstständig, sondern bedarf der expliziten Entwicklungshilfe durch Programme der UN, nationalstaatlicher Organe und transnationaler wie lokaler NGOs. ${ }^{44}$

Potentielle negative Effekte des Sports, die eintreten, wenn die Entwicklungshilfe zur Entfaltung der sportlichen Natur ausbleibt, werden nicht formuliert. Die in dieser diskursiven Formation unausgesprochenen, weniger inklusiven oder gar entzweienden Effekte lassen sich an einem Beispiel erahnen. Im Oktober 2009 nahm die UN-Vollversammlung eine Resolution im

44 Göttlich identifiziert eine solche Relativierung auch in sportverbandlichen Aussagen. Als der Bundestag des Deutschen Sportbundes 2004 eine neue Grundsatzerklärung mit dem Titel „Sport und Zuwanderung“ verabschiedet, schreibt er darin dem Sport nicht mehr per se eine integrative Funktion zu, sondern bezieht sich lediglich auf seine Integrationspotentiale, die es mithilfe von angeleiteten Angeboten und interkulturellen Schulungen für Multiplikator_innen zu aktivieren gelte (2008, 224ff.). 
Konsens an, in der sie die FIFA Weltmeisterschaft in Südafrika als Chance für sozioökonomische Entwicklung, Solidarität und Friedensförderung begrüßte. Daraufhin lobten die anwesenden südafrikanischen Delegierten die Weltmeisterschaft als Möglichkeit für alle Bürger innen, ungeachtet sozialer Ungleichheiten aufgrund der sozialen Herkunft, Hautfarbe, Ethnizität, Religion, des Geschlechts oder Alters nun Teil der globalen Fußball-Familie zu werden. Die hohen Eintrittspreise hinderten dann jedoch einen Großteil der Bevölkerung, an dieser „Familienfeier“ teilzunehmen. Wie bereits erwähnt, möchte ich keinesfalls die spezifische diskursive Konstitution des Sports mithilfe von Gegenbeispielen in Zweifel ziehen. Vielmehr dient dieses Beispiel dazu, die Frage nach Macht/Wissen-Regimen rund um den Sport aufzuwerfen und zu überlegen, wie diese das Sagbare festschreiben und andere mögliche Konstitutionen von Sport unsagbar werden lassen.

\subsection{3 „Fußball entzweit“ - eine Gegen-Aussage?}

Die Erinnerung an die WM in Südafrika wirft die Frage nach einer potentiellen Gegen-Aussage auf, welche die vergemeinschaftende Wirkung des Fußballs konsequent in Frage stellt und eine konträre Lesart anbietet. Eine Vielzahl sozialwissenschaftlicher Arbeiten analysiert, wie Machtverhältnisse entlang der Kategorien Geschlecht, Alter, Behinderung, Ethnizität, Klasse und sexuelle Orientierung die Teilhabe am Sport restringieren und sich über den Sport reproduzieren. So zeigt z.B. Degele (2013), dass Vergemeinschaftung im Fußball immer auch über Ab- und Ausgrenzung funktioniert.

Ein weiterer Aspekt zielt auf das Konfliktpotential des Sports. Zwar bringt der Sport Athlet innen zusammen, doch treffen diese immer auch als Konkurrent innen im Wettkampf aufeinander (vgl. Musil 1955, 825f.). Werte des Fair Plays stehen mit jenen des Kräfte- und Leistungsmessens und dem Streben nach dem Sieg in einem Spannungsverhältnis. Diese Kritik wurde deutlich in der Soziologie und der Sozialen Arbeit und im Anschluss an die Kritische Theorie geäußert. Theodor W. Adorno kritisierte das bürgerliche Sportverständnis sowie den Sport im Spätkapitalismus als Teil der Unterdrückungs- und Ausbeutungsmaschinerie, denn ,zum Sport gehört nicht nur der Drang, Gewalt anzutun, sondern auch der, selber zu parieren und zu leiden (2003, 80, zit. n. Krüger 2004, 24). Der moderne Wettkampfsport, so die Argumentation in der Sozialen Arbeit, vermittle Leistungsstreben und den Glauben an die Überlegenheit des Stärksten und strahle diese Prinzipien in andere gesellschaftliche Bereiche aus:

\footnotetext{
„'The winner takes it all' und, den Letzten beißen die Hunde' werden zu alltagskulturellen Etiketten eines gesellschaftlichen Ausleseprozesses, der Armut und Ungleichheit in der wettbewerbsorientierten Leistungsgesellschaft hervorbringt. [...] Das Lebensprinzip des Wettbewerbs wird weiter verinnerlicht, zum anerkannten Handlungsmodus im Alltag und im Sport.“(Michels 2016, 44f.)
} 
Ein anderer Kritikzweig fokussiert das Gewaltpotential innerhalb des Sports. Auf dieses verweist gegenwärtig im deutschsprachigen Bereich der Sportsoziologe Gunter Pilz beharrlich und zählt dazu nicht nur rivalisierende Hooligans und gesundheitsgefährdende Trainingsmethoden, sondern auch instrumentelle Gewalt. Damit bezeichnet er ein rationales, kalkuliertes und gesellschaftlich toleriertes Einsetzen von Gewalt - wie z.B. taktische Fouls - zum Zwecke übergeordneter Ziele, bei dem Verletzungen der Gegner_innen in Kauf genommen werden (2013, 12f.). Er argumentiert, dass der Sport an sich weder ein gewaltförderndes, exkludierendes noch ein integratives und präventives Wesen besitze, sondern es Aufgabe der Pädagogik sei, das eine zu fördern und das andere in Schach zu halten (ebd., 33ff.). ${ }^{45}$

Massenschlägereien in Fußballstadien, Rivalitäten zwischen Vereinen, harte Fouls, rassistische und homophobe Parolen und sexistische Stadionbanner werden als die dunkle Seite der Sportmedaille konstituiert. Beharrlich weisen Sozialwissenschaftler innen, aber auch Journalist innen und zivilgesellschaftliche Initiativen auf die Gewalt- und Exklusionseffekte des Sports hin, und sie werden durchaus von Sportverbänden und -vereinen sowie von politisch Verantwortlichen gehört, was sich z.B. in der Förderung von FanProjekten und in Anti-Rassismus-Kooperationen zwischen Verbänden und zivilgesellschaftlichen Initiativen zeigt. Jedoch sind sich Wissenschaften und politische wie verbandssportliche Institutionen erstaunlich einig darüber, dass es sich dabei lediglich um eine Seite der Medaille handle, die mit Maßnahmen verhindert werden könne und müsse.

In den Bereich des Inakzeptablen rückt somit die Äußerung, beim Fußball sowie beim Sport allgemein handle es sich überwiegend um eine Konflikte und Aggressionen schürende Tätigkeit, die Gesellschaften spalte und Menschen gegeneinander aufhetze sowie systematisch verschiedene gesellschaftliche Gruppen ausschließe. Verbände, Vereine und staatliche, inter/transnationale und zivilgesellschaftliche Akteur-Konstellationen bilden ein institutionelles Netz, das gemeinsam die Aussage „Fußball verbindet“" reproduziert und die Natur des Sports als Frieden und Integration fördernd konstituiert. Göttlich spricht daher in Bezug auf das Integrationsprogramm des Deutschen Sportbundes (jetzt DOSB) von einer „Strategie der Leerstelle“, die es ermöglicht, dass „,soziale Probleme im Sport nie dessen inhärenten Strukturen zugeschrieben, sondern stets auf Einwirkungen von außen zurückgeführt" werden $(2008,217)$. Festzuhalten bleibt, dass dadurch ein konsequentes Ausbuchstabieren der fußballerischen Dimensionen von Konkurrenz, Wettstreit und sportlichem Leistungsstreben in ihren gesamten gewaltförmi-

45 So beschreiben bspw. Ewing et al. (2002), dass Sport bei Jugendlichen zwar aggressives und gewaltförmiges Verhalten fördere, doch kommen sie immer wieder auf die positiven Lerneffekte (Erfolgsorientierung, hartes Arbeiten) zurück. Hansen et al. (2003) erfahren gar von Jugendlichen, dass (kompetitiver) Sport einer der wenigen Freizeitbereiche sei, in denen sie negative Gruppendynamiken erfuhren und wenig Empathie entwickeln konnten. 
gen und exkludierenden Wirkungen unmöglich wird und ein solches deutlich negatives und destruktives Verständnis von Fußball, wenn auch nicht unsagbar, so doch kaum akzeptabel ist. ${ }^{46}$ Stattdessen erlaubt diese Ausklammerung, dass hochkompetitive internationale Sportveranstaltungen zweifelsfrei als interkulturell-verbindende Begegnungen gelesen werden können.

\section{2 „Fußball ist unpolitisch!“}

Eng an die Aussage „Fußball verbindet“ ist die Konstitution von Sport als politisch neutral und unpolitisch geknüpft. Und dennoch ist diese Verknüpfung auf den ersten Blick wenig plausibel, wenn die enorme globalgesellschaftliche Wirkkraft des Sports einerseits, seine Unabhängigkeit und Unberührbarkeit von politischen, sozialen und ökonomischen Verhältnissen andererseits betont wird. Der Hessische Rundfunk befragte den Präsidenten des Internationalen Olympischen Komitees und damit mächtigsten Mann im weltweiten Sport, Thomas Bach, nach genau dieser paradox anmutenden Gleichzeitigkeit:

„Der Sport kann und muss politisch sein. Aber er muss politisch neutral sein. Nur wenn er politisch neutral ist, dann kann er seine positiven Wirkungen entfalten. Dann kann er für Kommunikation stehen, dann kann er für das Verbindende stehen. Dann kann Sport dazu beitragen, dass nicht Mauern errichtet, sondern dass Brücken gebaut werden. Aber Voraussetzung dafür ist seine politische Neutralität. Ohne die würde er dann zwischen den Fronten zerrieben.“ (Deutscher Olympischer Sportbund 2012b)

Bachs Antwort ist aufschlussreich in Bezug auf das Politik-Verständnis der Sportverbände. Politisch-Sein wird hier als aktives und dabei rein positives Einwirken auf andere gesellschaftliche Bereiche verstanden; als freie Handlungsentscheidung eines autonomen Subjekts auf Basis einer parteipolitisch und ideologisch neutralen Haltung. Dass Sport sich entschieden politisch neutral verhalten sowie Handlungsentscheidungen treffen könne, impliziert ein Verständnis von Sport als autonomen und unabhängigen Bereich jenseits

46 Ein weiterer Nicht-Diskurs ist bei den gesundheitsschädigenden Aspekten des Sports zu vermuten. Im Zuge der Verlagerung der Verantwortung für die menschliche Gesundheit weg von Medizin und Gesundheitswesen hin zum Individuum und seinen Handlungen, zum „präventiven Selbst“ (Lengwiler/Madarász 2010), wird Sport mehr und mehr durch Narrative der Gesundheitsförderung gerahmt. Die körperlichen Schäden, die sowohl der Leistungssport als auch der Amateursport verursachen, werden dabei häufig ausgeblendet. Regelmäßige und wiederholte Bänderrisse, Brüche und Gelenkschwierigkeiten, die im äußersten Fall zu völliger Sportunfähigkeit führen können, sind auch in einem Amateurfußballteam an der Tagesordnung. Dennoch hält sich die Erzählung von der gesundheitsfördernden Wirkung des Sports hartnäckig und Verletzungsrisiken und Überlastungserscheinungen werden als kleinere Nebenwirkungen bagatellisiert. 
politischer, ökonomischer, sozialer und anderer Verhältnisse. Der Sport ist demnach keinesfalls per se politisch bzw. Produkt politischer Verhältnisse.

\subsection{1 „Fußball ist unpolitisch“ im Verbandssport}

Das Narrativ vom politisch neutralen Sport ist so allgegenwärtig, dass es der Kulturwissenschaftler Matthias Marschik gar als „hegemoniales Modell des Sports" bezeichnet $(2004,117)$. Er verortet den Ursprung des in seinen Augen bis heute dominanten Bilds vom unpolitischen Sport im bürgerlichen Freizeitsport, der zum Ende des 19. Jahrhunderts von England aus Kontinentaleuropa erreichte. Sport, allem voran Fußball, galt als sinnfreies Freizeitvergnügen. Er folgte allein eigenständigen Werten und Normen des Spiels, der Bewegung und der Körper- und Charakterbildung (ebd., 115). Daher wurde er von den Einen als zu trivial verachtet und von den Anderen als reines Ideal fernab spalterischer Gesellschaftselemente gefeiert, sodass er sich sowohl oberhalb als auch unterhalb politischer Sphären konstituierte (Jackson/Haigh 2009). Damit konkurrierte das bürgerliche Sportverständnis mit der Turn- und Arbeitersportbewegung, die - wenn auch zu unterschiedlichen Zwecken - den Sport explizit in den Dienst der Politik zu stellen suchte. Im DFB wie auch in anderen Verbänden setzte sich jedoch früh das bürgerliche Postulat durch. Das Beharren auf politischer Neutralität und Unabhängigkeit seitens der Vereine und Verbände wurde durch die Erfahrungen mit dem Nationalsozialismus intensiviert und führte letztlich zur vollkommenen Verdrängung politisierender Sportverständnisse: So galt es nach 1945, eine erneute Indienstnahme des Sports zu Propaganda- und Disziplinierungszwecken zu verhindern, was sich zum einen - wie in Kapitel 4.3 ausgeführt - bis heute in der gesetzlichen Stärkung des Autonomieprinzips ausdrückt (Marschik 2004, 115; 2004a). ${ }^{47}$ Zum anderen verpflichtete sich der 1949 neugegründete DFB dem Prinzip der Einheitsorganisation und dem Verzicht auf weltanschauliche Bindungen (Hoffmann/Nendza 2011, 38).

Dieses bürgerliche Sportverständnis, welches sich bis heute über den Verbandssport und seine Statuten fest- und fortschreibt, konstituiert Fußball wie in Bachs Worten - als unpolitisch und meint damit parteipolitische und ideologische Neutralität. Gleichzeitig gesellt sich eine zweite, ergänzende Lesart dazu, die Unpolitisch-Sein als Nichtanfälligkeit des Sports gegenüber gesellschaftlichen Stratifizierungsprozessen versteht. Sport folgt demnach allein eigenlogischen Prinzipien wie Spiel, Leistung, Wettstreit und Fair Play

47 Interessant ist, dass gerade der Neutralitätsdiskurs vom NS-Regime genutzt wurde, um den Sport öffentlich als Beleg für die aufrechterhaltene Normalität anzuführen (Marschik 2004, 215). Das gegenwärtige Credo der politischen Neutralität führte bspw. dazu, dass das Internationale Olympische Komitee und fast die gesamte Presse die berühmte Solidaritätsgeste der Schwarzen US-amerikanischen Olympioniken John Carlos und Tommie Smith 1968 mit der Begründung verurteilten, sie würden die Spiele politisieren (Harvey et al. 2013, 1). 
und ist immun gegenüber den Auswirkungen sozioökonomischer, rassifizierter und vergeschlechtlichter Verhältnisse sowie vor ideologischer und parteipolitischer Instrumentalisierung beschützbar. Grundlegend für beide Lesarten ist die diskursive Konstituierung von Sport als autonomes, eigenlogisches System mit gänzlich eigenen Ordnungsprinzipien jenseits soziopolitischer und ökonomischer Ordnungen.

\subsection{2 „Fußball ist politisch!“-eine Gegen-Aussage}

In meiner Feldforschung beobachtete ich, wie die Mitglieder von DF immer wieder betonten, dass der Fußball doch politisch sei. Sie reihten sich damit in jene Argumentation ein, die z.B. das bürgerliche Sportverständnis pünktlich $\mathrm{zu}$ Megasportevents in autoritären Staaten herausgefordert. Die GegenAussage ,Sport ist politisch“ äußert sich nicht nur in Hinweisen auf die Indienstnahme des Sports für politische und ideologische Anliegen durch Regime und in Aufrufen zur politischen Stellungnahme von Verbänden und Athlet_innen gegenüber Menschenrechtsverletzungen ${ }^{48}$, sondern auch in der Thematisierung von Rassismus, Sexismus und Homophobie in Geschichte und Gegenwart einzelner Sportarten.

Die historischen Wurzeln der Aussage „Fußball ist politisch“ sind nicht klar auszumachen, doch ist anzunehmen, dass die enge Verzahnung des Turnens und des Arbeitersports mit Politik respektive Wirtschaft einen entscheidenden Beitrag geleistet hat. Sowohl die Arbeiter-, als auch der demokratisch orientierte Teil der Turnbewegung sahen im Sport ein geeignetes Mittel, um politische Ideologien und Verhältnisse zu thematisieren und in diese zwecks mehr oder weniger radikaler Veränderungen zu intervenieren. ${ }^{49}$ Ihre Bestrebungen endeten jedoch abrupt durch die Verbote und Restriktionen des NSRegimes in den 1930er Jahren und lebten nach 1945 nicht wieder auf (vgl.

48 Siehe hier die medial geführten Debatten um die Einmischung von Politiker_innen in den Sport sowie die Positionierungen von Sportler_innen zu politischen Themen. Berichte, Interviews und Kommentare arbeiten sich an der „Mär vom ach so unpolitischen Sport“ (Raz 2014, 30) ab und zeigen anhand aktueller wie historischer Beispiele die politische Einflussnahme auf und Instrumentalisierung von Sport. Eine Darstellung dieser Argumente würde weit über den Rahmen dieses Kapitels hinausreichen. Als eines von unzähligen Beispielen sei auf die Diskussion um einen möglichen Boykott der EM 2012 in der Ukraine verwiesen. Ausschlaggebend war damals der menschenrechtsverletzende Umgang mit der politischen Opposition, der sich in der Inhaftierung Julia Timoschenkos versinnbildlichte. Medien portraitieren die Diskussion und beteiligten sich an ihr (bspw. Gräff 2012; Lackner 2012).

49 Auch die Entwicklung des Turnens wurde von der Spannung zwischen politisierenden und neutralisierenden Bemühungen begleitet. Bausinger (2006, 153, Herv. i. Orig.) erwähnt das Treffen der Turnerschaft 1848 in Hanau. Bereits damals hieß die entscheidende Frage: ,politisch oder unpolitisch? Politisch - das meinte die demokratische Forderung. Unpolitisch hieß - wie oft, wenn dieses Etikett gebraucht wird -, dass man beim Bestehenden verharrte“. In Folge kam es zur Gründung des bürgerlich-nationalen Deutschen Turnerbundes und zur Abspaltung eines Demokratischen Turnerbundes. 
Harvey et al. 2013, 29). Nachdem das politische Verständnis von Sport nach 1945 vollständig aus dem deutschen Diskurs verdrängt worden war, dauerte es ein paar Jahrzehnte bis zur erneuten Politisierung des Sports im Zuge einer sich transnational formierenden und politisierenden Zivilgesellschaft. Unter dem diskursiven Bezug auf universelle Rechte forderten verschiedene soziale Gruppen in Form von Identitätspolitik gleiche Teilhabe am Sport. Im Gegensatz zur Arbeiter- und Turnbewegung wurden hier auch sportinterne Machtverhältnisse und sozioökonomische Auswirkungen auf den Sport problematisiert. Eine dieser neuen Gruppen waren Frauenbewegungen (vgl. Hargreaves 1994; Harvey et al. 2013).

Die Konstitution von Sport allgemein und Fußball im Besonderen als von rassistischen, homophoben und patriarchalen Strukturen durchkreuzt, basiert auf einem Verständnis von Sport als gesellschaftlich interdependent, wobei dem Fußball als populärster Sportart in Deutschland die größte Aufmerksamkeit zuteilwird. Entsprechend der thematischen Breite findet sich die Aussage in sehr verschiedenen zivilgesellschaftlichen Bereichen. Ich konzentriere mich aufgrund des Fokus der Arbeit vor allem auf ihr Auftreten im Kontext von Frauenbewegungen und feministischer Sportforschung. Hier ist es vor allem die Politisierung alles Privaten, die eine argumentative Anfechtung der bürgerlichen Neutralisierung des Sports ermöglicht. Es wird zu klären sein, wie der Hinweis auf soziale, geschlechterbezogene Exklusionen und patriarchale Gewalt im Sport als Argument für die politische Beschaffenheit des Fußballs herangezogen wird.

\subsection{3 „Fußball ist politisch!“ eine Gegen-Aussage der Frauenbewegungen}

Schon früh forderten Frauenrechtlerinnen Teilhabe am Sport (siehe Kap. 4.2.1). Ein prominentes Beispiel ist die französische Feministin Alice Milliat, die bereits in den 1920er Jahren gegen den Ausschluss von Frauen von den Olympischen Spielen protestierte. 1921 gründete sie schließlich die Fédération Sportive Féminine Internationale, die bis 1936 Olympische Spiele für Frauen organisierte. Milliat stellte schon damals eine klare Verbindung zwischen dem soziopolitischen Status der Frauen und ihrer sportlichen Exklusion her - so in einem Interview mit dem Frauenmagazin Independent Woman:

„Women's sports of all kinds are handicapped in my country by the lack of playing space. As we have no vote, we can not [sic] make our needs publicly felt, or bring pressure to bear in the right quarters. I always tell my girls that the vote is one of the things they will have to work for if France is to keep its place with other nations in the realm of feminine sport.“ (Milliat 1934, zit. n. Leigh/Bonin 1977, 76)

Milliat begründete die eingeschränkten Teilhabemöglichkeiten von Frauen mit ihrer politischen Benachteiligung: Indem Frauen das Wahlrecht verwei- 
gert wurde, konnten sie ihr Recht auf Sport nicht öffentlich und wirksam einfordern. Jean Harvey et al. schlussfolgern:

„For Milliat and her contemporaries, suffrage was as vital to bringing about acceptance of and recognition for women's sports as sport was for advancing women's rights in society more broadly.“ $(2013,54)$

Bereits damals wurde die Teilhabe von Frauen am Sport in einem breiteren sozialen Kontext verhandelt: Erste-Welle-Frauenrechtlerinnen konstituierten Sport und ihre Teilhabemöglichkeiten als Gradmesser für die gesamtgesellschaftliche Anerkennung von Frauen. ${ }^{50}$

Die Gründung der UN und der entsprechenden Frauenrechtskommission 1947 schuf zentrale institutionelle, rechtliche und argumentative Referenzpunkte (Harvey et al. 2013, 44). 1979 legten sie mit der Convention on the Elimination of All Forms of Discrimination against Women (CEDAW) das rechtliche Fundament für die Forderungen nach sportlicher Gleichberechtigung. Nur zwei Jahre später ratifiziert, stellt die CEDAW die ersten globalen und juristisch verbindlichen Prinzipien für Frauenrechte zur Verfügung und schließt dabei das Recht auf diskriminierungsfreies Sporttreiben ein:

Article 10

States Parties shall take all appropriate measures to eliminate discrimination against women in order to ensure to them equal rights with men in the field of education and in particular to ensure, on a basis of equality of men and women: [...]

(g) The same Opportunities to participate actively in sports and physical education; $[\cdots]$

Article 13

States Parties shall take all appropriate measures to eliminate discrimination against women in other areas of economic and social life in order to ensure, on a basis of equality of men and women, the same rights, in particular: [...]

(c) The right to participate in recreational activities, sports and all aspects of cultural life.

CEDAW identifiziert erstens eine systematische Diskriminierung von Frauen im Sport, indem sie Sport überhaupt zum Thema der UN erklärt, stellt zweitens juristische Werkzeuge als adäquate Instrumente zu deren Bekämpfung

50 Meine schlaglichtartigen Ausführungen zur sportbezogenen Frauenbewegung stützen sich auf Arbeiten zur internationalen Bewegung. Systematische Untersuchungen der Sportbezüge der ersten deutschen Frauenbewegungen sind mir nicht bekannt. Allein Pfister (1980) bietet eine Sammlung von frauenbewegten Streitschriften, die die sportliche Teilhabe einfordern. Auch die Einflüsse der zweiten deutschen Frauenbewegung auf den deutschen Fußball wurden kaum aufgearbeitet. Erst kürzlich erschien eine Sammlung erster historischer Spurensuchen (Thieler/Schibbe 2016). Gleichzeitig gilt es zu bedenken, dass nicht alle Frauen-Sport-Organisationen feministische Agenden hatten. Bspw. betreibt die 1949 gegründete und bis heute existierende International Association of Physical Education and Sport for Women and Girls zwar explizit Sportförderung von Frauen für Frauen, vermeidet jedoch eine Politisierung des Sports und weitreichendere gesellschaftliche Bezüge (Hargreaves 1999, 462f.). 
vor und ruft drittens nationalstaatliche Regierungen zur Beseitigung des Unrechts in die Pflicht. Dieses Politik-Dokument konstituiert den Sport damit als genuin politische Angelegenheit und die diskriminierungsfreie und gleiche Teilhabe am Sport als universelles Menschenrecht. ${ }^{51}$ Es kann demnach als Einspruch gegen das Autonomieprinzip gelesen werden, dem zufolge der Sport seine Angelegenheiten selbstständig und unabhängig von staatlichen Institutionen regeln soll und kann. Der institutionelle und rechtliche Rahmen der UN begünstigte auch die Entstehung einer internationalen Frauen-SportBewegung, die maßgeblich von Athletinnen, Wissenschaftlerinnen, Sportmedizinerinnen und Staats- und NGO-Angestellten aus dem globalen Norden getragen wurde und aus der zahlreiche Gremien, Organisationen und Netzwerke hervorgingen.

Der Slogan „Das Private ist politisch!“ der zweiten Frauenbewegung ab den 1960er Jahren forderte auch das bürgerliche Sportverständnis als unpolitisches Freizeitvergnügen und Sphäre jenseits von Politik, Arbeit und Ökonomie heraus. Jedoch rückte der Sport verhältnismäßig langsam ins Zentrum feministischer Politik, und Sportlerinnen unterstützten die feministische Sportpolitik nur verhalten. Dies änderte sich erst in den 1980er Jahren: "[S]ports are becoming part of a larger movement for female autonomy“ (Hargreaves 1994, 36), woran die enge Verzahnung von feministischer Bewegung und Wissenschaft maßgeblich beteiligt war. Viele der Gründerinnen und gegenwärtige Mitglieder internationaler Organisationen, wie WomenSport International oder International Association of Physical Education and Sport for Girls and Women, sind Sozial- oder Kulturwissenschaftlerinnen.

\subsection{4 „Fußball ist politisch!“ - eine Gegen-Aussage in den Wissenschaften}

Gleichzeitig forderten feministische Wissenschaftlerinnen ab den 1970er Jahren die männliche Dominanz in den Sportwissenschaften heraus (ebd., 25). $\mathrm{Zu}$ den Pionierinnen feministischer Sportstudien zählen unter anderem Jennifer Hargreaves, Nancy Theberge, Susan Birrell, Cheryl Cole und Ann M. Hall sowie die deutschsprachigen Sportsoziologinnen Gertrud Pfister, Marie-Luise Klein und Birgit Palzkill. Sie zeigen, dass Geschlecht nicht als erklärende Variabel für Sportpraktiken, sondern als Herrschaftsstruktur des kulturellen Phänomens Sport zu erfassen ist. Damit korrigieren sie nicht nur die geschlechtliche Leerstelle der Sportwissenschaften, sondern tragen auch dazu bei, Sport als ernstzunehmenden Gegenstand der Kultur- und Sozialwis-

51 Bereits 1978 deklarierte die UNESCO in der International Charter of Physical Education and Sport Sport als universelles Grundrecht. Ausführlich zu Sport und Menschenrechten siehe Donnelly (2009). 
senschaften wie Gender Studies zu etablieren. ${ }^{52}$ In diesen hat sich die Perspektive auf Sport als soziales und kulturelles Phänomen durchgesetzt, in dem gesellschaftlich, politisch und auch ökonomisch relevante Auseinandersetzungen ausgetragen und Verhältnisse stabilisiert oder auch herausgefordert werden. Entsprechend der globalen Popularität des Fußballs widerfährt diesem besonders große Aufmerksamkeit. Die sozial- und kulturwissenschaftlichen Argumentationen stützen sich dabei auf die besondere Rolle, die der Fußball als national bedeutsamste Sportart und „hegemoniale Sportkultur“ (Markovits/Hellerman 2004) in vielen europäischen und lateinamerikanischen Ländern gegenüber anderen Sportarten ausfüllt, und leiten daraus eine besonders restriktive und undurchlässige Re-Produktion heteronormativer zweigeschlechtlicher Ordnungen ab. ${ }^{53}$ Die wissenschaftliche Konstituierung des Sports bietet damit den zivilgesellschaftlichen Kräften wie der Frauenbewegungen Argumente und Legitimation für die Inklusion des Sports in ihr Credo „das Private ist politisch“. Dabei widerfährt dem Sport eine Politisierung in dem Sinne, dass seine interne Beschaffenheit als Produkt gesellschaftlicher Verhältnisse und nicht als diesen gegenüber immun verstanden wird. Durch die Bereitstellung international verbindlicher Frauenrechte werden die sportinternen Ungleichheiten als Form der geschlechterbezogenen Diskriminierung deutbar und zum Gegenstand internationaler und staatlicher Politiken und Verwaltungen erklärt.

Dieser sehr kurz gehaltene Ausflug in die Politisierung des Sports durch zivilgesellschaftliche und wissenschaftliche Akteur_innen macht deutlich, dass Fußball ein umkämpftes Terrain ist, auf dem Macht/Wissen-Regime um Deutungshoheit ringen. Wissenschaften legitimieren dabei die Politisierung des Fußballs. Eine über lange Zeit dominante, zeitweise gar exklusive bürgerliche Deutung konstituierte Fußball als unpolitisch und neutral und verbreite-

$52 \mathrm{Zu}$ feministischen Interventionen in die Sportwissenschaft siehe Hargreaves (1994), über die Verwobenheiten ihrer feministischen und wissenschaftlichen Tätigkeiten reflektieren Nancy Theberge, Celia Brackenridge und Kari Fasting ausführlich in Markula (2005).

53 Als Beispiel für diese Argumentation sei auf Müller $(2007 ; 2009)$ verwiesen. Zur Unmöglichkeit der Verkörperung des Nationalen durch Frauen im Fußball siehe Dietze (2012). Der nationale Körper wird im Fußball als männlich, heterosexuell und weiß normiert. In meinen Augen besitzt eine solche Theoretisierung gegenüber anderen Deutungsangeboten „Vernunft“ (Rabinow 2004), weshalb auch ich mich in dieser Arbeit auf sie stütze. Eine Objektivierung der eigenen theoretischen Werkzeuge, wie sie von Bourdieu (1993) gefordert und unter anderem in diesem Abschnitt angelegt ist, führt einmal mehr vor Augen, wie das Feld erst durch die Anwendung theoretischer Instrumente mit eigenen Fachgeschichten geschaffen wird. Uneinigkeit herrscht in der Forschung bezüglich der Frage, ob der „Fußball als Realitätsmodell“ (Theweleit 2004) und „Vergrößerungsspiegel der Unterschiede zwischen den Geschlechtern“ (Bromberger 2006, 42) oder aber als eigenlogische ,interessante gesellschaftliche Anomalie“ (Müller 2007, 133), als „male preserve“ (Dunning 1994) und ,,ausgewiesene[r] Schutzraum für moderne Vorstellungen der vormodernen männlichen Natur“ (Sülzle 2011, 252) verstanden werden soll. Die Diskussion, ob Sport Abbild von oder Gegenwelt zu anderen Gesellschaftsbereichen sei, wird auch in Bezug auf wirtschaftende und zivilisierungstheoretische Aspekte geführt, vgl. Alkemeyer (2006, 282). 
te sich innerhalb eines Netzes aus trans-/internationalen und staatlichen wie verbandssportlichen Institutionen, Leitlinien, Programmen und gesetzlichen Übereinkommen. Dieses Dispositiv um die sportliche Neutralität wurde jedoch immer wieder durch Politisierungsversuche herausgefordert, sodass es schließlich zu erodieren begann. Die politisierenden Gegen-Aussagen sind so heterogen und werden von vielen verschiedenen Institutionen und AkteurKonstellationen reproduziert, dass hier nur ein minimaler Ausschnitt skizziert werden konnte. Obwohl sie sich in Argumentationsweisen, Themen, Anliegen und Zielen unterscheiden, weisen sie auf ein geteiltes Aussagesystem hin, das Sport respektive Fußball als genuin politische Angelegenheiten sowie als soziokulturelles Phänomen konstituiert und das bürgerliche Sportverständnis problematisiert. Aufgrund dieser offenen Kontroverse ist Marschiks Diagnose von der Hegemonie der Neutralitätsaussage für die Gegenwart zu relativieren. Stattdessen gehe ich von einer Konjunktur der Aussagen und Aussagemöglichkeiten aus: Nach einigen Jahrzehnten der uneingeschränkten Akzeptabilität des bürgerlichen Verständnisses ermöglicht die gegenwärtige Friction öffentliche und plausibel erscheinende Problematisierungen; die politischen Dimensionen des Fußballs sind momentan verhandelbar und nicht zu einem Dispositiv gefestigt.

Für den Verlauf der Arbeit ist festzustellen, dass in Verbänden und Vereinen ein politisch neutrales Sportverständnis vorherrscht, das mehrere Jahrzehnte lang hegemonialen Status genoss und den Sport mit einer Aura des Unpolitischen versah, die noch immer nachwirkt. Dennoch konnten nicht zuletzt auch die Sozial- und Kulturwissenschaften in enger Zusammenarbeit mit zivilgesellschaftlichen Bewegungen und Organisationen eine GegenAussage etablieren und das vorherrschende Sportverständnis kritisieren. Dabei erfuhren sie entscheidende Legitimität durch den rechtlichinstitutionellen und argumentationsstrategischen Rahmen der UNFrauenrechtskommission. Die enge Verzahnung von Sport und sozioökonomischen wie politischen Verhältnissen wurde so (wieder) thematisierbar, sodass sich Funktionär_innen wie Thomas Bach nun immer wieder rechtfertigen müssen.

\section{3 „Fußball empowert Frauen!“}

„We empower girls and women through football“ - nicht nur bei der Weltsportministerkonferenz verwies DF auf die befähigende Wirkung; das Schlagwort „Empowerment“ zog sich wie ein roter Pfaden durch ihre Projektarbeit. Ausgehend von dieser Beobachtung, konnte ich die Aussage, Sport allgemein und männlich konnotierter Sportarten im Besonderen hätten emanzipatorische Wirkung auf Frauen, in zwei Bereichen feststellen. Den ersten 
Bereich bilden feministische, sozialwissenschaftliche Studien und damit eng verknüpfte Sportprojekte. Den zweiten Bereich bildet der Entwicklungssektor, in dem konkrete Sportprojekte mit dem Ziel der Frauenförderung, des Capacity Buildings und der Entwicklung von Zivilgesellschaft implementiert werden. ${ }^{54}$ Dabei ist jedoch zu beobachten, dass sich durch die unterschiedlichen kontextuellen Verwendungen und Bezüge unterschiedliche Bedeutungen in das Schlagwort einfalten. Aradhana Sharma spricht gar von „Empowerment Assemblages“ (2008, 1), um die Vielzahl von unterschiedlichen Ideen und institutionellen Praktiken zu fassen, die sich collage-artig zu einem lose zusammenhängenden Ensemble verdichten. Im Folgenden zeichne ich in Bezug auf den Sport verschiedene in den Begriff eingefaltete Bedeutungen nach. Ich greife die Aussage anhand von Selbstbeschreibungen von Vereinen, Sportprogramm-Broschüren oder Politik-Dokumenten. Dabei wird ersichtlich, wie der Topos eine Klammer um die verschiedenen Bedeutungen und entsprechenden Praktiken bildet und damit Kooperationen und AkteurKonstellationen hervorbringt.

\subsection{1 „Fußball empowert“ im Feminismus}

Sowohl sozialwissenschaftliche und feministische Ansätze zu Sport und Geschlecht/Sexualität sowie frauenbewegte und feministische Sportprojekte vor allem im englischsprachigen Kontext beziehen sich häufig auf „Empowerment". Im deutschsprachigen Kontext findet die feministische Auseinandersetzung mit Sport in den 1980er und 90er Jahren - wenn auch deutlich geringer (Rose 1992) - in frauen- und lesbenbewegten Sportinitiativen und programmatischen Schriften für eine feministische Bewegungs- und Sportkultur statt (Bischoff 1993; Kugelmann 1993). Aufgrund der engen Verzahnung der Geschlechterforschung mit den Frauenbewegungen und feministischen Aktionsformen scheint es hier ein ähnliches Verständnis von Empowerment zu geben. Auffällig ist, dass ich trotz der häufigen Verwendung keine Begriffserklärung finden konnte. Die Bedeutung lässt sich also nur anhand der konkreten Verwendung und kontextuellen Bezüge herausarbeiten.

Feministische sozialwissenschaftliche Studien zu Sport und Geschlecht thematisierten vorrangig in den 1980er und 90er Jahren Erfahrungen, die Frauen im Sport machen, auch unter dem Schlagwort Empowerment. Sie fokussierten hierbei den Körper und die Körpererfahrung vor dem Hintergrund der gesellschaftlichen Geschlechterordnung. Der Körper wird demnach als Austragungsort geschlechterpolitischer Kämpfe und als Mittel sowie

54 Einen dritten Bereich bilden Sportprojekte zur Integration in den Ländern des globalen Nordens und damit verbundene wissenschaftliche Begleitforschungen, die den Sport zur Stärkung marginalisierter Gruppen, u.a. Mädchen mit Migrationshintergrund, einsetzen. Da dieser jedoch für meinen Forschungskontext kaum relevant ist, spare ich ihn hier aus. 
Spielfeld patriarchaler Disziplinierung und Unterdrückung der Frauen theoretisiert (Birrell/Theberge 1994, 365). Young (1980) hat hierfür die theoretische Grundlage gelegt. Sie argumentiert, dass die Objektifizierung von Frauen in modernen, patriarchal organisierten Industriegesellschaften die eigene Wahrnehmung als handlungsfähige Subjekte durchkreuzt und sich in körperlicher Zurückhaltung und Hemmung und in durchbrochenem Raum- und Bewegungsverhalten in die weiblichen (Sport-)Körper einschreibt. Im Umkehrschluss wird gefolgert, dass sich die physische Kräftigung des Körpers und das Erlernen entschlossener, (selbst-)bestimmter Bewegungsabläufe im Sport auf die Wahrnehmung der eigenen Körperlichkeit auswirken:

„They change in confidence, in self-image, in interaction with others, and in the ways in which they carry themselves and move through the world [...] they think differently about who they are." (Bennett et al. 1987, 376)

Indem sie bewusst die Kontrolle über ihre Körper übernehmen, verwandeln Sportlerinnen diesen von einem Austragungsort der Unterdrückung in einen Ort des Widerstands gegen und der Befreiung von der männlichen Herrschaft:

„, $[\mathrm{R}]$ eclaiming physicality is a way for women to repossess themselves and intervene in their own behalf to counter restrictive patriarchal practices. Thus physical empowerment through sport can serve as a metaphor for personal empowerment." (Birrell/Theberge 1994, 366) $)^{55}$

Die körperliche Befähigung zu raumgreifendem Sporttreiben und physischer Kraft steht dabei nicht nur metaphorisch für mehr persönliche Stärke, sondern, so argumentieren z.B. Rosemary Deem und Sarah Gilroy, führt tatsächlich zu gesteigertem Selbstvertrauen und Durchsetzungsstärke (Gilroy 1989; Deem/Gilroy 1998).

Die Frage, wie diese veränderte Körperwahrnehmung durch Sport zu interpretieren ist, wird kontrovers diskutiert. Die einen, unter anderem in Ansätzen Young, verstehen weibliche Sport- und Körperpraktiken per se als transformativ und subversiv gegenüber dominanten Weiblichkeitsstereotypen und vermuten in der Aneignung eines kräftigen Körpers den Zugewinn von Selbstaffirmation und Agency. ${ }^{56}$ Andere, unter anderem Susan Bordo (2013), argumentieren dagegen, dass das Ideal eines definierten und sportlichen

55 Vgl. auch Theberge (1987, 365; 1994, 191), Broad (2001). Dworkin und Messner (2002) zeigen, wie die Idee des individuellen Empowerments von Frauen durch Sport von großen Sportartikelherstellern zu Werbezwecken eingesetzt wurde. Sie weisen darauf hin, dass es sich hierbei um individuelle, reproduktive Handlungsfähigkeit (,,reproductive agency“) handelt und nicht, wie im Falle von feministischen Initiativen - etwa der Gründung einer Frauensportliga - um widerständige Handlungsfähigkeit (,resistant agency“). Letztere ziele darauf ab, unterdrückende Strukturen zu verändern.

56 Kritisiert wurde diese Position für ihre Defizitorientierung, da sie Körper- und Sportverhalten von Jungen und Männern als Normalität impliziert, an der sich letztlich das Empowerment von Mädchen und Frauen orientiert (vgl. Strüver/Wucherpfennig 2012). 
weiblichen Körpers die heterosexistische Norm vom schwachen, zarten und passiven Frauenkörper abgelöst und nun denselben Zwangscharakter eingenommen hat. ,[B]odily ,empowerment" is merely a modern version of the ,docile body“", und zur Haus- und Lohnarbeit geselle sich nun noch der Imperativ zur Körperarbeit als dritte, tägliche Arbeitsschicht hinzu (Dworkin/Messner 2002, 23). Theberge bemüht sich um einen Kompromiss und unterscheidet schließlich zwischen den Sportarten und ihrer Organisation: Während Fitness einer Fortführung der Unterdrückung durch Sexualisierung entspricht, bieten ,männliche“ Sportarten Empowerment-Potentiale. Allerdings - so schränkt sie ein - nur dann, wenn sie sich von der reinen Befolgung des männlichen Sportmodells und deren Idealisierung von Wettkampf, Aggression und Stärke loslösten und den Sport im Sinne feministischer Visionen umgestalteten $(1987,389 ; 2003)$. Nach neuen Sport- und Bewegungsformen wurde - praktisch und theoretisch - im Rahmen der feministischen Bewegungs- und Sportkultur gesucht. Sport sollte sich, um befähigend zu wirken, an der Lebenswelt von Frauen orientieren und sich von einer auf Fortschritt, Instrumentalisierung und Manipulation basierenden männlichen Sportkultur abwenden (Kugelmann 1993). An dieser Stelle möchte ich die Debatte Empowerment vs. Disziplinierung sowie die Frage nach den transformativen Graden der Aneignung nicht weiter nachzeichnen, greife sie aber in Kapitel 11.1.2 und 17.2 wieder auf. Verwiesen sei auf aktuelle Untersuchungen, die von der Argumentation im Entweder-Oder-Schema abrücken und stattdessen die Gleichzeitigkeit von Subversion und Affirmation bestehender Geschlechterbilder, von Empowerment und Unterwerfung betonen (vgl. Faust/Assmann 2014). Entscheidend für den hiesigen Zusammenhang ist, dass feministische Ansätze mit „Empowerment“ den Prozess der durch Sporttreiben ausgelösten veränderten Körpererfahrung und Selbstwahrnehmung beschreiben und vor allem für Frauen in männlich konnotierten Sportarten gültig machen.

Die enge Verbindung von Frauenbewegung und Geschlechterforschung materialisiert sich unter anderem in der Gründung von Frauensportvereinen, die sich mit ebendem Spannungsverhältnis von Disziplinierung und Leistungsstreben auf der einen Seite und körperlicher Kräftigung und Stärkung des Selbstbewusstseins auf der anderen auseinandersetzen. Der DFC Kreuzberg, der Fußballverein von DF, ist nur eines der jüngsten Beispiele in der feministischen Berliner Sportlandschaft. Viele dieser Sportprojekte und vereine im deutschsprachigen Raum berufen sich nicht explizit auf Empowerment, jedoch lassen ihre Ziele und Programme auf ein ähnliches Körper-Sport-Verständnis schließen, wie es die US-amerikanischen Studien formulieren. So stellt sich das feministische Sportangebot SchokoSport des Kreuzberger Frauenzentrums Schokoladenfabrik e.V. interessierten Frauen wie folgt vor: 
„In unseren zwei Sportetagen geht es uns darum, sie [die Besucherinnen] in ihrer individuellen Bewegungsfähigkeit und ihrem körperlichen Selbstverständnis zu fördern. Sie entwickeln ein Gefühl für die eigenen Fähigkeiten und Grenzen, fernab von fremden Leistungsansprüchen.“ (Schokosport o.J.)

SchokoSport knüpft das Sportprogramm an die Idee der Körpererfahrung jenseits patriarchaler Objektifizierung sowie an den Gedanken der Selbstverteidigung und -behauptung. Dies schlägt sich im konkreten Angebot nieder: Es werden sowohl Kampfsport- und Selbstverteidigungskurse als auch Yoga und Pilates angeboten. Auch das Mädchensportprojekt Leyla rennt des FrauenLesbenTrans*-Sportvereins Seitenwechsel e.V. verweist ähnlich wie SchokoSport auf körperliche Gewalterfahrungen:

„Bei uns kannst du erleben, dass Sportlichkeit keine Voraussetzung für Sporttreiben ist. Im Vordergrund stehen zumeist der Spaß an der Bewegung und die Erfahrung, dass jede mit ihrer Persönlichkeit und ihrer Körperlichkeit willkommen ist. Unsere Trainerinnen sind pädagogisch und sportlich qualifizierte Frauen. Unser Ziel ist es weiter, zu vermitteln, dass Grenzen akzeptiert und Ängste und Gefühle ernst genommen werden. Nicht zuletzt im Wissen um die Gewalterfahrungen vieler Mädchen bieten unsere Sportangebote einen sicheren Rahmen, in dem sich diese Erfahrungen nicht wiederholen. Unser Anliegen ist es, durch aktive sportliche Freizeitgestaltung gezielt und umfassend zu fördern." (Seitenwechsel e.V. o.J.)

Zwar rekurriert Seitenwechsel hier nicht explizit auf den EmpowermentBegriff, jedoch steht das Programm im direkten Kontext des sportfeministischen Ansatzes des Trägervereins und addiert so das Gefühl von Sicherheit vor (sexueller, sexualisierter und patriarchaler) Gewalt und eine Bewusstwerdung der eigenen körperlichen Regungen zum feministischen Empowerment-Verständnis hinzu. Empowerment wird erreicht durch die Schaffung von sicheren, Gewalt ausschließenden Räumen. Gleichzeitig findet hier und bei SchokoSport - wie von Theberge und Kugelmann empfohlen - eine Umdeutung des als männlich gelesenen Sportverständnisses statt, das Leistung, Erfolg und Wettkampf als Grundelemente des Sports konstituiert. Diesem wird eine Sportpraxis jenseits des Bis-an-die-eigenen-GrenzenGehens und der Bevorzugung sportlich talentierter und trainierter Körper entgegengesetzt, das von den Bedürfnissen und Erfahrungen der Mädchen und Frauen ausgeht. Durch die Verbindung von sportlichen und pädagogischen Anliegen schließt sich der Zirkel von physischer und personaler Stärke.

Durch die Verzahnung wissenschaftlicher Theorien zu Sport und Körper mit konkreten Sportprojekten lässt sich ein feministisches Verständnis wie folgt - vereinfacht und auf den Hauptnenner reduziert - beschreiben: Empowerment meint die Veränderung des eigenen Körpergefühls und der Körperwahrnehmung durch Sport im Sinne einer Steigerung der physischen Stärke wie auch des Bewusstseins für die eigenen Grenzen. Diese körperliche Stärke mündet nicht nur in der Fähigkeit zur physischen Selbstverteidigung, sondern ebenfalls in personaler Stärke als Fähigkeit zur Selbstbehauptung, 
und ermöglicht sporttreibenden Frauen letztlich ein selbstbestimmteres Leben. Grundlage dafür ist ein sicherer Raum, in dem sich Mädchen und Frauen vom männlichen Verständnis von Sport und Körperlichkeit lösen, ein Bewusstsein für individuelle und kollektive Unterdrückung erlangen und sich darin in gegenseitiger Solidarität unterstützen können.

\subsection{2 „Fußball empowert“ in der Entwicklungspolitik}

Im entwicklungspolitischen Strang der diskursiven Formation wird der Begriff „Empowerment“ besonders prominent verwendet. Unter der Programmatik Sport for Development and Peace (SDP) schließt der Topos über den Entwicklungsbegriff an das bereits diskutierte Argument der verbindenden, friedensfördernden Kraft des Sports an. In dieser Programmatik laufen sodann zwei getrennte Entwicklungen zusammen und bringen ein spezifisches Verständnis von Empowerment hervor: Zum einen wurzelt der Empowerment-durch-Sport-Topos in der Identifizierung der Frau als entwicklungspolitische Zielgruppe durch die Entwicklungstheorien und -agenturen der 1970er Jahre. So erklärten die UN die Jahre zwischen 1976 und 1985 zur Dekade der Frau und machten fortan Mädchen und Frauen zu Zielobjekten der Entwicklungsprojekte (McDonald 2015). ${ }^{57}$ Zum anderen entdeckten die UN zu Beginn des 21. Jahrhunderts den Sport als Instrument der Entwicklungszusammenarbeit. Darnell verweist auf die Resolution 58/5 vom 17. November 2003 mit dem Titel „Sport as a means to promote education, health, development and peace", in der die UN unterschiedliche Stakeholder dazu auffordern, Sport und Bewegungsaktivitäten in ihre Entwicklungsprogramme und -leitfäden zu integrieren. Sport wird dabei als Mittel beworben, die Milleniumsentwicklungsziele zu erreichen und Partnerschaft und Kooperation zu fördern (2012, 12f.). Megan Chawansky und Lyndsay Hayhurst werten das Zusammentreffen von Sport als Entwicklungsinstrument und Mädchen und Frauen als entwicklungspolitische Zielobjekte als einen größeren Wandel im internationalen Entwicklungssektor, der ein „,girling“ of the SDP sector“ indiziere $(2015,877)$. Die entsprechenden Projekte prognostizieren positive Veränderungen durch die Kombination von Sporttrainings und sogenannten

$57 \mathrm{Zu}$ den verschiedenen Paradigmen der geschlechterbezogenen Entwicklungspolitik seit den 1970er Jahren - von Welfare-Ansätzen über Women in Development hin zu Gender and Development - siehe Sharma (2008, v.a. Kap. 1). Sie zeigt die verschiedenen und umstrittenen Bedeutungen von Empowerment in der Entwicklungspolitik und bemerkt, dass feministische und befreiungspädagogische Vorstellungen vom neoliberalen Entwicklungsdiskurs adaptiert und transformiert wurden und deutet Empowerment als Selbst-Technologie des Regierens. Alvarez beobachtet, dass das ,gendering of international regimes“ in den folgenden Jahrzehnten und nicht nur im EZ-Bereich zunahm und zu einer „flood of genderfocussed policies and programs" führte (1999, 191f.). 
life-skills-Bildungsangeboten zu Themen wie reproduktive Gesundheit, Hygiene, Kommunikation oder Finanzplanung (ebd., 878).

Zwar bilden die UN-Politiken nicht die Geburtsstunde des Sport-forDevelopment-Ansatzes, doch erhält dieser durch die UN-Dokumente (zusätzliche) Legitimität. Darnell verortet die Entstehung der Aussage, die Sport als soziale Kraft und transformative Kulturform benennt, weit zurück in der nordeuropäischen Geschichte. In der viktorianischen Epoche des 19. Jahrhunderts entstand der bürgerliche Imperativ zur Teilnahme am Sport als Weg zur Charakterbildung und Disziplinierung. Sport, nicht länger Raum für Ausschweifungen, Prasserei oder Weltflucht, galt fortan als vernünftige Freizeitgestaltung und Erholung samt Organisationsstrukturen, Kodifizierung und Wettkampf. Diese aus Großbritannien stammende Neudeutung des Sports wurde gegen Ende des 19. Jahrhunderts besonders in religiösen Reformbewegungen deutlich: Protestantische Reformer predigten auch in den Kolonien die Entwicklung und Disziplinierung des männlichen Körpers im Sinne einer Muscular Christianity. Zu Beginn des 20. Jahrhunderts ertönte zudem aus den Arbeiterbewegungen die Forderung nach Zugang zu sicheren Freizeitund Erholungsmöglichkeiten, die zusammen mit dem Interesse der Unternehmen an produktiven Arbeitskräften den Ausbau von Sport- und Bewegungsmöglichkeiten vorantrieben. Allen diesen unterschiedlichen Strömungen ist gemein, dass sie den Sport als bildend und sozial nachhaltig konstituieren, dessen Wirkung weit über das bloße Spiel hinausgeht (Darnell 2012, 12). Die darin enthaltene Zuschreibung eines erzieherischen, charakterbildenden Wertes habe ich bereits am Beispiel des Olympismus erwähnt, wo sie dann auch mit der integrativen Wirkung des Sports zusammengeführt wird. ${ }^{58}$

Im Fall der UN und deren konkreten Entwicklungsprogrammen ergibt sich in Ergänzung zur ebenfalls schon dargestellten friedensstiftenden Wirkung folgende Argumentation - vereinfacht paraphrasiert von Mary G. McDonald: Nur durch richtige Anleitung und das Bereitstellen von Sportund Bildungsangeboten würden Mädchen und Frauen in entsprechenden Regionen der Welt die ökonomischen und kulturellen Kompetenzen entwickeln, um Armut, Geschlechterstereotype und mangelnde Gesundheitsversorgung zu überwinden $(2015,915)$. Die UN sind durch die Schaffung eines United Nations Office on Sport for Development and Peace (UNOSDP) dabei zum wichtigen Akteur im Bereich Sport und Entwicklung geworden (Darnell 2012, 10). Zum Zeitpunkt meiner Forschung bekleidete der deutsche SPDPolitiker und ehemalige Manager des SV Werder Bremen Wilfried Lemke den Posten des Sonderberaters des UN-Generalsekretärs für Sport im Dienste von Frieden und Entwicklung. Zusammen mit dem IOC legitimieren und

58 Laut Coalter nimmt Sport aufgrund der ihm zugeschriebenen Fähigkeiten einen mythopoetischen Status ein: Diese Zuschreibungen sind eher unscharf und basieren auf idealistischen und populären Vorstellungen, sie werden reifiziert und verzerrt dargestellt und können so generalisierbare Bilder vom Sport evozieren $(2010,296)$. 
finanzieren sie viele Projekte, die vor allem von NGOs implementiert werden. Diese tragen Namen wie Right to Play, PLAY SOCCER Nonprofit International, Sports Sans Frontiers, Cross Cultural Project Association, Grassroots Soccer und Open Fun Football Schools. Viele dieser Namen sowie Lemkes Biografie als Fußballfunktionär weisen auf die prominente Rolle der Sportart Fußball hin.

Auch das AA sowie das BMZ haben die erzieherischen Aussagen zum Sport in ihre außenpolitischen Strategien integriert. Ihre Sportprogramme für sogenannte Entwicklungs- und Schwellenländer zielen ,als Bildungs- und Erziehungsinstrument" (BMZ o.J.) neben der Vermittlung eines positiven Deutschlandbildes darauf ab, durch Förderung sozialer Kompetenzen wie Teamfähigkeit, Disziplin und Fair Play „Hilfe zur Selbsthilfe“ zu leisten (AA 2012). Das BMZ erklärt seinen Sportfokus folgendermaßen:

„Seit mehr als 30 Jahren werden im Rahmen der deutschen Entwicklungszusammenarbeit Sportprojekte gefördert, um Menschen zu mobilisieren, zu sensibilisieren und um sie sozial und wirtschaftlich zu integrieren. Die bisherigen Erfahrungen haben gezeigt: Sport fördert wichtige Kompetenzen, regt zum Dialog an und kann wesentlich dazu beitragen, Entwicklungsziele zu erreichen. [...] Neben dem wichtigen Einsatz im Bildungsbereich kann, Sport für Entwicklung' mit Vorhaben in den Bereichen Gesundheitsförderung, Gewaltprävention, Gleichstellung der Geschlechter, gute Regierungsführung, Inklusion oder auch Umwelt kombiniert werden. [...] Zielgruppe der deutschen Aktivitäten sind vor allem Kinder und Jugendliche. Junge Menschen, die sonst nur Chancenlosigkeit kennen, sollen über Sport ein neues Lebensgefühl entwickeln. Besonders berücksichtigt werden dabei Mädchen und junge Frauen, die oft zusätzlich benachteiligt sind.“ (BMZ o.J.a)

Die spezifische Auswahl der weiblichen Zielgruppe wird unter der Überschrift „Selbstbestimmung und Inklusion. Fair Play nicht nur auf dem Sportplatz" so begründet:

„So kann Sport gerade Mädchen und junge Frauen in ihrem Selbstbewusstsein und innerhalb der Gemeinschaft stärken. In vielen Ländern sind Mädchen im öffentlichen Raum wenig sichtbar, und es ist schwierig für sie, Sportangebote wahrzunehmen. Daher achten wir in unseren Vorhaben darauf, diese Hälfte der Bevölkerung an Sportangeboten zu beteiligen und ihnen damit einen - in vielen Gesellschaften noch kaum genutzten - Weg zu mehr Selbstbestimmung zu eröffnen.“ (BMZ 2015, 17)

Hier wird zum einen ersichtlich, wie Sport durch die zugeschriebene Empowerment-Funktion zu einem sozialen Allheilmittel wird. Daniel Burdsey nennt dies eine, ,increasingly pervasive (and, at times, mistaken) rhetoric of football as a social panacea“ $(2008,270)$. Zum anderen schafft die deutsche Außen- und Entwicklungspolitik durch ihren Länderfokus Subjekte und Objekte des Empowermentprojekts: Projektkoordinator_innen und Geldgeber_innen des globalen Nordens empowern als bedürftig identifizierte Gruppen des globalen Südens bzw. postsowjetischer Länder zur Selbsthilfe. Nationale oder translokale NGOs sowie die staatliche GIZ übersetzen diese Politiken in konkrete Projekte. 
McDonald kommt anhand ihrer Untersuchung der US-amerikanischen Kampagne Empowering Women and Girls Through Sports zu einer Vielzahl an feministisch und postkolonial informierten Kritikpunkten, von denen auch einige meines Erachtens für den deutschen Kontext gültig gemacht werden können. Der erste Aspekt betrifft die Legitimierung eines neoliberalen Entwicklungsmodells durch die entwicklungstheoretische EmpowermentRhetorik. Westliche Unternehmen, die auch in Deutschland häufig Projekte finanzieren, NGOs, die UN und die Weltbank sowie staatliche Regierungsorgane konstituieren Mädchen und Frauen des globalen Südens als unternehmerische Subjekte, die fähig sind, sich selbst und ihre gesamten Familien und Communities $\mathrm{zu}$ mehr sozialer und ökonomischer Sicherheit zu verhelfen (McDonald 2015, 911; vgl. Hayhurst 2013; Sharma 2008). Der auf das Individuum gerichtete Fokus der Sportprogramme legitimiert das neoliberale Entwicklungsmodell und, so der zweite Kritikpunkt, überträgt die Verantwortung für die sozialen und ökonomischen Verhältnisse ganzer Gemeinschaften auf einzelne Mädchen und Frauen. Drittens ist das dabei imaginierte neoliberale Subjekt vergeschlechtlicht und konstituiert Mädchen und Frauen als „active subjects desiring to play sport but facing barrier to access“ und „agents of change“ (McDonald 2015, 915) - ein Bild, das an ein altbewährtes Weiblichkeitsstereotyp der Entwicklungsarbeit erinnert: die kümmernde, nährende, aufopferungsvolle, hart arbeitende Frau, die ihrer Familie, ihrer Community und sogar der gesamten Nation aus der Armut und zu ökonomischem Wohlstand verhilft (vgl. Cornwall et al. 2008, 8). Dass dieses Frauenbild zudem stark rassifiziert ist, machen Kritiken an dem innenpolitischen Integration-durch-Sport-Programm deutlich: Die Sportferne der Mädchen und Frauen wird mit den religiösen und/oder kulturellen Restriktionen und Eigenheiten der spezifischen Regionen begründet und diese damit als gegensätzlich zu den westlichen Länder behauptet. Emanzipation meint daher nicht nur die erfolgreiche Behauptung innerhalb männlicher Sportarten, sondern auch gegenüber der als frauensportfeindlich konstruierten Kultur (Klein 2011) ${ }^{59}$ Hier wird im Gegensatz zum feministischen Empowerment-Begriff die Kategorie Frau gespalten: in jene mit Emanzipationsbedarf und jene, die bereits emanzipiert sind. Empowerment orientiert sich hier nicht an den spezifischen Bedürfnissen und Erfahrungen, sondern ist ein bereits vordefiniertes Set an Handlungsmöglichkeiten, Geschlechterrollen und Lebensgestaltungen. Was durch diese Rahmung verschleiert wird, so der vierte Kritikpunkt, sind die globalen Machtungleichheiten. Anstatt diese als Ursachen zu

59 Klein widerlegt die monokausale Begründung der Sportferne aufgrund von Kultur/Religion durch den Verweis auf die Multikausalität und nennt vor allem strukturelle Gründe wie unzureichende Öffentlichkeitsarbeit und mangelnde Berücksichtigung der spezifischen Interessen, problematische öffentliche Zugänge der Sportstätten, weite Anreisen zu Wettkämpfen oder den Mangel an Trainerinnen sowie unterschiedliche Bedürfnisse und Sportpraktiken. Die Konstruktion als Problemgruppe bleibt dadurch unangetastet, lediglich wird ihre Kulturalisierung aufgehoben $(2011,127)$. 
adressieren, werden ökonomische und gesundheitliche Missstände sowie geringes Selbstwertgefühl bei den Individuen verortet und dort mittels Sport bekämpft (McDonald 2015, 916). Die historisch - etwa durch den Kolonialismus - gewachsenen Ungleichheiten schreiben sich so in jenen Sport-forDevelopment-Programmen fort. Denn, so belegt es auch die Länderauswahlliste von AA und BMZ, beziehen sie sich meist auf jene Länder des globalen Südens, in denen schon während der Kolonialzeit Sport im Sinne der Disziplinierung, (Um)Erziehung und vermeintlichen Zivilisierung eingesetzt wurde (Darnell 2012, 13) ${ }^{60}$ Empowerment im entwicklungspolitischen Diskurs meint, zugespitzt formuliert, häufig die einseitige Befähigung rassifizierter und vergeschlechtlicher Subjekte zur Selbstregierung (vgl. Sharma 2008).

Festzuhalten bleibt, dass von einem Aussagensystem innerhalb der gegenwärtigen diskursiven Formationen ausgegangen werden kann, das Sport im Allgemeinen und den Fußball als männliche Sportart im Besonderen als eine bildende und selbstbildende Tätigkeit konstituiert und unter dem prominenten Schlagwort Empowerment subsumiert. ${ }^{61}$ Dabei wird nicht zuletzt aufgrund der unterschiedlichen sprachlichen Kontexte mit jeweils eigenen SprechTraditionen mit unterschiedlichen Bezeichnungen auf die Aussage rekurriert: Während im feministisch-wissenschaftlichen Kontext von verändertem Körper-Selbst-Bewusstsein und Selbstbehauptung die Rede ist, wird in entwicklungspolitischen Kontexten von Befähigung und der Vermittlung von neoliberalen Kernkompetenzen gesprochen. Gemeinsam ist beiden Kontexten die Konstitution von Sport als körperliche Tätigkeit mit charakterbildenden Effekten speziell für als förderungsbedürftig identifizierte Mädchen und Frauen, die das eigentliche Sporttreiben weit überschreiten. Diese Repräsentation von Sport ist dabei eng an die Konzepte der liberalen Demokratie und pluralen Gesellschaft geknüpft, die unterschiedlichen individuellen und gruppenbezogenen Interessen gleiche Gültigkeit zusichern. Der moderne Sport mit seiner Sport-für-Alle-Philosophie wird darin als Fortschritt konstituiert, der die Befreiung immer weiterer sozialer Gruppen symbolisiert (Hargreaves 1994, 9).

Trotz der verschieden eichnungen, Bedeutungen und Praktiken ist in der Praxis zu, beobachten en Bez wi edie unterschiedlichen Stränge des Aussagensystems wieder miteinander verwachsen, etwa, wenn feministische Wissenschaftler_innen zusammen mit Akteur_innen internationaler Sport-forDevelopment-Organisationen Konferenzen organisieren. Es ist daher anzu-

60 Für eine Kritik am neo-kolonialen Impetus der Sport-for-Development-Programme, die indigene Sport- und Bewegungsspiele verdrängen, siehe Giulianotti (2004).

61 Im Verständnis von Empowerment als charakterliche Entfaltung verbergen sich wirkmächtige Sedimente des bürgerlichen Sportverständnisses. Dieses bezeichnete vor allem nach dem Zweiten Weltkrieg den Sport als Bereich, in dem man noch ,ganz Menschen' sein, seinen Spieltrieb ausleben könnte; er biete Ausgleich und Freiheit von einer Gesellschaft, die körperliche Bewegung immer mehr einschränke (Krüger 2004, 25). 
nehmen, dass der Begriff als verbindendes Element fungiert, unter dem verschiedene Bedeutungen, unterschiedliche Programme und Sportpraktiken zusammenkommen. Diese hohe semantische Flexibilität ermöglicht größtmögliche Schnittmengen und Synergien zwischen den einzelnen Institutionen und Akteur-Konstellationen und verdichtet sie zu einem Dispositiv: Für die Stabilität der Aussage „Fußball empowert Mädchen und Frauen“ spricht, dass sie trotz interner Differenzen und Kritiken sowohl vom Verbandssport, inter/transnationalen wie staatlichen Verwaltungen der Frauen- und Entwicklungspolitik sowie von feministischen Sportinitiativen und Sozial- wie Kulturwissenschaften geteilt wird. Die Konstitution von Fußball als sozialisierend-bildend und als befähigend und „empowernd“ fügt sich sowohl in das Sportverständnis der Verbände und Vereine als auch in frauenbewegte und feministische Forderungen und Programme ein und gibt gleichzeitig transnationalen und staatlichen Institutionen ein Politikinstrument an die Hand. Die Kritik am Empowerment-Begriff zielt zwar auf die postkolonialen Implikationen des Aussagensystems, stellt aber nicht die Konstitution des Sports als befähigend und charakterbildend in Frage. Diese bleibt merkwürdig unangetastet, sodass ich im Folgenden nach einer potentiellen Gegen-Aussage frage.

\subsection{3 „Sport hat keine charakterbildende Wirkung“ - eine Gegen- Aussage?}

„Sport hat keinerlei charakterbildende oder erzieherische Wirkung“ - wie vernünftig klingt ein solcher Satz? Nur wenige sind in ihren Schlussfolgerungen so radikal wie Christopher Stevenson: „There is no valid evidence that participation in sport causes any verifiable socialization effects", vielmehr gehörten diese Annahmen ,in the realm of ,belief' and should not be treated as ,fact"“ $(1975,299$, zit. n. Fletcher/St. Pierre 2014, 93). Pilz (2013), der bereits das Gewaltpotential des Sports ausformulierte, empfiehlt zwar mit Verweis auf Längsschnittstudien zur Zurückhaltung, sobald es um Aussagen über erzieherische und persönlichkeitsbildende Wirkungen von regelmäßigem Sporttreiben geht. Doch rät er in der gleichen Publikation immer wieder zur pädagogischen Begleitung des jugendlichen Sporttreibens zwecks Gewaltprävention und Entfaltung positiver sozialisierender Effekte. Bausinger hingegen bezweifelt nicht, dass der Sport bildende Wirkung habe, sondern warnt lediglich vor deren Überhöhung:

„Weniger auffällig, aber doch ziemlich häufig ist der Versuch, sportliche Leistung nicht nur als Teil der Persönlichkeitsentfaltung zu nehmen, sondern als Ferment der Persönlichkeitsbildung. Ich zweifle nicht an der Berechtigung dieses Ansatzes. Aber man sollte meines Erachtens die Fermentwirkung, die Übertragbarkeit sportlicher Leistungserfahrungen auf andere (Leistungs-)Bereiche nicht überschätzen: nicht jeder, dem nach dem Langlauf die Zunge heraushängt, ist deshalb schon Einstein.“ (2006, 134) 
Ob mit pädagogischer Nachhilfe oder aus sich selbst heraus - das Erlernen sozial relevanter und erwünschter Eigenschaften und Fähigkeiten durch den Sport sowie deren Übertragbarkeit auf andere Lebensbereiche scheinen weder Sport-, Sozial- und Kulturwissenschaften noch Verbandssport oder Politik grundsätzlich anzuzweifeln (vgl. Brunn 2016). Sollte die Übertragung nicht glücken, werden die Ursachen in den Begleitumständen gesucht - nicht jedoch im Wesen des Sports begründet. Die Behauptung, durch Sport würde man nichts außer Sportlichkeit - Technik, Regelwerk, Kondition, Geschick oder Schnelligkeit - lernen, und diese böte keinerlei Vorteile in anderen Lebensbereichen, scheint mir keine Akzeptabilität zu besitzen, sodass ich hier wie auch im Fall der verbindenden Kraft des Sports von dem Fehlen einer intelligiblen Gegen-Aussage ausgehe. Hier haben sich wirkmächtige Macht/Wissen-Regime gebildet, die verschiedene Akteur_innen, administrative Tätigkeiten, Politiken und Aussageereignisse verdichten und Sport allgemein und Fußball im Besonderen auf spezifische Weise hervorbringen. Natürlich ist dies eine vorsichtige Vermutung, die es weiterhin diskursanalytisch zu überprüfen gilt.

\section{Zwischenstand: ein Momentum für Fußball und Feminismus}

Das fünfte Kapitel widmete sich den gegenwärtigen diskursiven Dimensionen des Fußballs und analysierte, wie der Fußball als verbindende, integrative und charakterbildende, empowernde Tätigkeit imaginiert und konstituiert und zugleich seine politische Neutralität zunehmend in Frage gestellt wird. Dabei hat sich herauskristallisiert, dass die in diesem Moment produzierten Wahrheiten weit über den nationalen Kontext hinausgehen und über Differenzen hinweg sehr unterschiedliche Akteur-Konstellationen zu machtvollen Dispositiven verdichten. Vor dem Hintergrund der historischen Entwicklungen bilden die diskursiven Formierungen an der Schnittfläche von Fußball und Feminismus ein komplexes und spannungsreiches Gefüge, das produktiv sowohl konflikthaft als auch komplementierend - die männliche Dominanz im Fußball, die sich in der Kultur des Vereinswesens und Wettkampfsports festigt, hinterfragbar- und kritisierbar macht.

Der historische Blick hat gezeigt, wie die Geschlechterverhältnisse des Fußballs stets Konjunkturen der Stabilität und Durchlässigkeit durchliefen. Diese wurden nicht nur durch vereins- und verbandsinterne Kämpfe, etwa in Form des Widerstandes von Fußballerinnen gegen das Frauenfußballverbot oder gegen gesonderte Spielregeln, beeinflusst, sondern auch durch Entwicklungen in anderen gesellschaftlichen Bereichen wie der staatlichen Sportför- 
derung, der zivilgesellschaftlichen Frauenbewegungen oder der Marktwirtschaft. Der Diskurs um Empowerment durch Fußball bietet jenen feministischen und außen-, innen- und entwicklungspolitischen Projekten gegenwärtig die Basis sowie die Argumentationsressourcen, um sich trotz Differenzen zur Problematisierung der männlichen Vorherrschaft im Fußball zu verbinden. Zugleich versammelt der Diskurs um Fußball als verbindende und friedensfördernde Kraft die gleichen politischen Institutionen, Fußballvereine und -verbände sowie Soziale Arbeit und erlaubt die Instrumentalisierung des Fußballs für soziale und kulturelle Zwecke. Die Stabilität dieser Aussagensysteme zu einem Dispositiv und ihrer Ermöglichungen sowie die zunehmende Akzeptabilität der Aussage „Fußball ist politisch“ gilt es im Hinterkopf zu behalten, da sie Legitimation und Plausibilisierung feministischer Interventionen in den Fußball bereitstellen. Zugleich deute ich die (erneute) öffentliche Infragestellung der politischen Neutralität des Fußballs als Hinweis auf die gegenwärtige Instabilität des verbandssportlichen, bürgerlichen Dispositivs, das Fußball als autonomen und politisch neutralen Bereich konstituiert.

Diese Brüchigkeit einerseits und die Stabilisierungen andererseits ermöglichen es, dass die fußballerischen Geschlechterverhältnisse etwa um die Jahrtausendwende ihre Selbstverständlichkeit verlieren: Die internationale zweite Frauenbewegung sowie die Frauenrechtskommission der UN etablierten Frauenrechte und Gleichstellung als zentrale politische Themen, vor denen sich auch der Sport nicht verschließen konnte; Fußballerinnen forderten auch durch Regelverstöße ihre gleichberechtigte Teilhabe am Fußball ein; staatliche und inter-/transnationale Institutionen entdeckten die Frau als Zielgruppe sowie den Sport als Mittel ihrer Politiken; der DFB hob die Förderung des Frauenfußballs wie auch den Kampf gegen Diskriminierungen im Fußball auf seine Agenda und machte die Weltmeisterinnenschaft 2011 in Deutschland zu einem großen Spektakel; und bis heute entstehen immer mehr zivilgesellschaftliche Initiativen zur Stärkung von Frauen und zur Bekämpfung von Homophobie und Sexismus im Fußball.

Dieses plötzliche Aufeinandertreffen von gesellschaftlichen Bereichen und ihren historischen Sedimenten, differenten Diskursen, Rationalitäten und Akteur_innen habe ich mit Tsing als Friction theoretisiert (2005): als ein spannungsreiches und produktives Moment mit unklarem Ausgang. Das holprige Aufeinandertreffen von Fußball und Feminismus lässt Widersprüche sichtbar werden und unterbricht die alltägliche, reibungslose Produktion fußballerischer Geschlechterverhältnisse. Die Ermöglichungen, die aus den historischen Entwicklungen innerhalb der fußballerischen Geschlechterverhältnisse erwachsen sind, die diskursiven Formierungen sowie die Einflussmöglichkeiten anderer gesellschaftlicher Bereiche auf den Fußball lassen mich schlussfolgern, dass dieses spannungsreichen Aufeinandertreffen nun die feministische Problematisierung fußballerischer Geschlechterverhältnisse 
öffentlich und effektiv ermöglicht und plausibilisiert. Dabei treffen unterschiedliche Rationalitäten und Politiken aufeinander, die in sich selbst auch widersprüchlich sein können, wenn z.B. Feministinnen körperliche Selbstbehauptung und Selbstwertgefühl anstreben und internationale Entwicklungsprojekte unter dem gleichen Schlagwort des Empowerments das Erlernen neoliberaler Wirtschaftspraktiken forcieren. Dieses Aufeinandertreffen verläuft nicht unweigerlich konfrontativ, wie im Fall des Streits um die politische Verwobenheit des Sports. Oft werden widersprüchliche Logiken in alltäglichen Praktiken in Kompromisse überführt, kreativ miteinander verflochten und neu bzw. weiterentwickelt (vgl. Verran 2010).

Wenn ich im Folgenden diese Friction als günstiges bzw. begünstigendes Moment bezeichne, dann beziehe ich mich sowohl auf das produktive Aufeinandertreffen, das im Sinne eines Momentums Kräfte erzeugt und etwas in Bewegung setzt, als auch auf den zeitlich bestimmbaren Moment, in dem verschiedene begünstigender Umstände zusammentreffen und sich ein Möglichkeitsfenster eröffnet. Der Terminus des günstigen Moments soll nicht darüber hinwegtäuschen, dass jene ermöglichenden Umstände und erzeugten Bewegungen auch zugleich restringierend wirken. Wie ausgeführt, ist der Ausgang eines solchen Zusammentreffens nicht beliebig, vielmehr strukturieren historische Sedimente mögliche Pfade vor und grenzen sie ein. Damit zeichnet sich bereits ab, was ich später noch ausführlich untersuche: Jede Problematisierung impliziert bereits potentielle Lösungen und schließt andere aus (vgl. Klöppel 2010). Die Politisierung der fußballerischen Geschlechterverhältnisse konstituiert diese als Gegenstand staatlicher Verwaltungen und trans-/internationaler politischer Institutionen, die zivilgesellschaftliche Organisationen, Vereine und Verbände sodann mit der Umsetzung, sprich mit der Bearbeitung des Problems betrauen. Diese Bearbeitung verläuft entsprechend gültiger Paradigmen liberaler Geschlechterpolitik, die auf Gleichberechtigung und gleiche Teilhabe innerhalb der bestehenden Verbandsstrukturen abzielen. Andere Lösungen, wie z.B. die Etablierung eigenständiger Frauenverbände und Frauensportspiele fallen damit aus dem Set an Lösungen heraus. Innerhalb dieses produktiven und unvorhersehbaren, zugleich aber begrenzten und vorstrukturierten günstigen Moments können neue Akteur_innen, Diskurse und Politiken entstehen, sodass ich im Folgenden die Aufmerksamkeit darauf richte, wie sich eine Organisation als kompetente Bearbeiterin formiert (Teil II), um schließlich geschlechterpolitisch handelnd in das den Fußball zu intervenieren (Teil III). 


\section{Teil II: Die Formierung einer Organisation}

Im Frühjahr 2009 veranstaltet die Heinrich-Böll-Stiftung eine Diskussionsrunde zum Thema Fußball - Integration - Geschlechterrollen und lädt als Diskutant innen den damaligen Präsidenten des DFB und mächtigsten Mann im deutschen Fuß̄ßball, Theo Zwanziger, die Leiterin des Muslimischen Frauenbildungswerks Köln und Vorstandsmitglied der Türkisch-Islamischen Union der Anstalt für Religion, Ayten Kiliçarslan, die Publizistin Hilal Sezgin sowie den Grünen-Politiker Omid Nouripour ein. Anlass ist der kurz zuvor erschienene und auf der Berlinale mehrfach ausgezeichnete Dokumentarfilm Football Under Cover (Najafi/Assmann 2008), der eine internationale Fußballbegegnung dokumentiert: Ein multikulturelles Kreuzberger FrauenTeam reist in den Iran, um dort gegen die Frauen-Nationalmannschaft zu spielen. Der Clou dabei: Die iranischen Frauen durften aufgrund der strengen Geschlechterpolitik bislang noch kein einziges öffentliches Spiel im eigenen Land bestreiten. Der Film dokumentiert die lange und komplizierte Vorgeschichte, in der das ganze Projekt immer wieder zu scheitern drohte. Doch schließlich war es soweit: In langen Trikots und mit Kopftüchern liefen die Kreuzberger und Teheraner Spielerinnen auf den Rasen auf. Auf den Rängen wurden sie ausschließlich von Frauen beklatscht, denn heute wurden die Stadionregeln ausnahmsweise einmal umgedreht: Männer mussten draußen bleiben. Die Frauen auf den Rängen jubelten, sangen und feuerten die Teams an, und sie kritisierten in ihren Gesängen trotz der überall lauernden Sittenwächterinnen die Geschlechterpolitik des iranischen Regimes. Im anschließenden Gespräch in der Heinrich-Böll-Stiftung zeigen sich die Diskutant innen beeindruckt vom Film. In seinem Kommentar zum Film bemerkt Zwanziger: „,Da gibt es echte Kameradschaft und Völkerfreundschaft” und er appelliert: „Wir müssen diesen Sport dafür benutzen, von Rassismus und Diskriminierung weg zu kommen“. Mit auf dem Podium sitzen jetzt auch der iranisch-deutsche Regisseur und die deutsche Protagonistin des Films. Als Letztere von der Veranstaltung erfahren hatte, war sie aus allen Wolken gefallen. Noch vor einem Jahr hätte sie sich nicht träumen lassen, so viel Aufmerksamkeit für ihren Sport zu erhalten und gar den DFB-Präsidenten persönlich kennenzulernen. Auf dem Podium diskutiert sie gemeinsam mit den anderen Gästen über die Hindernisse, die Fußballerinnen aufgrund ihres Geschlechts im Iran, aber auch in Deutschland und anderswo in den Weg gelegt werden. Ein Thema ist dabei das gescheiterte Rückspiel: Als sich die Iranerinnen auf die Reise nach Berlin begeben wollten, wurde ihnen diese in letzter Minute mit dem Verweis auf technische Probleme verwehrt. Das geplante Rückspiel in Berlin musste kurzerhand ausfallen. Beim Stehempfang im Anschluss an die Discussion kommt die Kreuzberger Spielerin mit Zwanziger ins Gespräch und wird von ihm zu weiteren Aktivitäten ermutigt. ${ }^{62}$

Diese Situation suggeriert: Die Geschlechterverhältnisse des Fußballs sind ein Problem. Dieser Rückblick in die Anfangszeiten der Frauenfußballorganisation Discover Football zeigt, dass die Schlechterstellung von Frauen im Fußball zu einer öffentlich thematisierbaren Angelegenheit geworden war und Personen aus ganz unterschiedlichen Bereichen versammelte und Diskurse miteinander verknüpft. In dieser Veranstaltung materialisiert sich damit staltungsbericht der Heinrich-Böll-Stiftung (Gülfirat 2009). 
jenes Moment, das die Problematisierung der fußballerischen Geschlechterverhältnisse ermöglicht. Hier wird das Aufeinandertreffen der verschiedenen Entwicklungen, wie ich sie im vorangegangenen Teil nachzeichnete, in einer konkreten Situation als Friction (Tsing 2005) sichtbar: Eine politische Stiftung lud den höchsten Mann im deutschen Fußball, zivilgesellschaftliche Akteurinnen aus den Bereichen Kultur, Religion und Frauenpolitik sowie einen Außenpolitiker ein, damit sie gemeinsam über Frauenrechte und die gesellschaftliche Verantwortung des Fußballs diskutierten. Der damalige DFB-Präsident repräsentierte die Brüchigkeit in den fußballerischen Geschlechterverhältnissen, als er öffentlich Diskriminierung im Fußball tadelte und darunter - wie der Kontext der Veranstaltung vermuten lässt - auch (Hetero-)Sexismus subsumierte. Dass auch die Politik den Fußball als Thema für sich entdeckt hatten, wird in der Anwesenheit des Außenpolitikers Nouripour ersichtlich, der am Ende der Veranstaltung sogar seine Hilfsbereitschaft ausdrückte, um den Iranerinnen die Reise zum Rückspiel nach Berlin zu ermöglichen. In der gemeinsamen Diskussion bestätigten die Diskutant innen die verbindende und charakterbildende Wirkung des Fußballs und stellten zugleich seine politische Neutralität in Frage, indem sie die fußballerischen Geschlechterverhältnisse zu einem Politikum erklärten, sie als Problem benannten, das der Bearbeitung durch eine Koalition aus Politik und Verwaltung, Verbandsfußball und Zivilgesellschaft bedürfe. Da ich den diskursiven Kontext bereits ausführlich in Kapitel 5 beleuchtet habe, gehe ich nicht weiter auf die Inhalte der Diskussion ein. Stattdessen gilt meine Aufmerksamkeit der Berliner Spielerin und Protagonistin des Dokumentarfilms, die mich zur ihren Kreuzberger Teamkolleginnen und schließlich zur der aus diesem Team heraus gegründeten Frauenfußball- und Frauenrechtsorganisation Discover Football (DF) führt. Ich frage in diesem Teil danach, wie sich aus dem günstigen Moment heraus eine Organisation formiert und an der komplexen Schnittstelle von Fußball und Feminismus als Bearbeiterin der Geschlechterverhältnisse legitimiert. Dem stelle ich Überlegungen voran, wie sich zivilgesellschaftliche Organisationen theoretisieren lassen.

\section{Theoretische Ausgangspunkte: Organisationen und ihre multiplen Gestalten}

Die beschriebene Situation in der Heinrich-Böll-Stiftung als Materialisierung der Friction um Fußball und Feminismus zu verstehen, ermöglicht, ihre produktiven Effekte zu fassen. Ich verstehe die Entstehung von zivilgesellschaftlichen Organisationen, die die verschiedenen Dimensionen dieses Moments auf unterschiedliche Weise miteinander verknüpfen und an der Bearbeitung 
der Geschlechterverhältnisse mitwirken, als einen möglichen produktiven Effekt. Organisationen, auch wenn sie sich mit dem Versprechen formieren Lösungen zu bieten, erscheinen so nicht als Problemlöserinnen, sondern sind analytisch als Orte und Praktiken des Problematisierens zu denken (vgl. Hodžić 2017, xi). Indem ich zeige, was der beschriebenen Situation vorausging und was auf sie folgte, stelle ich DF als Beispiel für eine solch neu entstandene Organisation vor und untersuche, wie sie zu einer gewissen Stabilität gelangte. Die Beschreibung und Analyse der Entstehung und der gegenwärtigen Arbeitspraxis von DF dient zugleich einem ausführlichen Einblick in mein Forschungsfeld.

Ich verfolge dabei die Frage, wie sich eine Organisation innerhalb eines günstigen, aber komplexen Moments als kompetente Bearbeiterin der Geschlechterverhältnisse formiert. Dementsprechend liegt der Fokus auf den Praktiken der Herstellung von Kompetenz und Gestalt. Die bisherigen Ausführungen haben nahegelegt, dass eine Vielzahl von verbandsfußballerischen, staatlich-verwaltenden wie auch zivilgesellschaftlichen Institutionen mit unterschiedlichen Problemauffassungen, Zielen und Lösungsvorstellungen an der Problematisierung beteiligt sind. Ich gehe also davon aus, dass sich hier verschiedene Wissensbestände und damit unterschiedliche Vorstellungen, was eine kompetente Organisation ausmacht, begegnen. Mit dem Begriff der landscape of practices bieten Beverly und Etienne Wenger-Trayner (2015) ein Konzept zur Beschreibung der aufeinandertreffenden Erwartungen und Anforderungen, mit denen eine Organisation konfrontiert wird und im Rahmen derer sie sich formiert und bewegt. Um als kompetente und legitime Problembearbeiterin innerhalb der Landschaft aus unterschiedlichen communities of practice - oder in der Sprache internationaler Organisationen: Stakeholder $^{63}$ - anerkannt und damit handlungsfähig zu werden, bedarf es des Erlernens und Versammelns heterogenen Wissens. Wissen bezeichnet hier das inkorporierte Know-how in Form von vorreflektivem Können und Verstehen, wie es Praxistheorien als grundlegend für das erfolgreiche Navigieren im Alltag ausformulieren:

„Jede Praktik und jeder Komplex von Praktiken - vom Zähneputzen bis zur Führung eines Unternehmens, von der Partnerschaft bis zur Verhandlung zwischen Konfliktparteien - bringt sehr spezifische Formen eines praktischen Wissens zum Ausdruck und setzt dieses bei den Trägern der Praktik voraus. Beim Vollzug einer Praktik

„Stakeholder“ bezeichnet all jene Personen oder Personengruppen, die ein deutliches Interesse an einem bestimmten Prozess und seines Ausgangs haben, da auch für sie etwas (nicht unbedingt ein materieller Einsatz) auf dem Spiel steht. Im Folgenden verwende ich diesen Begriff aus Mangel an adäquaten deutschen Alternativen und beziehe mich damit auf verschiedene, hier als communities of practice beschriebenen Institutionen, Öffentlichkeiten und Personengruppen. Die Stärke des Stakeholder-Begriffs liegt darin, dass er die Teilhabe verschiedener Akteur innen an Prozessen betont und damit dem hier beschriebenen Moment des Versammelns gerecht wird. Mein Fokus liegt dabei auf den jeweiligen Kompetenz- und Anerkennungsregimen und weniger auf den einzelnen Interessen. 
kommen implizite soziale Kriterien zum Einsatz, mit denen sich die Akteure in der jeweiligen Praktik eine entsprechende ,Sinnwelt' schaffen, in denen Gegenstände und Personen eine implizit gewusste Bedeutung besitzen, und mit denen sie umgehen, um routinemäßig angemessen zu handeln." (Reckwitz 2003, 292) ${ }^{64}$

Wissen ist dabei generativ für soziale Praktiken - für beobachtbare, routinierte Handlungsvollzüge wie für nach innen gerichtete Prozesse des Fühlens, Planens, Erinnerns oder Reflektierens (ebd., 296). In Zusammenführung mit dem emergenten Charakter der Friction verstehe ich jene landscapes nicht als fest und unbeweglich, sondern als komplexe Gefüge, die stets in Bewegung sind, sodass das entsprechende Wissen auch mit Unsicherheiten und Unvorhersehbarkeiten umgehen muss (vgl. Vigh 2009). Ich frage im Folgenden also, welches Wissen erlernt und angewendet wird und wie dieses die Organisation innerhalb einer sich wandelnden Landschaft mittels sozialer Praktiken als kompetent konstituiert.

Diese Perspektive deutet bereits ein prozessuales Verständnis von Organisationen an, welches ich zunächst ausgehend von Beobachtungen in meinem Forschungsfeld ausbuchstabieren möchte. Während meiner Forschung bemerkte ich, wie schwer es allen Mitgliedern fiel, eine klassifizierende Bezeichnung für Discover Football zu finden: So war von „Projekt“, „Verein“, „NGO“, „Organisation“ oder „Initiative“ die Rede. In den meisten Fällen wurde schlicht der Eigenname verwendet. Ähnlich den Selbstbeschreibungen meiner Forschungspartner_innen hat auch die Forschungsliteratur immer wieder den Versuch unternommen, Formen zivilgesellschaftlicher Zusammenschlüsse zu klassifizieren - als soziale Bewegungen, Nichtregierungsorganisationen (NGOs), transnationale Netzwerke oder temporäre Proteste. ${ }^{65}$ Arjun Appadurai bemerkt jedoch, dass es sich bei den ,emergent social forms“ einer globalen Zivilgesellschaft um hybride Formen - ,part movements, part networks, part organizations“ - handele (2000, 15; vgl. Edelman 2005, 29). Aus den progressiven Bewegungen der letzten 200 Jahre, wie der ersten Frauenrechts-, der Arbeiter- und der Bürgerrechtsbewegung, haben

64 Der Wissensbegriff der Praxistheorie unterscheidet sich vom Foucault'schen Wissensbegriff, wie er im vorangegangenen Kapitel kurz Erwähnung fand. Foucault beschreibt mit Wissen ,alle Erkenntnisverfahren und -wirkungen [...], die in einem bestimmten Moment und in einem bestimmten Gebiet akzeptabel sind" (Foucault 1992, 32). Der Wissensbegriff hat bei Foucault eine rein methodologisch-analytische Funktion, er will kein empirisches Phänomen beschreiben, sondern analytisch fassbar machen, was zu einem gewissen Zeitpunkt als akzeptiert, als wahr gilt (ebd., 32f.). Der Wissensbegriff der Praxistheorie hingegen bezieht sich nicht nur auf das Gespür für Richtiges und Falsches, sondern auch auf die inkorporierten Fähigkeiten, Handlungsroutinen vorreflektiv auszuführen.

$65 \mathrm{Zu}$ einer aktuellen europäisch-ethnologischen Diskussion netzwerkförmiger zivilgesellschaftlicher Vergemeinschaftungen siehe Schönberger (2014). Er beschreibt diese neue Form von Protesten als Korrelate der Arbeits- und Lebensbedingungen im Postfordismus mit flachen Hierarchien, demokratischer und bürokratiearmer Organisation, die aus eher flüchtigen und weniger nachhaltigen Projekten bestehen und sich damit von den identitätspolitischen Bewegungen der 1960er Jahre unterscheiden. 
sich in den letzten Jahrzehnten in Form von NGOs neue Institutionen gebildet. Aber auch unter diesem Akronym versammelt sich eine Vielfalt an Organisationsformen und -strukturen sowie an Zivilgesellschaft-StaatBeziehungen mit sehr unterschiedlichen Einflussmöglichkeiten und Aktionsradien (Appadurai 2000, 15). Sie haben komplexe Beziehungen mit dem Staat, der Öffentlichkeit, anderen internationalen zivilgesellschaftlichen Institutionen und lokalen Communities; manchmal greifen sie staatliche Politiken und Institutionen direkt und konfrontativ an, manchmal kooperieren sie mit ihnen; manche von ihnen können viel ökonomisches Kapital versammeln, hunderte Hauptamtliche in vielen verschiedenen Ländern beschäftigen und zu einflussreichen politischen Kräften avancieren, andere hingegen arbeiten ehrenamtlich, haben kaum Einfluss, aber genießen lokale Legitimität (Hilhorst 2003, 15). Aufgrund des hybriden Charakters gegenwärtiger Organisationsformen verzichte ich auf eine weitere Einordnung entlang soziologischer Kategorien und bezeichne DF lediglich als zivilgesellschaftliche Organisation, da diese Bezeichnung recht offen gefasst ist und gleichzeitig auf eine gewisse Stabilität verweist. Als zivilgesellschaftlich beschreibe ich jene Ebene zwischen Staat, inter- und transnationalen Regierungsorganen einerseits und Familie bzw. Haushalt andererseits, auf der Vereine und Verbände, nicht-staatliche und Non-Profit-Organisationen und Interessensinitiativen angesiedelt sind. ${ }^{66}$

Der Verzicht auf weitere Klassifikationen ebnet sodann den Weg für ein prozessuales Verständnis von Organisationen, mit dem die Unschärfen, Gleichzeitigkeiten und Widersprüche innerhalb und zwischen den Klassifikationen analytisch fruchtbar gemacht werden können. Wie DF zwischen unterschiedlichen Selbstbezeichnungen hin- und herwechselte, so positionieren sich auch andere Organisation situativ unterschiedlich (vgl. Sharma 2014, 98f.). Organisationen verstehe ich daher im Anschluss an Hilhorst nicht als Entitäten mit festen Strukturen, die es nur zu beschreiben gilt, sondern als kontinuierliche Prozesse des Sich-Organisierens und Sich-Formierens. In den Fokus rückt dann das Wirken von Diskursen und lokalen, nationalen und globalen Verflechtungen innerhalb von Organisationen sowie auf ihre Gestalten, Werte und Praktiken (Hilhorst 2003, 215). Gerade in jenem günstigen Moment, in dem verschiedene Kompetenzregime unterschiedliche, teils widersprüchliche Erwartungen und Aufforderungen formulieren, gehe ich da-

66 Eine klare Trennung von Staat und nicht-staatlichen Organisationsformen ist auf-grund der rahmengestaltenden staatlichen Kompetenzen wie Vereinsrecht und Förderpolitiken nicht haltbar. Umstritten ist auch, inwiefern Markt und Unternehmen dem zivilgesellschaftlichen Bereich zugeordnet oder aus ihm ausgeklammert werden sollen (Edelman 2005, 30). Ich schließe mich der andernorts vorgeschlagenen Aus-klammerung an, um analytisch zwischen marktwirtschaftlicher und zivilgesellschaftlicher Logik trennen zu können, und verstehe Zivilgesellschaft ausschließlich als einen Bereich des Vereins- und Verbandswesens, nicht-staatlicher und Non-Profit-Organisationen und Interessensinitiativen. Zur anthropologischen Auseinandersetzung mit dem Begriff der Zivilgesellschaft siehe Ferguson (2004). 
von aus, dass eine Organisationsformierung nicht auf eine einheitliche, stabile Gestalt hinausläuft.

Im Folgenden untersuche ich die Praktiken der Organisationsformierung ausgehend von meiner Feldforschung bei Discover Football. Ich frage, wie welche Praktiken des Sich-Organisierens situativ und entsprechend der unterschiedlichen Kompetenzregime spezifisch Organisationsgestalten hervorbringen - „how ,NGO-ing' is done“ (Hilhorst 2003, 5). ${ }^{67}$ Dafür folgt jedes Kapitel den Aktivist_innen hin zu einem für ihren Alltag relevanten Stakeholder und diskutiert die verschiedenen Kompetenzanforderungen und die darauf reagierenden Organisationspraktiken. Ich schließe mit Überlegungen, die Konstitution als kompetente Problembearbeiterin als einen kontinuierlichen Prozess des Sich-Organisierens in jener heterogenen Landschaft $\mathrm{zu}$ verstehen, in dem zivilgesellschaftliche Akteure mannigfaltige Gestalten situativ annehmen und zwischen ihnen in einem solchen Radius changieren, dass sie zugleich eine gewisse Stabilität erlangen.

\section{Rechtskräftiger Verein und verlässliche Partnerorganisation - Verwaltungen und Projektförderung}

Staatliche Verwaltungen und Fördereinrichtungen, wie beispielsweise Stiftungen, formulieren entsprechend den Förderauflagen und Verwaltungsprozessen spezifische Anforderungen an Organisationen. Hier zeige ich, wie sich die Fußballerinnen Schritt für Schritt entlang dieser Anerkennungslogik und immer in Rücksprache mit eigenen Idealen als rechtsfähiger Verein (8.1) und verlässliche Partnerorganisation (8.2) formierten, und analysiere diese Praktiken schließlich als Produkte eines modernisiert-bürgerlichen Habitus (8.3). Eng mit der Institutionalisierung und Professionalisierung der Organisationsstruktur und Arbeitsweise verknüpft ist auch eine inhaltliche Politisierung, auf die ich in Kapitel 11.1.1 eingehe.

67 Diese Fragestellung wird in Institutionenanalysen unter dem Stichwort der Accountability diskutiert. Brown et al. (2012) bemerken, dass alle NGOs mit Anforderungen vieler verschiedener Akteur innen - Gründer innen und Spender innen, Vorstand und Mitarbeitende, Regierungsorgane, andere NGOs und lokale Gruppen - sowie nationalen Erwartungen, Förderauflagen und vorherrschenden Wahrheiten umgehen müssen. 


\subsection{Ein Verein werden: „Es ist ja nicht nur Glaubwürdigkeit"“}

Zum Zeitpunkt der Veranstaltung in der Heinrich-Böll-Stiftung waren bereits drei Jahre seit dem Fußballspiel gegen das iranische Team vergangen. Inzwischen hatten sich eine feste Narration der fußballerischen Begegnung in Teheran und eine Idee für ein weiteres Projekt entwickelt. Als ich Birgit, eines der Gründungsmitglieder, nach den Anfängen fragte, leitete sie ihre Erzählung wie folgt ein: „Also, ich erzähl das immer so gleich.“ (Birgit, Interview, Dez. 2013) Diese eröffnenden Worte sowie die Beobachtung, dass die Gründungsgeschichte bei vielen und verschiedenen Anlässen immer wieder erzählt und direkt neuen Mitgliedern vermittelt wurde, gibt den Hinweis, dass sie einer festen Narration folgte und eine identitätsstiftende Funktion erfüllte. Dies disqualifiziert sie jedoch nicht als Quelle, um etwas über die Vorgeschichte zu erfahren. ${ }^{68} \mathrm{Im}$ Rahmen des im November 2012 organisierten Expertinnenforums mit Funktionärinnen und engagierten Spielerinnen aus der MENA-Region besuchte DF mit den Teilnehmerinnen einen Workshop zum Thema Fundraising durch Crowdfunding. Dort lernten wir, dass eine Gründungsgeschichte einer Organisation zu einem individuellen Gesicht verhilft, es macht das oft abstrakt formulierte Anliegen greifbar und potentiellen Spender_innen ein Identifikationsangebot. $\mathrm{Zu}$ diesem Zeitpunkt hatte sich die Geschichte der Iranreise bereits als feste Narration, von selbst ${ }^{6}$ - also ohne explizite, strategische Intention - etabliert und bildete erste Fundamente einer sich im Laufe der Jahre weiter stabilisierenden Identität.

\subsubsection{Eine einmalige Gelegenheit}

In einem Zeitschrifteninterview erzählte Birgit die Entstehung des FrauenFußball-Kultur-Festivals wie folgt:

„Das Turnier [das Festival] ergibt sich aus der Vorgeschichte. Es begann ganz klein: Wir haben (um Sponsoren zu suchen) einen Kurzfilm über unser Team gemacht. Doch dann lief alles ganz anders, der Film lief im Rahmen der Berlinale mit anderen Filmen zum Thema ,Fußball und Kultur' und das mit den Sponsoren wurde nicht weiter verfolgt. Bei dieser Veranstaltung gab's nur einen anderen Film mit Frauenfußball, der war iranisch, und wir haben uns nach der Vorführung mit dem Regisseur unterhalten und erfahren, dass die Frau aus seinem Film die Kapitänin der Nationalmannschaft ist und das Team noch nie gegen ein anderes Team gespielt hat, aber regelmäßig trainiert. Wir haben sofort gesagt, dass wir kommen und gegen sie spielen und wussten nicht, von was wir da sprachen. Das Spiel zu organisieren hat mehr als ein Jahr gedauert, aber was wir erlebt haben, hat alle unsere Träume überboten. Es chungsgegenstand als auch Informationsquelle siehe Breidenstein et al. (2015, 83f.). 
war erstens ein tolles Spiel und zweitens eine unglaubliche Erfahrung. Frauen dürfen in Iran nicht ins Stadion, doch an diesem Tag war die Regel andersrum. Auf dem Platz, an der Linie, auf der Tribüne, im VIP-Bereich, alle waren Frauen und haben gezeigt, dass sie wissen, wie man sich im Stadion verhält. Und alle waren gleich nervös, wir natürlich, die iranischen Spielerinnen auch, aber auch die Schiedsrichterin, die bisher nur in der Halle gepfiffen hat, und die Journalistinnen und leider auch die Sicherheitskräfte [...]. Wir haben im Laufe des Prozesses Spielerinnen kennengelernt, mit denen wir erstaunlich viel gemeinsam hatten, obwohl wir in so unterschiedlichen Bedingungen leben und eben Fußball spielen. Es war von Anfang an ein Projekt mit Hin- und Rückspiel. Doch das Rückspiel wurde enttäuschender Weise am Tag vorher abgesagt, die Iranerinnen waren schon am Flughafen, als die Reise nicht weiterging, wir erfuhren, dass sie wegen technischer Probleme nicht ausreisen dürfen. Die Berichterstattung wechselte vom Sport- oder Kulturteil in den Politikteil. Und wir hatten zu viel erlebt, um aufzuhören. Und außerdem hatten wir auch einen Film über das Spiel gemacht und hatten plötzlich Aufmerksamkeit und die Möglichkeit, Unterstützung zu bekommen. Denn bis dahin ging es auf eigenes Risiko und eigenes Geld. Wir wollten so etwas wie die Absage nicht wieder erleben und die Idee vom Turnier [dem Festival] entstand.“ (E-Mail, 16.7.2013)

Wie in dem Interview endete auch bei anderen Gelegenheiten die Narration mit der Initiative zu einem neuen Projekt, dem Festival. Durch ethnografische Interviews erfuhr ich, wie es weiterging. Die Vorführung des Dokumentarfilms Football Under Cover in Kinos und auf internationalen Festivals wurde zum entscheidenden Ereignis. Die Dokumentation löste ein großes Medienecho aus und plötzlich sahen sich die Fußballerinnen im Fokus öffentlicher Aufmerksamkeit. Dies geschah zu einem Zeitpunkt, als Zwanziger Präsident des DFB war und sich bereits mehrmals öffentlich als großer Fan und Förderer des Frauenfußballs bekannt hatte. Nun stand er vor der Aufgabe, gemeinsam mit seinem Verband die Weltmeisterinnenschaft 2011 in Deutschland auszutragen.

Nach dem abgesagten Rückspiel traf sich eine Handvoll der Spielerinnen, um über ein Folgeprojekt nachzudenken. Birgit erinnerte sich, wie sich durch die ungewohnte Aufmerksamkeit eine einmalige Gelegenheit bot:

„Und dann gab's eine Veranstaltung in der Heinrich-Böll-Stiftung, wo Theo Zwanziger einen Kommentar zu Football Under Cover geben sollte. Da dachten wir: ,Das kann ja gar nicht sein!' Aber haben dann wahnsinnig viele Hoffnungen darein gelegt und haben gedacht: ,Vielleicht fragt er ja, was unser nächstes Projekt ist'. Ist ja immer, wenn man irgendwo ist und über was spricht, dann wird man ja immer über die Zukunft gefragt. Und dann haben wir uns vorher ein paar Mal getroffen und haben uns überlegt, was unser nächstes Projekt sein könnte und vielleicht geht da ja was.“ (Birgit, Interview, Dez. 2013)

Mit Blick auf das kulturelle Rahmenprogramm der anstehenden WM planten sie das erste Frauen-Fußball-Kultur-Festival, in dessen Mittelpunkt ein Fußballturnier mit sozial und politisch engagierten Frauenteams aus der ganzen Welt stand, und stellten diese Projektidee dem damaligen DFB-Präsidenten auf der Stiftungsveranstaltung vor: 
„Und er hat gefragt, ob wir noch ein anderes Projekt haben. Da haben wir gesagt: ,Ja, so ein Turnier". Da hat er gesagt: ,Das machen wir über die Kulturstiftung [des DFB]. Und wir sollen uns dann noch mal an ihn wenden. Und so kam es; wir hatten total viel Lust, was zu machen, und die Möglichkeiten wurden immer besser." (ebd.)

Die Spielerinnen wussten, sie müssten die unerwartete Chance nutzen. Die Erfolge des professionellen Frauenfußballs sowie die Bestrebungen des Dachverbands, sich mit einer gelungenen Sportgroßveranstaltung zu profilieren, entfalteten hier Breitenwirkung, die bis auf die Amateurebene ausstrahlte. Die günstigen Umstände ließen die Idee zu einem größeren Folgeprojekt entstehen und die Spielerinnen setzten auf den geschickten Gebrauch der Zeit und machten einen Schritt nach dem anderen.

\subsubsection{Schritt für Schritt planen}

Auf die Entwicklung der Idee folgte 2009 die konkrete Planung. Auch hier wurde Zeit als bestimmender Faktor deutlich, wie Annike, ein anderes Gründungsmitglied, erinnerte:

„Es sollte einen Bezug geben zu Football Under Cover, weil der Film auch in dem Moment im Kino war. Es war einfach sehr präsent und viele haben davon gehört und die Verbindung sollte klar sein. [...] Sylvia hat das erst gesagt, dann hat das allen eingeleuchtet und dann hat sie als erstes ,Discover Football' vorgeschlagen. [...] Danach haben wir noch mal noch so überlegt, aber eigentlich gar nicht mehr lange, weil wir das Gefühl hatten, wir brauchen jetzt ganz bald was. Also vielleicht so eine halbe Stunde. Und ich hab in der Woche die Domäne www.discoverfootball.de schon mal klargemacht. Dass uns das jetzt niemand mehr wegnehmen kann." (Annike, Interview, 21.11.2013)

Annike und Sylvia verwiesen beide auf die Dringlichkeit des richtigen Augenblicks: Die Aufmerksamkeit durch den Film vergrößerte den Spielraum, der jedoch durch die Schnelllebigkeit der Ereignisse gefährdet war. Der nächste Schritt musste schnell erfolgen und durch die Namensgebung - von „Football Under Cover“ zu „Discover Football“ - wurde die zeitliche Abfolge in einen Sinnzusammenhang von Iranspiel, Dokumentarfilm und Folgeprojekt verwandelt. Als kurz darauf die finanzielle Förderung durch die DFBKulturstiftung in Aussicht stand, folgte die Vereinsgründung:

„Auf jeden Fall war es klar, wenn wir mit Finanzen zu tun haben und irgendwie glaubwürdig sein wollen - es ist ja nicht nur Glaubwürdigkeit -, wir brauchen unsere eigenen Strukturen. Und dann haben wir unseren Verein gegründet, in dem wir Geld selbstständig verwalten können.“ (Birgit, Interview, Dez. 2013)

Die Entwicklung und Vorstellung neuer Projektideen, deren Kohärenz schaffende Benennung sowie die Vereinsgründung unter dem Namen „Fußball 
und Begegnung e.V. " ${ }^{69}$ waren taktische Reaktionen auf sich plötzlich öffnende Möglichkeitsräume und von diesen abhängig. Ihre Glaubwürdigkeit als kompetente Veranstalterin eines Fußballfestivals schien zunächst von der Organisationsform und der damit zusammenhängenden Fähigkeit der Mittelverwaltung abzuhängen. So verwandelte der Status als eingetragener Verein die Gruppe von Spielerinnen in eine juristische Person und rechtsfähige Körperschaft und der Gemeinnützigkeitsstatus befähigte zum Empfangen und Verwalten öffentlicher Gelder.

\subsubsection{Organisation-Sein lernen}

Die Formierung als kompetente Organisation gegenüber öffentlichen Fördereinrichtungen, die mit der Durchführung öffentlicher Aufgaben betraut werden kann, erscheint als ein gradueller Lernprozess: Ob sich intuitiv eine Gründungsnarration etablierte, ob die Spielerinnen aufgrund vorheriger Erfahrungen nun nach einer eigenständigen Rechtsform strebten oder ob sie auf Erfahrungen mit dem Ablauf von Veranstaltungen zurückgriffen (,Ist ja immer, wenn man irgendwo ist und über was spricht, dann wird man ja immer über die Zukunft gefragt"): Sie wandten praktisches Wissen an und lernten Neues dazu. ${ }^{70}$

„Doch es ist ja nicht nur Glaubwürdigkeit“, sondern auch die Schaffung von ,eigenen Strukturen“: In den schnellen und taktischen Schritten waren bereits stabilisierende Effekte enthalten. Annike sicherte die InternetDomäne, die Vereinsgründung ermöglichte die Akquise und Verwaltung von Geldern über einen längeren, wenn auch aufgrund der Projektierung ${ }^{71}$ der Arbeit begrenzten Zeitraum, die Namensgebung und die Etablierung einer eigenen Geschichte bildeten Grundsteine für eine Gruppenidentität.

Dieses taktische, schrittweise Vorgehen im Zusammenspiel mit den begünstigenden Umständen der gegenwärtigen Friction brachte die Gruppe als gemeinnützigen eingetragenen Verein hervor: die personellen Entwicklungen innerhalb des DFB, die bevorstehende Weltmeisterinnenschaft und die damit

69 „Fußball und Begegnung“ ist der offizielle Vereinsname, „DISCOVER FOOTBALL“ bezeichnete ursprünglich das konkrete Festival-Projekt, hat sich jedoch im Sprachgebrauch als Gesamtbezeichnung durchgesetzt.

70 Mekata (2000) beschreibt eine ähnliche Entwicklung: Die International Campaign to Ban Landmines konnte, nachdem sie mit dem Friedensnobelpreis ausgezeichnet wurde, erst ein Jahr später das Preisgeld ausbezahlt bekommen, da sie als loses, horizontales Netzwerk nirgendwo offiziell registriert war und weder Bankkonto noch Adresse besaß.

71 Der bevorzugte Fördermodus öffentlicher Geldgeber ist die finanzielle Unterstützung von konkreten Projekten statt längerfristiger Investitionen in organisatorische Infrastrukturen, denn dies verspricht schnellere und besser sichtbare Ergebnisse. Organisationen finden sich dann mit Lohnzahlungen, Verwaltungs- und Mietkosten allein gelassen (Thayer 2010, 129). Projektierung erschwert zudem die Entwicklung und Verfolgung langfristiger Ziele und umfassender sozialer Transformationen (Appadurai 2004, 73). 
einhergehende zunehmende Förderung des Frauenfußballs sowie damit verbundener kulturell-gesellschaftlicher Veranstaltungen; der Erfolg des Dokumentarfilms, der aktuelle globalpolitische wie frauenrechtliche Themen verhandelte und mit dem Fußball verknüpfte; sowie das mediale Interesse und die Aufmerksamkeit von Politiker_innen und verschiedenen zivilgesellschaftlichen Akteur_innen.

Dass sich eine Problembearbeiterin in der Gestalt einer gemeinnützigen, nicht-staatlichen Organisation formierte, erscheint zudem vor dem Hintergrund der veränderten Regierungsweise der öffentlichen Reformverwaltung plausibel. Seit den 1980er Jahren ist in Deutschland - wenn auch nicht so ausgeprägt wie z.B. in Großbritannien - eine Entwicklung in der Beziehung zwischen staatlichen Verwaltungsorganen und Zivilgesellschaft zu beobachten: Staatliche Verwaltungen ziehen sich zunehmend aus der Erfüllung öffentlicher Aufgaben zurück und übertragen diese zivilgesellschaftlichen und marktwirtschaftlichen Kräften. Diese Entwicklung wird vom Paradigma neoliberaler Ökonomie sowie liberaler demokratischer Theorie angeleitet. Im Sinne neoliberaler Ökonomie gelten marktwirtschaftliche und private Initiativen als wirksamere Mechanismen, wirtschaftliches Wachstum zu ermöglichen und Menschen mit den nötigen Leistungen $\mathrm{zu}$ versorgen. Wo die Marktmechanismen nicht greifen, werden gemeinnützige, nicht profitorientierte Organisationen als Überbringerinnen von Sozialleistungen favorisiert (Edwards/Hulme 1996, 4). So zieht sich der Staat von seiner Aufgabe zurück, sozialen Ungleichheiten durch Umverteilung entgegenzuwirken (Speed 2005, 35 ), und NGOs ,have become normalized as key players in national and global politics“" (Bernal/Grewal 2014, 1). Zugleich hat sich unter dem Paradigma liberaldemokratischer Theorie und mit dem Ende des Kalten Krieges eine Sicht auf NGOs und Graswurzelorganisationen (NGO, GRO) als Motoren der Demokratisierung und einer erstarkenden Zivilgesellschaft etabliert:

„NGOs and GROs are supposed to act as counter-weight to state power - protecting human rights, opening up channels of communication and participation, providing training grounds for activists and promoting pluralism." (Edwards/Hulme 1996, 4)

Aktivist_innen begrüßen gemeinnützige, nichtstaatliche Organisationen als potentielle neue Instrumente, mit denen dominante politische Orthodoxien durch alternative Ideen und neue Formen von Entwicklung, advocacy und kollektiven Aktionen herausgefordert werden können. Und internationale Entwicklungsinstitutionen wie die Weltbank haben diese als flexiblere Instrumente des Good Governance entdeckt, auf die sie Serviceleistungen auslagern können (Schuller/Lewis 2017, 635f.). Von Staaten wird erwartet, dass sie diese Regierungsformen durch die Schaffung entsprechender Rahmenbedingungen und die Bereitstellung finanzieller Mittel ermöglichen und ihre eigenen (sichtbaren) Interventionen minimieren (Edwards/Hulme 1996, 4). Die veränderten Bedingungen führten in den 1990er Jahren zu vielen Neugründungen und einer Stärkung bereits bestehender zivilgesellschaftlicher 
Organisationen, sodass Fisher für diese Zeit gar von einer ,associational revolution" spricht $(1997,441) .^{72}$ Dieses institutionelle Umfeld formuliert auch gegenwärtig Normen und gibt Strukturen vor, die Organisationsformierung entlang von Institutionalisierungs- und Professionalisierungsprozessen begünstigen, die ich im Folgenden beleuchte (vgl. Lang 2013, 72ff.).

\subsection{Arbeit institutionalisieren: „Ihr macht das unglaublich professionell"6}

Von Beginn an hatte sich die Gruppe dem Ideal der Basisdemokratie verschrieben, was in ihrem Fall bedeutete, dass Entscheidungen immer von allen gemeinsam im Plenum getroffen werden sollten und sich die Stellung der zwei bis drei hauptamtlichen Projektkoordinator_innen nicht von denen der Ehrenamtlichen unterschied. Kompetenzen, Verantwortungen und Zuständigkeiten waren auf unterschiedliche Mitglieder - unabhängig ob ehrenamtlich oder angestellt - verteilt. Auch als ich 2012 dazu kam, wurde mir diese flache Hierarchie und Gemeinschaftsverantwortung als Grundsatz des $\mathrm{Zu}$ sammenarbeitens vermittelt. Zwar investierten die Projektkoordinatorinnen mehr Arbeitszeit als die Ehrenamtlichen, jedoch wurden sämtliche als wichtig oder strittig erachteten Angelegenheiten im Plenum ausführlich diskutiert und gemeinsam entschieden. Dennoch hatte sich die Arbeitsweise in den fünf Jahren seit der Vereinsgründung langsam und kontinuierlich entsprechend externen und internen Umständen und Anforderungen verändert: Während die aus Projektmitteln finanzierten Koordinationsstellen in den Anfangsjahren mehrmals neu besetzt wurden, blieben sie bald von denselben zwei Personen besetzt. Gleichzeitig kam es unter den Ehrenamtlichen immer wieder zu Fluktuation - einige beendeten ihr Engagement aufgrund beruflicher Veränderungen, neuer Interessen, Unzufriedenheit mit den Gruppendynamiken oder des Wegzugs aus Berlin; Neue kamen dazu.

\subsubsection{Zuständigkeiten verteilen}

Mit der Verstetigung der Stellenbesetzung ging auch eine Veränderung im Arbeitsmodus der Organisation einher, wie ich von den Projektkoordinatorinnen erfuhr. Simone erklärte:

72 Unter dem Stichwort ,closing und shrinking spaces“ wird aktuell die weltweite Beschränkung zivilgesellschaftlicher Handlungsspielräume durch Regierungen diskutiert. Siehe bspw. Heinrich-Böll-Stiftung (2016). Von dieser Entwicklung war zur Zeit meiner Forschung in Deutschland (noch) nichts zu spüren. 
,[E]s hat sich auf jeden Fall geändert, finde ich, dass wir jetzt viel mehr Verantwortung tragen. Am Anfang [...] haben die Ehrenamtlichen viel mehr verantwortliche Sachen übernommen und waren viel mehr involviert. Ich glaube, weil es auch sonst nicht gelaufen wäre. Und heute gibt's halt jetzt schon so lange ein Büro mit zwei Leuten. Und früher haben sich, glaube ich, auch Leute viel mehr in andere Dinge reingedacht und die mitentschieden und heute machen wir ziemlich viel selbstständig.“ (Simone, Interview, Dez. 2013)

Saskia, die andere Projektkoordinatorin, deutete den Prozess als ,, Teil von so einer Professionalisierung, dass es eben klar ist, dass bestimmte Zuständigkeiten halt nicht von allen mitgetragen werden müssen ". Was sie als Professionalisierung begriff, umfasst die veränderte Arbeitsstruktur im Sinne der Verteilung von Zuständigkeiten und Verantwortungen, der Verstetigung von Arbeitsprozessen sowie der Bündelung von Wissen bei einzelnen Personen. In diesem Sinne nahm das, was ursprünglich als spontane Organisationsgruppe entstand, Züge von institutionalisierter Vereinsarbeit an. Wenn Simone dies damit begründet, dass es ,sonst nicht gelaufen wäre“, dann verweist sie damit auf zwei interdependente Prozesse der Professionalisierung: Zum einen luden die bürokratischen Pflichten eines eingetragenen, gemeinnützigen Vereins, die mit steigender Projektzahl wuchsen, DF auch mehr Verantwortung und Rechenschaftspflichten auf, sodass die Bündelung von Kompetenzen und Zuständigkeiten bei einzelnen Personen verlässliches Erledigen versprach. Zum anderen veränderte sich die Mitgliederstruktur: Viele beendeten ihr Studium, hatten durch feste Arbeitszeiten weniger Freizeit und konnten sich eher punktuell denn kontinuierlich engagieren, sodass klare, vom Büropersonal koordinierte Zuständigkeiten effizienteres Arbeiten ermöglichten.

Mit diesen Bündelungen von Zuständigkeiten, Wissen und Fähigkeiten etablierten sich auch informelle Hierarchien. Denn, wie Jo Freeman mit Blick auf radikal-feministische Frauengruppen in den USA moniert: ,'Structureles-

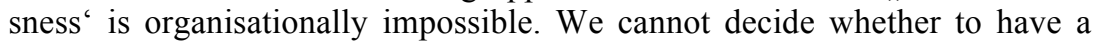
structured or structureless group; only whether or not to have a formally structured one.“ (1971, o.S.) Der Verzicht auf formelle Hierarchien führt nur zur Entstehung informeller, intransparenter Strukturen (ebd.), was sich auch bei DF andeutete. Hinter den Idealen von Egalität und Partizipation sicherte das Wissen darüber, was das Wesen von DF ausmache, bzw. das Einschätzungsvermögen darüber, was gut für DF sei, einzelnen Personen, die besonders lange oder intensiv mitarbeiteten, mehr Einflussmöglichkeiten.

\subsubsection{Professionell auftreten}

Die Institutionalisierung von Zuständigkeiten versprach nicht nur den Anforderungen an verlässliche und beständige Verwaltungsarbeit entsprechen zu können, sondern ermöglichte auch ein ,pprofessionelles“ öffentliches Auftreten. Eine geschickte Koordination der individuellen Fähigkeiten und Experti- 
sen kam der Projektarbeit zugute und resultierte in einem professionelleren Arbeitsmodus und Auftreten. Diese Entwicklung lobte 2015 der damalige Bundesminister des Inneren, Thomas de Maizière (CDU), als er, wie bereits fünf Jahre zuvor, das Frauen-Fußball-Kultur-Festival eröffnete:

„Ich war vor 5 Jahren schon mal hier, da war ich schon mal Innenminister. Und dieses Sportevent war ganz in den Anfängen, ein bisschen provisorisch aber liebevoll, was meistens heißt noch nicht so professionell. Aber es [die Botschaft, dass Fußball verbindet] kam rüber.“ (Feldnotiz 30.6.2015)

Der Minister verwies darauf, dass mit der internen Institutionalisierung von Zuständigkeiten eine nach außen hin sichtbare Professionalisierung einherging. Auch der damaligen Chefredakteurin der Tageszeitung taz fiel diese Professionalisierung auf, als sie 2015 eine von DF mitorganisierte Podiumsveranstaltung moderierte:

„Ihr macht das unglaublich professionell, wie ihr E-Mails beantwortet, Kooperationen macht, die T-Shirts gestaltet. Da können sich hauptamtlich organisierte Vereine eine Scheibe von abschneiden." (Feldnotiz, 5.6.2015)

Professionalisierung meint nicht nur die strukturelle Anpassung der Arbeitsorganisation an die Bedingungen des Vereinsrechts und der öffentlichen Projektförderung, sondern auch einen sichtbaren Gestaltwandel weg vom Provisorischen und Improvisierten hin zu gekonntem Design, routinierter Verwaltungsarbeit und Veranstaltungsorganisation. ${ }^{73}$ Professionalität wird so als Produkt diverser Wissenspraktiken - vom T-Shirt-Designen bis zur adäquaten Kommunikation - erkennbar.

\subsubsection{Kulturelles Kapital}

Ein Blick auf die Bildungshintergründe der Mitglieder zeigt, welches Wissen bei DF zusammenfloss und wie dieses die Organisation als professionell gegenüber öffentlichen Fördereinrichtungen - das Bundesinnenministerium zählt zu einem der Hauptförderer von DF - und Kooperationspartner_innen die $t a z$ war bereits mehrfach Medienpartnerin - organisierte. Während meiner Forschung setzte sich die Gruppe aus anfänglich drei, später dann zwei Projektkoordinatorinnen und insgesamt 24 Ehrenamtlichen zusammen. Während vor meiner Zeit eine der Koordinationsstellen von einem Mann besetzt war, hatten sie während meiner Forschung durchgehend Frauen inne. In Phasen erhöhter Arbeitsintensität, etwa in den Monaten vor und während eines groBen Projekts, wurden Ehrenamtliche als studentische Hilfskräfte eingestellt oder als Honorarkräfte für besonders hohen Arbeitsaufwand entlohnt. Von

73 Das ,liebevolle“, sprich improvisierende Auftreten ist inzwischen in einem kohärenten und professionell entworfenen DIY-Design aufgegangen und damit zu einer ästhetischen Strategie geworden. 
den Mitgliedern verfügten 17 bereits über einen Universitäts-, eine über einen Fachhochschulabschluss und vier befanden sich noch in ihrem Bachelorstudium. Die große Mehrheit studierte Fächer der Kultur- und Sozialwissenschaften, nur eine hatte ein naturwissenschaftlich-mathematisches Studium abgeschlossen und zwei hatten kreativ-künstlerische Studiengänge gewählt. Die meisten waren viel gereist und sprachen mehrere Sprachen. Bei der Berufswahl bevorzugte die Mehrheit der Berufstätigen soziale Berufe oder die Tätigkeit in politischen Institutionen. Darüber hinaus gab es eine selbstständige Grafikdesignerin, eine Filmemacherin, eine wissenschaftliche Mitarbeiterin, eine Mitarbeiterin in einem großen, privatwirtschaftlichen Unternehmen und mit mir zwei Doktorandinnen. Unter den Ehrenamtlichen befand sich ein Mann. Alle anderen waren Frauen und Personen, die weiblich sozialisiert wurden, nun aber geschlechtliche Zuschreibungen vermeiden wollten. ${ }^{74}$

Der Arbeitsalltag bei DF lief vor allem im angemieteten Büroraum ab. Hier arbeiteten die Projektkoordinatorinnen täglich, hier fanden die regelmäBigen Plena statt, hier trafen sich meist die einzelnen Arbeitsgruppen für die Vorbereitung der Projekte. Ab und an besuchte ich die Mitarbeiterinnen spontan außerhalb offiziell angesetzter Treffen und fand dort häufig auch andere Ehrenamtliche vor. Dies machte mir immer wieder die vielen parallelen Arbeitsabläufe bewusst, die permanent in Kleingruppen verfolgt wurden.

Dieser kurze Einblick in die Mitgliederprofile und Arbeitsweisen der Organisation lässt bereits das breite Spektrum der verfügbaren Fähigkeiten erahnen. Die meisten DF-Mitglieder hatten sich durch Studium und Praktika, Nebenjobs oder Ehrenämter in Parteien oder Vereinen gute Artikulationsfähigkeiten und Wissen über komplexe politische Zusammenhänge angeeignet. Einige kannten sich detailliert in aktuellen feministischen Diskussionen und Politiken aus. Andere verfügten über großes Organisations- und Verhandlungsgeschick und wussten, wie politische und administrative Organe und Prozesse funktionierten. Wieder andere brachten gestalterische und technische Fähigkeiten bei Filmschnitt, Fotografie, Grafik und Videodreh mit, die eine professionelle Außendarstellung ermöglichten. Bourdieu hat für solche Formen methodischen Wissens den Begriff des inkorporierten kulturellen Kapitals geprägt, dessen Akkumulation Zeit kostet und personengebunden ist (1983, 185ff.; 1992a, 53). Zwar können Einzelne ihr persönliches Können und ihre Bildungstitel nicht an andere ausleihen oder gar weitergeben, doch

74 Siehe für zwei exemplarische Lebensläufe die Porträts von Gerit und Thea in Kap. 12. Um der geschlechtlichen Heterogenität der Gruppe gerecht zu werden, verwende ich im Folgenden den Unterstrich (gender gap). In manchen Situationen wechsle ich in die ausschließlich weibliche oder männliche Schreibweise, um zu verdeutlichen, dass diese innerhalb diskursiver und rechtlicher Kontexte stattfanden, die eindeutige Zuordnungen erforderten und in denen die Akteur_innen diesen nachkamen. Dabei schließe ich jedoch nicht aus, dass sich die entsprechenden Personen dabei jenseits der Zweigeschlechtlichkeit verorteten. Vielmehr versuche ich durch die wechselnde Schreibweise dem Konstruktionscharakter von Geschlecht gerecht werden. 
können sie sie in den Dienst der Gruppe stellen, da sie jene Sprache des ,university-policy nexus" (Appadurai 2000, 17) beherrschen, in der globale Probleme, Projekte und Politiken artikuliert werden. ${ }^{75}$ Aufgrund ihres hohen kulturellen Kapitals konnten die Mitglieder von DF wortwörtlich mitreden. Der Einsatz kulturellen Kapitals bei DF macht deutlich, dass es sich hierbei nicht nur um die Beherrschung entsprechenden Vokabulars zur Benennung politischer Ziele und Mittel handelt, sondern ebenfalls um die Art der Kommunikation - gewählte Kanäle, Anredeformen, den ,richtige Ton“ und angemessene Zeitpunkte - wie auch der Repräsentation.

\subsubsection{Vom kulturellen zum ökonomischen Kapital}

Das Erlernen und der Einsatz des adäquaten Vokabulars und Stils ermöglichen die Konversion von kulturellem in ökonomisches Kapital, wie folgendes Beispiel zeigt: Während der Vorbereitungen für das Festival 2013 wollte DF die Botschaften der Länder der teilnehmenden Teams um materielle Unterstützung bitten. Dafür wurde ein Brief formuliert und über den EMailverteiler an alle Mitglieder der entsprechenden Arbeitsgruppe zur Absegnung verschickt. Hier ein Auszug aus dieser E-Mail:

„[...] im Anhang schicke ich euch den Brief [...]. Damit unser Schreiben so offiziell und, wichtig' wie möglich klingt, soll es auf jeden Fall von der ,ranghöchsten' Person von DF unterschrieben sein - was natürlich bei unserer Art der Arbeit ein bisschen schwer zu definieren ist. Aber am Besten unterschreibt Saskia [eine der Projektkoordinatorinnen] als ,Projektmanagerin', oder gibt es im Verein noch einen Vorstand, der/die vielleicht sogar nen Doktortitel oder so was hat?! Zudem würde ich schon vorschlagen, dass auch die jeweilige Teambetreuerin das Schreiben unterzeichnet, damit klar wird, wer wirklich zuständig ist.“ (E-Mail, 23.05.2013)

75 Kulturelles Kapital materialisiert sich in Form von Büchern, Lexika oder Maschinen und institutionalisiert sich in Bildungstiteln und Zeugnissen. Bourdieu denkt den Kapitalbegriff instrumentell: Kapital dient der unmittelbaren Verfolgung von Interessen (1983; 1992a). Hier wie im weiteren Verlauf wird ersichtlich, dass kulturelles, ökonomisches, soziales und sportliches Kapital zwar immer wieder instrumentell eingesetzt wurde - etwa für das Schreiben von Anträgen -, aber zugleich auch mittelbare, unbeabsichtigte Effekte erzielte, bspw. wenn soziale Kontakte mit dem Ziel gegenseitiger Solidarität intensiviert wurden, zugleich aber das Verfügen über ein weltweites Netzwerk als wertvolle Ressource auf dem „social movement market“ (Thayer 2010, 130) funktioniert. Kulturelles Kapital ist zugleich erlernbar. DF fungierte für die Mitglieder als ein solcher Lernort. Waren sie häufig als Studentinnen mit wenig Vorkenntnissen gestartet, eigneten sie sich viele zentrale Fähigkeiten bei DF an. So professionalisierten sich Mitglieder und Organisation gegenseitig. Kulturelles Kapital kann nicht delegiert, wohl aber vererbt werden. Bourdieu argumentiert, dass der Erwerb kulturellen Kapitals maßgeblich von dem in der Familie bereits vorhandenen Kapital abhängt (1983, 187). Für die Mitglieder von DF gilt, dass die meisten aus bildungsbürgerlich geprägten Familien der Mittelschicht kamen. In wenigen Fällen waren sie in ihren Familien die erste Person mit Hochschulabschluss. 
Diese E-Mail verdeutlicht nicht nur, wie professionelles Auftreten als kompetente Partnerorganisation eine hierarchische Organisationsweise begünstigt, sondern auch, wie personengebundenes kulturelles Kapital (hier der Doktortitel) in den Dienst der gesamten Organisation gestellt wurde und in ökonomisches Kapital konvertiert werden sollte. DF finanzierte die Projektarbeit vorrangig maus öffentlichen Fördergeldern. $\mathrm{Zu}$ den größten und regelmäßigsten Förderern zählten das BMI, AA, die DFB-Kulturstiftung und gelegentlich auch die EU. Immer wieder beteiligten sich auch politische und private Stiftungen. Gelegentlich erhielt DF Spenden von Einzelpersonen oder politischen Organen, wie beispielsweise Bundestagsfraktionen, oder wurde mit Preisen ausgezeichnet. Zudem stellten Sponsoren Sachmittel zur Verfügung. Um an diese Förderungen zu gelangen, waren Kenntnisse über den Umgang mit Bürokratien, wie Abrechnungen und Mittelverwaltungen, die Kompetenz, komplexe Anträge im typischen „Sprech“ politischer Institutionen zu verfassen und die eigene Arbeit entsprechend den Preisausschreibungen darzustellen, die Fähigkeit und die Redegewandtheit, auf öffentlichen Podien und vor der Presse für die eigenen Anliegen zu werben, sowie die gestalterischen und technischen Fähigkeiten für professionelle Außendarstellung notwendig. Auf diesem Wege wurde kulturelles in ökonomisches Kapital konvertiert. ${ }^{76}$ Es sind diese Einrichtungen öffentlicher Verwaltung und politischer sowie zivilgesellschaftlicher Institutionen, die DF als kompetente und verlässliche zivilgesellschaftliche Organisation anerkannten und damit die Projektarbeit entscheidend mit ermöglichten.

Die obige E-Mail macht zudem deutlich, dass die Formierung als professionelle Organisation immer auch ein taktisches Balancieren zwischen eigenen ideellen Präferenzen und den Anforderungen der Umstände beinhaltete. Jens Adam (2016) hat hierfür den Begriff des ,utopisch unterlegten Pragmatismus" vorgeschlagen, der mir auch für den hiesigen Kontext passend erscheint. Er verdeutlicht, dass neben der pragmatischen Bereitschaft, sich entsprechend externer Bedingungen zu verändern, Kompromisse und Zugeständnisse immer nur im Rahmen des utopisch Vertretbaren geschehen. Gerade die utopischen und idealistischen Untermalungen ermöglichen eine gewisse Stabilität der Arbeitsweise, da sie den Spielraum für strategische und pragmatische Anpassungen begrenzen.

76 Die Wechselkurse zwischen den Kapitalsorten ist dabei mit Verlusten - „Schwundkosten“ (Bourdieu 1983, 198) - verbunden: Nicht selten waren investierte Zeit und Arbeit umsonst, und ein Antrag wurde abgelehnt oder ein Sponsorengesuch blieb unbeantwortet. Hieran wird ersichtlich, dass die Konversion der Kapitalien in ökonomisches Kapital, die letztendlich die Umsetzung der Projektarbeit erst ermöglicht, immer den Faktoren Zeit und Arbeit unterliegt und der hohe symbolische Wert des kulturellen und sozialen Kapitals nicht in vergleichbar hohes ökonomisches Kapital konvertiert werden kann. 


\subsection{Sich engagieren: „Selbstständige Köpfe und kräftige Hände“6}

Die spezifischen Bildungs- und Berufsprofile der Mitglieder, ihre Fähigkeiten und erlerntes Wissen zusammen mit der idealistischen Untermalung in Form von Basisdemokratie und Hierarchiefreiheit weisen auf ein spezifisches gesellschaftliches Milieu hin. Als inkorporiertes kulturelles Kapital geronnen die oben beschriebenen Fähigkeiten sowie die Ideale und Moralvorstellungen zum Habitus der einzelnen Mitglieder und der gesamten Gruppe: „Inkorporiertes Kapital ist ein Besitztum, das zu einem festen Bestandteil der ,Person', zum Habitus geworden ist; aus ,Haben' ist ,Sein' geworden.“ (Bourdieu 1983, 187) Mit dem Konzept des Habitus beschreibt Bourdieu das verkörperte Scharnier zwischen sozialer Struktur und Praxis. Als strukturierte Struktur (opus operatum) schreiben sich gesellschaftliche Logiken und Differenzierungen in Praxissinn und Körper der Akteur innen ein. Als strukturierende Struktur (modus operandi) generiert der Habitus soziale Praktiken und macht Akteur_innen so zu Reproduzent_innen sozialer Ordnungen. Der Habitus ermöglicht nicht nur Distinktionen gegenüber anderen Milieus, sondern auch Anerkennung von jenen, die die ihn anleitenden Logiken und Anerkennungsregime teilen. Habitus wie Kapital sind dabei immer relational zu anderen Habitusformen und sozialen Positionen zu denken (Bourdieu 1997, 277286). Der Habitus äußerte sich bei DF zweifelsohne in Kleidung und Frisuren, Wohnstilen und Beziehungsformen, in Ess- und Ausgehkultur, in Freizeitgestaltung sowie Sprech- und Arbeitsformen. Anhand Letzterer zeige ich im Folgenden, wie sich DF als bürgerschaftliche und bürgerliche Organisation generierte und stabilisierte.

\subsubsection{Modernisiert bürgerlicher Habitus}

Im Zuge der Vorbereitungen für das Festival 2015 wurden neue Ehrenamtliche gesucht und als „selbstständige Köpfe und kräftige Hände“ angesprochen:

„Ob du gerne Strategien entwickelst, übersetzt, dich für Bühnenprogramme, Frauen*kämpfe oder Fußballregeln begeisterst, oder ob du technisch interessiert bist wenn du Lust auf Arbeit im Kollektiv hast, bist du herzlich willkommen!" (E-Mail, 11.3.2015)

Der bei DF vorherrschende Arbeitsmodus erforderte Schnelligkeit, zupackende Eigeninitiative und Selbstständigkeit. Außerhalb der Arbeitstreffen wurden Augen und Ohren nach Fördermöglichkeiten, Projektpartnerschaften oder interessanten Kontakten offen gehalten, neue Ideen entwickelt und 
Netzwerke aufgebaut, die dann in die Vereinsarbeit eingebracht wurden. ${ }^{77}$ Plötzliche Entwicklungen innerhalb der komplexen landscape of practice erforderten, schnell zu reagieren. So musste auf die Einladung in die Heinrich-Böll-Stiftung zügig die Idee eines Folgeprojekts entwickelt werden, und als sich z.B. unerwartet ein Profifußballer als homosexuell outete und eine Stellungnahme in sozialen Medien die Möglichkeit bot, sich in dieser Debatte $\mathrm{zu}$ verorten, war Eile geboten. Es ist das Wissen um diese ,certain uncertainty“ (Vigh 2009, 422), mit der zivilgesellschaftliche, projektfinanzierte Initiativen im Kontext der öffentlichen Reformverwaltung konfrontiert werden, das sich in der alltäglichen Arbeitsweise niederschlug. Diese spezifische Arbeitsweise reproduzierte eine milieubezogene Homogenität, prägte den gruppenspezifischen Habitus und musste von neuen Mitgliedern erlernt werden, sodass die Mitarbeit eine Vertrautheit mit oder ein Feingespür für diesen Habitus voraussetzte.

Diese spezifische Arbeitsweise, die Bildungshintergründe in Form von Bildungstiteln, erlernten Fähigkeiten und Auslandsreisen sowie die generelle Bereitschaft zu und Wertschätzung von sozialem und politischem Engagement fügen sich in das von Wolfgang Kaschuba als „bürgerlich“ beschriebene Kulturmodell:

„Eben dies meint ,Bürgerlichkeit', verstanden als ein sozial bestimmter und kulturell geformter Habitus: ein in sich zwar vielfach abgestuftes und variiertes, in seinen Grundzügen jedoch verbindliches Kulturmodell, das entscheidende Momente sozialer Identität in sich birgt. Es vermittelt bürgerliches Selbstverständnis und Selbstbewußtsein, definiert durch den Gebrauch materieller Güter, durch den Bezug zu ideellen Werten, durch die Benutzung kultureller Verhaltensmuster, die zusammengenommen ein lebensweltliches Ensemble bilden.“ $(1995,101)$

Während sich Kaschuba auf historische Untersuchungen zum Bürgertum im 19. Jahrhundert bezieht, zeigt Stefan Wellgrafs Ethnografie zu Haupt- und Gymnasialschüler_innen sowohl die Aktualität und Stabilität der wesentlichen Grundzüge dieses Habitus als auch Spuren zeitlichen Wandels. Wellgraf beschreibt das sozialpolitische Engagement, die Betonung von Kreativität und Individualität bei Berliner Gymnasiast_innen im Vergleich zu Hauptschüler_innen als modernisierte Bürgerlichkeit, ,die bereits Elemente der linksalternativen Gegenbewegung der 1970er Jahre integriert" hat (2012, 151); eine Beschreibung, die - wenn auch in einer anderen Altersgruppe - zu

77 Wie selbstverständlich und habituell verankert dieser Arbeitsmodus war, wurde deutlich, als die Gruppe im Rahmen eines Fundraising-Workshops ihre bisherige Fundraising-Arbeit reflektieren sollte. In meinen Feldnotizen hielt ich unser Ergebnis fest: „Was die Fundraising-Strukturen angeht, so kommen wir überein, dass man bei uns zwar die Tätigkeiten nicht personell klar zuordnen könne. [....] Und das Fundraising sei so in der Gesamtgruppe verankert, dass Jede ihre Augen offenhielte und aufgreife und weiterleite, was zufällig kommt.“ (Feldnotiz, 21.4.2013) 
großen Teilen auf die Mitglieder von DF zutrifft. ${ }^{78}$ Schnell und spontan Aufgaben erledigen, an langen und diskussionsreichen Plenumstreffen unter der Woche teilnehmen sowie ehrenamtlich mehrtägige Projekte durchführen zu können, steht nur jenen Personen offen, die die benötigte Zeit haben, wie Studierende, Halbzeitkräfte und Selbstständige, aber auch Beschäftigte, die über die soziale Sicherheit verfügen, sich Auszeiten von der Lohnarbeit zu nehmen. So verbinden sich sozioökonomische Dispositionen mit einem akademischen Bildungsmilieu zu einem modernisiert-bürgerlichen Gruppenhabitus, der die Mitglieder mit dem nötigen emotional-motivationalen Wissen ausstattet, ehrenamtliches gesellschaftspolitisches Engagement als wichtig und sinnvoll $\mathrm{zu}$ evaluieren und damit verbundene Lebensgestaltungen $\mathrm{zu}$ wählen. Über den Habitus stabilisierte sich die Gruppe trotz interner Varianzen, und die Herausbildung einer Arbeitsweise sicherte das Gelingen ihrer Vorhaben. Zugleich organisierte sie sich so als Teil einer politisch engagierten und bürgerlich geprägten Zivilgesellschaft, die weit über die Grenzen Berlins hinausreicht.

\subsubsection{Weiß sein}

Diese interne Ähnlichkeit der Mitglieder bezieht sich auch auf ihre ethnischen Hintergründe, sodass vermutet werden kann, dass diese modernisierte Bürgerlichkeit nicht allein ein klassenspezifischer, sondern auch ein rassifizierter Habitus ist. Während der Gruppe die von ihnen reproduzierten Ausschlüsse bestimmter sozialer Gruppen durch die akademisierte Arbeitsweise durchaus bewusst waren und sie diese immer wieder selbstkritisch kommentierten, schien ihnen die interne Homogenität in Bezug auf Ethnizität weniger leicht erklärbar. Keines der langjährigen Mitglieder hatte einen Migrationshintergrund, erst später kamen Ehrenamtliche aus Großbritannien und den

78 Wellgraf (2012) zufolge dient das soziale Engagement der Schüler_innen während und nach dem Abitur vor allem der beruflichen Qualifikation und Selbstoptimierung. Für die Mitglieder von DF gilt dies nicht gleichermaßen. Sicherlich dient das spezifische Engagement der habituellen Selbst-Versicherung, wertet den eigenen Lebenslauf auf und begünstigt teilweise berufliche Perspektiven. Doch nicht selten wird die zeitliche Dosis an Engagement, die selbstoptimierend wirkt, von vielen weit überschritten. Sie verzichten zugunsten des Engagements auf Freizeit, Vollberuflichkeit oder einen schnellen Studienabschluss. Zwischen Selbstoptimierung und -aufopferung liegen vermutlich nur ein paar Wochenstunden. Dennoch ist die objektive Mehrung des eigenen Kapitals nicht zu übersehen. Bernal/Grewal bemerken mit Blick auf internationale feministische NGOs, dass durch ihre Arbeit häufig das eigene Personal gestärkt würde und weniger die lokalen Frauen, die laut Projektzielen die eigentliche Zielgruppe darstellten: „One of the accomplishments of the NGO form is creating a sector of ,empowered" middle-class women workers in the first world and the global South.“ (2014a, 307) Zum Habitus von NGO-Aktivist_innen siehe Lang $(2013,72)$. Zur Verwobenheit von Mittelschicht und politischem Protest siehe McAdam (1989), Gunn/Bell (2003), Ibrahim (2013). Zugleich zeigt Schöneberger (2014), dass diese Milieuspezifik nicht für alle Formen politischen Engagements gilt. 
USA hinzu. Stets war die Organisationsgruppe als rein weiße Gruppe erkennbar. Anja Weiß untersucht die Frage, warum explizit antirassistische Gruppen keine interne Heterogenität herzustellen vermögen. Mit Bourdieus Konzept der symbolischen Macht argumentiert sie, dass „sich kulturell erzeugte symbolische Unterschiede zu einer Ressource verfestigen“ (2001, 86), indem sie zu unterschiedlichen Handlungsstrategien gerinnen und sich in stabilen Ungleichheitsbeziehungen zementieren. Weiß versteht diese Ressource als Kapitalsorte, wonach sich ein Ungleichheitsverhältnis zwischen von Rassismus Betroffenen und Nicht-Betroffenen ergibt (ebd., 90). ${ }^{79} \mathrm{Ob}-$ gleich DF keine explizit antirassistische Organisation war, zeigten sich die Mitglieder sensibel gegenüber Eurozentrismus, globalen, postkolonialen Ungleichheiten und gesellschaftlichem Rassismus und drückten immer wieder ihren Wunsch aus, diese Differenzierungsmechanismen nicht zu reproduzieren oder gar zu verstärken. Dass es dennoch zu rassistischen Effekten kommen kann, erklärt Weiß mit dem Habitus als ,,verinnerlichte, verkörperlichte und praktische Perspektive auf die Welt" (ebd., 97), die nicht bewusst geplant ist, sondern aus den spezifischen Erfahrungen und erlebten Möglichkeiten resultiert, die Weiße als rassistisch Nicht-Betroffene machen. In diesen schreiben sich rassistische Ungleichheitsstrukturen ein und können so von den Akteur_innen trotz der Bemühungen, rassistische Diskurse aktiv zu vermeiden, mittels Praktiken unbemerkt reproduziert werden. Dies äußert sich konkret in feinen Unterschieden in den Sprech- und Diskussionsweisen über manche Themen, in der Bewertung und Gewichtung einzelner Aspekte oder in den Umgangsweisen mit einzelnen Personen. Weiß' Analyse zeichnet also den Habitus jener Akteur_innen, die selbst nicht von Rassismus betroffen sind, für die Gruppenhomogenität verantwortlich.

Hieran wird ersichtlich, wie der Habitus der Mitglieder eine Arbeitsweise hervorbrachte und DF als eine modernisiert-bürgerliche, weiße Gruppe stabilisierte. Da es, außer „wenn jemand jetzt rechts ist oder total sexistisch“ (Feldnotiz, Mai 2013), keine expliziten Ausschlüsse von Personengruppen und keinen offensichtlichen oder ideologisch begründeten Klassismus oder Rassismus gab, kann die Distinktion gegenüber anderen sozialen und rassifizierten Milieus und Gruppen in den "Strukturen, Routinen und Diskursen“ (Weiß 2013, 17) der alltäglichen Arbeitspraxis vermutet werden. Zugleich ermöglichte die häufige Thematisierung des eigenen Weiß-Seins sowie das hohe Bewusstsein für Rassismen, Sexismen und Heterosexismen sich in einer spezifischen politisch-ideologischen und intellektuellen Szene innerhalb einer translokalen sowie städtischen Zivilgesellschaft verortete. 


\section{Städtische Sportveranstaltung und feministische Initiative - Stadt und Szene}

Der modernisiert-bürgerliche Habitus ist nicht nur typisch für eine transnationale Zivilgesellschaft, sondern auch Produkt einer stadträumlichen Umwelt, in der er gleichzeitig eine Lokalisierung ermöglicht. DF war in der Groß- und Hauptstadt Berlin ansässig. Hier fand die alltägliche Vereinsarbeit statt, hier wurden die meisten Projekte durchgeführt, hier interagierte die Gruppe mit anderen Institutionen und hier materialisierten sich die diskursiven Formationen des Fußballs sowie die politischen Ideale und Moralvorstellungen. Durch Verortung in Szene und Stadtteil konstituierte sich DF als feministische Initiative (9.1) und wurde durch erfolgreiche stadträumliche Navigation zur festen Sportveranstalterin im Bezirk (9.2).

\subsection{Sich stadträumlich verorten: „In the heart of Kreuzberg“6}

Bürgerlichkeit als differenziertes Kulturmodell ist eng an die Stadt als Sozialraum gebunden:

„Die Stadt als Wiege von Sozialität wie von Individualität - und eine sozial gebundene Individualität wiederum als Kern von Bürgerlichkeit: Diese beiden Bilder verbinden sich hier in genuiner Weise." (Kaschuba 2010, 86)

Es ist also jene urbane Öffentlichkeit, die die Herausbildung eines modernisierten bürgerlichen Habitus erst ermöglicht, ihn mitprägt und ihm Variationsmöglichkeiten bietet. Die explizite Verortung im Berliner Stadtraum machte aus dem frauen- und sportpolitischen Projekt weit mehr als eine bloße ehrenamtliche Tätigkeit, es war Teil eines progressiven, urbanen Lebensstils und Lebensgefühls, wie Dominik Kleinen und Cornelia Kühn formulieren:

„Urbanität steht für Dynamiken und Entwicklungsprozesse, für Gestaltungsfreiräume und Kreativität und für einen neuen Modus des Lebens und Arbeitens, der sich bewusst von einer lokal verankerten und oft als provinziell wahrgenommenen Lebensweise abgrenzen will.“ $(2016,7)$

Auf die Distinktionseffekte des bürgerlichen, urbanen Habitus werde ich später in Bezug zum Verbandsfußball eingehen. Zunächst frage ich nach der Formierung der Organisation im spezifischen Stadtraum, denn wie kaum eine 
andere soziale Umwelt erlaubt die Großstadt die Ausdifferenzierung in Szenen mit eigenen Stilen. ${ }^{80}$

\subsubsection{Inmitten Kreuzbergs politischer Utopien}

Barbara Lang hat gezeigt, wie sich Szenen an Stadtteilen orientieren:

„Berlin-Kreuzberg: Räumliche Materialisation des Chaos und Aussteiger-Utopie, Alternative zur bundesrepublikanischen Norm und bunte Exotik, spannender Erlebnisraum und schließlich Zentrum der neuen Metropole Berlin. Aus diesen Bildern von und Diskursen über den Bezirk konstituiert sich das Bedeutungssystem ,Kreuzberg'." $(1998,182)$

Mit diesen Worten fasst sie den „Mythos Kreuzberg“ zusammen und umschreibt damit die städtische Bedeutungslandschaft, in der sich spezifische Lebensstile verorten und herausbilden. In den zwei Jahren meiner Forschung musste das DF-Büro drei Mal umziehen, wobei nur Büroräume in Kreuzberg und ab 2014 im angrenzenden nördlichen Neukölln, das durch seine geläufige Bezeichnung als Kreuzkölln bereits eine Nähe zu Kreuzberg behauptet, in Frage kamen. Und DF bewarb das Frauen-Fußball-Kultur-Festival international als ,in the heart of Kreuzberg“ (Discover Football 2013a) stattfindend. Vor dem Hintergrund von Langs Untersuchung lässt sich erahnen, dass es sich hierbei nicht nur um eine territoriale Verortung handelte, sondern auch um eine ideelle. Auch wenn das vom „Mythos Kreuzberg“ transportierte Stadteilimage als exotische Enklave für alternative Lebensentwürfe, Aussteigertum und politischen Widerstand in den Jahren nach der Wende einem drastischen kulturellen Wandel und Gentrifizierungsprozessen unterlag, scheint dieser Mythos weiterhin ein „Fernweh nach Utopia“ bei jenen zu wecken, die nach politischen Alternativen und „Räumen, die sich durch ihre spezifische Anti-Struktur charakterisieren“ (Lang 1998, 237), streben und sich in verschiedene Subkulturen und Szenen ausdifferenzieren. ${ }^{81}$

80 Da Szenen auf eine größtmögliche Zahl an Menschen angewiesen sind, die ihren Lebensstil teilen, sind sie häufig an Städte gebunden (Haunss 2004, 257). Urbane Szenen im Sinne posttraditionaler Vergemeinschaftungen zeichnen sich als durch milieu- und schichtübergreifende Zusammenschlüsse von Personen aus (vgl. Schwanhäußer 2010). Ich verstehe mit Schwanhäußer Szene als Vergemeinschaftung, die auf geteiltem Wissen über Codes und der Annahme gemeinsamer gesellschaftspolitischer Überzeugungen basiert und sich an konkreten Orten und mithilfe bestimmter Symbole manifestiert. Es ist nicht notwendig, dass sich alle Szenemitglieder kennen, sondern dass sie sich erkennen können.

81 Ich schließe nicht aus, dass ein solches Frauenfußballprojekt nicht auch in anderen Städten oder Stadtteilen möglich wäre. Doch vermute ich, dass der ideelle Stadtraum die Entstehung und Umsetzung utopisch anmutender Ideen begünstigt. 


\subsubsection{Szenisch-spezifische Arbeits- und Kommunikationsstile}

Neben Basisdemokratie ist auch ein bestimmter Kommunikationsstil Ausdruck der urban-szenischen Ausdifferenzierung und ideellen Ausrichtung des Gruppenhabitus. In ihren lokalen szenespezifischen Bezügen wiesen die einzelnen Mitglieder eine erstaunliche Heterogenität auf: Einige verorteten sich eher im links-radikalen oder radikal-feministischen Bereich, andere in einem grün-alternativen oder sozialdemokratischen Milieu, und einige führten Lebensstile des kreativen Kulturprekariats. ${ }^{82}$ Dennoch schienen sie immer wieder einen gemeinsamen Nenner $\mathrm{zu}$ finden, den ich hier als linksalternative Bewegungsszene umschreibe. Mit dem Begriff der Bewegungsszene beschreibt Sebastian Haunss den Nexus aus sozialer Bewegung und urbaner Szene als Ort, an dem verschiedene Subkulturen und Lebensstile in einer sozialen Bewegung aufeinandertreffen und sich gegenseitig beeinflussen. So ist die Szeneinfrastruktur zum einen das Ergebnis von Bewegungsaktivitäten, zum anderen dient sie den politischen Aktivitäten als Mobilisierungsrepertoire $(2004,255)$. Auch DF bildete einen solchen Nexus aus urbaner, links-alternativer Szene und politischen Aktivitäten, der sich beispielsweise in von allen frequentierten Lokalen, in gemeinsamen politischen Einschätzungen und einer spezifischen Sprechweise ausdrückt. Erinnert sei an den Aufruf zur ehrenamtlichen Mitarbeit und die spezifische Schreibweise von „Frauen*kämpfe“: Das Sternchen $(*)$ entspricht der Markierung einer Fußnote, in der ein konstruktivistisches Verständnis der Kategorie Frau erklärt und diese dadurch auch für andere nicht-binärgeschlechtliche Positionen geöffnet werden soll. Da die Fußnote in der E-Mail jedoch ausblieb, verwies die Markierung auf Wissen, welches nicht erklärt werden musste, sondern vorausgesetzt werden konnte. Es ist das Wissen über eine zur Norm geronnene Vorstellung korrekten Sprechens und Denkens über Geschlecht, die auf intellektuellen Diskursen über Geschlecht basiert und damit einen hohen Bildungsgrad in bestimmten Fächern als kommunikative Grundlage voraussetzt. So gilt auch hier: „Wer die kulturellen Regeln nicht beherrscht, wird durch sie ausgeschlossen" (Kaschuba 1995, 100) oder eines Besseren belehrt. Wie in der Thematisierung des eigenen Weiß-Seins anklang, gesellte sich zu Geschlecht und Sexualität auch race als relevante Kategorie sozialer Ungleichheit sowie Vorstellungen eines richtigen Umgangs mit dem eigenen Weiß-Sein.

82 Diese Diversität führte selbstverständlich immer wieder zu Meinungsverschiedenheiten und Diskussionen. Bemerkenswert ist, dass diese Heterogenität in der alltäglichen Projektarbeit trotz mancher Unstimmigkeiten viele Schnittmengen und Konsensfähigkeiten aufwies. Dies stützt die Annahme von einem gemeinsamen Nenner, der nicht genau gefasst werden kann und von einem Mitglied mir einmal als ,,irgendwie so links“ sowohl in Bezug auf politische Meinungen als auch städtische Szenen beschrieben wurde. 
Wie in Kapitel 2.3.3 erwähnt, formulierte das spezifisch städtischszenische Umfeld im Einklang mit den eigenen Idealen der Aktivist_innen den moralischen Imperativ, Ungleichheiten und ausschließende Normativitäten in der aktivistischen Arbeit zu vermeiden. Die Einhaltung dieser kulturellen Regeln ermöglichte auch die Anerkennung von einem spezifischen ,politischen beziehungsweise politisierten Feld zivilgesellschaftlichen Aktivismus und beruflicher Tätigkeit", das sich spätestens mit der Institutionalisierung der Gender Studies und dem Eintritt der Absolvent innen in zivilgesellschaftliche Arbeitsfelder etabliert hatte (Binder 2013, $\overline{8}$ ). Dadurch, dass die dort vorherrschenden Vorstellungen von moralisch gutem Aktivismus mit den eigenen Überzeugungen überlappten, organisierte sich DF innerhalb dieser urbanen, feministischen und links-intellektuellen Bewegungsszene als feministische Initiative, die die richtige Sprache beherrschte und entsprechendes intellektuelles und aktivistisches Wissen besaß.

\subsection{Stadträumlich navigieren: „DF ist doch eine Institution hier"6}

Die stadträumlichen Bezüge der gesamten Gruppe wie auch der einzelnen Mitglieder generierten ein spezifisches urbanes Wissen, das weit über den Stadtteil hinausreichte. Es navigierte sie durch die Dichte der Stadt, machte Räume und Anordnungen identifizier- und unterscheidbar (vgl. Kaschuba 2014, 36). Ihre ,complex geography of knowledge“ (ebd., 36) schloss universitäre und staatlich-verwaltende Einrichtungen samt ihrer Expert innen und Themen, andere gesellschaftspolitische Initiativen und Medienschaffende sowie Fußballvereine und deren jeweilige Reputationen ein. ${ }^{83}$

\subsubsection{Das Berlin des feministischen Fußballs}

Das urbane Wissen befähigte die Mitglieder, sich souverän im Stadtraum zu bewegen und ihre Anliegen an die richtigen Stellen zu adressieren. Ihre Bewegungen in der Stadt zeigten, wie sich die Friction auch im Stadtraum materialisierte. So lernte ich durch die teilnehmende Beobachtung nicht nur die unterschiedlichen Sportstätten und -vereine des Landes kennen, sondern ich verinnerlichte auch den Stadtplan eines frauenpolitischen Fußballs. Tagsüber

83 Kaschuba beschreibt urbanes Wissen als historisch basiertes, lokales Wissen, das sowohl in städtischen Räumen als auch in der Gesellschaft aufzufinden ist $(2014,36 \mathrm{f}$.). DF und der DFC Kreuzberg als lokaler Verein sind in diesem Sinne „knowledge communities“, in denen dieses Wissen aufbewahrt, aktualisiert und praktiziert wird. 
führte die Mitarbeit bei DF in Wahlkreisbüros, zu Kultur- und Religionsvereinen, Infoveranstaltungen in Sportheimen, in die Geschäftsstelle des Fußballverbandes, in Ministerien und sogar ins Bundeskanzleramt. Für Interviews wurden die Redaktionen von Radiosendern aufgesucht, zur Besprechung von Kooperationen fuhren Mitglieder in die Bundeszentralen politischer Stiftungen und immer wieder wurde beim Bezirkssportamt über die Nutzung von Fußballplätzen diskutiert. Während so tagsüber kulturelle, sportliche, verwaltende, regierende und gesellschaftspolitische Institutionen und ihre konkreten Orte miteinander zu einer frauen- und sportpolitischen Karte Berlins verknüpft wurden, addierten sich am Abend noch szenespezifische Orte und ihre Bedeutungen hinzu. Durch die Ernennung einer bestimmten Bar zum inoffiziellen Vereinsheim und als häufiger Ort für lokale DFVeranstaltungen, durch die Wahl einer anderen Kneipe als Ort für Solidaritätspartys sowie den internen Austausch über Ausgehmöglichkeiten gerieten Orte in den Sinnzusammenhang einer links-alternativen, feministischen und queeren Freizeit- und Ausgehkultur, die sich dem Frauenfußball gegenüber aufgeschlossen zeigte. ${ }^{84}$ Solch eine Karte des urbanen Wissens verdeutlicht, wie die verschiedenen räumlichen Wege die Stadt und die unterschiedlichen Facetten von DF zu einem Sinnzusammenhang verknüpften: Das urbane Wissen ermöglichte DF, sich effizient im szenisch-subkulturellen Milieu, im organisierten Vereinsfußball wie auch in der Politik- und Kulturlandschaft zu bewegen. Dadurch, dass DF die vielen verschiedenen Institutionen mit ihren unterschiedlichen Agenden, politischen Ansichten, Zielen und Arbeitsweisen durch die Bindeglieder Geschlechterpolitik, Feminismus und Frauenfußball verknüpfte, entstand über die Differenzen hinweg eine kohärente Karte eines feministischen, fußballerischen Berlins, die aber gerade aufgrund der Unterschiede immer auch spannungsreich war.

\subsubsection{Eine etablierte Sportveranstaltung werden}

Gleichzeitig brachte diese Vielzahl von Bezügen DF als lokal situierte Sportveranstalterin hervor. Während sich DF 2010, 2011 und 2013 an die Bezirks-

84 Zum problematischen Verhältnis von links-politischen Milieus und Fußball siehe ausführlich Kuhn (2014). In einem ethnografischen Interview beschrieb Kerstin das Verhältnis von Antifa-Arbeit und Fußballspielen: „In [Heimatstadt] da waren Jugendantifa und Fußball noch Parallelwelten, die sich nicht geschnitten haben, weil als Mädchen Fußball zu spielen in linken Kreisen zumindest cool war und eher die Jungs dann ein Problem hatten und sich vom Sport distanzierten, weil man wollte ja nicht so ein Prollo sein." (Kerstin, Interview, 19.6.2013) Interessant ist, dass die weibliche Fußballpraxis in linken Milieus Ansehen genießt, während die männliche Fußballpraxis - vermutlich aufgrund der Assoziation zu proletenhaftem, rassistischem und heterosexistischem Männlichkeitsgehabe - abgewertet wird. Zur Entfernung der intellektuellen Linken von und ihrer Ablehnung gegenüber dem Proletariat siehe bspw. Baron (2016), Eribon (2016). 
verwaltung mit der Bitte um ein Stadion für die bevorstehenden Festivals wandte, war dies 2015 umkehrt: Diesmal fragte die Bezirksverwaltung bei DF an, wann genau in diesem Jahr das Festival stattfände und welches Stadion sie gerne nutzen würden. Und 2016 unterhielt ich mich im Anschluss an eine Informationsveranstaltung des Bezirkssportbundes mit dem Bezirksrat für Wirtschaft, Schule und Sport. Er fragte sodann, wann denn das internationale Frauen-Fußball-Kultur-Festival in diesem Jahr stattfinden würde. Als eine ebenfalls anwesende Mitarbeiterin des Bezirkssportamtes ergänzte, dass sie alles daransetze, das Stadion rechtzeitig für das Festival in Stand zu setzen, betonte der Stadtrat, dass es stattfinden müsse. Die Veranstaltung sei schließlich zu einer festen Institution des Bezirks geworden. Der städtische Bezug, der die Interaktion mit vielen lokalen Akteur_innen beinhaltete und durch das urbane Wissen ermöglicht wurde, hatte DF als feste Initiative und Organisatorin regelmäßiger Veranstaltungen in der Berliner Sport- und Freizeitlandschaft sowie in einer links-alternativen und feministischen Szene etabliert, mit der im wahrsten Sinne des Wortes gerechnet wurde.

\section{Fußballverein und Amateurin - Verbandsfußball}

Während DF von einer städtischen Szene, einer lokalpolitischen und zivilgesellschaftlichen Öffentlichkeit aufgrund des bürgerlich-intellektuellen, linksalternativen Habitus Anerkennung als sport- und frauenpolitisches Projekt zugesichert wurde, gestaltete sich die Beziehung zum lokalen Verbandsfußball gerade aufgrund dieser habituellen Dimension ambivalent. Wie in der historischen Entwicklung der Vereinskultur gezeigt, war auch der Berliner Fußball-Verband durch eine klare Verteilung von Ämtern und Zuständigkeiten geprägt und dem Leistungsprimat verschrieben (Kap. 4.1). Während sich DF durch seine Organisationsform als Alternative zum Verbandsfußball und dem Klischee der Vereinsmeierei präsentierte und damit Differenz zum lokalen Fußballverband schaffte (8.1), gliederte sich die Organisation gleichzeitig durch die Annahme des Leistungsprimats und die Formierung als Fußballverein in den Verbandsfußball ein (8.2). 


\subsection{Eine Alternative bieten: „Selbstorganisiert und transparent"6}

Das Ideal der Basisdemokratie und Hierarchiefreiheit war auch ein gewollter Gegenentwurf zu den internen Hierarchien und der Ämterverteilung in traditionellen Vereinen und Verbänden des Fußballs, wie sie viele Spielerinnen noch aus ihrer Kindheit und Jugend kannten. Und obwohl sich bestimmte Arbeitsweisen verstetigten und in Zuständigkeiten aufgliederten, wurde an anderen Stellen weiterhin auf geteilter Verantwortung bestanden. So auch im eigenen Fußballverein. 2012 gründete DF einen Frauenfußballverein, den Discover Football Club Kreuzberg, der seitdem die sportlich aktive Unterabteilung des Gesamtvereins bildete. Zwei Jahre später zählte er circa 80 Mitglieder zwischen 20 und 50 Jahren. In den Jahren meiner Forschung hatte der DFC Kreuzberg zwei bis vier Teams für den regulären Spielbetrieb gemeldet, darunter mindestens ein Kleinfeld- und ein Großfeldteam und später auch eine offene Trainingsgruppe, die unregelmäßig Freundschaftsspiele bestritt und an privat organisierten Turnieren teilnahm.

\subsubsection{Gegen das Klischee der Vereinsmeierei}

Bei einem konstituierenden Treffen 2012 wurde über die Ausrichtung des Vereins gesprochen. Anwesend waren neben den Vereinsgründerinnen aus den Reihen von DF auch neue Gesichter - Anfängerinnen wie erfahrene Spielerinnen - die künftig beim DFC Kreuzberg spielen wollten.

Die DF-Frauen hatten für den gesamten DFC eine Philosophie geplant: Jede darf spielen, wenn sie will, egal wie gut oder schlecht; wir entscheiden Vereinsbelange gemeinsam; Aufgaben werden ehrenamtlich verteilt und erledigt. Die Organisation lag bis dahin weitestgehend auf den Schultern von Saskia, der hauptamtlichen Projektkoordinatorin, und sie wollte diese Aufgabe gerne abgeben, sodass nun Ämter wie Kassenwartin, Kontaktperson für die Bezirksverwaltung oder Zuständige für Spielerinnenpässe unter den anwesenden Frauen verteilt wurden. (Erinnerungsprotokoll, 26.12.2012)

Auch einige Jahre später präsentierte sich der Verein auf seiner Website als „selbstorganisierte und transparente Alternative“ zu herkömmlichen Vereinen, welche die „bestehenden von Männern dominierten Strukturen“ des Fußballs ,aufzubrechen“ versucht (DFC Kreuzberg o.J.; siehe Kap. 4.1.3 und 4.2). Selbstorganisation als Besonderheit des Vereins hervorzuheben, ist zunächst merkwürdig, da die Selbstverwaltung - und eben nicht die Steuerung durch den Staat - fest zum Kern des gesamten Vereinswesens zählt und kein Alleinstellungsmerkmal darstellt. Hier meint der Begriff in Verbindung mit dem Verweis auf Transparenz, dass Verantwortungs- und Machtbünde- 
lungen bei Vorständen vermieden werden sollten und stattdessen die Organisation der anfallenden Aufgaben von allen gemeinsam gemeistert wurde und für alle nachvollziehbar war. Nadine zog eine solche Organisationsform und frauenpolitische Begründung - denn ihrer Erfahrungen nach waren es vor allem Männer, die Verantwortungen in Vereinen bündeln und Machtpositionen besetzen - den traditionellen Fußballvereinen vor, wie sie sie im Laufe ihrer Fußballkarriere schon häufig erlebt hatte:

„Und von daher bin ich dann [durch den DFC] wieder in den Vereinssport reingekommen und fand's aber angenehm, weil's eben nicht so die üblichen Vereinsstrukturen waren. Also erstens waren wir ja von Anfang an relativ Viele, so gab es überhaupt diese Möglichkeit zu sagen ,Ich komm'oder ,Ich komm nicht zum Spiel', die kannte ich aus den anderen Vereinen nicht. Gut, mittlerweile gibt's die auch nicht mehr so wirklich, aber es hat sich ja schon wieder so einiges geändert. Aber es war halt anfangs ja schon einiges sehr locker und eben viel selbstorganisiert. Die Leute waren ja eben auch andere als die, die ich so aus den, ich sag mal ,normalen' Fußballvereinen kannte, und so fand ich es dann irgendwie doch nett wieder im Verein zu spielen." (Nadine, Interview, 11.9.2013)

„Normale“ Vereine bedeuteten für Nadine einengende Verpflichtungen, wenig Wahlfreiheit, die Unterordnung unter Vereinszweck und Wettkampfprimat; schließlich war es unmöglich, einmal nicht am Ligaspiel teilzunehmen. Im DFC Kreuzberg fand sie einen Fußballverein, der „Lockerheit“ an die Stelle von Zwang, Inklusion an die Stelle von Leistungsselektion und geteilte Verantwortung an die Stelle von intransparenten Hierarchien setzen und so möglichst vielen Frauen eine Sportangebot machen wollte. ${ }^{85}$ Mit der Wahl des Vereinsnamens knüpft DF zwar zum einen an die Stadtteilgebundenheit der klassischen Lokalvereine an (vgl. Lindner/Breuer 1978). Zum anderen löst die englische Bezeichnung und Aussprache „Club“ anstelle von „Verein“ den Lokalbezug vom Klischee der traditionalen, konservativen ,Vereinsmeierei $^{6}$. Der Verein zeigte sich stattdessen als weltgewandte und kulturell offene Alternative - Attribute, die bereits im Mythos Kreuzberg angelegt sind, der immer auch von der Zuwanderungsgeschichte des Stadtteils gespeist wird.

Hier fanden also zum einen eine gewollte Abgrenzung von der ,normalen" Vereinskultur und der Versuch statt, sich als explizit frauenfördernde, hierarchiearme, weltgewandte und zwanglose Alternative in der lokalen Fußballlandschaft $\mathrm{zu}$ etablieren. Zum anderen brachte der bürgerlichintellektuelle Habitus nicht nur diesen ideellen Gegenentwurf hervor, sondern führte durch Lebensstile und Arbeitsweisen zu Distinktion gegenüber dem Verbandsfußball.

85 Bausinger deutet die interne Protesthaltung gegenüber herkömmlichen Vereinen bei einer gleichzeitigen äußeren Angleichungen in Form von Vereinsgründung und Ämterverteilungen als Ausdruck einer ,weitergehenden Krise des Vereins“: Die Organisationsform Verein verliere zusehends ihre Attraktivität $(2006,51)$. 


\subsubsection{Habituelle Distinktion}

Zwei weitere Beispiele zeigen, wie der Habitus von DF mit der Er- und Anerkennungsstruktur des Verbandsfußballs in Konflikt gerät. Um Einfluss auf den lokalen Fußball zu nehmen, entsandte DF seit 2012 eine Spielerin in die Arbeitsgemeinschaft Mädchen- und Frauenfußball des Berliner FußballVerbandes (BFV). Zu Beginn wechselten sich die Mitglieder entsprechend ihrer möglichst durchlässigen Organisationsform und nach dem Credo, dass sich alle für alles verantwortlich fühlen sollten, in der Teilnahme ab und informierten sich anschließend gegenseitig über die Geschehnisse. Die AG hingegen wünschte sich klare Zuständigkeiten und konstante Verantwortliche, sodass dieses Amt schließlich von Nadine fest übernommen wurde. Jene AG lud schließlich ihre Mitglieder zum gemeinsamen Weihnachtsessen ein. Die Organisatorin des Weihnachtsessens bat die Gäste in einer E-Mail im Vorfeld, zwischen Gans, Ente und Kassler auszuwählen. In einer späteren EMail bat sie um Bestätigung der Essenswahl. Dieser E-Mail enthielt eine zweispaltige Liste mit den Namen der Mitglieder und ihrer Essenswahl. Während die ersten acht Namen die Gans gewählt hatten, hatte einzig Nadine um vegetarisches Essen gebeten und fiel so mit dem Eintrag „vegetarisch“ wortwörtlich aus der Reihe. Als es sich schließlich als unmöglich erwies, dem Extrawunsch nachzukommen, sagte die Vertreterin von DF genervt ihre Teilnahme ab. Das Weihnachtsessen fand ohne sie statt.

Das Beispiel zeigt, wie verbandsfußballerischer und modernisiertbürgerlicher Habitus hier in Form von Organisationsweisen und Essenspräferenzen konfligieren, mal in Kompromissen zueinander finden und mal ein Zusammenkommen verunmöglichen. Der Verband erwartete feste Zuständigkeiten und Ämterverteilung, denen er durch erlesene Speisen zu einem feierlichen Anlass Anerkennung entgegenbringen wollte. Wie sich gerade auch in Essenspräferenzen klassen- und milieuspezifische Habitusformen ausdrücken, hat Bourdieu ausführlich gezeigt (1997, 288ff.), sodass hier der Vegetarismus als bürgerlicher, links-alternativer Gegenentwurf zum Habitus des Verbandsfußballs verstanden werden kann. ${ }^{86}$

Im Lichte der beschriebenen Entwicklungen des Verbandsfußballs und der Geschlechterverhältnisse erscheinen diese Beispiele in einem größeren Zusammenhang. Im Fußball sind vereinskulturelle Aspekte wie Traditionsbewusstsein und Ämterprestige, aber auch eine spezifische, als proletarisch imaginierte Männlichkeit anerkennungsrelevant. Der bürgerlichakademische, weibliche Habitus läuft dieser Anerkennungslogik entgegen, sodass die Lebens-, Arbeits- und Kommunikationsstile beim Verbandsfußball auf Unverständnis stießen und teilweise Irritation auslösten, was die Formie-

$86 \mathrm{Zu}$ überlegen ist, ob der strikte, ethisch motivierte Vegetarismus als links-alternativer, bürgerlicher Distinktionsmechanismus nicht bereits ausgedient hat und an seine Stelle eine pragmatische, an Gesundheit orientierte Flexibilität getreten ist (vgl. bspw. Gruber 2013). 
rung von DF als kompetente Problembearbeiterin erschwerte. Dieses oppositionelle Verhältnis war durchaus gewollt und notwendig, schließlich wurden die Fußballverbände als Hauptproduzenten der fußballerischen Geschlechterungerechtigkeit identifiziert und adressiert. Die Anerkennung von anderen Stakeholdern und damit die Chancen auf Koalitionen hingen auch von der erkennbaren Abgrenzung vom Verbandsfußball ab. ${ }^{87}$ Jedoch - wie James Ferguson und Akhil Gupta über die Beziehung zwischen Staat und NGO schreiben: „sometimes rivals; sometimes servants; sometimes watchdogs; sometimes parasites" $(2002,994)$ - war auch diese Beziehung nur partiell und situativ oppositionell und enthielt ebenso kooperative Momente.

\subsection{Sich eingliedern: „Den Wettkampf ernst nehmen“}

Die Einladungen des lokalen Fußballverbandes an der Arbeitsgemeinschaft Mädchen- und Frauenfußball teilzunehmen, bei den regelmäßig stattfindenden Tagungen zum Mädchen- und Frauenfußball einen Input zu geben oder sich beim Tag des Ehrenamts mit einem Stand zu präsentieren, zeigten, dass DF Anerkennung und Mitspracherecht nicht grundsätzlich verweigert wurden. Ich gehe davon aus, dass sich DF mit der Gründung des eigenen Fußballvereins in die Organisationsstrukturen des Verbandes eingliederte und so trotz der habituellen Differenzen entsprechend der demokratischen Grundprinzipien des Vereinswesens ein Mitspracherecht erhielt. Mit dem DFC Kreuzberg, der am regulären, verbandlich organisierten Spielbetrieb teilnahm, war auch die Trägerorganisation DF Mitglied in den entsprechenden Dachverbänden, sodass sie sich über die fußballerische Praxis als kompetente Mitspielerin im Verbandsfußball organisierte.

Sportliche Fähigkeiten sind in besonderem Maße als verkörpertes Wissen $\mathrm{zu}$ betrachten, da sie Bewegungen ermöglichen, die vorreflektiv vollzogen werden müssen, um zu gelingen. Meine Analyse dieses spezifischen Körperwissens stützt sich neben Beobachtungen vor allem auf meine Teilnahme und das Erlernen des Fußballspiels. Als Anfängerin erlebte ich, wie sich das Wissen um richtige Bewegungsabläufe, Körperhaltungen und Raumverhalten nach und nach in meinen eigenen Körper einschrieb und automatisierte. Dabei ist jedoch zu beachten, wie Juliane Müller beschreibt, dass das Erlernen nur teilweise nachahmend und im Stillen abläuft, vielmehr zeichnen sich Trainings gerade auch durch Erklären, Analysieren und Verbessern aus

87 Als vor einigen Jahren ein bekannter Fußball-Fan-Aktivist, Publizist und Künstler für eine Festanstellung zum Weltfußballverband wechselte, wurde dies unter Fußballaktivist_innen sowie innerhalb von DF überrascht bis skeptisch diskutiert. Auch NGOs und Netzwerke, die besonders eng mit den Verbänden zusammenarbeiten, geraten schnell unter Verdacht, von diesen kooptiert zu werden. 
(2013, 168ff.). Gerade das reflektiv vermittelte Wissen zielt auf jene Fähigkeiten, die sich objektiv messen lassen: erfolgreiches Zweikampfverhalten, die richtige Torschusstechnik oder Schnelligkeit. Darüber hinaus beobachtete und erlernte ich aber noch weitere Praktiken, welche nicht objektiv gemessen und als entscheidend für den Spielausgang gewertet werden, jedoch zu einer kompetenten Performanz dazugehören. Diese umfassen vor allem, so mein Eindruck, die ungeschriebenen Regeln, Stile und Feinheiten bei der Aufführung von Emotionen.

\subsubsection{Objektivierbares fußballerisches Können}

Da die Organisationsgruppe mit Ausnahme vereinzelter Freundschaftsspiele selbst nicht gemeinsam und regelmäßig spielte, konnte ihr messbares fußballerisches Können nur selten beobachtet, sondern vor allem anhand folgender Kriterien erfragt werden: „Wie lange spielst du schon? Wie hoch/in welcher Liga hast du schon gespielt?" Unter den Mitgliedern der Organisation befanden sich Frauen, die seit frühester Kindheit Fußball spielten. Einige von ihnen hatten in ihrer Jugend in Auswahlmannschaften trainiert und eine professionelle Fußballkarriere erwogen, wozu es jedoch aus unterschiedlichen Gründen nicht kam. Andere begannen in der Jugend oder im Erwachsenenalter mit dem Fußballspielen, nachdem sie bereits andere Sportarten intensiv betrieben hatten. Auch gab es Mitglieder, die abgesehen von besonderen Gelegenheiten nicht Fußball spielten und stattdessen andere Sportarten betrieben. Ähnliche Fußballbiografien wiesen die Spielerinnen des DFC Kreuzbergs auf: Einige kamen als Anfängerinnen zum DFC Kreuzberg, andere spielten bereits seit mehreren Jahrzehnten. Das fußballerische Können einzelner Spielerinnen und Teams wie auch des gesamten Vereins ließ sich leicht mithilfe der Auswertung gewonnener Zweikämpfe oder verwandelter Torchancen einzelner Spielerinnen und anhand von Torbilanzen, gesammelten Punktzahlen und dem Stand in den Tabellen ablesen. Dabei fielen die Teams des DFC Kreuzberg im Spielbetrieb weder als besonders schlecht noch als die Leistungsstärksten auf. Da sie zudem keine Mädchenabteilung hatten und damit keinen potentiellen Nachwuchs für den Profifußball bereitstellen konnten, befanden sie sich eindeutig auf der Ebene des Amateurfußballs, der jedoch ebenfalls kompetitiv ausgerichtet ist und dem Leistungsprimat folgt. Ähnliches galt für die Projektarbeit: $\mathrm{Zu}$ den Festivals wurden seltener Profispielerinnen eingeladen, da der Schwerpunkt auf Teams mit gesellschaftspolitischen Ausrichtungen lag. ${ }^{88}$

88 Die Unterscheidung zwischen Profi- und Amateurfußball ist für den Frauenfußball generell schwierig, da hier selbst hochklassige Spielerinnen nicht oder nur schlecht bezahlt werden und damit - im Gegensatz zum Männerfußball - ihren Sport nicht als Profession ausüben können. Für viele andere Länder gilt dies ebenfalls. Zwar nehmen auch Nationalspielerin- 
Sportliche Betätigung und Körperbildung fungieren, so Bourdieu, ebenfalls als klassenspezifische Distinktionspraktiken (1997, 333ff.), sodass sich auch körperlich-sportliche Dispositionen und Fähigkeiten als Kapitalsorte fassen lassen. Körperliches Kapital beschreibt Robert Gugutzer als „ein Instrumentarium, das in gesellschaftlichen Handlungsbereichen eingesetzt werden kann, um soziale Gewinne wie beispielsweise Anerkennung, Ansehen, materielle oder immaterielle Erfolge zu erzielen" (2004, 67f.). Wie die anderen Kapitalformen kann auch körperliches Kapital entsprechend gültiger Anerkennungslogik als symbolisches Kapital fungieren, es ist ebenfalls das Produkt akkumulierter Arbeit, Mühe und Aufmerksamkeit (ebd., 69). Durch sein sportliches Kapital und die Anerkennung des Wettkampfprinzips gliederte sich der DFC Kreuzberg als DFs fußballerisch aktive Unterabteilung in die Logik des lokalen Verbandsfußballs ein und wurde von diesem als regulärer Mitspieler anerkannt.

Neben dem oben bereits ausgeführten differenten Habitus sowie dem Geschlecht relativierte auch DFs Status als Neuling unter alteingesessenen Vereinen in der Vereinslandschaft diese Anerkennung. Auf nationaler Ebene verschob sich die Wertigkeit nochmals. Ist der lokale Fußballverband vor allem für den Amateursport zuständig, so konzentrieren sich der Bundesverband DFB sowie die internationalen Dachverbände UEFA und FIFA auf den Profibereich. Die fußballerischen Fähigkeiten und der Amateurstatus eröffneten so auf lokaler, jedoch nicht auf nationaler Ebene Einflussmöglichkeiten (siehe Kap. 16.6.2).

\subsubsection{Emotionspraktiken als fußballerisches Können}

Fußballerisches Körperwissen beinhaltet nicht nur, die geschriebenen Regeln des Sports zu beherrschen und seinen technisch-taktischen, reflektiv vermittelten Anforderungen gerecht zu werden, sondern umfasst auch entsprechende Emotionspraktiken ${ }^{89}$, die auf ungeschriebene Regeln des richtigen Spielens verweisen (vgl. Faust/Heissenberger 2016a; 2016b). Indem diese Praktiken überzeugend übernommen werden, kommunizieren sie die Verbundenheit der Spielerin mit dem Fußball und ermöglichen ihre Er- und Anerkennung als kompetente Mitspielerin.

nen z.B. aus Ägypten und Jordanien am Festival teil, doch sind die Bedingungen ihrer Fußballpraxis trotz herausragender Leistung nicht mit dem Profifußball der Männer gleichzusetzen, noch können sie hierüber ihren Lebensunterhalt bestreiten.

89 Zum Begriff der Emotionspraktiken siehe Scheer $(2011 ; 2012)$ und daran anknüpfend Bareither (2014; 2016). Ich verwende den Begriff hier, da er das Zusammenspiel von innerem Erleben und äußerem Ausdruck sowie deren historisch-soziale Gewordenheit und soziale Produktivität zu fassen vermag. Wie ich aus eigenen Erfahrungen auf dem Spielfeld zu allgemeinen Aussagen über Emotionspraktiken gekommen bin, schildere ich in Faust/Heissenberger (2016) und Faust (2017a). 
Zuerst ist hier der Umgang mit Schmerzen und Verletzungen zu nennen. Ging eine Spielerin im Zweikampf oder nach einem Foul zu Boden oder bekam den Ball in die Magengrube, ins Gesicht oder vor den Solarplexus, so verzog sie für einen kurzen Augenblick das Gesicht und spielte schnell weiter. Kam es zu Stürzen oder Zusammenstößen, rappelte sich die Spielerin auf und lief mit konzentrierter Miene ins Spielgeschehen zurück. Geschah dies während des Trainings, also in einer weniger ernsten und zeitlich entspannten Situation, reagierten gerade erfahrene Spielerinnen mit clowneskem Verhalten, mit dem sie sich über sich selbst und ihr Ungeschick lustig machten. Waren Verletzung oder Schmerzen jedoch so groß, dass konzentriertes Weiterspielen und Rumalbern unmöglich waren, ging sie zur Erholung an den Spielfeldrand, um möglichst in wenigen Minuten wieder einsatzfähig zu sein. Kleinere Verletzungen aber auch Bänderrisse, Zerrungen und Brüche kamen häufig vor. Sie wurden von den Mitspielerinnen mit Mitleid und Interesse am Heilungsprozess begleitet, doch gleichzeitig als selbstverständlich hingenommen. Selbstverständlichkeit, clowneskes Verhalten oder schnelles Weiterspielen kommunizieren den Schmerz und die Verletzungen als unwichtige Begleiterscheinungen, die nicht als gravierend erlebt werden und die es zugunsten des Sports in Kauf zu nehmen gilt. ${ }^{90}$

Die Emotionspraktiken des Umgangs mit Schmerz stellen die Härte des eigenen Körpers dar und weisen zweitens auf Verletzungsbereitschaft als fußballerisches Kapital hin. Heissenberger (2010) hat für den MännerAmateurfußball herausgearbeitet, dass der individuelle Spielerkörper dem Teamkörper untergeordnet wird. Gleiches gilt für den Frauen-Amateurfußball und bedeutet nicht nur die Selbstverständlichkeit und das Herunterspielen von Schmerzen und Verletzungen, sondern auch diese aktiv zu riskieren. Im Spiel gilt es, aggressiv und kampfeslustig zu sein und den eigenen Körper in den Dienst des Teams zu stellen. Als Anfängerin auf dem Spielfeld kostete es mich immer wieder große Überwindung, meinen Körper aktiv in die Schussbahn der Gegnerin zu stellen und so den Torschuss auch mit meinem Bauch, meinem Kopf oder Oberschenkel schmerzvoll abzuwehren. Auch fiel es Anfängerinnen wie mir schwer, entschlossen auf die Angreiferin zuzugehen,

90 Auch im Männerfußball geht es darum, körperliche Härte zu demonstrieren. Ausnahme bilden jene Szenen aus dem professionellen Männerfußball, wo übertrieben wirkende Schmerzdarstellungen taktisch eingesetzt werden. Sowohl in den Ligaspielen des DFC als auch bei den internationalen Projekten von DF waren solche taktisch eingesetzten Emotionspraktiken selten. $\mathrm{Zu}$ vermuten ist, dass das historische Bild der schwachen und anmutigen Frau, die für eine solch harte Sportart nicht geeignet ist, als ,controlling image“ (Hill Collins 1990, 69) auf die Emotionspraktiken der Spielerinnen wirkt. Das Bild stellt eine Stereotypenfalle dar, die das Mitspielrecht gefährdet. Zur Darstellung einer spezifischen Weiblichkeit in Abgrenzung zu anderen Weiblichkeiten siehe Kap. 17.4.3 Zu clowneskem Umgang mit Sportverletzungen siehe Berner (2016). Er zeigt, wie Skateboarder scherzhaft auf schwere Stürze reagieren und die an den Härte-Imperativ geknüpfte Maskulinität parodieren. 
sich mit dem Körper zwischen sie und den Ball zu drängen und Verletzungen auf beiden Seiten zu riskieren. Rufe von Trainer_innen und Mitspielerinnen signalisierten jedoch stets, dass genau dieses Verhalten gewünscht war.

Eng mit diesen Emotionspraktiken der Darstellung von Schmerz und Kampfeswillen verknüpft ist drittens die ungeschriebene Regel des spielerischen Ernstes. Es sind weniger das tatsächliche spielerische Können als vielmehr der Einsatz und das Bemühen, die gefordert und mit Anerkennung von Teamkolleginnen belohnt werden. In einem kollaborativen Gespräch kam die Sprache auf die für alle geltenden Spielregeln:

Julie erinnert sich an die Zeit, in der sie sehr leistungsorientiert spielte, und wendet ein: „Aber da gibt es auch noch subtilere Sachen. Zum Beispiel, wenn eine aus dem Team den Wettkampf nicht ernst nimmt, ist das ein totaler Mangel an Respekt, sowohl dem eigenen Team als auch den Gegnerinnen gegenüber, und sogar gegenüber dem Wettkampf.“ Nadine pflichtet ihr bei: „Wenn eine nur 50 Prozent gibt, den Ball verliert und dann nicht richtig kämpft, um ihn wiederzubekommen, dann hab ich keinen Spaß. Manche sagen ja, das hier sei nur Spaß, und strengen sich dann nicht an, aber ich verlier gerade dann den Spaß, wenn sich welche nicht anstrengen." Auch Laura stimmt $\mathrm{zu}$, die drei scheinen sich einig zu sein. (Kollaboratives Treffen, 18.12.2015)

Julie deutete damit auf das unausgesprochene Einverständnis aller Beteiligten hin, dem Wettkampf mit Ernsthaftigkeit zu begegnen. Ein Verstoß wurde als Respektlosigkeit gegenüber den Spielerinnen, sogar gegenüber den Gegnerinnen und dem Fußball selbst verstanden. ${ }^{91}$ Alle drei hatten in unterschiedlich hohen Ligen gespielt und großen Gefallen am Wettkämpferischen. Sie hatten diese ungeschriebene Regel internalisiert, was sich durch die Empörung ob der Respektlosigkeit und den Spaßverlust ausdrückte, die bei einem Regelverstoß eintraten. Gleichzeitig bemerkte Nadine, dass andere Spielerinnen diese Norm ebenfalls mit Verweis auf Spaß nicht teilten. Für Nadine war der Spaß unmittelbar an das Wettkämpferische und den Ernst des Spiels gebunden. Für andere Spielerinnen bestimmte der Spaß hingegen die Grenzen der körperlichen Opferbereitschaft und sie waren nicht bereit, für ein Hobby in großem Maße ihre körperliche Unversehrtheit zu riskieren. Vielmehr folgten sie dem Freizeit- und Erholungsgedanken, der auf Regeneration und Stressabbau statt auf Leistungsmessen und -steigerung abzielt. Dieser Konflikt verweist auf die Spannung im Amateurfußball, in dem Freizeit und Wettkampf unmittelbar miteinander verknüpft sind, und die immer wieder in unterschiedlichen Situationen und Formen auftrat. So beobachtet auch Heissenberger ausgehend von seiner Feldforschung bei einem schwulen* Freizeit-

91 Diese Wahrnehmung hängt auch immer von dem jeweiligen Spieltyp und der Spielklasse ab: Bei Trainingsspielen herrscht weniger Ehrgeiz als bei Freundschaftsspielen; bei letzteren wiederum weniger als bei Ligaspielen. Gleichzeitig bewerteten Spielerinnen die verschiedenen Spieltypen als unterschiedlich ernst, je nachdem, auf welchem kompetitiven Niveau sie früher spielten. 
team, dass mangelnde Ernsthaftigkeit und das Ausbleiben entsprechender Gefühlspraktiken - etwa Niedergeschlagenheit nach einem verlorenen Spiel sanft sanktioniert werden, denn schließlich werde ,eine Gefühlregel gebrochen" $(2018,221)$. Vor dem Hintergrund des vorherrschenden Primats des Wettkampfs (siehe Kap. 4.1.2) nehme ich daher an, dass auch im Amateurfußball die Emotionspraktiken der Ernsthaftigkeit, des Kampfeswillen und der Risikobereitschaft als quasi-selbstverständlich gelten, weshalb weniger ambitioniertes Freizeitkicken weniger Anerkennung erfährt.

Fußballerischer Ernst artikuliert sich nicht nur im riskanten Körpereinsatz und in der Bereitschaft, an die Grenzen von Kondition und Kraft zu gehen, sondern ebenfalls in Jubel und Freude. Dabei gilt: je brisanter das Spiel und je ungewisser sein Ausgang, desto größer die Freude über einen Treffer. Während der Festivals war auf dem Rasen zu beobachten, wie sich Spielerinnen nach einem Tor in die Arme fielen, wie sich zwei Spielerinnen nach erfolgreichem Zusammenspiel mit den Händen routiniert abklatschten, wie die Torhüterin der Mittelfeldspielerin nach einer gekonnten Finte ein Lob zurief oder wie sich das Turniersiegerinnenteam nach Abpfiff zu einem unentwirrbaren, lachenden Knäuel auf dem Rasen stapelte oder sich tanzend und jubelnd gegenseitig mit Wasser bespritzte. ${ }^{92}$ Das Gegenbild dazu waren Körperhaltungen, die Niedergeschlagenheit demonstrierten, enttäuschte oder genervte Gesichtsausdrücke, kurze und grimmige Meinungsäußerungen sowie Kraftausdrücke und Flüche nach einer verpatzten Situation, einer Niederlage und einem schlechten Spiel. ${ }^{93}$ Die Gesten der Freude, des Lobs, des Stolzes und Jubelns scheinen dabei deckungsgleich mit jenen des MännerProfifußballs zu sein und kommunizieren die Spannung, Relevanz und den Ernst des Spiels.

Das fußballspezifische Wissen - sei es in Form des eigenen Spiels oder der Organisation von internationalen Fußballveranstaltungen - brachte DF als fußballerisch kompetente Amateurin hervor, die dem Fußball mit Ernsthaftigkeit begegnete und damit ihr Verbunden-Sein mit ihm glaubwürdig kommunizierte. Dabei konstituierte sich DF über die differenten Arbeits-, Organisations- und Sprechweisen und die Distanzierung vom Klischee der Vereinskultur und vom Leistungsprinzip einerseits als Alternative zum „normalen“ Verbandsfußball. Andererseits erspielte sich DF durch die Eingliederung in den organisierten Spielbetrieb Mitgestaltungsmöglichkeiten innerhalb des Verbandes - auch wenn diese durch habituelle Distinktion, Geschlecht und den Status als Neuling in der Berliner Fußballlandschaft eingeschränkt wur-

92 Die Intensität des Jubels ist nicht nur von der Wichtigkeit und Spannung des Spiels abhängig, sondern vermutlich auch von der Größe des und der Unterstützung durch das Publikum, sodass davon auszugehen ist, dass sich Freude, Euphorie und Jubel der Spielerinnen und Zuschauer innen gegenseitig anstecken und intensivieren.

93 An anderer Stelle habe ich nachgezeichnet, was ein schönes Spiel ausmacht und wie sich dieses eher am Verlauf des Spiels denn am Ausgang misst (Faust 2017a). 
den. Dies bedeutete aber auch, dass DF das Leistungsprimat und die Konzeption des Fußballs als Wettkampf anerkannte und - mit Modifikationen (siehe Kap. 17.2) - übernahm. In dieser janusköpfigen Gestalt gegenüber den Fußballverbänden - als einerseits angrenzend und kritisierend und andererseits eingliedernd und mitredend - verdichtet sich jenes spannungsreiche Verhältnis zwischen Leistungsprimat und Feminismus, das in dieser Arbeit noch häufig thematisiert werden wird.

\section{Expertin und Betroffene - Social Movement Market}

Zur fußballerischen Expertise addierte sich Expertinnenwissen im Bereich Frauenrechte, das DF gegenüber feministischen Organisationen, sportpolitischen Initiativen, Medien, Sport-for-Development-NGOs und Fördereinrichtungen artikulierte. Dieses Konglomerat aus unterschiedlichen Stakeholdern und Beziehungen bezeichnet Thayer als "social movement market“ (2010, 130) und kennzeichnet damit die durch die weltweiten neoliberalen Reformen in den 1990er Jahren aufgetretenen Veränderungen. Ähnlich wie internationale Unternehmen müssen heutzutage auch NGOs um knappe Ressourcen konkurrieren, um das eigene Überleben $\mathrm{zu}$ sichern. Bürokratisierung und Professionalisierung sind die Folge (siehe Kap. 8.2). Auch die Beziehungen der NGOs untereinander werden zunehmend kommodifziert: Workshops, Publikationen, Expertisen, Beratungsangebote und investierte Zeit werden mehr und mehr zu Waren des Tausches statt frei verfügbare Produkte der Solidarität. Projektideen werden nicht mehr nur allein auf ihre politische Relevanz und Dringlichkeit hin geprüft, sondern auch auf ihr Potential, Fördergelder einzuwerben. Thayer beschreibt diese Entwicklungen als die größte gegenwärtige Bedrohung für solidarische Allianzen und Kooperationen zwischen Feministinnen - sowohl auf zivilgesellschaftlicher Ebene als auch zwischen ausführender Organisation und internationaler oder staatlicher Förderinstitution $(2010,164)$. Auf diesem social movement market konstituierte sich auch DF als kompetente NGO, um im Wettstreit um knappe Ressourcen das eigene Überleben zu sichern, dabei aber Solidarität sowie altruistische und feministische Prinzipien im Rahmen des Möglichen zu bewahren. Durch Anknüpfungen an die diskursive Formation des Fußballs (11.1), durch die Verwaltung eines komplexen Beziehungsnetzes (11.2), durch das Erarbeiten und Präsentieren von Expertisen (11.3) sowie das Bevorzugen von Erfahrungen gegenüber quantifizierenden Wissensformaten (11.4) organisierte sich DF als internationale Expertin für Fußball und Frauenrechte wie auch als feministische Graswurzelinitiative. 


\subsection{Diskursiv manövrieren: „So ein roter Faden“}

In Kapitel 5 habe ich verschiedene diskursive Formierungen und die sich durch sie versammelnden Akteur innen dargestellt. Nun zeige ich, wie die Mitglieder durch das Anknüpfen an sowie das Manövrieren durch verschiedene Aussagen zu Expertinnen im Bereich Fußball und Frauenrechte wurden. Dabei wird ersichtlich, dass sie dominante Aussagen affirmierten und sich zugleich zwischen konkurrierenden Diskursen situativ entsprechend eigenen Idealen, Moralvorstellungen und Notwendigkeiten hin und her bewegten.

\subsubsection{Fußball ist politisch!}

Im bereits zitierten Interview mit einem feministischen Magazin erinnerte sich Birgit, wie mit der Absage des Rückspiels durch das Teheraner Regime die Berichterstattung über das Fußballspiel vom Sport- in den Politikteil der Zeitungen wechselte. Die Journalist_innen rückten so den Einfluss staatlicher Regime auf sowie die gesellschaftspolitische Relevanz von Sport in den Fokus. Sie stellten das Ereignis in jenen Sinnzusammenhang, der Fußball als gesellschaftlich interdependenten Bereich versteht. Mit dem Ressortwechsel zeichneten sie auch eine Entwicklung vor, die DF ein paar Jahre nach dem gescheiterten Rückspiel vollziehen sollte: die Schwerpunktverschiebung von den sportlichen hin zu den politischen Facetten des Fußballs. Das erste Festival fand im Sommer 2010 statt, das zweite 2011 im Rampenlicht der WM. Linda erinnerte diese Phase ohne konkrete politische Bezüge:

„Da am Anfang, würde ich sagen, waren wir eigentlich noch krass unpolitisch. Ganz am Anfang ging es ja bei Discover Football nie -, also wir haben uns gar nicht offen die Frage gestellt: ,Was ist eigentlich los mit Frauenrechten?' Oder dieses ,[Fußball als] Spiegel der Gesellschaft' und dass die Frauen sich da was erkämpfen. Sondern ganz am Anfang war es wirklich nur: ,Ach, der Iran durfte nicht kommen und irgendwie wär's jetzt cool, wenn jetzt andere Teams aus anderen Ländern kommen. Das wär mal voll schön zu sehen, was die so machen. ' [...] Es war ja jetzt noch nicht irgendwie so ein roter Faden: ,Was wollen wir damit jetzt überhaupt?' Oder: ,Wen sollten wir anschreiben und einladen?' [...] manchmal hab ich auch das Gefühl, dass wir vielleicht durch die Teams teilweise auch politisiert wurden, weil die uns so viel aus ihren Ländern und von ihrer Arbeit vor Ort erzählt haben.“ (Linda, Interview, 25.9.2013)

Dieser Prozess der „Politisierung“ meint einen stärkeren Fokus auf die fußballerischen Geschlechterverhältnisse und deren Analyse im Kontext der gesamtgesellschaftlichen und rechtlichen Situation von Frauen. Er spiegelte sich in den seit 2012 dazugekommenen Projekten, ihrer inhaltlichen Ausgestaltung sowie in der Zusammensetzung der Gruppe wieder. Bis zum Verfassen dieser Arbeit wuchs die Projektarbeit rasant: 2013, 2015 und 2016 fanden 
wieder Festivals statt, die jeweils unter globalpolitischen Bezügen standen. 2013 wurden unter dem Motto „One Language“ Teams aus Ländern des sogenannten arabischen Frühlings eingeladen; 2015 thematisierte das Motto „Beyond (b)orders“ gesellschaftliche und geografische Grenzen; und 2016 nahmen sie unter dem Titel „Home Game“ Bezug auf die aktuellen Fluchtbewegungen. Das Festival beinhaltete ab 2013 eine zweieinhalbtägige Workshop-Reihe, in der gesellschaftspolitische Themen erörtert und praktisch behandelt wurden. Während meiner Forschung fanden zudem vier internationale Konferenzen mit engagierten Spielerinnen, Trainerinnen, Funktionärinnen und Aktivistinnen statt. Es wurde eine Deklaration erarbeitet, die von nationalen und internationalen Verbände den konsequenten Einsatz für den Frauenfußball, für Geschlechtergerechtigkeit und gegen Diskriminierungen forderte (siehe Kap. 16), sowie ein Handbuch veröffentlicht, in dem internationale Teilnehmerinnen von den Schwierigkeiten, als Frauen Fußball zu spielen, berichteten und ihre Strategien zur Bewältigung der Hindernisse vorstellten. 2012 reiste die Organisationsgruppe zur EM der Männer nach Polen und in die Ukraine sowie 2014 zur WM der Männer nach Brasilien und führte dort jeweils Mädchenfußballprogramme, Podiumsdiskussionen, Workshops und Turniere durch. Zwischen den großen Projekten organisierte DF kleinere, lokale Informations- und Kulturveranstaltungen, wie Podiumsdiskussionen oder Filmvorführungen. Während in den Anfangsjahren Männer selbstverständlich Mitglied werden konnten und eine der Projektkoordinationsstellen zeitweise von einem Mann besetzt war, wurde entsprechend des zunehmenden Fokus auf Geschlechterverhältnisse und der Frage nach dem richtigen feministischen Vorgehen später kontrovers diskutiert, ob und wenn ja, in welchem Ausmaß Männer teilnehmen und DF repräsentieren sollten.

Der von Linda als Politisierung beschriebene Prozess wurde auch von Saskia, die 2011 als Ehrenamtliche des zweiten Festivals dazukam, als Veränderung wahrgenommen:

„Ich glaub, seit ich dabei bin, war das schon in diesem Umbruchsprozess. Da waren so Leute, für die es teilweise auch einfach nervig war, wenn ständig so feministische oder politische Themen reinkamen, die eher so das Gefühl hatten: ,Wir wollen doch hauptsächlich was mit Fußball machen. 'Aber ich glaub, seit ich dabei bin, waren auf jeden Fall auch schon Stimmen da, die das klarer als ,es geht um Gleichstellung ' und irgendwie ,wir wollen was Politisches machen' geframet haben. [...] Einen richtigen Bruch oder so kann ich nicht erinnern." (Saskia, Interview, Dez. 2013)

Diese langsame Veränderung ging auch mit einer zunehmenden Orientierung hin zu Politiken der Geschlechtergerechtigkeit und Frauenförderung sowie der Antidiskriminierung einher. Wenn Linda die Politisierung als die Entstehung eines ,roten Fadens“ beschrieb, wies sie auch auf den Kohärenz schaffenden und stabilisierenden Effekt der diskursiven Anknüpfung hin. Die sich Schritt für Schritt vollziehende Politisierung ist als Produkt von drei vermutlich interdependenten Prozessen zu verstehen: Erstens war sie, wie von Linda 
beschrieben, auf die Auseinandersetzung mit anderen Fußballspielerinnen und deren Geschichten zurückzuführen. Zweitens veränderten die sozialwissenschaftlichen Bildungshintergründe und politisch-ideologischen Haltungen der Neuhinzukommenden die Themensetzungen innerhalb der Gruppe. Drittens verlangte auch die gesellschaftliche Kontroverse um die politische Neutralität des Fußballs nach einer klaren Stellungnahme von einer Frauenfußballorganisation. Organisationen absorbieren nicht wahllos jegliche Diskurse - diskursive Anknüpfungen und Abgrenzungen implizieren vielmehr die bewusste, wenn auch begrenzte Auswahl zwischen Bedeutungen und die Art und Weise ihrer Aneignung (Thayer 2010, 6). Zudem zeigt Lindas Hinweis, dass mit der Politisierung eine Auswahl der künftig einzuladenden Personen einherging, dass eine diskursive Stellungnahme und Stabilisierung auch bedeutet, sich mit der soziomateriellen Umwelt in Beziehung zu setzen und entsprechend der politischen Haltungen zu bestimmten Akteur_innen Nähe und zu anderen Distanz zu schaffen. Mit der Positionierung gegen das bürgerliche Postulat vom unpolitischen Sport distanzierte sich DF vom Verbandsfußball, der die Trennung von Staat und Sport fordert und die sportliche Neutralität gegenüber politischen Interessen postuliert.

Zugleich rückte DF mit der Politisierung des Fußballs in die Nähe feministischer Argumentationen, die bislang als privat Erachtetes in den öffentlichen Fokus stellen und als Effekte und Spielfelder von Herrschaftsverhältnissen verstehen (siehe 4.2). Als ich Laura im Interview danach fragte, was sie genau als politische Dimension von DF wahrnahm, knüpfte sie an feministische Diskussionen an:

„Das Zusammenbringen von Fußball bzw. Fußball als Mittel - und das hab ich über die Turniere einfach so wahrgenommen - für sowohl genderpolitisches Engagement also Frauenrechte: was dürfen Frauen nicht, was sollte möglich sein? - als auch, naja, territorial irgendwie: Wie verhalten wir uns als, oder was ist unsere Rolle als weiße deutsche privilegierte - oder vielleicht auch nicht privilegierte - Gruppe im Verhältnis zu Gruppen, Teams aus anderen Regionen, anderen Ländern? Also so einen globalpolitischen Aspekt." (Laura, Interview, 6.1.2014)

Geschlechterverhältnisse wurden hier als machtvolle Arrangements verstanden, Geschlechterrollen hinterfragt, spezifische Rechte eingefordert und Schwarze und postkoloniale feministische Kritik an weißen Frauenbewegungen berücksichtigt. Im Gegensatz zu dieser herrschaftskritischen Deutung beobachtet Thayer eine Entpolitisierung von Geschlecht im Zuge internationaler Entwicklungsarbeit, die Gender vom feministischen Projekt radikaler sozialer Transformationen loslöste und zu einer ,aesthetic variable used to measure policy impact" stilisierte $(2010,70)$. Die Anknüpfung an die diskursive Politisierung des Fußballs durch DF beinhaltete eine Re-Politisierung von Geschlecht im Kontext translokaler Projekte. Dies sicherte zum einen die Glaubwürdigkeit als feministische Sportinitiative vor wissenschaftlichen wie aktivistischen Feminismen innerhalb der Stadtlandschaft als auch einer inter- 
nationalen Frauen-Sport-Bewegung sowie einer Positionierung innerhalb einer gesamtgesellschaftlichen Debatte um Fußball. Die Formation des Fußballs als gesellschaftlich interdependentem Bereich ermöglichte zum anderen die Politisierung der weiblichen Fußballpraxis, wie DF unter anderem in einem Förderantrag argumentierte:

„In den Kämpfen von Frauen für ihr Recht, Fußball zu spielen, spiegeln sich gesellschaftliche Auseinandersetzungen um Geschlechterbilder und Frauenrechte wider. Die einzelnen Initiativen und Vereine müssen sich immer wieder mit einem frauenfeindlichen Umfeld sowie Vorurteilen und Klischees gegenüber Frauenfußball auseinandersetzen. Auch im familiären Umfeld der Spielerinnen bestehen oft Vorbehalte, diese würden durch die Teilnahme an einem als männlich wahrgenommenen Sport an Weiblichkeit und sozialer Akzeptanz verlieren. Allen Hindernissen zum Trotz spielen viele, gerade junge Frauen Fußball und erobern sich so gesellschaftliche Räume, die aus traditionellen Gründen Männern vorbehalten sind. Sie stellen damit soziale Geschlechterrollen in Frage.“ (Antrag Festival 2013)

Das Fußballspielen sowie die Organisation von Fußballveranstaltungen wurden dadurch $\mathrm{zu}$ gesellschaftspolitischen und nicht länger rein sportlichen Angelegenheiten. Mit dieser Übertragung organisierte sich DF als gesellschaftspolitische Kraft an der Schnittstelle von Frauenpolitik und Fußball.

Während DF so den Anschluss an feministische und emanzipative Frauenfußballprojekte fand, blieben Schulterschlüsse mit anderen verbandsfußball-kritischen Initiativen jedoch aus. In Kapitel 4.1.3 habe ich skizziert, wie der Herrschaftsanspruch der Fußballverbände gegenwärtig aus der Fanszene sowie einer breiteren Öffentlichkeit durch Kritik an Menschenrechtsverletzungen, Korruption und Kommerzialisierung im Männerfußball fundamental angegriffen wird - eine Infragestellung, die jenes günstige Moment koproduzierte, das DF entstehen ließ. Dennoch ergab sich hier nicht das, was Mouffe als „Äquivalenzkette“ beschreibt: „Die Herstellung eines gemeinsamen Willens" über differente und teils widersprüchliche Forderungen hinweg zwecks Infragestellung der Machtverhältnisse $(2014,118)$. Dies ist zum einen sicherlich dem Unwillen der Aktivist_innen geschuldet, sich mit jener männerdominierten Kultur intensiver auseinanderzusetzen, wie sie auch in kritischen Fanprojekten des Männerfußballs zu finden ist. Zum anderen kann auch vermutet werden, dass die neoliberale, politische Mobilisierung von „Gender“ durch die Bereitstellung finanzieller Mittel, wie von Thayer (2010) beschrieben, dazu führt, dass sich um diese Kategorie Netzwerke zwecks geschlechterpolitischer Reformierung des Fußball bilden. Für breitere Bündnisse hingegen, die eine strukturelle Umarbeitung der Machtverhältnisse im Fußball anstreben, lassen sich wohl nur schwerlich Ressourcen akquirieren, sodass dieses Vorhaben das Überleben einer von Projektmitteln abhängigen Organisation schlichtweg gefährden würde. 


\subsubsection{Fußball empowert!}

DF schloss die Aussage von der empowernden Wirkung des Fußballs unmittelbar an die Politisierung des weiblichen Fußballspiels an. Dadurch, dass Fußball als Abbild gesellschaftlicher Geschlechterverhältnisse verstanden wurde, könnten die bestärkenden Erfahrungen von Frauen auf dem Spielfeld auch in andere Lebensbereiche mitgenommen werden:

„Gleichzeitig kann aber gerade das Fußballspielen dazu beitragen, Mädchen und Frauen zu stärken: Im Team erfährt jede Solidarität, der Sport stärkt das Selbstbewusstsein und Mädchen und Frauen lernen hier, sich durchzusetzen. Fußball kann somit ein Weg zu Selbstbestimmung und sozialem Aufstieg sein. Frauen auf dem Fußballplatz tragen aber auch dazu bei, dass sich die Wahrnehmung von Geschlechterrollen in der Gesellschaft verändert und Frauen als aktiver Teil der Gesellschaft sichtbar werden.“ (Discover Football 2013, 4)

Ähnlich wie der integrationspolitische und entwicklungspolitische Sport-forDevelopment-Ansatz unterstrich auch DF die befähigende Wirkung des Fußballs und konstituierte Fußballerinnen als Akteurinnen gesellschaftlichen Wandels: Fußballspielen wirke emanzipatorisch auf die Spielerinnen, sie würden durch ihre vergeschlechtlichte Sportpraxis selbst zu Akteurinnen gesellschaftlichen Wandels. Während eine Politisierung des Fußballs vor allem Anknüpfungen an feministische und gesellschaftspolitische Debatten ermöglicht, stellt dieser nächste argumentative Schritt eine Verbindung zur geschlechterfokussierten Sportpolitik und zu jener Außen-, Innen- und Entwicklungspolitik her, die Frauen und Mädchen als Zielgruppe und Sport als Instrument identifizierten. Rabinow (1989, n. Riles 2007, 13) bemerkt, dass es typisch ist für die klassische, modernistische Vision von der Macht des Wissens, dass Probleme außerhalb ihrer selbst gelöst werden. Die Vorstellung, dass der Fußball systempolitische und ökonomische Probleme lösen könne, griff auch DF in einem Förderantrag auf:

„Allen Hindernissen zum Trotz spielen viele, gerade junge Frauen Fußball und erobern sich so gesellschaftliche Räume, die aus traditionellen Gründen Männern vorbehalten sind. Sie stellen damit soziale Geschlechterrollen in Frage. [...] Fußball kann darüber hinaus eine emanzipatorische Wirkung für Mädchen und junge Frauen entfalten, indem er geschützte Räume für eine selbstbestimmte Entwicklung schafft, Selbstvertrauen stärkt und die gesellschaftliche Partizipation von Mädchen und Frauen fördert. Die gesellschaftliche Mitbestimmung und Mitwirkung von Mädchen und Frauen ist zugleich essentiell für eine nachhaltige Entwicklung zu mehr Demokratie.“ (Antrag Festival 2013)

Wie bereits gezeigt, formulieren öffentliche Einrichtungen spezifische Arbeitsweisen und interne Strukturen als Kompetenzkriterien und begünstigen damit die Formierung als eingetragener, gemeinnütziger Verein. Hier werden nun die inhaltlichen Kriterien für eine kompetente Organisation erkennbar: Um der Gemeinnützigkeit zu entsprechen, müssen sie überzeugend Gutes 
tun. Das Akronym NGO ist daher nicht lediglich das abgekürzte, englische Äquivalent zum eingetragenen Verein - beide sind schließlich gemeinnützig, nicht-profitorientiert und nichtstaatlich -, sondern ein Label, das sich Organisationen durch überzeugende Vermittlung, Gutes zu tun und nicht von kommerziellen, parteipolitischen oder persönlichen Interessen geleitet zu sein, aneignen können (Hilhorst 2003, 215). Die diskursiven Anknüpfungen an die Aussagen über die empowernde und verbindende Wirkung des Fußballs sind daher auch Praktiken der Formierung als kompetente Organisation, in diesem Falle als NGO, die für als benachteiligt identifizierte Gruppen und für übergeordnete Politikziele - Menschenrechte, Demokratie und Zivilgesellschaft, sozioökonomische Entwicklung - Gutes tut.

Mit der Nähe zum internationalen NGO-Sektor und dem dort virulenten Empowerment-Dispositiv entstand eine Spannung mit dem feministischaktivistischen internationalen und szenisch-städtischem Umfeld und eigenen feministischen Überzeugen. Das Empowerment-Verständnis der staatlichen Außen- und Entwicklungspolitik, internationaler NGOs und Entwicklungsagenturen wird von feministischen und postkolonialen Akteur_innen unter anderem aufgrund des inhärenten Eurozentrismus sowie der neoliberalen Verlagerung der Verantwortung weg von Gesellschaft und Politik auf ein rassifiziertes, weibliches Individuum kritisiert (siehe Kap. 5.3.2). Diese Kritik war den Mitgliedern bestens vertraut, wie in folgender E-Mail deutlich wird: Hier reagierte Birgit auf den Entwurf einer Pressemitteilung zur Ankündigung des bevorstehenden Festivals, der in einer vorangegangenen EMail herumgeschickt worden war, mit einem Korrekturvorschlag:

„Hallo,

ich finde es sehr schön, habe aber einen Veränderungsvorschlag: ,Eines Sports, der lange Zeit von Frauen nicht ausgeübt werden durfte und bis heute in manchen Teilen der Welt nicht anerkannt und akzeptiert ist'. Hier fänd ich es besser, wenn wir das Problem, dass Frauenfußball nicht anerkannt/akzeptiert wird, nicht auf einige Teile der Welt schieben, ich denke da z.B. an Rudi Assauer, der mir gesagt hat, Frauen ab 20 müssen sich was anderes suchen, Frauenfußball sieht einfach nicht gut aus, oder den kleinen Jungen am Fußballplatz, der letzte Woche zu Annike gesagt hat: ,Ich bin besser, Du bist kacke'. :)

Mein Vorschlag: ,Eines Sports, der lange Zeit von Frauen nicht ausgeübt werden durfte und bis heute nicht immer anerkannt und akzeptiert ist".

Liebe Grüße, Birgit“ (E-Mail, Juni 2013)

Über die fußballerischen Geschlechterverhältnisse löste Birgit den Empowerment-Begriff von seiner eurozentrischen Implikation. Da Fußball, so die Argumentation auch in anderen Situationen, auch in vermeintlich entwickelten Ländern eine Männerdomäne sei, erführen Fußballerinnen auch hier Ausgrenzung und Diskriminierung. ${ }^{94}$ Eine Unterscheidung in benachteiligte

94 Eine Ausnahme von der Verallgemeinerung stellen die USA dar, in denen Fußball hauptsächlich von Frauen und (lateinamerikanischen) Immigrant_innen gespielt wird. Fußball 
und Empowerment-bedürftige Frauen des globalen Südens und gleichberechtigte, souveräne und emanzipierte Frauen des globalen Nordens sei in Bezug auf die fußballerischen Geschlechterverhältnisse nicht haltbar. Anstatt unilinearer Befähigung bedeutet Empowerment dann, sich gegenseitig im sportund frauenpolitischen Engagement zu bestärken und voneinander zu lernen.

Zugleich behielten die Aktivist_innen bestimmte Dimensionen des neoliberalen Empowerment-Verständnisses bei, wenn sie v.a. auf individuelle Befähigung fokussierten und Stärke und Durchsetzungsvermögen zu erstrebenswerten Charaktereigenschaften erklärten. Anders als es manche feministische Initiativen anstreben, war der Ausgang von Körper- und Bewegungserfahrung und gegenseitiger Befähigung nicht offen - womöglich hätte er zu einer Abkehr vom Ideal der (Durchsetzungs-)Stärke geführt -, sondern immer schon in Form von starken Körpern und Charakteren vorgegeben.

Diese partielle Abweichung von einigen feministischen Ansätzen und die Annäherung an neoliberale Entwicklungsansätze sowie an das verbandsfußballerische Leistungsprimat brachte eine hybride Form von Empowerment hervor, die Anschlüsse an verschiedene Stakeholder ermöglichte. Sie bot eine Schnittfläche zwischen DF, feministischen (Sport-)Bewegungen und entwicklungspolitischen Institutionen, ohne zugleich die Anknüpfung an den Verbandsfußball zu riskieren. In globalen Begegnungen kann es immer wieder zu terminologischen Überschneidungen kommen, wenn mehrere Parteien denselben Formulierungen unterschiedliche Bedeutungen beimessen. Die temporäre und vergängliche Übereinkunft des Miteinandersprechens beschreibt Tsing als ,awkward engagement“ $(2005$, xi) und macht damit deutlich, dass Ausdrücke Kooperationen und Koalitionen über Differenzen hinweg ermöglichen. Empowerment fungiert dabei als mobilisierende Metapher:

„Policy discourse generates mobilizing metaphors [...] whose vagueness, ambiguity and lack of conceptual precision is required to conceal ideological differences, to allow compromise and the enrolment of different interests, to build coalitions, to distribute agency and to multiply criteria of success within project systems." (Mosse 2004, 663)

Hier ermöglichte die semantische Flexibilität sowohl die Akquise öffentlicher Fördergelder als auch den Anschluss an feministische und frauenbewegte, lokale wie translokale Initiativen, Unterstützer_innen und Partnerorganisationen. ${ }^{95}$ Aufgrund der unterschiedlichen Bedeutungen des EmpowertmentBegriffs entstanden jedoch immer wieder Widersprüche, die im weiteren Verlauf der Arbeit noch diskutiert werden.

gilt hier als weibliche Sportart, wohingegen andere Sportarten wie Basketball und American Football deutlich mehr Prestige genießen und als Praxen der Einübung und Demonstration von Männlichkeit fungieren.

95 Thayer weist darauf hin, dass solche Umdeutungen und Aneignungen von global dominanten Diskursen im Lokalen auch immer eine Modifikation der globalen Kräfte und Machtverhältnisse beinhaltet $(2010,3 \mathrm{f}$.). 
Die schrittweise Anknüpfung an die diskursiven Formationen des Fußballs als politisch und empowernd verlief dabei keinesfalls willkürlich, sondern innerhalb eines von Werten und Überzeugungen eingegrenzten, idealistisch unterlegten Rahmens. Von öffentlichen Fördergeldern abhängige NGOs laufen prinzipiell Gefahr, Glaubwürdigkeit und Legitimität einzubüßen, wenn ihnen von Seiten der lokalen Bevölkerung und/oder der Zielgruppen ihrer Projekte unterstellt wird, sie würden zugunsten der Förderrichtlinien, sprache und -schwerpunkte ihre eigenen Ideale und moralische Überzeugungen aufgeben (Parks 2008, 218). Die erfolgreiche Formierung als kompetente und zugleich glaubwürdige Organisation erforderte also nicht nur, die heterogenen Kompetenz- und Anerkennungsregime erfolgreich zu antizipieren und durch sie durch zu navigieren, sondern sie auch beständig auszubalancieren und auszutarieren. Dem Glaubwürdigkeitsverlust, der durch die Anknüpfung an den entwicklungspolitischen Diskurs drohte, begegnete DF mit der partiellen Umdeutung des Empowerment-Narrativs. Diese Umdeutung verstehe ich daher als eine Praktik des Balancierens zwischen und Austarierens von Anerkennungslogiken unterschiedlicher Stakeholde.

\subsubsection{Fußball verbindet!}

Während sich der politisierende und der Empowerment-Topos erst im Laufe der Jahre herausgebildet hatten, bezog sich die Gruppe von Beginn an auf die integrative Wirkung des Fußballs (siehe Kap. 5.1). Birgit erinnerte, dass es ihnen beim ersten Festival 2010 vor allem darum gegangen sei, die weltweite Vielfalt des Frauenfußballs zu entdecken, ungewöhnliche Begegnungen zu ermöglichen und unterschiedliche Kulturen zusammenzubringen: „Egal was das heißt. Aber so möglichst breit und gucken, was passiert" (Birgit, Interview, Dez. 2013). Auch 2013 wurde die interkulturell-verbindende Dimension des Fußballs im Förderantrag hervorgehoben:

„DISCOVER FOOTBALL 2013 - Frauenrechte- und FrauenfußballBegegnungswoche ermöglicht außergewöhnliche Begegnungen, die Förderung von interkultureller Verständigung und Empowerment von jungen Frauen. Vom 21. - 28. Juli 2013 werden jeweils elf junge Fußballspielerinnen mit je 2 BetreuerInnen aus dem Libanon, Palästina, Jordanien und Polen/Ukraine zu einer Begegnungswoche nach Berlin eingeladen, wo sie mit den Spielerinnen von Fußball und Begegnung e.V. zusammentreffen. Die gemeinsame Leidenschaft für den Fußball - und häufig auch die Erfahrung gegen gesellschaftliche Hindernisse ankämpfen zu müssen - verbindet die Jugendlichen." (Antragsentwurf 2013)

Hier wie auch in anderen Anträgen griff DF auf jenen Diskurs zurück, den EU, UN und staatliche Organe sowie die Fußballverbände teilen. Der Vereinsname „Fußball und Begegnung“, die Bezeichnung des Festivals als internationale „Begegnungswoche“ und die Mottos „one language“ und „Beyond (b)orders" knüpften an die diskursive Formation des Fußballs als verbindend, 
völkerverständigend und friedensfördernd an. Im Abschlussbericht resümierte DF unter anderem:

„Das Aufsplitten der angereisten Teams in verschiedene Workshopgruppen, in welchen sie mit anderen angereisten Frauen neu durchmischt wurden, legte den Grundstein für einen Esprit der Verständigung, des Austauschs und des Fairplay. Innerhalb von 2,5 Workshop-Tagen entwickelten sich beeindruckende Eigendynamiken und eine große Intimität zwischen den Spieler_innen aus den verschiedenen Ländern.“ (Abschlussbericht 2014)

Mit konkreten Maßnahmen wie Workshops und einem Mischen der angereisten Spielerinnen zu multi-lingualen Teams kamen die Organisator_innen dem pädagogischen Imperativ zur Förderung des verbindenden Potentials des Fußballs nach, wie er im herrschenden Dispositiv enthalten ist. In einer Podiumsdiskussion fragte Marta von DF zwei der angereisten Trainerinnen nach dieser Erfahrung:

Marta: „Wie hat das mit der Kommunikation geklappt?“

Mei: „Am Anfang war es eine Herausforderung, wenn nicht dieselben Sprachen im Team gesprochen wurden. Aber wir haben gelernt, unsere Körpersprache sowie Zeichnungen zu nutzen. In meinem Team hatte ich Spielerinnen aus Tansania und hab ein paar tansanische Worte gelernt. ,Kappakappa' heißt ,Druck' [pressure].“ (Tansanische Spielerinnen im Publikum applaudieren)

Marta (an die zweite Trainerin gewandt): „Denkst du, dass in dieser Woche aus jeder Gruppe auch ein Team werden kann?“

Ruth: „Ich denke, das ist die große Herausforderung für die Trainer_innen und das ist viel wichtiger als der Ausgang des Spiels. In unserem Projekt in Argentinien ist Teamwork das wichtigste, dafür machen wir viele Teambuilding-Übungen. Wenn wir Brücken zu unseren Teamkameradinnen bauen, macht uns das stärker." (Feldnotiz, 28.6.2015; Übersetzung FF)

In diesem Dialog konstituierte sich der Fußball als eben jenes Mittel, um seinem exkludierenden Potential zu begegnen: Zuerst wurden durch die Aufsplittung der Teams potentiell konflikthafte Elemente verstärkt, um diese sodann durch genuin fußballerische Aktivitäten wie Teambuilding zu überwinden. Das Erleben der Überwindung kultureller und politischer Grenzen machte diese als kulturalisierte oder politisch instrumentalisierte Konstruktionen erkennbar. Bei der Abschlussfeier 2016 bemerkte eine afghanische Spielerin auf die Frage, was für sie das Besondere der Festivalwoche gewesen sei, dass sie gemeinsam mit iranischen Spielerinnen in einem Team gespielt habe. Ihre Regierungen seien verfeindet, und beide Seiten seien mit gegenseitigen negativen Vorurteilen angereist. Durch das gemeinsame Spielen habe sie die iranischen Frauen als ,amazing women“ kennengelernt. Indem DF solche Begegnungen ermöglichte, aber auch, etwa bei der Abschlussfeier und Podiumsdiskussion, öffentlichkeitswirksam inszenierte, knüpfte DF an die von Sozialpädagogik, Außenpolitik und Entwicklungszu- 
sammenarbeit sowie Sportverbänden vertretene Aussage „Fußball verbindet“ an. DF zeigte sich so vor einer heterogenen Verbandsfußball- und Sportpolitiklandschaft als kompetente Projektorganisatorin zur Entfesselung der positiven Potentiale des Fußballs zum Zwecke übergeordneter Politikziele.

\subsubsection{Plausibel problematisieren}

Zusammen genommen ergaben die drei diskursiven Anknüpfungen eine kohärente Problematisierung der fußballerischen Geschlechterverhältnisse. Daran anknüpfende Projektentwürfe unterbreiteten mit interkulturellen Begegnungen und Vernetzung sowie Formaten von Wissensvermittlung und -austausch plausible Lösungsvorschläge. Ramya Ramanath und Alnoor Ebrahim beschreiben ein solches Deutungsangebot gesellschaftlicher $\mathrm{Zu}$ sammenhänge und akuter Herausforderungen seitens NGOs als ,theory of change“: ,a framing of the root problem to be addressed and the pathway or steps required to ameliorate that problem" $(2010,33)$. Der E-Mail-Austausch über die genauen Formulierungen in der Pressemitteilung verdeutlicht, dass eine solche Rahmung und Problematisierung von Situationen und Ereignissen immer ein Prozess des Benennens und sprachlichen Fixierens ist und reflexive Elemente enthält (vgl. Binder 2014, 365). Wie in dem Begriff der theory anklingt, werden dabei einzelne Ereignisse mit anderen in Verbindung gesetzt und ihre Bedeutungen über die konkrete Situation hinaus ausgedehnt (vgl. Eyerman 2006, 196). Dabei wird einerseits an bestehende Macht/Wissen-Formationen angeknüpft, andererseits werden auch die Veränderungen in den Kräfteverhältnissen genutzt und zu neuen Fragestellungen und Argumenten synthetisiert (Klöppel 2010, 259). Indem sie strategisch an dominante Diskurse anknüpfen, zugleich etablierte Diskurse kritisieren und alternative und neue Deutungen entwickeln, verschaffen sich Aktivist_innen auf breiter Basis Gehör (vgl. Bergman et al. 2012, 114). Durch die wiederholte Ausformulierung ihrer Problemdefinition und Lösungsvorschläge arbeiten sie mit an der Identifizierung von Problemen und schlagen im Dialog mit der sie umgebenden landscape of practice aus öffentlichen Förderinstitutionen, lokalen zivilgesellschaftlichen Unterstützter_innen, internationalen wissenschaftlich-aktivistischen Debatten und Rahmenbedingungen öffentlicher Verwaltungen konkrete Bearbeitungsweisen vor. Dabei handelt es sich keinesfalls um eine lineare, von Anfang an durchgeplante Strategie; vielmehr korrigieren und ändern sie ihre Strategien und Taktiken entsprechend der Kontexte kontinuierlich (vgl. Ramanath 2007; Ramanath/Ebrahim 2010).

Seit der Gründung hatte DF bei immer mehr staatlichen und städtischen Verwaltungsinstitutionen sowie zivilgesellschaftlichen Fördereinrichtungen nach Förder- und Kooperationsmöglichkeiten gesucht, sodass DF diskursiv durch eine Vielzahl von unterschiedlichen Förderschwerpunkten und -zielen 
navigieren musste. Während kaum Institutionen reine Sportveranstaltungen unterstützten, begünstigten die Förderrichtlinien eine Betonung der emanzipativen, sozial-integrativen oder (inter-)kulturellen Projektdimensionen. Durch die diskursiven Anknüpfungen generierte sich DF vor lokalen, nationalen und internationalen Fördereinrichtungen, die den Sport als Mittel zu Integration, Frieden oder Entwicklung verstanden, als potentielle Partnerorganisation. Die Aktivist_innen rahmten ihre Tätigkeiten damit nicht mehr nur - wie noch zu Beginn - als buntes interkulturelles Frauenfußballfest, sondern auch als frauen- und gesellschaftspolitisch relevantes Projekt. Zur bereits beschriebenen Professionalisierung und Institutionalisierung der Arbeitsweise und Organisationsstruktur gesellte sich der schrittweise Prozess der Politisierung der Inhalte und Ziele - von interkultureller Begegnung hin zu Frauenrechten, Geschlechtergerechtigkeit und Antidiskriminierung - und verliehe der Projektarbeit auch eine moralische Dimension.

Plausibilisierung und Autorisierung eines Deutungsrahmens beinhaltet jedoch, dass sich bestimmte Deutungen von Ereignissen und die Weise, wie sie kausal miteinander in Verbindung gesetzt werden, behaupten und andere Interpretationen verstummen. Im Interview mit einem der Mitglieder wurde nicht nur der hegemoniale Status der Aussage „Fußball empowert“ ersichtlich, sondern auch, wie die diskursiven Anknüpfungen in die Organisationsgruppe hineinwirkten und dort über Sag- und Unsagbares entschieden:

„Ich hab manchmal so ein Problem mit diesem ,Fußball macht Frauen stark' und "Selbstbewusstsein durch den Sport", also diese ganzen Stichpunkte, die wir sagen, und die zum Beispiel [eine andere Frauen- und Sport-NGO] auch sagt und die auch alle stimmen. Aber ich nehme mich selbst als das genaue Gegenteil wahr und hab so das Gefühl: ,Okay, bei mir hat's nicht gewirkt und ich bin nicht so, aber allgemein hat Sport eine positive Wirkung'،

Die Wirkmächtigkeit der diskursiven Formation wird darin deutlich, dass die Fußballerin das Ausbleiben eines bestärkenden Gefühls als schambehaftetes, persönliches Defizit empfand, anstatt die Gültigkeit der Aussage „Fußball empowert" in Zweifel zu ziehen. Zugleich nahm sie die Äußerungen als Stichpunkte wahr und machte damit deutlich, dass diese eine Komplexität reduzierende Abstraktion darstellen. Durch Reduktion und Nicht-konkretWerden können sich möglichst viele nuancierte Erfahrungen in einer Aussage versammeln, verunmöglichen aber - vielleicht gerade durch die Menge der versammelten Erfahrungen oder ihrer bereits zur Formelhaftigkeit geronnenen Stabilität - die Entstehung einer intelligiblen Gegen-Aussage. Durch die verschiedenen diskursiven Anknüpfungen vergrößerte DF die Anknüpfungspunkte an die unterschiedlichen Ziele und Schwerpunkte der Geberinstitutionen und minderte zugleich jene Zukunftsunsicherheit, die aufgrund der sich kontinuierlich verändernden Förderprioritäten ständig bestand. Zugleich fanden so jene Erfahrungen, die nicht mit den dominanten - oder zumindest 
intelligiblen - diskursiven Formationen von Fußball und Geschlecht kompatibel sind, kaum Eingang.

\subsubsection{Sport politisieren, Politisches versportlichen}

Eine möglichst breite Anerkennung und Unterstützung DFs - sowohl seitens politischer, zivilgesellschaftlicher als auch verbandssportlicher Institutionen - wurde nicht nur durch die Art und Breite der Anknüpfungen erzielt, sondern auch durch die damit verknüpften Felder Sport und Entwicklungs-, Außen- und Geschlechterpolitik. Einerseits ermöglichte die Politisierung des Fußballs die Anschlüsse an und Unterstützungen durch verbandssportferne zivilgesellschaftliche Institutionen wie politische Stiftungen, Medien und feministische Initiativen. Dennoch scheint es auf den ersten Blick verwunderlich, dass DF darüber hinaus Würdigung und Anerkennung von fast allen parteipolitischen Lagern erhielt: Politiker innen sowohl von CDU, SPD, der Grünen und der Linken reagierten mit Begeisterung auf die Projekte und unterstützten sie entweder als Einzelpersonen oder durch Fraktionsspenden, Empfehlungen oder Kontaktvermittlungen. Während die Verknüpfung von sportlichen Mitteln mit gesellschaftspolitischen Zielen diese Unterstützung erst ermöglichte, möchte ich hier argumentieren, dass es gerade die Versportlichung und damit einhergehende Entschärfung politischer Inhalte ist, die die Breite der Anerkennung ermöglichte. Die Übertragung von Aufgaben der Friedensförderung und Emanzipation auf den Fußball verlagert diese zum einen auf eine vorrangig körperliche Aktivität, die sich dem Streit über ideologische und praktische Details entzieht. Zum anderen transferiert sie die Verhandlung politischer Themen von der engen und parteipolitisch umkämpften Arena der Außen- und Geschlechterpolitik in einen vermeintlich anderen Gesellschaftsbereich. Dieser andere Bereich ist nicht nur die beliebteste Sportart in Deutschland und damit besonders konsensfähig, sondern besitzt auch die Aura des Spielerischen und des Vergnüglichen und genießt den Ruf parteipolitischer Neutralität. Dies mildert die parteipolitische Brisanz und den Streitwert von Themen, wie die Implementierung von Frauenrechten und der angemessene Umgang mit Diskriminierungen. Bemerkenswert hieran ist, dass die erfolgreiche Politisierung und Problematisierung fußballerischer Geschlechterverhältnisse damit auf der Konstitution des Fußballs als jenseits politischer Interessen gelagert basieren. Diese Gleichzeitigkeit von Politisierung und Entpolitisierung mag als Widerspruch erscheinen. Im Alltag von DF gestaltete sie sich jedoch als produktives Spannungsverhältnis, das von den Aktivist_innen nicht als Antagonismus wahrgenommen wurde. Vielmehr situierten sie ihre Arbeit darin und nutzten beide Facetten zu ihrem Vorteil. 


\subsection{Strategisch essentialisieren: „Das ist nicht die politische Realität"6}

Die Anknüpfung DFs an bestehende diskursive Formationen basierte auch auf der Annahme der sozialen Kategorie Frau. Diese erfordert nicht nur die Eingliederung in einen intelligiblen Diskurs der Zweigeschlechtlichkeit, sondern bietet die Möglichkeit, auf konkrete Gesetze und Rechte und damit verbundene Normen und moralische Imperative sowie geschlechterzentrierte Förderprogramme zurückzugreifen und durch sie Legitimität, finanzielle und argumentative Ressourcen zu akquirieren (vgl. Katzenstein 1998, 79ff.). Rosalind P. Petchesky verweist darauf, dass Menschenrechte ein „discursive field of power relations within the domain of racialized, gendered global capital in its present form" sind $(2003,22)$. Die Forderungen und Politiken, die sich auf die vom Menschenrechtsdiskurs zur Verfügung gestellten Identitäten beziehen, können daher immer nur innerhalb dieser Machtbeziehungen geschehen, sie werden von diesen gar hervorgebracht und durchzogen. Feminist_innen müssen damit auf die ein oder andere Art umgehen (ebd., 23) und finden sich, wie Luca im folgenden Beispiel, nicht selten vor inneren Konflikten wieder:

„Ich bin da schon auch in einem inneren Konflikt. [...] Ich hab mich dafür entschieden, erst mal ich will Mädchen supporten, ihnen alle Möglichkeiten geben und da auch Sachen für schaffen, dass sie alles machen können, was sie wollen und ihnen dabei helfen. Aber ich sehe schon selbst, in meiner anderen Welt, die es ja auch gibt und die auch total wichtig für mich ist, denk ich gar nicht zweigeschlechtlich. Aber das ist nicht die politische Realität und das ist auch nicht die gesellschaftspolitische Form, in der wir leben. Und das ist für mich, glaub ich, so der wichtigste Punkt. Wenn es möglich wäre, würde ich Fußballtraining gerne auch für FLTI-Kinder anbieten.“ (Luca, Interview, 3.1.2014) ${ }^{96}$

Luca sprach hier nicht nur von der politischen Arena, die der Menschenrechtsdiskurs strukturiert, sondern von einer gesamten gesellschaftspolitischen Realität, die auch den Verbandsfußball einschloss. „The sport arena can be described as a distinctly sexed arena, in which sport participation in general is organized by the contestants' sex“" (Eng 2008, 117). Im Verbandsfußball gilt: Beantragt eine Person einen Pass, um am Spielbetrieb teilzunehmen, muss sie sich mit einem Kreuz auf dem Formular zwischen „,männlich“ und „weiblich“ entscheiden. Vereine können Teams entweder im Männer- oder Frauenfußball melden und Geschlechtstests im professionellen Fußball sichern Eindeutigkeit. ${ }^{97}$ Die gesamte Organisation des Spielbetriebs, die Diskussionen um Inklusion und gerechte Teilhabe, Vielfalt und Diskriminierung im Sport verlaufen entlang der Zweigeschlechtlichkeit.

96 FLTI steht für Frauen, Lesben, transsexuelle/-gender und intergeschlechtliche Personen.

$97 \mathrm{Zu}$ Geschlechtstests im Profisport siehe Jakubowska (2014), Müller (2016). 


\subsubsection{Jenseits der Heteronormativität}

Im Zuge der Politisierung äußerten viele Mitglieder das Anliegen, Diskriminierung aufgrund sexueller Orientierung stärker zu adressieren und in der Projektarbeit der engen Verbindung von Sport und sexueller Orientierung gerecht zu werden. Dies resultierte auf der Vereinsvollversammlung Anfang 2013 in der Satzungserweiterung, die nun ,die Bekämpfung von Ungleichheiten und strukturellen Diskriminierungen jeglicher Art wie Rassismus, Sexismus, Handicapism, Klassismus, Lookism, sexuelle Orientierung“ zu einem weiteren Vereinszweck erhob. Diese Erweiterung war auch durch den Wunsch motiviert, einen Gegenentwurf zu Satzungen anderer Vereine und Verbände zu schaffen, in denen Homophobie meist hinter der politisch weniger brisanten Formel ,und andere Diskriminierungsformen“ verschwand.

Die erfolgreiche Inklusion nicht-heterosexueller Erfahrungen durch die Benennung von Homophobie als Diskriminierungsform war nicht allein eine Frage der eigenen Überzeugungen, sondern hing maßgeblich von rechtlichen Rahmenbedingungen sowie von gesellschaftlichen Situationen innerhalb der internationalen Landschaft ab, in der DF agierte, wie eine Diskussion über den Themenschwerpunkt für ein bevorstehendes Expertinnenforum veranschaulicht. Es standen verschiedene Themenvorschläge im Raum, unter anderem die Verbesserung fußballinterner Strukturen oder die Adressierung von Diskriminierungen.

Thomas: „Wir müssen erst wissen, wen wir einladen, und dann erst das Thema wählen. In manchen Ländern ist LGBTI wichtig, in anderen sind Fußballstrukturen wichtig."

Nadine: „Für mich lässt sich das aber nicht trennen, das geht zusammen. Es ist eine Realität, dass Fußball und Homosexualität eng verbunden sind, aber man muss die natürlich auch nicht immer thematisieren."

Simone: „Wenn wir jetzt den Schwerpunkt des Projekts auf europäische Länder legen, darf man das Thema nicht ausklammern. Man darf nicht vergessen, dass es fast nur Lesben waren, die den Fußball in Deutschland hochgebracht haben.“

Birgit: „Frauenfußball ist sowieso schon mit so einem Stigma belegt. Ich fürchte, dass die Spielerinnen, die eh schon dadurch in ihrer Gesellschaft auffallen, weil sie Fußball spielen, sich dann nicht trauen würden, uns zu kontaktieren, wenn wir LGBTI so deutlich thematisieren. Dabei wär es für sie total wichtig, dabei zu sein.“

Saskia: „Aber so verschließt man sich anderen. Man schließt die aus oder spricht sie jedenfalls nicht an, für die das Thema wichtiger ist.“

Linda: „Ich hab halt Angst, dass wir für das Thema zu trampelig sind und Menschen dadurch in Gefahr bringen. Homosexualität wird eh im Frauenfußball subtil verhandelt. Bisher haben wir kein einziges Team getroffen, in dem das nicht Thema war. Die 
Spielerinnen tauschen sich dann bilateral aus und wir können es informell thematisieren ohne das offiziell in das Programm zu schreiben. “98

Hier stand einerseits der Wunsch zur Verhandlung, die enge Verwobenheit von Fußball und sexueller Orientierung sowie die Erfahrungen lesbischer Frauen im Fußball angemessen einzubeziehen. Auf der anderen Seite standen die rechtlichen Restriktionen und sozialen Stigmata, mit denen Homosexualität in vielen Ländern belegt ist und mit denen eine international ausgerichtete Organisation umgehen musste. Lindas Sorge, ,dass wir für das Thema zu trampelig sind“, bezieht sich auf die Brisanz des Themas, da aufgrund der rigiden Anti-Homosexuellen-Gesetze Fußballerinnen in manchen Ländern durch einen Kontakt mit den Themen Homophobie und Homosexualität in ernsthafte Gefahren geraten können. Die Thematisierung von Lesbenfeindlichkeit - im Unterschied zur Thematisierung von Frauenfeindlichkeit - ist aufgrund restriktiver Gesetze oder inkonsequenter Strafverfolgung von Hassverbrechen sowie starker gesellschaftlicher Stigmatisierungen mit viel höheren Risiken verbunden. Während sich DF vor der heterogenen Landschaft strategisch essentialisierend als Frauenfußball- und Frauenrechtsorganisation organisierte und somit auf argumentative und rechtliche Ressourcen wie Frauenrechte zurückgreifen konnte, erforderte die Formation als Organisation, die für die Anerkennung sexueller Vielfalt im Fußball und gegen Diskriminierung aufgrund sexueller Orientierung kämpfte, ein deutlich vorsichtigeres Vorgehen (siehe Kap.16.3). ${ }^{99}$

Mary Bernstein spricht ausgehend von der Beobachtung, dass Aktivist innen ihre Erfahrungen nie unreflektiert und essentialisierend in politische Strategien übersetzen, von ,identity deployment“ $(2005$, 62). Damit fasst sie, wie Kollektividentitäten strategisch eingesetzt werden, und macht für den hiesigen Kontext deutlich, dass Identitätspolitik immer das situative und taktische Anwenden und Aktualisieren kollektiver Identitäten bedeutet.

98 Da solche Diskussionen häufiger geführt wurden, habe ich hier zur klareren Darstellung der Argumente zwei Diskussionen miteinander verflochten (Feldnotiz, 20.6.2013 und 17.2.2013). Dass sich keine dialogartige Diskussion und direkten Bezüge entsponnen, sondern nacheinander jedes Mitglied seine Ansichten darlegte, ist der Tatsache geschuldet, dass Redelisten den Ablauf der Diskussion regelten.

99 Erst kürzlich fanden die Belange von LGBTI-Personen Eingang in den Menschenrechtsdiskurs der Vereinten Nationen: 2011, 2014 und 2016 adressierten schrittweise Resolutionen die weltweite Gewalt und Diskriminierung aufgrund sexueller Orientierung und Geschlechtsidentität (Ausserer 2016). Die Dokumente scheinen längst nicht die gleiche normative Autorität zu besitzen wie die Frauenrechtskonvention. Auch zeichnet sich kaum Veränderung im Verbandsport ab. LGBTI-Sportgruppen organisieren sich meist unabhängig von den Dachverbänden (vgl. Heissenberger 2018). Zu Queer Theory und Sport siehe Scraton/Flinthoff (2002), Caudwell (2006). Für DF bedeutete dies, dass ihr Engagement in dem Bereich weniger publik wurde; bspw. beteiligten sie sich an dem Netzwerk, das die Umsetzung der Yogyakarta-Prinzipien, die die Anwendung der Menschenrechte in Bezug auf Geschlechtsidentität und sexuelle Orientierung ermöglichen sollen, durch die Bundesregierung begleitete. 
Das realisierte sich bei DF dadurch, dass keine Grundsatzentscheidungen getroffen, sondern Möglichkeiten situativ eruiert, Kompromisse eingegangen und ausprobiert wurden. Das Changieren zwischen mannigfaltigen Gestalten, zwischen Explikation oder Implikation sexueller Vielfalt, zeigt den Prozess des Sich-Organisierens als schrittweises Vortasten entlang eines ,utopisch unterlegten Pragmatismus“ (Adam 2016).

\subsubsection{Durch das Dilemma der Zweigeschlechtlichkeit manövrieren}

Während eine Erweiterung der Kategorie Frau in Hinblick auf sexuelle Vielfalt nicht immer unproblematisch, jedoch partiell möglich war, gestaltete sich eine konsequente Entfernung vom Konstrukt der Zweigeschlechtlichkeit als unmöglich. Luca verwies auf die gesellschaftliche Realität und den daraus resultierenden inneren Konflikt zwischen Real- und Idealpolitik: Am liebsten wäre Luca ein Fußball, in dem nicht nur Frauen, sondern auch Lesben, transgender/-sexuellen und intergeschlechtlichen Personen intelligible Subjektpositionen und allgemein anerkannte Rechte zur Verfügung stünden. Die Organisator_innen standen damit wieder vor einem Dilemma: Die Bedingungen eines politisch intelligiblen Diskurses erforderten einen Kompromiss zwischen moralisch Vorstellungen vom richtigen Vorgehen und idealistischen Visionen einerseits und einem pragmatischen, identitätspolitischen Vorgehen andererseits. Dies wurde ersichtlich, als die Arbeitsgruppe, die das Handbuch „Frauen Macht Fußball. Erfahrungsberichte aus über 20 Ländern“ erstellte, die Schreibweise in der deutschen Ausgabe diskutierte. Im Raum stand die Frage, ob der sogenannte Gender-Gap verwendet werden sollte, der nach dem männlichen Genus-Suffix einen Unterstrich einfügt und dann das weibliche anhängt, um sowohl beide Geschlechter als auch weitere, nichtbinärgeschlechtliche Positionen zu inkludieren.

Saskia: „Konsequenter wäre es, das Binnen-I zu verwenden. Der Gender-Gap ist scheinheilig, weil wir in unserem ganzen Denken und Schreiben zweigeschlechtlich denken - außer vielleicht ein oder zwei.“ Ich frage nach: „Naja, klar, wir reden von Frauenfußball, aber das ist ja die offizielle Bezeichnung. Denken wir wirklich so zweigeschlechtlich?“ Saskia erklärt: „Aber wir reden immer von Mädchen und Frauen, die sich in der Männerwelt durchsetzen. Es geht nicht, wir schreiben Gender-Gap und eigentlich kämpfen wir für Frauenrechte." Martina merkt an, dass wir zwar immer Mädchen und Frauen ansprechen würden, aber ja nicht wissen könnten, wie sie sich selbst verstehen. Melanie schlägt vor, bei Frauen und Mädchen immer die weibliche Form zu verwenden und wenn wir von allen Menschen sprechen, den GenderGap zu verwenden. Martina stimmt dem schließlich zu, auch wenn es unlogisch sei. Ich merke an, dass wir so ja alle transgender/-sexuelle und intergeschlechtlichen Personen ausschließen. Martina und Saskia sind für Melanies Vorschlag. Melanie: „Wir sollten das an irgendeiner Stelle erklären. Vielleicht mit einer Fußnote einmal am Anfang, in der wir erklären, warum wir das so verwenden und wen wir meinen.“ Ich wende ein, dass Fußnoten zu wissenschaftlich seien und wir damit viele Leute 
abschrecken würden. Das Handbuch solle einfach zu lesen sein. Saskia schlägt etwas später einen Infokasten am Rande der Einleitung vor, der erklärt, dass wir uns an Frauen und Mädchen auf der ganzen Welt richten.(Feldnotiz, 9.4.2013)

Die taktisch notwendigen Anknüpfungen an die Identitätskategorie der Frauenrechte wie auch an die Aussage „Fußball empowert“" ließen das gleichzeitige Streben nach einem nicht-binärgeschlechtlichen Fußball ,scheinheilig“ und ,unlogisch“ erscheinen. Dieses Dilemma ist nicht aufzulösen, sondern nur zu manövrieren. Die Arbeitsgruppe entschied sich schließlich für einen Infokasten, der das Dilemma transparent machte:

\section{„Notiz zu unserer Verwendung des Gender Gaps und den Begriffen, Mäd- chen/Frauen'}

Wir haben uns entschieden, in der deutschen Fassung dieses Handbuchs den sogenannten Gender Gap zu verwenden, der über die Grenzen der Zweigeschlechtlichkeit hinausweist, während andererseits in diesem Buch immer wieder die Rede von Mädchen und Frauen ist. Diese Inkonsistenz ist unserem Bedürfnis geschuldet, sowohl die Möglichkeit einer Gesellschaft mitzudenken, in der geschlechtliche Vielfalt gedacht und gelebt wird, als auch von den gegebenen Verhältnissen auszugehen, in denen Menschen auf und jenseits des Fußballfeldes strikt in Männer und Frauen unterteilt werden und als Mädchen und Frauen Benachteiligungen erfahren." (Discover Football 2013, 2)

Dieser Infokasten wie auch die bereits zweimal thematisierte Schreibweise „Frauen*“ verstehe ich daher als Praktik des Manövrierens zwischen den einzelnen Kompetenzregimen: Unterstrich und Sternchen signalisierten das Bemühen um möglichst umfassende Inklusion und Repräsentation. Gleichzeitig verdeutlichten sie die Unumgänglichkeit eines ,strategic use of a positivist essentialism“ (Spivak 1996, 214). Gayatri C. Spivak (1996) formuliert mit dem Konzept des strategischen Essentialismus, wie Kollektivkategorien beschworen und zugleich theoretisch als unhaltbar kritisiert werden. Sternchen und Unterstrich übten diese theoretische Kritik, sodass sich DF damit vor den eigenen Mitgliedern selbst sowie zahlreichen lokalen und weltweiten Fußballer_innen und Aktivist_innen als queer-feministisch und politisch informiert legitimierte. ${ }^{100}$ Zugleich verschaffte die Reartikulation der zweigeschlechtlichen Kategorien die Anerkennung als Frauenrechts- und Frauenfußballorganisation seitens staatlicher Verwaltungen, frauenrechtlich argumentierender Förderprogramme sowie des Verbandsfußballs.

100 Degele versteht die spezifische Sprech- und Schreibweise auch als anerkennungsrelevantes Handeln nach Normen politischer Korrektheit: Dadurch positioniert sich die Sprecher_in vor relevanten anderen in der Hoffnung, deren Anerkennung zu erlangen (2013, 67f.). Die Schreibweise weist auch auf den Imperativ politischer Korrektheit in der Gruppe hin. 


\subsection{Netzwerk verwalten: „Beziehungsarbeit“}

Die Anerkennung als verlässliche, professionelle und glaubwürdige Expertin für Frauenrechte und Fußball basierte nicht allein darauf, diskursiv an die heterogenen Stakeholder anzuknüpfen, sondern auch darauf, diese taktisch miteinander zu verknüpfen. Die Aktivist_innen hatten seit DFs Gründung vielzählige Kontakte mit einzelnen Politiker_innen und Verwaltungsangestellten in der Lokal- und Bundespolitik, in Fraktionen, Ministerien und Ämtern, mit Stiftungen, Kulturvereinen, feministischen (Sport-)Initiativen und Netzwerken, zu Wissenschaftler_innen und Medienschaffenden geknüpft. International standen sie mit Frauenrechtsaktivistinnen und -journalistinnen, Fußballerinnen, Funktionärinnen, Graswurzelfußballprojekten, internationalen Sport-for-Development- und Frauenrechts-NGOs und sozialpolitisch engagierten Fußballvereinen im Austausch. Die Vernetzung mit den Fußballverbänden fiel aufgrund der bereits beschriebenen Differenz deutlich schwerer und wurde wenig forciert, dennoch unterstützten auch hier einzelne Persönlichkeiten und Funktionär_innen die Ziele von DF. Mit Bourdieus Konzept des sozialen Kapitals lässt sich dieses Beziehungsnetz als Ressource untersuchen:

„Das Sozialkapital ist die Gesamtheit der aktuellen und potentiellen Ressourcen, die mit dem Besitz eines dauerhaften Netzes von mehr oder weniger institutionalisierten Beziehungen gegenseitigen Kennens oder Anerkennens verbunden sind; oder, anders ausgedrückt, es handelt sich dabei um Ressourcen, die auf der Zugehörigkeit zu einer Gruppe beruhen." (Bourdieu 1983, 191; Herv. i. Orig.)

Der Umfang des Sozialkapitals bildet sich aus der Ausdehnung des tatsächlich mobilisierbaren Beziehungsnetze und aus dem Umfang des Gesamtkapitals der Mitglieder im Beziehungsnetz (ebd. 1983, 192; 1992a, 63f.). Es geht also nicht nur um die einzelnen Personen im Beziehungsnetz, sondern auch um die Ressourcen, die sie zur Verfügung stellen können. Sei es, dass ein Verweis auf die prominenten Netzwerkmitglieder das Renommee DFs steigerte, ein Bundespolitiker den Kontakt zu einem großen Unternehmen und zukünftigen Sponsor herstellte, dass ein Empfehlungsschreiben des UNSondergesandten für Sport einem Antrag auf finanzielle Förderung beigelegt werden konnte oder dass ein Mitglied einen Technikverleih kannte, der kostengünstig technisches Equipment zur Verfügung stellte. So ließ sich soziales in ökonomisches und symbolisches Kapital konvertieren und die Umsetzung der Projekte sicherstellen. 


\subsubsection{Kontakte pflegen}

Eine solche Konvertierbarkeit hängt jedoch in großem Maße vom geschickten Verwalten des Sozialkapitals ab, denn soziales Kapital funktioniert in Form gegenseitiger Versicherung und der Vergabe von Kreditwürdigkeit (ebd., 191). Durch aktive „Beziehungsarbeit“ (Bourdieu 1992a, 67) in Form von „materiellen und/oder symbolischen Tauschbeziehungen“" (ebd. 1983, 191), wie das Verschicken von Newslettern, die öffentliche Nennung ihrer Förderer, durch Kooperationsangebote und Weihnachtskarten sowie die Pflege einer Facebook-Seite und dadurch die Versorgung der vielen Freunde mit Informationen, zollte DF ihrerseits Anerkennung. Zugleich fungierten die einzelnen Mitglieder als Delegierte des sozialen Kapitals der Organisation, denn im Gegensatz zum kulturellen ist soziales Kapital nicht körpergebunden (Bourdieu 1992a, 68). Mitglieder nahmen deutschlandweit wie international an Konferenzen zu den Themen Geschlecht und Empowerment, Menschenrechte und Antidiskriminierung sowie Integration und soziale Verantwortung im Sport und Fußball teil, wie z.B. an einem Round Table des asiatischen Fußballverbandes im jordanischen Amman, an der Weltsportministerkonferenz in Berlin oder an dem Symposium für Sport und Menschenrechte der Akademie für Fußballkultur in Nürnberg. Sie besuchten Netzwerktreffen verschiedener Zusammenschlüsse zivilgesellschaftlicher, sportbezogener Netzwerke wie Football Against Racism in Europe (FARE) und European Gays and Lesbian Sports Federation (EGLSF). Sie reisten auf Einladung von Partner_innen in andere Länder und Regionen zu Freundschaftsspielen, präsentierten dort eine mobile Ausstellung oder gaben einen Workshop. So war es aufgrund der relativ hohen Mitgliederzahl möglich, an vielen Stellen und auf verschiedenen Ebenen gleichzeitig Beziehungsarbeit zu leisten.

\subsubsection{Kontakte verknüpfen}

Das geschickte Verwalten des Sozialkapitals ging über die bloße Beziehungsarbeit in Form der Aufrechterhaltung oder Intensivierung bilateraler Kontakte hinaus und umfasste auch multilaterales Verknüpfen verschiedener Kontakte miteinander. Diese Kontaktverwaltung geschah ebenfalls in einem beständigen Balanceakt zwischen verschiedenen Erwartungen und Anerkennungslogiken. Zum einen erforderten die eigenen feministischen, solidarischen Ziele und Überzeugungen, dass vor allem jenen internationalen Teilnehmer_innen auf der fußballerischen und frauenpolitischen Graswurzelebene größtmögliche Unterstützung durch die Vermittlung von Kontakten ermöglicht wurde - sei es in Form von potentiellen Förderinstitutionen oder verbündeten Mitstreiterinnen. In diesem Sinne bemühte sich DF um eine beständige Verbesserung der Netzwerkarbeit durch Internetblogs, Newsletter 
und direkte Kontaktvermittlungen. Zum anderen hing ihre Legitimität als Expertin für Frauenrechte und Fußball auch von ihrer Scharnierfunktion zwischen lokaler und (trans-)nationaler Ebene ab (vgl. Thayer 2010a, 217). $\mathrm{Da}$ es mehrere (trans-/inter-)nationale Regierungsinstitutionen, wie AA, BMZ und UN, und (internationale) NGOs vor allem im globalen Norden gab, die mithilfe des Frauensports soziale und politische Ziele häufig mit Bezug zum globalen Süden verfolgten, waren Kontakte zu dort engagierten Frauen gefragt. Als DF 2013 im Nachklang des sogenannten arabischen Frühlings ein Expertinnenforum mit Frauen aus der MENA-Region organisierte, notierte ich über den Bewerbungs- und Auswahlprozess der Teilnehmenden:

Es dauerte eine ganze Weile, bis Bewerbungen eintrudelten. Wir mussten den Bewerbungsschluss 2 Mal nach hinten verschieben. Viele Bewerbungen kamen über die NGOs Cross Cultural Projects Association und Right to Play. Wir entschieden uns dafür, tatsächlich nur Personen aus der Region aufzunehmen und Anfragen z.B. aus den USA oder aus dem Kosovo abzulehnen. Auch dann mussten wir uns noch entscheiden, da wir nur 15 Plätze vergeben konnten, aber mehr Bewerberinnen hatten. Besonders bedeutend war die Bewerbung von Sarah aus Saudi Arabien, und bei allen öffentlichkeitswirksamen Gelegenheiten wiesen wir darauf hin. (Feldnotiz, 3.1.2013)

So galten Kontakte zu Frauen in Krisengebieten wie Afghanistan oder Libyen oder in Ländern mit einem besonderen Defizit an (der Umsetzung von) Frauenrechten, wie Saudi Arabien oder Pakistan, als besonders wertvoll. Die Bedeutung, die Sarahs Bewerbung beigemessen wurde, speiste sich auch aus der Annahme, dass sie von dieser internationalen Begegnung besonders viel profitieren würde, indem sie sich hilfreich vernetzen könnte. Gleichzeitig wurde der Wert erkannt, den dieser seltene Kontakt für die öffentlichkeitswirksame Erscheinung von DF als Expertin und Vermittlerin besaß. Der symbolische Wert eines Kontakts hing von der politischen, frauenrechtlichen und kulturellen Situation in den einzelnen Ländern ab. Dass diese Strategie funktionierte, belegt die recht große mediale Resonanz auf die Teilnahme der saudischen Spielerin. NGOs gelten gerade aufgrund ihrer Kontakte zur und ihrer Kompetenz auf lokaler Ebene als wichtige und wertvolle Partnerinnen für Politikprogramme (Edwards/Hulme 1996b). „Localness“ wird dabei zu einer gewichtigen Legitimität- und Argumentationsressource (Forbes 1999).

Paradoxerweise gerät dieser Status jedoch bei allzu erfolgreicher Kontaktvermittlung in Gefahr: Als z.B. ein Vertreter einer öffentlichen Fördereinrichtung auf einer von DF organisierten Veranstaltung den Kontakt zu einer Teilnehmerin aus einem südasiatischen Land suchte, um mit ihr mögliche Kooperationen zu besprechen, reagierten Mitglieder von DF irritiert, da sie in die Planung selbst nicht miteinbezogen wurden. Das Verwalten der Beziehungen innerhalb machtvoll und marktförmig strukturierter Landschaften muss immer mit Fingerspitzengefühl geschehen, um einerseits gegenseitige solidarische Unterstützungen im Sinne des übergeordneten politischen Ziels und Ideals zu ermöglichen, andererseits aber nicht den Wert des eigenen 
Sozialkapitals unbedacht zu verschenken und sich als Bindeglied und Expertin überflüssig zu machen.

Hier wird die Fragilität sozialen Kapitals auf zweierlei Weise deutlich: Erstens erfordert die Aufrechterhaltung des Netzwerks und damit der Erhalt seines symbolischen Werts kontinuierliche Beziehungsarbeit. Zweitens kann der Wert des Sozialkapitals durch mangelnde Behutsamkeit verloren gehen. Die Arbeit am Sozialkapital erfordert ein genaues Überprüfen der Kontakte, der Arten der Beziehung sowie der zu erbringenden und einzufordernden Tauschgaben, um die eigene Anerkennung als Expertin nicht aufs Spiel zu setzen. Nur durch beständige Aushandlung der Beziehungen und Tauschgaben kann ein Netz aus heterogenen Akteur_innen mit unterschiedlichen Kapitalvolumen dem Anspruch gegenseitiger Solidarität und Unterstützung sowie gleichzeitig dem Bestreben, den eigenen symbolischen Wert möglichst hoch $\mathrm{zu}$ halten, gerecht werden. Denn gerade auf den symbolischen Wert dieses Netzwerks kommt es dann an, wenn ökonomische Ressourcen akquiriert werden müssen. Das geschickte Verwalten der Kontakte bedeutet also, permanent zwischen der Gestalt der internationalen Expertinnen-NGO und der Gestalt der feministisch-solidarischen Aktivistin zu balancieren.

\subsection{Expertise schaffen: „Mit differenziertem Wissen auftrumpfen"}

Die Mitglieder von DF wurden häufig von privaten und politischen Stiftungen und Bildungseinrichtungen zu Konferenzen, Netzwerkveranstaltungen oder Diskussionsrunden als Zuhörer_innen, Workshop-Leiter_innen, Diskutant_innen oder Sprecher_innen eingeladen:

Zusammen mit Martina reise ich mit dem Zug nach Leipzig. DF wurde von einer Stiftung zum Zwecke der partnerschaftlichen Entwicklung Afrikas zu einem großen Kongress eingeladen. Da ein thematischer und methodischer Schwerpunkt der Stiftungsarbeit auf dem Sport lag, sollte sich DF hier neben vielen anderen sportbezogenen Initiativen vorstellen. Beim Plenum wurde, nachdem ich meine Bereitschaft erklärt hatte, entschieden, dass ich die Präsentation halten und von Martina als Unterstützung begleitet werden sollte. Bei anderen Anlässen hatte ich gelernt, dass zu solchen Gelegenheiten stets die Gründungsgeschichte [siehe Kap. 8.1.1] erzählt wurde und dass dies in einer "Wir"-Perspektive geschah, die die Erzählerin zum Teil der Iranreisegruppe werden ließ und ihr damit die Teilhabe an der kollektiven Erfahrung der Gruppe ermöglichte. Nun stand ich also vor circa 30 Zuhörer_innen, hinter mir liefen Powerpoint-Folien mit Bildern und Stichpunkten an der Wañ vorbei, die die internationale Projektarbeit von DF dokumentierten, und ich erzählte, wie die damalige fußballerische Begegnung im Iran die Erfahrung ermöglichte, dass mit Fußball nationale, sprachliche und kulturelle Grenzen überwunden werden könnten. Ein kurzer, musikunterlegter Imagefilm über das Frauen-Fußball-Kultur-Festival vermittelte 
eine Stimmung von Gemeinschaft und Leidenschaft für Fußball und Frauenkämpfe unter den Teilnehmerinnen. Anschließend applaudierte das Publikum und das Mikro wurde weitergereicht an eine Vertreterin einer anderen Sport-for-Development-NGO. Diese visualisierte auf ihren Powerpoint-Folien die vielen Projekte, führte Charts zur Dokumentation ihrer Aktivitäten an und bewies mithilfe vieler Zahlen die Reichweite ihrer Arbeit. Der Unterschied zwischen unseren Präsentationen hätte kaum größer sein können. Nach dem Ende des Panels wandte sich Martina mir zu und lobte mich, wie es unter DF-Mitgliedern üblich ist, für meinen Auftritt. Es sei sehr gut gewesen, wie ich DF präsentiert hätte und gerade im Kontrast zu der anderen, unbestreitbar größeren NGO seien wir viel sympathischer, lebendiger und einprägsamer rübergekommen. (Erinnerungsprotokolle 3.9.2015; 1.3.2013)

An diesem Ereignis fällt zunächst auf, dass Erfahrung die Projektaktivitäten legitimierte und den Expertinnenstatus begründete. Sowohl die eigenen als auch die Erfahrungen der teilnehmenden Frauen begründeten immer wieder die Deutung von Fußball als verbindend, empowernd und geschlechterpolitisch. Silvy Chakkalakal stellt das anthropologische Paradigma der Erfahrung in historischer Tiefe dar und zeigt, wie Erfahrung ab dem 18. Jahrhundert in anthropologischen Debatten als zentrale Methode des Erkenntnisprozesses erachtet wurde:

„Erfahrung bedeutete nun eine Sammlung von (körperlich-seelischen) Eindrücken. Damit machten Anthropologen sie zur Grundlage der menschlichen Wissensproduktion und Verstandestätigkeit. Die aufklärerische Devise lautete: ohne Erfahrung keine Vernunft!“ (Chakkalakal 2014, 56)

Indem die Mitglieder auf ihre eigenen Erfahrungen verwiesen und damit ihre geschlechter- und sportpolitischen Tätigkeiten begründeten, knüpften sie an diese abendländisch-aufklärerische Tradition des epistemologischen Umgangs mit Erfahrung an. Sie addierten ihre eigenen mit den Erfahrungen ihrer Projektpartnerinnen und deuteten diese durch gesellschaftliche und sozialwissenschaftliche Theorien über Geschlecht und Sport. So übersetzten sie Erfahrung in Wissen und schließlich - da dieses Wissen nicht allen zugänglich war - in Expertise auf dem Gebiet von Fußball und Frauenrechten. Sonia Alvarez erklärt die Wertigkeit diese Expertise aus den neoliberalen Rahmenbedingungen: Um Gender in Entwicklungspolitiken und -programmen berücksichtigen zu können, greifen Staaten und internationale Regierungsorganisationen vermehrt auf feministische NGOs und ihre ,Gender-Expertise“ zurück (1999, 192). Auch Saskia beobachtete diese Wertigkeit:

Ich erzähle, dass ich gerade an einem Kapitel schreibe, wo es um die Herstellung von Gemeinschaft gehe. Ich müsse dabei an eine kritische Bemerkung von einem anderen Mitglied denken, das mal meinte, dass wir ja auch viele Frauen nicht beachten würden, die was anderes sagen würden, als wir annehmen würden. Wir gehen ein paar Beispiele durch. [...] Saskia ergänzt: Das stimme so nun auch nicht, zum Teil würden wir deren Aussagen auch aufgreifen und sie Teil unserer Expertise werden lassen. So könnten wir dann an anderen Stellen durch differenziertes Wissen über den weltweiten Frauenfußball auftrumpfen und sagen: „Aber in Jordanien ist es zum Beispiel so 
und so und in Palästina so und so". Dadurch würde unsere Expertise gestärkt und wir könnten noch mehr wie Expertinnen wirken. (Feldnotiz, 12.11.2014)

Ich gehe in den Kapiteln 13.1.2, 16.3.2 und 17.5.3 auf den Umgang mit abweichenden Situationsdeutungen ein. An dieser Stelle ist Saskias Hinweis relevant, dass sich der Expertinnenstatus auch durch differenziertes Wissen über lokale Kontexte und die vielen verschiedenen Realitäten des Frauenfußballs generierte, das dann in entsprechenden Momenten vor jenen, die nicht über dieses Wissen verfügten, artikuliert werden konnte. Erfahrungsaustausch und die Kontakte zu denjenigen, die diese Erfahrungen machten, waren nicht nur erklärter Selbstzweck der Arbeit von DF, sondern fungierten auch als „exchangeable commodity“ (Thayer 2010a, 217), deren geschicktes Verwalten und Austauschen Legitimität und Glaubwürdigkeit sicherte. ${ }^{101}$ Das zitierte Erinnerungsprotokoll vom gemeinsamen Konferenzbesuch zeigt zugleich zwei verschiedene Arten, in Expertise übersetzte Erfahrungen zu präsentieren, und damit auch zwei Weisen, sich als Expertin zu organisieren. Im Folgenden wird ersichtlich, wie sich DF über die gewählte Darstellungsart auch von anderen NGOs abgrenzte.

\subsection{Erfahrung privilegieren: „Wir sind viel sympathischer"6}

Durch den Bezug auf eigene Erfahrungen als Argumentationsstrategie formierte sich DF als glaubwürdige, , authentische' Organisation, deren Mitglieder das, wovon sie sprachen, selbst erlebt hatten. Zwar steht dieser Erfahrungsbezug in einer abendländisch-aufklärerischen Tradition der Erkenntnisgewinnung, doch weicht die Darstellung der Erfahrung vom Pfad der Rationalisierung $a b$. Während sich die andere NGO auf dem Leipziger Kongress mithilfe von Statistiken und Impactanalysen als kompetente NGO und breitenwirksame Partnerin legitimierte (vgl. Mosse 2004, 460), nutzte DF Authentifizierung, Emotionalisierung und Personalisierung - hier sei an die ständige Erzählung der hoch emotionalen Gründungsgeschichte erinnert -, um als ,liebevoll“ (Innenminister) und „enthusiastisch“ (Selbstbeschreibung) wahrgenommen zu werden. ${ }^{102}$ Dass Martina und ich diese Selbstdarstellung

101 Zur Genealogie von Erfahrungspolitik und der Verallgemeinerbarkeit von Erfahrung siehe Kap. 16.2 und 16.3. Tsing bemerkt, dass „traveling stories“ bei ihrem Transfer vom einen kulturellen und politischen Kontext in einen anderen die Form von Allegorien annehmen. Aktivist_innen tragen Geschichten über Regionen und Genres, um sie in neue institutionelle Kontexte einzubetten, in denen sie sich verbreiten und wirken können $(2005,234)$.

102 Thayer zeigt, wie kapitalistische Marktlogik Effizienz und Ergebnisse von NGOs als quantifizierbare und linear auf ein Ergebnis zulaufende Module konzipiert. Die quantifizierende Darstellung ihrer Rechenschaftspflicht orientiert sich eher an den Kriterien der Geberinsti- 
begrüßten, zeigt, dass wir mit unserem Engagement bei DF ein spezifisches Bild von internationalen NGOs zu vermeiden suchten. Dieses Bild ist stark von feministischen und postkolonialen Diskussionen geprägt, die unter den Schlagworten Post-Development und NGOization einen internationalen Entwicklungsdiskurs und die entsprechenden Agenturen und Praktiken als imperialistisch, eurozentrisch und die ökonomischen, ökologischen, menschenrechtlichen wie gesundheitlichen Probleme entpolitisierend kritisieren (vgl. Ferguson 1990; Escobar 1997; Ziai 2006). Saida Hodžić spricht in diesem Zusammenhang vom „NGOization paradigm“ (2014, 224). Sie erfasst damit, wie feministische Ethnografien die Zunahme von NGOs mit geschlechterund frauenthematischen Schwerpunkten eher normativ als analytisch diskutieren. Die Ethnografien beschreiben die wachsende Zahl an NGOs als Bedrohung für feministische Initiativen und Aktionsformen, denn mit ihnen erhielten Förderpolitiken, vereinheitlichte Organisationsstrukturen und universalisierende Diskurse über den modernen Staat Einzug in die widerständigen Räume der Zivilgesellschaft; die feministisch-widerständigen Bewegungen würden NGOisiert (ebd., 244). So kritisieren Autor_innen mit dem Konzept der NGOization den Wandel von widerständigen feministischen Bewegungen zum bürokratischen und institutionalisierten ,well-mannered, reasonable, salaried, 9-to-5 job“ (Roy 2014). In der Folge würden Frauen außerhalb des professionalisierten NGO-Umfeldes keinen Zugang mehr finden (Simpson 2009, 144; vgl. Hodžić 2014, 245), und Prioritäten würden sich zunehmend von den Belangen der lokalen Frauen entfernen und Aktivitäten in verengte, staatszentrierte Strategien verwandelt, die mehr der patriarchalen Logik denn einer alternativen feministischen Perspektive entsprächen (Siliman 1999, 40; vgl. Hodžić 2014, 246). Die Studien bewerten die Abkehr von Erfahrung als politischer Strategie als einschneidenden Effekt der Bürokratisierung, Professionalisierung und Institutionalisierung (Lang 1997, 116). Feministische wie Post-Development-Ansätze konstituieren damit eine Opposition zwischen zivilgesellschaftlichen Organisationsformen mit lokalem Aktivismus auf der einen und NGOs auf der anderen Seite. Aktivismus wird nostalgisch als "'below', closer to the ground, more authentic, and more 'rooted" idealisiert (Ferguson/Gupta 2002, 982) und NGOs als kooptierte Regierungsinstrumente des Entwicklungsapparats und neoliberaler Reformen abgewertet (Hodžić 2014, 224).103 Hodžić argumentiert, dass sich das

tutionen, denn an den Kriterien der lokalen Bevölkerung und Zielgruppe der Politiken (2010a, 220f). Siehe hierzu auch Edwards/Hulme (1996a). An dieser Stelle ist zu überlegen, ob sich in DFs Moralisierung und Emotionalisierung nicht etwa ein aktueller Trend in der internationalen Frauenrechtsarbeit abzeichnet, den Bernal/Grewal beobachteten: weg vom technokratischen Diskurs um Entwicklung und Empowerment und hin zu moralischen Begriffen und emotionalen Appellen im Namen von Frauen (2014a, 308).

103 Hodžić zeigt, dass das Narrativ auf drei verschiedenen Strängen kritischer Wissenschaft basiert (2014, 226f.): Erstens beinhaltet es eine Erweiterung der Post-Development-Kritik Escobars am Macht/Wissens-Regime des Entwicklungsdiskurses, welche nun auf feministi- 
NGOization-Paradigma inzwischen zu einem Masternarrativ entwickelt, das nun in einem normativen Sinne feministisches Wissen über zivilgesellschaftliche Organisationen anleitet (ebd., 222).

Als Celia und ich uns 2015 am Flughafen trafen, um die Teilnehmenden des bevorstehenden Festivals zu empfangen, ereignete sich folgende Szene:

Sowohl das Team aus Burkina Faso als auch aus Kamerun kamen etwa zeitglich am selben Terminal an. Als Celia und ich das Gate erreichten, aus dem gleich das ruandische Team kommen sollte, sprachen uns zwei ältere, weiße Damen an. Sie trugen TShirts von der deutsch-kamerunischen NGO, zu der das Team gehörte, und wollten das Team ebenfalls empfangen. Später gesellten sich noch mehr NGO-Angehörige hinzu. Besonders fiel mir ein großer weißer Mann in Afrika-Muster-Hemd auf. Als wir weitergingen, regte sich Celia ein wenig auf: So eine Frau habe sie heute schon mal getroffen, die ganz euphorisch rief: ,We are a German NGO' und, so entnahm ich ihrem Zitat, dabei „German“ besonders betonte. Celia und ich rollten mit den Augen und gingen dann weiter, um das Team aus Burkina Faso in Empfang zu nehmen. (Feldnotiz, 29.6.2015)

Celias und meine Abneigung gegen die westlichen Repräsentant_innen transnationaler NGOs basierte auf unserer Vorstellung von bilateralen, paternalistischen Beziehungen zwischen der gebenden deutschen und der empfangenden und ausführenden kamerunischen Seite, die sich hinter der philanthropischen Haltung verbergen mochten und letztlich globale Ungleichheitsstrukturen festigen würden. Unsere Abneigung resultierte nicht zuletzt aus unserer Auseinandersetzung mit postkolonialen und feministischen Kritiken während unseres Studiums und in politisch engagierten Milieus. Mit Ian Hacking lassen sich solche wissenschaftlichen Klassifizierungen zivilgesellschaftlicher Akteur innen darauf hin befragen, wie sie neue Subjekte schaffen - hier die Unterscheidung zwischen NGO-Bürokrat_in ${ }^{104}$ und Aktivist in als zivilgesellschaftliche Unterkategorien - und wie die klassifizierten Personen nun mit den Klassifikationen interagieren. Das Phänomen, dass die wissenschaftliche Klassifikation selbst im Feld wirkmächtig wird, bezeichnet Hacking als „looping effect“ $(2007,286)$. Bei DF war zu beobachten, wie das NGOization-Paradigma und die ihm inhärenten Klassifikationen im Feld von Relevanz

sche NGOs übertragen wird. Zweitens ist ihm die u.a. von Brown (1995) artikulierte Skepsis gegenüber Allianzen mit jenen (staatlichen) Institutionen eingeschrieben, die selbst Elemente der männlichen Herrschaft beinhalten und so letztendlich nur reformerische und keine emanzipatorischen Politiken produzieren. Drittens greift es auf die Kritik an der akademischen Institutionalisierung des Feminismus zurück, wonach der Feminismus seine kritische Schärfe durch die Umwandlung in eine universitäre Karriere verlor.

104 Riles beschreibt die in den 1980er Jahren aufstrebenden neuen Mitarbeiterinnen in pazifischen Frauenrechtsorganisationen, die die Aktivistinnen der Gründungsjahre ergänzten oder ablösten, wie folgt: „Educated professionals and persons with institutional experience in fields unrelated to women and activism, much more skilled in interfacing with aid agencies on these organizations' terms, accustomed to travel and life overseas, knowledgeable about the procedures of the UN and other international institutions, and less interested in the overt politicization of causes that had animated the YWCA." $(2007,32)$ 
waren und die Selbstdarstellung, die Beziehungen zu anderen NGOS sowie die eigene Verortung innerhalb einer internationalen NGO-Landschaft anleiteten. DF wurde zwar von einem social movement market in die Kategorie der NGO gedrängt, stand dieser aber selbst skeptisch gegenüber. Am Flughafen drückten wir diese Skepsis aus: Anstatt den Kontakt zur anderen NGO zu intensivieren, wandten wir uns einem anderen Gate $\mathrm{zu}$, aus dem gleich ein frauenpolitisch aktives Fußballteam kommen würde. Die Formierung als Aktivist_innen betont die Verschmelzung des Privaten und Politischen in Lebensstilen und hebt die Verflochtenheit von persönlichen Erfahrungen mit politischen Überzeugungen als Motiv des Handelns hervor. ${ }^{105}$ Die Gestalt der Aktivistin ist damit auch eine moralisch aufgeladene Identitätskategorie, da sie in einem spezifischen Umfeld ,gutes' politisches Handeln repräsentiert. Auf dem Leipziger Kongress grenzten wir uns in diesem Sinne durch unsere spezifische Selbstdarstellung als „sympathisch“, „lebendig“, selbst betroffen und erfahrungsbasiert sowie den Verzicht auf Fakten und Quantifizierungen mittels Analysen, Evaluationen und Statistiken vom Bild der distanzierten, allein aus professionellen Motiven handelnden Bürokrat_innen ab.

Bei näherem Hinsehen ist diese Abgrenzung nicht nur den eigenen Idealen, sondern auch den Rahmenbedingungen geschuldet, da DF schlicht nicht die personellen und finanziellen Ressourcen zur Quantifizierung und Verwissenschaftlichung zur Verfügung standen. Aufgrund der Hochkonjunktur von erfahrungsbasierter Argumentation und Politik in liberalen Demokratien boten visualisierte und narrativierte Erfahrung dennoch ein zugängliches „,investment capital“, das es mit evidenz- und faktenbasierten Strategien aufnehmen konnte (Scott 1991; Phipps 2016; siehe Kap. 16). Ich greife die Formierung als Aktivist innen später wieder auf, da sie nicht nur die Distanzierung von großen NGOs und eine spezifische Positionierung innerhalb eines social movement markets bedeutete, sondern DF auch innerhalb einer transnationalen Graswurzelbewegung situierte. Hier gilt festzuhalten: Die situative Abgrenzung von internationalen Entwicklungsorganisationen und die Konstitution als feministische, aktivistische Alternative auf Graswurzelebene folgte sowohl internen moralischen Vorstellungen von translokalen feministischen Beziehungen als auch einem pragmatischen Umgang mit den finanziell und personell begrenzten Mitteln. Das Changieren zwischen den Gestalten zwischen Expert_innen-NGO und Aktivistin - ermöglichte strategische Anschlüsse an verschiedene Stakeholder, brachte aber auch Spannungen mit. 


\section{Aktivistin und Fußballerin - die Mitgliederschaft}

Sozialwissenschaftliche Bewegungsforschungen untersuchen diskursive Anknüpfungen und Umdeutungen durch Protestbewegungen als ,collective action frames" (Benford/Snow 2000) auch auf ihre interne Mobilisierungsund Vergemeinschaftungsfunktion hin. Dies aufgreifend zeige ich, wie eine solche diskursive Problematisierung der fußballerischen Geschlechterverhältnisse eine Interpretation der eigenen Erfahrungen und eine Auseinandersetzung mit der zugewiesenen Subjektposition ermöglichte und damit einen geteilten Wissensbestand schuf, auf dessen Basis sich DF als kohärente Gruppe organisierte und vergemeinschaftete. Die Mitglieder hatten eine gemeinsame affirmative Subjektposition entwickelt (10.1), die aufgrund gruppeninterner Heterogenität immer wieder verhandelt wurde (10.2). Bevor ich diese Vergemeinschaftungsprozesse nachzeichne und zeige, wie sich DF gegenüber den eigenen Mitgliedern als aktivistische, fußballaffine Initiative auswies, stelle ich anhand zweier Portraits dar, aus welchen verschiedenen Motiven und Hintergründen die Mitglieder von DF zusammenkamen. Thea und Gerit sind fiktionale Charaktere, die ich zur Verdichtung und Exemplifizierung aus den biografischen Interviews mit den Mitgliedern DFs geschaffen habe. Während Gerit mit dem Verbandsfußball aufwuchs und bestens mit dessen Logik vertraut ist, wird Thea von gesellschaftspolitischen und feministischen Motiven zu DF geleitet. In der Empirie sind selbstverständlich (mindestens) diese beiden Logiken in den einzelnen Akteurinnen eng miteinander verflochten. ${ }^{106}$

\subsubsection{Portrait 1: Gerit}

Gerit kommt 1982 in einer mittelgroßen westdeutschen Stadt zur Welt. Sie kann kaum laufen, da kickt sie schon mit ihren älteren Brüdern und Nachbarskindern bei jeder Gelegenheit im Wendehammer am Ende ihrer Straße. Mit sechs Jahren zieht sie mit ihrer Familie für ein Jahr nach Israel. Es ist ein Sommer mit Europameisterschaft, und die ermöglicht ihr, trotz Sprachbarrieren die ersehnten Freundschaften zu schließen: „Erstens, war das die Möglichkeit, mit den anderen Kindern zu spielen. Zweitens war, wenn Deutschland gespielt hat, das ein Grund von anderen Kindern zum Fernsehen eingeladen zu werden." Zurück in Deutschland wird der Pausenhof zum Fußballfeld, und als einziges Mädchen spielt Gerit in der Fußball-AG der Grund-

106 Neben analytischen und argumentativen Zwecken dient dieses Vorgehen auch der Anonymisierung von Personen bei gleichzeitigem Einblick in persönliche Erfahrungen und Empfindungen. $\mathrm{Zu}$ composite person als Werkzeug semi-fiktionaler Ethnografie siehe Humphreys/Watson $(2009,49)$. 
schule. Sie erinnert sich an das erste Spiel mit ihren Schulkameraden: „Das war für mich so ein Initiationsritus, weil ich wurde als Letzte gewählt, und Sebi, der total unsportlich war, wurde noch eins vor mir gewählt." Die Vorurteile, die ihre Teamkollegen ihr gegenüber haben, lösen sich auf, sobald sie ihr Können unter Beweis stellt. Für sie wird Gerit zu einem ganz normalen Fußballer, ihr Mädchen-Sein wird temporär unsichtbar. Einmal staunte ein Mitspieler über ihre Schussstärke: „Boah, der Geri, der hat 'nen Bums!“ Es ist schließlich auch die Mutter eines Klassenkameraden, die sie mit in ihren ersten Verein nimmt. Fortan trainiert sie als einziges Mädchen auch im lokalen Fußballverein mit. In den Augen der Gegner, gegen die sie bei Turnieren oder Freundschaftsspielen antreten, sticht sie als einziges Mädchen weiterhin heraus. „Es gab einerseits so: ,Iiieh, Mädchen!' Das ist ja auch so eine Zeit. Dann gab's aber auch ganz viel solche Kommentare wie: ,Wenn du schaffst, mir den Ball abzunehmen, dann verlieb ich mich in dich." "Ihre Mitspieler wissen diesen Moment anfänglicher Verunsicherung geschickt zum Vorteil umzudeuten: „Tobi, der war zum Beispiel so voll ein Guter, der hat immer zu mir gesagt: ,Du musst das voll ausnutzen, wenn die anderen Jungs von den anderen Teams über dich denken, du seist voll schlecht. Ich würd das voll ausnutzen, das ist voll dein Vorteil! ““ In diesen Jahren bietet ihr der Fußball eine Gegenwelt zum Leistungsdruck der Schule.

Mit 13 wird Gerit signalisiert, dass sie nicht mehr lange bei den Jungs mitspielen könne. Sie muss sich bereits in der Schiedsrichterkabine getrennt von ihrem Team umziehen. „In dem Verein gab es natürlich eine ganz funktionierende Jugendabteilung für die Jungen und dann für die Herren, das war alles organisiert, und man kommt dann halt einfach in die anderen Altersklassenmannschaften und dann gibt es Trainer und alles ist gut. Aber für die Mädchen gab es so was einfach nicht. [...] Deswegen war es halt so: ,Okay es ist vorbei, jetzt dürft ihr nicht mehr bei den Jungs spielen'." Sie wechselt auf Einladung einer Trainerin in ein weiter entferntes Mädchenteam.

Mit der Pubertät verstärkt sich das Gefühl, im Fußball in eine Gegenwelt einzutauchen. Plötzlich werden die Klassenkameradinnen komisch, sie beginnen, sich für Jungs und Mode zu interessieren, und auch das familiäre Umfeld formuliert Erwartungen an ihre Geschlechterperformanz. „Beim Fußball ging's halt darum, also aus meiner Position zumindest, laut zu sein, gut $\mathrm{zu}$ sein, mehr inhaltliche Positionen zu haben. Alle hatten irgendwie Fußballsachen an oder einen Trainingsanzug, und dieser ganze Schnickes drumherum war so irrelevant, und auch diese ganze Jungswelt war dann so draußen im Mädchenfußball." Ihren Eltern, die sie lieber im Tennisverein oder Geigenunterricht gesehen hätten, missfällt ihr Hobby. Auch wenn sie sie nicht aktiv hindern, verweigern sie ihr doch die Unterstützung. Der deutlich längere Anfahrtsweg und auch die anfallenden Materialkosten für Ausrüstung können nur mithilfe der engagierten Trainerin gemeistert werden. Gerit lässt sich nicht entmutigen und nimmt sogar an verschiedenen Auswahltrainings 
teil, schafft jedoch trotz intensiven Trainings den Sprung in den professionellen Sport nicht.

Zum Ende der Gymnasialzeit trifft sie endgültig die Entscheidung, Fußball nur noch als Hobby zu betreiben und sich nun vorrangig auf das Studium der Soziologie und Geschichte zu konzentrieren. Sie zieht nach Berlin. Es ist die Zeit der Bildungsstreiks Mitte der Nullerjahre, und Gerit beginnt, sich vermehrt für Politik zu interessieren: „,[Ich hab an] Unistreiks teilgenommen und hab dann auch das Thema Gender und Queer für mich entdeckt. Aber da gab's halt nie so eine Verbindung [zum Fußball]. Das waren immer so zwei Sachen." Ihr politisches und wissenschaftliches Interesse führt sie zu einer Konferenz zu Gender und Diversity der Gesellschaft für Internationale $\mathrm{Zu}$ sammenarbeit, wo zwei Mitglieder die Arbeit von DF präsentieren. Hier bietet sich für Gerit erstmals eine Verknüpfung der beiden Bereiche an, die sie bislang immer als getrennte, aber wesentliche Interessengebiete erlebte. Die Verknüpfung von Geschlechterpolitik und Fußball ,hat mir die Theorien für das gegeben, was ich zum Teil eben auch praktisch erfahren habe. Also so Geschlechterkonstruktionen, und was jetzt von wem erwartet wird und von wem nicht.“ Als Volunteer hilft sie 2011 beim Festival mit und steigt im Anschluss als Ehrenamtliche in die Organisationsgruppe ein.

\subsubsection{Portrait 2: Thea}

1989 geboren, wächst Thea in einer mitteldeutschen Kleinstadt als einzige Tochter einer alleinerziehenden Mutter auf. In der dörflich geprägten Umgebung tanzen sie aus der Reihe: ,Sie war mit noch einer anderen Mutter zusammen die einzige, die alleinerziehend war und alles andere waren angeblich heile Familien mit Vater, Mutter, Kind, die natürlich auch nicht so heil waren, aber nach außen eben so, wie's sein soll. Und es war halt schon so, dass sie immer einfach dafür kritisiert wurde und dass auch immer Fehler gesucht wurden." Thea erinnert den Lebensweg ihrer Mutter als Bruch mit Normen und gleichzeitig als Vorbild: „Was ich auch auf jeden Fall wieder von meiner Mama mitgekriegt habe, ist so ein bestimmtes Bild von einem Lebensweg oder einem möglichen Lebensweg von einer Frau, sodass es nicht Kinderkriegen, Kochen und Putzen ist oder sein muss, sondern ganz viel bedeutet und auf jeden Fall auch Ausbildung und Studium und so weiter. Und das hab ich auch von ihr so völlig selbstverständlich mitgekriegt, dass das eben klar ist, dass Frauen genauso beruflich aktiv sein können.“

Trotz Berufstätigkeit unterstützt die Mutter Theas frühe Leidenschaft für Sport, sodass sie schon in der zweiten Klasse ein Leichtathletiktraining beginnt. Ihre Mutter fährt sie zu den Trainings und schaut auch bei ihren späteren Wettkämpfen zu. Im Laufe ihrer Pubertät ebbt die Faszination des Leistungssports $a b$, mehr und mehr entwickelt sie eine Abneigung gegen den 
Leistungsdruck, und Freundschaften und andere Freizeitgestaltungen rücken in den Mittelpunkt. Als sie mit vierzehn Jahren Freundschaften zu älteren Jugendlichen knüpft, die in linken Jugendorganisationen aktiv sind, interessiert sie sich zunehmend auch für Politik: „Ich bin als Jugendliche jedes Wochenende auf eine Demo gefahren. Wir sind bis nach Nürnberg runter oder Wunsiedel. Oder nach Hamburg und Berlin auf Demos gefahren und haben Veranstaltungsreihen organisiert. Einmal oder zweimal die Woche hatten wir Plenum und wir haben uns irgendwie ständig gesehen." Ihre Mutter, selbst Mitglied einer politischen Partei, unterstützt auch dieses Interesse. Erst als sie in die gymnasiale Oberstufe kommt, erwacht - nicht zuletzt angeregt durch den Schulsport - ihr Interesse am Sport erneut, und gemeinsam mit zwei Freundinnen gründet sie ein eigenes Basketballteam. Nach dem Abitur beginnt sie ein Graphikdesign-Studium an der Kunsthochschule Leipzig. Doch dieses enttäuscht ihre Erwartungen, und zu ihren Kommiliton_innen findet sie keinen Anschluss. Schließlich bricht sie das Studium ab, es zieht sie nach Berlin, wo sie sich für ein Magisterstudium der Islamwissenschaft und Gender Studies einschreibt. Hier trifft sie auf ähnliche Menschen, wie sie sie noch aus ihrer politisch aktiven Schulzeit kennt, und findet schnell Kontakt zur linksalternativen und queer-feministischen Szene. Sie engagiert sich in verschiedenen Kollektiven, arbeitet ehrenamtlich in einem Projekt für geflüchtete Frauen aus dem arabischsprachigen Raum, und während eines Praktikums im Libanon 2011 begleitet sie eine Arbeitskollegin zum Fußballspielen, das von der dortigen Expat-Community organisiert wird. Sie steht das erste Mal auf einem Fußballplatz. Zurück in Berlin besucht sie fortan das Frauenfußballtraining des Unisports.

2013 erreicht sie über den E-Mailverteiler der Gender Studies ein Aufruf, in dem eine Frauenfußball- und Frauenrechtsorganisation Ehrenamtliche für die Organisation eines Festivals mit Fußballerinnen aus der MENA-Region suchte. Da sie ,in der Zeit tatsächlich nach ehrenamtlichen Sachen gesucht“ hatte, schließt sie sich der Gruppe an und sympathisiert mit Themen und Arbeitsweisen von DF: „Regelmäßige Treffen und die Art, Sachen zu planen, und die Entscheidungsfindung, dass versucht wird, das basisdemokratisch zu machen, und dass sich jeder einbringen kann, dass es nicht darum geht, was man vorher schon gemacht hat, was man kann, dass man halt kein Experte sein muss. Dass man halt Freiheit hat sich einzubringen. Und das dann in Verbindung mit Fußball und Frauenrechten, fand ich ein gutes Projekt. Deswegen bin ich dann dabeigeblieben." 


\subsection{Subjektivität affirmieren: „Female football activist“}

Wie Thea und Gerit brachten die Mitglieder unterschiedliche Interessen an und Erfahrungen mit Fußball und Feminismus mit. Ich frage daher, wie unter den Mitgliedern eine Subjektposition geschaffen wurde, die Unterschiede vereint und es ermöglicht, als einheitliche Organisation mit gemeinsamem Ziel aufzutreten.

Ich habe bereits gezeigt, wie DF schrittweise eine plausible Problematisierung der fußballerischen Geschlechterverhältnisse erarbeitete. Indem sich die Mitglieder untereinander und mit ihren internationalen Teilnehmerinnen über Erlebnisse als Frauen im Fußball austauschten, generierten sie eine plausible Deutung der Wirklichkeit. Ich erachte in Anlehnung an Joan Scott (2012) weder Erfahrung noch den wirklichkeitsdeutenden Rahmen (collective action frame) als vorrangig, sondern als gegenseitig konstituierend. Das bedeutet, dass die Deutung der eigenen Erfahrungen mithilfe eines kollektiven Diskurses wiederum das eigene Erinnern und Erleben anleitet, sodass die zu Portraits verdichteten biografischen Erzählungen als Produkte der spezifischen Problematisierung zu verstehen sind. Dadurch, dass das eigene Erleben entsprechend etablierter Diskurse und Erzählstrukturen erinnert und erzählt wurde, konstituierten sich die Erfahrungen der Mitglieder als ähnlich und boten damit einen geteilten Wissensbestand als Basis von Gemeinschaft. ${ }^{107}$

Noch zu Beginn meiner Forschung verwiesen die Mitglieder mit sehr unterschiedlichen Bezeichnungen aufeinander: als „Aktive“108, „Teamkolleg_innen“, „Freund_innen“, „Fußballenthusiast_innen“ oder „Kolleg_innen". Auf den T-Shirts, die die Mitglieder während der Festivals trugen, kennzeichnete sie schlicht das Wort „crew“ als Organisator_innen und gegenüber Externen umschrieben sie sich mit ,die Anderen bei DF“. Mit Blick auf die Entwicklung der Organisation von einer FußballfestivalOrganisationsgruppe hin zu einer Frauenrechtsorganisation mit ihren struktur- und formgebenden Prozessen verstehe ich diese Beobachtung auch als Hinweis darauf, dass sich damals noch kein einheitlicher Deutungsrahmen und keine kollektive Identität etabliert hatten. Dies geschah erst im Zuge der

107 Scott erinnert daran, dass Identitätskategorien - denn auch die marginalisierte Fußballerin ist innerhalb feministischer Diskurse eine Identitätskategorie - nie vor ihrer Anrufung existieren. Vielmehr werden Teile unserer physischen Körper und kulturelle Aspekte retrospektiv an die Identitäten gekoppelt und durch die Referenz auf die Kategorie eine überzeitliche ,illusory sameness“" zwischen Personen geschaffen $(2012,46)$. Cheah verweist auf die Rolle von Menschenrechtsdiskursen dabei. Die Subjekte des Menschenrechtsdiskurses existieren nicht a priori: „They are constituted as an institutionally recognizable collective, which they were previously not, so that they can have leverage as the subjects/objects of institutional decision making“. (2006, 168)

108 Diese Bezeichnung ist abgeleitet von der Unterscheidung zwischen aktiven und (temporär) inaktiven Mitgliedern und erschließt sich nur den Gruppenmitgliedern selbst. 
Formierung als nicht-staatliche Organisation und der Politisierung der Themen. Als DF 2014 schließlich ein Projekt in Brasilien durchführte, prangten auf der Rückseite der dafür entworfenen T-Shirts die Worte „female football activist“". Für die Frage nach den Formierungen als kompetente Organisation vor den eigenen Mitgliedern ist an dieser selbstgewählten und expressiven Subjektposition vor allem die Synthese aus den drei Facetten Frau, Fußballerin und Aktivistin relevant.

\subsubsection{Die Vereinbarkeit von Fußball und Weiblichkeit}

Johanna Kösters und ich haben herausgearbeitet, wie DF durch die Kommunikation der Problemdefinition eine Subjektposition kreierte, auf die sich die Mitglieder affirmativ beziehen konnten (Faust/Kösters 2016). Indem sie die ausgrenzenden und verletzenden Erfahrungen, die viele Fußballerinnen im Laufe ihrer Sportbiografien immer wieder machten, als Effekte struktureller Geschlechterungleichheiten und der heterosexistischen Ordnung des Fußballs statt als individuelle Reaktionen auf persönliches Versagen und Fehlentwicklungen deuteten, sahen sie das eigene Erlebten als kollektiv und sozialstrukturell bedingt. ${ }^{109}$ Darin hatten sich bereits die Debatten der Geschlechterforschung der letzten Dekaden eingeschrieben: „Frau/Fußballerin“ meinte nicht eine biologische, sondern eine soziale Kategorie, die, obwohl sie als kontingentes und soziales Konstrukt der Geschlechterverhältnisse galt, im Alltag der Spielerinnen so machtvoll wirkte, dass sie als zentraler Referenzpunkt für die Erfahrungen und Aktivitäten plausibel wurde. ${ }^{110}$

Der explizite Verweis auf die eigene Geschlechtlichkeit in Verbindung mit Fußball transformierte die Erfahrung von der Unvereinbarkeit von Fußball und Weiblichkeit, die viele Spielerinnen als ein ständiges Dilemma erfahren hatten: Wie Gerit durch die mangelnde elterliche Unterstützung und

109 Siehe hierzu die ethnografische Studie von Lau (2011) über eine US-amerikanische Schwarze Fitnessbewegung. $\mathrm{Zu}$ negativen Gefühlen und geschlechtlicher NichtKonformität als strukturell und nicht persönlich bedingt, siehe Ahmed (2004, 172; 2010).

110 Viele Mitglieder bei DF mussten diesen Deutungsrahmen erst lernen. Thomas beschrieb: „Es gibt aber auch Discover-Football-Mitglieder, die aus einer linkspolitischen feministischen Richtung kommen, in deren Denkstrukturen muss ich erst mal einsteigen. Und auch diese ganzen Gender-Begriffe, die bei allen Mitgliedern geläufig waren, die waren für mich absolutes Neuland“ (Interview, Thomas, 28.1.2014). Und Linda erinnert sich: „Wer weiß, aber ohne DF [hätte ich] vielleicht nie diese ganzen männlichen Strukturen oder die gesellschaftlichen Strukturen, wie sie halt so sind, wie sie sind, die hätte ich vielleicht ohne DF einfach gar nicht als das erkannt, was sie sind. Vielleicht wär ich dann eher zu so einer Frau geworden, die auch ja klassisch ist, die sich halt irgendwie alleine durchgekämpft hat und die dann an einer Karrierestufe ist und sich dann auch denkt: ,Warum, was habt ihr denn? Hat doch alles geklappt.' Und die halt gar nicht merkt, dass sie viel mehr kämpfen musste als Männer.“ (Linda, Interview, 25.9.2013) Neue Mitglieder transformierten ihre Erfahrungen so, dass sie ins Wissensrepertoire von DF passten (vgl. Wenger 2008, 138). 
das ausgedrückte Missfallen der Mutter, war auch anderen vermittelt worden, dass es sich für Mädchen und junge Frauen nicht schickte, Fußball zu spielen, weshalb einige versuchten, ihr sportliches Hobby zu verstecken. Mit ,Kampflesbe ' und ,Mannweib' drohten Stigmatisierungen, sollten sie traditionelle Weiblichkeiten zurückweisen und die männlich konnotierten Praktiken des Fußballs kompetent ausführen. Beim Fußballspielen selbst hingegen sahen sie sich aufgefordert, feminine Geschlechterperformanzen zu vermeiden, da sie sonst als ungeeignet oder unpassend für den Sport wahrgenommen wurden (Faust/Kösters 2016, 75). Bei Gerit war es die einsetzende Pubertät, also der sichtbare Prozess des Zur-Frau-Werdens, der ihren Status als regulärer Mitspieler jäh aufkündigt. Der Selbstentwurf als female footballer ermöglichte, zwei vermeintlich unvereinbare identitäre Facetten zu vereinen, eine positive Subjektposition $\mathrm{zu}$ imaginieren und negativen Stereotypisierungen selbstbewusst zu begegnen. ${ }^{111}$

\subsubsection{Die Vereinbarkeit von Fußball und Aktivismus}

Auch die Verbindung von Fußball und Aktivismus ist nicht selbstverständlich. So wie Gerit Fußball und Hochschulpolitik als zwei getrennte Bereiche wahrnimmt, erlebten auch andere ihr Fußballhobby als von ihren gesellschaftspolitischen Interessen losgelöst. Im Gegensatz dazu betonte die Bezeichnung als football activist die Politisierung des Fußballs und übertrug damit die moralisch aufgeladene Identität als Aktivistin auch auf das mit Leidenschaft ausgeübte Hobby. Durch die Politisierung des weiblichen Fußballspiels innerhalb des Deutungsrahmens bei DF wurde die eigene sportliche Betätigung zu einem Akt des Normverstoßes und des Widerstandes gegen gesellschaftliche Geschlechterbilder. Durch die Schlussfolgerung, dass Frauen im Fußball Selbstbehauptung und Durchsetzungsfähigkeit erlernten und damit aktiv die Geschlechterverhältnisse modellierten, verstanden sie das eigene Engagement für den Frauenfußball als gesellschaftspolitischen Aktivismus (ebd., 77) und boten eine ,liberating new synthesis of the personal and the political“" (Kauffman 1990, 67, zit. n. Fisher 1997, 458). Fußballerin-

111 Zum Zwang, sich im Fußball als kompetent zu beweisen sowie für eine ausführliche Beschreibung dieser Fußball-Weiblichkeit siehe Kap. 17.2.2, 17.4 und 17.6.3 Zur Unvereinbarkeit von Fußball und Weiblichkeit siehe Cox/Thompson (2000, 10f), Sobiech (2011, 58), Degele (2012). Z.B. berichtete Birgit, wie sie sich nicht traute, in der Straßenbahn den Kicker zu lesen, und stets lange Hosen trug, um ihre muskulösen Waden zu verstecken. Zur subjektivierenden Wirkung des Stereotyps der ,mannish lesbian“ (Newton 1989) auf Fußballerinnen bzw. Frauen in männlich konnotierten Sportarten siehe Palzkill (1995), Cox/Thompson (2000), Broad (2001), Griffin (2002), Pfister/Fasting (2004), Grimm (2016). Sie zeigen auch, wie viele Sportlerinnen in Abgrenzung zu und in Auseinandersetzung mit Heteronormativität eigene, selbstbewusste Selbstbilder entwerfen, die auf unterschiedliche Weisen Weiblichkeit, sexuelle Orientierungen und Sportlichkeit vereinen. Siehe Spivak (1996) zur identitätspolitischen Strategie einer positiven Subjektposition. 
nen wurden damit sowohl als Benachteiligte der patriarchalen Struktur als auch als potentielle Akteurinnen ihrer eigenen Befreiung angesprochen (vgl. Thayer 2010, 62).

In die andere Richtung boten sich so auch jenen Identifikationsmöglichkeiten, die wie Thea erst seit kurzem oder gar nicht Fußball spielten und aufgrund ihres frauenpolitischen Interesses zu DF gekommen waren. Die Verknüpfung von Geschlechterpolitik und Fußball baute die Brücke, über die sie sich dem Frauenfußball näherten und nun ihre neue sportliche Leidenschaft oder zumindest ihre Unterstützung ausdrücken konnten.

Es ist kein Zufall, dass die Mitglieder die feministische Problematisierung des Fußballs als Aktivismus beschrieben. Im Zusammenspiel mit der vom NGOization-Paradigma (vgl. Hodžić 2014) angeleiteten Positionierung auf Graswurzelebene verdichtete sich politisches Engagement innerhalb der urbanen Bewegungsszene und dem Image des Stadtteils zu einem attraktiven, da moralisch guten Lebensstil. ${ }^{112}$ Am Beispiel von DF wird ersichtlich, wie Organisationsform - aktivistische Initiative statt bürokratische NGO - und kollektive Identität verflochten sein können, sodass politisches Handeln, sportliches Hobby, Leidenschaft sowie vergeschlechtlichtes Selbstbild zu einem Lebensstil verschmelzen. Diese Symbiose half DF zum einen, die Motivation der einzelnen Mitglieder aufrechtzuerhalten und damit den $\mathrm{Zu}-$ sammenhalt als Organisation zu ermöglichen. Zum anderen bot die Subjektposition eine kollektive Identität, die Ähnlichkeit zwischen den Mitgliedern herstellte und damit ein kohärentes Auftreten und Forderungen-Stellen nach außen ermöglichte. Die kollektive Subjektivierung als Frauenfußballaktivistin ermöglichte dann, als eine einheitliche, handlungsfähige Person, die einen gemeinsamen Willen und ein geteiltes Bewusstsein besitzt, aufzutreten (Phillips 2010, 64).

\subsection{Identitäten verhandeln: „Frauen und Lesben“}

Neben den befreienden und selbstaffirmativen Effekten beinhaltet die Stabilisierung einer intern mobilisierenden und extern mobilisierbaren Identität stets die Auswahl von Erfahrungen und die Verunmöglichung alternativer, gar

112 Eng verflochten, aber nicht identisch ist die Subjektposition der Feministin. Diese Selbstbezeichnung wird zwar von den einzelnen Mitgliedern immer wieder verwendet, jedoch selten öffentlich als Gruppenidentität präsentiert. Katzenstein argumentiert, dass Frauen in männlich dominierten Bereichen wie dem US-Militär bereits mit so großen Vorbehalten gegenüber ihrem Geschlecht konfrontiert werden, dass ein Bekenntnis zum Feminismus unmöglich erscheint: ,there is little reason to be feminist when it is bad enough [...] to be women“ (1998, 86). Im Vergleich zum feministischen Subjekt erscheint die Aktivistin deutlich weniger kontrovers. 
konträrer Wirklichkeitsdeutungen. ${ }^{113}$ Denn die Verschmelzung der multiplen Verortungen und Perspektiven zu einer Kollektividentität beinhaltet auch das Glätten interner Differenzen (Phillips 2010).

\subsubsection{Humorvoller Umgang mit Differenz}

Während die Besetzung der Subjektposition der Aktivistin von allen Mitgliedern begrüßt wurde, löste die Subjektivierung als Frau immer wieder Diskussionen aus, denn schließlich bedürfen Sichtbarkeit und Unsichtbarkeit von Erfahrungen in heterogenen Gruppen ständiger Aushandlungsprozesse vor dem Hintergrund vergeschlechtlichter gesellschaftlicher Machtverhältnisse, in denen die Grenzen immer wieder verschoben werden. So arbeitete ich 2012 an mehreren Texten mit, mit denen sich DF um ausgeschriebene Preise bewerben wollte. Während ich in einem Entwurf stets von „Frauen“ sprach, ergänzte Nadine an den entsprechenden Stellen „und Lesben“ und machte damit erstens deutlich, dass die Kategorie Frau vorrangig heterosexuell gedacht wird und dass es einer expliziten Benennung sexueller Vielfalt bedarf, um dieser Annahme entgegenzuwirken. Zweitens implizierte sie, dass Erfahrungen lesbischer und heterosexueller Fußballerinnen nicht identisch seien, und unternahm den Versuch, Ersteren zu mehr Sichtbarkeit zu verhelfen.

Mit der Politisierung ging daher nicht nur eine Fokusverschiebung von „hauptsächlich was mit Fußball“ (Saskia) hin zu Geschlechterpolitiken einher, sondern - mit zeitlicher Verzögerung - auch eine Auseinandersetzung mit der gruppeninternen sexuellen Vielfalt. Kerstin erinnerte sich, dass die Anfänge von DF von heterosexuellen Frauen geprägt waren. Innerhalb DFs spiegelte sich so die gesellschaftliche heteronormative Ordnung: Heterosexualität wird so lange als gültig vorausgesetzt und durchzieht das Miteinander als unausgesprochene Selbstverständlichkeit, wie sie nicht aktiv in Zweifel gezogen und ihr andere Erfahrungen entgegengestellt werden (vgl. Valentine 1993). Den Schwierigkeiten und Spannungen, die entstehen, wenn innerhalb der Subjektposition Frau mehrere Erfahrungen gleichberechtigt nebeneinanderstehen sollen - heterosexuelle, lesbische und queere -, begegnete die Gruppe mit vielen Diskussionen, vor allem aber mit Humor.

Im Frühsommer 2013 wurden wir von einer ehemaligen Festival-Teilnehmerin in ihre Heimatstadt in Serbien eingeladen, um dort ein Freundschaftsspiel gegen das lokale Frauenteam auszuspielen und den Film Football Under Cover vorzuführen. [...] An einem Nachmittag bat unsere Gastgeberin darum, dass ein paar von uns sie zu einem kurzen Treffen mit einer lokalen Autorität begleiten würden. Bei dieser Person handelte es sich um die Direktorin der örtlichen Schule, die unseren Besuch unterstützt hatte. Nun sollten wir sie begrüßen und uns herzlich bedanken. So richtig schien keine Lust zu haben, dafür auf die bevorstehende Freizeit zu verzichten. Schließlich erklär-

113 Siehe den Überblick über die Theoretisierungen von Identitätspolitik bei Bernstein (2005). 
ten Annike, Ariane, Melanie und ich uns bereit. Ich setzte ein gespielt repräsentatives Lächeln auf und sagte: „Na gut, dann sind wir jetzt die Delegation der Herzen.“ Die anderen lachten und griffen diese Bezeichnung sofort albernd auf. Fortan etablierte sich die „Delegation der Herzen“ als Bezeichnung für jene Personen und kleinere Gruppen, die repräsentative Aufgaben übernahmen bzw. die aufgrund ihrer heteronormativen Erscheinungen als für diese geeignet erschienen. Kurze Zeit später tauchte ein zweiter Begriff als Gegenpol zur Delegation der Herzen auf, die „QueerFraktion", mit dem fortan augenzwinkernd jene Personen und Gruppen bezeichnet wurden, deren Auftreten nicht als heteronormativ-repräsentativ gedeutet wurde. (Feldnotiz, Mai 2013)

Mit der „Delegation der Herzen“ und der „Queer-Fraktion“ entstanden zwei scherzhafte Begriffe, um interne Differenzen und Spannungen zu benennen und mit ihnen umzugehen. Humor bot, vorausgesetzt die internen Beziehungen waren freundschaftlich und nicht konfliktreich (vgl. Heissenberger 2018, 220), einen aushaltbaren Umgang mit einem ernsten Thema - denn hier ging es um nicht weniger als den möglichen Ausschluss aus der Subjektposition und damit aus der Mitgliederschaft. ${ }^{114}$

Zudem gab die Verknüpfung der sexuellen Identitätsdarstellungen mit Zuständigkeiten allen das Gefühl, für die Gruppe nützlich zu sein: Wenn es darum ging, bei Veranstaltungen mit vielen verschiedenen Personen zu netzwerken, wurde vorher auch mit vielen Scherzen überlegt, wer sich wem annähern sollte. Neben bereits bestehenden persönlichen Kontakten und freien Kapazitäten basierte die Aufgabenverteilung auch auf Mutmaßungen über die entsprechenden Erwartungen der Stakeholder an Repräsentant innen von DF. Während davon ausgegangen wurde, dass die meisten staatlichadministrativen Institutionen eine heteronormative Anerkennungslogik verfolgten, wurde z.B. bei internationalen LGBTI-Netzwerken diese nicht erwartet. Dementsprechend bot die Heterogenität der Mitglieder in Hinblick auf ihre geschlechtlichen und sexuellen Identitätsdarstellungen die Möglichkeit, mehreren Kompetenzregimen gleichzeitig zu entsprechen.

Schließlich wurde die Verknüpfung von Zuständigkeiten und Identitäten in Form von Fraktionen und Delegationen als relativ durchlässig gelebt. Während manchmal Zuständigkeiten entsprechend der zugeschriebenen oder dargestellten sexuellen Orientierung verteilt wurden, waren diese nicht immer

114 Ein Gruppenausschluss ist auch auf persönlicher Ebene bedrohlich: DF war weit mehr als ein Arbeitszusammenhang: Die Mitglieder verbanden persönliche Freundschaften und verbrachten auch ihre Freizeiten zusammen. Gould zeigt, welche sozialen und emotionalen Funktionen jenseits politischer Interessenartikulation die Gruppen von ACT UP für die Aktivist_innen erfüllten $(2009,193)$. Zu Freundschaften in politischen Gruppen siehe Roth (2006). Schütte (1996) untersuchte Scherzkommunikation bei Orchestermusikern, also bei einer Gruppe von Menschen, die zur Kooperation verpflichtet sind. Ihm zufolge kann mittels Frotzelein und Witzen die ernsthafte Bearbeitung von Spannung und somit auch die Verkomplizierung des Miteinanders umgangen werden. Auch DF etablierte mit dem Scherzen einen positiven Kommunikationsmodus, der allen Beteiligten vermittelte, dass trotz Meinungsverschiedenheiten und Differenzen Interaktion möglich war. 
verbindlich, sondern wurden in anderen Momenten nach persönlichen Präferenzen, Kapazitäten und Kompetenzen vergeben. Auch auf andere Weise wurden die „Delegation der Herzen“ und die „Queer Fraktion“ nicht als geschlossene Kategorien sexueller Orientierung gehandhabt, sondern als humorvolle Bezeichnung für Geschlechterperformanzen, die innerhalb entsprechender Umfelder - heteronomativ, schwul-lesbisch oder queer - irritationsfrei bestehen:

Für die Auslosung der Gruppen, in denen das bevorstehende Turnier ausgespielt werden soll, treffen wir uns am frühen Abend in einem Biergarten. Die Auslosung soll auf Video aufgezeichnet, ins Internet gestellt und damit den bald anreisenden Teams zugänglich gemacht werden. Simone hatte in einer E-Mail gebeten, dass alle für das Video ihr T-Shirt mit dem DF-Logo vorne drauf tragen sollten. Als ich ankomme, sind schon einige da, doch ich bin die einzige, die tatsächlich das DF-T-Shirt trägt, alle anderen bringen Erklärungen und Ausreden vor. Simone sagt augenzwinkernd zu mir: „Deinen Platz in der Delegation der Herzen hast du sicher. Saskia ist raus.“ (Feldnotiz, 6.6.2013)

Bei einem Plenumstreffen sprechen wir über den bevorstehenden Empfang im Rahmen der Weltsportministerkonferenz. [...] Saskia fragt Nadine, was sie anziehen würde. Nadine schaut an sich runter: „Na, diese Hose oder so eine andere.“ Da ich vom heutigen Tag auf der Konferenz einen Blazer dabei habe, biete ich ihr diesen an. Sie grinst skeptisch und probiert ihn an. Lachend wendet sie sich zu Simone: „Auch ich kann eine Prinzessin sein!“” (Feldnotiz, 28.5.2013)

Die Durchlässigkeit der Kategorien wurde deutlich, als sich Saskia durch ein Nicht-Erfüllen der gefällig-femininen Repräsentationsfunktion ihre Zugehörigkeit verspielte und Nadine ein feminin-repräsentatives Kleidungsstück überzog. Die Zugehörigkeit zu einer Kategorie konnte mit der vergeschlechtlichten Performanz, seien es Kleidung oder politische Ansichten, wechseln und basierte damit nicht exklusiv auf sexueller Orientierung. Die Unterkategorien des weiblichen Subjekts wurden so nicht als essentialisierte Kategorien und damit unüberwindbare Gräben verhandelt, sondern tatsächlich als Fraktionen und Delegationen, denen man sich anschließen konnte. Das Vorgehen, Identitäten gruppenintern de-essentialisierend als fluide und instabil $\mathrm{zu}$ behandeln und gleichzeitig nach außen hin strategisch zu essentialisieren, erforderte ein ständiges Hin-und-her-Bewegen zwischen unterschiedlichen Argumentations- und Deutungsweisen. Gerade weil der diskursive und rechtliche Kontext essentialisierende Erfahrungspolitik erforderte, bestand die Gefahr, dass sich auch intern Kategorien verfestigten und damit Differenzen festschrieben, statt weiterhin als kontingente Effekte heteronormativer Ordnungen kritisiert zu werden.

Festzuhalten ist, dass sich Humor als Modus des Umgangs mit dem ernsten und spannungsreichen Thema interner Differenz herausgebildet hatte. Die Ambiguität des Humors ermöglicht es, potentiell konfliktive Anliegen zu thematisieren und als ernst zu vermitteln, dabei jedoch den Grad der Ernsthaftigkeit so offen und unscharf zu halten, dass ihn alle in ihrem Sinne deuten 
können. Dieses Vage-Halten macht zusammen mit der humorvollen Überspitzung Differenzen und Spannungen aushaltbar und vermeidet unüberbrückbare Konflikte oder gar Ausschlüsse (Günther 1996; Schütte 1996). Zudem verweisen Frotzeleien, wie sie Saskia, Nadine und mir wiederfuhren, auf geteilte Wissensbestände und Interaktionsgeschichten. Die Möglichkeit der Äußerung spielerisch-ernster Kritik ohne Aufforderung zur Veränderung basiert auf einer vertrauensvollen Beziehung und bestärkt die Intimität zwischen den Beteiligten (Günther 1996), denn schließlich funktionieren Running Gags und Insiderwitze selbstreferentiell und setzen einigermaßen dauerhafte Gruppenkonstellationen voraus (Heissenberger 2018, 214). Über den Humor konstituierte sich die Gruppe somit in Bezug auf sexuelle Orientierung als intern divers und konnte diese Diversität nicht nur als potentielle Konfliktquelle, sondern auch als nützliche Ressource verhandeln.

\subsection{2 „I started kicking in my mother's stomach“}

Bei DF trafen Frauen wie Thea, die ein Interesse an Politik mit Sportbezug hatten, auf Personen wie Gerit, die ihren Sport politischer gestalten wollten. Wurde die Identifikation als Aktivistin von allen begrüßt, so musste nicht nur die gemeinsame Identifikation als Frau, sondern auch als Fußballerin ausgehandelt werden. Während sich Mitglieder wie Gerit und Laura aufgrund ihrer Fußballbiografie tiefgehend mit der Subjektposition der Fußballerin identifizierten, bedurfte es bei Mitgliedern wie Thea aktiver Identifikationsanstrengungen. Diese sahen z.B. so aus, dass die meisten neuen Mitglieder, die bislang keinen oder nur einen sehr sporadischen Bezug zum Fußball hatten, alsbald die Trainings des DFC Kreuzbergs besuchten und sich im Fußballspielen ausprobierten. Für jene, die nicht spielten, bot die Politisierung des Fußballs den Bezugsrahmen zur Subjektposition: Ihr feministisches Streben nach umfassender Gerechtigkeit begründete ihr Engagement für den Fußball. Diese breiten Identifikationsmöglichkeiten funktionierten so lange, wie die Subjektposition der female football activist nicht zu eng definiert wurde, wie folgendes Beispiel zeigt. Für das Festival 2013 hatte DF eine Aussage einer früheren Teilnehmerin aus dem Libanon aufgegriffen und in großen weißen Lettern auf hellblaue und hellgrüne T-Shirts gedruckt: „I started kicking in my mother's stomach“. Monate danach erinnerte ich mich wieder an meine eigene Reaktion auf den Merchandise-Artikel:

Meine Reaktion war damals, dass ich es super fand, aber mich selbst nicht traute, es zu kaufen und zu tragen, weil ich mir darin lächerlich vorkam. Ich fühlte mich, als würde ich lügen und mir eine Fußballerinnenidentität anziehen, die ich überhaupt nicht verkörperte, da ich Anfängerin sei und nicht seit Kindesalter spielte. Nachdem ich dies notiert habe, rufe ich bei Linda an, um zu fragen, ob sie das T-Shirt besäße. Sie bejaht die Frage und ahnt sofort, worauf ich hinauswill, denn sie fügt an: ,Obwohl ich nicht gut Fußball spiele." Sie erklärt, dass das Shirt durchaus viele Diskussionen 
in der Gruppe ausgelöst habe. Das T-Shirt sei ein Politikum, auch innerhalb von DF. Eine andere wolle es z.B. nicht tragen, da sie denke, nicht gut genug Fußball zu spielen. „Natürlich“, wendet Linda ein, ,es hat schon was von Angeben, aber das ist mir dann egal.“ (Feldnotiz, 6.6.2014)

Was Linda als Angeben und ich als Lügen empfand, verweist auf eine Diskrepanz zwischen Wahrnehmung und Behauptung der eigenen fußballerischen Fähigkeit. Daraus lässt sich schließen, dass die Subjektposition der Fußballerin den Mitgliedern so lange eine gemeinsame Identität ermöglichte, wie sie nicht auf eine lebenslange Fußballkariere und entsprechende spielerische Stärke reduziert wurde. Kollektive Subjektivitäten wirken nur dann affirmierend und mobilisierend, und formen aus heterogenen Personen eine Mitgliederschaft, wenn sie eine gewisse Offenheit für Differenzen bieten und diese gleichzeitig als durchlässig praktizieren. Da auch innerhalb von und durch Gruppen hindurch gesellschaftliche Machtverhältnisse wirken, erfordert diese Offenheit ein ständiges Thematisieren und Aushandeln. Konflikte und Spannungen um sowie ein humorvoller Umgang mit Inklusion von Erfahrungen und Identitätsfacetten sind daher nicht Ausnahmen und temporäre Erscheinungen in heterogenen Gruppen, sondern erscheinen hier als dauerhafter Modus der Formierung einer Mitgliederschaft.

\section{Mitstreitende und Lernende - weltweite Frauenfußballinitiativen}

Nachdem die Ausschreibungen für das Festival über Goethe-Institute, Botschaften, Stiftungen, Fußballverbände und -netzwerke, Universitäten und NGOs in die ganze Welt verschickt worden waren, wählten die Aktivist_innen anhand der eingegangenen Bewerbungsbögen acht Teams aus: ein im indischen Exil lebendes tibetisches Team; ein zu einer deutsch-ruandischen NGO gehöriges Team aus Lehrerinnen; ein sich für Frauen-Empowerment stark machendes, am regulären Ligabetrieb teilnehmendes tunesisches Team; ein professionelles Team aus Jordanien, das seine Spielerinnen auch außerhalb des Fußballplatzes besonders stark fördert; ein argentinisches Team, das in Armenvierteln Fußballprojekte durchführt und die sozial benachteiligten Spielerinnen in allen Lebensfragen unterstützt; ein Team einer kambodschanischen NGO; ein südafrikanisches Team lesbischer Aktivistinnen, die Hassverbrechen öffentlich anprangerten, sowie ein Heimteam bestehend aus Berliner Spielerinnen des eigenen Fußballvereins, des Geflüchteten-Projekts Champions ohne Grenzen und des Projekts Frau am Ball für Frauen mit und ohne Behinderung. Ein paar Wochen vor dem Festival verschickte DF eine E-Mail an die zukünftigen Gäste mit allen relevanten Infos. Neben praktischen Reisehinweisen hieß es darin:

„DISCOVER FOOTBALL is a non-profit organization and almost the entire team is working on a voluntary basis. Therefore, the DISCOVER FOOTBALL festival 2013 needs the support and commitment of each and every participant to become a real 
success. As your hosts we hope that you will spend a fantastic week with us and with each other. Due to our limited financial and personal capacities, please excuse if not everything is perfect. So take initiative, help each other and look after each other because DISCOVER FOOTBALL 2013 is a group project [...] Before fixing the definite program, we want to encourage you to contribute to its realization by offering workshop units yourself. [...].“

Schließlich ist es so weit: An einem sommerlich heißen Sonntagmorgen im Berliner Willy-Kressmann-Stadion sitzen alle Teilnehmenden auf der langen Tribüne und eine Handvoll Organisator_innen übernehmen die offizielle Begrüßung ein. Noch ist nicht alles aufgebaut und dekoriert, noch ist kein Publikum da, denn die offizielle Eröffnung ist erst am Dienstag. Die nächsten Tage sind dem gegenseitigen Kennenlernen und einem Workshop-Programm in Kleingruppen gewidmet. Nach interaktiven Kennenlernspielen finden sich die Workshop-Gruppen zusammen; so auch das Grüppchen um Thea und Gerit, das in den nächsten Tagen über Fußball und Feminismen diskutieren soll. Die beiden begrüßen ihre acht Teilnehmer_innen auf Englisch und erklären den Ablauf: „In den nächsten zwei Stunden lernēn wir uns besser kennen, und nach der Mittagspause fahren wir gemeinsam in einen Seminarraum, wo wir auch die nächsten Tage zusammen arbeiten werden." Eine Freiwillige flüstert den zwei Argentinierinnen die spanische Übersetzung zu. Eine jordanische Spielerin übersetzt für die beiden tunesischen Spielerinnen ins Arabische. In einer schattigen Ecke nimmt die Gruppe in einem Kreis auf der Tartanbahn Platz. Dort liegen bereits Filzstifte und Packpapier bereit, und nach einer Vorstellungsrunde bekommen alle die Aufgabe, in Paaren ihre Lebenswege, einen sogenannten river of life, aufzumalen und anschließend den anderen vorzustellen. 45 Minuten später beginnt Catalina aus Argentinien: Sie erzählt, wie sie als Jugendliche Drogen nahm, kriminell und aggressiv war. Sie weint. Erst durch den Kontakt zu ihrer jetzigen Fußballtrainerin und zum Verein, der gleichzeitig ein soziales Projekt in den Armenvierteln von Buenos Aires betreibt, konnte sie sich davon lösen. Sie wolle nun versuchen, für andere Mädchen und Jungen ein gutes Vorbild zu sein und diese mithilfe des Fußballs von Drogen und Kriminalität wegzuholen. Alle um sie herum sind sichtlich gerührt und applaudieren, als sie fertig ist. Als nächste präsentiert Gerit ihren Lebensweg und erzählt, wie sie als Kind mit dem Fußballspielen begann, doch ihre Eltern immer dagegen waren und ihr vorwarfen, kein richtiges Mädchen zu sein. Sie erklärt, dass sie nun bei DF sei, weil sie fände, dass diese Geschlechterrollen unfair seien und dass Fußball immer auch ein politisches Thema sei. Nach ihr ist Qamar aus Jordanien an der Reihe. Auch sie spielt schon, gefördert von ihrem Vater, seit ihrer Kindheit Fußball. Nun sei sie sogar in der Nationalmannschaft. Ihr sei es wichtig, dass Fußball und Politik getrennt blieben. Fußball sei wichtig für Mädchen und gut für die Entwicklung zu einer starken Persönlichkeit, und daher wolle sie ihre Leidenschaft weitergeben. Vom Verband und der jordanischen Regierung würde sie dabei nur Unterstützung erhalten. Rabia aus Tunesien nickt bestätigend. Sie wirft ein, dass immer alle denken würden, Musliminnen seien nicht emanzipiert, aber das stimme gar nicht: „Western women are not more emancipated than Muslim women!“ Danach berichtet Yeshe, der Trainer des tibetischen Teams, von der Flucht seiner Eltern aus China, und wie schwierig es sowohl für ihn als Spieler in einem Männerteam als auch für sein Frauenteam sei, an Wettbewerben teilzunehmen. Für das Frauenteam war es die erste Auslandsreise überhaupt, da sie aufgrund ihres Flüchtlingsstatus nur unter deutlich erschwerten Bedingungen und mit Sondergenehmigungen reisen können. Als alle ihre Lebenswege vorgestellt hatten, erinnern Gerit und Thea noch einmal an Zeit und Treffpunkt für das Nachmittagsprogramm. Dann ist Mittagspause. 
Als Thea und Gerit nach der Mittagspause am vereinbarten Treffpunkt erscheinen, fehlen die Jordanierinnen und Tunesierinnen. Als sie auch nach 20 Minuten nicht auftauchen, macht sich die verkleinerte Gruppe auf den Weg ins Hostel und trifft dort prompt die anderen vier verschlafen und müde an. Gab es ein Missverständnis? Thea bittet sie, sich fertig zu machen und mitzukommen und nach 10 Minuten zieht die ganze Truppe ab in Richtung Seminarraum. Dort angekommen fragen die zwei, was los gewesen sei. Qamar erklärt in einem leicht genervten Ton, es sei Ramadan und sie würden fasten. Ihre jordanische Teamkollegin ergänzt: Es sei auch so schrecklich heiß, und sie hätten alle von der gestrigen Reise noch einen Jetlag. In dem kleinen Hostel-Zimmer sei es so stickig gewesen, dass sie sich nicht richtig erholen konnten. Sie albert müde herum: Viel lieber würde sie jetzt mit ihrer playstation spielen. Thea ist enttäuscht und noch immer verärgert: „Ihr hättet uns ja Bescheid sagen können! Ihr könnt doch nicht einfach weggehen. Die Teilnahme an den Workshops gehört zum Programm dazu. Das ist auch eine Auflage der Geldgeber, die hier alles bezahlen. Außerdem ist DF ja auch ein politisches Projekt. Es geht nicht nur um Fußball und Wettkampf.“ Gerit hingegen hat Mitleid mit den erschöpft wirkenden Teilnehmenden und versucht zu vermitteln: „Wir finden es auch total blöd, dass das Festival ausgerechnet während Ramadan stattfindet. Aber wir hatten einfach keine andere Möglichkeit, denn zum einen macht es nur Sinn, das parallel zur WM zu machen, sonst interessieren sich die Medien überhaupt nicht für uns. Und außerdem können wir das Stadion immer nur in der Sommerpause haben, wenn keine anderen Spiele hier drin stattfinden." Qamar, Rabia und die anderen zeigen Verständnis, aber betonen auch, wie anstrengend es für sie sei. Auch die, die nicht fasten, wirken von der Hitze und den Strapazen der Reise erschöpft. Schließlich einigen sich alle darauf, den Workshop am nächsten Morgen ausfallen zu lassen, dafür aber heute Nachmittag weiterzumachen. Als schließlich das Nachmittagsprogramm vorüber ist und die Teilnehmenden zusammenpacken, schnappt sich Catalina den Fußball, der vorher für ein Spiel benutzt worden war, und beginnt damit zu dribbeln. Gerit stürzt auf sie zu und täuscht an, ihr den Ball abzunehmen. Catalina reagiert blitzschnell und kappt den Ball in die andere Richtung, Gerit bedrängt sie von hinten. Die beiden schreien und lachen. Rabia bietet sich sofort als Anspielstation an und Catalina spielt ab. Gerit hastet dem Ball hinterher und geht nun auf Rabia los. Das Ganze geht eine Weile so, bis die anderen den Raum verlassen haben und sie folgen müssen. Unten wartet ein Kleinbus, der die Teilnehmenden zurück ins Hostel bringt.

Diese Verdichtung einer typischen Situation, wie sie sowohl beim Festival als auch in Ausschnitten bei den Expertinnenforen vorkommen konnte, zeigt die Komplexität eines Moments, in dem viele Personen aus dem Fußball mit unterschiedlichen Erwartungen und aus unterschiedlichsten Lebenssituationen zusammenkommen. Sie verweist zudem auf das Spannungsfeld zwischen den Erwartungen der Teilnehmenden, logistisch-organisatorischen Rahmenbedingungen, förderpolitischen Auflagen und eigenen Ansprüchen, in dem DF in solchen Situationen manövrieren musste. Im Folgenden zeige ich, dass die glaubwürdige Formierung als kompetente Problembearbeiterin vor einer internationalen Frauen-Sport-Bewegung darauf basierte, dass DF eine Auswahl zwischen den Teilnehmenden und damit auch zwischen Kompetenzkriterien traf (13.1). Das Festival fand vor dem Hintergrund komplexer globaler Machtverhältnisse statt. Dadurch, dass diese Machtverhältnisse zunächst im 
Kontext der translokalen Begegnung verkompliziert wurden (13.2) und DF versuchte, bestehende Asymmetrien zwischen sich als Gastgeberin des globalen Nordens und den zumeist aus dem globalen Süden kommenden Teilnehmenden zu verkleinern und Ähnlichkeit untereinander herzustellen (13.3), zeigte sich die Organisation nicht als besserwissende und vorbildhafte NGO, sondern als betroffene und lernende Mitstreiterin.

\subsection{Erwartungen auswählen: „Why do you consider football important as a woman*?"}

Von den Projektteilnehmenden als einem Stakeholder mit kohärentem Kompetenzregime zu sprechen, ist aufgrund der vielen Unterschiede kaum möglich. Grob lassen sie sich unterscheiden in (1) Mitarbeitende lokaler oder internationaler Sport-for-Development-NGOs oder (2) deren Zielgruppen in Gestalt lokaler Frauen, die an entsprechenden Programmen teilnahmen und ein Fußball-Team formten; (3) in Frauenteams regulärer Fußballvereine, die wettkampforientiert am organisierten Ligabetrieb teilnahmen und darüber hinaus auf verschiedene Weise und in verschiedener Intensität Mädchen und Frauen förderten; (4) in Mitarbeitende lokaler, sozialpolitisch ausgerichteter Fußballprojekte, die sich neben dem regelmäßigen Spielen in der Gesellschaft und für unterschiedliche Themen engagierten, und (5) in Fußballteams, die aufgrund menschenrechtlich oder politisch heikler Lage mit besonders großen Schwierigkeiten umgehen mussten. Auch wenn jedes Team ein bestimmtes öffentliches Selbstverständnis und eine formulierte Agenda vorweisen konnte, wurde doch jede einzelne Spielerin aus eigenen Motiven zum Fußballspielen getrieben: Den einen war die gesellschaftliche Bedeutung des Fußballs wichtig, manche wollten über den Fußball etwas verändern, andere mochten vorrangig ihre Leistungen messen und wieder andere bevorzugten schlicht das freizeitliche und spielerische Beisammensein. Die zum Festival anreisenden Spielerinnen waren weiblich, wurden aber teilweise von männlichen Trainern oder Teambetreuern begleitet. Die Expertinnenforen sprachen ausschließlich Einzelpersonen an: Frauenfußballjournalistinnen, Menschenrechts- und Fußballaktivistinnen, Schiedsrichterinnen, Trainerinnen und Funktionärinnen aus nationalen Verbänden, die in ihren Bewerbungen ihren Einsatz für Frauen und den Frauenfußball darlegten und begründeten.

\subsubsection{Die verschiedenen Erwartungen der Teilnehmenden}

Die Heterogenität der Teilnehmenden an DF-Projekten drückte sich nicht nur in den verschiedenen Motiven für und Modi des Fußballspielens aus, sondern 
auch in unterschiedlichen sozioökonomischen Lebensbedingungen, Sprachkenntnissen und Bildungschancen - vor allem aber in verschiedenartigen Erwartungen an das Projekt und die Organisator_innen. ${ }^{115}$ Während einige berufsbedingt häufige international reisten, verließen andere zum ersten Mal ihr Land. Während einige studierten, der Mittelschicht entstammten oder auf berufliche Erfolge im sportlichen, medizinischen oder internationalen NGOBereich blickten, hatten andere Teams Schwierigkeiten, die sportliche Mindestausrüstung zu finanzieren. Dementsprechend divers und widersprüchlich waren auch die Erwartungen an DF als Gastgeberin und Organisatorin. Einige Spielerinnen und Teams rechneten entsprechend ihrer Vorstellung von Gastfreundschaft und deutscher Organisation und westlichem Wohlstand mit perfektem Service, einer reibungslosen Veranstaltung und hohen Versorgungs- und Unterbringungsstandards. Diese Erwartung wurde von DF bereits in der vorausgegangenen E-Mail antizipiert und korrigiert und unter anderem von Qamars Teamkollegin geäußert, die entsprechend der FIFA-Standards eine Playstation im Hostel vermisste (siehe Vignette). ${ }^{116}$ Andere waren kritisch gegenüber Bevormundung und entwicklungspolitischem Paternalismus und beharrten auf der Achtung ihrer kulturellen oder religiösen Regeln und/oder wiesen die dem entwicklungspolitischen Empowerment-Begriff inhärente Asymmetrie zwischen entwickelten und nicht-entwickelten, westlichen und nicht-westlichen Frauen vehement zurück. Rabia äußerte mit ihrem Widerstand gegen das stereotype Bild von Musliminnen die Erwartung, nicht auf Basis von Vorurteilen und Unwissenheit kategorisiert und bevormundet zu werden. Wohlwissend, dass sich Asymmetrien nicht auflösen ließen, erwartete sie, dass DF den Paternalismus zumindest in der direkten Begegnung nicht fortschreiben würde.

\subsubsection{Erwartungen auswählen}

Aufgrund der Menge unterschiedlicher und teils widersprüchlicher Erwartungen war es für DF unmöglich, allen zu entsprechen. Eine Eingrenzung der

115 Förderprogramme entschieden mit über die geografische Herkunft der Teilnehmenden. Der Schwerpunkt des AA auf Transformationsländer veranlasste, dass zu einem Festival und Expertinnenforum vor allem Teams aus Nordafrika und dem Nahen Osten eingeladen wurden. Die Sportförderung des AA im Rahmen der Auswärtigen Kulturpolitik fokussiert hingegen die Länder der sogenannten DAC-Liste (Entwicklungs- und Schwellenländer sowie einige postsowjetische Staaten), und die EU ist vor allem an europäischen und angrenzenden Ländern interessiert. Die Organisator_innen passten sich diesen Anforderungen an, versuchten aber dieser Gewichtung entgegenzuwirken, indem sie über andere Kanäle Reiseund Unterbringungskosten für Teams aus den nicht abgedeckten Ländern einwarben oder mit den Förderern um Ausnahmen verhandelten.

116 So zeigten sich Spielerinnen z.B. empört, als sie nach dem Frühstück im Hostel dort entsprechend der Hausregeln ihren Tisch abwischen sollten, oder waren enttäuscht, dass sie in Mehrbettzimmern untergebracht wurden. 
Erwartungen war erforderlich. Die Auswahl, welchen Erwartungen DF entsprechen konnte und welchen nicht, wurde durch den engen finanziellen, materiellen und personellen Spielraum sowie die eigenen Ideale vorstrukturiert. Allein aufgrund mangelnder finanzieller Möglichkeiten konnte DF weder Hotelübernachtungen, Shuttleservice noch ein variationsreiches Catering bieten. Da sie auf ehrenamtliche Übersetzer_innen und Helfer_innen angewiesen waren, mussten Teilnehmende oft selbst aushelfen. Zugleich war das Aushelfen und Mitanpacken aller entsprechend dem links-liberalen, bürgerlichen Ideal des ,group projects“, in dem sich alle für das Gelingen verantwortlich fühlten, gewollt. Die eigenen in einer Berliner Bewegungsszene verankerten Moralvorstellungen verboten es, als besserwissende Expert innen mit einem maßgeschneiderten Programm aufzutreten, das die Teilnehmenden nur durchlaufen müssten; vielmehr strebten die Aktivist_innen nach einer „Begegnung auf Augenhöhe“.

Üm zwischen den heterogenen Erwartungen, die an sie gerichtet wurden, auszuwählen, hatte sich ein bestimmtes Vorgehen etabliert. Dies implizierte erstens die Aufforderung zur ausführlichen Bewerbung, in der bereits die Motivation zur Teilnahme, Details zum Engagement und die Einstellung zum Fußball abgefragt wurden. So bat DF die Teilnehmenden des Festivals 2015 mit dem Motto „Beyond (b)orders“ auf folgende Fragen zu antworten:

Have you already been playing football? If yes, for how long?

Why are you interested in women's football?

Why do you consider football important as a woman*?

Which borders have you faced in your activities?

Has football helped you to overcome limits? If yes, how?

What is your main interest in the DISCOVER FOOTBALL festival?

What do you want to contribute to the women's football community at the festival?

What has been your best experience in football as a woman*?

Die Auswahl verlief sodann entlang verschiedener Kriterien: Erstens wurden jene Bewerbungen favorisiert, die eine feministische oder allgemeine gesellschaftspolitische Ausrichtung erkennen ließen und in denen Frauen als Protagonistinnen auftraten. Zweitens spielte der politische und frauenrechtliche Kontext eine Rolle, sodass im Sinne des Projektnamens (Discover Football = Entdecke den Fußball) und der Formierung als Expertin mit wichtigen Lokalkontakten Bewerbungen aus jenen Ländern begrüßt wurden, in denen die frauenrechtliche Situation besonders prekär war und es kaum frauenfußballerische Infrastrukturen gab. Und drittens wurde auf den fußballerischen Ernst geachtet. Genuin fußballerische Projekte und Initiativen wurden z.B. entwicklungspolitischen Projekten vorgezogen, die den Fußball lediglich als spielerisches Mittel neben vielen weiteren einsetzten. Da die Bewerbungen immer nur begrenzt Aufschluss gaben, meist von einer Person für das gesamte Team ausgefüllt wurden und auch die Imaginationen der Organisationsgruppe beim Lesen der Bewerbungen mitwirkten, trafen in den konkreten 
Begegnungen immer noch sehr unterschiedliche Erwartungen aufeinander. Hier entschieden dann habituelle Nähe und persönliche Sympathie, geteilte Ansichten von Feminismus und Projektgestaltung darüber, ob Kontakte vertieft wurden und einzelne Personen oder ganze Teams zu Folgeprojekten eingeladen oder gar gemeinsame Projekte entwickelt wurden.

Auswahlprozesse erscheinen zunächst als ein aktives Tun auf Seiten der Auswählenden und ein passives Erleben auf Seiten der Auszuwählenden. In der Praxis gestaltete sich der Prozess jedoch reziprok. Hier ist es Qamar, die die Sichtweise auf die frauenpolitische Situation des Fußballs nicht teilte und eine gemeinsame Problemdefinition zurückwies. Bei anderer Gelegenheit beobachtete ich, wie sich ein Team dem Rahmenprogramm entzog und fast ausschließlich für die Turnierspiele erschien. Während manche Teams oder einzelne Frauen Interesse an zukünftigen Partnerschaften zeigten oder sich für Folgeprojekte bewarben, mieden andere das eingerichtete Planungstreffen oder bewarben sich in den kommenden Jahren nicht wieder. Ich gehe davon aus, dass alle Teilnehmenden selbst in heterogene Landschaften eingebunden waren und pragmatisch zwischen diversen Kompetenzregimen navigieren mussten, um ihr Fortbestehen und ihre Handlungsspielräume zu erhalten. Wenn Qamar die gemeinsame Problemdefinition ablehnte und das Engagement ihres nationalen Verbands für den Frauenfußball lobte, dann womöglich deshalb, weil eine konfrontative Strategie für sie als Spielerin eines Erstligisten wenig hilfreich gewesen wäre und sie auf die gute Zusammenarbeit mit dem Verband angewiesen war. Und wenn sich ein Team dem gemeinschaftlichen Rahmenprogramm entzog und alle Energie auf die Turnierspiele richtete, dann vielleicht deshalb, weil die Spielerinnen den Erwartungen an sie als leistungsstarke und sogar international erfolgreiche Athletinnen entsprechen mussten, um so ihr Sporttreiben legitimieren zu können. So wurden, wie in Qamars Fall, Meinungsverschiedenheiten ausgehalten und Erwartungen enttäuscht: Thea vermisste die Partizipationsfreude einiger Teilnehmenden, und diese wiederum ihr gewünschtes $\mathrm{Ma}$ an Erholungsphasen. In den meisten Fällen fanden die Beteiligten allerdings, wie in der Vignette oben, durch einen Kompromiss zueinander. ${ }^{117}$

Die verschiedenen Auswahlpraktiken halfen DF, durch die heterogene Erwartungslandschaft zu manövrieren und dabei die Personen, Initiativen und Teams als relevante Stakeholder auszuwählen, deren Erwartungen mit den eigenen Idealen und Rahmenbedingungen kompatibel schienen. Hierbei handelte es sich vor allem um jene Einzelpersonen oder Teams, die die Problemdefinition vom Fußball als männliche Sportart und der Benachteiligung von Frauen teilten, partnerschaftliche Zusammenarbeit paternalistischen NordSüd-Beziehungen vorzogen sowie mit partizipativer Projektgestaltung und Do-it-yourself-Kultur vertraut waren. In diesen habituellen Dimensionen des

117 Natürlich gab es immer wieder Teilnehmende, die mit den geschlossenen Kompromissen nicht einverstanden waren. $\mathrm{Zu}$ Ausweichmöglichkeiten siehe Kap. 17.5.3. 
reziproken Auswahlprozesses zeichneten sich intersektionale Dimensionen $\mathrm{ab}$, wenn die Organisierenden und Teilnehmenden ähnliche Bildungswege, die Vertrautheiten mit Gender-Diskursen einer globalen Zivilgesellschaft, translokale Mobilitätserfahrungen oder eine aktivistische Einstellung teilten. Um von den ausgewählten Teilnehmerinnen als kompetent anerkannt zu werden, was in diesem Fall bedeutete, sich glaubwürdig als von der eigenen Betroffenheit motivierte Aktivistin und Mitstreiterin zu formieren, verkomplizierte DF die präsenten Machtbeziehungen (13.2) und bemühte sich, Asymmetrien zu verkleinern und Ähnlichkeiten herzustellen (13.3).

\subsection{Machtbeziehungen verkomplizieren: ,Western women are not more emancipated than Muslim women!“}

Die Analyse der Vignette im vorangegangenen Abschnitt zeigt, dass translokale feministische Projekte immer vor dem Hintergrund ungleicher Zugänge $\mathrm{zu}$ Ressourcen und asymmetrischer globaler Machtverhältnisse stattfinden und zugleich von gegenseitiger Solidarität, Aufgeschlossenheit und Kompromissbereitschaft geprägt sein können (vgl. Thayer 2010, 7). Als Rabia anmerkt, dass westliche Frauen nicht emanzipierter seien als muslimische, sprach sie aus, was durch DFs diskursiven Bezug auf den EmpowermentBegriff sowie die Konstituierung als Expertin und NGO in dieser Situation bislang unausgesprochen im Raum stand. Durch die Anknüpfung an diese Diskurse konnte DF die finanziellen Mittel einwerben, um das Festival umzusetzen, das Programm zu gestalten und den Teams Flug-, Verpflegungsund Unterbringungskosten $\mathrm{zu}$ bezahlen. Diskurse um Fußball und Empowerment, materialisiert in Förderpolitiken und -kriterien, Projektanträgen, Programmentwürfen und -durchführung, strukturierten die konkrete Begegnung zwischen DF und den anreisenden Teilnehmenden vor. So wurde auch finanziell schwachen Teams die weite Reise und die Teilnahme an einem internationalen Turnier ermöglicht, und so ließen sich die sozioökonomischen Differenzen zwischen den Teilnehmenden temporär minimieren. ${ }^{118}$ Gleichzeitig wurde DF in die Position versetzt, die Teilnehmenden auszuwählen und darüber zu entscheiden, was diese eine knappe Woche lang machten, aßen, tranken, wo sie schliefen und wann sie sich wie wohin bewegten, was sie lernen und worüber sie diskutieren sollten. Dadurch verfestigte sich ein offensichtlich asymmetrisches Machtverhältnis zwischen Orga-

118 Wenn bspw. ein Team keine Fußballschuhe besaß, kaufte DF Stollenschuhe aus projektungebundenen Mitteln, sodass das Team nicht im Nachteil war. Gleichzeitig kehrte dies auch die materiellen Ungleichheitsverhältnisse zwischen DF und Teilnehmenden hervor. 
nisierenden und Gastgebenden einerseits und Teilnehmenden und Gästen andererseits, mit dem die nun Aufeinandertreffenden umgehen mussten.

Als ich in meiner Supervisionsgruppe zur ethnografischen Deutung jene Feldnotiz vorlegte, auf der die obige Vignette unter anderem basiert und in der auch ich eine organisatorische Rolle einnahm, diskutierten meine Kolleg_innen meine ängstliche Reaktion auf Rabias Bemerkung, dass westlichen Frauen nicht emanzipierter seien als muslimische, sowie meine Irritation über das Fernbleiben der Spielerinnen. Sie vermuteten dahinter Scham und Enttäuschung als Reaktion auf das plötzlich sichtbar werdende Machtgefälle: Das Fernbleiben und der Widerstand der Spielerinnen machten die einflussreiche Position von uns Organisierenden und damit unsere machtvolle Position innerhalb des translokalen Gefüges deutlich. Unser eigenes Weiß-Sein und die damit verbundenen Einfluss- und Entscheidungsmöglichkeiten wurden spürbar. Was durch die Aktualisierung dieses Machtverhältnisses - denn die verschwundenen Teilnehmerinnen wurden aufgesucht und aus dem Hostel abgeholt - in Gefahr geriet, war nicht nur die Anerkennung als linke, feministische Initiative von Seiten der städtischen Bewegungsszene, sondern auch die Legitimation des Engagements vor den eigenen Moralvorstellungen, das sich durch aktivistische „Augenhöhe“ statt entwicklungspolitischem Paternalismus auszeichnen sollte. Bevor ich im nächsten Unterkapitel die Bemühungen darstelle, diese Asymmetrien entsprechend der eigenen Moral und der Erwartungen der Teilnehmenden zu verkleinern, betrachte ich zunächst diese von Ungleichheiten durchdrungene Beziehung genauer. Denn der Zeitpunkt des Festivals (im Ramadan), die Gestaltung des Programms, das Fernbleiben der Spielerinnen nach der Mittagspause sowie ihre unterschiedlichen Lebenswege weisen auf deutlich komplexere Machtverflechtungen hin, als sie allein die Vorstellung einer eindimensionalen Hierarchie erfassen würde.

\subsubsection{Abhängigkeitsverhältnisse transparent machen}

Thea reagierte auf den Vorwurf der Teilnehmerin, dass religiöse Bedürfnisse wie das Ramadan-Fasten nicht beachtet würden, mit dem Verweis, dass ihr als Organisatorin die Hände gebunden seien: Erstens fand zeitgleich die Weltmeisterinnenschaft statt, sodass auch das Festival und seine Teilnehmerinnen von der gesteigerten medialen Aufmerksamkeit profitieren würden. Zweitens hing die Verfügbarkeit des Stadions von den Schulferien und der Saisonpause des Verbandsfußballs sowie der Schonfrist für den Rasen ab. Das Ausweichen auf einen anderen Platz hätte eine Abwertung der Turnierspiele bedeutet, da diese mit dem qualitativ schlechteren Kunstrasen ausge- 
stattet waren. ${ }^{119}$ Und drittens hätten hohe Regenwahrscheinlichkeit und früh eintretende Dunkelheit das Festival zu einem früheren oder späteren Zeitpunkt stark beeinträchtigt. Die Terminentscheidung was daher keine freie, sondern ein Kompromiss.

Auch Programmgestaltung und -fülle waren Produkte des Manövrierens zwischen den verschiedenen Anforderungen. So zeigte Thea einerseits, dass die vorangestellten Workshop-Tage ein Amalgam aus Förderauflagen und eigenen Idealen darstellten, die dann den Verhandlungsspielraum mit den Teilnehmenden absteckten. ${ }^{120}$ Andererseits machte Thea durch den Verweis auf Förderauflagen den politisch-verwaltenden Staat sichtbar, der sich im Zuge des New Public Management hinter nichtstaatlichen Organisationen versteckt. Internationale Machtasymmetrien und die sie ko-produzierenden Regierungsorgane werden auf diese Weise verschleiert. Der Rückzug des sichtbaren Staates lässt ausführende Organisationen vor den Teilnehmenden als die einzig verantwortlichen Entscheidungsträgerinnen erscheinen, die über große Mengen an finanziellen Mitteln zu verfügen scheinen - schließlich übernahmen sie wie im Falle von DF Flug-, Verpflegungs- und Unterbringungskosten und konnten ein großes Festival finanzieren. Dass das Budget genau berechnet und an einzelne Posten gebunden war, war für die Teilnehmenden nicht transparent, sodass es für einige unverständlich erschien, warum sie kein Trinkwasser aus Flaschen verschwenden sollten oder warum DF Bitten nach finanzieller Unterstützung in den Heimatländern nicht nachkommen konnte. ${ }^{121}$ Theas Ansprache und die vorausgegangene E-Mail versuchten, das Bild der wohlhabenden deutschen NGO, die frei über ein großes Budget verfügt, zu revidieren, und machten die für viele Teilnehmenden bislang unsichtbaren infrastrukturellen Handlungsrestriktionen und förderpolitischen Auflagen teilweise transparent. DF zeigte sich dadurch als ebenfalls in komplexe Machtverhältnisse und Abhängigkeiten involviert.

119 Zur technischen und symbolischen Bedeutung des Spieluntergrundes siehe die Diskussionen, die 2015 um die Frauen-WM in Kanada entbrannten, die auf Kunstrasen ausgespielt wurde (vgl. Fisher/Davis 2016). Um den fastenden Teilnehmenden entgegenzukommen, traf DF verschiedene Vorkehrungen, unter anderem erklärte sich das Hostel bereit, Frühstück bereits vor Sonnenaufgang anzubieten, und alkoholische Getränke wurden im Stadion an einer gesonderten Bar ausgeschenkt.

120 Diese relationale Position DFs innerhalb globaler machtvoller Verflechtungen kam auch im Umgang mit den verschiedenen Stakeholdern zum Ausdruck: Während DF die Möglichkeit hatte, zwischen den Teilnehmenden auszuwählen, besaß DF nur sehr geringen Spielraum, um entlang eigener Ideale die Förderinstitutionen auszusuchen und gegebenenfalls auf unliebsame zu verzichten.

121 Jenen Teilnehmenden, die selbst für internationale NGOs arbeiteten, waren diese bürokratischen Restriktionen bekannt, und sie hatten Verständnis. 


\subsubsection{Intersektionale Verkomplizierung}

Auch ein intersektionaler Blick zeigt, dass die Beziehung zwischen DF und Teilnehmerinnen vielschichtig ist und die in dieser Begegnung wirkenden Machtverhältnisse kompliziert sind. Einige Teilnehmerinnen entstammten den Mittelschichten ihrer Heimatländer und widersprachen mit ihren gehobenen Lebensstilen, ihrem Auftreten und ihren Forderungen entschieden der Kategorie der zu empowernden Frau aus dem globalen Süden. So war nicht nur Qamars Teamkollegin von der Unterbringung enttäuscht. Während eines Expertinnenforums brachte ich eine Teilnehmerin ins Hotel und in ihr Zimmer. Beim Anblick des kleinen Zimmers mit bunter Tapete und Nippes in den Regalen in der familiär geführten Pension rümpfte sie die Nase und runzelte die Stirn. Eine andere Spielerin fragte besorgt nach den hygienischen Standards, war sie doch aus ihrem arabischen Heimatland anderes gewohnt. Während einige bei Expertinnenforen im Kostüm erschienen, traten die Organisator_innen in Jeans oder Jogginghose auf. Der links-alternative Lebensstil der Organisator_innen, eine aktivistische Ästhetik und ihr kreativer Umgang mit begrenzten Ressourcen, die sich in einer DIY-Kultur, in der Wahl der Räumlichkeiten oder eben in der Kleidung ausdrückten, lösten bei manchen Teilnehmerinnen zunächst Irritationen aus. Einerseits trafen so gut situierte, teils beruflich erfolgreiche Frauen auf links-alternative, teils noch studierende junge Frauen, sodass sich in der Interaktion die Machtverhältnisse intersektional verkomplizierten und die Asymmetrie durch Klassen- und Milieuspezifika ihre Eindeutigkeit verlor. Andererseits wurde auch die Heterogenität unter den Teilnehmenden sichtbar: Während die einen in ihrer Freizeit in Berlin mit einem großzügigen Taschengeld shoppen gingen, konnten sich die anderen kaum Souvenirs leisten. Während die einen auf umliegende Restaurants ausweichen konnten, wenn ihnen das von DF organisierte Catering nicht schmeckte, aßen andere jede Mahlzeit auf dem Festivalgelände. Während einige in modischer Kleidung großer Sportartikelhersteller umherliefen, trugen andere vor allem die von DF bereitgestellten Trikots oder die Trainingsanzüge ihres Vereins. Hieran zeigt sich, dass die teilnehmenden Frauen keinesfalls eine homogene Gruppe bildeten, wie es eine verengte Perspektive auf ein eindimensionales Machtverhältnis zwischen globalem Norden und Süden suggerieren könnte. Sozioökonomische Klassen- und Milieuunterschiede traten ebenfalls wirkmächtig in Erscheinung und verkomplizierten die Beziehung zwischen den organisierenden Gastgeber_innen und den teilnehmenden Gästen sowie unter den Teilnehmenden. 


\subsubsection{Aufeinander angewiesen sein}

Das Fernbleiben nach der Mittagspause macht zudem die gewichtige Position deutlich, die Teilnehmende innerhalb eines Projektes bekleiden, das auf Partizipation zielt. Das Gelingen des Festivals hing, wie bereits die vorausgegangene E-Mail signalisierte, maßgeblich davon ab, dass alle mitmachten und die Vorgaben und den Zeitplan einhielten. Ein Workshop, der mit interaktiven Methoden statt frontalem Unterricht arbeitet, braucht Beteiligung und Diskussionsbeiträge, vor allem aber braucht er Teilnehmende. Indem diese schlicht nicht erschienen, wiesen sie die Organisator_innen auf die gegenseitige Abhängigkeit hin. Wenn DF vor Förderern mit der verbindenden Wirkung des Fußballs sowie den emanzipativen Effekten des gegenseitigen Austausches argumentierte und diese in Abschlussberichten belegte, wurde ihr Status als kompetente Ausführerin entsprechender Politiken durch das Fernbleiben der Spielerinnen gefährdet. Nicht nur waren die Teilnehmenden auf DF angewiesen, um an diesem Festival zu partizipieren, auch DF hing von den Teilnehmenden ab, um das Projekt erfolgreich durchzuführen und sich damit auch vor anderen Stakeholdern zu legitimieren. ${ }^{122}$

Die Machtverhältnisse, in die solche internationalen Begegnungen eingebunden waren, sind deutlich komplexer als es auf den ersten Blick erscheinen mag. Weder handelt es sich um ein eindimensionales Herrschaftsverhältnis, in dem die einen über die anderen bestimmen oder unilateral Ressourcen fließen, noch um eine vollkommen symmetrische, zwanglose Beziehung, in der sich alle mit gleichen Ressourcen, Rechten und Handlungsmöglichkeiten „auf Augenhöhe“ begegnen. Gäste und Gastgebende, Teilnehmende und Organisierende, , westliche" und ,muslimische" Frauen waren auf verschiedene Arten aufeinander angewiesen und handelten dieses Verhältnis reziprok, wenn auch in ungleicher Verflochtenheit und mit ungleich großen Spielräumen aus. Innerhalb dieser vielfältigen Verflechtungen organisierte sich DF vor den ausgewählten Teilnehmenden als kompetente Mitstreiterin - nicht als paternalistische Besserwissende. Die Glaubwürdigkeit dieser Organisationsgestalt basierte neben der Verkomplizierung der Machtverhältnisse auf DFs Bemühungen, Ähnlichkeit herzustellen und bestehende Asymmetrien zu verkleinern.

122 Siehe Thayer (2010) für eine ähnliche Beziehung zwischen ländlicher Frauenbewegung und urbaner NGO in Brasilien sowie Sperling et al. (2001) für die Kooperation zwischen Feministinnen in den USA und Russland. 


\section{3 Ähnlichkeiten herstellen: „Wir hatten ganz ähnliche Erfahrungen“"}

Während sich die Machtbeziehungen also vielschichtig gestalteten, versuchte $\mathrm{DF}$, die bestehenden Asymmetrien zu verkleinern und das Ideal einer „Begegnung auf Augenhöhe“" zu erreichen. Dies geschah erstens über das Teilen von Erfahrungen, das durch die Aktualisierung der Subjektposition der Fußballerin ermöglicht wurde; zweitens über die Betonung der Fußballleidenschaft; und drittens dadurch, dass sich die Organisator_innen selbst als Lernende und die Teilnehmerinnen als Expertinnen adressierten.

\subsubsection{Erfahrungen theoretisch deuten, Ähnlichkeiten fühlen}

Manche der ausgewählten Teilnehmerinnen erhielten keine Visa und konnten letztlich nicht anreisen; andere mussten lange Kontrollen bei der Einreise akzeptieren. Die Formierung der von DF als Mitstreitende „auf Augenhöhe“ - und nicht als besserwissende Lehrende - geschah stets im Kontext global ungleich verteilter Ressourcen und Privilegien. Es war nicht zu übersehen, dass bestimmte Differenzen - seien sie durch Grenzregime oder ökonomische Ungleichheit bedingt - nicht überwunden oder minimiert werden können, wenn Menschen aus unterschiedlichen Regionen und sozioökonomischen Verhältnissen, mit diversen Hintergründen und rechtlichen Status aufeinandertreffen.

Postkoloniale und Schwarze feministische Wissenschaftlerinnen haben die Schwierigkeit konstatiert, heterogene Erfahrungen von Frauen zum Zwecke translokaler Bündnisse zu verallgemeinern, und die Idee einer global sisterhood kritisiert, in der differente Erfahrungen marginalisierter Frauen zugunsten einer ,illusory sameness“ unsichtbar gemachten werden (Scott 2012, 46). Chandra Mohanty warnt vor der Annahme einer „cross-culturally singular, monolithic notion of patriarchy or male dominance“, die weltweit gleiche Erfahrungen produziere (2003, 502). DF sah sich vor der Herausforderung, einerseits diese Kritik ernst zu nehmen und andererseits translokale Bündnisse zum Zwecke gegenseitiger Solidarität und eines größtmöglichen politischen Gewichts zu schmieden, in denen DF selbst die Rolle als privilegierte westeuropäische Initiatorin mit Zugang zu Fördergeldern innehatte. DF formulierte für diese translokalen Beziehungen im Sinne ihrer moralischen Vorstellungen von Aktivismus das Ideal einer „Begegnung auf Augenhöhe“. Um diese Augenhöhe herzustellen, verwiesen sie auf die eigenen Diskriminierungserfahrungen: Gerit hob in ihrem Lebensweg die Vorurteile und starren Geschlechterrollen hervor, mit denen sie als Fußballerin konfrontiert worden war. Sie revidierte das von Rabia bereits kritisierte Bild, das westli- 
che Frauen als emanzipiert, den deutschen Fußball als Vorbild für Gleichberechtigung und eine deutsche Gesellschaft ohne geschlechtliche Benachteiligungen darstellt.

Für die Herstellung von Augenhöhe und die Formierung als betroffene Mitstreiterin nutzte DF Erfahrung als Ausgangspunkt, wie ein kollaboratives Treffen verdeutlicht:

Als wir beim Punkt angelangen, wie DF argumentiert, ein translokales Netzwerk trotz globaler Ungleichheiten herstellen zu können, bemerkt Laura: „Vielleicht sind es nicht die gleichen Erfahrungen, aber ähnliche Quellen der Erfahrung“ und meint Sexismus und Homophobie als universelle Strukturen im Fußball. [...] Nadine ist sich nicht ganz sicher: „Das sind schon Ähnlichkeiten in unseren Erfahrungen, die uns verbinden. Aber wie wir schließlich zusammenkommen, das ist nicht gleich, wir haben unterschiedliche Ressourcen und so." (Kollaboratives Treffen, 18.12.2015)

Wenn Laura einwandte, dass Frauen im Fußball weltweit nicht die gleichen Erfahrungen machen, dann meinte sie damit, dass die Diskriminierungen von Fußballerinnen in Deutschland - etwa durch geringe und stereotype mediale Repräsentation, Heteronormativierung oder abwertende Kommentare am Spielfeldrand - nicht gleichzusetzen seien mit den Erfahrungen von Spielerinnen in Ländern mit deutlich schlechteren frauenrechtlichen oder sportpolitischen Ausgangssituationen, in denen Frauen zum Teil nur unter besonderen Sicherheitsvorkehrungen trainieren können. Indem sie die Berichte der Spielerinnen und eigene Erfahrungen mithilfe von strukturalistischer, feministischer Theorie deutete und so von einer universellen sexistischen und homophoben Struktur im weltweiten Fußball ausging, identifizierte sie einen gemeinsamen Ursprung der Probleme und schuf die Grundlage für Bündnisbildung: Zwar kämpften Frauen weltweit mit unterschiedlichen Erscheinungsformen eines globalen Problems, jedoch konnten sie sich im Kampf gegen die Ursachen verbünden. Nadine ging noch einen Schritt weiter, als sie auch die Möglichkeit ähnlicher Erfahrungen erwähnte. Auch sie hielt gleiche Erfahrungen für unmöglich, doch hatte sie in den Jahren bei DF immer wieder erlebt, dass sich die Erfahrungsberichte trotz ungleich verteilter Ressourcen ähnelten. Die Organisator_innen näherten sich einer „Begegnung auf Augenhöhe" dadurch an, dass sie theoretisches feministisches Wissen auf eigene Erlebnisse sowie auf die Berichte anderer Spielerinnen anwandten. Die Annahme universeller Strukturen ermöglichte, hinter den sichtbaren lokalen Unterschieden Gemeinsamkeiten zu vermuten und sich darüber als ähnliche, aber nicht gleiche Mitstreiterin zu organisieren.

Da Erfahrungen und nicht etwa politische Ziele oder Strategien als Ausgangspunkt für die Herstellung von Augenhöhe fungierten, galt es, diese zu artikulieren. Hierfür griffen die Organisator_innen auf die Methode des river of life zurück, bei der die Teilnehmenden und die Organisierenden mit bunten Stiften ihre Lebenswege in Form eines Flusses auf Plakate malten und entlang des Flussverlaufs Lebensabschnitte, prägende Erlebnisse und wichtige 
Beziehungen markierten. Diese Lebenswege wurden anschließend den anderen präsentiert. Wenn, wie in der Vignette, Catalina von der Gewalt erzählte, die sie in einem argentinischen Armenviertel erlebt hatte, und danach Gerit von der mangelnden Unterstützung ihrer Eltern berichtete, ist die Diskrepanz zwischen den Erlebnissen nicht zu übersehen. Dennoch beharrten die Organisatorinnen darauf, im Austausch mit den Teilnehmerinnen Ähnlichkeit und Nähe zu spüren. Und auch die Teilnehmerinnen reagierten oft erstaunt auf die Berichte von DF-Mitgliedern über eigene verletzende und ausschließende Erfahrungen und wandten ein, dass dies ihr Bild vom deutschen Fußball veränderte. Danach befragt, was sie aus diesen Projekt-Begegnungen mitnähmen, antworteten einige: „We learned that we are not alone“, oder „this is what unites us". Wie wird in der individuellen Begegnung trotz strukturell sichtbarer Asymmetrien Ähnlichkeit erfahren?

Ariane erzählte mir, dass sie sich Safee aus Tunesien sehr nahe gefühlt hatte. Als ich per E-Mail nachfragte, antwortete sie folgendermaßen:

„In dem Jahr, als Safee bei DF war, hatte ich tatsächlich zu ihr eine ganz enge Verbindung, mit den anderen Tunesierinnen hingegen hatte ich kaum etwas zu tun. Und es ging darauf zurück, dass wir einen Workshop gemeinsam besucht haben, in dem wir u.a. diesen river of life gemalt und uns gegenseitig vorgestellt haben. Ich weiß tatsächlich interessanterweise gar nicht mehr genau, worum es in dieser einen Episode ging, war es ihr Vater? Ich dachte irgendwie, es war vielleicht ein Polizist? Irgendjemand, der sie zurechtgewiesen hat. Auf jeden Fall war es ein Einschnitt, die Erfahrung, dass ihr das Fußballspielen verboten wird, dass sie nicht dabei sein darf. Es war eine dieser typischen Geschichten, die in jedem Frauenfußballlebenslauf vorkommen und immer eine entscheidende Rolle spielen. Beim Erzählen selbst habe ich sie ja zunächst gar nicht verstanden, erst später in der Übersetzung; was mich sehr berührt hat, war zu sehen, wie die Gefühle bei ihr wieder hochkommen, wie sie gezittert hat beim Erzählen - dieser emotionale Gehalt ihrer Geschichte. Ich habe dann ihre zitternde Hand genommen und das war der Anfang unserer Freundschaft. Als ich meinen Fußball-river of life vorgestellt habe, war sie sehr erstaunt, dass es auch in Deutschland Grenzen gab, und wir hatten teilweise ganz ähnliche Erfahrungen in dem Sinne, dass wir beide als Kinder größtenteils auf der Straße gespielt haben, mit Jungs.“ (E-Mail, 9.9.2016)

Ariane sah in dem geschilderten Zurechtweisung eine jener von Nadine beschriebenen ,ähnlichen Erfahrung“ und damit ein wiederkehrendes Moment. Dass sie vergaß, was Safee tatsächlich berichtete, verweist darauf, dass Ariane Nähe und Ähnlichkeit weniger über das genaue Geschehen und den entsprechenden Kontext wahrnahm, also über all das, in dem sich gravierende Unterschiede zum eigenen Erleben wiedergefunden hätten, als vielmehr über die emotionale Reaktion, den „emotionale Gehalt“ ihrer Erzählung: Es war Safees Ausdruck von Angst und Anspannung, der Ariane veranlasste, auf Basis eigener Erlebnisse mitzufühlen und Empathie zu signalisieren. Die interaktive Narration und Darstellung von Erfahrungen und Emotionen ließen zwischen Ariane und Safee das Gefühl von Ähnlichkeit entstehen, die sich 
auf die gefühlte Dissonanz „between whom one feels oneself to be and the conditions of possibility for a liveable life" bezog (Hemmings 2012, 149).

Ich möchte Gefühle von Zurückweisung und Exklusion, an die sich Safee, Nadine und Ariane erinnerten, nicht als Ausdruck einer authentischen, vordiskursiven und damit ,reinen "Erfahrung fehldeuten. Vielmehr sind sie als Produkte eines feministischen Bewusstseins zu verstehen, das eine entsprechende Deutung des Erlebten erst ermöglicht (vgl. Hemmings 2012). Es war jenes feministische Bewusstsein in Form des strukturtheoretischen Deutungsrahmens, der es Ariane ermöglichte, das eigene Erlebte mit Safees Erzählung zu verknüpfen, und Ähnlichkeit affektiv spürbar machte. Durch die ritualisierte und emotionale Narration von dissonanten Erfahrung und ihre Verknüpfung mithilfe des feministischer Deutungsrahmens versuchte DF in der interpersonellen Begegnung Ähnlichkeiten herzustellen und sich darüber vor den Teilnehmenden als organisierende, aber zugleich betroffene Mitstreiterin auf Augenhöhe zu zeigen.

Ein erneuter Blick auf den vorausgegangenen Auswahlprozess macht sodann deutlich, dass die Herstellung von Ähnlichkeit und Augenhöhe auch den Ausschluss all jener Personen und Erfahrungen bedeutete, die dieses Bewusstsein - hier verstanden als eine ganz spezifische Problemdeutung nicht teilten, von der dominanten Narration abweichen oder ihr widersprachen (vgl. Phipps 2016, 131). Augenhöhe ist damit nicht die Voraussetzung translokaler Begegnungen, sondern das Produkt asymmetrischer Beziehungen und Prozesse der Ein- und Ausschließung.

\subsubsection{Geteilte Fußballbegeisterung}

Ariane erinnerte sich daran, dass die gefühlte Nähe auch durch den gemeinsamen Fußballbezug entstand. Ähnlichkeit zwischen Organisierenden und Teilnehmenden wurde nicht nur durch Erinnerungen an und Aktualisierungen von diskriminierenden, dissonanten Erfahrungen hergestellt, sondern ebenfalls durch die Verständigung auf die gemeinsame Fußballleidenschaft. Bei einem kollaborativen Treffen wies mich Kerstin darauf hin, dass es gerade das Einhalten der fußballerischen Regeln sei, die das Entstehen von Gemeinschaftlichkeit begünstige. ${ }^{123}$ Als Catalina, Gerit und Rabia in der Vignette um den Ball kämpften oder als Ariane und Safee berichteten, wie sie als Kinder mit den Jungens auf der Straße gespielt hatten, taten sie dies entsprechend der geschriebenen sowie der Gefühlsregeln des globalisierten Fußballs (siehe Kap. 10.2). Hier wie auch bei zahllosen anderen Gelegenheiten versicherten sie sich ihrer fußballerischen Kompetenz und Leidenschaft, indem sie dem

123 Klein/Meuser untermauern Kerstins Beobachtung wissenschaftlich: Sie argumentieren, dass um den Fußball als hochritualisierte Praxis institutionalisierte, aber auch flüchtige Sinnund Wertegemeinschaften, die sogenannten Fußballgemeinden, entstehen (2008, 13f.). 
Sport mit dem gebührenden Ernst begegneten: In fast jedem gemalten Lebensweg sowohl der Organisator_innen als auch der Teilnehmer_innen nahm der Fußball eine herausragende Rolle ein. Während des Festivals saßen Teilnehmer innen und Organisator innen zusammen auf der Tribüne, fachsimpelten über das gerade stattfindende Spiel und fieberten mit den spielenden Teams mit. Sie tauschten sich darüber aus, wie lange sie schon Fußball spielten, welche Wege ihre Fußballkarrieren nahmen und auf welcher Position sie spielten. Fielen in einem Team Spielerinnen aufgrund von Verletzungen oder Krankheiten plötzlich aus, freuten sich die Organisatorinnen, aushelfen zu dürfen. Und jene Teilnehmerinnen und Organisator innen, die selbst kaum oder nicht Fußball spielten, betonten die Wichtigkeit des Fußballs, wollten sich bei nächster Gelegenheit selbst auf dem Spielfeld ausprobieren oder wurden am Spielfeldrand zu großen Fans.

Als Organisator_innen hatten die Mitglieder von DF die Wahl, wie zentral sie den Fußball platzieren wollten. Sogenannte Plus-sport-Projekte setzen den Sport im Sinne des Sport for Development als zusätzliches Instrument für übergeordnete Entwicklungsziele ein (Coalter 2010, 298). Im Gegensatz dazu rückte DF den Fußball in den Mittelpunkt und kommunizierte immer wieder die eigene Fußballbegeisterung als Quelle ihrer Motivation. Sie hielten sich an die gleichen fußballerischen Regeln wie die Teilnehmenden und traten als leidenschaftliche Spieler_innen statt sportferne, organisierende Bürokrat_innen auf, sodass sie die dem Sport-for-Development- und Empowerment-Diskurs inhärente Differenz verkleinerten und gemeinsame Leidenschaft und geteilte Ziele demonstrierten.

\subsubsection{Gegenseitiges Lernen}

Einen Plus-sport-Ansatz verfolgt z.B. die norwegische NGO Right to Play mit ihren weltweiten Projekten. Darnell interviewte junge Erwachsene aus dem globalen Norden, die ein Praktikum in einem der Projekte im Ausland absolvierten, und identifiziert in ihren Aussagen die Konstruktionen von komplementären Subjektivitäten: ,[W]hiteness as a subject position of benevolence, rationality and expertise, [is] confirmed in opposition to marginalised, unsophisticated and appreciative bodies of colour." (2011, 560) Dies sind genau jene oppositionellen Subjektivitäten globaler Empowerment- und Entwicklungs-Programme, die Rabia kritisierte und von denen sich auch DF distanzierte (siehe Kap. 5.3.2). In meiner Zeit bei DF erlebte ich es jedoch nicht, dass dieser Vorwurf an DF gerichtet wurde - weder direkt in abschlieBenden Feedback-Runden, noch in privaten Einzelgesprächen, noch in anonymen Evaluationsbögen, obwohl diese durchaus zur Kritik genutzt wurden. Zwar wurde stets mehr auf Partizipation in der Projektplanung gedrängt, doch blieb eine Kritik an der möglichen Reproduktion oppositioneller Sub- 
jektpositionen und des entwicklungspolitischen Empowerment-Diskurses aus. Hingegen bemerkte z.B. eine Teilnehmerin, selbst lokale Mitarbeiterin einer halbstaatlichen Entwicklungsorganisation in einem arabischen Land, dass es bei den DF-Projekten nicht so sei und DF „different" sei. Sie hatte zuvor nicht nur beobachtet, wie DF-Mitglieder die eigenen dissonanten Erfahrungen artikulierten und von der eigenen Fußballbegeisterung motiviert wurden, sondern erlebte die Organisator_innen auch in der Rolle der Lernenden: In nicht-perfektem Englisch moderierten sie Workshops, nahmen selbst an den Gruppenarbeitsphasen teil und setzten sich beim Mittagessen mit den Teilnehmerinnen zusammen, anstatt sich zurückzuziehen. DF bemühte sich auch durch eine Umkehrung der implizierten Expertin-Lernenden-Beziehung „Augenhöhe“ herzustellen. Sowohl an den Workshops im Zuge des Festivals als auch an den Expertinnenforen nahmen immer DF-Mitglieder selbst teil, wenn sie nicht gerade in die Organisation eingebunden waren. Sie diskutierten mit und hatten selbst den starken Wunsch, die übrigen Teilnehmenden besser kennenzulernen und von ihren Erfahrungen zu lernen. Auf diese Weise konstituierten sie sich vor den Teilnehmenden selbst als Lernende. Zugleich sprachen sie die Teilnehmenden, wie im Veranstaltungstitel (,Expert Seminar") und in der Ausschreibung, immer wieder als Wissende an.

Auf die Forderung nach verstärkter Partizipation reagierten die Mitglieder von DF, indem sie nach und nach versuchten, die Teilnehmenden bei der inhaltlichen Gestaltung stärker miteinzubeziehen. Während das Programm des ersten Expertinnenforums noch gänzlich von DF gestaltet worden war und viele externe Referentinnen eingeladen wurden, entwickelten sich in den Folgejahren viele verschiedene Methoden und Formate, um dem Wissen und den Anliegen der Teilnehmenden mehr Platz zu geben, wie World Cafés, Podiumsdiskussionen, das Erarbeiten einer Deklaration und vor allem unzählige offene Diskussionsmöglichkeiten. Zwar gab DF weiterhin den materiellen, zeitlichen, räumlichen und thematischen Rahmen vor, doch wurden Teilnehmende im Vorfeld verstärkt in die Programmgestaltung einbezogen, zu Inputs ermutigt und ihre Themenwünsche abgefragt. Zeitlich enge Planung, begrenzte Kapazitäten, ungleich verteilte Gelder, große geografische Distanzen und wenig Wissen übereinander erschwerten jedoch die Einbeziehung.

Trotz der Bemühungen bemängelten 2014 die Teilnehmenden des Expertinnenforums die Kurzfristigkeit der Planung und ihrer Teilnahmebestätigung. Die Umsetzung partizipativer Projekte in dem Sinne, dass Teilnehmerinnen als gleichberechtige Partnerinnen und nicht bloße Rezipientinnen agieren und so auch den für sie relevanten Kompetenzkriterien entsprechen können, scheitert nicht selten an den Rahmenbedingungen jener Förderprogramme, die Partizipation als Leitwert verankert haben. Die Projektierung der Arbeit in Form zeitlich begrenzter und kurzfristig freigegebener Gelder sowie die geringe Förderung von institutionellen und Personalkosten machten langfristige Planungen unmöglich und erschwerten verlässliche und dauerhafte 
Zusammenarbeit. Die Bedingung, dass jeder Förderantrag bereits eine detaillierte Projektskizze enthalten musste, schränkte die Offenheit gegenüber den Beiträgen und Gestaltungswünschen der Teilnehmenden ein. Und der Wunsch der Förderer nach quantitativ messbaren Erfolgen implizierte, dass Projekte möglichst viele Personen erreichen sollten, sodass dauerhafte $\mathrm{Zu}-$ sammenarbeit mit einzelnen Personen erschwert wurde. Durch ihre Kritik enttarnten die Teilnehmerinnen die Diskrepanz zwischen Versprechen und Realität der Förderprogramme. Partizipation generierte unter den Bedingungen der Projektierung von Arbeit vor allem als Mitmachen jedoch kaum als Mitbestimmen (vgl. Hamm 2013, 57). ${ }^{124}$

\subsubsection{Gutes tun: solidarscher Aktivismus statt 9-to-5-Job}

DFs Distanzierung von großen, bürokratischen NGOs hingewiesen spielte auch gegenüber den Teilnehmerinnen eine Rolle. Als ich Annike im Sommer 2016 von meiner Beobachtung erzählte, dass sich DF von bestimmten Bildern internationaler NGOs abgrenze, erinnerte sie ein Gespräch, dass sie auf einem Festival mit einer Spielerin aus Libyen geführt hatte:

„Da hab ich noch mal gemerkt, wie wichtig es ist, dass wir nicht so werden, wie die großen Sport-for-Development-NGOs. Faizah hat erzählt, dass Discover Football ein riesiges Thema für ihr Team ist, weil wir bis jetzt die einzigen sind, durch die sie die Chance haben, zu spielen, als Fußballerinnen aufzutreten. Aber dass sich ihre Gespräche komplett verändert haben, nachdem sie uns zum ersten Mal gesehen haben. Es ging weniger um unsere Arbeit und was wir bieten, sondern wohl nur noch: ,Hast du gesehen, wie jung die sind!', ,Hast du die Gesichter gesehen? They are just like us!" Weil wir halt nicht so eine gesettelte Organisation sind. Klar, die [NGOs] brennen auch für ihre Arbeit. Aber es ist halt Arbeit. Die sind erwachsen und haben Büros und sind professionell. Die wollen den anderen helfen und was Gutes tun. Und bei uns ist das Freizeit und Leidenschaft und wir machen das mehr aus Solidarität und weil uns das selbst ja eben auch betrifft und uns hilft. Damit beeinflussen wir ihre Leben [der Libyerinnen] nicht nur durch das, was wir für sie tun, sondern noch mehr dadurch, wer wir sind: junge Frauen, die sich einfach für das einsetzen, was sie wollen, und dadurch am Ende ganz viel erreichen können. So kamen die zu dem Schluss, dass sie das auch können, wenn wir das können. Und jetzt werden zwei der Spielerinnen versuchen, Positionen im Verband zu bekommen. Das machen sie, weil sie uns kennengelernt haben." (E-Mail, 24.9.2016)

Annikes Aussage bestätigt, wie wirkmächtig das NGOization-Paradigma in der Unterscheidung zwischen Aktivismus und Entwicklungsbürokratie ist und DFs Positionierung auf Seiten des Aktivismus anleitete. DF konstituierte sich vor den Teilnehmenden als fußballbegeisterte, aktivistische Initiative,

$124 \mathrm{Zu}$ Partizipation als mobilisierende Metapher in der projektierten Entwicklungsarbeit und die sie ermöglichenden Koalitionen, ihre inneren Ambivalenzen und Widersprüchlichkeiten siehe Mosse (2005). 
die das, wovon sie sprach, selbst erlebt hatte und die die angestrebten gesellschaftspolitischen Veränderungen selbst tangierte. In diesem Kontext erschien sie weiterhin als wohltätige Organisation, die Gutes tut. Jedoch wurden nicht nur die Teilnehmenden, sondern die Organisatorinnen selbst $\mathrm{zu}$ Empfängerinnen ihrer Wohltaten. Indem die Mitglieder vorrangig in ihrer Freizeit für DF arbeiteten, jugendlich-sportlich auftraten und auf weitergehende Professionalisierung und Institutionalisierung verzichteten, wehrten sie das Erscheinungsbild einer NGO ab, die die Probleme anderer routiniert, technisch und rational bearbeitet. DF konstituierte sich so entsprechend der Kompetenzkriterien einer transnationalen Landschaft aus feministischen Frauenfußballinitiativen als Teil einer gemeinsamen Bewegung und versuchte, die Spannung zwischen Solidarität und Ungleichheit, die einem translokalen feministischen Projekt, das sich auch auf einem humanitären neoliberalen social movement market legitimieren muss, innewohnt, ein Stück weit in Richtung Solidarität zu verschieden.

\section{Zwischenstand: Durch multiple Anforderungen navigieren}

In diesem zweiten Teil habe ich die Entstehung und Formierungen einer Organisation als unvorhersehbaren, doch durch historische Pfade und gegenwärtige Machtbeziehungen vorstrukturierten Effekt eines günstigen Moments untersucht. Ich habe auf dem Argument aufgebaut, dass zu einem spezifischen Zeitpunkt die Problematisierung fußballerischer Geschlechterverhältnisse möglich wird und diese Problematisierung heterogene Institutionen, diskursive Aussagen, Personen, Politiken und Verwaltungsprozesse über Differenzen hinweg versammelt und neue Akteur_innen und Praktiken produziert. Anhand von DF habe ich in diesem Kapitel die Formierungen einer solchen neuen Akteurin nachgezeichnet. Dabei zeigte sich, dass die verschiedenen Gestalten einer Organisation wie DF als Resultate eines beständigen Prozesses des Sich-Organisierens entsprechend differenter, versammelter Kompetenzregime verstanden werden können. Organisationen erscheinen daher nicht als statische und klar umrissene, klassifizierbare Gebilde und Strukturen, sondern können als mannigfaltige Prozesse verstanden werden. Ich habe dabei folgende relevante Stakeholder identifiziert, die jeweils eigene Kompetenzkriterien an eine Organisation richten: Staatliche Sportförderungen (auf Bundes- und Lokalebene sowie auf verschiedene Ressorts verteilt) sowie zivilgesellschaftliche Fördereinrichtungen (wie politische und private Stiftungen) erwarten eine verlässliche Partnerorganisation, die aufgrund ihrer modernen Bürokratie sowie gemeinsamer politischer und/oder gesellschaftli- 
cher Ziele mit Aufgaben des Gemeinwohls betraut werden kann. Vor diesen Stakeholdern, die das finanzielle Überleben sichern, formierte sich DF als professionell arbeitender, rechtsfähig eingetragener Verein und internationale Expertinnen-NGO im Bereich Gender, Frauenrechte und Frauenfußball. Vor dem städtischen Umfeld, in dem DF lokalisiert war und aus dem sich materielle und personelle Unterstützung sowie Moralvorstellungen vom ,richtigen“ politischen Handeln speiste, wurde einerseits die Erwartung einer linksalternativen und feministischen Szene an sie gerichtet, eine Alternative zum kritisierten Verbandsfußball sowie zu entwicklungspolitischen ExpertinnenNGOs zu bieten. Andererseits zählten sportpolitische Institutionen, wie das Bezirkssportamt, auf DF als regelmäßige Sportveranstalterin im Stadtteil. In diesem Kontext formierte sich DF als feministische, aktivistische und weltgewandte Alternative $\mathrm{zu}$ traditionellen Fußballvereinen und als MitOrganisatorin des städtischen Kultur- und Sportprogramms, die das Image des Stadtteils als bunt, weltoffen und progressiv mitprägte. Die Formierung gegenüber dem Verbandsfußball war durch gleichzeitige Abgrenzung und Eingliederung geprägt. Zum einen spielte er in den Problematisierungen eine zentrale Rolle, wenn er als Ort und Reproduzent der kritisierten Geschlechterverhältnisse benannt wurde, sodass die Formierung als glaubwürdige Frauenrechts- und Frauenfußballinitiative immer eine oppositionelle Distanz zum Verbandsfußball einforderte. Zum anderen verlangte die effektive Adressierung der Politiken und der Wunsch, dass sich etwas im Verbandsfußball veränderte, kooperatives Vorgehen, sodass der Verbandsfußball ebenfalls $\mathrm{zu}$ einem relevanten Anerkennungsregime wurde. Während sich der nationale Dachverband vorrangig auf den Profifußball konzentrierte, setzte der lokale Fußballverband die Eingliederung in das Leistungs- und Wettkampfprinzip als Mitsprachekriterium voraus. Durch die Gründung eines eigenen Amateur-Fußballvereins, der den spielerischen Ernst anerkannte, organisierte sich DF trotz vieler Differenzen als fußballerisches Verbandsmitglied. Auch die internationalen Teilnehmer_innen der von DF durchgeführten Projekte bildeten einen relevanten Stakeholder. Die meisten waren skeptisch gegenüber dem den Expertinnenstatus mitbegründenden entwicklungspolitischen Diskurs, der sie selbst als entwicklungsbedürftige Zielgruppen subjektivierte. Sie erwarteten von den Organisator_innen auf Augenhöhe behandelt zu werden und formulierten damit Anforderungen, denen sich die Aktivist innen selbst aufgrund eigener feministischer Moralvorstellungen verpflichtet sahen. Vor sich selbst und den Teilnehmenden konstituierte sich DF als aktivistische Graswurzelinitiative, die durch Herzblut, Fußballleidenschaft und den Willen, gemeinsam etwas im Fußball zu verändern, angetrieben wurde und sich in der neu geschaffenen Subjektposition der female football activist verdichtete. Bevor die forschungsleitende Frage mithilfe dieser Beobachtungen weiter konkretisiert werden kann, gilt es, das günstige Moment anhand von sechs Punkten weiter auszuleuchten. 
(1) Die Problematisierung fußballerischer Geschlechterverhältnisse versammelt eine Vielzahl unterschiedlicher, nicht nur verbandssportlicher Institutionen und Personengruppen mit jeweils eigenen Praktiken, Argumenten, Moralvorstellungen und Konventionen auf lokaler, nationaler und internationaler Ebene und aus unterschiedlichen sozialen Feldern. Dies gibt Anlass, das rechtlich verankerte, bürgerliche Credo von der relativen Autonomie des Sports, wonach dieser für die Regelung seiner Belange allein selbst zuständig sei, mit Blick auf die tatsächliche Praxis zu überdenken (siehe Kap. 4.3.1). Tatsächlich sind es eine Vielzahl von Akteur_innen auch außerhalb der Verbände, die mit unterschiedlichen Motiven, Zielen und Instrumenten sich zur Bearbeitung der fußballerischen Geschlechterverhältnisse mal lose, mal in enger Kooperation versammeln.

(2) Die vielzähligen Stakeholder bilden eine heterogene, teils widersprüchliche und diskontinuierliche, sich immer wieder verändernde Landschaft aus Anforderungen und Erwartungen an eine zivilgesellschaftliche Organisation, die sie mit der Bearbeitung der Geschlechterverhältnisse betrauen. Diese beziehen sich sowohl auf organisationsinterne Strukturen, Arbeitsweisen und Inhalte als auch Beziehungen zu den einzelnen Stakeholdern. Eine Organisation, die sich als kompetente Problembearbeiterin legitimieren will, muss sich entsprechend strukturell, inhaltlich und relational formieren. Diese Differenzen und Widersprüche werden durch Unterschiede unter den Mitgliedern in Bezug auf politische Überzeugungen, Lebenswelten und geschlechtliche wie sexuelle Identitäten multipliziert (vgl. Hilhorst 2003, 217). Als besonders spannungsreich haben sich hier bereits die Beziehungen zwischen der Gestalt der Aktivistin und der Expertinnen-NGO sowie zwischen der Formierung als feministisch-solidarisches Fußballprojekt und dem verbandsfußballerischen Leistungsprimat angedeutet. Edwards und Hulme schlussfolgern daraus:

„Because stakeholder perceptions inevitably differ and NGOs are subject to so many conflicting pressures, the dilemmas of accountability identified above cannot be ,solved" - they have to be managed." (1996a, 223, Herv. i. Orig.)

Sich-Organisieren meint also nicht das Auflösen von Widersprüchen und Dilemmata, sondern das geschickte Navigieren zwischen den verschiedenen Regimen.

Henrik Vigh $(2006,2009)$ hat den Begriff der „social navigation“ analytisch geschärft und theoretisiert. Ausgehend von seiner Forschung zum Umgang junger Männer mit den alltäglichen Auswirkungen von sozio-politischer Instabilität, Armut und Konflikt in Guinea-Bissau und in Anlehnung an die ursprüngliche Wortbedeutung (navigare = lat. segeln, Schiffsführung) konkretisiert er soziale Navigation als "Motion within a moving environment“ (2009, 425). Er argumentiert, dass diese beweglichen Umgebungen nicht nur in solch konflikthaften Konstellationen wie in Guinea-Bissau zu finden sind, sondern ebenfalls die Alltage in gegenwärtigen europäischen Gesellschaften, 
geprägt durch Wirtschaftskrisen, Klimawandel und Globalisierung, entscheidend gestalten. Flüchtigkeit und Unvorhersehbarkeit schienen auch das günstige Moment auszuzeichnen, war doch nicht ersichtlich, ob sich das Möglichkeitsfenster weiter öffnen oder vielleicht bald schließen würde, sodass DF situativ reagierte, sich auf neue Gegebenheiten einstellte und dabei nach bestem Wissen und Gewissen zukünftige Veränderungen, Chancen und Schwierigkeiten antizipierte (vgl. ebd., 240). Daher scheint es mir hilfreich, auch die Bewegungen, die DF vollzog, um sich innerhalb der heterogenen Landschaft als kompetente Organisation zu formieren und zu legitimieren, als Navigation, bzw. unterschiedliche Navigationspraktiken zu verstehen. Navigieren ist dabei immer zugleich auf den aktuellen Moment als auch eine zukünftige Vorstellung des sozio-politischer Arrangements gerichtet:

„[N]avigation entails simultaneously moving toward a distant future location or condition (that is, movement toward future positions and possibilities), and making one's way across immediate and proximate oncoming changes and forces of the near future." (Vigh 2009, 429)

Mit Blick auf die in diesem Kapitel beschriebenen Praktiken der Organisationsformierung kann nun ergänzt werden, dass soziales Navigieren unterschiedliche Bewegungen beinhaltet. Navigieren erfordert, sich beständig neu $\mathrm{zu}$ formieren und zwischen diesen multiplen Gestalten situativ zu changieren. Das Formieren und Changieren geschieht dabei innerhalb machtvoll strukturierter Verhältnisse. So führen z.B. die Förderbedingungen im Kontext der öffentlichen Reformverwaltung, die nichtstaatliche Organisationen mit der Durchführung sozialer Aufgaben betraut, dazu, dass die für das Fortbestehen von NGOs notwendigen Ressourcen nur durch Kompromisse bei den eigenen Projektvorstellungen erlangt werden können; und Anforderungen staatlicher und verbandsfußballerischer Verwaltung an professionelle Organisationsstrukturen konterkarieren organisationsinterne Ideale geteilter Verantwortung und Zuständigkeiten. Nicht jede Organisationgestalt wurde von den Aktivist_innen freudig begrüßt: Während die Klassifizierung als NGO Skepsis hervorrief, erschien die Gestalt der Aktivistin und Frauenrechtsexpertin deutlich attraktiver; und die Eingliederung in den Wettkampfbetrieb wurde als Bedrohung für die feministische Solidarität und gegenseitige Befähigung mit Skepsis beobachtet. In diesen multizentrischen, doch nicht herrschaftsfreien Gefügen ermöglichte eine „messy reality of multiple identities“ (Chhachhi/Pittin 1999, 71) das strategische Koalieren und letztlich das Überleben der Organisation. Das Navigieren entlang externer wie interner Variablen beinhaltet daher nicht nur den Wechsel zwischen Gestalten, sondern ebenfalls das In-Einklang-Bringen, Ausbalancieren oder schlicht Aushalten von Widersprüchen. Organisationen wie DF erscheinen durch diese Praktiken als ,amalgam of different discourses, relations and ambitions“ (Hilhorst 2003, 217). 
(3) Diese Formierungen geschehen nicht entlang einer festen Agenda und einer intentional ausgearbeiteten Strategie, sondern erfolgen schrittweise im Dialog mit internen Gegebenheiten sowie mit einer sich ebenfalls ständig wandelnden Landschaft aus vielen verschiedenen Stakeholdern. Organisationen erscheinen dabei nicht als zielgerichtet handelnde politische Subjekte, die mithilfe rational abgewogener Mittel formulierte Ziele kontinuierlich verfolgen. Organisation-Sein bzw. Sich-Organisieren bedeutet vielmehr die beständige Anpassung an und das Abstimmen mit verschiedenen Diskursen, rechtlich-institutionellen Rahmenbedingungen, heterogenen Erwartungen an Organisationsstrukturen und moralischen Vorstellungen von Beziehungsgestaltungen und Politikzielen entlang eines ,utopisch unterlegten Pragmatismus“ (Adam 2016) und unter geschicktem Einsatz des eigenen Wissens. Soziales Navigieren ist demnach, wie es Vigh beschreibt, keineswegs auf den unmittelbaren Moment beschränkt, auch wenn es wie spontane Manöver erscheinen mag: ,,[I]t is in fact equally directed towards both the near and the distant future as the practice of moving along an envisioned, yet frail and tentative, trajectory in an unstable environment" (Vigh 2009, 424). Genau wie Anpassungen und Veränderungen verstehe ich diese Beweglichkeit keinesfalls als Opportunismus. Moralische und utopische Leitplanken und Ziele stecken den Navigationsradius $a b$ und ziehen die definitorische Grenze zwischen Beweglichkeit und Beliebigkeit. Beweglichkeit erscheint mir vor diesem Hintergrund als zentraler Modus der Organisationsformierungen in heterogenen landscapes of practice.

(4) Gleichzeitig war zu beobachten, dass sich seit der Gründung von DF Organisationsstrukturen und Arbeitsweisen, Argumente und Wissensbestände stabilisierten. ${ }^{125}$ Stabilität gewährleistet Wiedererkennungswert und Berechenbarkeit. Während die Stabilisierung in Form von Vereinsgründung und Institutionalisierung Verlässlichkeit gegenüber Förderinstitutionen kommunizierte, stellte das Beharren auf aktivistischen Idealen und die Ablehnung weiterer Prozesse der NGOisierung DF als glaubwürdige Partnerin und Mitstreiterin gegenüber Fußballerinnen aus verschiedensten Ländern, feministischen Fußballinitiativen und der eigenen Mitgliedschaft dar. Politisierung der Inhalte, Vorgehensweisen und Ziele sowie Professionalisierung der internen Struktur und Arbeitsweise bilden zwei parallele, aber miteinander verknüpfte Prozesse des Sich-Organisierens. Mit der Zunahme an relevanten Kompetenzregimen erweiterte sich das Themenspektrum - Frauenrechte und Geschlechterverhältnisse gesellten sich zum interkulturellen Fußballfest - und wurde zugleich spezialisiert, sodass ein Expertinnenstatus gesichert wurde. Es ist also nicht allein die flexible Anpassungs- und Navigationsfähigkeit, die das Überleben als Organisation sowie die Anerkennung in einer multizentri-

125 Ramanath (2007) erklärt die sogenannte Pfadabhängigkeit von NGOs vor allem mit dem Beharren auf den Gründungswerten, eingespielten Routinen und stets beschränkten Ressourcen. Eine völlige Anpassung an institutionelle Bedingungen wird so verhindert. 
schen Landschaft ermöglicht, sondern ebenso die Her- und Darstellung eines bestimmten Maßes an Stabilität (vgl. Bernal/Grewal 2014a, 304). Nur durch ein ausgeglichenes Zusammenspiel von Flexibilität und Stabilität gelingt die Anerkennung als kompetente Organisation.

(5) Navigieren, Anpassen und Stabilisierung gehen mit verschiedenen Kosten einher. Mit der zunehmenden Komplexität der Landschaft, in der DF navigierte, stiegen nicht nur die Kooperationsmöglichkeiten, sondern auch Konfliktpotentiale und Kompromisszwänge. Die Unmöglichkeit langfristiger Planung, die Abhängigkeit von Projektförderungen und die Ungewissheit über zukünftige förderpolitische Konjunkturen erschwerten die Nachhaltigkeit der Projekte sowie die berufliche Zukunftssicherheit für die Angestellten. Während die steigende Professionalität der ehrenamtlichen wie bezahlten Arbeit einen größeren Bekanntheitsgrad und Ansehen ermöglichte, führte sie auch $\mathrm{zu}$ erhöhtem Arbeitsaufwand und zeitlichen Belastungen unter den Ehrenamtlichen. Während die Institutionalisierung von Arbeit verlässlichere und erfolgreichere Kooperationen versprach, mussten die Projektkoordinatorinnen zugleich ein höheres $\mathrm{Ma} ß$ an Verantwortung tragen. Während identitätspolitische Strategien mehr Koalitionsmöglichkeiten und Argumentationsressourcen boten, blieben Ideale der Inklusion und queeren, nichtzweigeschlechtlichen Politiken auf der Strecke. Während eine Übernahme entwicklungs- und außenpolitischer Sprachnormen die Anerkennung als glaubwürdige Partnerorganisation versprach, mussten zugleich Abstriche bei den Wünschen und Anliegen der Teilnehmenden gemacht werden.

(6) Die Praxis des Problematisierens beinhaltet das Versammeln differenter Akteur_innen und Praktiken an konkreten Orten. Mit Tsing (2005) lassen sich die Formierungen einer Organisation als Produkte der spezifischen Friction verstehen, die jenes Momentum für das Zusammenspiel von Feminismus und Fußball eröffnete. Daran anschließend hat meine Analyse gezeigt, dass dieser Prozess das konstante Navigieren zwischen den differenten Stakeholdern beinhaltet und Organisationen damit entscheidend dazu beitragen, diese Versammlungen produktiv zu halten. Problematisierungen werden also zum einen durch das Versammeln differenter Akteur innen ermöglicht, zum anderen produzieren sie neue Relationen. Hilhorst verweist darauf, dass Organisationen mithilfe multipler Gestalten nicht nur durch die verschiedenen Stakeholder hindurch navigieren, sondern diese zugleich miteinander verbinden: ,[T] hey link a variety of domains that, at most, partially overlap, have a knowledge advantage over their stakeholders that enhance their power." (2003, 219; ähnlich Wenger 2008, 8) Vereinsgründung und Satzungsfestlegung, regelmäßige Projekt- und Beziehungsarbeit sowie die Verstetigung von Beziehungen und Arbeitsprozessen institutionalisieren die Berührungen, Spannungen und Relationen des günstigen Moments innerhalb des relativ stabilen Rahmens der Vereinsarbeit. Was in der eingangs erwähnten Veranstaltung der Heinrich-Böll-Stiftung als ein kurzzeitiges Aufeinandertreffen an 
einem konkreten Ort erschien, wurde durch die Organisationsformierungen und das kontinuierliche geschlechterpolitische, fußballerische Engagement der Flüchtigkeit entrissen und stabilisiert. Organisationen wie DF fungieren aus diesen Versammlungen heraus als jene benötigten Bindeglieder, die Beziehungen knüpfen, relevante Akteur_innen, Rationalitäten und Praktiken über Differenzen hinweg miteinander verknüpfen und Prozesse der Problematisierung am Laufen halten. Es ist also zum einen die Komplexität der sich ständig verändernden Landschaft, die die Akteur_innen navigieren lässt (vgl. Vigh 2006; 2009). Zum anderen führen jene Navigationspraktiken zu neuen Bewegungen in der Landschaft und lassen neue Arrangements entstehen.

Auf Basis dieser ersten Befunde kann nun die Frage für den nächsten Teil dieser Arbeit zugespitzt werden. Während hier umrissen wurde, wie sich eine kompetente Problembearbeiterin formiert und legitimiert, rücken im Folgenden die konkreten Praktiken der Problematisierung in den Fokus. Es gilt nun zu fragen, wie DF situativ entlang differenter Anerkennungslogiken in die Geschlechterverhältnisse intervenierte und welche Effekte sich innerhalb der multizentrischen Landschaft entfalteten. 


\section{Teil III: Geschlechterpolitische Interventionen}

Anfang 2013 treffen sich die Mitglieder von DF außerhalb des regulären Rhythmus der Plena, um gemeinsam über die Ziele von DF nachzudenken, sich über die einzelnen Vorstellungen, Wünsche und Visionen auszutauschen und Strategien für die Zukunft zu entwickeln. Birgit hatte im Vorfeld angemerkt, wie wichtig sie es fände, dass wir eine gemeinsame Identität hätten, und damit auf die vielen verschiedenen Bedeutungen, die DF für die einzelnen Mitglieder habe, und die daraus resultierenden unterschiedlichen Ziele und Vorstellungen von Projekten angespielt. Nadine, Birgit und Luca hatten das eintägige Treffen vorbereitet. Nach einer Anfangsrunde, in der alle ihre Erwartungen äußerten, und einem Brainstorming über die persönlichen Hoffnungen für und Erwartungen an die Zukunft von DF steht der Punkt „Identität” auf der Tagesordnung. Gemeinsam erarbeiten wir zwei Fragen, zu denen wir der Reihe nach Stellung beziehen wollen: Warum ist DF wichtig? Welches sind unsere Ziele?

Saskia beginnt: „Also für mich steht im Mittelpunkt, was wir ja auch immer sagen, dass wir Frauen im Fußball eine Stimme geben und sie sichtbar machen." Als Ziele nennt sie Geschlechtergleichstellung und Frauenrechte und fügt hinzu: „Wir sind schon einmalig, weil es diese direkte Verknüpfung zwischen Fußball und AntiSexismus sonst nicht gibt." Als großes übergeordnetes Ziel der Arbeit von DF formuliert sie die Gleichberechtigung für alle und überlegt: „Vielleicht müssen wir Schritte dahin formulieren." Linda ist als Nächste an der Reihe und betont, wir sollten weiterhin Bewusstsein schaffen für die „Grenzen der Frauen“ und diese Grenzen problematisieren. Es gäbe schließlich noch immer keine Gleichberechtigung, auch nicht im Fußball: „Wir legen den Finger in die Wunde“. Und mit Blick auf die weltweite Projektarbeit: „Wir sind nicht von oben herab; wir haben keine Weltstrategie, sondern gehen immer von den einzelnen Vereinen und Projekten aus." Nadine hakt hier nach: „Empowerment?” - Linda bejaht und Birgit fügt hinzu: „Praktisch, nicht theoretisch.” Linda fährt fort, dass es ein Ziel sein könnte, irgendwann genauso ernst genommen zu werden wie andere Initiativen, die nun von internationalen Verbänden für deren Nachhaltigkeitsarbeit beauftragt werden. Und irgendwann könnten wir so ein großes Netzwerk sein, sodass wir international mit einer Stimme sprechen und Forderungen stellen könnten. Sie sieht schließlich das übergeordnete Ziel, für das DF kämpfen solle, darin, dass der Frauenfußball irgendwann genauso ernst genommen werden würde wie der Männerfußball. Annike macht weiter und zählt die Relevanz von DF auf: „Es geht darum, eine bessere Welt mitzugestalten und das umzusetzen, woran man glaubt, also den Fußball inklusiver machen: ,Football is for everyone!' Wir stärken den Frauenfußball in verschiedenen Ländern und machen auch Friedensarbeit durch die internationalen Begegnungen und den Austausch." Dann ergänzt sie: „Netzwerke finde ich auch sehr wichtig, das macht sonst niemand.“ Birgit knüpft nun an Saskia und Linda an: „Stimme geben und sichtbar machen! Meistens sind Organisationen ja so von oben herab. Wir gucken da eher von unten, von der Spielerinnenperspektive und nicht wie Funktionärinnen; aber auch mit dem Anspruch, das selber zu machen." Sie fügt grinsend hinzu: ,Wir sind das gute Gewissen des Frauenfußballs." Thomas macht weiter und bemerkt, dass es um den Gedanken gehe, dass Fußball für alle sei. Daher würden wir zu den internationalen Veranstaltungen nicht nur verbandlich organisierte Teams einladen, sondern auch private Initiativen. Es gäbe einen common sense im Frauenfußball, über den Gleichberechtigung gefördert werden könne. Mit Blick auf die lokale Arbeit formuliert er das Ziel, mehr Mädchen für den Fußball zu begeistern: „DF geht zur Basis, von unten, nicht von oben, und wir sagen: 
,Du darfst auch gegen den Ball treten, das ist dein gutes Recht." Simone ist nun an der Reihe und betont, dass für sie immer der Aspekt wichtig war, dass DF Frauen im Fußball sichtbar mache. Sie bemerkt, dass wir versäumt hätten, Ziele richtig zu benennen, und dass wir wissen sollten, welches Potential wir hätten. Sie schlägt als nächsten Schritt vor, ein Papier mit unseren konkreten Zielen zu formulieren. Nun wurde schon viel gesagt, sodass Nadine nur noch hinzufügt, dass DF Bewusstsein für die diskriminierenden Stereotype im Frauenfußball schaffen und thematisieren solle, was im Fußball mit der Identität und den Körpern der Spielerinnen passiere: „Wir sollten unsere eigenen Erfahrungen hervorheben." Nachdem sich nun alle geäußert haben, ist die Diskussion eröffnet. Linda spricht als Erste die unterschiedlichen Ziele und deren Implikationen an: ,Welches Konzept fahren wir? Ich finde DF so toll wegen des Titels [,DISCOVER FOOTBALL - female perspectives“]. Ich hätte nix unterschrieben, wenn es hieße ,Wir kämpfen gegen Diskriminierung in der Ukraine". Am besten ist das naive Herangehen: ,Wie ist der Fußball bei euch?', und nicht mit konkreten Zielen herangehen. Das ist so kulturalistisch. Sondern: ,Entdecke den Fußball!', und dann strukturiert Beschwerden von Frauen aus anderen Ländern sammeln. Wir sind das Ohr an der Basis!“ Saskia wendet skeptisch ein: „Ich weiß nicht, wie lange das trägt. Für den Anfang ja. Aber was machen wir damit?" Die Diskussion geht noch eine ganze Weile weiter, jedoch werden keine konkreten Zukunftsstrategien formuliert, sondern unterschiedliche Themensetzungen und Vorgehensweisen diskutiert. (Feldnotiz, 17.2.2013)

In den vorangegangenen Kapiteln habe ich gezeigt, wie an den Schnittstellen von Verbandsfußball, nationaler, inter-/transnationaler Sport- und Entwicklungspolitik, Frauenbewegungen und Frauensport sowie Zivilgesellschaft in den Bereichen Geschlechterpolitik, Sport und Entwicklungszusammenarbeit ein Moment entsteht, das die fußballerischen Geschlechterverhältnisse problematisierbar macht und die Entstehung einer zivilgesellschaftlichen Organisation als Problembearbeiterin ermöglicht. In dieser Feldnotiz wird ersichtlich, wie das Aufeinandertreffen diskursiver Formationen, internationaler Rechte und Normen und frauenbewegter und feministischer Politiken Praktiken der geschlechterpolitischen Problematisierung hervorbringt sowie verschiedene Stakeholder zu Adressaten oder Kooperationspartnern macht: Weltweite Frauenfußballinitiativen werden im Netzwerk zu Bündnispartnerinnen, mit denen zusammen Kritik formuliert und Verbände und Sportpolitik adressiert werden; internationale Menschen- bzw. Frauenrechtsregime und Normen stellen dafür mit ,Frauenrechten“ und „Gleichstellung“ den rechtlichen und argumentativen Rahmen bereit; „Empowerment" bietet die mobilisierende Metapher, die den eigenen Ansatz an internationale Sport-, Frauenrechts- und Entwicklungsprogramme anschließt; der Verbandssport ist nicht nur der Adressaten für Beschwerden und Forderungen nach Gleichberechtigung, sondern zugleich Kooperationspartner zur Umsetzung entsprechender Programme; und das öffentlich geweckte Bewusstsein für geschlechterbezogene Ungleichheit im Fußball soll mit Sichtbarkeitspolitik und Bewusstseinsbildung weiter geschärft und mobilisiert werden. Als Linda sagte, sie wolle den Finger in die Wunde legen und die Grenzen, die Frauen im Fußball gesetzt werden, kritisieren, oder Annike die Wichtigkeit betonte, eine bessere 
Welt mitzugestalten, äußerten sie Kritik an Bisherigem und die Notwendigkeit zur breiteren, gesellschaftlichen Veränderung. Es sind jene ,unhintergehbaren Momente des Dissens und Widerstreits, des Ereignisses, der Unterbrechung und Instituierung" (Bröckling/Feustel 2012, 8), in denen das Politische seinen Ausdruck findet. Mir erscheint es daher plausibel, die in der Zukunftsdiskussion angeklungenen unterschiedlichen Problematisierungsweisen als politisches, genauer gesagt als geschlechterpolitisches Handeln zu untersuchen.

Ich frage in diesem Teil also nach den konkreten Vorgehensweisen der feministischen Interventionen in die fußballerischen Geschlechterverhältnisse, ihren Bedingungen, Effekten und Wegen, die durch das Moment ermöglicht werden. Eine politikethnologische und feministisch informierte Perspektive kann diese als unterschiedliche Formen zivilgesellschaftlichen geschlechterpolitischen Handelns kontextualisieren und in ihren historischen und gegenwärtigen Relationen, ihren Möglichkeitsbedingungen und Effekten analysieren. Dieser Analyse möchte ich zunächst Überlegungen voranstellen, wie politisches Handeln innerhalb spätkapitalistischer Demokratien konzipiert werden kann.

\section{Theoretische Ausgangspunkte: politisches Handeln und Topografien der Macht}

Die mit Ulrich Bröckling und Robert Feustel bewusst offen gehaltene Begriffsannäherung an die politischen Dimensionen zivilgesellschaftlichen Handelns trifft den Kern des Problematisierungskonzepts. Sie fasst jene Momente, in denen ,ein unproblematisches Erfahrungsfeld oder eine Reihe von Praktiken, die als selbstverständlich akzeptiert wurden, die vertraut und ,unausgesprochen' sind, also außer Frage stehen, zum Problem werden, Diskussionen und Debatten hervorruft, neue Reaktionen anregt und eine Krise der bisherigen stillschweigenden Verhaltensweisen, Gewohnheiten, Praktiken und Institutionen bewirkt" (Foucault 1996, 78). Doch wie ist politisches Handeln in jenem gesellschaftspolitischen Gefüge, das ich in Teil II analytisch als landscape of practice (Wenger-Trayner/Wenger-Trayner 2015) gefasst habe, zu theoretisieren? Anstatt eines klaren Zentrums versammelt sich hier eine Vielzahl mehr oder weniger einflussreicher Institutionen und Akteursgruppen zur Bearbeitung der Geschlechterverhältnisse. Wenn die Mitglieder von der „Basis“, von einem „Oben“ und einem „Unten“ sprachen, zeichnet sich jedoch ab, dass sie diese Landschaft als vertikales Machtgefüge wahrnahmen. Ein ähnliches Machtverständnis leitet auch verschiedene politikethnologische Theoretisierungen von der Beziehung zwischen Zivilgesell- 
schaft und staatlichen, trans-/internationalen und ökonomischen Regierungsinstitutionen sowie von zivilgesellschaftlichem politischem Handeln (Ferguson/Gupta 1997; 2002). Doch eignen sich die para-ethnografische Selbstverortung der Aktivist_innen, die ihrer präferierten Formierung auf Graswurzelebene ähneln, und die entsprechenden ethnologischen Theoretisierungen von Macht und politischem Handeln, um die heterogene Landschaft spätkapitalistischer Demokratien und die darin verorteten zivilgesellschaftlichen geschlechterpolitischen Praktiken zu verstehen? Um dies herauszufinden, verfolge ich dieses vertikale Machtverständnis zunächst zurück, indem ich die politikethnologische Auseinandersetzung mit politischem Handeln schematisch nachzeichne.

Zivilgesellschaftliche Akteur innen, die sich zur Artikulation eines gesellschaftlichen oder politischen $\bar{D}$ issens kollektiv organisieren und für diese Arbeit von Interesse sind, werden vermehrt ab den 1970er Jahren zum Untersuchungsgegenstand der Ethnologien. ${ }^{26}$ Sowohl die Anthropology of Social Movements (Edelman 2001) als auch die Anthropology of NGOs (Mertz/Timmer 2010; Schuller/Lewis 2017) rücken zwar die Frage nach politischem Handeln nicht in den Vordergrund, verhandeln sie aber häufig durch die unterschiedlichen theoretischen Rahmungen mit. In den 1970er Jahren entwickeln sich in der US-amerikanischen und europäischen Ethnologie und eng an die sozialwissenschaftliche Bewegungsforschung angelehnt neue Ansätze als Antworten auf die politischen Bewegungen des vorangegangenen Jahrzehnts. Marxistische Ansätze hatten sich als unzureichend erwiesen, um die sozialen Bewegungen dieser Dekade zu erfassen, deren Führungsriegen - anders als zu Zeiten der Arbeiterbewegung - meist bürgerlichen Schichten entstammten und klassenübergreifend Anhänger innen vereinten. Im Gegensatz zur Hochzeit der Arbeiterbewegung verlaufen die zentralen Konfliktlinien postindustrieller Gesellschaften nicht länger zwischen Arbeit und Kapital, sondern entlang von Lebensweisen, Verhaltensformen und Bedürfnissen. Der New-Social-Movement-Ansatz in Europa untersucht diese neuen politischen Phänomene als Identitätspolitiken (Edelman 2001, 288). Soziale Konflikthaftigkeiten werden dabei nicht allein entlang von Klassenunterschieden analysiert, sondern entlang vieler verschiedener Relationen wie Rassismus, Patriarchat, Naturbeherrschung, Heteronormativität und Kolonialismus, die neue multiple Subjektpositionen hervorbringen

126 Edelman $(2001,285)$ bemerkt, dass politisches Handeln, das sich oberhalb alltäglicher, unorganisierter Widerstände und unterhalb großer gesellschaftlicher Transformationen und Revolutionen abspielt, lange unbeachtet blieb. Die politikethnologische Aufmerksamkeit galt zunächst den alltäglichen Mikropraktiken widerständigen Handelns. Er begründet dies erstens mit der akademischen Arbeitsteilung, die das ethnologische Interesse auf Bauern, die städtischen Armen, ethnische Minderheiten und religiöse Sekten lenkte und andere Mobilisierungstypen der Soziologie, Politik- und Geschichtswissenschaft überließ. Zweitens verweist er auf die ethnologische Schwerpunktsetzung auf das Alltägliche und Mikropolitische als Gegensatz zum organisierten Widerstand. 
(vgl. Edelman 2001, 289; Laclau/Mouffe 2001; Day 2004, 722). Der in den USA entwickelte Resource-Mobilization-Ansatz fokussiert hingegen eher die Organisationsformen und -strategien sozialer Bewegungen und analysiert diese als Interessenspolitiken sozial vernetzter Gruppen statt als Identitätspolitiken benachteiligter gesellschaftlicher Gruppen (Edelman 2001, 289).

$\mathrm{Zu}$ der Erforschung sozialer Bewegungen tritt in den 1990er Jahren im Zuge der sprunghaften Zunahme von NGOs eine eher organisationsethnologisch ausgerichtete Anthropology of NGOs hinzu (Schuller/Lewis 2017). ${ }^{127}$ Sie diskutiert und bewertet die Beziehung zwischen zivilgesellschaftlichen Non-Profit-Organisationen und Regierungsorganen unterschiedlich. Während die einen in zivilgesellschaftlichen Organisationen eine effektive, da flexible, unbürokratische und innovationsfreudige Alternative zu staatlichen Organen sehen ${ }^{128}$, heben andere, wie z.B. William Fisher (1997), Sonia Alvarez (1999) und Jael Siliman (1999), die Kooption der Organisationen durch staatliche Regierungstechnologien im Zuge neoliberaler Reformen hervor und sehen NGOs als verlängerten Arm transnationaler Regierungsformen (Ferguson/Gupta 2002; Schuller/Lewis 2017). Besonders prägend ist hierbei der in den frühen 1990er Jahren entwickelte Post-Development-Ansatz. In Anknüpfung an Foucaults Diskurstheorie und im Lichte der Kritik am hegemonialen Entwicklungsapparat wird der Staat - und in Zeiten der Globalisierung auch transnationale Regierungsinstitutionen wie die Weltbank, die UN, der Internationale Währungsfond und internationale Unternehmen - als Träger von Souveränität und Autorität konzipiert, der in der Lage ist, Macht von ,oben' über zivilgesellschaftliche Akteur_innen auf der Graswurzelebene auszuüben (Gupta/Ferguson 1997; Ferguson/Gupta 2002). Zivilgesellschaftliche und vor allem lokale, aber translokal vernetzte Vereinigungen werden demgegenüber als transformative Kräfte begrüßt, die es vermögen, dem neoliberalen Entwicklungsdiskurs alternative und auf lokalem Wissen basierende Entwicklungskonzepte und -praktiken entgegenzusetzen (Esteva/Suri Prakash 1997, 282; Kothari 1997, 146; Rahnema 1997, 395f.). Ihnen werden wiederum jene zivilgesellschaftlichen Organisationen als ,anti-politics machine“

127 Schuller/Lewis resümieren, dass die Ethnologie im Gegensatz zu Nachbardisziplinen wie der Politikwissenschaft und Soziologie und trotz der bereits in den 1960er Jahren geäußerten Aufforderung des studying up - der Untersuchung von Organisationen und Institutionen - zögerlich auf den neuen Forschungsgegenstand reagierte. Sie begründen dies mit der Bevorzugung von Forschungen auf Community-Ebene. So entstanden Arbeiten, die die Graswurzelperspektive der lokalen Bevölkerung auf Präsenz und Wirkung von NGOs einnehmen, jedoch nicht den Blick in die Organisationen richten (2017, 637). Ausnahmen bilden hier bspw. die Ethnografien von Hilhorst (2003), Mosse (2005), Riles (2007). Zur Zurückhaltung der Ethnologie gegenüber Expertinnenkulturen siehe Rabinow et al. (2008).

128 Für einen Überblick über diese Studien siehe Fisher (1997, 444). 
(Ferguson 1990) gegenübergestellt, die im Auftrag von bzw. finanziert durch Regierungen agieren. ${ }^{129}$

Diese binären Gegenüberstellungen greifen auch feministische Ansätze auf und diskutieren mit dem Konzept der NGOisierung die Entstehung von NGOs aus Frauenbewegungen heraus als potentielle Depolitisierung feministischer Kämpfe (Alvarez 1999). Verträge mit staatlichen Organen, so die Argumentation, behindern und kompromittieren letztlich die Fähigkeit von NGOs, auf feministische Reformen hinzuwirken (Bernal/Grewal 2014b). Die Konzentration auf globale Machtstrukturen und weltweite neoliberale Regierungsformen lässt organisierte zivilgesellschaftliche Aktivitäten weniger als politisches, sprich widerständiges, denn als Regierungshandeln erscheinen, welchem mit Foucault prinzipiell skeptisch gegenüberzutreten ist (vgl. Sharma 2008, xix).

Progressives - eben nicht kooptiertes - politisches, zivilgesellschaftliches Handeln erscheint in dieser Perspektive als ,grounded“, als im Lokalen wurzelnde interessengeleitete oder pluralistische Politik - wie in der liberalen politischen Theorie - oder als klassenkämpferische Positionskämpfe in Anknüpfung an Marx und Gramsci (Ferguson/Gupta 2002, 983). Widerständiges Handeln wird romantisierend außerhalb von Macht verortet (Abu-Lughod 1990) und als bürokratischen Regierungsformen zeitlich vorgängig konstituiert, sodass zivilgesellschaftlicher Alltag und staatliches Regieren als eigentlich getrennte Bereiche anstatt als immer schon miteinander verflochten erscheinen (Hodžić 2017, 5). Wenn lokales widerständiges Handeln als genuin politisch gedacht und zugleich jene Aktivitäten im Einklang mit regierenden Institutionen als kooptierte Anti-Politik theoretisiert werden, droht dieser Perspektive ein „fetishism of ,grassroots““ (Bernal/Grewal 2014a, 305). ${ }^{130}$

129 Der Vorwurf der Anti-Politik speist sich bei Autoren wie Ferguson (1990) und Escobar (1997) aus der Beobachtung, dass Entwicklungsorganisationen ihre Themen und Projekte als technische deuten und damit technisch und nicht politisch bearbeitbar machen. McRobbie (2009) argumentiert ähnlich mit Blick auf die neoliberale Vereinnahmung des Feminismus, die im Kontext der reflexiven Moderne nach und nach frühere feministische Errungenschaften abbaut und Frauen in die flexible Ökonomie des Postfordismus integriert. Newman bemerkt kritisch, dass diese Argumentationen die Handlungsfähigkeit von Kapitalismus oder Neoliberalismus nicht in Frage stellen, sondern als unproblematische und nuancenfreie Kategorien verwenden. Während politischen Bewegungen, wie dem Feminismus, Flexibilität und damit letztlich auch Kooptierbarkeit zugeschrieben werden, werden diese Eigenschaften beim Kapitalismus nicht vermutet $(2014,138)$.

130 Für diese Tendenzen finden sich unterschiedliche Erklärungsversuche. Fisher sieht in diesen normativen Tendenzen die Kontroverse zwischen jenen, die alternative Entwicklungsmodelle favorisieren, und jenen, die das Entwicklungsprojekt prinzipiell anzweifeln, widergespiegelt $(1997,446)$. Analytisch wie aktivistisch engagierten sich Ethnolog_innen vorrangig in Graswurzelbewegungen, da diesen unterstellt wurde, im Interesse der Marginalisierten und Machtlosen zu handeln, wohingegen die Motive formeller NGOs als , unrein' galten (Schuller/Lewis 2017, 637). Bernal/Grewal argumentieren hingegen, dass die kontroversen Perspektiven auf NGOs - als neoliberale Agenten, als Graswurzel- 
Die Beziehungen zwischen Regierungsinstitutionen, NGOs und sozialen Bewegungen werden dabei als ein Ringen gedacht, in dem Erstere jeweils drohen, Letztere zu kooptieren. Ob die jeweiligen feministischen Organisationen zur Artikulation des Dissens fähig sind und ihr Handeln damit politisch oder etwa anti-politisch ist, scheint hierbei vorrangig von ihrer Position innerhalb einer vertikalen Machtarchitektur von Staat beziehungsweise trans/internationaler Regierungsinstitutionen einerseits und Zivilgesellschaft andererseits abzuhängen.

Mit diesem kurzen Ausflug in frühere ethnologische Ansätze zu zivilgesellschaftlichem politischem und vor allem geschlechterpolitischem Handeln will ich nicht die Bandbreite und Differenziertheit der Argumentationen wiedergeben, die die Forschungen selbstverständlich aufweisen, sondern auf Tendenzen aufmerksam machen, die gesellschaftspolitische Konstellationen vertikal in ein Macht ausübendes, regierendes Oben und ein regiertes oder gegebenenfalls widerständiges Unten teilen und Eingang in der Selbstpositionierung in meinem Feld gefunden hatten. Entsprechende Studien haben gewiss zu wichtigen Erkenntnissen über koloniale, post-koloniale, patriarchale und imperialistische Ungleichheitsstrukturen und Regierungsprozesse geführt. Dennoch bezweifel ich, dass eine solche Machttopografie auch auf die hiesige Konstellation übertragbar ist.

Wie ich in Teil I und II gezeigt habe, wirkt eine Vielzahl von Stakeholdern - staatliche Verwaltungseinrichtungen, verbandssportliche Institutionen, große und kleine NGOs sowie transnationale Organe - auf die fußballerischen Geschlechterverhältnisse ein. Ein herrschendes Oben ist in multizentrischen Landschaften liberaldemokratischer, spätkapitalistischer Gesellschaften nicht identifizierbar. So kritisieren James Ferguson und Akhil Gupta (2002) die Idee einer vertikalen Machttopografie und die damit verbundene konzeptionelle Dichotomie zwischen Staat und Zivilgesellschaft, in der der Staat als nach unten eindringendes oder planendes Machtzentrum gedacht wird:

„Traditional leftist conceptions of progressive politics in the Third World (to which many anthropologists, including myself, have long subscribed) have almost always rested on one or another version of the vertical topography of power [...]. ,Local ${ }^{\text {‘ }}$ people in ,communities' and their , authentic' leaders and representatives who organize ,at the grass-roots', in this view, are locked in the struggle with a repressive state representing (on some complex combination) both imperial capitalism and the local dominant class. The familiar themes here are those of resistance from below and repression from above, always accompanied by the danger of co-optation, as the leaders of today's struggle become the elite against whom one must struggle." (Ferguson 2004, 394)

Ich schließe mich daher jenen Ansätzen an, die zivilgesellschaftliches politisches Handeln nicht entlang der Widerstand-Kooptions-Dichotomie denken

Alternativen zum Staat oder als lokale Zivilgesellschaft - der Unschärfe der Rubrik NGO selbst geschuldet sind $(2014,3)$. 
(bspw. Mosse 2004; PoLAR 2010; Bernal/Grewal 2014b). ${ }^{131}$ In diesem Sinne lässt sich mit Ferguson und mit Blick auf (trans-)nationale Regierungsformen in Zeiten öffentlicher Reformverwaltung paradigmatisch fragen:

„Are we ready for social movements that fight not ,from below' but ,across', using their ,foreign policy" to fight struggles not against ,the state" but against the hydraheaded transnational apparatus of banks, international agencies and market institutions [and federations, FF] through which contemporary capitalist [and patriarchal, FF] domination functions?" (Ferguson 2004, 394)

Ob hydraköpfig oder multizentrisch - diese mehrdimensionale Machttopografie ordnet zivilgesellschaftliche und Regierungsorganisationen nicht länger vertikal an, sondern macht sie zu "horizontal contemporaries“ (Ferguson/Gupta 2002, 994; vgl. Abu-Lughod 1990). Diese horizontale Machttopografie fügt sich in das Konzept einer landscape of practice aus komplexen, sich stets verändernden, dennoch machtvoll strukturierten Beziehungen (vgl. Wenger-Trayner/Wenger-Trayner 2015, 15). Organisationen wie DF sind diesen multizentrischen Gefügen längst mit ihren Strategien begegnet, wenn sie den Dissens sowohl konfrontativ als auch kooperativ artikulieren. Sie sind auf öffentliche Förderungen angewiesen und führen im Auftrag staatlicher Einrichtungen sport- und entwicklungspolitische Projekte durch, sie kooperieren mit Verbänden, argumentieren im Einklang mit internationalen Rechten, formulieren zugleich eigene Utopien, beteiligen sich an Protesten und kritisieren öffentlich die verbandlichen Institutionen. Am Beispiel DFs wird so verständlich, warum neuere Ansätze NGOs als hybride Organisationen und ihr Handeln, das nicht nur machtvolle Diskurse befeuert, sondern diese auch untergräbt, als deutlich ambivalenter beschreiben (vgl. Mertz/Timmer 2010, 171; Hodžić 2014). ${ }^{132}$

Ein theoretisches Verständnis dieses geschlechterpolitischen Handelns sollte der Hybridität, Mehrdimensionalität und augenscheinlichen Widersprüchlichkeit mit entsprechenden konzeptuellen Werkzeugen gerecht werden (Ferguson 2004, 394). Ich folge daher dem Vorschlag von Lila Abu-

131 Newman $(2014,139 f$.) identifiziert auch in der gegenwärtigen Konjunktur der Bewegungsforschung ein Machtverständnis, das klar zwischen Macht und oppositioneller Konfrontation trennt. Aufschlussreich sind in diesem Zusammenhang auch die kritischen Diskussionen der Widerstandstheorien, z.B. Abu-Lughod (1990), Brown (1996), Ortner (2006, Kap. 3). Im Anschluss an Abu-Lughods Kritik, dass Widerstand in der Forschung romantisiert würde, kann vermutet werden, dass dem Widerstandskonzept - ähnlich wie dem NGOisierungskonzept - auch normative und wissensanleitende Tendenzen eingeschrieben wurden.

132 Hilhorst (2003) und Ramanath (2007) zeigen, dass Kooperation mit öffentlich-staatlichen Institution nicht unweigerlich Kooption bedeutet. Die Beziehung zwischen NGOs und Geldgebern ist durch Feilschen und Verhandeln und nicht durch einseitige Anpassung geprägt. Hodžić schlägt anknüpfend an Haraway eine posthumanistische Wendung vor, die sämtliche zivilgesellschaftliche Organisationen als cyborgartige Verschmelzungen denkt: Auch wenn NGOs die illegitimen Kinder von „bureaucratic, top-driven, and masculinist neoliberal states“ seien, sind sie ihrem Ursprung gegenüber doch häufig äußerst untreu und die Effekte ihrer feministischen Politiken nicht vorherbestimmt $(2014,231)$. 
Lughod (1990), gesellschaftspolitische Gefüge konsequent durch Foucaults Machtkonzept zu denken. Foucault entfaltet in „Der Wille zum Wissen“ (2014) ein Verständnis von Macht als multizentrischem Gefüge, das nicht nur negativ - verbietend, unterdrückend oder verleugnend - wirkt, sondern ebenfalls positiv - durch Diskurse, Freude und Wissen. Widerstand lässt sich so nie außerhalb beziehungsweise in binärer Opposition zur Macht, sondern nur in gegenseitiger Verstrickung denken (Abu-Lughod 1990, 42). Politische Macht ist nicht etwas, dem es zu widerstehen gilt oder das einem aufgezwungen wird, sondern etwas, das überhaupt erst die Grundlage jeglichen politischen Handelns bildet. Macht ist mobil und wirkt durch Institutionen, Praktiken, Programme und Projekte sowie durch die Beziehungen zwischen ihnen (Rose/Miller 1992, 173). Ein solches Verständnis ermöglicht es, Macht horizontal und zugleich dezentral zu denken: Sie ist nicht länger auf eine bestimmte Sphäre oder ein Zentrum beschränkt und wird nicht von oben nach unten ausgeübt, sondern setzt netzförmig alle Akteur innen miteinander in Beziehung bzw. bringt diese als solche erst hervor. Damit sind nicht nur zivilgesellschaftliche Organisationen, sondern auch Regierungsinstitutionen selbst als Effekt und nicht als Ursprung von Macht denkbar (ebd.). Die unterschiedlichen Formen von Machtbeziehungen und ,the ways in which these forms may actually be working simultaneously, in concert or at crosspurpose" werden untersuchbar (Abu-Lughod 1990, 48). Hegemoniale Positionen artikulieren sich dabei durch eine Vielzahl von partiellen Zwängen, Dispositionen, Dringlichkeiten und Möglichkeiten und sind zugleich anfällig für Widersprüche und Bruchstellen (vgl. Newman 2014, 135f.). Entsprechend lassen sich die vielen verschiedenen Stakeholder - die eigenen Mitglieder genauso wie die fördernden Verwaltungseinrichtungen, die lokale Bewegungsszene genauso wie der Verbandsfußball, internationale Frauenfußballinitiativen genauso wie nationale und internationale Regierungsinstitutionen - als Knotenpunkte machtvoller Relationen denken.

Dieses netzförmige Machtgefüge erscheint im Kontext des günstigen Moments als temporäre, emergente Assemblage, als Prozess, und nicht als festes, zeitloses System. Widerstand und Vereinnahmung sind demnach nicht länger sich gegenseitig ausschließende, sondern potentiell gleichzeitige Effekte, die sich entlang der verschiedenen Relationen situativ entfalten können. So werden sowohl dissidenter Protest, ziviler Ungehorsam, Petitionen und Direct Actions als auch Kooperationen mit regierenden Institutionen und die vielen Nuancen in Form von kreativer Aneignung, subtiler Umdeutung, vorsichtiger Distanzierung, taktischer oder affinitätsbasierter Partnerschaft als Artikulationen geschlechterpolitischer Problematisierungen fassbar, und ihre Gleichzeitigkeit sowie das Navigieren zwischen ihnen werden als elementarer Modus geschlechterpolitischen Handelns erkennbar. Das Adjektiv ,politisch“ wird so explizit nicht evaluierend und synonym für ,widerständig“ verwendet, sondern beschreibend für all jene Praktiken der Auseinanderset- 
zung über die Frage nach der Art und Weise des gesellschaftlichen Zusammenlebens. $\mathrm{Zu}$ fragen ist also nicht, $o b$ tatsächlich politisch gehandelt wird, sondern wie. Meine Aufmerksamkeit gilt dabei den vielfältigen Effekten in Form von Subjektivierungen, Bekräftigungen oder Umarbeitungen hegemonialer Formationen, den Ermöglichungen und Verunmöglichungen. ${ }^{133}$

Im Folgenden untersuche ich die geschlechterpolitischen Vorgehensweisen von DF. Ich überführe dabei Bezeichnungen aus dem Feld in analytische Perspektiven. In der Zukunftsdiskussion erwähnten die Mitglieder die Wichtigkeit, mit vielen Fußballerinnen gemeinsam die Stimme zu erheben und Forderungen zu stellen. Nadine betonte die Rolle, die die eigenen Erfahrungen dabei spielen sollten. Zugleich wurde der Wunsch, eine bessere Welt mitzugestalten und die eigene Vision selbst umzusetzen, in anderen Situationen auch als „Räume eröffnen“ bezeichnet und eng mit dem Anliegen, Sichtbarkeit zu schaffen, verknüpft. Eine Überführung dieser empirischen Begriffe in analytische Konzepte bedeutet, dass ich sie von ihrer Verwendung im Feld löse, mithilfe theoretischer Überlegungen neu beleuchte und schließlich in entgegengesetzter Richtung analyseleitend statt deskriptiv nutze.

Dabei kristallisieren sich zwei verschiedene Vorgehensweisen heraus, die diesen dritten Teil gliedern: Stimme erheben und Forderungen stellen einerseits und alternative Räume präfigurieren andererseits. Diese werden gegenwärtig wieder im feministische Sport mit der Frage „Nischen und Alternativen schaffen oder rein in die Institutionen?" diskutiert (Seitenwechsel e.V. 2018). Dabei unterscheiden sich die Vorgehensweisen wesentlich in ihrem Verhältnis zur politischen Öffentlichkeit, in ihrem Umgang mit bewegungsinternen Differenzen sowie ihren geschlechterpolitischen Effekten des Gesehen- beziehungsweisen Gehört-Werdens. Im 16. Kapitel untersuche ich zunächst das Stellen von Forderungen darauf hin, wie aus den vielen verschiedenen „gesammelten Beschwerden“ und unterschiedlichen „Erfahrungen“ eine gemeinsame „Stimme“ geformt wird. Im 17. Kapitel betrachte ich das Präfigurieren fußballerischer Alternativen durch Raumkonzepte und zeige, wie mittels Raumgestaltung ,eine bessere Welt“ im Sinne eines geschlechtergerechteren Fußballs hergestellt wird. Abschließend diskutiere ich, was die Gleichzeitigkeit dieser beiden Strategien, ihre unterschiedlichen Bedingungen und Effekte für die Konzeption politischen Handelns in spätkapitalistischen Demokratien bedeutet.

133 Dies entspricht einer kritischen Haltung im Sinne Foucaults, die nach Machteffekten und Kontingenzen fragt, anstatt normativ zu beurteilen (Foucault 1992; siehe Kap. 2.3.3). Ferguson (in Schouten 2009, o.S.) erinnert an das Interview, das Dreyfus und Rabinow mit Foucault führten, und paraphrasiert es zugespitzt, um die kritische Haltung der Ethnologie gegenüber Entwicklungsinstitutionen deutlich zu machen ,The interviewer said: 'You think everything is bad'. Foucault immediately responded by saying, 'No, No. I don't say that everything is bad. I say that everything is dangerous.' That's very different. Because when things are dangerous, we have to watch them closely. We have to attend to them. We have to see what are they doing. Where are they leading us astray? Where are the dangers?" 


\section{Forderungen stellen}

Im Dezember 2013 organisierte DF die viertägige DISCOVER FOOTBALL Conference 2013 on Women's Football in Central Asia and Europe und lud dafür Fußballerinnen, Trainerinnen, Funktionärinnen, Sportjournalistinnen und Menschenrechtsaktivistinnen aus den Regionen nach Berlin ein. 2012 hatte DF erstmals mit dem Expert Seminar on Women's Football in the MENA Region das Projektformat Expertinnenforum ausprobiert und dann beim Festival 2013 mit einer zweieinhalbtägigen Workshop-Reihe ein ähnliches Format durchgeführt. Diese Projekte entsprachen der zunehmenden Politisierung von DF und bewährten sich, da in ihnen konkret und intensiv an der Identifikation von Hindernissen und an Lösungsvorschlägen gearbeitet werden konnte. Saskia bemerkte in der diesen dritten Teil einleitenden Zukunftsdiskussion, es sei eine Hauptaufgabe von DF, „Frauen im Fußball eine Stimme zu geben“, und bezog sich damit auf DFs Bestreben, Frauen eine Plattform zu bieten, auf der ihre Erfahrungen öffentlich hörbar würden. In der Zukunftsdiskussion überlegte Linda, dass DFs Vorgehen so weiterentwickelt werden sollte, dass ,wir international mit einer Stimme sprechen und Forderungen stellen könnten“, und Simone schlug ein „Papier, das konkrete Ziele benennt", vor. In diesem Sinne sollte während des Expertinnenforums im Winter 2013 eine Deklaration formuliert werden. Gemeinsam mit den 23 Teilnehmerinnen unter anderem aus Spanien, der Türkei, Kirgisistan, Bulgarien, Aserbaidschan, Ungarn, Mazedonien und der Ukraine sollte während des viertägigen Projekts ein Forderungskatalog zur Verbesserung der Situation des Frauenfußballs in den betreffenden Regionen erarbeitet werden, der von allen unterschrieben und an die verantwortlichen Personen in den Fußballverbänden und der Sportpolitik übergeben werden sollte.

Das Erheben von Forderungen ist mit der Vorstellung von einer Öffentlichkeit verbunden, an die sich die transformativen und reformerischen Vorschläge richten. Die Adressierung der Verbände und Sportpolitik durch einen Forderungskatalog verstehe ich in Anlehnung an Michael Warner (2002) als diskursive und textuelle Praktik, die entsprechend einer Imagination von Öffentlichkeit diese aktiv herstellt. Öffentlichkeit ist daher kein präexistenter gesellschaftlicher Raum, sondern eine Vielzahl diskursiver Bereiche, die sich immer wieder selbst organisieren (ebd., 414f.). Bereits die Wahl des Vorgehens - Forderungen stellen - deutet die Idee und Herstellung einer spezifisch liberaldemokratischen öffentlichen Sphäre an, die nicht globale Gültigkeit besitzt (Butler 2013). Das Vorgehen gewinnt seine Plausibilität vor allem aus der Spezifik spätindustrieller, wohlfahrtsstaatlicher Demokratien Westeuropas, die auf der Vorstellung basieren, dass alle Menschen zwar Individuen, jedoch im Sinne eines universellen Humanismus gleich und gleichwertig sind. Jedem Individuum kann so die Freiheit zugestanden werden, sich selbst 
mit den Mitteln seines Verstandes eine Meinung zu bilden und diese ungehindert zu äußern, wobei zunächst jede Meinung den gleichen Wert hat (Hickel 2012, 2; Butler 2013). Jürgen Habermas hat sich dieser Öffentlichkeit ausführlich gewidmet und sie als Produkt der bürgerlichen Gesellschaft beschrieben (vgl. Habermas 1962; Kaschuba 1995). Gerade in der feministischen Kritik an der von Habermas präferierten Öffentlichkeit wird ersichtlich, dass Mitsprache- und Partizipationsmöglichkeiten nicht nur von der Klassen-, sondern auch Geschlechtszugehörigkeit abhängen. Aus historischer Perspektive wird gezeigt, dass Frauen durch informelle Mitsprachekriterien ausgeschlossen wurden: Bestimmte Belange wurden inhaltlich als privat und politisch nicht relevant klassifiziert und damit der öffentlichen Verhandlung und einer politisch-rechtlichen Bearbeitung entzogen; diese erforderten einen tugendhaften, rationalen und nicht emotionalen Sprachstil, wie er v.a. Frauen zugeschrieben wurde. So konstituierte sich die öffentliche Sphäre als männlich: Weiblichkeit und Öffentlichkeit erschienen als Widersprüche, und der Ausschluss von Frauen, aber auch von rassifizierten ,Anderen', aus dieser als legitim (Fraser 1997, 73):

„[D]iscursive interaction within the bourgeois public sphere was governed by protocols of style and decorum that were themselves correlates and markers of status inequality. These functioned informally to marginalize women, people of color, and members of the plebeian classes and to prevent them from participating as peers." (Ebd., 77)

Linda knüpfte in der Zukunftsdiskussion das Ziel, als Organisation ernstgenommen zu werden, an das Sprechen mit einer gemeinsamen und kohärenten Stimme, und Simone schlug vor, diese Stimme formal in ein „Papier“ und inhaltlich in konkrete Ziele zu übersetzen. Mit dem Genre der Deklaration und der Herstellung einer einheitlichen Stimme griff DF die modalen und formalen Kriterien dieser Öffentlichkeit auf. Während des Expertinnenforums sollte die Deklaration inhaltlich mit Forderungen gefüllt werden, die, um von der antizipierten Öffentlichkeit als verallgemeinerbare politische Interessen ernstgenommen zu werden, den Legitimitäts- und Relevanzkriterien würden entsprechen müssen.

Im Folgenden frage ich nach diesem Prozess der modalen, inhaltlichen und formalen Formierung einer gemeinsamen Stimme als spezifisches geschlechterpolitisches Vorgehen, das einer situierten Imagination von Öffentlichkeit folgt. Zwischen der von Saskia beschriebenen Vielzahl unterschiedlicher Stimmen und der von Linda vorgeschlagenen Einstimmigkeit liegt ein Prozess, an dessen Anfang die vielfältigen Erfahrungen von Frauen im Fußball im Rahmen von DF-Projekten artikuliert wurden (siehe Kap. 13.3.1) und der schließlich in einen Forderungskatalog mündete. Dies wirft die Frage auf, wie in einer internationalen Begegnung unter Frauen, die unterschiedliche soziale Positionen in ihren jeweiligen nationalen Kontexten bekleiden und in unterschiedlichen politischen Öffentlichkeiten agieren, kohärente und ratio- 
nale Einstimmigkeit hergestellt werden kann. Ich beginne mit der Frage nach dem Status von Emotionen (16.1) und der Rolle von Erfahrungen (16.2). Anschließend zeichne ich nach, wie von diesen ausgehend politische Problembestimmungen und verallgemeinerbare Interessen abstrahiert (16.3), in plausible sportpolitische Forderungen übersetzt (16.4) und in eine entsprechende inhaltliche wie ästhetische Form gegossen werden (16.5). Abschließend frage ich nach der Hörbarkeit dieser Stimme und ende mit Überlegungen zu möglichen und unmöglichen Wegen der Problematisierung (16.6). Dabei analysiere ich besonders, wie spezifische Vorstellungen politischer Öffentlichkeit in diesen Prozess Eingang fanden, während für andere kein Platz blieb.

\subsection{Emotional Stellung beziehen: „Figo, we will kill you“}

Für den ersten Tag des Expertinnenforums hat sich Laura ein besonderes Format ausgedacht: Zusammen mit den Teilnehmerinnen möchte sie eine Podiumsdiskussion nachspielen, in der alle in fremde Rollen schlüpfen. Sie selbst wird die Moderation übernehmen, sechs Teilnehmerinnen stellen die Podiumsgäste dar, von denen jede eine spezifische Haltung zum Frauenfußball verkörpert. Die restlichen Teilnehmerinnen bilden das Publikum. [...] Dann eröffnet die Moderatorin das Panel: „Pünktlich zur WM der Männer in Brasilien möchten wir heute über den Frauenfußball diskutieren. Eine feministische Gruppe hat kürzlich ,Claim the pitch!' ausgerufen und fordert nun, dass fortan mit einem pinken Ball gespielt werden solle. Wir haben hier unterschiedliche Perspektiven auf diese Forderung versammelt. Ist Fußball männlich und hat Fußball etwas mit Politik zu tun? Auf dem Podium begrüße ich Gregory Kraus, ehemaliger Profispieler und nun bei der FIFA verantwortlich für den Frauenfußball; Marta aus Brasilien und aktuelle Weltfußballerin; Hillary, eine konservative Politikerin aus den USA; Michael, ein Marketingmanager; der ehemalige Profifußballer Figo und Rosa Lichtenstein, eine der feministischen Aktivistinnen." Während sie Marta vorstellt, jubeln ein paar der Zuschauerinnen.

Gregory: „Als ich jung war, spielten nur Männer Fußball. Da war damals viel mehr Power und Leidenschaft dahinter. Meine Tochter spielt selbst Fußball, ich glaube da zwar nicht so dran, aber ich denke, dass wir das ändern müssen. Ich arbeite in der Entwicklungsabteilung und will den Frauenfußball fördern. Aber das mit dem pinken Ball, ich denke, das wäre zu hart. Wir sollten uns lieber nach weniger rigiden Wegen umschauen.“ Marta: „Früher gab es so viele soziale Stigmata. Ich unterstütze die feministische Kampagne vollends.“ Hillary: „Ich glaube daran, dass Gott Männer und Frauen als verschieden erschaffen hat. Frauen sollten eher zu Hause bleiben, kochen und gute Hausfrauen seien. Sie sollten nicht Fußball spielen, das ist was für Männer, denn die haben Muskeln und sind stark und schnell." Hillarys Bemerkung wird mit verächtlichen Geräuschen aus dem Publikum quittiert. Michael: „Mir persönlich hat Frauenfußball nie gefallen, meine Tochter soll auf keinen Fall damit ankommen. Aber dennoch sehe ich, dass Frauen im Fußball Karriere machen können. Meine Firma könnte sie sponsern, wir sollten in die Entwicklung von Fanshops investieren und auch sonst mehr Kapital in den Frauenfußball investieren.“ Figo: „Ich interessiere 
mich nicht wirklich für so Familiensachen, ich liebe den Fußball einfach zu sehr. Frauen gehören nicht ins Stadion, hier geht es doch nur um Politik und Geld, die haben doch eigentlich gar keine Ahnung vom Fußball." Aus dem Publikum kommen Buh-Rufe. Figo fährt fort: ,Wir sollten viel lieber mehr Pyrotechnik im Stadion haben als Frauen. Frauen wollen doch nur Geld abgreifen und es dann an andere Frauen weiterverteilen.“ Rosa wendet sich an Gregory von der FIFA: „Wir wollen den Ball nicht verändern, wir wollen, dass ihr die Farbe des Balls verändert. Die Qualität soll gleich bleiben. Ihr würdet dadurch ,Ja“ zum Frauenfußball sagen.“ Gregory: „Du bist zu spät dran. Außerdem ist der Ball ja bunt. Ihr hättet früher kommen müssen, der offizielle Ball hat schließlich eine Geschichte.“ Marta unterstützt Rosa: „Das Hauptproblem sind die gesellschaftlichen Einstellungen gegenüber Frauen. Niemand unterstützt diese Kampagne, obwohl alle sagen, sie unterstützten den Frauenfußball. Aber wenn wir sie dann ansprechen, sagen sie, sie hätten doch bereits genug Zugeständnisse gemacht. Die maskuline Kultur des Fußballs ist das Hauptproblem.“ Hillary: ,Jeder sollte die Möglichkeit haben, zu wählen und selbst zu entscheiden. Aber bei Fußball ist das was anderes. Da geht es um Kraft, Muskeln, Stärke. Frauen sind eher schüchtern, sie haben nicht so viele Muskeln, sie sind nicht so leistungsstark wie Männer. Und das geht letztlich auf Kosten des Ansehens und der Ehre unseres Landes." Auch Hillary wird jetzt ausgebuht. Die Diskussion geht noch ein paar Minuten in derselben Manier weiter. Immer wieder kommt es zu Zwischenrufen bei besonders provokanten Äußerungen, und Figo, aber auch Hillary und andere skeptische Kommentare gegen den Frauenfußball werden mit Rufen und Gesten disqualifiziert. Als nun alle um einen Schlusssatz gebeten werden, endet die Runde mit Figo: „Frauenfußball ist ein großer Fehler, ihr solltet euch vom Ball fernhalten.“ Eine aus dem Publikum ruft nun: „Figo, come over, we will kill you." Viele im Raum lachen. (Feldnotiz, 11.12.2013)

Freund und Feind waren eindeutig. Während klar Opposition gegen Figo und Hillary, gegen eine typische Macho-Haltung und eine konservative Rollenvorstellung bezogen wurde, waren Marta und Rosa Identifikationsfiguren. Zugleich verwies die Stille nach Michaels und Gregorys Äußerungen auf weniger eindeutige Stellungnahmen. Beide Charaktere förderten den Frauenfußball, jedoch nicht aus Gerechtigkeitsmotiven heraus. Michael trieb ein kommerzielles Interesse, Gregory bzw. die FIFA kamen dem öffentlich gespürten Druck, Frauenfußball nicht länger ignorieren zu können, durch finanzielle Unterstützung nach, verweigerten jedoch größere Konzessionen. Darin, dass sie weder einstimmig ausgebuht noch angefeuert wurden, zeigte sich die Unmöglichkeit, sie in einem Gut-Böse-Spektrum eindeutig einzuordnen. Die mobilisierten Emotionen bildeten eine moralische Skala von richtig bis falsch, die Platz für Ambivalenzen und Abwägungen ließ. So ermöglichte die gespielte Podiumsdiskussion, sich emotional, moralisch und körperlich mit den unterschiedlichen Haltungen zum Frauenfußball auseinanderzusetzen.

Das viertägige Expertinnenforum begann damit, Ängste, Wut oder Verletzungen in moralische Empörung zu transformieren sowie Sympathien für einen konkreten Lösungsweg - feministischen Aktivismus - und Skepsis und Ambivalenz gegenüber anderen Lösungswegen - Vermarktung und paternalistische, halbherzige Förderung - zu wecken. In den letzten zwei Jahrzehnten hat die sozialwissenschaftliche Forschung vermehrt auf die zentrale Rolle 
von Emotionen bei politischen Protesten und Mobilisierungen hingewiesen, galten Emotionen doch lange Jahre als Störfaktoren rationaler oppositioneller Politik, oder wurden dazu genutzt, soziale Bewegungen allgemein und Frauenbewegungen im Besonderen als nicht politikfähig zu disqualifizieren. ${ }^{134}$ Rebecca Allahyari weist darauf hin, dass Politik und Protest immer auch eine verkörperte Dimension besitzen. Moralische Haltungen können genauso wie Gefühle als verkörperte Gedanken verstanden werden, sodass Sachverhalte nicht nur kognitiv abgewogen und evaluiert, sondern auch emotional als richtig oder falsch gefühlt werden $(2001,196) .{ }^{135}$ Während des Expertinnenforums wurde über das Evozieren und Artikulieren von Emotionen moralisch Stellung bezogen, und die Teilnehmerinnen näherten sich einer gemeinsamen Positionierung an, die in der mehrheitlichen Abwertung von „Figos“ Meinung gipfelte.

Um die Zentralität moralischer Emotionen im Formierungsprozess einer rationalen und kohärenten Stimme zu verstehen, knüpfe ich an die Kritik der Geschlechterforschung an der dualistischen Gegenüberstellung von Emotionalität und Rationalität an. Feministische Geschlechterstudien zeigen, dass die konzeptuelle aufklärerische Gegenüberstellung von Emotion und Ratio und das daran anknüpfende Verständnis von Vernunft und Gerechtigkeit dazu führen, dass Frauen und rassifizierte ,Andere' häufig als unvernünftig kategorisiert und damit als weniger moralisch und nicht zu Urteilen fähig dargestellt werden (Ahmed 2004, 195). Zwar qualifiziert ein gewisser Grad an Emotionalität als Beleg für das eigene Involviert-Sein durchaus für politische Ämter, doch darf dieser nicht überschritten werden, da Emotionalität als nicht beherrschbares und handhabbares Risiko in politischen Prozessen gilt. Die dualistische Denkfigur delegitimierte Frauen und andere soziale Gruppen damit als zu emotional, um als Fürsprecher_innen für öffentliche Angelegenheiten fungieren zu können, und stabilisierte ihren Ausschluss von politischer Herrschaft (Binder 2009, 164). Der Beobachtung, dass Wahlkampfveranstaltungen und Parteitage kaum weniger emotional sind, zeigt, dass Emotionspraktiken deutlich vergeschlechtlicht sind. Ob sie als zu emotional oder als angemessen engagiert-involvierte Untermalung des Rationalen gelesen wer-

134 Goodwin et al. $(2001,13)$ bezeichnen dies als ,misleading opposition between emotions and rationality", denn auch die professionalisiertesten und bürokratischsten Oppositionsbewegungen haben emotionale Dimensionen, nur eben andere als die expressiven Protestformen. Die Opposition prägt auch die Forschung: Emotionen fanden spät Eingang in die Mobilisierungstheorien; in diesen dominierten der Ressourcen-Mobilisierungs-Ansatz, die diskursorientierte Frame-Analyse sowie der Political Opportunity-Ansatz. Siehe zum emotional turn in der Bewegungsforschung zudem Flam/King (2006), Jasper (2011).

135 Jasper (2011) unterscheidet hierfür moralische Emotionen von anderen Emotionstypen in sozialen Bewegungen. Moralische Emotionen umfassen jene zustimmenden oder missbilligenden Gefühle, die aus moralischen Prinzipien resultieren, bspw. Scham, Schuld, Stolz oder Wut. 
den, ist damit maßgeblich vom ausagierenden Körper abhängig. ${ }^{136}$ Obwohl das Expertinnenforum von Beginn an darauf ausgerichtet war, den Mitsprachekriterien dieser bügerlich-männlichen Öffentlichkeit zu entsprechen, wurden Emotionen nicht als irrationale Ablenkungen ausgeklammert, sondern gezielt evoziert. Sie halfen, einen moralischen Deutungsrahmen der Ereignisse um Fußball und Geschlecht abzustecken. Denn für Aktivist_innen besteht die Herausforderung, die vielen moralischen, kognitiven und emotionalen Haltungen innerhalb sozialer Bewegungen zusammenzufügen (Goodwin et al. 2001, 16). In Spaß und Spiel erfuhren sich die Teilnehmenden als Gruppe mit ähnlicher moralischer Haltung und Situationsdeutung. In geteilter Zustimmung, Ambivalenz, Empörung und Ablehnung erlebten sie sich als „epistemic community“ in Alison Assiters Sinne: als eine Gemeinschaft, die bestimmte politische Wissensbestände oder Werte teilt (2000; vgl. Stoetzler/Yuval-Davis 2002, 320). Emotionen erscheinen so zunächst nicht als irrationale Störfaktoren auf dem Weg zur Formulierung vernünftiger Forderungen, sondern als Wegbereiterinnen einer rationalen Einstimmigkeit.

Die Abwendung von einem dualistischen Denken legt es zudem nahe, auch die modale Formierung einer Stimme graduell zu denken. Dafür knüpfe ich an Albert Hirschmans Beobachtungen dazu an, wie Mitarbeitende von Unternehmen ihr Missfallen artikulieren. Voice, also das Erheben einer Stimme, bewegt sich dabei zwischen schwachem Flüstern oder Grummeln und lautstarkem Protest (1970,16). Mandy de Wilde (2015) zeigt anhand eines niederländischen Stadtteilentwicklungsprojekts, dass diese Grade vergeschlechtlicht und sozialstrukturell ausdifferenziert sind: Während lokale (post-)migrantische Frauen flüstern (whisper), erheben autochthone Männer ihre Stimmen zum lauten und trotzigen Protest (raising voice). ForderungenStellen und Stimme-Erheben verstehe ich daher als einen graduellen Prozess, in dem heterogene, emotionale und ,leise' Stimmen verschiedene modale, inhaltliche wie formale „Qualifizierungsstufen“ (Scheffer 2013, 95) hin zu einer kohärenten, rationalen, plausiblen und entsprechend ,lauten' Stimme durchlaufen. Dieser Qualifizierungsprozess beinhaltete folglich auch die Etablierung und Konkretisierung des epistemischen Rahmens, der sich zu Beginn aus eher vagen moralisch-emotionalen Haltungen zusammensetzte und im nächsten Schritt durch den Austausch von Erfahrungen weiter spezifiziert wurde.

136 Interessant ist die Parallele zum Fußball: Auch für diesen galten/gelten Frauen als zu emotional. Ein Blick in den Männerfußball mit samt dem Jubel, Ärger, der Aggressivität und Verzweiflung zeigt diesen jedoch als hoch emotionale Tätigkeit. 


\subsection{Erfahrungen mobilisieren: „What discouraged you?“}

„Main goals of the conference are to exchange our experiences with and learn about how to strengthen and empower girls and women in and through football and to develop strategies for

Asia and Europe."

In diesem Ausschnitt aus der Ausschreibung für das Expertinnenforum 2013 stellten die Organisator innen den Austausch persönlicher Erfahrungen als Schritt hin zur Entwicklung gemeinsamer und allgemein gültiger geschlechter- und sportpolitischer Forderungen dar. Diese Selbstverständlichkeit, auf persönlichen Erfahrungen aufzubauen, drückte sich auch in der Programmgestaltung aus. Die Podiumsdiskussion hatte nicht nur verschiedene geschlechter- und sportpolitische Einstellungen körperlich erfahrbar gemacht und die Teilnehmenden als Gruppe emotional zum Konflikt positioniert, sondern auch Erfahrungen aktualisiert. Die meisten der Anwesenden wurden durch die verschiedenen Aussagen - seien es „Figos“ abfällige Bemerkungen, „Hillarys“ Sittlichkeitsargumente, „Michaels“ desinteressierter Paternalismus oder „Martas“ Empörung - an eigene Erlebnisse erinnert.

Wie beim Festival wurden die Teilnehmerinnen aufgefordert, ihren fußballbezogenen Lebensweg mithilfe eines river of life vorzustellen. Melanie und Luca, die diese Workshop-Einheit leiteten, baten anschließend, er- und entmutigende Erfahrungen zu diskutieren. Eine Profispielerin berichtete davon, dass ein ziemlich gemeiner Trainer immer starken Leistungsdruck auf sie ausgeübt habe, nie sei sie gut genug gewesen. Von mehreren wurde Korruption als Demotivation und Hemmnis für die Entwicklung des Frauenfußballs genannt. Als Melanie schließlich nach den ermutigenden Erfahrungen fragte, erwähnten einige die Rolle positiver Vorbilder. Taliko, eine Menschenrechtsaktivistin aus dem Südkaukasus, erzählte, wie ermutigend es sei zu merken, dass sie in etwas gut sei und sich darin selbstverwirklichen könne. Soleiha aus Südasien ergänzte, dass die erfahrenen Diskriminierungen und Drohungen sie motivierten. Sie wolle sich mit Männern messen und zeigen, dass Frauen dasselbe könnten. Die einzelnen Erfahrungen wurden auf Karteikarten festgehalten und an eine Pinnwand geheftet.

Mit Methoden wie river of life ritualisierte DF den Erfahrungsaustausch als elementaren Bestandteil der Projektarbeit und realisierte damit Nadines präferiertes Vorgehen, die ,eigenen Erfahrungen hervorzuheben“. In den Kapiteln 11.4, 11.5 und 13.3.1 habe ich die abendländische Tradition des Erfahrungskonzepts beschrieben und gezeigt, wie Erfahrung und nicht etwa ein politisches Ziel oder Ideologie als Grundlage von Vergemeinschaftung und Gemeinsamkeit herangezogen wird. Hier wird nun ersichtlich, wie Erfahrung auch als politisch-strategisches Instrument und als Ausgangspunkt für politische Forderungen mobilisiert wird. Für mich stellten sich die Fragen, 
wie aus Erfahrung politische Strategien gewonnen werden und welche Umstände dies ermöglichen. Kurz: Wie wird das Private politisch?

\subsubsection{Erfahrung als Evidenz: Das Private wird politisch}

Die Mobilisierung von Erfahrung zu politischen Zwecken basiert auf einer langen Tradition der Authentisierung von persönlich Erlebtem. Insbesondere die zweite Frauenbewegung reagierte auf den herrschenden Empirismus, dem zufolge wissenschaftliches Wissen oder öffentlich-politische Problematisierungen unabhängig von den Erfahrungen und sozialen Positionen des erkennenden Subjekts produziert werden, und institutionalisierte nach und nach den Status von Erfahrung in der Produktion von Wissen (Phipps 2016, 304f.). Diese feministische, erfahrungsbezogene Politik reiht sich in ein westlichabendländisches Subjektverständnis ein: Das Konzept Erfahrung setzt die Existenz eines handlungsfähigen Individuums voraus, denn schließlich werden Erfahrungen erlebt, gemacht, gesammelt oder geteilt und es wird aus ihnen gelernt (Scott 1991, 782). Die Idee eines Individuums ist jedoch selbst Produkt einer spezifisch westlichen Vorstellung, geformt durch humanistische, aufklärerische und romantische Ideen von Identität und Selbst, wie sie von der Menschenrechtsaktivistin Taliko in ihrem Streben nach Selbstverwirklichung artikuliert wurden. Erst die Vorstellung von einem Subjekt, das durch Fühlen, Reflektieren, Erleben und Erleiden die äußere materielle Welt mit seiner inneren verknüpft, lässt die Argumentation entstehen, Erfahrung sei eine gültige und authentische Quelle für Wissen (Desjarlais 1997, 13).

Dieses abendländische Subjektverständnis trifft auf liberale, pluraldemokratische und rechtsstaatliche Vorstellungen von gleichen Rechten für alle sowie Schutz von Minderheiten vor Diskriminierungen (Hickel 2012, 2). Einerseits werden Erfahrungen zur legitimen Grundlage von und Begründung für politische Forderungen nach Gleichberechtigung. So konnte z.B. die zweite Frauenbewegung unter dem Slogan „Das Private ist politisch“ auf die Belange und Lebenssituationen von Frauen aufmerksam und diese zu öffentlichen Angelegenheiten machen. Andererseits spielt die Verknüpfung von Erfahrung und Liberalismus einer „,neoliberal commodification of firstperson narratives“ (Phipps 2016, 303) in die Hände. Neoliberalismus meint dabei nicht nur die ökonomischen Prinzipien des freien Marktes, des Abbaus staatlicher sozialer Sicherungssysteme und der Privatisierung, sondern ein ganzes Handlungs- und Denksystem, das umfassende soziale Probleme durch individualistische, eigenständige, anpassungsfähige und ökonomisch effiziente Subjekte lösen will (McDonald 2015, 911; Rottenberg 2014). Er verlangt nach Individuen, ,constructed around the principles of agency and selfgovernance“, strebend nach Selbstverwirklichung (Phipps 2016, 308). Wie David Harvey (2005) zeigt, wurde der liberale Wunsch nach individueller 
Freiheit, den viele soziale Bewegungen artikulieren, von neoliberalen Ideologien zum Zwecke radikaler Marktliberalisierung vereinnahmt. So teilen Neoliberalismus und Liberalfeminismus die Idee vom reflektiven, flexiblen, informierten und empowerten Subjekt, fokussieren sogenannte soft management skills, personenzentrierte Formen von Führung und die Investition in individuelle Bildung, Ausbildung und Persönlichkeitsentwicklung (Newman 2013, 213; vgl. Petchesky 2003, 24). Mit Blick auf das Expertinnenforum lässt sich daraus schlussfolgern, dass sich der Rückgriff der Aktivist_innen auf eine erfahrungsbasierte geschlechterpolitische Strategie, um häufig delegitimierten und überhörten Stimmen Gehör zu verschaffen, nicht außerhalb oder entgegen machtvoller Formationen vollzog, sondern durch sie ermöglicht wurde, schließlich ko-produzierte die Institutionalisierung des Liberalfeminismus jenes Moment und verschaffte Erfahrungspolitik Legitimität.

Die Artikulation von Erfahrungen bildet nach der emotional-moralischen Positionierung eine weitere Qualifizierungsstufe auf dem Weg hin zu einer gemeinsamen Stimme. Erfahrung, so führt Robert Desjarlais in Anlehnung an Martin Heidegger aus, bezeichnet ein transformatives, Spuren hinterlassendes Ereignis und unterscheidet sich so von trivialen, flüchtigen Geschehnissen (1997, 16). Was als Erfahrung, also als einschneidendes Erlebnis galt und ausgetauscht werden sollte, wurde durch den diskursiven und situativen Kontext vorstrukturiert: Die Ankündigung des Expertinnenforums mit den Wörtern „Empowerment", „Mädchen und Frauen“, „Fußball“, „Diskriminierung“ und ,inklusiv“" steckte im Zusammenspiel mit den Fragen nach ermutigenden und entmutigenden Erlebnissen den Deutungsrahmen dafür ab, was als Erfahrung galt. Durch den angeleiteten Erfahrungsaustausch konkretisierte sich die emotional und moralisch erfahrene epistemische Gemeinschaft inhaltlich. Gleichzeitig zeichnete sich sowohl in der Wahl des Vorgehens als auch in der Absteckung des Deutungsrahmens bereits die machtvolle Strukturierung dieses Formierungsprozesses ab, die Fragen an den Qualifizierungsprozess richtet: Mit dem Rückgriff auf Erfahrungspolitik knüpften die Organisator innen unbeabsichtigt erstens an eine spezifisch liberalfeministische Tradition demokratischer, westlicher Öffentlichkeit an, sodass sich hier die Frage nach der Verallgemeinerung dieses Vorgehens in einem translokalen Kontext stellt. Zweitens wirft Desjarlais' Unterscheidung zwischen trivialem Erlebnis und relevanter Erfahrung die Frage auf, wer in der Lage ist, den entsprechenden Deutungsrahmen zu setzen und damit zu entscheiden, was als politisch mobilisierbare Erfahrung gilt und was nicht. 


\subsection{Privates politisieren: „That is discrimination“"}

Nachdem die Teilnehmerinnen ihre Erlebnisse artikuliert hatten, wurden diese auf Karteikarten notiert und zu konkreten politischen Problembestimmungen abstrahiert. Die Politisierung des Privaten folgte dabei der Verwendungslogik von Karteikarten, die zu Verkürzungen des Erzählten auffordert: Ganze Sätze oder gar Erzählungen werden zu Stichpunkten oder Phrasen verknappt, Personalpronomen sowie narrativer Kontext entfallen und mit ihnen die Personen- und Situationsgebundenheit des Stichpunkts. Karteikarten mit der Aufschrift ,prejudice of society, family“ oder ,lack of support of family and friends" transformierten persönliche, alltägliche Erfahrungen in gesellschaftspolitisch relevante Angelegenheiten und machten Marginalisierungen und Benachteiligungen sichtbar. Dabei wurden die Erfahrungen jedoch ihrer historischen, biografischen und lokalen Kontexte entledigt (vgl. Scott 1991). Nach einer Weile hing eine kleine Wolke aus Karteikarten an einer Stellwand und ordnete „corruption inside the association“, „lack of money“, „lack of competition, games, clubs“, „lack of support of society, family“, ,prejudice of society, family“ und weitere Punkte einander zu.

Erzählen, zu Stichworten Verkürzen, Aufschreiben, Anheften und Anordnen stellen sich als konkretes Tun aller Beteiligten dar. Durch das gemeinsame Auswählen und Abwägen von Begriffen und das Aufschreiben falteten sich verschiedene Erfahrungen in die Karteikarten und verallgemeinerbaren Schlagworte ein und waren in den entsprechenden Problemdefinitionen weiterhin enthalten. Durch das Anordnen der Karteikarten rückten differente, situierte und zeitlich versetzte Erfahrungen zusammen. Auf diese Weise wirkten die Narration von Erfahrungen, das Aufschreiben der zentralen Schlagworte und ihr Arrangieren sowie die Materialität der Karteikarten zusammen und schlossen einzelne Erlebnisse in abstraktere Problembestimmungen ein. Indem die Erfahrungen dekontextualisiert zu einer Wolke aus verallgemeinerbaren Problemen arrangiert wurden, erschienen sie nicht länger als partikular, sondern als gemeinsam und geteilt und als auf eine bestimmte Art miteinander verbunden.

Die postkoloniale feministische Kritik an translokalen feministischen Bündnissen diskutiert ausführlich, was ich bereits in Bezug auf die Setzung des epistemischen Rahmens als machtvollen Prozess andeutete (siehe Kap. 13.1): Im Zuge der erfahrungspolitischen Entlokalisierung, Dehistorisierung und Neuanordnung von Erfahrungen werden Differenzen unter Frauen unsichtbar gemacht und marginalisierte Erfahrungen zugunsten einer einheitlichen Deutung vereinnahmt oder ausgeklammert (vgl. Phipps 2016). Diese Spannung zwischen Vereinheitlichung und Ausklammerung zwecks Herstellung einer kohärenten Stimme einerseits und Heterogenität der Erfahrungen 
andererseits lässt mich im Folgenden nach den Selbstverständlichkeiten und Machtverhältnissen in dieser internationale Begegnung fragen.

\subsubsection{Die Unmöglichkeit differenzfeministischer Problematisierung}

Nicht alle Erfahrungen ließen sich zu Problembestimmungen abstrahieren. Manche schafften es nicht auf eine Karteikarte, weil sie in der Gruppendiskussion nicht weiter verfolgt wurden und keine die Sprecherin ermutigte, sie zu notieren. Andere blieben als Karte an der Stellwand hängen und wurden im weiteren Verlauf nicht wieder aufgegriffen. Das Einfalten von Erfahrungen in die Begriffe bedeutet auch, zwischen Erfahrungen auszuwählen und einige irrelevant werden zu lassen. Politisieren wird dabei als machtvoll strukturierter Prozess erkennbar und lässt mich wieder fragen: Welche Erfahrungen werden mobilisiert und eingefaltet und vom wem? Und welcher Logik folgt diese Politisierung?

In einem anderen Workshop arbeiteten Kleingruppen zu konkreten Begriffen. Ich nahm am Tisch zum Thema Stereotype Platz. Nachdem Pavlinka, eine Sportjournalistin aus der Balkanregion, in einem kurzen Input erklärt hatte, wie die Vorurteile über Frauen im Fußball das Bild des Frauenfußballs formen und seine Entwicklung behindern, begannen wir zu diskutieren, welche Stereotype uns im Fußball begegnen. Nora, eine ehemalige Profispielerin aus Mitteleuropa und nun für verschiedene Verbände international als Mädchen-Trainerin tätig, berichtete, wie sehr sie das Stereotyp des Biertrinkens beim Fußball nerve. Es stoße immer wieder Eltern ab, wenn diese sähen, wie am Platz gesoffen werde. (Feldnotiz, 11.12.2013)

Nora sprach an, wie sehr die Inszenierung des Fußballs als archaischer Männerkult abschreckend auf Mädchen und Eltern wirkt. Im Fortlauf unseres Gesprächs fand Noras Bemerkung jedoch keine weitere Beachtung, es kreiste fortan nur noch um das Stereotyp von Fußballerinnen als ,Mannweiber'. Noras Beschwerde ebenso wie das geäußerte Leiden unter dem hohen Leistungsdruck und dem gemeinen Trainer oder die Beschwerden über ständige Verletzungen und Schmerzen treffen den Kern patriarchaler fußballerischer Kultur, denn sie problematisieren Leistungsprimat und Männlichkeitskult und halten ihnen als Frauen andere Bedürfnisse entgegen. Im Gegensatz zu den Erzählungen von Benachteiligungen und rigiden Geschlechterrollen schafften es diese Erzählungen nicht auf Karteikarten oder wurden nicht wieder aufgegriffen. Hinter diesem Auswahlprozess verbarg sich eine gleichheitsfeministische Selbstverständlichkeit, die Soleiha ausgesprochen hatte: Frauen sind zur selben fußballerischen Leistung fähig, wenn man sie nur lässt. Zwar wurden in internen Gesprächen bei DF immer wieder spezifische Bedürfnisse von Frauen und ein Missfallen an der Fußballkultur geäußert, dabei jedoch darauf geachtet, diese nicht zu verallgemeinern und schon gar nicht mit einer vermeintlichen weiblichen Natur zu begründen. Öffentlich vermied DF jegliche differenzfeministische Argumentation und Forderung, ohne dass es eine 
explizite Absprache gegeben hätte. ${ }^{137}$ Diese Haltung schien auch von vielen, aber nicht allen Teilnehmerinnen geteilt zu werden. Als die Arbeitsgruppe Noras Klage über den bierseligen Männerkult nicht weiter verfolgte, einigte sie sich stillschweigend darauf, die Gefahr zu vermeiden, biologistischen und essentialistischen Argumenten - die in Vergangenheit und Gegenwart stets den Ausschluss von Frauen aus dem Fußball rechtfertigten - ungewollt in die Hände zu spielen. Differenzfeministische Argumente drohten, durch das wirkmächtige ,controlling image“ (Hill Collins 1990, 69) des (zu) schwachen Geschlechts gedeutet zu werden und so das Gegenteil dessen zu bewirken, was die Gruppe eigentlich zum Ziel hatte. Jene machtvollen Diskurse und Praktiken, die über körperliche Leistung die Höherwertigkeit des Männlichen und die Unvereinbarkeit von Weiblichkeit und Fußball festigen und im Zentrum der geschlechterpolitischen Problematisierungen stehen, bilden die Bedingungen dessen, was als plausible Problemdefinition möglich wird: In einem Feld wie dem Verbandsfußball scheint dadurch, dass Geschlechterverhältnisse über Geschlechterdifferenzen legitimiert werden, eine gleichheitsfeministische Mobilisierung erfolgversprechender. Nicht in diesem Sinne politisierbare Erlebnisse werden ausgeklammert. Hier zeichnet sich bereits ab, was ich im Laufe diesen Kapitels weiter ausführen werde: Die heterogenen Erlebnisse der Teilnehmerinnen trafen auf das von Geschlechterpolitiken und Diskursen über Geschlecht und Sport vorstrukturierte Moment, das eingrenzt, welche Erfahrungen politisiert werden können und somit relevant und vernünftig sind, und welche als illegitim, unvernünftig oder schlicht nicht erfolgversprechend gelten und damit im Status des trivialen Erlebnis verweilen (vgl. Binder 2009, 165).

\subsubsection{Die Selbstverständlichkeit liberaldemokratischer Rechtsstaatlichkeit}

Der Rahmen plausibler Problematisierung wurde nicht nur durch die fußballerischen Geschlechterdiskurse selbst abgesteckt. Auch dominante Vorstellungen von politischer Öffentlichkeit und Rechtsstaatlichkeit entschieden mit darüber, welche Erfahrungen politisiert, trivialisiert oder zum Schweigen gebracht wurden. Am Beispiel des Diskriminierungsbegriffs wird zunächst ersichtlich, wie national-rechtliche Kontexte unterschiedliche Erfahrungen hervorbrachten:

137 Die gleichheitsfeministische Argumentation wurde besonders in einem Video-Trailer vom Festival 2012 deutlich, der mit der Aussage einer Teilnehmerin begann: „What men can do, women can do, too". Dadurch, dass die Stimme aus dem Off kam, wurde die Aussage paradigmatisch für die gesamte Veranstaltung und das zugrunde liegende Verständnis von der Durchlässigkeit von Geschlecht. Geschlechterunterschiede galten als allein sozial konstruiert, und die Gruppe begegnete biologistischen Argumenten, bspw. dass Frauen aufgrund ihrer Physis weniger schnell sprinten könnten, mit Ablehnung und Skepsis. 
Am Ende des ersten Tages kommt die Organisationsgruppe zusammen und bespricht den Tag. Simone moderiert: „Wie sieht es mit Blick auf die Deklaration aus, welche Probleme könnte es geben?“" Inka erzählt: „Ein Problem könnte von Wiktoria und Jekaterina kommen. Sie haben gesagt, in ihrem Land gäbe es keine Diskriminierung, keinen Rassismus und schon gar keine Homophobie. Es gibt ein Missverständnis darüber, was Diskriminierung ist. Sie sagen, Homosexualität sei in ihrem Land illegal. Da es keine Homosexuellen gäbe, gäbe es auch keine Diskriminierung. Sie würden bestimmt keine Deklaration unterschreiben, wo was mit Homophobie drin steht.“ Luisa ergänzt: „Die zwei Frauen vom [zentralasiatischer Binnenstaat] Verband sagen auch, bei ihnen gibt es keine Diskriminierung.“ Martina: „Soheila hat dem widersprochen und das in Frage gestellt.“ Inka: „Wir sollten diese Sichtweise [in den Workshops] auch hinterfragen, es geht hier ja schließlich auch darum, was zu lernen." [...] Luisa kommt später noch einmal auf eine der zentralasiatischen Funktionärinnen zu sprechen: „Nasgül war nicht einverstanden, das, was sie im Verband und ihrem Land erlebt, als Diskriminierung zu bezeichnen. Es bedeutet für sie, dass es dann eine Aggression gegen sie persönlich darstellt. Sie versteht nicht, dass Diskriminierung was Strukturelles meint. Und wenn sie es Diskriminierung nennt, werden Männer ihr gegenüber aggressiv.“ Martina ergänzt: „Aber sie hat verstanden, warum alle anderen es Diskriminierung nennen. Nur für sie ist es nicht effektiv, den Begriff zu benutzen. Ich bin sicher, dass sie sehr viel Diskriminierung erfährt.“ (Feldnotiz, 11.12.2013) ${ }^{138}$

Die Verbandsfunktionärinnen Wiktoria und Jekaterina hielten zunächst an der offiziellen Rhetorik ihres Verbands und der Öffentlichkeit ihres osteuropäischen Landes fest, die eine Politisierung von Marginalisierungserfahrungen aufgrund sexueller Orientierung mithilfe des Diskriminierungsbegriffs verunmöglichten. Nasgül, Juristin und eines der wenigen weiblichen Mitglieder im Exekutivkomitee ihres Landesverbands, verzichtete aus strategischen Gründen auf diesen politisch-rechtlichen Terminus zur Problembenennung, da er in ihrem Arbeitskontext nur noch mehr Aggression ihr gegenüber hervorrief und damit Möglichkeiten verschloss, statt zu öffnen. Ob Erlebnisse zu prägenden Diskriminierungserfahrungen oder aber zum trivialen Alltagsgeschehen wurden, schien maßgeblich von der unterschiedlichen rechtlichen Mobilisierbarkeit des Diskriminierungsbegriffs in den verschiedenen Ländern abzuhängen. Die Selbstbezeichnung als Diskriminierte ist nur in jenen liberaldemokratischen Kontexten sinnvoll, in denen Antidiskriminierungsnormen

138 Da DF eine Partnerin aus Palästina als Unterstützung und Workshop-Leitung eingeladen hatte, nahm diese auch an den Besprechungen des Organisationsteams teil. Daher fanden dieses wie auch viele weitere zitierte Gespräche auf Englisch statt und wurden von mir hier übersetzt. Während der gesamten Konferenz gab es eine Flüsterübersetzung für eine türkische Teilnehmerin sowie Simultandolmetschung für die russischen und ukrainischen Teilnehmerinnen. Immer wieder halfen sich Teilnehmende gegenseitig, wenn etwas nicht verstanden wurde oder ausgedrückt werden konnte. Interessant ist, dass ich die Einwände selbst nicht hörte, sie erreichten mich nur durch die Erzählung anderer DF-Mitgliedern, die wiederum nur mithilfe der zwei professionellen Dolmetscherinnen davon erfuhren. Dass die drei ihre Meinung anscheinend schnell änderten, wie im Verlauf des Kapitels gezeigt wird, kann auch darauf hindeuten, dass hier vielleicht ein Missverständnis vorlag bzw. ihre Äußerungen in einem Rahmen aus Vorwissen und Vorurteilen über Homosexualität und Homophobie in Osteuropa gedeutet wurden. 
existieren und rechtsstaatlich angewendet werden. Festzuhalten ist zunächst, dass verschiedene staatlich-rechtliche Rahmenbedingungen, aber sicherlich auch unterschiedliche kulturelle, soziale und ökonomische Bedingungen unterschiedliche Erfahrungen von Frauen im Fußball produzieren (vgl. Scott 1991, 783). Zusammen mit dominanten Diskursen um Fußball und Geschlecht entscheiden sie mit darüber, welche Erfahrungen politisiert werden können.

Doch wie wurde in dieser internationalen Begegnung, die dem Ziel verschrieben war, eine einheitliche Stimme zu schaffen, mit jenen Erfahrungen umgegangen, die sich zunächst einer Vereinheitlichung widersetzten? An Inkas Einwand, dass es auch darum gehe, etwas zu lernen, sowie an Luisas Erklärungsversuch, dass Nasgül den Diskriminierungsbegriff nicht verstehe, zeigt sich DFs Umgang mit den lokal produzierten Differenzen. Nasgüls, Wiktorias und Jekaterinas Deutungen ihrer Erfahrungen wurde von den DFMitgliedern als Resultat von Unwissen betrachtet. In Kapitel 11.1 habe ich ausführlich das bei DF gültige Wissen beschrieben und in Kapitel 13.3.1 gezeigt, wie unterschiedliche Erfahrungen mithilfe feministischer Theorien als Produkte universeller Ungleichheitsstrukturen verstanden und zu politischen Problemen verallgemeinert wurden. Luisas Bemerkung impliziert die Selbstverständlichkeit dieser feministischen Deutung und überführt sie sogleich in politisch-rechtliche Begrifflichkeiten. Diese Verknüpfung von persönlicher Erfahrung, feministischer Theorie und rechtlich-politischer Begrifflichkeit erschien den Organisator_innen vor dem Hintergrund gültiger und angewandter Antidiskriminierungsgesetze und institutionalisierter Gleichstellungspolitik in Deutschland plausibel und erfolgversprechend. Ich habe bereits auf die Situiertheit von Erfahrungspolitik in westlichen, liberalen Demokratien hingewiesen. Nun wird deutlich, dass die Mobilisierbarkeit von Erfahrung mithilfe konkreter politischer Begriffe eng an die entsprechenden rechtsstaatlichen Bedingungen geknüpft ist. Liberaldemokratische Rechtsstaatlichkeit hatte sich als unsichtbare Selbstverständlichkeit in das Vorgehen der Organisator_innen eingeschrieben, sodass sie die Erfahrungen von Wiktoria, Jekaterina und Nasgül als unplausibel zurückwiesen. ${ }^{139}$

In der Formierung einer rationalen Stimme wurden Differenzen zugunsten einer kohärenten Erfahrung von Frau-im-Fußball-Sein und einem kollektiven Wir ausgeklammert. Phipps weist mit der provokanten Frage „whose personal is more political?" darauf hin, dass auch innerhalb feministischer Öffentlichkeiten politische Kämpfe darum ausgetragen werden, wessen Erfahrungen als , authentischer' und ,richtiger' gelten. Ausklammerungen wer-

139 Scott (1991) bemerkt, dass die politische Mobilisierung von Erfahrungen häufig dazu führt, dass sie sich von ihrer ursprünglichen Intention, Diversität sichtbar zu machen und damit das Primat einer einzigen Wahrheit zu brechen, entfernt. Stattdessen funktioniert Erfahrungspolitik heute häufig nach dem gleichen Muster wie Evidenzpolitik, indem sie eine ,authentischere" Wahrheit proklamiert. 
den, so Phipps, aufgrund des internen Machtungleichgewichts möglich, das sich während des Expertinnenforums durch die Rollen als Organisierende und Gastgeber_innen einerseits und eingeladene Gäste andererseits ausdrückte und Ersteren durch Themensetzung und Programmgestaltung deutlich mehr Einflussmöglichkeiten einräumten $(2016,313)$.

Auch diesem DF-Projekt war ein Bewerbungs- und Auswahlprozess vorangegangen (siehe Kap. 13.1.2), der eine gewisse Ähnlichkeit in Bezug auf politisch-feministisches Vokabular unter den Anreisenden und Organisierenden sicherstellte. Während der Veranstaltung festigte sich nun eine epistemische Koalition aus Organisierenden und dem Großteil der Teilnehmerinnen, die die Idee liberaldemokratische Rechtsstaatlichkeit teilte. Dieser Koalition standen Frauen aus jenen Ländern gegenüber, in denen die liberale Ideologie nicht institutionalisiert war und keine argumentative Legitimität besaß. Dieses Ungleichgewicht unter den Anwesenden wurde besonders sichtbar, als die Erzählungen politisiert wurden und damit in den ,,marketplace of experience“ (Phipps 2016, 308) eintraten: jene Öffentlichkeit, in der Erfahrung als Kapital funktioniert und politischen Nutzen verspricht, wenn sie in einer ganz bestimmten Währung eingebracht wird (ebd., 313). Mit diesen internen Deutungskämpfen konkretisierte sich der epistemische Rahmen und die Zugehörigkeit zur erfahrenen Gemeinschaft wurde exklusiver. ${ }^{140}$

\subsubsection{Vereinnahmungen widerstehen}

Während Nasgül später keine weiteren Bedenken mehr bezüglich des Diskriminierungsbegriffs äußerte, war bis zum Ende des Expertinnenforums unklar, ob Wiktoria und Jekaterina für oder gegen den Begriff in der Deklaration stimmen würden. Konkret drehten sich die Befürchtungen um die Diskriminierungskategorie der sexuellen Orientierung. Am letzten Tag des Expertinnenforums wurde jeder Punkt im Detail besprochen und ein Konsens gesucht, sodass das Dokument am Abend unterzeichnet werden konnte:

Lieke von einem europäischen lesbisch-schwulen Sportverband bemerkt, dass von UEFA und AFC gefordert werden solle, sexuelle Orientierung als Diskriminierungskategorie explizit zu nennen. Später schlägt Thaliko vor, dass Geschlecht und sexuelle Orientierung bereits in dem Teil der Deklaration, in dem die Teilnehmenden ihr En-

140 Dass eine solche Exklusivität von DF selbst kritisch beobchtet wurde, zeichnet sich in der Zukunftsdiskussion ab. Linda lehnte es ab, eine „Weltstrategie“ zu implementieren und damit ein spezifisch westlich-feministisches Vorgehen als allgemeingültig zu erklären. Stattdessen erachtete sie es für richtig, ,immer von den einzelnen Vereinen und Projekten“, also den konkreten Situationen und Kontexten auszugehen. Wenn Saskia dieses Vorgehen daraufhin als „naiv“ bezeichnete und bezweifelte, dass dieses ,lange trägt“, antizipierte sie die Zwänge und Anforderungen, die von einer politischen Öffentlichkeit ausgehen. Dieses Vorgehen wird also von einer Spannung zwischen eigenen moralischen Idealen und dem pragmatischen Bemühen um Einflussnahme begleitet. 
gagement bekräftigen, ganz am Anfang der aufgelisteten Diskriminierungskategorien stehen sollen. Denn genau das sei doch der kontroverseste und schwierigste Punkt und daher auch die Stärke der Deklaration, dass sie als Erste dies öffentlich ausspreche und einfordere. Ich merke, wie sich das DF-Team freut. Wiktoria spricht für sich und ihre Kollegin. Für sie sei dieser Punkt problematisch, da es in ihrem Land rigide Gesetze gegen Homosexualität gebe. Ich bin einerseits erstaunt, klingen sie doch nun ganz anders, als es Inka noch vor ein paar Tagen beobachtet hatte. Zugleich fürchte ich, dass nun ein Einspruch kommt, und frage vorsichtig nach, was dies nun bedeute. Doch zu meiner Überraschung stellt sich heraus, dass es beide total gut fänden, den Aspekt hier so deutlich zu erwähnen und sexuelle Orientierung unbedingt explizit genannt haben wollen. Auch würden sie die Deklaration unterschreiben. Sie schlagen sogar noch vor zu fordern, dass vor jedem Fußballspiel die Diskriminierungskategorien öffentlich vorgelesen werden sollten. Dann kommen jedoch Einwände von drei anderen Teilnehmerinnen: Sie könnten so etwas nie unterschreiben, weil es in ihren Ländern verboten sei und eine Berührung mit dem Thema für sie drastische Konsequenzen bis hin zur Steinigung haben könnte. Jekatarina sagt irgendwann an die drei gewandt grinsend: „Have the guts“, und fordert sie auf, sich gegen ihre Verbände zu stellen. Eine der drei betont immer wieder, wie gerne sie unterschreiben würde und wie wichtig sie diese Forderung fände, aber ihr seien durch die zu erwartenden Restriktionen die Hände gebunden. Nach einer kurzen Diskussion wird sich darauf geeinigt, dass sie nicht unterschreiben werden, denn die Deklaration würde deutlich an Gewicht verlieren, wenn ,sexual orientation“ herausgenommen werden würde. (Feldnotiz, 14.12.2013)

Der Begriff der Diskriminierung und vor allem die Diskriminierungskategorie sexuelle Orientierung sind in manchen Ländern aufgrund der Gesetzeslage - hier vor allem in den Nicht-EU-Staaten - politisch nicht mobilisierbar. Manche Teilnehmenden hatten den Spielraum, ihn dennoch zum Zwecke der Einstimmigkeit zu übernehmen. In anderen staatlich-rechtlichen Kontexten wiederum ist die Begriffsverwendung nicht nur nicht erfolgversprechend, sondern gar existentiell bedrohlich. In der Diskussion werden erneut die Differenzen deutlich, die die unterschiedlichen rechtlichen Bedingungen in den einzelnen Ländern produzierten und die bei der Formierung einer gemeinsamen translokalen Stimme verhandelt wurden. Obwohl die politischen Begriffe an international geltende Menschenrechte anschlossen, wurden diese Rechte in den einzelnen Staaten unterschiedlich konsequent durchgesetzt oder erst gar nicht ratifiziert. In diesem Falle verhinderten die rechtlichen Kontexte eine Vereinheitlichung der Erfahrungen und die Problembenennung als „Homophobie“. Während Scott und Phipps vor dem Unsichtbarmachen von differenten Erfahrungen durch die machtvolle Vereinheitlichung und Vereinnahmung durch eine bestimmte Narration warnen, zeigt sich in diesem Formierungsprozess, dass manche Erfahrungen so prekär sind, dass sie dieser Vereinheitlichung widerstehen. Während die Artikulation von Erfahrungen in Bezug auf die rechtlich verankerte Identitätskategorie Frau - wenn auch unter der Bedingung der Vereinheitlichung - möglich ist, führt das Fehlen konsequent umgesetzter internationaler LGBTI-Rechte dazu, dass viele situierte Marginalisierungs- und Gewalterfahrungen erst gar nicht in eine öffentlich 
hörbare Stimme übersetzt werden können. Sie verweilen somit - denn drei Unterschriften fehlten im finalen Dokument der Deklaration - im Status des Nichtartikulierten und öffentlich Ungehörten.

\subsection{Plausible Lösungen vorschlagen: „IST-Beschreibung und SOLL-Forderung“"}

Während zu Beginn der Veranstaltung mit dem Evozieren von Emotionen und dem Erzählen von Erfahrungen Vergangenes in der Gegenwart aktualisiert und anschließend zu öffentlichen Angelegenheiten politisiert wurde, galt es in der nächsten Qualifizierungsstufe, konkrete Lösungen vorzuschlagen. Entsprechend dem gewählten Genre der Deklaration wurden diese in Form von Forderungen formuliert. Die Formulierung von Forderungen beinhaltet, die Gegenwart mit einer konkreten Zukunftsvision plausibel zu verknüpfen. Dafür musste zunächst ein detailliertes Verständnis der Gegenwart erarbeitet werde.

\subsubsection{Gegenwart erarbeiten}

Vor Beginn des Forums wurde die Deklaration in einer Arbeitsgruppe bestehend aus Inka, Luisa, Saskia und mir vorbereitet. Beim ersten Treffen planten wir den Aufbau des Dokuments und die notwendigen Arbeitsschritte, um dann in den wenigen Projekttagen ein Ergebnis erzielen zu können. Ich protokollierte unser Brainstorming:

Inka fasst zusammen, was bisher überlegt wurde: „Das Paper soll eine Bestandsaufnahme sein: Was gibt es in den Ländern für Richtlinien und wie werden die umgesetzt? Es soll an FIFA, UEFA und AFC (Asian Football Confederation) adressiert sein sowie an deren nationale Mitgliedsverbände." Der Workshop am Mittwoch um 14 Uhr solle der Einführung und weiteren Planung dienen, was die Deklaration will und wie sie aufgebaut sein würde. Inka erklärt, dass es gut wäre, wenn es Inputs zu den einzelnen Verbänden geben würde. So könnten wir deutlich machen, was bemängelt werden kann, welche Ziele es gibt und ob diese umsetzbar sind. Wir überlegen gemeinsam weiter: [...] Wie finden wir heraus, wie die Guidelines der Dachverbände in den einzelnen Ländern implementiert werden? Es wäre gut, wenn es in jedem Workshop eine Wand geben würde, die an die Deklaration erinnert und auf die die Teilnehmenden ihre Ideen, Kritiken und Vorschläge schreiben könnten. Diese Vorlage an der Wand solle genau wie die Deklaration in IST-Beschreibung und SOLL-Forderung aufgeteilt werden. Luisa erklärt: „Es geht auch darum, für uns eine Bestandsaufnahme zu machen.“ Und Inka ergänzt: „Bei SOLL geht es auch darum, was wäre wenn.“ [...] Untereinander teilen wir dann die Rechercheaufgaben auf: Inka recherchiert zur FIFA, Luisa zur AFC, Friederike zur UEFA: Was für Statuten gibt es zur Förderung von Mädchen und gegen Diskriminierung? Welche Programme gibt es? Haben sie sich zu 
etwas verpflichtet? Wer ist dort zuständig für Frauen und Diskriminierungsthemen? Wie viele Frauen gibt es in den Exekutivkomitees? Welche Anlaufstellen, an wen kann man sich mit welchen Belangen wenden (bspw. bei der Veruntreuung von geldern oder bei Diskriminierungsfällen), was kann von den Verbänden eingefordert werden? (Feldnotiz, 11.11.2013)

Die Arbeit an der Deklaration begann damit, dass wir nationale und internationale Verbände als konkrete Adressaten identifizierten. Indem wir diese als Verantwortliche anriefen, bestätigten wir die verbandsfußballerischen Institutionen in ihrer hegemonialen Position (vgl. Rose/Miller 1992; Day 2004). Hier zeigt sich, wie Macht, verstanden als netzwerkförmiges, polyzentrisches Geflecht, durch Konsens und Dissens zugleich wirkt: Erst durch die Anerkennung der Fußballverbände als herrschende Institutionen im Sinne des liberalfeministischen Wegs ,rein in die Institutionen“ bietet sich eine Adresse, an die die Beschwerden und Forderungen gerichtet werden können.

So wie es uns selbstverständlich erschien, diese Autorität anzuerkennen, so plausibel erschien es uns, als ersten Schritt Wissen über gegenwärtige Bedingungen, Verhältnisse und Politiken - „eine Bestandsaufnahme“ - zu sammeln, um konkrete Lösungsvorschläge unterbreiten zu können. Die Bestandsaufnahme konzentrierte sich auf rechtliche Rahmen und bürokratische Verfahren, wenn wir Antidiskriminierungsleitlinien, Förderprogramme, Selbstverpflichtungen und Ämterproporz recherchierten. Auf diese Weise identifizierten wir Verwaltung und Recht als zentrale Triebwerke der fußballerischen Geschlechterverhältnisse und ihrer Veränderung. Zusammen mit der politischen Problemdeutung stellte diese Idee von moderner und mächtiger Bürokratie und berechenbaren und einklagbaren Rechten das Wissen darüber bereit, „was bemängelt werden kann“, also welche Forderungen Vernunft besitzen, und „was umgesetzt werden kann“. Während des Expertinnenforums teilten wir das erarbeitete Gegenwartswissen mit den Teilnehmenden und steckten damit den Horizont dafür ab, was im Zuge der vier Projekttage gemeinsam als Forderungen entwickelt werden würde (vgl. Appadurai 2004, 61). Dabei geriet die spezifische Lokalität dieses Vorgehens, also seine Gebundenheit an rechtsstaatliche Bürokratien aus dem Blick.

\subsubsection{Mit Forderungen Gegenwart und Zukunft verknüpfen}

Wir beschlossen, die Deklaration in zwei Teile zu teilen. Der erste (IST) sollte das erarbeitete Gegenwartswissen auflisten und die bestehenden frauenfördernden und Diskriminierung bekämpfenden Maßnahmen begrüßen. Der zweite (SOLL) sollte mit weiterreichenden Forderungen daran anknüpfen. Als Inka betonte, es ginge mit der Arbeit an der Deklaration auch darum, „was wäre wenn“, spielte sie auf jene Zukunftsfähigkeit an, die Arjun Appadurai als Voraussetzung für die Formulierung von Wünschen und Forderungen identifiziert. Anknüpfend an Hirschmans Konzept von voice argu- 
mentiert Appadurai, dass es der Fähigkeit bedarf, eine gute oder bessere Zukunft zu denken und nach ihr zu streben, um die eigene Stimme zu erheben und zentrale gesellschaftliche Entwicklungen $\mathrm{zu}$ beeinflussen. Diese capacity to aspire beinhaltet, Forderungen und Kritiken entsprechend gültiger Ideologien, Doktrinen und Normen formulieren und in den lokal anerkannten und wirkungsvollen Formen vermitteln zu können (2004, 63ff.). Mir erscheint es sinnvoll, die Formierung einer Stimme als konkrete Forderungen mithilfe Appadurais Konzept zu untersuchen, denn so lässt sich einerseits eruieren, wie gegenwartsbezogene Problemdefinitionen mit Vorstellungen von einer besseren Zukunft verknüpft werden, und andererseits erkennen, wie diese Verknüpfungen situativ und kulturell verankert sind.

Voraussetzungen für die wirkungsvolle Artikulation einer Stimme sind die Fähigkeit, zwischen unmittelbaren und entfernten Zielen eine Verbindung herstellen zu können, die Erfahrung, den Weg zwischen eingesetzten Mitteln und angestrebten Zielen einschätzen zu können, und das Vermögen, materielle Dinge und unmittelbare Gelegenheiten für allgemeinere Möglichkeiten zu nutzen (Appadurai 2004, 63ff.). Kurz gesagt, die Formulierung erfolgreicher Forderungen basiert auf der Fähigkeit, Gegenwart und Zukunft plausibel und realistisch miteinander zu verknüpfen. Mit Blick auf die Deklarationsvorbereitungen lässt sich hinzufügen, dass die capacity to aspire auch auf detailliertem Wissen um aktuelle Regeln, Politiken, Prozesse und Verhältnisse basiert, denn Gegenwart und Zukunft lassen sich vor allem dann mittels Forderungen verbinden, je passgenauer Forderungen und Zukunftsentwürfe an bestehende Politiken anknüpfen. Forderungen als Beschreibung konkreter, realistischer und plausibler Wege stellen sodann die Bindeglieder zwischen Gegenwart und (angestrebter) Zukunft dar.

\subsubsection{Liberalfeministischen Pfaden folgen}

Zugleich betont Appadurai, dass die capacity to aspire nicht nur eine politische und öffentliche, sondern auch eine kulturelle Fähigkeit ist, und unterstreicht damit die Situiertheit von Zukunftsaspirationen $(2004,78)$. Die Verbindung von Gegenwartszustand und Zukunftsvision durch Forderungen ist also keine beliebige, die Raum für große Visionen und Utopien ließe, sondern ist eng an die Möglichkeitsbedingungen des günstigen Moments gebunden. Erinnert sei an Tsings Metapher der Friction als jenen Moment, in dem drehende Reifen auf eine Straße treffen $(2005,4)$ : Zwar eröffnet dieses $\mathrm{Zu}-$ sammentreffen Spannungen und Bewegung, jedoch sind diese durch die Materialität der Straße eingegrenzt. Mit Appadurai lässt sich diese Idee nun darum ergänzen, dass sich solche Momente lokal unterschiedlich materialisieren und damit unterschiedliche Pfade der Problematisierung eröffnen. Im Falle von DF hatten jene historischen geschlechterpolitischen Pfade, die jenes 
Momentum mit begünstigten, dazu beigetragen, dass den Aktivist innen einige Zukünfte realistischer und andere unrealistischer erschienen - wie sich im folgenden Beispiel zeigt. Auf dem letzten Plenum vor dem Expertinnenforum stellten Luisa, Inka und ich das Konzept zur Diskussion:

Linda schlägt fragend vor: „Kann man nicht die Forderungen auf einzelne Akteure zuschneiden? Ich mein, nicht gleich den Rücktritt von Blatter fordern, aber die sind so hierarchisch die Verbände, der hat schon krassen Einfluss. Und sagen, dass ist seine Schuld.“ Martina widerspricht: „Ich finde es besser die Positionen anzugreifen und nicht einzelne Personen." Simone geht noch einen Schritt weiter: „Wir kritisieren Strukturen und nicht Personen.“ Linda kommt mit einer neuen Idee: „Wir könnten die provokante Forderung stellen, dass die FIFA eine Doppelspitze haben sollte, eine Frau und einen Mann.“ Celia wendet ein: „Dann ist aber jede queere Politik dahin, wenn wir eine Mann-Frau-Doppelspitze fordern.“ Simone überlegt: „Oder eher eine Doppelspitze für verschiedene Positionen, eine für den Profisport und eine für Social Responsibility.“ Luca überlegt in eine ganz andere Richtung: „Wollen wir wirklich eine Doppelspitze oder nicht eigentlich einen eigenen Verband?" Saskia scheint die Diskussion jetzt etwas zu sehr ins Detail zu gehen und schlichtet: „Das ist ja nur die Diskussionsvorlage.“ Ich nehme skeptisch Bezug auf Luca: „Die Forderung nach dem eigenen Verband läuft den anderen Forderungen aber ja zuwider, denn die richten sich ja gerade an die Verbände." Linda greift die Idee enthusiastisch und zugleich humorvoll auf: „Damit hätten wir auch Presse: ,DF gründet...! ““ Nadine steigt auf ihren Humor ein: „Da könntest du dann auch Präsidentin werden!“ Linda grinst und seufzt: „Ja endlich!“”(Feldnotiz, 9.12.2013)

Celias Einwand und die Tatsache, dass er nicht weiter berücksichtigt wurde, zeigen die Unmöglichkeit intelligibler Politik jenseits der Zweigeschlechtlichkeit im Verbandssport (siehe Kap. 11.2). Doch auch innerhalb zweigeschlechtlich organisierter Frauensportpolitik erschienen einige Forderungen erfolgsversprechender als andere. Hier standen zwei zur Verhandlung: eine Doppelspitze oder ein eigener Verband. Die Idee einer Doppelspitze konnte sich schließlich durchsetzen, und DF wollte diese auf dem Expertinnenforum den Teilnehmenden vorschlagen - ich werde später noch genauer auf diese Forderung eingehen. Hier führe ich zunächst anhand der Idee eines eigenen Verbands aus, was ich bereits mit Blick auf differenzfeministische Argumentationen angedeutet habe: Geschlechterpolitische Pfadabhängigkeiten entscheiden mit über Plausibilität und Rationalität von Forderungen.

Um zu verstehen, welche Positionen hier miteinander verhandelt wurden, hilft ein Blick zurück in die Geschichte des Frauenfußballs. Lucas Forderung nach einem eigenen Verband ähnelt jenen Bestrebungen nach separaten Strukturen, Vereinen, Wettbewerben und Verbänden unabhängig vom Männersport, wie sie in der ersten Frauenbewegung zum Teil realisiert wurden. ${ }^{141}$ Als solche Bestrebungen im Laufe der 1960er Jahre wieder Aufwind bekamen und Spielerinnen europaweit eigene Verbände gegründeten und eigene

141 Siehe bspw. Leigh/Bonin (1977). Für weitere radikalfeministische Sportprojekte siehe Bryson (1994), Costas/Guthrie (1994). 
Wettbewerbe ausrichteten, hoben nach und nach viele westeuropäische Fußballverbände ihr Frauenfußballverbot auf und gliederten den Frauenspielbetrieb in ihre Strukturen ein (Meier 2004, 302; Degele 2012; siehe Kap. 4.2.4). Ich verstehe diese Entwicklung auch als das Ringen zweier verschiedener geschlechterpolitischer Ansätze um Plausibilität und Legitimität: Das Streben nach eigenen, separaten und unabhängigen Strukturen spiegelt radikalfeministische Forderungen, einen Widerstand gegen die männliche Kontrolle über den Sport und eine Abwendung von der herrschenden Ordnung wider, wohingegen das Streben nach inkludierter Gleichberechtigung dem sich damals durchsetzenden liberalfeministischen Ansatz entsprang. Der liberale Ansatz und in ihm vor allem jene auf Gleichheit und nicht auf Differenz zwischen den Geschlechtern basierende Argumente fordern seither gleichgestellte Teilhabe sowie gleiche Chancen und schlagen als Lösung für die als ungerecht identifizierten Geschlechterverhältnisse vor, jene Hindernisse reformerisch zu beseitigen oder zu kompensieren, die Frauen daran hindern, unter gleichen Bedingungen wie Männer ihre Leistung zu beweisen. „Aesthetically, the equal-rights mode of consciousness seeks duplication; politically, it seeks integration; psychically, it seeks assimilation" (Sandoval 2000, 55) Passend dazu ließ die Sport-für-alle-Philosophie des modernen Sports die Befreiung immer weiterer Frauen aus ihrer unverschuldeten Sportferne als Ziel selbstverständlich erscheinen und motivierte Sportpolitik und -verbände zu entsprechenden Maßnahmen der Frauensportförderung (Hargreaves 1994, 9\&27). In der Koalition aus Gleichheits- und Liberalfeminismus geriet die Kritik an der Verflochtenheit von Leistung und Männlichkeit aus dem Blick, sodass Integration in und Anerkennung durch die Verbandsstrukturen über die Darstellung von Leistung zu erreichen bzw. über das Argument der gleichen Leistungsfähigkeit rechtfertigbar schien.

Durch die Frauenrechtskonvention (CEDAW, 1979) und die Beijing Platform for Action (1995) erfuhren liberalfeministische Forderungen und Maßnahmen eine rapiden weltweiten Verbreitung (Krook 2006). Die Übersetzung universeller Frauenrechtsnormen in nationales und internationales Recht schuf die politischen Rahmenbedingungen für verstärkte feministische Proteste und Bewegungen und gibt ihnen bis heute eine Stimme, die sonst innerhalb der existierenden Institutionen nicht gehört werden würde. Der Erfolg von equal rights erschwert aber zugleich die Aufmerksamkeit für geschlechtliche Differenzen sowie Unterschiede innerhalb der Geschlechtskategorien und verfestigt die Unterscheidung zwischen legitimen Forderungen und jenen, die fortan als über das Ziel hinauszuschießend oder gar als indiskutabel gelten (Katzenstein 1998, 80f.; vgl. McRobbie 2009). Wenn Linda lachend anmerkte, dass das Streben nach einem Frauen-Verband viel Presseaufmerksamkeit auslösen würde, implizierte sie die Außergewöhnlichkeit dieser radikalfeministischen Idee. Das Herausfallen aus dem gewohnten Katalog geschlechterpolitischer Maßnahmen birgt das Risiko, über den Rahmen des als 
rational und diskutabel Geltenden hinaus zu fordern und in den Bereich des Irrationalen, Unrealistischen oder Unplausiblen eingeordnet zu werden. Aufzufallen und Aufmerksamkeit zu bekommen ist nicht gleichbedeutend damit, gehört zu werden. Hierin besteht eine beständige Gefahr für soziale Bewegungen: Wenn die kritisierten Herrschenden die Beschwerden und Forderungen als diffus, unrealistisch oder irrational diskreditieren, droht den sozialen Bewegungen Legitimitätsverlust, und die Chancen sinken, Reformen zu erwirken und Unterstützung für ihre Forderungen zu mobilisieren (Scholl 2013, 218). Dieses Risiko wohnt der Idee der Doppelspitze hingegen nicht inne, denn sie gliedert sich nahtlos in den intelligiblen liberalfeministischen Pfad ein; schließlich verlangt sie Korrekturen bestehender Strukturen und nicht Separation. Ein Konsens mit den dominanten Formen der Geschlechterpolitik über die Logik der Argumentation und die Wahl der Mittel erscheint als notwendiger und einzig erfolgsversprechender Weg, um sich Gehör zu verschaffen. Ich deute dies jedoch nicht in einem strukturdeterministischen Sinne als passives und automatisiertes Folgen vorgezeichneter Pfade - auch wenn sich das für viele Mitglieder in der Schnelligkeit und Dringlichkeit des Projektalltags häufig so anfühlte. Vielmehr zeigen Diskussionen und Entscheidungen, wie die dargestellten, dass es sich hierbei um ein aktives Tun handelt, das selbstkritische Diskussionen und bewusste Entscheidungen beinhaltet. ${ }^{142}$

Aber auch unter den Teilnehmerinnen des Expertinnenforums zeichnete sich $\mathrm{ab}$, dass die dominante Formation des Liberalismus für viele jene globalisierte Imagination bildete, ,through which collective patterns of dissent and new designs for collective life emerge" (Appadurai 2000, 6).

\subsubsection{Differenzen verhandeln}

Als DF schließlich die im Laufe des Expertinnenforums gesammelten Forderungen mit den Teilnehmenden verhandelte, traten die lokalen Differenzen wieder hervor und verdeutlichten die Situiertheit vermeintlich globalisierter geschlechterpolitischer Instrumente. Als ein DF-Mitglied während der bereits

142 Bei DF wurde die Frage, wie stark mit den kritisierten Institutionen zusammengearbeitet werden sollte, häufig diskutiert: Vor- und Nachteile wurden genau abgewogen, potentielle Handlungsrestriktionen sowie Chancen und Möglichkeiten antizipiert. Eines der zentralen Motive war finanzielle Sicherheit. Sich durch unplausible Forderungen oder utopische Projektideen beständig außerhalb der gültigen Plausibilitätskriterien zu positionieren, würde erstens das bislang Aufgebaute und Erreichte gefährden. Zweitens versprach finanzielle Stabilität die Möglichkeit, sich als Organisation zu behaupten und nach und nach mehr Mitspracherechte zu erhalten und zugleich der ständig drohenden Prekarität zu entkommen. Drittens vertraten die Mitglieder die Ansicht, dass Veränderungen ebenfalls von innen heraus erreicht werden könnten, dass sie als von den verbandsfußballerischen und sportpolitischen Institutionen anerkannte Organisation sogar mehr Einflussmöglichkeiten hätten denn als außenstehende. Sie entschieden sich damit bewusst, wenn auch nicht ohne Zwang für „Realpolitik“, also für Kooperation statt Konfrontation und für Reform statt Separation. 
ausschnitthaft vorgestellten Abschlussverhandlung die Idee einer Doppelspitze vorschlug, argumentierten mehrere Teilnehmerinnen dagegen: Diese würde schließlich auch doppelte Korruption mit sich bringen. Ähnliche Abwägungen wurden bei der Forderung nach Frauenquoten für die verbandlichen Gremien ersichtlich:

Von den Teilnehmenden kommt immer wieder der Wunsch, die Forderung nach Frauenquoten damit zu ergänzen, dass die Frauen auch qualifiziert seien sollen. Inka findet das nicht gut, denn schließlich sollten Frauen diese Ämter bekleiden, die dann eh schon qualifiziert seien. In Ungarn sei das zum Beispiel nicht so, bemerkt eine Journalistin, da käme dann einfach die Schwester vom Präsidenten in das Amt. Viele nicken zustimmend. Inka erwidert: „Aber es geht darum, dass alle qualifiziert sein müssen. Es stört mich, dass das hier nur von den Frauen gefordert wird.“ Wir kommen zu dem Konsens, dass stattdessen immer transparente Berufungsverfahren gefordert werden, sodass die Qualifikation der Personen nachverfolgt werden kann. (Feldnotiz,-14.12.2013)

Quoten und Doppelspitze als „global phenomenon“ (Krook 2006, 304) stehen im Einklang mit international verbreiteten Normen von Gleichberechtigung/stellung und angemessener Repräsentation unterschiedlicher sozialer Gruppen. Dennoch lehnten Teilnehmerinnen die aus Berlin kommenden Vorschläge mit dem Verweis auf Korruption ab oder wollten sie spezifizieren. Globalisierungstheorien haben darauf aufmerksam gemacht, dass globale Phänomene immer lokal spezifisch angeeignet werden und dass das Globale damit als vielfältig und nicht einheitlich gedacht werden muss (vgl. Robertson 1995). Für die Erarbeitung der Deklaration bedeutete dies, dass die meisten Teilnehmerinnen und Organisator_innen sich zwar in einer translokalen Zivilgesellschaft bewegten und über eine gemeinsame Sprache und ein geteiltes Wissen um Politiken und Instrumente verfügten, die Begriffe und Instrumente jedoch in den einzelnen lokalen und nationalen Kontexten in unterschiedliche politische Prozesse eingebunden waren und entsprechend unterschiedlich wirkten. In translokalen feministischen Begegnungen werden multiple „glokalisierte“ Versionen (Robertson 1995) des globalisierten Phänomens Liberalfeminismus verhandelt, die durch die Verknüpfung lokaler Erfahrungen mit globalisierten Frauenrechten unterschiedliche Wissensbestände produzieren (vgl. Wenger 2008, 141).

Der Widerstand einiger Teilnehmerinnen macht deutlich, was sich bereits abzeichnete: Es trafen zwei - und wahrscheinlich sogar mehr - Wissensbestände davon aufeinander, wie Verwaltung funktioniert. In der Forderung nach einer Doppelspitze sowie in der Annahme, dass Ämter generell nach Qualifikation vergeben werden, drückt sich eine Vertrautheit mit und ein Vertrauen in rationale Bürokratie aus, die bereits Max Weber (2002) als typische Herrschaftsform kapitalistischer, westlicher Nationalstaaten beschrieben hat und die sich durch Berechenbarkeit statt Willkür, professionelles Personal statt Korruption und Klientelismus auszeichnet. Die Selbstverständlichkeit bürokratischer Herrschaft und sicherlich auch die Unerfahrenheit mit ver- 
bandsinternen Prozessen veranlassten die Organisator_innen, verbandssportliche Institutionen als kohärente Entitäten zu begreifen, die allein durch Regelwerke, Gesetze und administrative Prozesse gesteuert würden - erinnert sei an die anfängliche „Bestandsaufnahme“ bestehender Regelwerke, bürokratischer Verfahren und Strukturen. Mit dem Verweis auf Korruption und Vetternwirtschaft stellten die Teilnehmerinnen - darunter viele Verbandsmitarbeiterinnen - dieses Verständnis vom Funktionieren von Verwaltungsprozessen in Frage und machten andere Erfahrung geltend: Die verbandsfußballerischen Verhältnisse schienen weniger von bürokratischen Strukturen und Regeln und mehr von einzelnen Personen und persönlichen Beziehungen abzuhängen; regierende Institutionen wurden nicht als entpersonalisierte Menge aus bürokratischen Prozessen, Ämtern und zugrundeliegenden Regeln betrachtet, sondern als Gefüge interpersoneller Beziehungen. ${ }^{143}$

Ich habe bereits gezeigt, dass dem Versuch translokaler identitätspolitischer Bündnisbildung die Gefahr der machtvollen Vereinnahmung marginalisierter Erfahrungen durch privilegiertere Frauen innewohnt und differente Erfahrungen zugunsten eines kohärenten Wissensbestands entsprechend der vorherrschenden Kräfteverteilung transformiert werden. Hemmings warnt davor, dass es in solchen Auseinandersetzungen leicht dazu kommen könne, dass die strukturell machtvollere Seite die Erfahrungen der anderen fehldeutet und die Unterschiede übersieht, um sie für die eigenen anschlussfähig zu machen $(2012,152)$. Und Sharma (2008) bemerkt, dass ein solches Vorgehen Hierarchien zwischen NGO-Mitarbeiter innen und Teilnehmenden festigen und damit die eigene auf Gleichheit abzielende Empowerment-Agenda konterkarieren kann. DF wusste um diese Kritik. Die daraus entstehende Angst $\mathrm{zu}$ vereinnahmen drückt sich in der Zukunftsdiskussion in Lindas Ablehnung einer Weltstrategie und Thomas und Birgits Wunsch, nicht „von oben herab“ vorzugehen, aus. Diese Kritik ließ die Organisator_innen Formate schaffen, die ihre eigenen Einflussmöglichkeiten minimierten und den Teilnehmenden Mitspracherechte einräumten, sodass schließlich in der Abschlussverhandlung alle Stimmen gleiches Gewicht erhielten. Weder Inkas Wunsch, auf Qualifizierungsforderungen zu verzichten, noch Lindas Vision einer Doppelspitze konnten sich gegen die lokalen Erfahrungen der Teilnehmenden mit Verwaltungsprozessen durchsetzen. Während Hemmings für feministische Bündnisse plädiert, die in affektiver Solidarität und der Anerkennung von Differenzen wurzeln, ist mit Blick auf die geschilderte Situation zu ergänzen, dass auch konkrete organisatorische Rahmen, Ablauf- und Abstimmungsverfahren einer ,cannibalisation of the other“" (Hemmings 2012, 152) entgegen-

143 Day erachtet es als typisch für Neue Soziale Bewegungen und jene politics of demand, dass sie die herrschenden Institutionen als feste Gefüge adressieren, anstatt sie als Geflecht interpersoneller Beziehungen zu betrachten $(2004,729)$. Die Verhandlung im Rahmen des Expertinnenforums macht deutlich, dass diese Form der Adressierung hegemonialer Strukturen einem ganz spezifischen historischen, politischen und rechtlichen Kontext entspringt. 
wirken können. Die Entwicklung eines Forderungenkatalogs ist unter diesen Umständen kein Aufoktroyieren politischer Imaginationen durch aktive Organisator_innen gegenüber passiven Teilnehmerinnen. Vielmehr zeichnen sich hier vielfältige Reaktionen, Aneignungen, Umdeutungen und Widerstände sowie unterschiedliche und ganz eigene Motive für kooperatives Verhalten auf Seiten der Teilnehmerinnen ab (vgl. Sperling et al. 2001; Mosse 2004). Indem diese Einwände gegen bestimmte Begriffe erhoben und einige geschlechterpolitische Instrumente ablehnten, ihnen erst nach gründlichen Abwägungen zustimmten oder nicht unterschrieben, nutzten sie ihre Möglichkeiten, sich dem sanften Zwang zu entziehen.

Festzuhalten ist, dass mit der Einrichtung interaktiver, demokratischer Formate der bis dahin hegemoniale epistemische Rahmen ein Stück weit herausgefordert und dadurch modifiziert wurde, dass sich Forderungen in die Deklaration einschrieben, die dem westlichen Politik- und Staatsverständnis zuwiderliefen. Die Formierung einer kohärenten Stimme aus sehr heterogenen Erfahrungen innerhalb eines ungleichen translokalen Kräfteverhältnisses kann also durchaus auch Züge einer „two-way interaction“ beinhalten (Wenger 2008, 138): Nicht nur bestimmt der dominante Wissensbestand darüber, wie Erfahrungen gedeutet werden. Auch können von diesem abweichende Erfahrungen, wenn sie von einer kritischen Masse vertreten werden, den gültigen Wissensbestand transformieren.

\subsection{Stimme formatieren: ,We, representatives of girls' and women's football"}

„Liebe Alle, ich sitze gerade an dem Paper, das ja eine Art Deklaration werden soll. [...] Wir orientieren uns bei Struktur [IST und SOLL sowie Selbstverpflichtungen] und Formulierung an offiziellen Deklarationen aus der Politik [...]." (E-Mail, $8.12 .2013)$

Diese E-Mail schickte ich als Teil der Arbeitsgruppe in der Vorbereitungsphase an die Organisationsgruppe. Bei der Wahl des Genres und der ästhetischen Form orientierten wir uns an der Folie politischer Dokumente nationaler und internationaler Regierungsinstitutionen. Modell stand unter anderem die Declaration of Berlin, die ein halbes Jahr zuvor auf der Weltsportministerkonferenz in Berlin verabschiedet worden war. Annelies Riles (2007) hat mit ihrer Untersuchung von NGO-Dokumenten gezeigt, dass politische Prozesse immer auch materialisierend, Form und Struktur gebend sind. Sabine Höhn (2013) argumentiert anhand ihrer Beobachtungen zum Gebrauch von Dokumenten in NGOs, dass diese ein Eigenleben entwickeln und nicht als passive Objekte, sondern als Aktanten des Aktivismus zu erachten sind. Ich 
frage daher, wie Form und Ästhetik des gewählten Genres den Inhalt ergänzen und plausibilisierend auf die Forderungen wirken.

Während des Expertinnenforums hängte die Arbeitsgruppe BlankoEntwürfe der Deklaration an Stellwänden aus. Wir hatten diese entsprechend der bewährten Struktur internationaler politischer Dokumente in drei Teile gegliedert, sodass in einer oberen Reihe drei Blätter nebeneinanderhingen. Jedes Din-A4-Blatt war mit einem englischen Satz und dessen russischer und deutscher Übersetzung überschrieben, die vorgaben, was in den freien Bereich darunter eingetragen werden sollte. Das erste Blatt rief die Teilnehmenden im Sinne der „Bestandsaufnahme“ des IST-Zustands dazu auf, jene Politiken, Programme und Maßnahmen aufzuzählen, die sie befürworteten: „We, representatives of girls' and women's football and signatories to this declaration, welcome and affirm“. Das zweite Blatt rief mit dem Satz „We, representatives of girls' and women's football and signatories to this declaration, call upon all stakeholder" dazu auf, konkrete Forderungen und Veränderungsvorschläge - SOLL - aufzuschreiben, die sich an Verbände und Vereine richteten. Das dritte Blatt schließlich ließ Platz für Selbstverpflichtungen. Unter den drei Sätzen war das jeweilige Blatt leer und unter ihm hing noch ein weiteres leeres weißes Blatt. Ein schwarzer dünner Rahmen signalisiert, dass diese Leere auszufüllen sei.

In dieser Form, deren Gliederung eine deutliche Nähe zur anvisierten Deklaration erkennen ließ, war Platz für ganze Sätze. Nachdem verschiedene Erfahrungen zunächst auf einzelne Begriffe oder Phrasen reduziert, in diese eingefaltet oder aus ihnen ausgeklammert worden waren, entfalteten sich die abstrahierten Problembestimmungen nun zu einer generalisierbaren, aber dennoch konkreten Forderung. Jene Spielerin, die zuvor noch ,lack of money" als entmutigende Erfahrung beschrieben und auf einer Karteikarte als generelles Problem notiert hatte, formulierte hier nun die Forderung nach staatlich finanzierten Stipendien für Sportlerinnen. Durch die vorangestellten Sätze, die stets mit einem kollektiven Wir begannen, war der Duktus und der Grad der Verallgemeinerung klar: Hier war kein Platz für Partikularforderungen, sondern nur für solche, die sich global stellen ließen.

Nachdem die weißen Blätter ausgefüllt worden waren, setzte sich die Arbeitsgruppe am Vorabend des letzten Tages zusammen und formulierte aus den dort notierten Sätzen sowie den im Verlauf der Workshops festgehaltenen Ideen einen ersten Entwurf. Bereits im Vorfeld hatte Simone die Grafikerin um ein Layout gebeten, das „eher offiziell aussehen“ sollte (E-Mail, 4.11.2013). Das Layout griff, genau wie Aufbau und Formulierung, ästhetisch offizielle politische Dokumente auf. Höhn beobachtet, dass Dokumente in NGOs ein selbstreferentielles System bilden, innerhalb dessen es höchst wichtig ist, dass jedes Dokument die formalen Eigenschaften des vorangegangenen wiederholt $(2013,111)$. Diese formalen Eigenschaften umfassen einen bestimmten Aufbau (IST, SOLL und Selbstverpflichtung), konkrete 
Formulierungen (,We, representatives of ...“) sowie eine bestimmte Ästhetik und bilden so das „,vertical skeleton“ (Riles 2007, 87) der rationalen Stimme.

Am letzten Seminartag wurden der von uns vorbereitete Entwurf, die konkreten Formulierungen und ihre Reihenfolge in einer knapp dreistündigen Diskussion mit allen Beteiligten im Detail besprochen, umformuliert und noch einmal neu angeordnet. Nach der Verhandlung des Dokuments zog sich die Arbeitsgruppe mit dem fixierten Inhalt zur Fertigstellung ins Büro zurück. Es galt, die von den Teilnehmerinnen aufgeschriebenen und von den Organisator_innen während der Workshops mitgeschriebenen Forderungen in die Formatvorlage einzufügen und in eine Reihenfolge zu bringen. Das Formulieren rationaler Forderungen wurde zu einer ,sorting exercise in which language was cut, arranged, or inserted to produce appropriate strings of words" (Riles 2007, 80). Unterstützung erhielten wir von einer befreundeten Dolmetscherin, die die sprachliche Fehlerfreiheit sicherstellen sollte. ${ }^{144}$ Unter dem hohen Zeitdruck kam immer wieder Verzweiflung über das Textverarbeitungsprogramm auf, wenn Nummerierungen und Formatierungen durcheinander gerieten, schließlich stellte die politische Öffentlichkeit auch formale Anforderungen: ,Demands should take the form of a list“" (Butler 2013, 8). Hier stand nichts Geringeres als das Grundgerüst der rationalen Stimme auf dem Spiel: Eine verzogene Formatierung und die Professionalität des Dokuments schien sich in Improvisation und die Legitimität der Forderungen in Lächerlichkeit zu wandeln. Öffentlichkeit versteht Rationalität also nicht nur in Bezug auf Inhalt (plausibel und realistisch) und Modus (nicht emotional), sondern ebenfalls in Bezug auf Genre, Struktur und Ästhetik. Eine rationale Stimme im Sinne generalisierter, also nicht persönlicher und partikularer, plausibler, also nicht überzogener oder unrealistischer, und kohärenter, also nicht in sich widersprüchlicher Forderungen wird damit von geschlechterpolitischen Pfadabhängigkeiten und staatlichen-rechtlichen Kontexten sowie internationalen Rechtsnormen vorstrukturiert und maßgeblich durch das Dokumenten-Genre, seine Form und Ästhetik hergestellt.

Am Abend wurde die erfolgreiche Formierung der Stimme in einem Restaurant gefeiert: Die auf schwerem weißem Papier gedruckte Deklaration wurde auf einem eigenen Tisch, geschmückt mit einer kleinen bunten Decke, ausgelegt. Ein schwarzer Tinten-Stift lag zur Unterzeichnung bereit. Einzeln wurde jede Teilnehmerin nach vorne gerufen, wo ihr ein DF-Mitglied eine Teilnahmeurkunde und ein Exemplar der Deklaration überreichte und wo sie anschließend das ausgelegte Papierdokument unterschreiben konnte. Mit diesem letzten Schritt der Unterschrift besiegelten die Teilnehmerinnen und Organisator_innen die Transformation ihrer multiplen Erfahrungen, unkonkreten emotionalen Haltungen und vielen Zukunftsaspirationen in eine gemeinsame, kohärente und plausible Stimme. Jede Teilnehmerin erhielt ein

144 Im Laufe der nächsten Monate halfen Partnerinnen von DF, die Deklaration ins Arabisch, Spanische und Russische zu übersetzen. 
ausgedrucktes Exemplar, und in den nächsten Wochen wurde die Deklaration als PDF auf die Homepage von DF gestellt, und die digitale Unterzeichnung durch eine Anwendung ermöglicht.

De Wilde verweist darauf, wie fragil jene Stimmen sind, die üblicherweise als emotional disqualifiziert und in den Bereich des Privaten gedrängt werden, nun aber durch besondere Anstrengungen zum Sprechen gebracht werden. Sie bemerkt, dass ihre Artikulation maßgeblich an die geschaffenen Räume und ihre Atmosphären geknüpft ist und mit dem Verlassen der Räume schwindet. Das Expertinnenforum wie auch andere Projekte von DF boten solche ,fragile public space, that die with the fading away of the sphere of belonging in which they come to life" $(2015,131)$ : Sie ermöglichen das Artikulieren von Erfahrungen, deren Abstraktion zu öffentlichen Problemen und das Formulieren konkreter Forderungen, sie schafften die Möglichkeit, die capacity to aspire zu erproben. Mit dem Auflösen des Raums am Ende der Veranstaltung drohten auch die Forderungen, Zukunftsvisionen und gemeinsamen Positionen zu schwinden. Die materialisierte Form sowie ihre virtuelle Entsprechung versuchten, die Stimme über den aktuellen Moment und Raum hinaus zu konservieren.

\subsection{Gehört werden: „Es ist einfach unser Recht"“}

Die inhaltliche, modale und formale Qualifizierung der Stimme ermöglicht, dass Forderungen gehört werden (vgl. Scheffer 2013). So wurde die Deklaration in den Folgemonaten an Funktionär_innen und lokale und nationale Politiker_innen übergeben und von diesen teilweise unterzeichnet. Nicht nur die Deklaration, auch andere Formate sollten DFs Forderungen Gehör verschaffen: Bei öffentlichen Veranstaltungen richteten Vertreter_innen von DF ihr Wort an Sportpolitiker_innen und Funktionär_innen, sie evaluierten ein Sportgroßereignis auf seine Gleichstellungseffekte, sie vertraten ihre Anliegen an einem Runden Tisch des Bezirkssportamts und sie verbündeten sich mit anderen lokalen Vereinen, um beim Berliner Fußballverband einen Antrag auf Einrichtung eines Mädchen- und Frauenausschusses zu stellen. Die Artikulation von Forderungen zeigt sich dabei als voraussetzungsvolles Vorgehen, dessen Erfolg mit den unterschiedlichen Wegen variierte.

\subsubsection{Voraussetzungen: Wissen, Artikulations- und Navigierfähigkeit}

Mit der Gründung eines eigenen Fußballvereins konzentrierten sich die Aktivist_innen ab 2012 auch auf die Berliner Sportpolitik und die Geschlechter- 
verhältnisse im lokalen Fußballverband. Kaum hatten sie den Verein gegründet, erlebten sie die Schwierigkeit, in einem der am dichtesten besiedelten Bezirke eine Trainingszeit auf den verhältnismäßig wenigen Fußballplätzen $\mathrm{zu}$ erhalten. Fortan verfolgte DF das Ziel der geschlechtergerechten Vergabe von Platzzeiten an Mädchen- und Frauenteams. Bei einer Podiumsdiskussion adressierte Linda einen SPD-Politiker, der unter anderem im Senat für Sport zuständig war:

„Ich möchte hier Marie Juchacz zitieren: „Ich möchte hier feststellen, und glaube damit im Einverständnis vieler zu sprechen, dass wir dieser Regierung nicht Dank schulden. Was diese Regierung getan hat, das war eine Selbstverständlichkeit: sie hat den Frauen gegeben, was ihnen bis dahin zu Unrecht vorenthalten worden ist.' Ebenso steht uns die Hälfte der Plätze zu. Das Argument, wir würden dann anderen ihre Platzzeiten wegnehmen, das geht einfach nicht. Man kann uns nicht erst verbieten, Fußball zu spielen, und dann sagen: ,Ach, jetzt spielen schon überall Männer, deswegen gibt es keinen Platz mehr für euch'. Frauen machen 50 Prozent der Bevölkerung aus, wir zahlen 50 Prozent der Steuern, es ist einfach unser Recht, dass wir auch 50 Prozent der Platzzeiten bekommen." (Feldnotiz, 5.6.2015)

Wie auch in der Deklaration diskutierte DF das Thema unter Gerechtigkeitsaspekten (,es ist einfach unser Recht") und mit liberalfeministischen Instrumenten (Quotierung der Plätze) sowie mit Rückgriff auf ein kollektives Wir, das in diesem Fall nicht nur gegenwärtige, sondern auch frühere Fußballerinnen einschloss. Mitgetragen wurden diese Forderungen vom lokalen Bezirkssportamt, an dessen „Runden Tisch für Mädchen- und Frauensport“ DF gemeinsam mit anderen bezirklichen Sportinitiativen teilnahm.

Appadurai zeigt, dass die Fähigkeit, Zukunft zu aspirieren und mittels politischer Aktionen einzufordern, zwischen Armen und Reichen ungleich verteilt ist (2004). Dies wird hier nun besonders deutlich: Lindas Bezug auf ein Recht im Sinne der Gerechtigkeit zwischen den Geschlechtern bildet auf Gegenwarts- und Geschichtswissen basierende Gegenargumente zum Senioritätsprinzip, das der SPD-Politiker vertrat und das vorsieht, dass denjenigen, die bislang den Platz bespielten, dieser nicht wieder weggenommen werden darf. Die Frauenquote als beliebtes Instrument im liberalfeministischen Werkzeugkasten stellt sodann den adäquaten politischen Lösungsweg dar. Der Verweis auf die Tradition dieser Forderungen durch das Zitat der sozialdemokratischen Frauenrechtlerin Marie Juchacz verleiht der Forderung zusätzliches moralisches Gewicht, gerade gegenüber einem Politiker derselben Partei.

145 Marie Juchacz, Frauensekretärin im SPD-Parteivorstand, hielt als erste Frau 1919 eine Rede in einem deutschen Parlament. Mit diesem Zitat erinnert Linda den SPD-Politiker an die frauenrechtliche Tradition seiner Partei, denn dieser hatte sich kurz zuvor gegen eine Quotierung von Plätzen ausgesprochen, da dies bedeuten würde, dort bereits trainierenden Jungen und Männern diese Plätze zu entziehen. 
In Verhandlungen mit öffentlichen Verwaltungen zu treten, erfordert bürokratische Navigationsfähigkeiten (vgl. Appadurai 2004); der öffentliche Auftritt und die Formulierung von Forderungen setzen das Gegenwarts- und Geschichtswissen um eigene Rechte und Gerechtigkeitsnormen voraus, und sie erfordern Artikulationsfähigkeit und ein entsprechendes politisches Vokabular. Wissen um konkrete politische Maßnahmen sowie grafische und ästhetische Fähigkeiten verleihen zusätzliches Gewicht. ${ }^{146}$ Hier zeigt sich erstens, wie das umfangreiche kulturelle Kapital von DF (siehe Kap. 8.2.4) zum Einsatz gebracht wurde, um die eigenen Forderungen hörbar zu machen. Zweitens ermöglichte das Stadtwissen der Aktivist_innen (siehe Kap. 9.2.1), räumlich und politisch-ideologisch durch die städtische Verwaltungslandschaft und Zivilgesellschaft $\mathrm{zu}$ navigieren, Forderungen an den richtigen Stellen mit den richtigen Argumenten zu platzieren und unter der Vielzahl von Aktuer_innen Mitstreitende zu identifizieren und Partnerschaften zu schließen. Die capacity to aspire umfasst, so möchte ich ergänzen, neben dem gekonnten Bewegen zwischen Rechtfertigungen, politischen Narrativen und Metaphern, Pfaden und abstrakteren Vorstellungen und Normen des sozialen Miteinanders auch konkretes Stadt- und Institutionenwissen. Dieses Wissen ermöglicht die geschickte Platzierung der Forderungen und strategische Partnerschaften und eröffnet damit Wege der Problematisierung. Ein solches Vorgehen erscheint weniger privilegierten Frauen im Fußball nur schwer zugänglich.

Zugleich, so machen sowohl Bourdieus Kapitaltheorie als auch Appadurais Beispiel deutlich, müssen diese Fähigkeiten, wie alle anderen auch, erlernt und regelmäßig geübt werden. Gerade marginalisierten Personen mangelt es an Gelegenheiten, Zukunftsvisionen zu entwickeln und zu diskutieren (Appadurai 2004, 69). Auch wenn die Funktionärinnen, Spielerinnen, Trainerinnen und Journalistinnen, die im Rahmen vom Expertinnenforum zusammenkamen, meist aus deutlich privilegierteren Lebens- und Bildungssituationen kamen als die von Appadurai begleiteten Slum Bewohner_innen, ist diese Beobachtung dennoch übertragbar. Zwar erforderte ihr alltäglicher Arbeitskontext ständig das Entwickeln von Zukunftsvisionen. Aufgrund der wenigen Frauen und der Marginalisierung geschlechterpolitischer Themen mangelte es jedoch häufig an Gelegenheiten, explizit feministische Zukünfte zu entwickeln. Im Gegensatz dazu bilden die von DF organisierten Expertinnenforen mit dem Ziel, „Austausch anzuregen“, jene Diskussionsräume, in denen Frauen die Möglichkeit erhalten, bestehende Verhältnisse zu kritisieren, Wissen über bürokratische Vorgehensweisen auszutauschen, Informationen über das Funktionieren von Verwaltungen zu erhalten, über mögliche

146 Merry et al. (2010) zeigen anhand US-amerikanischer Menschenrechtsorganisationen, wie (un)zugänglich das legale System der Menschenrechte ist. Hier wie dort erfordert die Mobilisierung des Rechts weitreichende Expertisen und breite Netzwerke. 
Zukünfte zu debattieren und die Fähigkeit, geschlechtergerechtere Verhältnisse zu aspirieren, erlernen und erproben können (vgl. Sharma 2014).

In diesem Sinne bietet das Vorgehen, Forderungen zu stellen, auch die Möglichkeit, die Beteiligten dahingehend zu befähigen, jene geschlechterpolitischen Instrumente zu nutzen, mit denen sie ihre marginalisierte Stellung und die sie benachteiligenden Verhältnisse anfechten und die entsprechenden verwaltenden Institutionen in die Pflicht nehmen können.

\subsubsection{Wege des Problematisierens}

Doch folgt auf Gehört-Werden nicht unmittelbar das Erhört-Werden. Nicht immer fanden DFs Forderungen Wiederhall und stießen Veränderungen an. Ich möchte hier die unterschiedlichen Wege skizzieren, um mit Forderungen die fußballerischen Geschlechterverhältnisse umzuarbeiten, und diskutieren, warum manche erfolgreicher waren als andere.

In den folgenden Monaten und Jahren wurde die Deklaration von DF und einigen Teilnehmerinnen an Verbandsfunktionär_innen und (Sport)Politiker_innen überreicht und von diesen zur Kenntnis genommen. Diskussionen oder gar Veränderungen blieben jedoch aus. Die Deklaration schien bei den nationalen und internationalen Dachverbänden kaum Gehör zu finden. Deutlich erfolgreicher verlief der Versuch auf lokaler Ebene: Nachdem die Forderung nach einem Mädchen- und Frauenfußballausschuss beim Berliner Fußballverband immer wieder abgelehnt worden waren, bildete sich 2017 eine Allianz aus Berliner (Frauen-)Fußballvereinen, um selbst auf dem Verbandstag des Berliner Fußballverbandes einen Antrag auf Satzungsänderungen zu stellen, die den Ausschuss ermöglichen sollte. Im letzten Moment reagierte der Verband auf diesen sanften Druck von außen und stellte auf dem Verbandstag selbst einen entsprechenden Antrag, der sodann ohne Gegenstimmen angenommen wurde. Dass DFs Forderungen beim lokalen Verband eher erhört wurden als bei nationalen und internationalen Dachverbänden, erklärt sich meiner Ansicht nach aus der Formierung als kompetente Amateurin und Eingliederung in den lokalen Amateurspielbetrieb sowie der gleichzeitigen Distanz zum professionellen und profitorientierten Leistungssport (siehe Kap. 10.2). Doch der lange Kampf für einen Mädchen- und Frauenausschuss zeigt auch, dass selbst auf Amateurebene Veränderungen nur mühsam zu erreichen sind. ${ }^{147}$ So habe ich bereits in Kapitel 4.1.3 auf

147 Diese Erfahrung machte auch Tanja Walther-Ahrens, die 2014 als erste Frau ins Präsidium des Berliner Fußball-Verbands berufen wurde und keine zwei Jahre später frustriert zurücktrat. Sie fühlte sich in einer „Alibi-Funktion“ und kritisierte: „Und wenn irgendwann sogar das Argument auftaucht, dass mehr Mädchen und Frauen, die Fußball spielen wollen, ja bedeuten, dass die Jungs und Männer keinen Platz mehr zum Spielen haben, bin ich mir nicht sicher, ob das ein Witz sein soll." (Zit. n. Rundfunk Berlin-Brandenburg 2015; vgl. Schrey 2015, 2:37). 
Bausingers Kritik an der Vereinskultur in Form von bürokratischer Überformung durch Statuten und Mitgliederverwaltung und der Übernahme alter Einflussstrukturen und innerer Rangordnungen hingewiesen. Hier wird nun ersichtlich, wie der Traditionalismus des Vereinswesens Veränderungen erschwert und Altes „mit Klauen und Zähnen“ (Bausinger 2006, 48) verteidigt wird.

Viel einfacher verlief der Weg über die lokale Sportpolitik, denn das Sportamt unterstützte DFs Forderung nach mehr Trainingszeiten mit großem Engagement. Diese strategische Koalition von DF und Bezirkssportamt zum Zwecke der Frauensportförderung wurde durch den Einzug feministischer Politiken in öffentliche Verwaltungen und durch DFs Formierung als lokale Sportveranstaltung ermöglicht: Nicht nur schuf DF ein lokales und gleichzeitig international ausgerichtetes Sportangebot und regelmäßige Sportfestivals, die sich in das Image des Stadtteils einfügten und dieses mit Leben füllten (siehe Kap. 9.2.2), DF entsprach auch dem bezirklichen Ansatz der Frauenförderung, der unter anderem mit einem Gender-Mainstreaming-Programm institutionalisiert worden war (vgl. Färber/Geppert 2004; Meyer 2012). ${ }^{148}$ Trotz der staatlich zugesicherten Autonomie der Sportvereine und -verbände (siehe Kap. 4.3.1) können sportpolitische Verwaltungs- und Reformprozesse über das Instrument der Sportförderung geschlechterpolitische Effekte auf den Verbandsfußball entfalten und das Geschlechterverhältnis verändern. Tatsächlich wurde im Frühsommer 2015 in der Bezirksverordnetenversammlung ein Antrag auf einen eigenen Sportplatz für Mädchen und Frauen eingereicht und dort wie auch medial intensiv diskutiert (vgl. Polat 2016).

Regierende Institutionen wie die öffentlichen Verwaltungen, aber auch die Fußballverbände erscheinen damit nicht als starre patriarchale Entitäten, sondern als Geflechte aus Prozessen, Personen und Beziehungen, die durch feministische Interventionen und strategische Kooperationen mal leichter, mal mühsamer umgearbeitet werden können (Woodward 2004; Mouffe 2014; Revillard/Bereni 2016). Janet Newman (2012) beschreibt z.B., wie Feministinnen als Mitarbeiterinnen in und Beraterinnen von Regierungs- und Verwaltungsorganen die Möglichkeiten der machtvoll strukturierten Räume nutzen und in ihrem Sinne umgestalten. In den Räumen des Bezirkssportamts entstand eine Friction zwischen Sportförderung und Frauenpolitik, und unter dem Dach des Verbandes versammelte sich eine Koalition aus feministische orientierten Vereinen zu einer kritischen Masse, sodass auf diesen Wegen feministische Gerechtigkeitsvorstellungen in berechenbare und rechtlichverbindliche Regelwerke übersetzt werden konnten und feministische Politiken Einzug in die öffentliche Verwaltung und den Verbandsfußball hielten.

148 An anderer Stelle habe ich ausführlich dargestellt, wie die bezirkliche Sportpolitik und Frauenförderung einen Weg für DF eröffnete, als Fußballerinnen Anerkennung und gleiche Mitspielrechte einzufordern (Faust 2017). 
Zurückkommend auf das dieses Kapitel leitende Interesse am Stellen von Forderungen als geschlechterpolitischen Handeln lässt sich zusammenfassen, dass dieses Vorgehen einen mehrstufigen Prozess der Herstellung einer kohärenten und rationalen Stimme beinhaltet. Problematisieren mittels Forderungen bedeutet, dass die Mitsprachebedingungen einer bürgerlichen Öffentlichkeit antizipiert und diesen entsprechend eine kohärente, rationale, realistische und plausible Stimme formiert wird. Anhand des Expertinnenforums vom Dezember 2013 habe ich gezeigt, wie DF dafür emotionale Stellungnahmen mobilisierte und eine gemeinsame moralische Positionierung anregte, Erfahrungen mobilisierte und zu politischen Problemen abstrahierte, und sodann Forderungen entwickelte und formatierte, die die aspirierte liberalfeministische Zukunftsvision zu erreichen versprachen.

Der Rationalitäts- und Kohärenzzwang der bürgerlichen Öffentlichkeit vor dem Hintergrund translokaler Ungleichheitsverhältnisse machten die Formulierung von Forderungen zu einem spannungsreichen Prozess, in dem verschiedene Machtrelationen sichtbar wurden. So strukturierten dominante Diskurse um Sport und Geschlecht vor, welche Erfahrungen sich zu Forderungen politisieren ließen und welche ausgeklammert wurden; die unterschiedlichen lokalen rechtsstaatliche und politische Kontexte entschieden mit darüber, wie Probleme benannt und welche geschlechterpolitischen Instrumente gefordert werden konnte. Der translokale Kontext des Expertinnenforums ließ die Situiertheit dieses Vorgehens sichtbar werden und zeigte, wie dominante Formationen durch NGO-Handeln regierten: In DFs Vorgehen hatten sich spezifische Vorstellungen von liberaler, bürgerlicher Öffentlichkeit und von bürokratischen, rechtsstaatlichen Demokratien sowie liberalfeministischen Instrumenten eingeschrieben. Diese dominante politische Imagination leitete maßgeblich die Projektentwicklung und das Vorgehen an. Während die Aktivist_innen aus ihrem Wunsch nach gleichberechtigter $\mathrm{Zu}$ sammenarbeit heraus auf größtmögliche Partizipation der Teilnehmenden setzten, eine Deklaration gemeinsam erarbeiten und selbst nur den Rahmen stellen wollten, geriet aus dem Blick, wie sich die Situiertheit bereits in den organisatorischen Rahmen eingeschrieben hatte: Dass es einer kohärenten Einstimmigkeit bedurfte, dass Erfahrungen relevant waren und dass diese gleichheits- und nicht differenzfeministisch gedeutet und das Leistungsprimat nicht in Frage gestellt wurde, dass Sportverbände als stabile Institutionen und nicht als Gefüge persönlicher Beziehungen adressiert wurden und liberalfeministische Forderungen nach Anerkennung und gleicher Teilhabe als einzige realistisch erschienen, galt unter den Organisator_innen und vielen Teilnehmenden als Selbstverständlichkeit. Vor diesem Hintergrund erscheint das Stellen von Forderungen als ein machtvoll strukturierter Prozess: Zum einen intervenierten sie so erfolgreich in die Geschlechterverhältnisse und konnten diese teilweise deutlich weiter verändern, als es von den Verbänden gewünscht gewesen wäre; zum anderen stabilisierten die Aktivist_innen domi- 
nante Konzepte des Liberalismus, der bürokratischen Herrschaft oder auch des Leistungsstrebens, indem sie als international agierende NGO die Politiken regierender westlicher Institutionen implementierten.

Nachdem ich in diesem Kapitel jene Vorgehensweise untersucht habe, die sich auf eine spezifische politische Öffentlichkeit bezog, konzentriere ich mich im folgenden Kapitel auf ein Vorgehen, das sich dieser Öffentlichkeit ein Stück weit entzieht. So entsteht Raum für radikalere, utopischere Umarbeitungen des Fußballs, während sich zugleich die Frage nach der breiten Wirksamkeit dieses Vorgehens stellt. 


\section{Alternativen präfigurieren}

Mit dem Fahrrad strample ich von der Katzbachstraße kommend den leichten Anstieg am Eingang des Kreuzberger Viktoriaparks hoch und biege in die Einfahrt zum WillyKressmann-Stadion ein. Das große Eisentor am Ende der langen Einfahrt ist geöffnet und eine Kabelleiste auf dem Boden deutet an, dass hier eine größere Veranstaltung stattfindet. Dann sehe ich auch das „DISCOVER FOOTBALL“-Plakat am Tor. Eine tätowierte Frau mit DF-Crew-Shirt kontrolliert den Eingang. Zu dem vertrauten Grün des Rasens, dem Steingrau des Bodens und der Tribüne und dem Karminrot der Tartanbahn gesellt sich eine für den Fußball untypische, hier jedoch omnipräsente Farbe: Pink. Entlang der Umzäunung der Sportfläche sowie an der großen Bühne neben dem Eingang hängen pinke Stoffstreifen schlaff in der brütenden Nachmittagshitze, die im Schatten gelegene Steintribüne ist mit Sitzkissen in sämtlichen rosaroten Tönen bequem gemacht worden und die Stühle vor der Bühne wurden noch in letzter Minute in pinker Farbe lackiert. Hinter der Bar räumen ein paar Helfer_innen im pinken Volunteer-T-Shirt Flaschen einer fair gehandelten Limonade, Säfte und Biere in die Kühlschränke, am Merchandise-Stand arrangiert Thea die bunten T-Shirts und pinken Stofftaschen, Panini-Hefte und Buttons ordentlich für den Verkauf und auf der Tribüne verfolgen etwa 60 Zuschauer innen das Spiel auf dem Platz. Ich gehe vorbei an der interaktiven Ausstellung, wo sich gerade vier Spielerinnen aus Tansania fotografieren lassen, um morgen bereits als Fotos selbst Ausstellungselemente zu sein, und setze mich zu Thea und einer Gruppe von Spielerinnen aus Argentinien, Brasilien und Griechenland auf die Steinstufen. Gerade rechtzeitig, denn keine Minute später fällt das erste Tor des Spiels: 1:0 für das Team in Grün. Das Grüne Team sowie die gesamte Tribüne jubeln. Unerwartet schwillt der Jubel an, als sogar das gegnerische Rote Team einen Freudentanz aufführt! So viel Fairness wird mit Applaus belohnt. Im Fortgang des Spiels wird die Überlegenheit des Grünen Teams immer deutlicher: Eingespielte Pässe und Laufwege, gekonntes Dribbling und entschlossene Torschüsse. Nach jedem Tor fallen sich die Spielerinnen jubelnd in die Arme, bei gekonnten Passkombinationen grinsen sie sich gegenseitig zu, und das Ausspielen der Gegnerinnen wird mit einem schelmischen Lächeln quittiert. Das Rote Team ist deutlich unterlegen, bei einigen klappt es mit der Ballannahme und dem Stellungsspiel noch nicht so recht. Das Publikum, vor allem andere Spielerinnen, Trainer innen sowie Freund innen und Bekannte der Organisator innen, versuchen mit Anfeuerungsrufen auszugleichen - „Red! Red! Red!“ - und belohnen die Grünen Tore dennoch mit begeistertem Applaus. Abpfiff, 4:0 für Grün. Aus den Lautsprechern ertönt Gerits Stimme. Sie kündigt die bevorstehende Veranstaltung an: „In zehn Minuten werden Spielerinnen aus Norwegen, Brasilien und Burkina Faso auf der Bühne über Erfahrungen als Frauen im Fußball und über die Arbeit ihrer Projekte berichten“.

Die Vignette schildert einen typischen Moment während des DF-Festivals. Auf die drei Workshop-Tage folgte das öffentliche Programm im Stadion mit Turnierspielen, Podiumsdiskussionen, Infoständen, Ausstellungstafeln und kulturellem Rahmenprogramm. Vieles war, verglichen mit Turniersituationen im Amateur- oder Profisport, ungewohnt: Eine Frauenstimme ertönte aus den Lautsprechern, die Farbe Pink schmückte das Stadion, konkurrierende Teams unterstützten sich gegenseitig, das Publikum war allparteilich und Spielerinnen am Mikrophon wurden um explizit politische Statements gebeten. Zu- 
gleich war vieles vertraut: Der Fokus lag auf dem Fußball, das Spiel auf dem Rasen folgte den gültigen Fußballregeln, und die Fußballerinnen wie das Publikum jubelten nach erfolgreichen Spielzügen. Durch eine spezifische Platzierung einzelner Elemente schien die Folie des internationalen Profifußballs der Männer in der Gestaltung des Stadionraums durch: MerchandiseStand, Panini-Hefte mit Stickern der Spielerinnen, Getränke- und Essensstände, Stadiondurchsagen und Spielkommentierung, große Banner an den Seitenrändern, Zuschauertribüne und ein zusätzliches Unterhaltungsprogramm glichen dem Eventcharakter von Fußballspielen und -turnieren der Männerprofis.

Auf den ersten Blick wird deutlich, dass es sich hier um eine Fußballveranstaltung handelt. Und doch ist etwas anders. Durch die Gestaltung des Stadionraums erfuhr eine Frauen-Amateurbegegnung eine unübliche Inszenierung. ${ }^{149}$ DF inszenierte eine Zukunft und damit das Ziel ihres feministischen Projektes so, wie es Linda in der Zukunftsdiskussion formuliert hatte: „dass der Frauenfußball irgendwann genauso ernst genommen werden wird wie der Männerfußball“. Zugleich modellierte DF die Folie des internationalen Männer-Profifußballs hin zu einem feministischen, solidarischen Gegenentwurf und realisierten damit, was Annike vorgeschlagen hatte: „Es geht darum, eine bessere Welt mitzugestalten und das umzusetzen, woran man glaubt.“ Die Umsetzung besserer Welten beziehungsweise die Darstellung konkreter politischer Alternativen und gesellschaftlicher Veränderungen durch Protestbewegungen und Alternativkulturen wird in der sozialwissenschaftlichen Bewegungsforschung als präfigurative Politik untersucht (bspw. Day 2004; Sharpe 2008, 227f.). Dadurch dass die Aktivist_innen den Gästen ihres Festivals eine andere Art, das soziale Miteinander im Fußball zu gestalten, präsentierten, ergänzten sie das Vorgehen, einflussreiche Institutionen mit Forderungen zu adressieren, und schufen ein fußballerisches Event entsprechend ihren eigenen Vorstellungen. ${ }^{150}$ Zukunft wurde nicht nur im Sinne einer capacity to aspire imaginiert und aspiriert, sondern performativ in der Gegenwart präsent und erlebbar gemacht (Anderson 2010, 783).

149 Bei Amateurbegegnungen, auch wenn sie in hohen Ligen stattfinden, ist gewöhnlich keine Presse anwesend, selbstredend gibt es auch keine Panini-Hefte, und das Publikum beschränkt sich in der Regel auf wenige Bekannte oder Verwandte.

150 Während Sharp (2008) ein politisch motiviertes Musikfestival als „leisure politics“, also als einen positiven und optimistischen Gegenentwurf zu den Figuren ,Widerstand“ oder , Rebellion' beschreibt, konzentriere ich mich nicht weiter auf die Verbindung von Freizeit und Politik, obwohl dies auch für mein Feld interessante Einsichten bringen würde. Zu ,,vergnügtem Protest“ als neue hybride Form zivilgesellschaftlichen Engagements siehe Betz (2016). Über die Festivalisierung von Politik, die politische Rolle internationaler Spektakel sowie die politischen Interessen hinter diesen siehe den Sammelband von Merkel (2014). Mit Sicherheit ist die Hochkonjunktur von Festivals und Events für die Realisierung von DFs Projekt wichtig, und vermutlich hat die Festivalisierung politischer Inhalte einen ähnlichen Effekt auf ihre Akzeptanz wie die in Kap. 11.1.5 beschriebene Versportlichung. 
Die räumliche Dimension solcher politischen Visionen und Gegenentwürfe wird mit Foucaults Begriff der Heterotopien greifbar, wodurch sich die Planung und Durchführung eines solchen Ereignisses als raumbezogenes politisches Handeln untersuchen lässt. Als Heterotopien bezeichnet Foucault „wirkliche Orte, wirksame Orte, die in die Einrichtung der Gesellschaft hineingezeichnet sind, sozusagen Gegenplatzierungen oder Widerlager, tatsächlich realisierte Utopien, in denen die wirklichen Plätze innerhalb der Kultur gleichzeitig repräsentiert, bestritten und gewendet sind" (1992a, 73). Präfigurative Politik im Sinne der Gestaltung konkreter Alternativen und Umsetzung eigener Zukunftsaspirationen lässt sich mit Foucault als „Kompensationsheterotopie" beschreiben, die die gesellschaftliche Funktion erfüllt, sich als Gegenraum zum herkömmlichen gesellschaftlichen Raum zu präsentieren (1992a, 45).151 Um zu untersuchen, wie genau Alternativen und aspirierte bessere Zukünfte präfiguriert werden, greife ich auf den Raum als beobachtungsleitende Kategorie zurück. Ich gehe dabei von der räumlichen Verfasstheit der Geschlechterverhältnisse sowie von Geschlechterpolitik als immer auch räumlicher Praxis aus. Ich betrachte im Folgenden das Frauen-FußballKultur-Festival von DF vor dem Hintergrund eines relationalen und prozessualen Raumverständnisses, wie es Martina Löw (2001) formuliert hat.

Der physische Raum wird dabei nicht lediglich als Materialisierung sozialer Ordnung, der Raumhandeln anleitet, sondern als zugleich durch Praktiken hervorgebracht gedacht. Das Verhältnis zwischen physischem und sozialem Raum erscheint damit als wechselseitig. So können die Effekte räumlichen Handelns auf soziale Ordnungen untersucht und die Gestaltung konkreter Orte als geschlechterpolitische Praxis, die auf die sozialräumlichen Geschlechterverhältnisse des Fußballs wirkt, gefasst werden.

Als zumeist geografisch lokalisierbare Materialisierung unterscheidet Löw Orte von Räumen (2001, 198f.). Gebaute Orte sind Resultate vergangener materieller und symbolischer Handlungen (ebd., 153), in ihnen verfestigen sich soziale Ordnungen und Kämpfe zu Arrangements oder Architekturen. Spacing und Synthese beschreiben dabei die Prozesse der aktiven Raumkonstitution: Indem Akteur innen aktiv Dinge und andere Menschen zueinander platzieren (Spacing), schaffen sie konkrete, gebaute Orte. Wahrnehmungs-, Vorstellungs- und Erinnerungsprozesse fügen die Anordnungen zu einem zusammengehörigen, sinnvollen Ensemble zusammen (Synthese) (ebd., 158ff.). Zugleich wirkt die Materialität auf die Praktiken der Anordnung und Deutung, sie bietet nicht nur Möglichkeiten, sondern setzt der Gestaltung und Umgestaltung auch Grenzen (vgl. Niewöhner 2014, 19). Mit der Betonung der prozessualen Dimension der Raumgestaltung durch materiell-

151 Foucault (1992a) begrenzt heterotopische Räume nicht auf politisch und gesellschaftlich visionäre Räume, sondern bezeichnet bspw. auch Gefängnisse als Heterotopien. Der Begriff des Gegenraums wird bei ihm also keinesfalls normativ gedacht im Sinne progressiver politischer Utopien. 
semiotisch affizierte Praktiken wird nicht nur die Reproduktion von Räumen und die in ihnen materialisierte soziale Ordnung, sondern ebenfalls ihre aktive Umgestaltung in Form von kreativen und transformativen Spacing- und Synthese-Prozessen greifbar. Präfiguratives politisches Handeln kann so als raumbezogenes Handeln durch konkrete Spacing- und Synthese-Praktiken gedacht werden, das stets eine materielle und eine symbolische Dimension besitzt.

Im Folgenden zeige ich, wie DF das Stadion mithilfe materiellsemiotischer Raumgestaltung als spezifische Hetereotopie konstituierte, seine dominanten Bedeutungen modifizierte und die aspirierte Zukunft eines feministischen Fußballs im Stadion körperlich erlebbar machte. Während beim Festival bestimmte Elemente des fußballerischen Raums wiederholt, andere in ihrer Zitation leicht abgeändert und wiederum andere völlig verfremdet wurden, wurde das Stadion als ein anderer fußballerischer Raum hervorgebracht. Mich interessiert dabei, wie das Festival als heterotopischer Gegenraum zum Alltag erlebbar gemacht wurde (17.1) und wie und in welchem Möglichkeitsrahmen dabei fußballerische Selbstverständlichkeiten - seien es der Leistungsgedanke (17.2), die politische Neutralität (17.3) oder die Geschlechterverhältnisse (17.4) - umgestaltet wurden. Mit Blick auf unterschiedliche Weisen des Raumerlebens kann ich anschließend Bedingungen für das Funktionieren heterotopischer Räume herausarbeiten (17.5). Abschließend diskutiere ich die mobilisierende und transformative Wirkung präfigurierender Politik (17.6).

\subsection{Raumzeitliche Gegenwelt aufbauen: „So ein Inselgefühl“"}

Nach Ende das Festival 2015 hielt ich meinen Gesamteindruck fest:

Die letzte Woche war fast surreal: durch die Hitze, die Schwüle, die Abgeschiedenheit und Abgeschlossenheit. Obwohl nicht immer alle Spieler_innen die ganze Zeit über im Stadion waren, [...] fühlt es sich für mich dennoch wie eine eigene, in sich abgeschlossene kleine Welt an, mit eigenen Regeln und einer besonderen Atmosphäre. Wie auch andere Crew-Mitglieder bemerkten: Die Zeit verging wie im Flug. Manche kamen bereits morgens für das Mädchencamp ins Stadion oder hatten gar die Nacht im Stadion verbracht, die Restlichen trudelten um die Mittagszeit oder am frühen Nachmittag ein. Auf einmal ist es drei Uhr, beim nächsten Blick auf die Uhr ist es neun und dann ist es auch schon elf und Zeit, sich auf den Heimweg zu machen; nur um zu schlafen, zu duschen, das Allernötigste zu erledigen und dann wieder ins Stadion zurückzukehren. Wenn die Hitze allzu unerträglich wurde, sprangen wir unter die Rasensprenger oder steckten den Kopf unter das kalte Wasser aus den Wasserhähnen der Stadiontoilette. Wie in Trance wandelte man zwei Mal täglich zu den aufgebauten Essensständen, die jeden Tag das Gleiche verkauften, und wählte eines der heißen, 
fettigen Gerichte aus. Die Zeit verflog, ohne dass ich irgendwas geschafft hätte, so mein Eindruck. Alles erinnerte mich an den Titel eines Films über einen bekannten Berliner Club: „Tage außerhalb der Zeit““. (Feldnotiz, 28.6.2015)

Während des Festivals hatte ich das Gefühl, dass die Stadionatmosphäre meine Wahrnehmung absorbierte und ich mich wie „in Trance“ durch die Woche bewegt hatte. Nicht nur fürchtete ich, durch die eigene Ergriffenheit auf meinem ethnografischen Auge zu erblinden, ich wurde auch ständig an die Ausschnitthaftigkeit meiner Perspektive erinnert: Vergeblich hatte ich versucht, alle Ereignisse und Eindrücke mit meinem Laptop festzuhalten. Ich machte während des Festivalprogramms am Nachmittag und Abend Notizen und ergänzte diese jeden Morgen zu Hause. Die schiere Menge an gleichzeitig stattfindenden kleinen Ereignissen und Interaktionen in einem großen Stadion, in dem sich stets zwischen 50 und 2.000 Personen aufhielten, ließ mich mit dem Beobachten und Aufschreiben nicht hinterherkommen. Meine Aufmerksamkeit konnte nie überall zugleich sein und meine Finger nicht so schnell tippen, wie meine Sinne Eindrücke aufnahmen.

Um den verzerrenden Effekten der schieren Reizüberflutung sowie der eigenen Ergriffenheit zu begegnen, hatte ich zwei Bekannte und erfahrene Ethnografinnen gebeten, mich ins Stadion zu begleiten und Feldnotizen zu schreiben. Beide kannten DF und waren mit dem Festivalformat und seinen Zielen vertraut, sodass sie bereits Vorwissen mitbrachten, jedoch nicht wie ich die Perspektive der Organisatorin hatte. Von Almut Sülzle erhielt ich folgende Feldnotiz:

\begin{abstract}
„Das Erste was ich sehe: ein Plakat, das mir den Weg weist und doch nicht, weil kein Pfeil drauf ist. Was auch nicht wundert, denn ich erinnere mich, dass es zwei Eingänge zum Gelände gibt. Ist also jeder Weg richtig? [...] Den Platz zu betreten ist ein Erlebnis. Die Sonne scheint und ich fühle mich sofort wohl. Die Hektik des Straßenverkehrs fällt sofort von mir ab. Der Raum ist weit, die Luft weich, buntes fröhliches Leben um mich ganz ohne die Anspannung, die ich sonst oft in Menschenmassen oder Fußballstadien spüre [...]. Ich habe das Wichtigste verpasst: Die Eröffnungsrede, die Politiker. Egal, das Schönste ist eh die Stimmung und das Beobachten. Ich versuche etwas zu essen zu holen. Stelle mich von rechts, von links, von rechts an. Immer mehr Spielerinnen mit ihren Märkchen drängen sich an den Stand. Anstatt ungeduldig zu werden, freu ich mich an der guten Laune und dem Trubel um mich herum. Essen also später, erst mal Freundinnen und Bekannte begrüßen [...].“ (Feldnotiz AS, 30.6.2015)
\end{abstract}

Die erhoffte Korrektur fand nicht statt, vielmehr wurde mein positiver Eindruck von einem entspannten und fröhlichen Raum bestätigt. Ich setzte auf die zweite Feldnotiz von Ulrike Richter, doch sie gestand mir, dass sie gerade gar nicht in der Lage sei, etwas zu formulieren:

„Ich komme hier gerade so richtig an und es macht sich bei mir so ein Inselgefühl breit, wie im Biotop. Es ist einfach Alles mit so viel Liebe gemacht, jedes Detail, auch die Namen der Teams, die passende Ausstellung dazu und um mich herum sind so viele verschiedene Sprachen. Ich fühl mich einfach total wohl und genieße die Atmosphäre drum herum." (Feldnotiz, 1.7.2015) 
Abgeschiedenheit, weiter Raum und Biotop, Tage außerhalb der Zeit wir alle erlebten das Stadion auf eine für einen zentralen städtischen Raum untypische Weise. Das Stadion ist ein öffentlicher Ort mitten im Zentrum eines der belebtesten Kieze Berlins, und doch wurde es hier als eine ,in sich abgeschlossene kleine Welt", eine „Insel“, auf der „Hektik und Trubel“ vergessen wurden, erlebt. Anstatt weiterhin meiner Wahrnehmung zu misstrauen, nehme ich unsere drei Eindrücke zum Ausgangspunkt, um den erlebten zeitlichen wie räumlichen Bruch mit dem Alltag und der umgebenen Stadtlandschaft näher zu untersuchen.

\subsubsection{Eine raumzeitliche Unterbrechung des Alltags}

Foucault bemerkt, dass Heterotopien häufig an Zeitschnitte gebunden sind und „die Menschen mit ihrer herkömmlichen Zeit brechen“" (1992a, 43). Dieser Bruch entstand beim Festival dadurch, dass Teilnehmende wie Organisierende ihre Alltage für eine Woche verließen und sich auf den vorgegebenen Zeitplan einließen. Wenn möglich, nahmen die Organisator_innen Urlaub von der Lohnarbeit, legten die Seminararbeit für die Uni beiseite und suspendierte alle sonstigen Verpflichtungen. Der reguläre Alltag schien für eine Woche stillzustehen. ${ }^{152}$ Die Architektur des Stadions verstärkte den erlebten Bruch mit dem Alltag. Eine hohe Umzäunung und dichte Büsche machten das Stadion von außen schwer einsehbar und hielten das Straßengeschehen fern, und die Tribünen lenkten die Aufmerksamkeit in die Mitte des Stadions und nicht an seine Ränder. Die Ausstellungselemente und die aufgebauten Infostände an den beiden Eingängen leiteten Besucher_innen in das Stadion hinein. Getränke- und Essensstände, Sanitäranlagen sowie ein in einer Umkleidekabine eingerichtetes Büro mit Internet ermöglichten, den ganzen Tag am Platz zu verweilen. Die Raumgestaltung ergänzte sich hier mit der Stadionarchitektur in der Schaffung einer von der Außenwelt zeitlich und räumlich getrennten Atmosphäre.

Auf fast schon surreale Weise wurde dieser zeiträumliche Bruch durch die Umweltbedingungen verstärkt. Sowohl 2013 als auch 2015 setzte mit Beginn des Festivals die heißeste Woche des Jahres ein. Was ich oben als „Trance“ beschrieb, meint die wetterbedingte Verstärkung der Raumwahrnehmung als zeiträumlichen Exzeptionalismus, da die Hitze die Sinne vernebelte. Mit Ende des Festivals änderte sich jedes Mal auch das Wetter abrupt,

152 Während die Teilnehmenden eine lange Reise, zumeist mit dem Flugzeug, antraten, wurde der Bruch bei den Organisierenden durch einen sich verändernden Arbeitsrhythmus eingeleitet: Vor dem Festival wurde der Takt der regelmäßigen Plena enger, die Zahl der täglichen E-Mails stieg an und die täglichen, drängenden Aufgaben wuchsen. Während dies hier auch als eine graduelle und spannungsaufbauende Phase des Eintritts in den heterotopischen Raum verstanden werden kann, so folgte durch wetterbedingte Veränderungen sowie die Abreise der Teams eine aktive Austrittsphase. 
so auch 2015, als sich mit dem Ende der Siegerinnenehrung die Hitze in einem Gewitter auflöste:

Gerade nachdem der letzte Pokal vergeben worden war, bemerke ich, wie sich am schon dunklen Himmel noch dunklere Wolken zusammenziehen. Jetzt geht es ziemlich schnell, der Wind wird immer stärker, die Planen und Sonnenschirme flattern in den Windböen. Der Druck, zum Ende der Veranstaltung zu kommen, bevor das Unwetter losgeht, liegt deutlich spürbar in der Luft. Kaum sind die letzten Worte gesprochen, springen wir auf und räumen alles zusammen. Wir schicken die Teams in den benachbarten Biergarten. So schnell haben wir noch nie zusammengepackt. Es blitzt. Die ganze Spannung und Hitze der letzten Tage ballt sich in den dunklen Wolken über uns. Wir bringen Pappkartons voller Merchandise-Artikel und Panini-Hefte, die pinken Kissen und die Ausstellungselemente in die Umkleidekabinen, hängen die Banner ab, klappen die Bierbänke zusammen und bringen die pinken Stühle unter das Vordach der Bühne. Müllsäcke werden zugezogen und auf einen Haufen geworfen. Einige räumen die technischen Bühnenelemente zusammen. Der Wind weht alles, was nicht niet- und nagelfest ist, über das Gelände. Irgendwann fallen die ersten Tropfen. Wir haben es geschafft. Im Regen schließen wir unsere Fahrräder auf und eilen ebenfalls rüber in die überdachten Räume des Biergartens, wo die Teams bereits die Tanzfläche gestürmt haben. (Feldnotiz, 7.7.2015)

Der plötzliche Wolkenbruch markierte das abrupte Ende der Festivalwoche: Die flimmernde Hitze und die warmen Sommerabende wurden durch Blitz, Donner und Regen für beendet erklärt. Bis zum nächsten Morgen blieben nur die großen Elemente wie Bühne und Bar sichtbar übrig, dann wurden auch sie abgebaut und abtransportiert. Architektur, aktive Raumgestaltung und Umweltbedingungen synthetisierten das Stadion zu einem zeiträumlich abgeschlossenen und abgetrennten Raum und kreierten im Zusammenspiel eine besondere Atmosphäre, die als zeiträumlicher Bruch mit dem Alltag, seinen Emotionen, Aufgaben und Pflichten erlebt werden konnte. Insofern Umwelt selbst als materiell-semiotische Akteurin und nicht lediglich als Ressource sichtbar wird (Haraway 1995), erscheint die Herstellung heterotopischer Räume als spannungsreiche Kooperation menschlicher und nichtmenschlicher Akteur_innen.

\subsubsection{Die präfigurative Funktion heterotopischer Räume}

Nach dem Festival 2016 schrieb mir Ariane, die selbst nicht in Berlin lebte, sondern nur für die einzelnen Projekte anreiste, eine E-Mail:

„Ich bin am Dienstag wieder abgereist, nachdem ich dieses Mal endlich zum Abbau geblieben bin. Das war sehr gut, sonst hatte ich immer Montag morgens um 4:30h den Zug nach Hause genommen und fühlte mich dann so rausgeworfen. Aber auch so war die Rückkehr nicht leicht, ich fühle mich nach DF immer so wahnsinnig fremd hier. Nosiba hat mir gestern geschrieben: ,Ya I get back home but I feel like there is some- 
thing wrong. I feel like this is not my life. "Das kann ich so gut nachempfinden, so geht es mir auch immer." (E-Mail, 9.9.2016)

Die Teilnehmerin Nosiba aus dem Sudan und die Organisatorin Ariane beschrieben beide den Bruch mit dem Alltag und die Flüchtigkeit des Ereignisses. Foucault hat diesen zeiträumlichen Bruch als Bedingung für das gesellschaftliche Funktionieren von Heterotopien beschrieben (1992a, 43). An Nosibas Nachricht und Arianes Bericht, dass sie die Rückkehr aus Berlin als „Rauswurf" in einen „falschen“ Alltag erlebten, lässt sich dieses Funktionieren als präfigurative Politik genauer verstehen: Mit dem heterotopischen Raum in Form des Festivals wurde eine Vision von einem feministisch, richtigen' fußballerischen Raum - im Gegensatz zum ,falschen' fußballerischen Raum, den sie aus ihrem Alltag gewöhnt waren - erschaffen und erlebbar gemacht. Zukunft wurde in die Gegenwart eingefaltet ,through a range of utopic sensibilities, skills and techniques“, die ,contestable visions of possible or not-yet spatial futures" aktualisierten (Anderson 2010, 703, Herv. i. Orig). Foucault betont, dass Heterotopien tief in die Gesellschaft eingelassen sind, nicht außerhalb, sondern nur in ihr existieren und stets auf sie bezogen sind (1992a, 41). Dem folgend erscheint es mir plausibel, die präfigurierte Vision fußballerischer Räume als eine vergegenwärtigte Zukunftsaspiration oder Gegenwelt zum gewohnten (fußballerischen) Alltag zu verstehen. Die Konstitution eines Gegenraums ist auf das Engste mit den herkömmlichen fußballerischen Räumen verflochten, denn es wird auf gewohnte Elemente entweder aneignend oder modifizierend - Bezug genommen sowie mit Selbstverständlichkeiten gebrochen.

Die Einlassung in die gesellschaftliche beziehungsweise fußballerische Ordnung bedeutet auch, dass diese die Begrenzungen des heterotopischen Raums mitbestimmt. Zum Festival 2013 reisten unter anderem Nationalspielerinnen aus Ägypten, Jordanien und Palästina an. Der einwöchige Turniermodus sah Spiele auf dem Kleinfeld vor, sodass ein verkleinertes Spielfeld auf dem Großfeld markiert werden musste. Allerdings wurde es den Organisator_innen untersagt, diese Markierung mit Farbe auf den Rasen zu zeichnen, denn diese würde die Spieler des in der folgenden Woche dort stattfindenden Landesligaspiels der Männer irritieren. Stattdessen wurde das Spielfeld mit orangenen Hütchen behelfsweise abgesteckt, die keine Spuren hinterließen. An dieser kleinen logistischen Angelegenheit zeigt sich nicht nur die Flüchtigkeit der Heterotopie, sondern auch, wie ihre Grenzen durch die Bedingungen jener Räume abgesteckt werden, zu denen sie eine Alternative darstellen.

153 Ariane bemerkte, dass sie durch das Abbauen den Bruch weniger abrupt erlebte. Teilnehmende, indem sie die langen Rückreisen antraten, Organisierende, indem sie abbauten, und beide gemeinsam, indem sie am letzten Abend im benachbarten Biergarten feierten, kehrten relativ langsam in ihre Alltage zurück. 
Durch den deutlichen Austritt aus dem Alltag und die klaren zeitlichen und räumlichen Begrenzungen wurde der präfigurierte Kontrast zum Alltag besonders intensiv erlebt. Darauf aufbauend befrage ich im Folgenden die drei Praktiken der Raumgestaltung - den Leistungsgedanken modifizieren, Fußball politisieren und Geschlechterverhältnisse umkehren - darauf, wie sie mit den Selbstverständlichkeiten fußballerischer Räume brechen und ein ,richtiges' Bild vom Fußball präfigurieren.

\subsection{Leistung modifizieren: „Es geht nicht nur um Fußball“}

Kräfte- und Leistungsmessen durch Wettkampf als zentrale Austragungsform des Verbandssports (siehe Kap. 4.1.2) sind dem Stadion als architektonischem und kulturellem Ort eingeschrieben. Stadien entstanden im antiken Griechenland als Orte zur Durchführung sportlicher Wettkämpfe. Dabei bezeichnete Stadion zunächst eine 600 Fuß lange Strecke und den entsprechenden läuferischen Wettkampf. Nach und nach bildete sich eine spezifische Architektur für die Wettkämpfe heraus, und der Begriff Stadion bezog sich nun auf die Bauwerke für das Publikum, während der Austragungsort der sportlichen Wettkämpfe als Dromos (Laufbahn) bezeichnet wurde (Kratzmüller 2005, 92). Ich gehe davon aus, dass diese Nutzungstradition, die auch die Anordnungen von menschlichen Körpern (Sportler_innen, Publikum, Kampfrichter_innen etc.) und Dingen (Startblöcke, Tribünen, Laufbahnen etc.) beinhaltet, das Stadion mit der Bedeutung des Leistungsprinzips kodiert und es als „Ort der permanenten Inszenierung und Dramatisierung von Überlegenheit und Scheitern, einer Zurschaustellung von Leistungsfähigkeit, trainierten Körpern, Männlichkeit und Dominanz" (von der Heyde/Kotthaus 2016, 3) konstituiert. Durch die primäre Nutzung der öffentlichen Sportstätten durch den Verbandssport und entlang dessen kodifizierter Regeln institutionalisiert und formalisiert sich der Wettbewerb als Format und Prozess des Messens und Belohnens von Leistung im Stadion. Während des Festivals machte sich eine Spannung zwischen fußballerischem Leistungs- und Wettkampfprinzip und dem feministischen Projekt der möglichst breiten Inklusion sowie gegenseitigen Bestärkung und Unterstützung bemerkbar. Diese Spannung tritt auch bei anderen alternativen Sportveranstaltungen auf, die das Leistungsprimat zugunsten gegenseitiger Solidarität zu brechen versuchen. Elspeth Probyn (2000) erinnert an den Gründer der Gay Games und ehemaligen Profisportler Tom Weddell, der den LGBTI-Sportspielen eine Umdeutung des Leistungsbegriffs zugrunde legte: Nicht länger ginge es um das gegenseitige Besiegen, sondern nur um die persönliche Bestleistung. In diesem Sinne könne jede_r siegen. Probyn kritisiert dies als rein rhetorische Verschiebung des Wettkampfs in eine andere Sphäre. Der intrapersonelle 
Wettkampf um Höchstleistung bliebe hinter dieser Beschönigung verborgen. Davide Sterchele und Chantal Saint-Blancat (2013) beobachten bei dem antirassistischen internationalen Fußballturnier Mondiali Antirazzisti in Italien, dass eine Abschwächung des Leistungsprinzips zugunsten gegenseitiger Solidarität und Gemeinschaft möglich ist, es aber direkter Eingriffe in das Reglement des Wettbewerbs bedarf: Die Organisator_innen begegneten dem sportlichen Ehrgeiz und damit einhergehenden Konflikten mit Regeländerungen. Halbfinale und Finale wurden z.B. als Elfmeter-Schießen ausgespielt, so dass Glück entscheidender wurde als Leistung, und der größte Pokal zeichnete nicht das Siegerteam, sondern jenes aus, das am besten den gesellschaftspolitischen Gedanken des Festivals lebte. Diese Beobachtungen deuten darauf hin, dass der Spannung zwischen Leistung und Solidarität auf rhetorischer Ebene nur schwer beizukommen ist und es strukturell-organisatorischer Veränderungen bedarf, die Menschen und Dinge im Raum umordnen und diese Umordnung explizit mit Bedeutung versehen.

\subsubsection{Vom Wettkampf zu Fairness und Solidarität}

Ebensolche strukturellen und organisatorischen Umgestaltungen nahm DF, angeregt durch feministische und sozialwissenschaftliche Kritik am Wettkämpferischen (siehe Kap. 4.2.4), mit der schrittweisen Implementierung neuer Spielregeln vor. Im Zentrum des Festivals stand schließlich ein fünftägiges Kleinfeldturnier, das den Regeln des globalen Verbandsfußballs folgte. Während der Festivalvorbereitungen 2013 wurden erste Änderungen in der Siegerinnenehrung geplant:

Auf einem der letzten Plena vor Beginn des Festivals wird der Turniermodus besprochen. Celia leitet diesen Punkt ein und fragt in die Runde, welche Preise neben der regulären Platzierung der teilnehmenden Teams ausgeschrieben werden sollen, also was gewürdigt werden solle. Sie hätten sich bereits in einer Kleingruppe darüber Gedanken gemacht und folgenden Vorschlag entwickelt: Schönstes Tor anstatt beste Torschützin, sensationellste Parade anstatt beste Torhüterin, Fair Play und Stimmungskanone. Annike hatte schon per Mail geschrieben, dass sie eine Kategorie gut fände, in der das Publikum entscheiden könne. Und dass sie auch die Kategorie „Beste Spielerin“ beibehalten möchte, da es immer die größte Auszeichnung sei. Thomas ergänzt, dass auch die Kategorie „,beste Torhüterin“ wichtig sei, um die Leistung zu würdigen. Gerade weil der Job der Torhüterin so unbeliebt sei. Luca wendet ein: „Ich finde es gut, den Leistungsaspekt etwas abzudämpfen. Das wird von Teams immer so ernst genommen, da ist es gut, dem was entgegenzusetzen.“ Simone stimmt zu: „Der Sinn ist ja auch, die zu würdigen, die nicht so gut spielen. Das ist ja auch ein soziales Projekt hier und es geht nicht nur um Fußball. So ehrt man eine einzelne Leistung und nicht die gesamte Fähigkeit, dann kann auch eine gewinnen, die sonst nicht so gut ist oder die früh [aus dem Turnier] rausgeflogen sind.“ Thomas gibt zu bedenken: „Jedes Spiel ist dazu da, gewonnen zu werden.“ Aber Linda wiederspricht: „Wir würden es uns schon wünschen, wenn es mehr miteinander und weniger gegeneinander wäre.“ (Feldnotiz, 26.6.2013) 
Celia versuchte mit ihrem Vorschlag die Aufmerksamkeit von der Gesamtleistung einer Spielerin hin zu einzelnen Aktionen zu lenken, sodass auch jene Aussichten auf Ehrungen hätten, die über weniger sportliches Kapital verfügten. Die Kategorien Stimmungskanone und Fair Play sollten zudem verdeutlichen, dass es, wie Simone sagte, „nicht nur um Fußball geht“. Das „soziale Projekt" drückte sich hier im Fair-Play-Gedanken sowie im Miteinander und der gemeinschaftlichen Stimmung aus. Der Vorschlag zielte darauf $a b$, der integrativ-solidarischen und kompetitiven Dimension gleiches Gewicht zu geben, also eine Balance herzustellen. Wie gravierend dieser Bruch mit den Gewohnheiten des Fußballs sein würde, deutet sich in Thomas und Annikes Bedenken: „Jedes Spiel ist dazu da, gewonnen zu werden“, und die Auszeichnung als beste Spielerin wird nicht nur erwartet, sondern auch als größte Ehrung verstanden. Dennoch einigte sich Gruppe schließlich auf den von Celia unterbreiteten Vorschlag.

Zwei Jahre später erfuhr der integrative und solidarische Gedanke noch stärkere Betonung. Anstatt sie gegeneinander antreten zu lassen, mischte DF die angereisten Teams neu. So spielten in einem Team bis zu vier verschiedene Nationalitäten, das jeweils von einem bi-nationalen Trainer_innentandem geleitet wurde. An die Stelle nationaler oder lokaler Bezüge im Teamnamen traten nun Farben, sodass im Sprachgebrauch Grün gegen Rot und nicht wie im Vorjahr etwa Ägypten gegen Jordanien antrat. Im Vorfeld konnten die Organisator_innen jedoch nicht die Spielstärken der Spielerinnen abschätzen, weshalb das Risiko bestand, ungleich starke Teams zu schaffen und dadurch Frustration auszulösen. Um Missmut und Enttäuschungen entgegenzuwirken, teilten sie den Teams frühzeitig diesen ungewöhnlichen Turniermodus mit. In der E-Mailkommunikation betonten sie, dass der Gedanke des Austauschs und des Brückenbauens im Vordergrund stehen sollte. Sie schickten ihnen die schriftlich fixierten und in verschiedene Sprachen übersetzten Turnierregeln samt Ablaufplan zu, in denen sie den emanzipativen Gedanken verankert hatten: Unter der Rubrik „Fußballregeln“ befanden sich die üblichen Regeln für ein Kleinfeldturnier. Daran schloss sich die Rubrik „DISCOVER FOOTBALL Regeln“ an, die besonders faires, sprich verletzungsrisikoarmes Spiel ohne Beleidigungen und eine gerechte Verteilung von Spieleinsätzen vorschrieb. Der zweite Punkt sollte sicherstellen, dass alle Spielerinnen in den neugemischten Teams genügend Spielmöglichkeiten erhielten und schwächere Spielerinnen nicht aus Ehrgeiz auf die Ersatzbank verbannte wurden. Zudem wurden Trainer_innen angehalten, auf dem Spielfeld aggressive oder verärgerte Spielerinnen kurzzeitig auszuwechseln, noch bevor die Schiedsrichterin eingreifen musste. Als ich im Zuge der Autorisierung von Zitaten Celia kontaktierte, ergänzte sie meine damalige Analyse und erklärte mir, es ginge darum, dass die Trainer_innen selbst die Initiative zum Fair Play ergriffen und dies nicht anderen überließen. Auch hier beinhalteten die veränderten Regeln eine teilweise Umgestaltung ge- 
wohnter Arrangements und Abläufe, indem sie Personen auf ungewohnte Weise zueinander in Beziehung setzten.

Ähnlich verhielt es sich schließlich bei der Siegerinnenehrung, die ungewöhnlicher Weise in der Ehrung des Fair-Play-Teams anstelle des Siegerinnen-Teams gipfelte. Das Fair-Play-Team war zuvor von allen Teilnehmenden und Organisator_innen per Stimmabgabe gewählt worden. Ausgezeichnet wurde jenes Team, das sich nach Niederlagen besonders fair zeigte, auf der Tribüne aktiv die anderen Teams anfeuerte, Lieder anstimmte und/oder am Abend für gute Stimmung sorgte. Die anschließende Ehrung verschob so das Ziel des Festivals von Sieg und Kräftemessen hin zu Fairness und Miteinander. Als fußballerische Leistung galten damit nicht allein physisches Können und Stärke, sondern solidarisches, sich gegenseitig unterstützendes Verhalten, kurz: Miteinander statt Gegeneinander.

Während in der Plenumsdiskussion zwar gemeinsam festgelegt wurde, inwieweit mit den Selbstverständlichkeiten des Fußballs gebrochen werden könne, reisten die Teilnehmerinnen zunächst mit anderen Vorstellungen und Erwartungen an. Naoual, die 2015 zum dritten Mal an einer DFVeranstaltung teilnahm, erzählte mir, wie sie den veränderten Turniermodus erlebte. In ihrem nordafrikanischen Heimatverein spielte sie im besten Team des Landes ausschließlich leistungsorientiert. Für sie sei das Mischen der Teams eine völlig neue Erfahrung: „When I play, I fight. That's how I am, I always fight. Usually, after we loose I get angry and sad. But here I am not angry and sad." (Feldnotiz, 2.7.2015) Nicht alle reagierten so positiv. Julie, die 2015 das Rote Team betreute und nach dem ersten Spiel feststellte, dass einige der Spielerinnen kaum oder gar keine Fußballerfahrung besaßen, berichtete im Plenum davon:

Sie habe sich in dem Moment auch Sorgen um die einzige brasilianische Spielerin im Team gemacht, denn diese würde sehr gut spielen. Sie habe vorher aber noch nie vor Publikum gespielt. Julie sei besorgt gewesen, ob diese nicht die Geduld verlieren würde, und habe sie gefragt, wie es ihr damit ginge. Im Anschluss habe Julie intensiv mit dem Team über das stark variierende Leistungsniveau geredet und jetzt sei alles in Ordnung. (Feldnotiz, 30.6.2015)

Julies Fall verdeutlicht, dass der Grad der Umgestaltbarkeit unterschiedlich eingeschätzt wurde: Während ein angereistes Team darin die Möglichkeit erkannte, als fußballerisch Unerfahrene teilnehmen zu können, reagierten andere Spielerinnen enttäuscht oder verärgert über die plötzliche Leistungsschwäche. Unzählige kleine Diskussionen am Spielfeldrand, erhitzte Gemüter auf dem Rasen, enttäuschte Mienen nach einer Niederlage oder Unzufriedenheit mit dem Los, einem leistungsschwachen Team zugeordnet worden $\mathrm{zu}$ sein, verdeutlichen die unterschiedlichen Erwartungen, die sie mit einer Fußballbegegnung unter dem Credo des Empowerment verknüpften. Julies Teamgespräch, die schriftliche Fixierung der Regeln und ihre Wiederholung durch Stadiondurchsagen sowie entschieden durchgreifende Schiedsrichte- 
rinnen zeigen, dass das von den Organisator_innen einmal festgelegte Ausmaß der Umgestaltbarkeit stets wiederholt und vermittelt werden musste.

Naouals Erzählung sowie die Enttäuschungen einiger Spielerinnen, wenn ihre Teamleistung unter ihrer eigenen Spielstärke lag, zeigten aber auch, wie die Verinnerlichung und Wirkmächtigkeit des fußballerischen Leistungsprimats über die eigentliche Spielsituation hinaus in die Begegnung abseits des Platzes wirken kann. Die dominanten Formationen des Verbandsfußballs und das damit einhergehende normative Urteil geben über den praktischen Sinn der Akteur_innen und die historische wie gegenwärtige materiell-semiotische Umwelt, die zu Raumnutzungsfähigkeit verschmelzen und Raumhandeln affizieren, die Grenzen der Umgestaltbarkeit vor. Wo genau allerdings diese Grenzen verlaufen, wurde keinesfalls von allen Beteiligten gleich eingeschätzt, sondern situativ und beständig untereinander ausgehandelt. So blieb letztlich die Spannung zwischen feministischen und verbandsfußballerischen Prinzipien bestehen und konnte nur mithilfe von Kompromissen ausbalanciert werden - etwa dadurch, dass bei der Bewerberinnenauswahl sowohl leistungsschwache als auch leistungsstarke Teams gewählt und die Turnierregeln immer wieder kommuniziert und auch mal Ausnahmen gemacht wurden. Welcher Kompromiss letztlich geschlossen wurde, war keinesfalls festgelegt. Er basierte auf einem schrittweisen Austesten und Verhandeln, sodass im Laufe der Jahre und in vielen kleinen Diskussionen und Missverständnissen innerhalb der Organisationsgruppe als auch mit Teilnehmenden nach und nach Regeländerungen erprobt wurden und neue Elemente hinzukamen.

Obwohl diese Veränderungen auch mal zu Missverständnissen und Irritation führten, zeigt Naouals Bemerkungen aber auch, dass die Veränderungen durchaus einen Effekt hatten: Zwar konnte sie selbst den Wettkampf-Modus nicht völlig abstellen, ihn aber wohlgleich zügeln und einen neuen emotionalen Umgang mit fußballerischen Begegnungen finden. Ich beobachtete, wie durch das Neumischen der Teams jede Spielerin angehalten wurde, ihre Loyalität aufzuteilen: Nicht nur fieberten sie für ihr eigenes, multi-nationales Team, sondern auch für all jene Teams, in denen ihre eigentlichen Teamkolleginnen nun spielten:

Das Spiel um Platz 3 zwischen Lila und Blau ist gerade durch Elfmeterschießen knapp für das Lila Team ausgegangen. Obwohl, wer hat noch mal gewonnen? Bei jedem Tor jubelte ich, bei jedem gehaltenen Schuss ebenfalls. Auch alle um mich herum. Die Argentinierinnen im Publikum jubelten den anderen Argentinierinnen im Blauen Team zu, die Griechinnen neben mir der griechische Spielerin im Lila Team. (Feldnotiz, 5.7.2015)

Die ergänzten Turnierregeln setzten dem Konkurrenzdenken, das sonst auch durch nationale Zugehörigkeiten verstärkt wird, Grenzen und machte eine andere Art fußballerischer Begegnung erfahrbar. 


\subsubsection{Entsportlichung? Das Dilemma des feministischen Fußballs}

Sterchele und Saint-Blancat beschreiben ähnliche Maßnahmen bei der Mondiali Antirazzisti. Sie deuten den Effekt des Mischens von Teams und Spielstärken, der Verflüssigung der Trennung zwischen Spielenden und Zuschauenden, die Polyzentrierung der Aufmerksamkeit durch viele verschiedene Partizipations- und Unterhaltungsangebote und des Herunterspielens des Wettkampfs als Entsportlichung $(2013,187)$. Unter de-sportization verstehen sie, „that sports are re-shaped (or de-shaped) into mere games and even less structured forms of play" (Sterchele 2015, 97). Die Wortwahl impliziert, dass damit nicht nur eine Umgestaltung und Umformung des Fußballs, sondern eine Deformierung und Verunstaltung im Sinne einer Verfremdung eines wesentlichen Elements - des Sportlich-Kompetitiven - einhergeht, sodass letztlich nur das Spielerische übrigbleibt. Vor dem Hintergrund, dass Kritik am Wettkämpferischen kaum Plausibilität besitzt, lese ich die Diagnose der Entsportlichung auch als eine moralische Wertung über den ,richtigen' Sport. Diese Wertung übernimmt die Anerkennungslogik und Deutungsweise des Verbandsfußballs, die Fußball als Wettkampfsport und die Praktiken des Leistungsvergleichs als neutral und von gesellschaftlichen Machtverhältnissen unbeeinflusst konstituieren (Kap. 4.2.2), und hinterfragt nicht die immer schon vergeschlechtlichten (und rassialisierten) Dimensionen von Leistung und Wettkampf. Diese in der Stadionarchitektur materialisierte Norm vom Fußball als kompetitives Kräftemessen wird durch Sozialisation im Vereinsfußball erlernt und steckte auch bei DF die Grenzen für mögliche feministische Umgestaltungen ab. Der praktische Sinn der Organisierenden und Teilnehmenden für dieses normative Primat des verbandlichen Wettkampffußballs kommt in der Diskussion um mögliche Auszeichnungen in Annikes und Thomas Bemerkungen zum Ausdruck, die die Selbstverständlichkeit des Gewinnen-Wollens betonten. Im biografischen Interview bemerkte Luca diese Selbstverständlichkeit bei sich selbst:

„Ich komm aus dem Leistungssportbereich und ich merke auch beim Fußball, wenn ich spiele, dass ich lieber mit Spielerinnen zusammen - im Punktspiel, im Training ist mir das völlig egal, da spiel ich mit allen Spielerinnen gerne zusammen - aber im Punktspiel da merk ich, leider ist mir das nicht egal. Und da geht's für mich darum, schönen Fußball zu spielen, und das kann ich persönlich besser mit Menschen, die es besser können. Und das find ich auch irgendwie nicht so cool. Halt eben ganz unfeministisch [...]." (Luca, Interview, 1.3.2014)

Luca verdeutlichte, wie sich der praktische Sinn durch die Sozialisation in den leistungsorientierten Sport in die Wahrnehmung des Sporttreibens eingeschrieben hatte: Schön ist der Fußball vor allem dann, wenn er erfolgreich gespielt wird; Spaß hat Luca am meisten, wenn die Teamleistung im Wettbewerb stimmt. In anderen Diskussionen wurde gegen manche Veränderungsvorschläge auch mit der Begründung ,aber das ist dann kein Fußball 
mehr" argumentiert. Und Kritik an möglicherweise nicht-feministischen oder exkludierenden Effekten des Leistungsmessens wurde mit einem hilflosen „das ist halt Fußball" begegnet.

Neben dem praktischen Sinn für die Grenzen der Umgestaltbarkeit verhindert auch die materiell-semiotische Umwelt des Stadions eine Entsportlichung, denn die spezifische Architektur ist „für die Disziplinierung des Blickes von ,Massen" entworfen“ (Marschik et al. 2005, 10). Zwar ermöglicht das Stadion aufgrund seiner räumlichen Größe eine Dezentrierung der Aufmerksamkeit durch spezifisches Anordnen und Hinzufügen von Dingen, doch strukturiert die Materialität wie auch die tradierte Bedeutung diese Anordnung und ihre Wahrnehmbarkeit vor: Statt die Aufmerksamkeit gleichmäßig und gleichzeitig auf Sport und Rahmenprogramm zu verteilen, wurden die Turnierspiele priorisiert. Die weitaus größte Fläche nahm der Rasenplatz ein, die siebenstufigen Steintribünen an den langen Seiten erforderten eine Sitzhaltung, die die Aufmerksamkeit auf das Leistungsmessen auf dem Platz leitete und Stadiondurchsagen kommentierten die körperliche Leistungen. Das Zusammenwirken des praktischen, über die Sportsozialisation erlernten Sinns mit der materiell-semiotischen Umwelt des Stadions generiert eine räumliche Fähigkeit, die eine Priorisierung und Zentralität des Fußballs erlaubt. Yi-Fu Tuan beschreibt „spatial ability“ als jenes inkorporierte Knowhow der Raumnutzung und des Navigierens im Raum, das immer auch die Fähigkeit impliziert, die Texturen von Räumen lesen zu können (2011, 74f.). Auf das Festival bezogen meint diese Fähigkeit, dass die Anwesenden die Bedeutung des Stadions als Raum des sportlichen Wettkampfes verinnerlicht hatten, es als Ort des Leistungsmessens lasen und gelernt hatten, es durch ihr Verhalten als solchen zu ko-produzieren.

Dieses verbandsfußballerische Wissen traf in der Vorbereitung und Durchführung sodann auf feministische Ideale von Solidarität, Begegnung und gegenseitiger Bestärkung. In Lucas Überlegungen wird der Konflikt zwischen Fußball und feministischem Empowerment deutlich. Diese Spannung wird auch in der Forschungsliteratur sowie in den Frauenbewegungen immer wieder unterschiedlich diskutiert und bewertet. Z.B. zieht Lois Bryson (1994) Parallelen zur Arbeitswelt und argumentiert, dass Frauen im Sport immer in der untergeordneten Position bleiben würden, da eine Anpassung an das Wettkämpferische eine Unterwerfung unter von Männern geschaffene und kontrollierte Bedingungen bedeutet. Differenzfeministisch schlägt sie, ausgehend von frauenspezifischen Interessen und Bedürfnissen, ein separates Sportsystem vor. Claudia Kugelmann (1993) plädiert für eine feministische Sport- und Bewegungskultur, die an den Lebenswelten von Frauen ansetzt und sich mit eigenen Körper- und Bewegungskonzepten grundlegend vom männlich geprägten Leistungs- und Optimierungsprinzip abwendet. Susan Birrell und Diana Richter (1994) argumentieren hingegen auf der Grundlage einer Untersuchung mit feministischen Softballerinnen für die Möglichkeit 
der feministischen Transformation und Aneignung wettkampforientierter Sportarten. Sie verstehen die Umarbeitung der sportlichen Prämissen im Softball als feministische Alternative zum Männersport, nicht weil diese typisch weiblich seien oder nur von Frauen umgesetzt werden könnte, sondern weil diese von Frauen umgesetzt wurden. Und aktuellere, durch Performativitätstheorien und Queer Theory beeinflusste Studien argumentieren für eine Aneignung männlicher Sportpraxen und eine Transgression der Geschlechternormen, wenn Athletinnen ,typisch männliche' Bewegungsabläufe kompetent aufsühren (vgl. Whitson 2002; Gugutzer 2011).

Die Fragen, ob es sich bei der Ausübung männlich geprägter Sportarten durch Frauen um Unterwerfung oder Aneignung handelt und ob es nicht einer eigenständigen Sportkultur bedürfe, können hier nicht abschließend beantwortet werden. Allerdings schließt sich die Frage an, wie eine resignifizierende und damit transgressive Wirkung mit Leistungsstärke verwoben ist, die ich in Kapitel 17.6.3 aufgreifen werde. DF selbst schloss sich jener queertheoretischen Deutung an, die innerhalb des szenisch-städtischen Umfelds von DF verbreitet war, und ließ sich z.B. das Motto „Claim the pitch“ auf TShirts und Sticker drucken. Wie beim Festival deutlich wurde, beinhaltete diese Beanspruchung und Aneignung des Fußballplatzes sowohl die Übernahme einiger bestehender Regeln als auch die Transformation anderer. So ermöglichte die Schaffung eines abgeschlossenen, separaten Raums, dass zeitweilig das Leistungsprimat dezentriert und zugunsten von Solidarität ausbalanciert, nicht jedoch völlig demontiert wurde.

\subsection{Fußball politisieren: „Es lässt sich mit Fußball gut Politik machen"}

Eng verbunden mit der Idee, Leistung im Sinne feministischer Solidarität zu modifizieren, ist die Strategie, Fußball zu politisieren. In Kapitel 11.1 habe ich bereits gezeigt, wie DF Fußball narrativ politisierte. Beim Festival wurde nicht nur durch diskursive, sondern auch mit raumgestaltenden Praktiken mit dem bürgerlichen Sportverständnis gebrochen, denn dieses Verständnis basiert darauf,

„dass zwar ein allgemeines Wissen über die politisch-ökonomische Bedeutung und Beeinflussung des Sports existiert, dass aber das einzelne Sportereignis so konstruiert, inszeniert und auch erlebt wird, als wäre Sport ein von der übrigen Welt abgekoppeltes Terrain“"(Marschik 2004, 111).

Eingezäunte, von der Straße kaum einsehbare und von Tribünen eingerahmte Stadien begünstigen das Inszenieren und Erleben des Sports als politisch neutralen und gesellschaftlich autonomen Ort und scheinen damit sowohl das 
bürgerliche Sportverständnis (siehe Kap. 5.2.1) als auch die gesetzlich festgeschriebene Autonomie des Verbandssports (siehe Kap. 4.3.1) zu materialisieren. Die Vignette zeigt jedoch, dass das Stadion beim Festival als politischer Ort gelebt wurde. Die Stadionsprecherin verwies neben den sportlichen auch auf die gesellschaftspolitischen Leistungen der Athletinnen, nach den Spielen wurden die Spielerinnen nicht von Eurosport, Kicker oder Sportschau nach dem Spielverlauf, sondern von Emma, der Deutschen Welle oder dem RBB nach der frauenrechtlichen Situation in ihren Heimatländern befragt, und auf Podien wurde nicht über Spielstrategien gefachsimpelt, sondern es wurde über die menschenrechtlichen und politischen Situationen in den Heimatländen und deren Auswirkungen auf den Frauenfußball diskutiert. Auf der Bühne rappte eine Künstlerin zum Abschluss des Tages gegen Homophobie, oder ein Film in Kooperation mit der Feminist Film Week porträtierte das Leben junger Frauen in einer Pariser Banlieue. Die Verknüpfung der sportlichen Praxis mit politischen, gesellschaftlichen und geschlechterpolitischen Themen synthetisierte das Stadion zu einem nicht allein sportlichen Raum. Seine Bedeutung wurde im Sinne von Simones Aussage „mehr als nur Fußball“ modifiziert: Während das bürgerliche Sportverständnis über die verbandsfußballerische Praxis Stadion und Fußballplatz als unpolitische Orte des Sports, Spiels und Spaßes institutionalisierte, unterbrach das Festival diese Raumkonstitution und politisierte Stadion und Fußballspiel.

\subsubsection{Politische Dimensionen explizit machen}

Bei der Politisierung des Stadions wurde die sportliche Arena keinesfalls beliebig mit politischen Themen aufgeladen. DF bemühte sich vielmehr um eine strenge Kontrolle der Inhalte und um eine Gestaltung des Raums im Sinne intendierter Politiken. Um Aufmerksamkeit auf das eigene Projekt zu lenken und den Teilnehmenden größtmögliche Wertschätzung entgegenzubringen, lud DF die Botschafter_innen der jeweiligen Länder sowie Lokalund Bundespolitiker_innen und leitende Verwaltungsbeamte ein, bat die deutschen Repräsentant_innen um Grußworte bei der Eröffnungsfeier und besuchte mit allen Teilnehmer innen das Kanzleramt für ein Gruppenfoto mit der Kanzlerin Angela Merkel. Diese personelle Versammlung - Politiker_innen, Diplomat_innen und Beamt_innen anstelle von Sportfunktionär_innen - verstehe ich zum einen als weiteren Versuch, das Festival nicht nur als Sportveranstaltung, sondern auch als geschlechter- und gesellschaftspolitisches Event zu inszenieren. Zum anderen lässt ein genauer Blick auf die Anordnung politischer Subjekte an einem Ort des Sports Rückschlüsse auf das spezifische Verhältnis zwischen bürgerlichem Sportverständnis und zivilgesellschaftlicher Politisierung zu. 
Die Inszenierung von Politik im Stadion ist keineswegs ein ungewohntes Phänomen, sondern besitzt eine lange Tradition (Petermandl 2005, 148). Marschik et al. merken in Bezug auf die entstehungsgeschichtliche Prägung des Stadions an, dass es lediglich ein ,vordergründig sportlicher, jedoch zugleich politischer, (zivil-)religiöser und nicht zuletzt ökonomischer Ort" sei $(2005,9)$. Politische Machtinszenierungen im Sport sind gerade deshalb so effektiv, weil sie unter dem Mantel politischer Neutralität firmieren können (ebd.; siehe Kap. 5.2.1). Politiker_innen signalisieren mit ihrer Anwesenheit bei Sportereignissen Volksnähe und gemeinsame Interessen. Markus Pinter zitiert in einem Artikel mit dem programmatischen Titel „Die Ehrentribüne als politische Bühne" die ehemalige Justizministerin Sabine LeutheusserSchnarrenberger (FDP):

„Keine Weltmeisterschaft ohne Politikerdelegation. [...] Es lässt sich mit Fußball gut Politik machen, nicht nur bei Weltmeisterschaften, auch auf kommunaler Ebene." (Leutheusser-Schnarrenberger 1997, 242, zit. n. Pinter 2005, 323)

Pinter (2005) führt eine Vielzahl jüngerer Beispiele an, in denen demokratisch gewählte Staatsoberhäupter sich und ihre Politiken inszenierten. Das Stadion wird dabei zum Vermittlungsort und Multiplikator von Politik.

Bei DF war zu beobachten, wie sich die Politiker innen durch die Anknüpfung an unterschiedliche, hier zusammenlaufende Themen inszenierten: Die einen betonten in ihren Reden die kulturelle Vielfalt und die entstehende Völkerverständigung, andere strichen den Kampf für Frauenrechte und gegen Diskriminierung hervor. Während das Stadion schon immer als Ort der Inszenierung von politischen Themen und Akteur_innen genutzt wurde, konnten so auch beim DF-Festival Politiker innen durch den öffentlichen Auftritt ihr symbolisches Kapital mehren. Im Umkehrschluss stieg aber auch das Kapital der Teilnehmenden und der Organisationsgruppe vor Ort wie auch in den Heimatländern, wenn sie z.B. ein Foto mit der Bundeskanzlerin vorweisen und damit die Relevanz ihrer Arbeit bekräftigen konnten. Während die politische Dimension des Stadions jedoch zumeist durch das Credo der politischen Neutralität verschleiert wird, meint der Prozess der Politisierung im Rahmen des Festivals ein Explizit-Machen der politischen Dimensionen.

In Kapitel 5.2 habe ich dargelegt, wie gegenwärtige Brüche im bürgerlichen Sportverständnis zu verzeichnen sind und feministische, antirassistische und andere Initiativen, Journalist_innen und Wissenschaftler_innen auf die soziopolitischen und ökonomischen Verflochtenheiten des Sports hinweisen und diese öffentlich anprangern. DF reihte sich in diese Initiativen ein, die den Mantel der Neutralität zu lüften versuchen, und nutzte das günstige Moment dadadurch, dass Politiker_innen nicht nur eine Bühne gegeben, sondern das sportliche Geschehen durch räumliche Anordnung und diskursive Formierung sicht- und hörbar politisiert wurde. 


\subsubsection{Politische Positionen auswählen}

Politisieren beinhaltete jedoch im Falle DFs nicht nur den räumlichen und diskursiven Prozess des Explizit-Machens, sondern auch die Auswahl politischer Inhalte. Dies war $2015 \mathrm{zu}$ beobachten, als der Bundesinnenminister während der Eröffnungsfeier konkret wurde. In seiner Rede lobte er DF und die Teilnehmenden. Anschließend kam es zum Gespräch zwischen der Moderatorin Ines Pohl und dem Minister:

Pohl: „Schön, dass heute hier eine Frau aus Afghanistan da ist. Andere konnten nicht kommen. Und das Team aus Uganda hat kein Visum gekommen. Das ist doof, oder?"“ Der Innenminister antwortet: „Es ist klar, dass Fußball auch genutzt wird, dass ein paar mit anderen Absichten kommen. Da muss man genau hinschauen!“ Er deutet damit an, dass die Spielerinnen einer ugandischen LGBTI-Organisation von den zuständigen deutschen Behörden verdächtigt wurden, nach dem Festival nicht nach Uganda zurückzukehren, und deshalb kein Visum erhielten. Ich schaue meine Sitznachbarin stirnrunzelnd an. Sie ruft ein lautes „Buuuuh“ in Richtung Bühne. (Feldnotiz, 30.6.2015)

Auch vom Innenminister wurde der Fußball politisiert, allerdings im Sinne einer Grenz- und Asylpolitik, die bei DF auf Ablehnung stieß. Ähnlich verhielt es sich in Bezug auf das Public Viewing: Wenn am Abend die Spiele der großen Fußballevents im Stadion übertragen wurden und sich der Platz vor der Bühne mit Fußballbegeisterten füllte, bat das Sicherheitspersonal an den Toren darum, schwarz-rot-goldene Elemente abzulegen oder unsichtbar $\mathrm{zu}$ tragen. Eine Politisierung des Fußballs im Sinne nationalistischer oder bereits Nationalitäten betonender Politik war unerwünscht. ${ }^{154}$ Es gilt also näher hinzuschauen: Die Anknüpfung an die Aussage „Fußball ist politisch“ meint nicht, dass ein vormals politikloser Raum mit politischer Bedeutung versehen wird. Vielmehr bedeutet sie hier ein Explizit-Machen seiner historischen und gegenwärtigen politischen Dimension sowie eine Selektion politischer Inhalte und Positionen dadurch, dass unerwünschte politische Verknüpfungen durch Raumgestaltung abgewehrt und eingedämmt werden. Politisierung meint nicht nur, wie es die Rhetorik der Aktivist_innen („Fußball ist politisch!“) suggeriert, eine Verknüpfung von Fußball und Politik, sondern das Auswählen politischer Aussagen und Überzeugungen.

154 Dieser Wunsch ist eng verknüpft mit anti-nationalistischen Ideen der lokalen Bewegungsszene, sodass hier erneut ersichtlich wird, wie die lokale, politisch-ideologische Verortung die Gestaltung der internationalen Fußballbegegnung beeinflusste. 


\subsection{Geschlechterverhältnisse umkehren: „Dein Chef hat mir das erlaubt"6}

Neben den politischen und leistungsorientierten Dimensionen wurden auch die geschlechtlichen mittels Raumgestaltung modifiziert. Gesellschaftliche Geschlechterverhältnisse und -kämpfe wirken in die Produktion von Räumen hinein. Caudwell macht dies für Sporträume und ihre konkreten Orte wie Fußballstadien und -plätze ${ }^{155}$ geltend und analysiert diese als hierarchisch strukturiert und entscheidend in die materielle und ideologische Produktion des Normativen und Herrschenden eingebunden (2011a, 214). Durch den hohen Grad an ritualisierten Handlungen auf und abseits des Platzes sowie einer Nutzungstradition, wie sie sich aus den verschiedenen in Kapitel 4.2 skizzierten Mechanismen der Exklusion von Frauen aus dem Fußball ergibt, institutionalisiert sich das Stadion als männlicher, heteronormativer Raum (vgl. Löw 2001, 162). ${ }^{156}$ Hier wird z.B. über homophobe und sexistische FanGesänge und Gesten eine als echt und archaisch imaginierte Männlichkeit kommuniziert, die das Stadion als heteronormativen Raum synthetisiert und erlebbar macht (Bromberger 2010; Caudwell 2011a; Sülzle 2011). In diesem Raumverhalten spiegelt sich die gesellschaftliche Konstruktion vom Fußball als „Arena der Männlichkeit“ (Kreisky/Spitaler 2006), in der Weiblichkeiten kein gleichberechtigter Platz eingeräumt wird. Zugleich kann das Stadion unterschiedlich erlebt werden: Während z.B. die Fankurve für die einen die Vertrautheit eines zweiten Wohnzimmers samt Fan-Familie bietet, stellt sie für andere eine bedrohliche Menschenmasse dar, von der verbale und physische Gewalt ausgehen kann und in der sie entsprechende Schutzstrategien entwickelt haben. Die durch das Spacing von Dingen und vergeschlechtlichten Körpern institutionalisierten und zu einem männlichen Raum synthetisierten Geschlechterverhältnisse wurden beim DF-Festival umgekehrt und ein konkreter Entwurf einer Fußball-Weiblichkeit in das Stadion eingeschrieben.

155 Ich betrachte die städtischen Fußballplätze als kleine Brüder der großen Stadien und mache das hier beschriebene Verhältnis zwischen Raum und Geschlecht auch für die von mir besuchten Plätze geltend. Dies scheint mir zulässig, da der Übergang von Fußballplatz zu Stadion ein gradueller ist und sich meist nur an ein paar Tribünenbänken entscheidet. Zudem fungieren Fußballplätze als Bühne und Proberaum, um den Stars und bedeutenden Spielen in den großen Arenen nachzueifern.

156 Grimm (2015) zeigt dies anhand eines Berliner Mädchenteams, das sich für ein Fußballturnier anmelden wollte. Mädchenteams wurden von dem privatwirtschaftlichen Veranstalter erst gar nicht mitgedacht und ihre Anmeldung entsprechend abgelehnt. Nur durch den Druck infolge eines offenen Briefs durfte das Team teilnehmen. Solche geografischen Ausdrucksformen von Macht im Fußballstadion beschreibt Bale als ,politics of territory“ (1993, 130), und feministische Forschungen zu Raum und Geschlecht weisen auf die Inkorporierung dieser Machtverhältnisse hin, die sich in der zögerlichen Nutzung oder gar dem Fernbleiben von Frauen von öffentlichen Orten äußern (vgl. Valentine 1989, 389; Skeggs 1999, 214; Feltz 2007). 


\subsubsection{Feminisierung des Fußballraums}

Bereits in der einleitenden Vignette wurde deutlich: Auf dem Festivalgelände wie auch auf der DF-Homepage und im Büro der Organisation dominierte die Farbe Pink als zentraler Bestandteil der corporate identity DFs. Ein Großteil der Dekoration war in Pinktönen gehalten, und das sich überall befindende Logo der Organisation war in Pink auf weißen Hintergrund gedruckt. Die Bedeutung der Farbe ist gegenwärtig vor allem eine vergeschlechtlichte: Pink markiert Weiblichkeit und setzt sie in Opposition zu (Hell-)Blau als Männlichkeitsmarker. Wie sehr diese Weiblichkeitsdarstellung mittels Farbwahl mit der Geschlechtlichkeit des Stadions brach, macht ein Blick auf den Umgang mit Rosa und Pink im Männerfußball deutlich. Beim SV Darmstadt 98 ist es z.B. bewährte Trainingspraxis, dass monatlich der unengagierteste Spieler damit bestraft wird, ein pinkes Trikot tragen zu müssen, auf dem hinten „Fehleinkauf“ und vorne „Tussi“" steht (Geukler-Palmert 2014). Zwar wird die Kausalität zwischen Weiblichkeit und Leistung offengehalten - ob die ,Verweiblichung' des Spielers zur schlechten Leistung führt oder ob seine Leistung als weiblich beschimpft wird, weil sie schlecht ist -, doch der dahinterstehende Zusammenhang, in dem Weiblichkeit mit schlechter Leistung gleichgesetzt und durch das Pink symbolisiert wird, ist unmissverständlich.

Sülzle beobachtet eine ähnliche Abwertung des Weiblichen im Fanblock des Männerfußballs. Die dort erwartete und selbstverständliche Geschlechterperformanz ist maskulin, sie grenzt sich von dem „Klischee einer rosazickigen Weiblichkeit" ab und alle aus, die mit diesem Klischee assoziiert werden - seien es „Tussis“ oder schwule Männer (2011, 217). Diese Grenzziehung wurde nicht nur von männlichen, sondern auch von weiblichen Fans betrieben, die sich so als echte Fans beweisen wollten. Umso deutlicher stach eine Frauen-Fan-Gruppe hervor, die mit pinken Bannern und Fahnen Weiblichkeit demonstrierte und sich zugleich durch gekonntes Fachsimpeln als echte Fans auszeichnete. Sülzle versteht dies als „Aufdeckung, verborgener“, nicht thematisierbarer Ausschlussmechanismen und gleichzeitig d[as] Übertreten und Verwischen dieser eben dadurch aufgezeigten Grenzen" (ebd., 338). Sie bezeichnet diese Taktik der Fans als ,vorweggenommenen Sexismus“, bei der es darum geht, ,aus der inneren Logik des Systems heraus verborgene (weil selbstverständliche und damit vermeintliche ungreifbare) Wahrheiten öffentlich zu machen und damit normalisierte und naturalisierte Aspekte gesellschaftlicher Verhältnisse sichtbar zu machen“ (ebd., 339). Auch für DF lässt sich diese Analyse geltend machen: So entlarvten etwa das Spiel mit einem pinken Ball oder in pinken Stutzen, die rosa-pinken Kissen auf der Tribüne und die magenta-farbige Stoffdekoration die Naturalisierung der Verbindung von Stadion und Männlichkeit und verwischte sie zugleich. Die Farbwahl zusammen mit den sich kompetent und selbstverständlich im Raum bewegenden und als weiblich lesbaren Körpern kreierten das Fußball- 
stadion als Raum, in dem Weiblichkeit und Fußball nicht länger einen Widerspruch darstellten. Um Fußball und Weiblichkeit kompatibel zu machen, bedurfte es jedoch eines ganz konkreten Weiblichkeitsentwurfes.

\subsubsection{Das Pink der female football activist}

Entlang der Farbwahl und mit Blick auf die Bedeutungs- und Verwendungsgeschichte der Farben Pink und Rosa lässt sich herausarbeiten, welche Weiblichkeit genau von DF her- und dargestellt wurde und wie sich diese Fußballweiblichkeit zu anderen Weiblichkeiten verhielt. Als ich 2012 zum ersten Mal das Büro der Organisation betrat, war ich überrascht und verwirrt, da ich Pink stets mit einer hyper-femininen Weiblichkeit, nicht jedoch mit Feministinnen und noch weniger mit Fußballerinnen assoziiert hatte. Ingrid Tomkowiak (2014) erinnert jedoch daran, dass Farben in unterschiedlichen Kontexten auch unterschiedliche Bedeutungen tragen. Die Assoziation von Pink/Rosa mit Mädchen und Blau/Hellblau mit Jungen etablierte sich in den 1920er Jahren und erlebte in den letzten Jahrzehnten eine deutliche Intensivierung, die sich in Form von Barbie, Hello Kitty und Prinzessin Lilliefee konsumieren lässt (ebd., 181f). ${ }^{157}$ Einen solchen Gebrauch der Farbe Pink bezeichnet Dominique Grisard als „Princess Culture“, die eine ganz bestimmte Weiblichkeit signalisiere: „Pretty and decorative; light-skinned; as well as just and good" $(2015,29)$. Die pinke Prinzessinnenkultur ruft Mädchen zu: „You too can become a princess" (ebd.). Vor allem die erste Bedeutung verweist auf die Objektifizierung von Frauen als dekorativ und positioniert die Farbe Pink damit diametral entgegen den feministischen Forderungen nach weiblicher Selbstbestimmung.

Die Farbe Pink erfuhr jedoch ab den 1960er Jahren im Zuge kritischer Auseinandersetzungen mit Heteronormativität und Lesben- und Schwulenfeindlichkeit immer wieder provokante Umdeutungen. Etwa wenn das nationalsozialistische Symbol des rosa Winkels von der AIDS-Bewegung ACT UP angeeignet oder vom Filmemacher Rosa von Praunheim zum Künstlernamen gemacht wurde (Tomkowiak 2014, 188f.). Auch die männlichen Fußballer der Anti-Homophobie-Kampagne The Justin Campaign tragen pinke Trikots (Caudwell 2011a, 132). DF bezog sich im Gebrauch von Pink zwar auf die hyper-feminine Prinzessinnenkultur und nicht auf die nationalsozialistische Verfolgung Homosexueller, reihte sich so aber ein in die „kulturellen Auseinandersetzungen um Geschlechterrollen und sexuelle Identitäten“, die die Bedeutung der Farbe vervielfältigten (Tomkowiak 2014, 192).

157 Tomkowiak (2014) unterscheidet in ihrem historischen Blick auf die Farbbedeutung nicht zwischen Rosa und Pink. Ich stütze mich hingegen nur auf die Bedeutungen der Farbe Pink, da sie deutlich greller ist, sich so vom zarten Rosa unterscheidet und andere Assoziationen ermöglicht. Dieser Unterschied ist für den hier untersuchten Kontext zentral. 
In einem Interview erzählte die Grafikerin von der Entstehung des Logos als erstem Schritt zu einem corporate design:

\begin{abstract}
„Das ist ein Prozess, der intuitiv passiert. Es hat so irgendwie gestimmt. Ich hatte etwas anderes im Kopf, dass ich so was, so ein bisschen Sauberes, bisschen Schickes und relativ Cooles machen wollte. Eigentlich so, wie ich gerne gehabt hätte, dass man Frauenfußball assoziiert, und [...] im Endeffekt hab ich das dann durch dieses Pink erreicht.“
\end{abstract}

Die Farbwahl erscheint als intuitiver Prozess; die Grafikerin präfigurierte mit der Verbindung von Fußball und Pink ein Bild des Frauenfußballs, wie sie es sich selbst von den Massenmedien wünschte. Dafür griff sie die Geschlechtlichkeit bewusst auf, anstatt auf eine möglichst große Unsichtbarkeit der Weiblichkeit zu setzen und wendete sich damit nicht nur gegen die fußballerische Weiblichkeitskritik, sondern ebenfalls gegen eine bestimmte Geschlechterpolitik. Denn im Gegensatz zur patriarchatskritischen Pädagogik, die genderfreie Erziehung und Unisex-Kleidung bevorzugt (Tomkowiak 2014, 182) und sich an einem Feminismus orientiert, der Femininität als Produkt und Spielfeld des Patriarchats deutet, betont das Pink durch die Herausstellung einer bestimmten Weiblichkeit die Geschlechterunterschiede. Das Pink signalisiert die Vereinbarkeit von Weiblichkeit und Fußball, wie sie die Subjektposition der female football activist (siehe Kap. 12.1) für sich beansprucht: „You too - as a footballer - can be a woman!“ Umgekehrt richteten sie sich mit der Auflösung des Widerspruchs und der Inszenierung als Frauensport auch gegen ein Bild vom Fußball als reinem Männersport. Simone bemerkte in einem Workshop, in dem sie DFs Medienarbeit vor internationalen Teilnehmerinnen vorstellte: „We use pictures from men's soccer with typical gestures and resignify the images by displaying women." (Feldnotiz, 8.4.2015) Indem eindeutig weibliche Körper sich selbstverständlich und gekonnt im Fußball bewegen, vermitteln sie die Vereinbarkeit von Weiblichkeit mit Fußball und richten sich an all jene Mädchen und Frauen, die diesen Sport als unpassend erachten: „You too - as a woman - can be a footballer!“

Zugleich ist diese fußballerische Weiblichkeit keinesfalls beliebig, sondern entwirft innerhalb eines von Geschlechterstereotypen vorstrukturieren Rahmens eine ganz spezifische Vorstellung. Die Begriffe „cool“, „schick“ und ,sauber“, die die Grafikerin wählte, sind der Fußballsprache entnommen und bezeichnen einerseits ein weitsichtiges, passgenaues und nicht hysterisch-hektisches, ein robustes und nicht zimperliches Spiel, das andererseits schick und nicht proletenhaft-aggressiv oder pöbelnd ist, das sauber verläuft und keiner taktischen Fouls und sogenannter Schwalben bedarf. So bewahrt die female football activist zum einen Distanz zu spezifischen Weiblichkeitsbildern. Zum anderen grenzt sie sich von einer proletarisch und archaisch inszenierten Fußball-Männlichkeit ab, die zunehmend wegen der vielen Tricksereien und Unsportlichkeiten kritisiert wird, und festigten damit die Distanz zum Verbandsfußball (siehe Kap. 10.1). Durch diese Abgrenzung 
präsentiert sich der Frauenfußball als die fußballerische Alternative, die all das bietet, was durch die zunehmende Kommerzialisierung und Professionalisierung des Männerfußballs oder die überzogene Inszenierung als Männerkult verlorengegangen scheint, jedoch auf der körperlich-sportlichen Leistungsfähigkeit der Spielerin basiert.

\subsubsection{Dosierungsanleitung für eine subversive Wirkung}

Nur eine geschickte Dosierung der Farbe verhinderte, dass die entworfene Weiblichkeit in die Stereotypenfallen geriet, die für Frauen im Fußball bereitstehen. Zum einen umschifften die Aktivist_innen durch die pinke Unterstreichung der Weiblichkeit das Stereotype des fußballerischen ,Mannweibs'. Zum anderen mussten sie auf dem schmalen Grat zwischen hyper-femininen Weiblichkeitsstereotypen einerseits und subversiv wirkender Bedeutungsverschiebung andererseits balancieren. Ein genauer Blick ins Willy-KressmannStadion zeigt die bewusste Dosierung der Farbe. So durften der Ball, Stutzen und Schnürsenkel zwar pink sein, Trikots jedoch nicht; die T-Shirts am Merchandise-Stand variierten zwischen vielen Farben, doch ein pinkes suchte man hier vergeblich, nur das DF-Logo war pink. Pink diente als begleitendes und wiederkehrendes Accessoire in Form von Stoffbeuteln und Buttons, Ohrsteckern oder als dekorative Stofffetzen, Kissen und Schriftzüge.

Das Klischee der „Tussi“" und der „rosa-zickigen Weiblichkeit" sowie die Gefahr, als „sexy Kickerin“ (Schaaf 2013) kommodifiziert und objektifiziert $\mathrm{zu}$ werden, stehen für Frauen im Fußball stets parat. Gerade im Zuge der Frauen-WM 2011 in Deutschland nahmen die medialen und verbandsfußballerischen Bestrebungen zu, Fußballerinnen medial und kommerziell zu vermarkten und sie dafür gezielt feminin und heterosexuell $\mathrm{zu}$ inszenieren (Schaaf/Nieland 2011; 2011a). So ermöglichte die Verquickung von Markt und Profifußball (bspw. durch eine Fußball-Barbie oder Home- und BeautyStories in Frauen-Magazinen) eine zahlenmäßige Zunahme von Frauen im Fußball und erhöhte die mediale Repräsentation des Frauen-Profisports (siehe Kap. 4.2.5; 4.3.2). Das auch durch die WM entstandene günstige Moment ermöglichte jedoch nicht nur die Entstehung von DF, sondern stellte zugleich auch Weichen für mögliche Geschlechterdarstellungen, die auch auf der Amateurebene spürbar waren. Daniela Schaaf und Jörg-Uwe Nieland deuten diese Heterosexualisierungs- und Feminisierungsbestrebungen als „,der Widerspenstigen Zähmung“ (2011) - als Versuche, den Fußballerinnenkörper seines transgressiven Potentials zu entledigen. ${ }^{158}$ Auch frühere geschlechtertheoretische Sportstudien beschreiben heteronormativ-gefällige Weiblichkeiten im Sport als „female apologetic“ (vgl. Sabo 1993; Broad 2001) beziehungsweise „lesbian apologetic“ (Griffin 1998, 68), mit denen sich Sportle- 
rinnen für ihre Teilnahme an männlichen Sportarten und der damit verbundenen unerhörten Geschlechter- und Sexualitätsdarstellung entschuldigten. ${ }^{159}$

Bei DF drückte sich die Auseinandersetzung mit der feministischen sowie der fußballerischen Weiblichkeitskritik in einer genauen Dosierung aus. Als vor dem Festival 2013 die Grafikerin Entwürfe für den Flyer an die Organisationsgruppe schickte, antwortete unter anderem Thomas folgendermaßen:

„Die Flyer sind alle 3 toll! Meine Favoriten sind die beiden mit Fußballrasen im Hintergrund. Persönlich finde ich pinke Stutzen und pinke Schrift etwas viel - kann aber auch an meinem Bildschirm liegen. (Gibt es evtl. auch einen schlammverschmierten Stutzen? [...]“" (E-Mail, 7.5.2013)

Pink wurde gezielt mit grünem Rasen und schmutzverschmierten Stutzen ausgeglichen - Symbole, die die fußballerische Ernsthaftigkeit dieser Weiblichkeit demonstrieren. Die Farbe erzielte ihre Wirkung, den fußballerischen Raum zu feminisieren und seine Fußball-Männlichkeit in Zweifel zu ziehen, durch den wohldosierten, gezielten Einsatz. Bei zu viel Pink hätte die Gefahr bestanden, in die Stereotypenfalle zu geraten und damit das Anliegen belächelbar und sich selbst objektifizierbar zu machen. ${ }^{160}$

Die Abwendung von Sexualisierung und Objektifizierung bedeutet daher nicht nur die Auseinandersetzung mit Frauen-exkludierenden Kräften, sondern auch eine Distanzierung von jenen Prozessen, die das günstige Moment miterzeugten. Während Feminisierung und Sexualisierung auf professioneller Ebene - in Maßen - mitgetragen oder gar gefördert werden, um die Geschlechterverhältnisse des Fußballs zu bearbeiten, so geht die Abkehr von der Sexualisierung auch mit einer Distanzierung vom professionellen Frauenfußball und seinen Verwobenheiten mit marktwirtschaftlichen Anerkennungspraktiken einher.

\subsubsection{Geschlechterrollen umdrehen}

Die Aktivist innen enthoben nicht nur das Stadion raumgestalterisch seiner männlichen Konnotation, sie kehrten auch durch eine Rollenumverteilung die fußballerischen Geschlechterverhältnisse um:

Kurz vor dem Finalspiel fährt ein Auto auf das Festivalgelände, obwohl dies nicht gestattet ist. Als der Fahrer, ein Mitarbeiter einer Botschaft, aussteigt, geht Simone

159 Dass diese wissenschaftlichen Klassifizierungen auch wieder in den Sport zurückwirken, zeigt Caudwell (2007) anhand eines lesbischen Sportteams: Durch die Deutung von Femininität als Spielball des Patriarchats wurden lesbische Femme-ininitäten innerhalb des Teams gegenüber der butch marginalisiert.

160 Sexualisierung und Objektifizierung wurde auf dem Festival auch dadurch verhindert, dass bestimmten Personen der Zutritt zum Gelände verwehrt blieb. Z.B. wurde eine Gruppe junger Männer abgewiesen, nachdem diese sich laut gefragt hatten, ob wohl Spielerinnen dabei wären, die in ihr „Beuteschema“ passten. 
auf ihn zu und sagt, er könne hier nicht parken. Er sagt, er dürfe alles und: „Dein Chef hat mir das erlaubt." Simone weist ihn entschieden darauf hin, dass es hier keinen Chef gäbe und sie gerade verantwortlich sei. (Feldnotiz, 30.7.2013)

Simone ist durch Funkgerät und Crew-Shirt als eine verantwortliche Person auf dem Gelände markiert. Dennoch wird sie von dem Mann als junge Frau in Freizeitkleidung nicht als Verantwortliche wahrgenommen. ${ }^{161}$ Die Geschlechterhierarchie im Stadion sieht als Protagonisten, Entscheidungs- und Würdenträger vor allem Männer vor. Frauen dürfen zwar anwesend sein, werden aber vorrangig in begleitenden und kümmernden Rollen imaginiert und häufig auf diese verwiesen: Die Mutter, die die Trikots wäscht und Pflaster auf die aufgeschürften Knie klebt, die anfeuernde Freundin am Spielfeldrand oder die Ehefrau, die im Kartenhäuschen Tickets verkauft (vgl. Selmer 2005; Sülzle 2014). Stefanie Schütze und Julia Haß hingegen zeigen, dass die Schaffung alternativer fußballerischer Räume unabhängig von den organisierten Ligen es ermöglicht, Geschlechterrollen partiell und temporär umzukehren (Schütze/Haß 2017; Haß 2017). Dadurch, dass DF gezielt Frauen in die repräsentativen und sichtbaren Verantwortungspositionen setzte, wurde die Geschlechterhierarchie im Stadion unterbrochen: Frauen entschieden und verantworteten, auf der Bühne traten Künstlerinnen auf und die Hauptrollen in den aufgeführten Filmen spielten stets Mädchen und Frauen, die Stimmen aus den Lautsprechern waren weibliche ${ }^{162}$, und die Sticker für das PaniniHeft zierten weibliche Gesichter. Für viele der beteiligten Männer bedeutete dies den Entzug ihrer gewohnten Zuständigkeiten und Aufgaben. Zwar durften männliche Trainer oder Teambetreuer anreisen und Männer in der Organisationsgruppe oder als Volunteers ${ }^{163}$ mitmachen, jedoch wurden ihnen stets Nebenrollen zugewiesen.

Beim Festival 2015 beobachtete ich ein Trainer_innentandem, das aus einer Frau und einem Mann bestand:

Ganz links hinter der Spielfeldbegrenzung, aber noch vor der Sandgrube, stand schon das Blaue Team bereit. Die beiden Teambetreuerinnen sowie die verletzte Catalina auf Krücken waren auch dabei. Yeshe hält immer noch eine Ansprache. ,Was ist mit der anderen Trainerin? Kommt sie auch zu Wort oder dominiert der Mann?`, frage ich

161 Solche Erfahrungen waren nicht selten. So reisten wir z.B. als Gruppe zu einer serbischen Partnerorganisation. Wurden wir jemandem vorgestellt, bekamen wir Frauen ein freundliches Nicken und der einzige Mann in unserer Gruppe, der als Partner mitgereist war und sich eher im Hintergrund hielt, wurde mit Handschlag begrüßt und als Verantwortlicher angesprochen. Junge Frauen schienen nicht als Verantwortliche in Frage zu kommen.

162 Wie sehr dies Proteste auslösen kann, wurde 2016 deutlich, als bei der Männer-EM eine Kommentatorin ein Spiel im öffentlich-rechtlichen Fernsehen begleitete (vgl. Haas 2016).

163 Im Sprachgebrauch bei DF wird zwischen „Ehrenamtlichen“ und „Volunteers“ unterschieden. Die „Ehrenamtlichen“ sind jene Personen, die das ganze Jahr über ehrenamtlich bei DF mitarbeiten. Die „Volunteers“ sind die freiwilligen Helfer innen, die nur an der Durchführung des Festivals beteiligt sind, dort kleinere Hilfsarbeiten übernehmen und keine organisatorische Verantwortung tragen, für das Gelingen aber unabdingbar sind. 
mich. Bereits gestern hatte ich so etwas aus dem Mund einer der Organisator_innen gehört. Später frage ich eine der beiden Teambetreuerinnen. Sie bestätigt und beschwichtigt zugleich: Es habe bereits Gespräche mit Yeshe gegeben und sie würden sich weiterhin darum kümmern. (Feldnotiz, 1.7.2015)

In Extremfällen, anders als beim einsichtigen Yeshe, konnten solche Einschränkungen und Zurechtweisungen in Konflikten münden, wie beim Festival 2013:

Das tunesische Team bringt den Präsidenten des Vereins und den Trainer mit, beides Männer. Beide nehmen an dem Workshop teil, der von Celia geleitet wird. Zugleich ist Celia auch eine von zwei Betreuerinnen des tunesischen Teams. Beim Plenum während des Festivals beklagt Celia, dass beide wohl sehr dominant auftreten würden, sie wollten zu allem was sagen, redeten viel zu lang und dominierten so den ganzen Workshop. Celia reagierte darauf genervt und nahm sie einfach irgendwann nicht mehr dran. Auch außerhalb der Workshops fielen die beiden Männer auf. So beschwerte sich der Präsident des Vereins bei den Teambetreuerinnen, wenn diese etwas mit den Spielerinnen vereinbarten, ohne ihn vorher zu fragen. Als z.B. eine der Spielerinnen der deutschen Presse ein Interview gab, ohne dass er vorher gefragt wurde, schrie er die Teambetreuerinnen an. Die beiden Männer warfen ihnen vor, sie zu diskriminieren. (Erinnerungsprotokoll, 30.7.2013)

Der Vorwurf der Diskriminierung traf bei den DF-Mitgliedern auf einen epistemischen Rahmen, in dem er keine Gültigkeit besaß. Dieser machte den Diskriminierungsbegriff nur für strukturell benachteiligte Gruppen geltend, zu der Männer aufgrund universeller patriarchaler Strukturen per se nicht zählten. Die Aktivist_innen sahen in dem Vorwurf eine argumentative Strategie, dem Entzug gewohnter Privilegien zu begegnen, und fanden sich in der Zurecht- und Zurückweisung bestätigt. Zuspruch erfuhren sie dabei von den tunesischen Spielerinnen: Bereits während des Festivals beklagten diese die Bevormundung und Unehrlichkeit ihrer männlichen Begleiter, und nach ihrer Rückkehr aus Berlin gingen sie bei offiziellen Stellen gegen ihren Trainer vor. Die Anschuldigungen der Männer weisen aber auch darauf hin, dass diese Umkehrung immer einen Eingriff in die internen Konstellationen der angereisten Teams beinhaltete.

DF versuchte gezielt durch Spacing und Synthese von Personen, Aufgaben und materieller Umwelt, das Stadion als nicht-heterosexistischen und nicht-männlichen Ort zu konstituieren. Die Praktiken basierten darauf, dass die Organisator innen von Beginn an die Gestaltungshoheit über den Raum besaßen: Celia konnte als Workshop-Leiterin den Redefluss der Männer unterbrechen und in die internen Konstellationen des Teams eingreifen, die Teambetreuerinnen konnten den dominierenden männlichen Trainer zurechtweisen, als Organisator_innen konnten sie entscheiden, wer auf den Podien sprach. Ihre einflussreiche Position als Organisierende und Gestaltende wurde genutzt, um vorherrschende Ungleichheitsverhältnisse temporär umzukehren und damit einen fußballerischen Raum entsprechend ihrer feministischen Überzeugungen zu präfigurieren. Dieses räumliche geschlechter- 
politische Handeln funktionierte jedoch nur, wenn jene Ungleichheit zwischen Teilnehmenden und Gastgebenden, die DF in anderen Momenten mit großen Anstrengungen $\mathrm{zu}$ minimieren versuchte (siehe Kap. 13.3), ausgespielt wurde. Transnationale feministische Begegnungen und Bündnisse können sich temporär und in persönlichen Kontakten dem Ideal der Augenhöhe zwar annähern, der organisatorische und finanzielle Rahmen, der diese Begnungen mittels Projektförderung erst ermöglicht, kehrt die intersektional verkomplizierten Ungleichheiten jedoch immer wieder hervor.

\subsection{Multiple Räume erleben: „Überhaupt keinen Zugang finden"}

Bislang galt meine Aufmerksamkeit vorranging der Frage, wie die gestalterischen Praktiken auf den Raum wirken, die Wahrnehmung der verschiedenen Personen im Raum blieb dabei weitestgehend unbeachtet. Aus der Subjektperspektive betrachtet können am selben Ort dieselben Menschen, Dinge und Umwelten zu unterschiedlichen Räumen synthetisiert werden (Löw 2001, 201). Der Blick vom Subjekt aus lässt die Frage nach dem unterschiedlichen Erleben und Wahrnehmen der Heterotopie zu (vgl. Rolshoven 2012, 164). Ich gehe davon aus, dass im Zuge präfigurativer Politiken multiple Räume erlebt und gelebt werden, die zwar in hohem Maße miteinander korrespondieren, aber dennoch eigene Qualitäten für die wahrnehmenden Subjekte besitzen. Im Folgenden diskutiere ich die unterschiedlichen Räume, die sich im Stadion entfalteten, und was diese letztlich für das Funktionieren von Heterotopien im Sinne präfigurativer Alternativen bedeuten.

\subsubsection{Das Festival als verschlossener Raum}

In ihrer Notiz vom Eröffnungsabend, die mir die Kulturanthropologin Almut Sülzle zur Verfügung gestellt hatte, wird die Verwirrung ob des Eingangs zum Festivalgelände deutlich:

„Ein Plakat, das mir den Weg weist und doch nicht, weil kein Pfeil drauf ist. Was auch nicht wundert, denn ich erinnere mich, dass es zwei Eingänge zum Gelänge gibt. Ist also jeder Weg richtig?“" (Feldnotiz AS, 30.6.2015)

Auffällig ist, dass eine öffentliche und gewollt öffentlichkeitswirksame Veranstaltung schwer zu finden ist. ${ }^{164}$ Und tatsächlich: Die pinken Pfeile, die wir

164 Im Vorfeld wurde die Veranstaltung über Plakate, Billboards, Flyer und Stadtmagazine in der Stadt beworben. Zeitungen und Radiosender berichteten vom Eröffnungstag. Um das Stadion herum war dann die Werbung tatsächlich sehr spärlich. 
mit Kreide auf den Weg gemalt hatten, waren schnell verblasst; zwar verkündete ein Plakat am Haupteingang die Veranstaltung, jedoch war dieser zur Straße gelegene Eingang die meiste Zeit verschlossen; der Zugang erfolgte vorrangig über den im umliegenden Park versteckten zweiten Eingang. Almut fand den Weg, weil sie ihn kannte.

Foucault bemerkt, dass Heterotopien immer Öffnungen und Schließungen voraussetzen und nicht allen unmittelbar zugänglich sind (1992a, 44). Beim Festival waren mehrere verschachtelte Schließungen zu beobachten: Neben der versteckten Lage und den mangelnden Hinweisen, die das Festival für Laufpublikum fast unsichtbar machten, erschloss es sich auch für jene, die hineingefunden hatten, nur schwer:

Am frühen Nachmittag entdecke ich einen Mann, der vor zwei Jahren als Volunteer beim Festival aushalf und nun anscheinend als Besucher vorbeigekommen ist. Er läuft alleine rum, setzt sich irgendwann auf die Tribüne und schaut dem Spiel zu. Ich spreche ihn an, ob ich ihm ein paar Fragen für meine Arbeit stellen könnte. Er hatte sich vor zwei Jahren als Volunteer gemeldet, weil er über einen Aufruf der Jusos vom Festival gehört hatte und ein DF-Mitglied flüchtig von der Filmhochschule kannte. [...] Dieses Jahr sei er spontan vorbeigekommen, weil das Wetter so schön sei. Aber in diesem Jahr gefalle es ihm nicht so gut. Er würde überhaupt keinen Zugang zu den einzelnen Teams bekommen, da die nun gemischt seien und man gar nicht wisse, wer da nun gegen wen spiele. (Feldnotiz, 2.7.2015)

Der ehemalige Volunteer bemängelte den fehlenden Zugang für ein externes Publikum durch die raumgestaltenden Praktiken wie das Mischen der Teams. Als Externer ging für ihn dadurch ein wesentlicher Teil der Spannung des Fußballs verloren, da der Wettkampf nicht mehr zwischen klar identifizierbaren Gruppen stattfand. Das Mischen der Teams entzog dem Publikum die Identifikationsmöglichkeiten - gleich, ob diese auf nationalen Stereotypen oder Wissen über Städte, Projekte oder Regionen basierten. Zugleich fehlte es an wissensvermittelnden Praktiken, die das Geschehen auf dem Platz lesbar machten. Erst als mich 2015 eine Besucherin fast schon verzweifelt fragte, wo denn mal ein Programmplan zu finden sei, wurde mir bewusst, wie schwer sich der Raum einigen externen Gästen erschloss. Während für mich als Organisatorin und für die Teilnehmenden jederzeit transparent war, was wann stattfand, und wir bereits alle am ersten Tag die Anlage kennengelernt und den Sinn der einzelnen Elemente verstanden hatten, fehlte es sowohl an Hinweisschildern als auch an leicht zugänglichen Erläuterungen zu den gravierenden Umgestaltungen: Ablaufplan und Hintergrundinfos versteckten sich in dem käuflich zu erwerbenden Panini-Heft und wurden nur gelegentlich von Stadionsprecherin oder Bühnenmoderatorin erklärt.

Mit dem Begriff des „discerning eye“ $(2011,192)$ fasst Tuan, dass zu Räumen synthetisierte Architekturen nicht von allen lesbar sind, sondern es eines bestimmten Wissens bedarf, um sie zu dechiffrieren. Was Tuan auf die historische Lesbarkeit moderner Städte bezieht, ist meines Erachtens mithilfe seiner Unterscheidung zwischen „spatial ability“ und „spatial knowledge“ 
(2011, 74f.) auch auf die gegenwärtige, situative Umgestaltung vertrauter Räume zu übertragen: Aufgrund des Bruchs mit Selbstverständlichkeiten funktionierte das inkorporierte räumliche Know-how (spatial ability), das sonst die Navigation durch und das Lesen der Stadionlandschaft ermöglichte, nur begrenzt. Die Umgestaltung des Stadions erforderte ein Wissen über diese neugestaltete materiell-semiotische Umwelt, das die inkorporierten Fähigkeiten ergänzte und auch korrigierte. Hierbei handelte es sich um verbalisiertes, symbolisch und assoziativ vermittelbares Raumwissen (spatial knowledge) und nicht um eine schrittweise, vorreflektive Ansozialisierung, da diese schon aufgrund der kurzen Dauer des Festivals nicht möglich ist. So waren Lesbarkeit und Erleben des Stadions beim Festival von dem Wissen um die Absichten, Hintergründe und Abläufe des Projekts abhängig. Für jene, die dieses Wissen nicht besaßen, blieb das Festival also ein schwer zugänglicher, rätselhafter und kaum zu erschließender Raum. ${ }^{165}$

\subsubsection{Das Festival als Arbeitsraum}

Auf dem letzten Plenumstreffen vor dem Festival teilten die Koordinatorinnen Wochenpläne an alle Ehrenamtlichen aus. Diese Pläne zusammen mit den Vorbereitungstreffen und den unzähligen E-Mails vermittelten das Raumwissen, das den Mitgliedern den heterotopischen Raum öffnete. 2013 umfasste dieser Plan eine kleingedruckte 15-seitige Tabelle, die die einzelnen Aufgaben und Arbeitsschritte in sechs Spalten auflistete. Dieser Plan hielt teilweise im Viertelstundentakt fest, wer zu welchem Zeitpunkt an welchem Ort im Stadion welche Tätigkeit ausführen sollte. Er zerlegte die Intention des Festivals, das gesamte raumgestaltende Konzept und die Mittel zur politischen Zielerreichung in viele einzelne Arbeitsschritte und leitete die Bewegung der Organisierenden im Raum an. Da diese durch die Vorbereitungen mit Intention, Zwecken und Zielen des Festivals vertraut waren und somit über ein discerning eye verfügten, vermochten sie, die einzelnen raumgestaltenden Handlungen in einen übergeordneten Sinnzusammenhang einzuordnen: „Kopfhörer austeilen“ z.B. war nicht einfach ein Handgriff, sondern ein wichtiger Baustein zur Herstellung ,einer besseren Welt" (Annike) und gewann damit die Relevanz einer politischen Tätigkeit.

165 Ähnliches kann bei Podiumsdiskussionen beobachtet werden, die einerseits während des Festivals, andererseits im Rahmen von Expertinnenforen an öffentlichen Orten als intendiert öffentlichkeitswirksame Veranstaltungen durchgeführt wurden. Riles bemerkt die große Beliebtheit solcher Veranstaltungen bei NGOs, um Informationen ,nach draußen “ zu bringen, beobachtete aber zugleich, dass sich im Publikum immer dieselben Leute befinden und die Diskussionen voll mit Akronymen und Querverweisen sind, die sich ,Outsidern nicht erschließen. Sie deutet diese offiziell öffentlichkeitswirksamen Formate als ,simulation of anonymous communication" $(2007,51)$. 
Zugleich ermöglichte die Aufteilung und Anleitung der Bewegungen im Raum ein spezifisches Raumleben und -erleben. Auch wenn alle Organisator_innen im Anschluss an das Festival betonten, wie gut es ihnen gefallen habe, wie schön sie die Woche erlebt hätten und gar von der Stimmung überwältigt worden seien, meldeten viele auch Stress und Überlastung zurück. Während Teilnehmende und Publikum im Schatten auf der Tribüne entspannten, fanden die vielbeschäftigten Ehrenamtlichen und Koordinatorinnen nur selten eine ruhige Minute. Abends, als das Publikum das OpenAir-Kino genoss, versammelte sich das Organisationsteam zu einer Rekapitulation des Tages und einem Ausblick auf den bevorstehenden in einer ungestörten Ecke. Wenn die Teilnehmenden bereits ins Hostel gegangen waren, räumten sie auf, oder hatten am nächsten Morgen schon alles vorbereitet, wenn die Teilnehmenden eintrafen. Für die Organisator_innen wurde das „Biotop“ (Ulrike Richter) zu einem Arbeitsraum, der neben viel Freude auch mit Hektik, Anstrengung und Erschöpfung verbunden war.

\subsubsection{Das Festival als unpassender Raum}

Auch die Teams bekamen detaillierte Ablaufpläne, die sie durch die Festivalwoche navigierten und sie das Stadion als Gegenraum leben und wahrnehmen ließen. Hierin waren nicht die einzelnen Organisationsschritte vermerkt, sondern die Programmpunkte: Kulturprogramm, Ausflüge, Botschaftsbesuche, Workshops und Turnierspiele. Er war deutlich kürzer als der Wochenplan der Organisierenden und passte im Querformat auf eine DINA4-Seite. Auf einer zweiten Seite war ein Ausschnitt eines Stadtplans von Berlin gedruckt, der den Weg zwischen Hostel, Stadion und Wäscherei vorzeichnete, die entsprechenden Adressen sowie eine Notrufnummer auflistete und den Teams mitteilte:

\section{"Dear participants,}

The entire program is obligatory. During the workshop program your team will be divided into different subgroups. Sunday morning you will get to know your individual workshop group that will inform you about the location. You always have to take into account the distance from the hostel to the locations (stadium/workshop/embassy, etc.): You always meet with your team assistance about 30 minutes before the event mentioned in the schedule starts!!!" (Festival Wochenplan 2013)

Diesen Plan zusammen mit Erklärungen über die Absichten, den Ablauf und die politischen Ziele des Festivals verstehe ich ebenfalls als Navigations- und Lesehilfen zur Dechiffrierung des Raums. Sie leiteten die Bewegung und die Handlungen der Teilnehmenden zeitlich und räumlich an und wirkten damit ebenfalls an der Gestaltung des Stadions mit. Dadurch, dass den Teilnehmenden das entsprechende Raumwissen vermittelt wurde, wurden die Wege durch und Tätigkeiten im Stadion mit Bedeutung versehen. 
Zugleich war das zur Entzifferung des Raums nötige Wissen um Absichten, Hintergründe, Abläufe und Ästhetik des Projekts eng an die ästhetischen, politischen und organisatorischen Praxen der Bewegungsszene (siehe Kap. 9.1.2) gebunden, in der sich DF lokal verortete, die aber weit über die Grenzen der Stadt hinausreicht. Sei es die Do-it-yourself-Ästhetik der Stadiondekoration, das Format Workshop, der Fokus auf Erfahrungspolitik oder der Wunsch nach aktiver Partizipation und Verantwortlichkeit aller Teilnehmenden. Viele Teilnehmende waren aufgrund ihres Vorwissens und ihrer eigenen wissenschaftlichen oder aktivistischen Beschäftigungen mit diesen Praktiken vertraut oder eigneten sie sich schnell an. Sie teilten die Inhalte, die Form des gemeinschaftlichen Miteinanders, die Idee des Erfahrungsaustauschs und der öffentlichen Meinungsäußerung sowie Werte gegenseitiger Toleranz. Sie konnten den präfigurierten Raum lesen und halfen mit, die Bedeutungen zu verbreiten. Auf diese Weise wurden auch die Teilnehmenden zu Eingeweihten, die in der Lage waren, die Textur des Raums zu lesen und den evozierten Gegenraum nicht nur wahrzunehmen, sondern auch mitzugestalten.

An der Situiertheit dieses Wissens lässt sich aber auch erahnen, dass auch die spezifische Qualität der Gegenwelt und der Bruch mit Selbstverständlichkeiten nicht von allen Teilnehmenden gleich erlebt wurden. So gab es Teilnehmende, die sich mit der präfigurierten Alternative nicht einverstanden zeigten. Erinnert sei an Qamar (Kap. 13.1.2), die entschieden jene Benachteiligung und strukturelle Diskriminierung zurückwies, wie sie von DF und vielen Teilnehmerinnen immer wieder bemängelt wurde und sich z.B. in der Umkehrung der Geschlechterrollen zeigte. Andere Spielerinnen wiederum irritierte die offene Thematisierung und Sichtbarkeit weiblicher Homosexualität, wenn anwesende Frauen Zärtlichkeiten austauschten. Unstimmigkeiten, Widersprüche und Missmut wurden jedoch kaum öffentlich geäußert, sondern erreichten die Organisator_innen meist nur über Dritte und Hörensagen. Sei es die Sichtbarkeit sexueller Vielfalt, die mit der Heteronormativität des Fußballstadions brach, sei es das Missfallen an der Zuweisung zur Rolle der Fußballerin als diskriminiertes Subjekt und am Eingriff in die internen Strukturen der Teams oder aber der Wille, am kompetitiven Charakter festzuhalten und sich den integrativen Elementen zugunsten besserer Leistung zu entziehen. In Kapitel 13.3 habe ich dies dahingehend gedeutet, dass die Teilnehmenden ebenfalls entsprechend heterogenen Kompetenzregimen agierten, z.B. wenn für Qamar die Konfrontation mit den Verbänden wenig erfolgsversprechend erschien und sie deshalb öffentliche, harsche Kritik ablehnte, oder wenn sich die eigene sportliche Aktivität nur durch erbrachte Leistungen oder betonte Heterosexualität zu Hause legitimieren ließ.

In anderen Fällen ergaben die Umgestaltungen der dominanten Bedeutungen schlicht keinen Sinn. Ich erlebte beim Festival 2013, dass Teilnehmerinnen, die noch vor wenigen Monaten auf den Straßen gegen die autokratische Herrschaft in ihren Ländern protestiert hatten, die Beschreibung ,poli- 
tisch“ sowohl für ihre zivilgesellschaftlichen Aktivitäten als auch ihre sportliche Frauenförderung zurückwiesen. Politik war etwas, das , die da oben“ taten und negativ behaftet war. In den äußersten Fällen konnte Politisch-Sein im Sinne einer Einmischung in staatliche Politik auch negative Konsequenzen nach sich ziehen. Myra Marx Ferree und Carol McClourg Mueller erklären eine solche Zurückweisung des Politik-Begriffs unter Aktivistinnen mit seiner vergeschlechtlichten Dimension und der Aufteilung gesellschaftlicher Sphären. So fällt es Graswurzelaktivistinnen häufig schwer, ihre Aktivitäten auf Community-Ebene als, ,politisch“ zu bezeichnen, denn dieses Attribut sei für männlich dominierte, formale Institutionen - gewählte Ämter, Verwaltungs- und offizielle Führungspositionen - reserviert (2007, 589). ${ }^{166}$ Diese Erklärung mag auf manche Kontexte zutreffen, jedoch weist sie Tendenzen auf, die Phipps innerhalb feministischer Öffentlichkeiten kritisiert, wenn Feministinnen nicht die gleichen Deutungen teilen und die einen bei den anderen ein Marx'sches falsches Bewusstsein diagnostizieren (2016, 313). Ich halte es eher für plausibel, hinter der Verwendung des Politikbegriffs bei DF und der Zurückweisung durch manche Teilnehmerinnen einen Unterschied in den politisch-zivilgesellschaftlichen Systemen und entsprechenden Praktiken zu vermuten. Während es in liberaldemokratischen Staaten völlig plausibel ist, zivilgesellschaftliche Aktivitäten aufgrund ihrer Einflussmöglichkeiten auf Regierungspersonal und -organe als politisch zu bezeichnen, so existieren in autokratisch regierten Staaten weder ein solches Zivilgesellschaftsverständnis noch entsprechende demokratische Einflussmöglichkeiten. Das damit verbundene Debattieren in Gruppen, das Bilden von Netzwerken zu konkreten Themen und das strategische Planen auf Graswurzelebene identifizieren Valerie Sperling el al. daher als spezifische Techniken in „Western societies long accustomed to self-organized local groups“, die in anderen Kotexten nicht selbstverständlich existieren oder sinnvoll sind (2001, 1172). In diesem Sinne beinhaltete die Politisierung des Fußballs eine konkrete Vorstellung von aktiver Zivilgesellschaft und Politisch-Sein. Wenn die verschiedenen Praktiken der Umgestaltung mit den lokalen Kontexten und Bedingungen der Teilnehmerinnen konfligierten, wurde das Festival von jenen Teilnehmenden weniger als „bessere Welt" (Annike), sondern als unpassender, vielleicht anstößiger Raum erlebt, der, wenn sie sich zu sehr auf ihn einließen, ihre eigenen Agenden konterkarieren konnte.

166 Zur Zurückweisung des Politikbegriffs unter Aktivistinnen siehe Sperling et al. (2001), Helms (2014). 


\subsubsection{Voraussetzungen: Schließungen, Ausweichmöglichkeiten und viel Arbeit}

Was bedeutet es für das Funktionieren der Heterotopie, wenn sich aus Subjektperspektive am Ort des Stadions multiple Räume entfalten? Ich meine, dass diese verschieden erlebten und gelebten Räume zum einen die Effekte der oben beschriebenen raumschaffenden Praktiken sind und zum anderen daran mitwirken, die Heterotopie für die Dauer des Projektes aufrechtzuerhalten. Das Präfigurieren einer feministischen Zukunftsvision durch die Umdeutung von Leistung und die Umkehrungen geschlechtlicher und politischer Dimensionen des Fußballs führten dazu, dass der Raum für Externe schwer zugänglich und häufig unverständlich war, für die Organisierenden permanente Arbeit, Hektik und manchmal Überforderung bedeutete und von manchen Teilnehmenden als anstößig, unerhört oder unpassend erlebt wurde. Während es verständlich ist, dass das beständige Brechen mit den Selbstverständlichkeiten des Fußballs kontinuierliche Arbeit und Anstrengung seitens der Organisierenden bedarf, so erscheinen Schließungen und Irritationen für ein Projekt, das entsprechend der pluralistisch-liberalen Überzeugung der Organisator_innen die größtmögliche Inklusion sozialer Gruppen wünscht, zunächst als unliebsame Machteffekte. Aus meinen Beobachtungen schlussfolgere ich jedoch, dass es gerade diese partiellen Ein- und Ausschließungen sowie Ausweichmöglichkeiten sind, die den heterotopischen Charakter ermöglichten. Erstens verstärkte die Abwesenheit fremden Publikums das Gefühl der Abgeschlossenheit und damit zugleich der empfundenen Freiheit der Personen im Raum. Zweitens bot die Zwanglosigkeit der Veranstaltung den Spielerinnen Ausweichmöglichkeiten und Gelegenheiten zu widersprechen, wenn sie mit den präfigurierten Alternativen nicht einverstanden waren. Auch wenn die Organisierenden die Teilnahme an den Workshops als verpflichtend erklärten, gab es zum einen weder Kontrollen noch Sanktionen beim Fernbleiben und zum anderen akzeptierte Ausweichmöglichkeiten: Krankheiten, Unwohlsein und Verletzungen sowie Verweise auf kulturelle oder religiöse Bedürfnisse standen als legitime Entschuldigungen zur Verfügung, mit denen sich Teilnehmende zeitweise jenen für sie unpassenden Elementen entziehen konnten, ohne vom gesamten Projekt ausgeschlossen zu werden. So mussten sie nicht zu Ungunsten ihrer eigenen Ziele und Projekte agieren, während die anderen Teilnehmenden und Organisator_innen keine allzu großen Kompromisse eingehen mussten. ${ }^{167}$

Ich möchte daher den Stadionraum entgegen seiner geläufigen Wahrnehmung nicht als öffentlichen, sondern als lediglich partikularen öffentlichen

167 Siehe Kap. 13.2; die Organisator_innen stellten mit der Zeit fest, dass ihre Gäste auch Sightseeing machen und shoppen gehen wollten und richteten explizite Freizeitfenster. Diese Bedürfnisse hätten aber nicht das Fernbleiben von festen Programmpunkten legitimiert. Als völlig inakzeptable galt Desinteresse an den politischen Inhalten. 
Ort verstehen, an dem durch subtile Praktiken der Öffnung und Schließung eine feministische Gegenöffentlichkeit entstehen kann (vgl. Schuster 2008, 136). ${ }^{168}$ Am Beispiel des DF-Festivals bestätigt sich, dass die heteropische Kompensationsfunktion im Sinne der Herstellung und Vorstellbarmachung konkreter gesellschaftlicher Alternativen vor allem funktioniert, wenn Einund Ausschließungen stattfinden, also ein interner Kreis von einem externen abgegrenzt und unterschieden und damit Gegenöffentlichkeit ermöglicht wird (Foucault 1992a, 44). Dass diese Gegenöffentlichkeit für den gesetzten Zeitraum fortbesteht und der Bruch mit den Selbstverständlichkeiten verständlich wird, bedarf dabei der unermüdlichen Arbeit und Aufmerksamkeit der Organisator_innen.

\subsection{Gesehen werden und mobilisieren: „It is a thin line”}

Abschließend frage ich nach den geschlechterpolitischen Effekten der präfigurativen Politik vor dem Hintergrund zunehmender Routine. Ich unterscheide dabei zwei Dimensionen der Wirksamkeit: Mobilisierung, gerichtet an die bewegungsinterne Öffentlichkeit, und Resignifikation, gerichtet an eine breitere Öffentlichkeit.

\subsubsection{Mobilisieren und motivieren}

Das Erlebbachmachen alternativer Zukünfte zielte auf eine gegenseitige Bestärkung, Motivierung und Mobilisierung der engagierten Fußballerinnen und Aktivistinnen. Es richtete sich sowohl an die Teilnehmenden als auch die Organisierenden. Jene Teilnehmenden, die sich mit den umgestaltenden Praktiken einverstanden zeigten, beschrieben das Festival häufig als eine großartige Erfahrung. Sie erlebten den Fußball auf eine ungewohnte, aber positive Weise, wie es z.B. eine libanesische Spielerin im Interview mit dem Kamerateam von DF ausdrückte: „At Discover Football, what I really felt was I am free, I am free to do whatever I want". Und Naoual und Katalina bemerkten: „You learn that you are not alone“, und zeigten so, wie das einwöchige Projekt die Teilnehmerinnen in ihrem Engagement bestärkte. Ähnlich wie die Libyerinnen, die sich zukünftig im Fußballverband engagieren zu wollten (Kap. 13.3.4), begannen Spielerinnen aus Tunesien nach ihrer Rückkehr aktiv gegen ihren autoritären Trainer vorzugehen und auf seine Absetzung hinzu-

168 Schuster greift Ipsens Begriff der „partikular geprägten öffentlichen Orte“ auf $(2008,136)$. Ipsen grenzt partikulare von ,allgemeinen Orten“ dadurch ab, dass sie zwar prinzipiell allen sozialen Gruppen offenstehen, faktisch jedoch einer bestimmten Gruppe zugeordnet werden können. Deren Verhaltensmuster verdichten sich dort zu Lebensstilen (2002, 238). 
wirken. Spielerinnen wurden durch das Projekt ermutigt, selbst aktiver zu werden und sich gegen Ungerechtigkeiten aufzulehnen. Diese mobilisierenden Effekte des präfigurativen Vorgehens gründen laut Anderson in der Verknüpfung von Gegenwart und Zukunft:

„In the enactment of better worlds, the future is constantly being folded into the here and now; a desired future may act as a spur to action in the present, for example, or action in the present may bring back memories of long-forgotten hoped-for futures." $(2010,778)$

Gerade das körperliche Erlebbarmachen von Zukunftsvisionen und umgearbeiteten Normen in der Gegenwart vermag zu bewirken, so lässt sich mit Nina Schuster ergänzen, dass sich diese in die Körper der Beteiligten einschreiben $(2008,140)$.

Über die gegenseitige Motivation hinaus bot das Festival vielen die Möglichkeit, das erste Mal nach Europa oder überhaupt ins Ausland zu reisen. Und für einige war es die erste Teilnahme an einem internationalen Fußballturnier. Plötzlich interessierten sich deutsche und ausländische Journalist_innen für sie, die Botschafter_innen ihrer Länder wohnten der Eröffnungsfeier bei und die deutsche Bundeskanzlerin empfing sie für ein gemeinsames Foto. Die Einladung nach Deutschland und die dort erfahrene Anerkennung wurden auch in ihren Heimatländern wahrgenommen und verhalfen ihrer sportlichen und sozialpolitischen Arbeit dort zu mehr Wertschätzung. Spielerinnen knüpften untereinander Kontakte und bauten Freundschaften auf. In Einzelfällen unterstützten sie sich später auch ganz praktisch durch die Vermittlung von Jobs oder Kontakten.

Auch die Organisator_innen, die sich selbst als Lernende verstanden, fühlten sich durch das Festival in ihrem Engagement bestärkt und motiviert. Annike erinnerte sich an das erste Festival 2010:

„2010 hat das erste Mal Discover Football stattgefunden und da haben wir seit 2008 dran gearbeitet, also zwei Jahre. Das heißt, ja auch eigentlich nicht so lang, das kam mir wahnsinnig lang vor. Vielleicht weil es einfach so unklar war. Ich hatte ja keine Vorstellung davon, was passieren würde. Jetzt ist es was anderes, weil ich weiß ja, was ich zu erwarten habe. Damals war es so sehr wackelig und man konnte sich das nicht so richtig vorstellen und das war total surreal, als die ersten Teams ankamen. Das war absolut surreal, die ganze Woche war surreal, das war abgehoben. Oder es war schon so verrückt, dass dann einfach wirklich die Nationalmannschaft aus Afghanistan da aufgelaufen ist. Das war sooo -, ich hab eigentlich in der Woche vom ersten Discover Football bis zum letzten Tag nicht kapiert, dass es echt passiert. Also dass zum Beispiel Afghanistan und Sambia, dass die da waren. Das war irgendwie so auf eine Art unvorstellbar. Die halt echt kennenzulernen und da zu haben und dass dieses Turnier, an dem wir so lang so gefeilt hatten, dass das halt echt passiert ist. In dem ersten Jahr war das ganz krass. Und dass die da echt gespielt haben und dass die das ernst genommen haben, und dass, wenn man dann ein Konzept plant, dass das dann echt passiert. Im ersten Jahr war es auch noch jeden Tag fast 40 Grad und es war so eine ganz surreale Woche, dieses Jahr war es wieder so heiß. Aber es war halt, also es 
war total surreal, die Spielerinnen zu sehen, und es hat mich wahnsinnig bewegt." (Annike, Interview, 21.11.2013; Betonungen i. Orig.)

Anhand Annikes Schilderung wird deutlich, wie organisatorische (Un-)Erfahrenheit und Raumerleben zusammenhängen, wenn sich die Organisierenden selbst als Betroffene und damit als Zielgruppe ihrer eigenen Projekte positionierten. Neben den Raumgestaltungen und Umweltbedingungen bewegte Annike auch das Gefühl, etwas so Großes möglich gemacht zu haben. Die Neuheit, die Unvorhersehbarkeit und das Risiko, eine große Veranstaltung zu organisieren, ließen die tatsächliche Realisierung beim ersten Mal besonders eindrucksvoll erscheinen. In ihrer Beschreibung des Erlebens als „surreal“ deutet sich eine Raumwahrnehmung als Gegenentwurf zu „realen“ fußballerischen Räumen an. Die mobilisierende Wirkung präfigurativer Politiken funktioniert daher auch über Raumwahrnehmung und das Erleben von Atmosphäre. Deborah Gould untersucht die mobilisierende Funktion von Emotionen bei den AIDS-Aktivist innen von ACT UP und beobachtet, dass die Beteiligten zwar schon mit den Zielen sympathisierten, es jedoch die Erfahrung des aktiven körperlichen Teilnehmens und Tuns ist, die die Identifikation mit der Bewegung und den Mitstreitenden verstärkt und ihre Einsatzbereitschaft erhöht $(2009,210)$. In Annikes Bemerkung, wie sehr sie die Stimmung beeinflusste, wird deutlich, dass das Raumerleben nicht nur Anstrengung und Stress erforderte, sondern zugleich motivierte und zu weiterem Einsatz bewegte.

\subsubsection{Routine: bedrohte Wirksamkeit}

Indem sich die Organisierenden selbst als Lernende und Betroffene erfuhren, die durch die internationale Begegnung ebenfalls Bestärkung und Rückhalt erfuhren, erlebten sie sich und die Teilnehmenden als einer gemeinsamen sozialen Kategorie - Frau im Fußball - zugehörig. Ersichtlich wird hieran einerseits erneut, wie die bereits in Kapitel 13.3 dargestellte Augenhöhe hergestellt wurde. Andererseits zeichnet sich ab, wie abhängig diese erlebte Ähnlichkeit von Raumerleben und Atmosphäre war.

Das Raumerleben änderte sich mit zunehmender Routine. Die Organisator_innen waren aufgrund der positiven Erfahrungen und Rückmeldungen motiviert, das Festival regelmäßig zu wiederholen. Der Ablauf routinisierte und professionalisierte sich dabei. Was früher drei Projektkoordinatorinnen erledigten, konnte bald eine fast allein stemmen, und die logistischen und organisatorischen Schritte waren inzwischen bekannt und erprobt. Im Gegensatz zum ersten Festival erlebte Annike das zweite schon anders:

„Irgendwie hat mich das zweite Turnier nicht sehr berührt. Wobei ich nicht verstehen konnte, weshalb eigentlich nicht. Es lief zwar glatter, oder auch dadurch, dass es alles 
schon mal gab, war es einfacher zu organisieren, aber es hatte nicht so diesen totalen Glanz, dieses Abgehobene.“ (Annike, Interview, 21.11.2013)

Die organisatorische Unerfahrenheit und die Unvorhersehbarkeit wichen einem ,glatten“ Ablauf. Annike vermisste den „Glanz“. Gleichzeitig bemerkte sie die sich einstellende Routine. Die motivierenden, mobilisierenden und bestärkenden Effekte für die Organisierenden drohten mit zunehmender Institutionalisierung und Professionalisierung von DF nachzulassen. Denn die einkehrende Routine bezog sich nicht nur auf den Organisationsablauf, sondern auch darauf, dass es das spezifische Raumerleben „schon mal gab“ und es sie nicht mehr plötzlich überfiel. Damit bedeuten Professionalisierung und Institutionalisierung nicht nur schnelles, verlässliches und vorrauschauendes Erledigen von Aufgaben der Planung und Durchführung, sondern auch ein Schwinden von Unvorhergesehenem und von Überraschungsmomenten, die Raumerleben und Atmosphäre plan- und erwartbar werden lassen. ${ }^{169}$ Sozialwissenschaftliche Forschungen zu sozialen Bewegungen haben darauf hingewiesen, dass Emotionen nicht nur für Mobilisierung und Fortbestand von Protesten zentral sind, sondern auch für ihr Abschwächen und Verschwinden mitverantwortlich sind, wenn etwa Erschöpfung und Frustration das Engagement beenden (Goodwin et al. 2001, 21). Diesen Beobachtungen möchte ich hinzufügen, dass auch die Routinisierung präfigurativer Politiken die Mobilisierungsfunktion einschränken kann: Ein beflügelndes und motivierendes Raumerleben kann sich in einen mess- und planbaren Faktor verwandeln, den es durch Einsatz und Anstrengung zu erarbeiten gilt. Anhand des DF-Festivals lässt sich vermuten, dass präfiguratives politisches Handeln mittels Hetereotopien sein volles Funktionieren nicht nur durch einen Bruch mit der herkömmlichen Zeit entfaltet, sondern auch dadurch, dass dieser Bruch und die durch ihn evozierte Raumwahrnehmung nicht zur Routine gerinnen, sondern stets Elemente des Unerwartbaren, Unvorhersehbaren und Überraschenden behalten.

In den Kapiteln 8.1 und 8.2 habe ich die zunehmende Routine des Aktivismus vor dem Hintergrund organisationsinterner Verstetigungs- und Institutionalisierungsprozesse erklärt. Nun wird ersichtlich, dass nicht nur organisationsinterne Strukturen, sondern ebenfalls politische Praktiken Produkt dieser Prozesse sind. Dies zeigt sich auch im Fortgang jener Zukunftsdiskus-

169 Diese Atmosphäre hatte bereits einen Namen erhalten, mit dem alle etwas verbinden konnten: „DF-Stimmung“. So bemerkte Saskia ein paar Tage, bevor im Winter 2012 die Teilnehmerinnen eines Expertinnenforums anreisten, dass es ja schön wäre, wenn wir an dem Ankunftsabend bei einem gemeinsamen Essen in einem kleinen Lokal „DF-Stimmung“ verbreiten würden. Diese Atmosphäre war planbar: An diesem Abend wurden freundliche Eröffnungsworte gesprochen, alle Teilnehmerinnen persönlich vorgestellt und begrüßt und an den Tischen Kennenlernspiele durchgeführt. Die Organisator_innen saßen nicht separat, sondern nahmen einzeln an den Tischen mit den Teilnehmenden Platz. 
sion, aus der ich zu Beginn dieses dritten Teils zitiert habe. Bald widmete sie sich der Frage nach den konkreten nächsten Schritten:

Als nächsten Punkt machen wir einen Zeitstrahl. Jede soll sich überlegen, wie DF in ein, in drei und in fünf Jahren aussehen könnte, bzw. was dann passiert. Annike stellt die Ergebnisse an der Flipchart dar. Nach einer kurzen Zeit, in der jede für sich nachdenkt, machen wir eine Runde. [...] Annike sagt, dass Kontinuität wichtig sei. [...] Und wir sollten einen festen Rahmen für Formate finden und sie dann häufiger machen. [...] Als Linda an der Reihe ist, bemerkt sie, dass wir alle zwei Jahre ein großes Event machen und so in Berlin sichtbar sein sollten. Im Anschluss daran könnten wir ein Expertinnenforum machen. In einen Jahr sollten wir nach Deutschland einladen, im nächsten Jahr selbst ins Ausland gehen, so würden wir uns Glaubwürdigkeit bei unseren Partnern schaffen. (Feldnotiz, 17.2.2013)

So ist auch die Verfestigung von Projektformaten ein Produkt der Formierung als glaubwürdige Organisation vor heterogenen Kompetenzregimen und trägt letztlich zum Überleben der Organisation bei. Zugleich stellt die Routinisierung mit Blick auf Mobilisierung und Motivierung eine Gefahr erstens für das Fortbestehen dar, wenn Stress oder Arbeitsroutinen die Freude und den Enthusiasmus überlagern. Und zweitens droht die mühsam hergestellte Augenhöhe zwischen Organisierenden und Teilnehmenden $\mathrm{zu}$ schwinden. Wenn die mobilisierenden und bestärkenden - sprich: empowernden - Effekte bei den Organisierenden selbst ausbleiben, werden sie von Lernenden zu Lehrenden, von Überraschten zu Planenden und von Mitgerissenen zu Diensleister_innen eines bestärkenden und motivierenden Raumerlebens, und die Teilnehmerinnen geraten in die alleinige Rolle der Empfängerinnen. Mit zunehmender Routinisierung droht dieses Vorgehen nahe an das abgelehnte neoliberale und eurozentrische Verständnis von Empowerment als einseitiger Befähigung zur Selbstregierung und die entsprechenden Praktiken der Entwicklungshilfe zu rücken (vgl. Darnell 2012, 70; siehe Kap. 5.3.2; 11.1.2).

Hier wird ein paradox anmutender Effekt der Institutionalisierung deutlich: Mit der Verfestigung des Vorgehens drohte einer der Politikzwecke Mobilisierung, Motivierung und gegenseitige Bestärkung auch unter den DFMitgliedern - verloren zu gehen und die mühsam hergestellte Balance zwischen Entwicklungspolitik und nicht-bevormundendem Feminismus aus dem Gleichgewicht zu geraten.

\subsubsection{Resignifizieren und gesehen werden}

Simone betonte in der Zukunftsdiskussion die Wichtigkeit, Frauen im Fußball sichtbar zu machen. Mit dem Festival sowie mit anderen Projekten wollte DF in dominante Darstellungen von Frauen und Frauenfußball eingreifen und differenziertere Bilder zeigen. Während das Festival aufgrund der verschachtelten Ausschließungen nur wenig externes Publikum anlockte, versuchten die Organisatior_innen mit gezielter Presse- und Öffentlichkeitsar- 
beit dem Zweck der Sichtbarkeit näherzukommen. Sie strebten damit jene resignifizierende Wirkung an, die Butler (1997) als transgressiv beschreibt: Wenn gültige Normen zitiert, aber dabei entscheidende Elemente performativ umgearbeitet werden, führen sie das vermeintliche Ideal selbst als Konstrukt vor. In diesem Sinne wohnt der Präfiguration einer feministischen fußballerischen Alternative, die dennoch unverkennbare Ähnlichkeit zum vermeintlichen Original des Männerfußballs aufweist, das Potential inne, die Geschlechterverhältnisse des Fußballs als kontingent zu entlarven und zu destabilisieren.

Solche performativitätstheoretischen Überlegungen lassen jedoch die Frage nach der Rezeption des Präfigurierten weitgehend unbeachtet (vgl. Brill 2009, 111). Hingegen kann eine praxistheoretische Betrachtung konkreter Prozesse des Rezipierens von Botschaften ergänzen und differenziertere Einsichten in die Wirkung dieses geschlechterpolitischen Vorgehens geben. Präfigurieren als politisches Vorgehen impliziert neben den unmittelbar beteiligten Personen ein Gegenüber, das das Dargestellte sieht (Day 2004; Anderson 2010; Butler 2013). Stuart Halls Begriffe des En- und Dekodierens ermöglichen, das Erstellen, Verbreiten und Lesen der gesendeten Bilder als situierte Praktiken zu untersuchen, die immer an die Positionen der Sendenden und Rezipierenden, bzw. an deren Beziehung gebunden sind $(2006,166)$.

Anhand der Feminisierung des Fußballs habe ich bereits auf eine mögliche Diskrepanz zwischen intendierter/gesendeter und rezipierter Bedeutung hingewiesen. Hier trat nun eine weitere Stereotypenfalle zutage: Ähnlich wie differenzbasierte, geschlechterpolitische Forderungen droht auch eine allzu radikale Abwendung von Leistung zugunsten von Solidarität und Inklusion, dominante Weiblichkeitsstereotype zu befeuern und damit die Basis für die Marginalisierung von Frauen im Fußball zu bestätigen (vgl. Bryson 1994): Biologische, biologisierte oder soziale Argumente attestierten historisch und gegenwärtig die Unvereinbarkeit von Frauen und Fußball und sprachen Frauen die Fähigkeit ab, ,richtigen“, sprich Männerfußball zu spielen. Diese Stereotypisierung droht dann, wenn das Zeigen von Leistung, Erfolg und schönen Spielzügen nicht mehr möglich ist, weil Solidarität und Inklusion dazu führen, dass unerfahrene und erfahrene Spielerinnen gleich viele Spieleinsätze erhalten oder die neu durchmischten Teams nicht eingespielt sind. Dieses Dilemma wurde bei der Auswahl der Teilnehmerinnen während eines Plenumstreffens offensichtlich. Birgit gab zu bedenken, dass das soziale Engagement der Teams im Vordergrund stünde, die sportliche Leistung aber dennoch für den politischen Zweck unabdingbar sei: „Wer nicht gut spielt, kann auch keine Räume erobern. Es funktioniert alles nur, wenn man auch gut spielt.“ (Erinnerungsprotokoll, 26.12.2012) Als ich Monate später mit Saskia darüber sprach, bekräftigte sie dies: „Und es stimmt ja auch, wenn sie [Birgit] sagt, dass es Frauen nicht wirklich empowert, wenn die am Spielfeldrand denken, wie schlecht die spielen." (Feldnotiz, 29.5.2013) Birgit und Saskia 
verdeutlichten damit die Schwierigkeit, jenem dekodierenden Publikum am Spielfeldrand oder vor den Fernsehern, die über kein discerning eye verfügten, die dargestellte fußballerische Alternative zu vermitteln. Sie befürchteten gar gegenteilige Effekte: Wenn das Spiel nicht als feministische Alternative zum Männerfußball dekodiert, sondern als Bestätigung der weiblichen Leistungsschwäche gelesen werden würde, wäre das Ziel verfehlt, einen anderen Fußball zu imaginieren und damit den spielenden Frauen Anerkennung und Wertschätzung zu ermöglichen. Über diesen praxistheoretischen Blick zeigt sich so eine gefährliche Nähe zwischen queertheoretischer Deutung (siehe Kap. 17.2.2) und fußballerischer Hegemonie der Männlichkeit. Denn Aneignung und Resignifiaktion funktionieren nur durch die Übernahme jenes Leistungsvertsändnisses, das körperliche wie charakterliche männliche Eigenschaften als Norm setzt.

Die Selbstverständlichkeit des Leistungsprimats, mit der NichtEingeweihte die Bilder von DF rezipierten, wurde durch kulturalisierende Lesarten verkompliziert, wie ein Ereignis im Vorfeld des Festivals 2013 andeutet:

Am Nachmittag haben wir einen Fototermin für ein Gruppenbild vor einer der großen Billboard-Tafeln mit unserem Festival-Plakat. Als ich ankomme, sind schon fast alle da. Ich begrüße alle nacheinander. Annike erzählt, dass wir auf das Cover eines der großen Stadtmagazine kommen würden. Sie ist skeptisch, denn die Redaktion hätte von ihr ein Foto vom damaligen Spiel gegen die iranische Nationalmannschaft angefragt. Sie wollte, und das habe sie explizit gesagt, ein Bild einer „Verschleierten“, also einer Spielerin mit muslimischem Kopftuch. Annike zweifelt, sie findet es nicht gut. Ich zeige ebenfalls Missmut. Wir wägen ab, ob wir dieses Stereotyp zugunsten der gewonnenen Aufmerksamkeit bedienen sollen. Annike scheint dagegen zu sein. (Erinnerungsprotokoll, 11.7.2013)

Annike und mir missfiel die Anfrage der Redaktion, da wir vermuteten, dass das Bild schließlich in einem anderen diskursiven Kontext weiterverbreitet und dekodiert werden würde, als DF es intendierte. Simone erklärte mir DFs Intention, Fotos der internationalen Teilnehmerinnen mit sichtbaren phänotypischen und kulturellen Markern zu verbreiten: „Ich denke immer, dass es so was vermittelt wie: ,Es gibt starke Frauen überall auf der Welt.' Und vielleicht auch in Regionen, wo jetzt Leute hier damit nicht gerechnet haben." (Interview, Simone, Dez. 2013) Mit der Verbreitung von Bildern, die die weltweite Diversität im Frauenfußball darstellen, wollte DF jenen Diskurs kontern, der nicht-westliche Frauen als weniger emanzipiert und damit als schwach und abhängig imaginiert. ${ }^{170}$ Aus der Wortwahl des Stadtmagazins

170 Das muslimische Kopftuch erhielt in diesem Kontext eine spezifische Bedeutung, denn das Tragen des Kopftuchs auf dem Fußballplatz wurde lange von der FIFA verboten. Die frühere jordanische Nationaltorhüterin und Teilnehmerin an DF-Projekten focht das Verbot erfolgreich an. DF verbreitete ihre Erzählung als Beispiel für den erfolgreichen Kampf einer Frau gegen den mächtigen Verband und für ihre Rechte. Das Kopftuch wurde so bei DF zu einem Symbol für ,starke Frauen". 
hingegen schlossen wir, dass sie das Bild in genau jenem unerwünschten Kontext, der sich entlang islamophober Topoi weiter ausdifferenziert, verbreiten würde. Gegenüber den Teilnehmerinnen eines Expertinnenforums erklärte Simone dies so: „In Germany, media is interested in special pictures. They want pictures of women with headscarves. It is a thin line." (Feldnotiz, 8.4.2015) Das Kopftuch fungiert in diesem diskursiven Kontext, so kann mit Sara Ahmed geschlussfolgert werden, als Metonymie, sodass bestimmte Körper mit Begriffen verbunden werden und gar ein Kausalitätsargument zwischen den Begriffen „Islam“ und „unemanzipiert“ impliziert wird, ohne dass es einer expliziten Erklärung bedarf. Die Kraft der Metonymie liegt gerade darin, dass sie diese Verbindungen zwischen Körpern und Begriffen auch dann aufrechterhält, wenn eigentlich genau ihr Gegenteil dargestellt oder gesagt wird (Ahmed 2004, 76). Mit Blick auf den Fußball vermute ich auch für die Begriffe „Fußball“ und „Leistung“ bzw. „Frauenfußball“ und „leistungsschwach“ eine Metonymie, die mit „Islam“ und „unemanzipiert“ interdependent verwoben ist, sodass Kulturalisierung und Leistungsprimat die Grenzen des Sichtbar-Machens ziehen.

Präfigurieren als geschlechterpolitisches Problematisieren wird zwar einerseits durch das günstige Moment ermöglicht, wenn sich Medien interessiert zeigen, Fördereinrichtungen entsprechende Programme zwecks Empowerment, Sportförderung oder internationaler Begegnung bereitstellen, sich zahlreiche internationale Frauenfußball-Initiativen angesprochen fühlen, lokale wie nationale Politiker_innen hierin die Chance zur Inszenierung ihrer Politik sehen oder die Stadtverwaltung ein solches Festival willkommen heißt. Andererseits stößt auch das Präfigurieren von Alternativen, das sich anders als das Stellen von Forderungen nicht gänzlich den Kriterien einer dominanten Öffentlichkeit anpassen, sondern Utopien entwerfen, Normen umarbeiten und eigene Kriterien aufstellen will, sowohl in seiner motivierenden als auch seiner resignifizierenden Wirkung immer wieder an Grenzen. Denn auch dieses Vorgehen ist tief in jene Verhältnisse eingelassen, die es kritisieren und verändern möchte. Für die Organisator_innen bedeutet dies, dass sie immer wieder zwischen zwei konfligierenden Vorstellungen davon, was „Gutes tun“ genau beinhaltet, navigieren mussten: Jene Frauen zum Fußball ermutigen, die sonst womöglich vor Kompetitivität und Leistungsdruck zurückschrecken und ein möglichst diverses und breites Solidaritätsnetzwerk zu bauen, oder möglichst guten Frauenfußball zeigen, die Vereinbarkeit von Weiblichkeit und Fußball demonstrieren und für Anerkennung streiten? 


\section{Zwischenstand: Sich zwischen multiple Strategien und Positionen bewegen}

Ich habe in dieser Arbeit argumentiert, dass sich an der Schnittstelle von Fußball und Geschlechterpolitik heterogene Institutionen, diskursive Aussagen, Personen, Politiken und Verwaltungsprozesse über Differenzen hinweg versammeln, ihr spannungsreiches Aufeinandertreffen die Problematisierung der fußballerischen Geschlechterverhältnisse ermöglicht und neue Akteur_innen und Praktiken produziert. Während im zweiten Teil die Entstehung der Frauenfußball- und Frauenrechtsorganisation Discover Football als solch eine neue Akteurin untersucht wurde, widmete sich der dritte Teil den Praktiken der Problematisierung. Dabei habe ich auf ein Verständnis politischen Handelns zurückgegriffen, das dem Beziehungsgeflecht aus multiplen Stakeholdern als einer horizontalen Machttopografie gerecht wurde.

Ich habe anhand von DF gezeigt, wie zivilgesellschaftliche Akteur_innen die verbandsfußballerischen Geschlechterverhältnisse kritisieren und umarbeiten: Die Organisation richtete sich mit Forderungen an die Dachverbände und schmiedete internationale Koalitionen mit Fußball-Aktivistinnen, um den Forderungen stärkeres Gewicht zu geben; sie kooperierte mit der staatlichen Sportförderung zwecks Einflussnahme auf die Anerkennungsbedingungen des Verbandsfußballs; sie organisierte Fußballveranstaltungen und gründete einen eigenen Fußballverein, um Frauen selbst geschlechtergerechtere Angebote zu machen; sie modellierte die politische, geschlechtliche und wettkämpferische Dimension des Fußballs in ihrem Sinne um und sie mobilisierte weltweit Fußballerinnen zu verstärktem frauenrechtlichem Engagement im Fußball. Bevor ich die einzelnen Zwischenergebnisse zusammenführe, möchte ich resümieren, was diese Beobachtungen denn genau für die Frage bedeuten, wie politisches Handeln durch ein günstiges Moment ermöglicht wurde.

(1) Discover Footballs geschlechterpolitische Interventionen wurden durch das Aufeinandertreffen verschiedener politischer Entwicklungen ermöglicht. Nachdem die Außenpolitik Sport als Mittel des Empowerments von Frauen aus dem globalen Süden sowie der internationalen Friedensförderung entdeckt hatte, stellte sie die Mittel für die von DF organisierten internationalen Fußball-Begegnungsprojekte bereit; da liberalfeministische Forderungen bereits Einzug in die städtische Verwaltung erhalten hatten, wurden DFs Vorhaben und Forderungen entscheidend durch die lokale staatliche Sportförderung unterstützt; und ein breites Bewusstsein für die Benachteiligung von Frauen im Fußball ermöglichte den Zusammenschluss mit anderen lokalen wie auch internationalen Frauenfußballinitiativen, um die Verbände mit Forderungen nach paritätischer Repräsentation unter Druck zu setzen. Diese günstigen Umstände eröffneten verschiedene Möglichkeiten des Problematisierens, die DF ergriff, um durch Forderungen und Präfigurationen von 
Alternativen dem abstrakten Ziel der Geschlechtergerechtigkeit näher zu kommen. Dabei hat sich gezeigt, dass nicht nur die gewählten Strategien, sondern auch die mit ihnen verknüpften Zukunftsvisionen Produkte jener Friction sind, die die begünstigenden Umstände entstehen ließ. Die abstrakte feministische Vision von einem geschlechtergerechten Fußball konkretisierte sich erst im Zuge der Umsetzung der Strategien und war ihnen nicht vorgängig. Wie und welche Zukunft imaginiert und aspiriert werden kann, hängt entscheidend von den politischen Instrumenten und Strategien ab.

(2) Das Einfordern sowie das Darstellen eines geschlechtergerechten Fußballs habe ich als zwei verschiedene Vorgehensweisen untersucht, die sich in ihrem Verhältnis zur politischen Öffentlichkeit, in ihrem Umgang mit bewegungsinternen Differenzen, ihren aspirierten feministischen Zukünften, ihrem Verhältnis zum verbandsfußballerischen Leistungsprimat sowie ihren geschlechterpolitischen Effekten wesentlich unterscheiden. Das Stellen von Forderungen antizipiert die Kriterien einer bürgerlichen, männlich dominierten, liberaldemokratischen Öffentlichkeit und formt entsprechend den Anforderungen der Einstimmigkeit, Rationalität und Plausibilität eine hörbare Stimme. Durch das Ungleichgewicht zwischen Organisierenden und Teilnehmenden wurden lokale, nationale und politische Differenzen teilweise unsichtbar, und in die vermeintlich universell anwendbaren Forderungen schrieb sich eine situierte Politik ein. Diese Politik orientierte sich an der antizipierten Öffentlichkeit, die keinesfalls global war, sondern in Form von liberaldemokratischer Rechtsstaatlichkeit und bürokratischer Verlässlichkeit imaginiert wurde, wie sie die Organisator_innen aus Deutschland kannten. Zugleich habe ich gezeigt, dass den vereinheitlichenden und vereinnahmenden Tendenzen durch demokratische Elemente entgegengewirkt werden konnte, sodass auch nicht-hegemoniale Erfahrungen Einzug in die Forderungen fanden. Während also manche Forderungen auf Basis unterschiedlicher Erfahrungen mit Rechtsstaatlichkeit und Bürokratie miteinander konkurrierten, herrschte aufgrund der globalen Verbreitung und Institutionalisierung liberalfeministischer Argumente und Instrumente mit wenigen Ausnahmen Konsens über die aspirierte Zukunft. Das Ziel der Geschlechtergerechtigkeit versprach sich in Gleichstellung, gleichwertiger Anerkennung und paritätischer Repräsentation in den bestehenden Verbandsstrukturen zu realisieren. Legitimiert wurde dieses Vorgehen durch das gleichheitsfeministische Postulat, wonach Frauen zur gleichen sportlichen Leistung fähig seien, bislang nur durch rechtliche, kulturelle, soziale und ökonomische Restriktionen daran gehindert werden. Während dadurch die Geschlechterverhältnisse des Verbandsfußballs mit Instrumenten wie Quoten, transparenten Verfahren und Diskriminierungsbekämpfung reformerisch bearbeitet wurden, blieb jedoch der Herrschaftsanspruch der Verbände und mit ihm das sportliche Leistungsprimat unangetastet. Hier zeigt sich, dass der liberalfeministische und gleichheitsfeministische Weg zunächst eine - vermutlich ungewollte - Komplizen- 
schaft mit der Hegemonie des Männlichen eingeht: Durch die Anerkennung des und das Streben nach Anerkennung durch das verbandliche Leistungsverständnis fügt sich der liberalfeministische Weg zunächst jener Deutung von sportlicher Leistung, der der männliche, junge, fitte und weiße Körper als Norm eingeschrieben ist. Diese Fügsamkeit wurde mit Blick auf die Hoffnung, später die Verhältnisse ,von innen heraus` zu verändern, akzeptiert. Die geschlechterpolitische Wirkung dieses Vorgehens erstreckt sich dabei nicht nur auf die quantitative Umarbeitung der Geschlechterverhältnisse sowie die möglichen schrittweisen qualitativen Veränderungen von innen heraus. Wie ich gezeigt habe, erfordert das Stellen von plausiblen, rationalen und realistischen Forderungen ein hohes $\mathrm{Ma} ß$ an Artikulationsfähigkeit und Wissen und steht somit nur wenigen privilegierten Frauen zur Verfügung (vgl. Appadurai 2014). Dieses Vorgehen erzielte also auch dahingehend geschlechterpolitische Wirkung, als dass sehr unterschiedliche Frauen jene politische Fähigkeit, öffentlich und überzeugend für eigene Belange und Interessen einzutreten, gemeinsam erlernen und erproben konnten.

Präfiguratives Vorgehen wendet sich hingegen von einer breiteren Öffentlichkeit und ihren Mitsprachebedingungen $\mathrm{ab}$ und einer zeiträumlich begrenzten bewegungsinternen Gegenöffentlichkeit zu. So umgeht es die Adressierung regierender Institutionen und unterbricht damit die Anerkennung und Reproduktion der verbandsfußballerischen Autorität (vgl. Day 2004, 734). Dadurch entstand Raum, um utopische Visionen von Geschlechtergerechtigkeit und radikalere Kritik an den fußballerischen Verhältnissen zu artikulieren - in Form einer teilweisen Umarbeitung des Leistungsprinzips, der qualitativen und quantitativen Umkehrung der Geschlechterverhältnisse und einer entschiedenen Politisierung des Fußballs. Geschlechtergerechtigkeit wurde hier weniger in Form von Gleichheit und Gleichstellung konkretisiert, sondern näherte sich radikalfeministischen Ideen von separaten, von Frauen bestimmten Sporträumen an, in denen sich Sportpraktiken an den Lebensrealitäten von Frauen orientieren und feministische Prinzipien von Solidarität und Ermächtigung im Mittelpunkt stehen. Diese Vision wich von historisch sedimentierten liberalfeministischen Pfaden ab, denn sie strebte nicht nach Gleichstellung innerhalb und Anerkennung durch bestehende Strukturen, sondern präfiguriert einen Fußball jenseits der männlich dominierte Verbandskultur. Da hier kein Zwang zur Einstimmigkeit herrschte, entstand Platz für die heterogenen Erfahrungen der vielen verschiedenen Frauen. Gleichzeitig führten die radikalen Brüche mit fußballerischen Selbstverständlichkeiten immer wieder zu Irritationen und Missverständnis und konnten im äußersten Fall dazu führen, dass sich Teilnehmerinnen aus Angst, in ihren eigenen Kontexten an Legitimität und Unterstützung zu verlieren, abwandten. Das Funktionieren präfigurierender Politik basiert also nicht nur auf impliziten und expliziten Ausschlüssen einer breiten Öffentlichkeit, sondern auch darauf, dass Ausweichmöglichkeiten zur Verfügung gestellt werden und kein 
Mitmach-Zwang herrscht. Zugleich hat meine Analyse gezeigt, dass es ständiges Eingreifen durch die Organisierenden erforderte, um immer wieder auf die Veränderungen und ihre Einhaltung hinzuweisen, sodass ähnlich wie beim Stellen von Forderungen die Ungleichheiten zwischen den organisierenden, weißen Frauen des globalen Nordens und den Teilnehmerinnen, zumeist aus dem globalen Süden, deutlich spürbar wurden. Unter diesen Umständen entfaltete die körperliche Erlebbarmachung feministischer Utopien in der Gegenwart motivierende und mobilisierende Wirkung auf die Beteiligten. Die Umgestaltungen verschoben bewegungsintern die Möglichkeiten dessen, was in Bezug auf Wettkampf und Geschlechterverhältnisse vorstellbar war, und ermutigten, selbst geschlechterpolitisch tätig zu werden bzw. mit dem Engagement weiterzumachen. Doch auch die präfigurierte Utopie ist letztlich tief in die Verhältnisse eingelassen, die sie kritisiert und die die Grenzen der möglichen Umarbeitungen abstecken. Will sie auch jenseits der Gegenöffentlichkeit geschlechterpolitisch wirken und mittels Bedeutungsverschiebungen Bewegung in die verbandsfußballerischen Geschlechterverhältnisse bringen, muss sie sich an den Rezeptionsweisen einer breiteren Öffentlichkeit orientieren. Andernfalls läuft diese Strategie Gefahr, dass ihre radikalen Umarbeitungen nicht ernst genommen oder missverstanden werden. So finden sich Aktivist_innen vor Dilemmata wieder, die letztlich nicht gelöst, sondern nur temporär überbrückt oder ausgehalten werden können - etwa dann, wenn eine Abkehr vom reinen Leistungsmessen drohte als Bestätigung der weiblichen Unfähigkeit zum Fußballspielen gelesen zu werden. Der Bruch mit Selbstverständlichkeiten riskiert in jene Falle zu treten, die das Stellen von Forderungen umgeht, denn je radikaler die Umgestaltungen, desto weniger verständlich sind sie für eine breite Öffentlichkeit.

Die Problematisierung der verbandsfußballerischen Geschlechterverhältnisse stellte sich somit als ein nuancenreicher Prozess dar, der zwischen widerständigen und regierenden Facetten auch strategische Kooperationen, geschickte Aushandlungen, eigenwillige Aneignungen und partielle Umdeutungen sowie vielzählige Kompromisse beinhaltete. Wobei Kompromisse als temporäre Einverständnisse innerhalb fortdauernder agonistischer Relationen verstanden werden können (Mouffe 2000,16), die, so möchte ich anfügen, die geschlechterpolitischen Problematisierungen produktiv halten.

(3) Ich habe das geschlechterpolitische Vorgehen vor dem Hintergrund eines horizontalen, asymmetrischen Machtverständnisses untersucht. So konnte ich herausarbeiten, dass sich die unterschiedlichen Formen politischen Handelns nicht entlang einer eindimensionalen Gegenüberstellung von widerständig und kooptiert-regierend analysieren lassen. Vielmehr wirken hier vielzählige Machtrelationen auf unterschiedliche Weise. So habe ich gezeigt, wie beide Strategien das begünstigende Moment nutzten und die fußballerischen Geschlechterverhältnisse effektiv problematisierten und bearbeiteten, dabei aber in unterschiedlichem Maße jeweils verschiedene Machtverhältnis- 
se situativ stabilisierten. Während manche Verhältnisse, wie die politische Formation des Liberalismus, häufig unbemerkt reproduziert wurden, wurden andere Machtverhältnisse, etwa über das neoliberale und individualistische Empowerment-Verständnis oder das fußballerische Leistungsprimat, situativ umgearbeitet. Beide Strategien produzieren Subjekte, die sich sowohl dem sanften und befähigenden Zwang gängiger politischer Prozeduren, Paradigmen und Kriterien fügen (vgl. Brown 1995; Foucault 2014), als auch als widerspenstige ,active, sometimes dissident, political actors“ auftreten, die verwaltende und regierende Institutionen kritisieren und umarbeiten (Sharma 2008, 196; vgl. Hodžićs 2014; Leve 2014): Wenn Frauen mit solcher Motivation aus den Projekten gingen, dass sie sich fortan gegen die autoritäre Strukturen auflehnten, oder wenn die Aktivist innen mit anderen Fußballerinnen den Verband dazu drängten, endlich einen Mädchen- und Frauenausschuss einzurichten, dann wurden Veränderungen angestoßen, die weit über jene anfängliche Bereitschaft der Verbände zur Förderung des Frauenfußballs hinausgingen und damit die Spielräume des günstigen Moments erweiterten. Keines der beiden Vorgehen ist damit außerhalb von Machtverhältnissen verortet und damit vermeintlich ,reiner' oder ,feministischer' als das andere. Die Frage, die gegenwärtig wieder im feministischen Sport diskutiert wird, ob der politische Weg in "Nischen und Alternativen“ oder ,hinein in die Institutionen" führen solle (Seitenwechsel e.V. 2018), kann daher nicht allgemein und abschließend beantwortet, sondern nur anhand der konkreten und unterschiedlichen Zielsetzungen entschieden werden.

(4) Die analytische Gegenüberstellung der beiden Strategien hat wesentliche Unterschiede in ihren feministischen Positionen und Zielen sichtbar gemacht. Auffallend ist daher, dass sie im aktivistischen Alltag von DF miteinander verwoben waren, ohne als prinzipiell widersprüchlich erlebt zu werden. Nicht nur wechselten sich die Strategien mit den unterschiedlichen Projekten turnusmäßig ab, auch fanden sich innerhalb einzelner Projekte präfigurierende und fordernde Elemente. Etwa, wenn beim Festival Organisator innen und Teilnehmerinnen vor Journalist innen die gleiche Leistungsfähigkeit von Frauen betonten, zugleich aber mit ihren veränderten Spielregeln den Wettkampfgedanken umarbeiteten; wenn bei Expertinnenforen nur Frauen eingeladen und diese damit als verantwortliche und entscheidende Akteurinnen angerufen wurden; wenn sie dabei von einem eigenen Frauen-Verband träumten, sodann aber die Repräsentation in den bestehenden Verbänden forderten; oder wenn DF durch die Gründung ihres eigenen FrauenFußballvereins selbst ein Vereinsleben umsetzte, das die Belange von Frauen an erste Stelle stellte und das Leistungsprimat abschwächte, aber zugleich beim Fußballverband die Gründung einer gleichberechtigten Mädchen- und Frauenabteilung einforderte. Für die Mitglieder von DF war es selbstverständlich, realpolitische Forderungen zu stellen und mit verwaltenden Institutionen, wie Sportpolitik und Verbänden, zusammenzuarbeiten, auf Reformen 
zu hoffen und im nächsten Moment eine radikale feministische Umarbeitung des Fußballs zu entwerfen und konfrontativ die Verbände anzugreifen. In der Praxis bewegten sich die Aktivist_innen situativ zwischen den verschiedenen Visionen, Strategien und damit verknüpften politischen Modi hin und her, ohne dass dies als Widersprüchlichkeit erlebt wurde. Solche „cruising mobilites" (Sandoval 2000, 61) zwischen Instrumenten, Positionen, Ästhetiken und Zukünften verstehe ich mit Chela Sandoval (2000) selbst als zentralen Aspekt feministischen politischen Handelns. Sandoval beschreibt eine solche taktische Beweglichkeit zwischen verschiedenen feministischen Positionen unter US-amerikanischen Dritte-Welt-Feministinnen der 1960er und 70er Jahren, die beständig zwischen liberalen, marxistischen, sozialistischen und radikalen Strategien und Positionen hin und her wechselten. Während die verschiedenen Positionen im weißen Feminismus als ideologisch verankert und daher als miteinander unvereinbare Strategien galten, wurden sie von Feministinnen of Color taktisch eingesetzt, um die geringen Handlungsspielräume zu nutzen. Sandoval schlägt daher vor, das ,movement ,between and among“ ideological positionings" (ebd., 58) selbst als zentrales feministisches Bewusstsein zu fassen, das es vermag, auf die sich ständig wandelnden Machtformationen des Spätkapitalismus und der Postmoderne zu reagieren:

„When enacted in dialectical relation to one another and not as separated ideologies, each oppositional mode of consciousness, each ideology-praxis, is transformed into tactical weaponry for intervening in shifting currents of power." (Sandoval 2000, 57)

Ich möchte mich Sandoval anschließen und diese Beobachtung für das geschlechterpolitische Handeln von DF geltend machen. Dieses ist jedoch in einem zeitlichen Kontext angesiedelt, in dem die Errungenschaften der Frauenbewegungen bereits institutionelle Veränderungen erwirkt haben, sodass die Mobilität sich nicht nur auf unterschiedliche feministische Positionen innerhalb der Protestbewegung, sondern ebenfalls über Zivilgesellschaft und verwaltende Institutionen hinweg erstreckt. Eine solche grenzüberschreitende Beweglichkeit untersucht Newman $(2012$; 2013; 2014) anhand von Feministinnen in Großbritannien. Viele der Frauen, die außer-institutionell für Frauenrechte gekämpft hatten, übernahmen bald Ämter in Verwaltung und Regierung und versuchten, diese von innen heraus feministisch umzugestalten. Dafür wechselten sie ständig zwischen verschiedenen Taktiken.

„[B]order work also linked, inside“ and ,outside“ rationalities, connecting political activism to governmental power, community activism to state work, social movements to professional and organizational change projects, and so on." (Newman 2014, 139)

Mit dieser taktischen Mobilität überschritten sie nicht nur ständig die Grenzen zwischen Graswurzelebene und staatlichen Verwaltungen, zwischen Protestbewegung und Staatsfeminismus, sondern verbanden zugleich die verschiedenen Rationalitäten vermittelnd miteinander (Newman 2012; 2013; 
2014). Mit Sandoval und Newman verstehe ich die Gleichzeitigkeit der unterschiedlichen Vorgehensweisen, Instrumenten, Positionen und daran geknüpfte Zukunftsvisionen von Geschlechtergerechtigkeit sowie die Mobilität zwischen ihnen und über verschiedene politische Ebenen hinweg als zentralen Modus geschlechterpolitischen Handelns in komplexen Gefügen.

(5) Noch einmal möchte ich den Blick zurück auf die Zukunftsdiskussion zu Beginn dieses dritten Teils lenken. Die Selbstbeschreibungen und Positionierungen der Aktivist_innen waren einer vertikalen Machttopografie verhaftet: Sie sahen sich mit der „Basis“ und einem „Unten“ verbunden und traten einem „Oben“ skeptisch gegenüber. Diese Positionierung entspricht der präferierten Formierung als Graswurzelaktivistin (siehe Kap. 12; 13), sodass hier der Schluss nahe liegt, dass eine vertikale Machttopografie selbst als normatives Konzept im Feld wirkmächtig wurde. Als Birgit grinsend anmerkte, DF sei „das gute Gewissen des Frauenfußballs“, und Linda „das Ohr an der Basis“ sein und den „Finger in der Wunde“ legen wollte, zeichnete sich in der Verwendung dieser romantisierenden Allgemeinplätze Wissen um Machttopografien sowie entsprechende widerständige politische Praktiken ab. In Kapitel 13.5 habe ich mithilfe von Hodžićs NGOisierungs-Paradigma (2014) argumentiert, dass die Formierung von Organisationen durch das Wissen um wissenschaftliche Klassifizierungen von NGO und Graswurzel-Aktivismus angeleitet wird und sich ursprünglich analytische Kategorien zu normativen verfestigt haben. Sodann habe ich in Kapitel 15 auf ein dualistisches Verständnis politischen Handelns verwiesen, das tendenziell Widerstand auf Graswurzelebene romantisiert (Abu-Lughod 1990). Mit Blick durch Hackings looping effect (2007) auf die Positionierung von DF lässt sich nun schlussfolgern, dass nicht nur das NGOisierungs-Paradigma, sondern auch die vertikale Machttopografie im Feld normativ wirksam sind und die Positionierung als spezifisch politisch Handelnde anleiten. In Kapitel 2.3 habe ich argumentiert, dass in Forschungsfeldern, die beständig Bezug auf ihr eigenes Tun nehmen, die Gefahr besteht, die Selbstdeutungen des Felds zu übernehmen und damit letztlich nur eine reflexive Doppelung statt ethnografische Einsicht zu produzieren. Die Positionierung auf Graswurzelebene und die damit einhergehende Verortung entlang der Widerstand-Regierung-Dichotomie ist daher nicht mit einer para-ethnografischen Beschreibung zu verwechseln, sondern ist selbst strategische geschlechterpolitische Praxis. Formieren und Handeln sind damit zwei aufeinander bezogene, jedoch nicht unweigerlich kongruente Facetten geschlechterpolitischer Problematisierungen, die sich durch ständige Beweglichkeit auszeichnen und so den vielzähligen und sehr unterschiedlichen Bedingungen und Kompetenzerwartungen sowie den eigenen moralischen Idealen begegnen. Zum Schluss werde ich nun eruieren, wie Beweglichkeit als Modus des Problematisierens konzeptualisiert werden kann und was ein solches Fazit in jenen reflexiven Feldern macht, die mithilfe paraethnografischer Konzepte auf sich selbst Bezug nehmen. 


\section{Zum Schluss}

„Nach dem Spiel ist vor dem Spiel“ - was für ein Fußballturnier gilt, trifft auch auf wissenschaftliches Arbeiten zu. Jede ethnologische Erkundung mündet unweigerlich in viele neue Fragen. Das Ziel dieser Ethnografie war die Exploration feministischer Interventionen in den Fußball in ihren historischen, diskursiven und vor allem gegenwärtig praktischen Dimensionen. Ein solcher Ansatz erlaubt kein endgültiges Fazit - stattdessen führe ich im Folgenden die Ergebnisse der drei Teile dieser Arbeit zusammen. Ich diskutiere anschließend die zukünftigen Entwicklungen des Fußballfeminismus sowie zentrale Forschungsperspektiven für die ethnologische Sport-, Politik- und Geschlechterforschung an der Schnittstelle von Fußball und Feminismus. Daran anknüpfend eruiere ich, wie die Analysen dieser Arbeit über das konkrete Forschungsfeld hinaus für die politikethnologische Forschung fruchtbar gemacht werden können, und schlage vor, Beweglichkeit durch das Konzept der politischen Navigation als zentralen Modus geschlechterpolitischer Interventionen zu denken. Ich schließe mit einem Antwortvorschlag auf die Frage, was solche Erkentnisse in den politisch aktiven Feldern bewegen können, in und mit denen sie generiert wurden.

Wie werden fußballerische Geschlechterverhältnisse problematisiert? Diese forschungsleitende Frage habe ich in einem Dreischritt untersucht, wobei ich mit jedem Schritt ein separates theoretisches Interesse verfolgte. Erstens habe ich danach gefragt, wie etwas so Selbstverständliches wie die Geschlechterverhältnisse im Fußball in Zweifel gezogen werden kann; zweitens habe ich anhand der Frauenfußballorganisation Discover Football untersucht, wie sich eine Organisation formiert, um von allen Beteiligten als kompetente Problembearbeiterin anerkannt zu werden; und drittens habe ich eruiert, wie diese nichtstaatliche Organisation die fußballerischen Geschlechterverhältnisse bearbeitet.

Im ersten Teil habe ich die Schnittstelle von Fußball und Feminismus mithilfe Tsings Konzepts der Friction als produktives, dynamisches Aufeinandertreffen historischer Entwicklungen und diskursiver Formationen untersucht. Ich habe argumentiert, dass dieses Aufeinandertreffen ein Momentum für die geschlechterpolitische Problematisierung des Fußballs erzeugte, denn plötzlich galt es als plausibel, die Benachteiligungen und Herabwürdigungen von Frauen im Fußball öffentlich anzuprangern und Geschlechtergerechtigkeit einzufordern. Verschiedene um die Jahrtausendwende schließlich aufeinandertreffende Entwicklungen und Umstände ermöglichten es, dass die fußballerischen Geschlechterverhältnisse instabil und damit problematisierbar geworden waren: Die internationale zweite Frauenbewegung sowie die Frauenrechtskommission der UN hatten Frauenrechte und Geschlechtergerechtigkeit als zentrale politische Themen etabliert, denen sich der Sport nun nicht 
verschließen konnte; Frauen forderten seit den Anfängen des Fußballs und teilweise durch Regelverstöße ihre Teilhabe ein, und die deutsche Nationalmannschaft feierte internationale Erfolge; staatliche sowie inter- und transnationale Institutionen hatten die Frau als Zielgruppe sowie den Sport als Mittel ihrer Politiken entdeckt; der DFB schrieb sich zu Beginn des neuen Jahrtausends die Förderung des Frauenfußballs sowie den Kampf gegen Diskriminierungen auf seine Agenda und machte die Weltmeisterinnenschaft 2011 in Deutschland zu einem großen Spektakel; und nicht zuletzt hatte die Kritik an Korruption, Menschenrechtsverletzungen und Kommerzialisierung den Machtanspruch der Verbände erodieren lassen. Die stabilen, diskursiven Formierungen des Fußballs als verbindend-integrative und charakterbildendempowernde Tätigkeit vereinten über Differenzen hinweg Verbandsfußball, Wissenschaften, feministische Sportprojekte, staatliche Entwicklungs- und Außenpolitik sowie inter-/transnationale Entwicklungsinstitutionen. Die Diskurse stellten die argumentativen Ressourcen bereit, um die gesellschaftsund geschlechterpolitische Relevanz und Förderungswürdigkeit des weiblichen Sporttreibens zu betonen. Zum einen konnte nun argumentiert werden, dass weibliches Sporttreiben wichtige charakterbildende Effekte im Sinne von Emanzipation und Entwicklung ermöglichte, zum anderen galt dieses Sporttreiben, gerade in internationalen Kontexten, als entwicklungs- und außenpolitisch effektives Instrument der Völkerverständigung und der Entwicklung. Diese argumentativen Ressourcen erfuhren dadurch Unterstützung, dass das bürgerliche Sportverständnis vom unpolitischen Sport ins Wanken geraten war. Die diskursive Formierung des Fußballs als von politischen, v.a. geschlechtlichen Machtverhältnissen durchkreuzt, konstituierte die fußballerischen Geschlechterverhältnisse zugleich als eine frauenrechtliche Angelegenheit und verlieh feministischen Forderungen nach sportlicher Partizipation und der Bekämpfung von Ungleichbehandlung Nachdruck. Diese spezifische diskursive Formierung des Fußballs verband innen-, entwicklungs- und außenpolitische Institutionen, NGOs, feministische Initiativen und Fußballverbände auf spannungsreiche Art miteinander. In diesem Sinne habe ich argumentiert, dass Problematisierungen auch als Versammlungen gedacht werden können, die verschiedene Institutionen, Politiken und Interessen zusammenbringen, um gemeinsam ein Problem zu bearbeiten.

Dieses produktive Aufeinandertreffen habe ich im zweiten Teil untersucht und die Entstehung einer Organisation als Produkt dieser Friction gedeutet. Entsprechend dem heterogenen Gemenge aus Akteur_innen und Institutionen samt unterschiedlicher Erwartungen und Anerkennungskritierien (landscape of practice) habe ich die Frage verfolgt, wie sich eine Organisation als kompetent formiert, um mit der Bearbeitung der fußballerischen Geschlechterverhältnisse betraut $\mathrm{zu}$ werden. Aus praxistheoretischer Perspektive habe ich zivilgesellschaftliche Organisationen als kontinuierliche Prozesse der Formierung untersucht und argumentiert, dass diese, wollen sie als legitime 
politische Kräfte in der heterogenen Landschaft spätkapitalistischer demokratischer Regierungsverhältnisse anerkannt werden, geschickt zwischen verschiedenen Gestalten changieren müssen. So konnte ich bei Discover Football erleben, wie die Gruppe junger Frauen gegenüber staatlich-verwaltenden Institutionen, transnationalen Regierungsorganisationen und NGOs sowie zivilgesellschaftlichen Fördereinrichtungen als verlässliche, professionelle Partnerorganisation und Expertin im Bereich Fußball und Gender auftrat. Vor der links-alternativen städtischen Bewegungsszene präsentierte sie sich zugleich als feministische, kämpferische Alternative zum Verbandsfußball. Und sportpolitische Institutionen, wie das Bezirkssportamt, zollten ihnen als regelmäßige Sportveranstalterin im Stadtteil Anerkennung und Förderung. Gegenüber den Fußballverbänden traten die Aktivist innen nicht nur konfrontativ als Kritikerinnen auf, sondern stellten zugleich durch ihre Teilnahme am organisierten Wettbewerb ihre sportliche Ernsthaftigkeit unter Beweis und gliederten sich so in die verbandliche Organisationsstruktur ein. Während sie in einer internationalen Förderlandschaft als Frauenrechts- und Fußballexpertinnen auftraten, betonten sie gegenüber weltweiten FrauenfußballInitiativen und feministischen Aktivistinnen ihren Status als Graswurzelorganisation, die aus den Erfahrungen von eigener Betroffenheit heraus „von unten" aktivistisch die Verhältnisse anprangerte - eine Gestalt, die sich in der Subjektposition der female football activist verdichtete und den Mitgliedern ein affirmatives Identifikationsangebot machte. Daraus habe ich geschlussfolgert, dass das kompetente und glaubwürde Sich-Organisieren ein permanentes Navigieren durch verschiedene Diskurse, rechtlich-institutionelle Rahmenbedingungen, heterogene Erwartungen und moralische Vorstellungen sowie unterschiedliche Politikziele entlang eigener moralischer Leitplanken und geleitet von utopischen Visionen erfordert. Dabei wird das eigene Wissen geschickt zum Einsatz gebracht, um situative zwischen multiplen Gestalten entsprechend den heterogenen, teils widersprüchlichen Anforderungen zu changieren.

Allerdings ging die Gleichzeitigkeit multipler Gestalten nicht ohne Widersprüche und Dilemmata einher, und es hatten sich zwei zentrale Spannungslinien herauskristallisiert: Erstens stellte das Verhältnis zwischen feministisch-fußballerischer Graswurzelinitiative einerseits und ExpertinnenNGO andererseits die Aktivist innen immer wieder vor das Dilemma, globale Ungleichheitsstrukturen nicht zugunsten gegenseitiger Solidarität und gleichberechtigter Partizipation auflösen zu können, solange ihr eigenes organisatorisches Überleben durch entwicklungs- und außenpolitische Sportfor-Development-Politiken gesichert wurde. Als nichtstaatliche, gemeinnützige und transnational agierende Organisation unterlag sie der Marktförmigkeit, die im Zuge öffentlicher Reformverwaltung feministische Mitstreiterinnen in Konkurrentinnen um Ressourcen verwandelt. Während das Anknüpfen an entwicklungspolitische Empowerment-Politiken den Zugang zu Ressour- 
cen sicherte, entstanden dadurch in den internationalen Begegnungsprojekten ungewollte Hierarchien und Ungleichheiten zwischen den Fußballerinnen und Organisatorinnen aus dem globalen Norden und den teilnehmenden Spielerinnen aus dem globalen Süden. Zweitens konnte die Diskrepanz zwischen feministischer fußballerischer Alternative und fußballerischem Leistungsprimat nicht gänzlich aufgelöst werden; solange die Fußballverbände als Adressaten der Forderungen und als Kooperationspartner zur Umsetzung geschlechterpolitischer Maßnahmen adressiert wurden, bedeutete eine völlige Abkehr von Leistungsstreben und Wettkampf zugunsten von Solidarität, Emanzipation und Inklusion, die eigene Glaubwürdigkeit zu riskieren, Weiblichkeitsstereotypen in die Hände zu spielen und damit letztlich die bestehenden Verhältnisse zu stabilisieren. Diese Dilemmata konnten nicht gelöst, sondern nur temporär in Einklang gebracht, ausbalanciert oder schlicht ausgehalten werden. Changieren, Ausbalancieren und In-Einklang-Bringen habe ich in Anlehnung an Vigh (2009) als Praktiken sozialer Navigation gedeutet. Ich habe vorgeschlagen, Navigieren als zentralen Modus zivilgesellschaftlicher Organisationen zu denken, die sich im Rahmen neoliberaler öffentlicher Reformverwaltungen und vor dem Hintergrund komplexer und schwer vorhersehbarer Möglichkeitsräume als geeignete Akteurinnen anbieten, um Politiken für gegenwärtige soziale Probleme zu implementieren.

Im dritten Teil habe ich mich den geschlechterpolitischen Vorgehensweisen von Discover Football zugewandt. Mein Interesse galt dabei der Frage, wie sich geschlechterpolitisches Handeln zivilgesellschaftlicher Akteur_innen in jenen multizentrischen Gefügen spätkapitalistischer Demokratien $\bar{j}$ enseits einer vertikalen Machttopographie aus widerständigem Graswurzelaktivismus, kooptiertem NGO-Handeln und machtvoll regierenden Institutionen denken lässt. Ich habe hierfür auf ein Foucaultsches Machtverständnis zurückgegriffen, um das multizentrische Geflecht aus machtvollen Beziehungen zu konzeptualisieren. Somit habe ich Problematisieren als geschlechterpolitisches Handeln untersucht, das sich durch Hybridität, Nuancenreichtum und Mehrdimensionalität auszeichnet. Ausgehend von den geschlechterpolitischen Strategien von Discover Football habe ich zu Analysezwecken zwischen zwei Vorgehensweisen - das Stellen von Forderungen und das Präfigurieren von Alternativen - anhand ihres Verhältnisses zur politischen Öffentlichkeit, ihres Umgangs mit translokalen Differenzen, ihrer aspirierten $\mathrm{Zu}-$ künfte sowie ihrer Beziehungen zu den Prinzipien des Verbandsfußballs unterschieden. Das Stellen von Forderungen setzte voraus, eine rationale, vernünftige und in sich kohärente Stimme entlang der Mitsprachekriterien einer bürgerlichen politischen Öffentlichkeit herzustellen - ein Prozess, der wenig Raum für heterogene Erfahrungen ließ und liberalfeministischen Instrumente als globale Strategien imaginierte. Dabei wurde leicht übersehen, dass diese Instrumente auf das engste mit bürokratischer Rechtsstaatlichkeit und liberal-pluralistischer Demokratie verbunden waren, wie sie im lokalen 
Kontext von DF selbstverständlich in den staatlich-rechtlichen Kontexten mancher Teilnehmerinnen jedoch nicht gegeben waren. Zugleich eröffnete diese Vorgehensweise Mitsprache- und Gestaltungsmöglichkeiten, die die fußballerischen Geschlechterverhältnisse schrittweise im reformerischen Sinne umarbeiten konnten. Während so die Herrschaftsweise der Verbände kritisiert und umgearbeitet werden konnte, blieb ihr Herrschaftsanspruch, und mit ihm das Leistungsprimat, zunächst unangetastet - versprach doch die Möglichkeit, Strukturen ,von innen heraus' zu verändern, befriedigende Ergebnisse in Sinne liberalfeministischer Vorstellung von Gleichstellung und Anerkennung.

Im Gegensatz dazu richtete sich das Präfigurieren von Alternativen an eine bewegungsinterne Öffentlichkeit und ließ daher mehr Platz für translokale Differenzen und für jene utopischen und idealistischen Visionen, die andernfalls als unvernünftig verworfen worden wären. Präfigurative Politiken konnten eine feministische Vision des Fußballs in der Gegenwart körperlich erlebbar machen, die mit vielen Selbstverständlichkeiten brach und die leistungsbezogenen, vergeschlechtlichten und politischen Dimensionen des Fußball radikal modifizierte. Anders als das Stellen von reformerischen Forderungen umging das Präfigurieren von Alternativen den Herrschaftsanspruch der Verbände und schuf zeiträumlich begrenzte, separate Räume. Durch die Erlebbarmachung dieser radikalfeministische Vision eines eigenständigen, anderen Fußballs entfaltete dieses Vorgehen innerhalb der angesprochenen Gegenöffentlichkeit mobilisierende und motivierende Wirkung und kreierte neue Zukunftsimaginationen, riskierte aber zugleich, von der breiten Öffentlichkeit nicht verstanden zu werden, sodass die resignifizierende Wirkung zunächst auf einen kleinen Kreis beschränkt blieb. Denn die politischmännliche Öffentlichkeit rezipierte die feministischen Fußballpraktiken entsprechend der naturalisierten Verwobenheit von Leistung und Geschlecht, sodass eine rigide feministische Kritik an Leistung und Wettkampf letztlich drohte, als Beleg für die weibliche Unsportlichkeit gelesen zu werden. Um öffentliche Anerkennung zu erhalten, mussten sich Fußballerinnen letztlich mittels sportlicher Leistung als gleichberechtigt beweisen und sich damit einem Leistungsverständnis unterwerfen, dem der männliche, junge, weiße Körper als Norm eingeschrieben ist. Während in beiden Strategien also wieder jene zentralen Spannungslinien zwischen Feminismus und NGO-Handeln einerseits und Feminismus und Fußball andererseits zutage traten, stach zugleich die Gleichzeitigkeit der beiden Vorgehensweisen, die sich doch grundlegend in ihren feministischen Positionen und ihrem Umgang mit der verbandsfußballerischen Hegemonie unterschieden, ins Auge. Aus der Gleichzeitigkeit dieser beiden Strategien und in Anlehnung an die Arbeiten von Sandoval (2000) und Newman $(2012 ; 2013 ; 2014)$ habe ich geschlussfolgert, dass politisches Handeln die ständige Mobilität zwischen geschlechterpolitischen Strategien, Positionen, Instrumenten und Zukünften sowie die Fähig- 
keit, zwischen diesen zu vermitteln und sie in den eigenen Vorgehensweisen miteinander zu verbinden, beinhaltet.

\subsection{Quo vadis Fußballfeminismus?}

Meine Arbeit hat die Möglichkeiten und Bedingungen für geschlechterpolitische Umarbeitungen des Fußballs herausgearbeitet. Doch wie geht es weiter? Ausgehend von meiner Analyse kann ich hier nur spekulieren, welcher Feminismus im Fußball in Zukunft möglich und effektiv sein wird. Ich habe gezeigt, dass radikale feministische Kritik an den Grundfesten verbandsfußballerischer Herrschaft und ihrer Deutung über die Art und Weise des Fußballspielens in Nischen verweilt. Jene feministischen Sportprojekte der 1980er Jahre, die entschieden mit Kräftemessen und Körpermanipulation brachen und sich entschlossen hatten, nicht den Weg in die Institutionen zu gehen, bestehen zwar weiterhin, haben aber kaum Einfluss auf die öffentliche Diskussion um Geschlechtergerechtigkeit im Sport. Diese wird gegenwärtig von liberalfeministischen Forderungen, wie jenen nach gleicher Bezahlung und angemessener medialer Repräsentation mit Verweis auf gleiche Leistung und Gleichwertigkeit der weiblichen Athletinnen, dominiert. Zu vermuten ist daher, dass es zukünftig nicht etwa zur zahlreichen Gründung von Frauenverbänden und feministischen Sportveranstaltungen kommt, sondern Frauen vermehrt für paritätische Repräsentation in, Gleichbehandlung und Anerkennung durch die bestehenden Verbände streiten werden. Ob diese dann die über den Fußball bestimmenden Institutionen von innen heraus umarbeiten und ihre patriarchalen Instrumente geschlechtlich umkodieren oder aber sich diesen anpassen, bleibt abzuwarten.

Aus dem explorativen Charakter meines Vorgehens haben sich neben der Frage nach zukünftigen Entwicklungen noch weitere Forschungsperspektiven für die Ethnologie und Geschlechterforschung herauskristallisiert. Ich habe die feministische Problematisierung des Fußballs in ihren vielzähligen historischen, diskursiven, politischen und sozialen Relationen anhand eines kleinen Ausschnitts aus der sozialen Welt nachgezeichnet. Diese Perspektive ist unweigerlich partiell, sodass diese Arbeit viele Aspekte von kulturanthropologischem Interesse zwar berührt hat, jedoch nicht weiter verfolgen konnte. Als Impulse für weiterführende Forschungen möchte ich zwei mir besonders wichtig erscheinende Forschungsperspektiven hier nennen:

(1) Während die Geschichte des Frauenfußballs in verschiedenen europäischen Ländern hervorragend rekonstruiert worden ist, ist die Schnittstelle zwischen Feminismus und Fußball aus historischer Perspektive gerade für Deutschland wenig erforscht. Welchen Einfluss hatten Argumente, Kritiken und Theorien der ersten und zweiten Frauenbewegungen auf den Sport? 
(Wie) kamen Sportlerinnen mit feministischen Gedanken in Kontakt? Welchen Einfluss übten Frauenrechtlerinnen auf die Sportverbände aus? Allein Pfister (1980) bietet eine Sammlung von sportbezogenen Streitschriften aus der ersten Frauenbewegung. Und Kerstin Thieler und Laura Schibbe (2016) versammeln einzelne historische Erkundungen zur Auseinandersetzung mit Sport innerhalb der zweiten Frauenbewegung. Tiefergehende Forschungen sind meines Erachtens deshalb relevant, da sie die geschlechterpolitischen Entwicklungen des Sports als relationale Prozesse, eingebettet in politische, soziale und kulturelle Zusammenhänge, erfassen und damit auch die Wirkungen der Frauenbewegungen in jenen Kontexten sichtbar machen würden, die sich selbst als politisch unberührbar verstehen.

(2) In dieser Arbeit ist das komplizierte Verhältnis zwischen Leistungsprimat und Feminismus immer wieder angeklungen. Zukünftige Arbeiten sollten dieses Verhältnis anhand u.a. folgender Fragen weiter ausleuchten: Welche Spannungen, aber auch Komplizenschaften mit der Männlichkeit des Fußball und den Instrumenten patriarchaler Herrschaft ergeben sich aus der liberalfeministischen Anerkennung des Leistungsprimats und dem Streben nach Partizipation und Gleichstellung? Welche Möglichkeiten der Umarbeitung von Fußballverbänden und -vereinen aus ihrem Inneren heraus bieten sich den liberalfeministischen Instrumenten im Detail und welche Effekte haben sie? Wie sind fußballerisches Leistungsprimat und eine Neoliberalisierung des Feminismus verwoben, bzw. (wie) widersteht der Fußball als Teamsport einer neoliberalen Vereinnahmung, die auf Vereinzelung und Individualismus basiert? Welche unliebsamen Nähen entstehen zwischen einer queertheoretischen Perspektive, die die Resignifikation der Geschlechternormen durch sportlich-kompetente weibliche und queere Körper betont, und einem neoliberalen Imperativ der individuellen Verantwortung sowie einer patriachialen Aufforderung sich beweisen zu müssen? Meines Erachtens bietet der Sport aufgrund der Zentralität körperlicher Leistung ein besonders geeignetes Forschungsfeld, um die spannungsreichen Beziehungen zwischen sozialen und biologischen Körpern, unterschiedlichen Feminismen und neoliberalem Leistungsprimat und Individualismus genauer zu beleuchten.

\subsection{Politische Navigationen}

Die ethnografische Erkundung der Prakitken einer Frauenfußball- und Frauenrechtsorganisation erlaubt jedoch nicht nur weiterreichende Aussagen über und Fragen an die Schnittstelle von Fußball und Feminimus, sondern ermöglicht auch feldübergreifende konzeptuelle Überlegungen zur ethnologischen Auseinandersetzung mit politischem Handeln. Dafür möchte ich noch einmal zu der zentralen Frage zurückkommen, wie Geschlechterverhältnisse in ge- 
genwärtigen spätkapitalistischen Demokratien problematisiert werden. Sowohl in Teil II als auch Teil III dieser Arbeit hat sich Beweglichkeit als zentraler Modus des Problematisierens herauskristallisiert: sei es das Navigieren durch heterogene Anerkennungsregime mithilfe des Changierens zwischen Gestalten und des Ausbalancierens von Widersprüchen und Spannungen, oder die grenzüberschreitende und verbindende Mobilität zwischen Strategien, Positionen und Visionen. Letztlich ist zu berücksichtigen, dass Organisationsformierungen und Strategien auf das Engste miteinander verflochten sind. So ermöglichte das Formulieren rationaler Forderungen sowohl von internationalen NGOs, der Sportpolitik als auch den Verbänden als Expertinnen für Fußball und Frauenrechte ernst genommen zu werden und gleichzeitig Netzwerke zu anderen engagierten Frauen in Entscheidungspositionen des Fußballs und der Sportpolitik zu knüpfen. Über das Präfigurieren von Alternativen hingegen konnten sich die Organisator_innen als Graswurzelaktivist innen erleben und gemeinsam mit anderen Fußballerinnen eine Frauenfußball-Bewegung erfahren, die die fußballerischen Verhältnisse radikal anzweifelte. Ausgehend von dieser engen Verwobenheit von Organisationsformierung und strategischem Vorgehen schlage ich vor, Beweglichkeit zusammengefasst als zentralen Modus der Infragestellung, Bearbeitung und Veränderung fußballerischer Geschlechterverhältnisse in den komplexen, asymmetrischen Gefügen spätkapitalistischer Demokratien zu verstehen.

Eine solche Beweglichkeit heben bereits Anthropologinnen wie Amrita Chhachhin und Renée Pittin (1999), Dorothea Hilhorst (2003) und Aradhana Sharma (2014) anhand von Studien zu Entwicklungsprojekten hervor. Sie zeigen, dass das beständige Changieren zwischen multiplen Gestalten elementarer Modus von NGOs ist, um sich in komplexen ökonomischen und gesellschaftspolitischen Gefügen zu legitimieren und Handlungsspielräume zu schaffen. Justyna Struzik (2019) hat anhand der polnischen LGBTI- und AIDS-Bewegung gezeigt, wie Aktivist_innen flexibel und mithilfe verschiedener sozialer Rollen durch politisch restriktive Bedingungen manövrieren. Zugleich haben feministische und postkoloniale Studien wie jene von Chela Sandoval (2009) und Janet Newman (2012) zu sozialen Bewegungen oder feministischen Interventionen gezeigt, dass Aktivistinnen zwischen verschiedenen Taktiken und ideologischen Positionen wechseln und diese eklektisch anwenden, um die beschränkten Handlungsräume zu nutzen und die Verhältnisse zu bearbeiten. Diesen Studien ist gemein, dass sie sich von einer (dominanten) normativen Perspektive auf Fragen des Politisch-Seins und -Handelns lösen und stattdessen prozessual das Changieren, Balancieren und Manövrieren, die Metamorphosen und grenzüberschreitende Mobilität analysieren. Diese Praktiken vereint der Aspekt der Beweglichkeit in einem begrenzten und strukturierten Raum - der wiederum mal größere Schritte und strategische Planung erlaubt, mal taktisch-schnelle Reaktionen, spontane Richtungswechsel oder vorsichtiges Aus- und Vortasten erfordert. 
Mit Vighs Konzept der Social Navigation (2009, vgl. Kap. 14) kann diese Beweglichkeit konzeptuell weiter geschärft werden. Social Navigation basiert auf der Beobachtung der Praktiken von Individuen, mit denen diese auf sich rapide wandelnde Umstände, soziale, politische und ökonomische Unsicherheiten und Instabilitäten reagieren. Navigieren beinhaltet dabei die Antizipation zukünftiger Veränderungen, obwohl diese aufgrund der Komplexität nie verlässlich prognostiziert werden können. In diesem Sinne umfasst das Konzept sowohl spontane und situative Manöver im Sinne schneller Reaktionen, als auch vorausschauende Bewegungen, die sich an der Imagination einer besseren Zukunft orientieren. Die Stärke dieses Konzepts liegt aber vor allem darin, dass es sowohl die soziale Umwelt als auch die sozialen Praktiken als dynamisch greifen kann; es richtet den Fokus auf die Interaktion zwischen den Bewegungen von Akteur_innen und den Bewegungen der sozialen Formationen, Strukturen und Verhältnisse. So gelingt es zu zeigen, wie Akteur_innen ihre Strategien entsprechend sozialer Veränderungen, Instabilität und Unsicherheit anpassen oder neu ausrichten (ebd.).

Mir erscheint es vor dem Hintergrund meiner eigenen Beobachtungen, wie auch der ethnologischen Beobachtungen zu Aktivismus und NGOs sinnvoll, das Social Navigation-Konzept nicht nur für die Analyse von individuellem Handeln, sondern auch im Sinne politischer Navigation für die Untersuchung von Organisationen und ihren Praktiken der Beweglichkeit fruchtbar $\mathrm{zu}$ machen. So hat meine Analyse gezeigt, wie die Fußball-Aktivist_innen auf die kaum vorherzusehenden Dynamiken und die Komplexität des günstigen Moments beweglich und pragmatisch, doch von einer geschlechterpolitischen Vision geleitet reagierten. Die Übertragung auf politische Kontexte, also auf jene, die danach streben, Bewegung in die sozialen und politischen Umwelten zu bringen, ermöglicht eine weitere Verfeinerung des Navigationskonzepts. Während Vigh die Interaktion zwischen Akteur_innen und sozialer Umwelt mit Blick auf die Akteur_innen untersuchte, lässt sich für politische Felder fragen, welche Bewegungen in den sozialen Verhältnissen angestoßen werden. So hat meine Arbeit gezeigt, wie die Aktivist_innen mit ihrem alltäglichen Handeln die Verhältnisse in neue Bewegungen versetzten, und zwar indem sie mit dem Changieren zwischen den Gestalten, dem Ausbalancieren von Widersprüchen neue Akteur-Konstellationen schufen und mit dem Wechsel zwischen verschiedenen Strategien und feministischen Positionen geschlechterpolitische Veränderungen in Gang setzten.

Zugleich - dies wird gerade dann besonders deutlich, wenn man Praktiken des Navigierens in politischen Feldern untersucht - verlaufen diese Bewegungen keinesfalls willkürlich. Ihr Radius ist nicht nur durch die Möglichkeitsbedingungen der begünstigenden und einschränkenden Umstände und ihre historisch sedimentierten Pfade begrenzt, sondern sie werden auch von ideologischen und moralischen Leitplanken flankiert, und von abstrakten 
Visionen geleitet. Sie stecken den Navigationsradius ab und ziehen die definitorische Grenze zwischen Beweglichkeit und Beliebigkeit.

Die ethnologische Auseinandersetzung mit Problematisierungen gesellschaftspolitischer Verhältnisse gewinnt durch die konzeptuelle Schärfung dieser Beweglichkeit ein analytisches Instrument, das zivilgesellschaftliches Handeln als relational zu den dynamischen Verhältnissen, in die es eingelassen ist, greifen kann und das zugleich erfasst, wie sich politische Praktiken und soziale, ökonomische, geschlechtliche und politische Verhältnisse wechselseitig konstituieren.

\subsection{Kritik und die Lücken im paraethnografischen Wissen}

Doch was kann solch ein wissenschaftliches Wissen in den Feldern, in und mit denen es generiert wird, tun (siehe Kap. 2.3.3)? Und welcher Arbeitsauftrag ergibt sich daraus für zukünftige Forschungen in, mit und durch politisch aktive Felder?

Ich habe ausführlich beschrieben, wie sich DF mithilfe feministischer Kritik am Leitungsprimat und an globalen Machtverhältnissen, aktivistischer Moralvorstellungen vom ,guten' (sprich: widerständigen) Handeln, erfahrungspolitischer Strategien sowie Wissen um staatliche Politiken in Bezug auf Sport und Geschlecht eine Bricolage aus Wissenspraktiken zusammenstellte. Gerade jener Wissensbestand an der Schnittstelle von feministischer Wissenschaft und Aktivismus diente DF dazu, das eigene Tun immer wieder selbstkritisch zu befragen und damit auch eine Distanz zu diesem aufzubauen. Dies wurde einmal mehr im Zuge der Vorbereitungen für das Festival 2013 sichtbar. Teilnehmen sollte auch ein Heimteam bestehend aus Berliner Spielerinnen. In den entsprechenden Vorbereitungen wurde nun die Leistungsschwäche des Teams deutlich. Das mangelnde Können und der fehlende Ehrgeiz wurden von manchen Organisator_innen kritisch kommentiert, und sie versuchten, diese mit intensiven Trainingseinheiten auszugleichen. Mit Simone scherzte ich über diese Vorbereitungstrainings: „Das wird jetzt aber einen großen Riss in meine Dissertation reißen, wenn ihr euch hier als so ehrgeizig entpuppt.“ Simone lachte und stieg feixend ein: „Mist, wir tun hier halt immer nur so." (Feldnotiz, 28.5.2013) Simone und ich nahmen hier Bezug auf die in dieser Arbeit ausführlich beschriebene Spannung zwischen Feminismus und fußballerischem Leistungsprimat, die auch in der Formierung und den Vorgehensweisen von DF nie aufgelöst werden konnte. In diesem Moment stand zum einen DFs Gestalt als solidarisches, frauenförderndes und inklusiv-emanzipatives feministisches Projekt, zum anderen ihre Bestrebungen durch gute fußballerische Leistung die Gleichwertigkeit von Frauen 
zu beweisen, auf dem Spiel. Unser Scherzen war der Versuch dieses Dilemma auszuhalten.

Sei es Simones ironische Bemerkung, dass der Fokus auf gute sportliche Leistung das emanzipative Ziel konterkariere, sei es Annikes Distanzierung von den großen, professionalisierten NGOs (Kap. 13.4.4), sei es die Selbstverortung ,unten“ und die Abneigung gegenüber einem Vorgehen ,von oben herab" (Teil III) oder Celias und meine Ablehnung paternalistischer Entwicklungshilfe (Kap. 11.5) - in diesen reflexiven Bezugnahmen auf die eigene Position, Gestalt und das eigene Handeln wird ein normatives Wissen erkennbar. Anhand des NGOization-Paradigmas (Hodžić 2014), der Romantisierung von Widerständigkeit (Abu-Lughood 1990) und des vertikalen Machtkonzepts (Ferguson/Gupta 2002) habe ich gezeigt, wie spezifisches sozialwissenschaftliches Wissen im Feld normativ angeeignet wurde und die Formierungen und Positionierungen der Aktivist innen und ihr Verständnis politischen Handelns entsprechend formte: Ideale von aktivistischen Graswurzelinitiativen, netzwerkförmigen Partnerschaften auf Augenhöhe, linksfeministischen Alternativen und einem widerständigen „Unten“, das fest in einer politischen Identität und Überzeugung wurzelte, standen Vorstellungen von professionellen NGOs, paternalistischen Geber-Nehmer-Beziehungen, traditionellen Fußballvereinen, patriarchalen, neoliberalen Verwaltungen und einem machtvollen „Oben“ gegenüber.

In Simones und meinem Scherzen fällt jedoch auf, dass wir nicht nur reflexiv Bezug auf DFs Formierung als feministische Alternative zum leistungsgetriebenen Wettkampfsport nahmen. Auch thematisierten wir die Vielgestaltigkeit und die multiplen Strategien - allerdings mit eindeutig negativer Bewertung: DF würde immer nur so feministisch-emanzipativ tun, hätte sich in Wirklichkeit aber dem Leistungsprimat gebeugt und würde sich nun als ehrgeizig entpuppen. Damit bemängelten wir eine wahrgenommene Diskrepanz zwischen idealistischem Anspruch und tatsächlichem Tun.

Ich habe anhand der alltäglichen Praxis von DF ausführlich gezeigt, dass effektives Problematisieren in komplexen gesellschaftspolitischen Gefügen erfordert, sich flexibel und pragmatisch und zugleich von normativen Leitplanken geleitet zu bewegen, zwischen Gestalten zu changieren und zwischen verschiedenen feministischen Strategien, Positionen und Visionen zu wechseln. DFs Mitglieder vollzogen jenen ,process of metamorphosis“, den Sandoval $(2000,61)$ als zentral für feministische oppositionelle Politik in der Postmoderne beschreibt, im Alltag wie selbstverständlich. Durch das ihnen zur Verfügung stehende normativen Wissen erschien diese Beweglichkeit jedoch als Unaufrichtigkeit und Täuschung. Im Wissenskontext von DF galten Abstriche bei den eigenen Idealen, Kooperationen mit sowie Zugeständnisse an regierende Institutionen und ihre Politiken und Prinzipien als unliebsame, aber entschuldbare Kompromisse mit äußeren Zwänge, nicht jedoch als zentrale Eigenschaften politischen Handelns in spätkapitalistischen Demokra- 
tien. ,Richtiges‘ Handeln im Sinne von Glaubwürdigkeit und Aufrichtigkeit hingegen war eng mit dem kompromisslosen Festhalten an eigenen Überzeugungen und einer Skepsis gegenüber dem „Oben“ verbunden: mit Standhaftigkeit statt Beweglichkeit. Sandoval (2000) zeigt, wie jene Beweglichkeit zwischen politischen Gestalten, Positionen und Instrumenten als ,activity of the trickster", „masquerade“ oder gar als „,betrayal“ und „disloyalty“ (2000, 57, 61) innerhalb der Frauenbewegung abgewertet wurde. Auch hier hatten wissenschaftliche Kategorisierungen und Periodisierungen von Positionen, Kritiken und Theorien dazu geführt, dass diese von den meist weißen Aktivist_innen selbst als sich gegenseitig ausschließend wahrgenommen wurden. Während Aktivist_innen of colour beständig zwischen unterschiedlichen Positionen und Strategien wechselten, um die geringen Spielräume zu nutzen, wurde ihnen von Seiten weißer Feministinnen Betrug und illoyales Verhalten vorgeworden.

Mit Islam verstehe ich die Inkongruenz zwischen den Wissenspraktiken der Selbstthematisierung und den Praktiken der geschlechterpolitischen Interventionen als Auslassung bzw. als Lücke, die von den Aktivist_innen nicht nur selbst produziert, sondern auch bemerkt worden war (Islam 2015, 243). Simones und mein Scherzen war ein Versuch, diese Leerstelle zu thematisieren. Ob Betrug oder So-tun-als-ob: Was uns fehlte, war ein entsprechendes Wissen und Vokabular, um Beweglichkeit als Modus, die begrenzten Möglichkeiten zu nutzen, reflexiv zu affirmieren. Stattdessen verfügten wir nur über jene Perspektive, die die Verbreitung von NGOs samt ihrer komplexen Kooperationen mit anderen Institutionen und den Aufstieg von Frauen zu Expertinnen negativ beäugte (Bernal/Grewal 2014b, 305).

Ich habe gezeigt, wie zwischen Erwartungen, Handlungszwängen und Kompetenzregimen navigiert wurde, und die entstehenden Spannungen nur mittels Kompromissen ausbalanciert, nicht jedoch völlig aufgelöst werden konnten. Indem ich auf Klassifizierungen entlang von Organisationstypologien verzichtet, und die vom Feld beschriebene vertikale Machttopografie angezweifelt habe, habe ich zeigen können, dass Problematisieren in heterogenen Landschaften bedeutet, sich flexibel und pragmatisch und zugleich von normativen Leitplanken geführt zu bewegen, zwischen Gestalten zu changieren und durch verschiedene Kompetenzregime, -kriterien und Erwartungen $\mathrm{zu}$ navigieren. Das ist keineswegs als opportunistisches Verhalten oder gar Kooptierung durch Stakeholder, wie z.B. staatliche Verwaltungen, fehl zu deuten, sondern vielmehr eine Strategie, das Überleben als Organisation zu sichern, und eine elementare Taktik politischen Handelns in komplexen gesellschaftspolitischen Landschaften.

Ich habe in Kapitel 2.3.3, anschließend an Foucault (1992) und Butler (2001), argumentiert, dass es Aufgabe wissenschaftlicher Kritik ist, das paraethnografischen Wissen des Feldes zu ergänzen und aufzuzeigen, warum gegenwärtige Zustände im Zusammenspiel mit ihren ökonomischen, sozia- 
len, kulturellen und politischen Relationen so und nicht anders gewachsen sind und durch Wissensbestände als richtig oder falsch bewertet werden. So kann, mit Foucault (1992) gesprochen, auf gefährliche Verstrickungen mit unliebsamen Machtverhältnissen hingewiesen, und im Sinne politisch engagierten Forschens das Repertoire an Denk- und Handlungsmöglichkeiten im Feld erweitert werden (vgl. Calhoun 2008, xx).

Mit dieser Arbeit möchte ich nicht nur die komplexen Möglichkeitsbedingungen, Wissensbestände und Machteffekten im aktivistischen Alltag von DF aufzeigen, sondern auch begriffliches Instrumentarium anbieten, das den längst gängigen Umgang mit multizentrischen, dynamischen Machtgefügen klar beschreibbar macht und den normativen Selbstbezügen ein neues Vokabular entgegensetzt. Zukünftige politikethnologische, feministische und organisationsethnologische Forschungen in NGOs, sozialen Bewegungen und Protestformen können hier anschließen und die zivilgesellschaftlichpolitischen Navigationen, die Metamorphosen und grenzüberschreitenden Bewegungen genauer untersuchen und theoretisieren.

Ein solch analytisch geschärftes Begriffsinstrumentarium kann gegenwärtige politische Kämpfe differenziert beschreiben und zu ihrem Verstehen beitragen. Gerade in der aktuellen Situation, in der sich Proteste und Bewegungen formieren und Parteien gründen, die aufmerksamkeitserregend die Position der Marginalisierten besetzen, sich lautstark als von dominanten Diskursen Zum-Schweigen-Gebrachte präsentieren, zugleich antiemanzipatorische Politik betreiben und gruppenbezogene Menschenfeindlichkeit kultivieren, bedarf es solch differenzierender Konzepte. Diese müssen sowohl Formierungen von Organisationen, Protesten und Initiativen als auch deren Vorgehensweisen und Positionen als unterschiedliche, aber aufeinander bezogene Dimensionen gesellschaftlicher Problematisierungen fassen. Das bedeutet erstens, zwischen dem, wie sie sich formieren, und dem, was sie tun, zu unterscheiden. Zweitens verlangt es, die Beziehung zwischen diesen zu unterscheidenden Ebenen als aufeinander bezogen, aber potentiell inkongruent $\mathrm{zu}$ untersuchen und machtanalytisch in ihren historischen, gesellschaftlichen und diskursiven Relationen zu kontextualisieren. So können die notwendigen Unterschiede zwischen emanzipativen und progressiven Bewegungen einerseits und reaktionären, rechtspopulistischen und antiaufklärerischen Protesten andererseits beleuchtet werden. Indem ein solches Begriffsinstrumentarium auf normative Metanarrative verzichtet bzw. selbst zu Untersuchungsgegenständen macht, gelingt es, mit Dreyfus und Rabinow über Foucault hinauszugehen: Es gilt nicht nur, auf mögliche Gefahren hinzuweisen, sondern auch zu zeigen, was mache Art der Problematisierung „gefährlicher macht als eine andere“ (Dreyfus et al. 1994, 307). 


\section{Dank}

„That knowledge is situated means, that knowing and thinking are inconceivable without a multitude of relations that also make possible the worlds we think in." (Puig de la Bellacasa 2012, 198) Oder, angelehnt an DISCOVER FOOTBALL: It needs a team to write a PhD thesis. Ich möchte mich hiermit bei all denen bedanken, die die Gedanken und Argumente dieser Arbeit ermöglichten, ihre Fertigstellung inhaltlich, organisatorisch oder emotional begleiteten und dafür sorgten, dass die Promotionszeit vor allem durch Freude am Lernen und Denken geprägt war - und mich bei denen entschuldigen, die ich aus Platzgründen hier nicht namentlich erwähnen kann. An erster Stelle gilt mein großer Dank den Frauen von DISCOVER FOOTBALL für ihr Vertrauen und ihre Bereitschaft, mich als Ehrenamtliche und Ethnografin Teil ihrer Gruppe werden zu lassen. Meiner Erstgutachterin Beate Binder danke ich für ihre weitsichtige inhaltliche und konzeptuelle Betreuung sowie für ihre unermüdlichen Bemühungen, feministische Kritiken und Ideale auf sämtlichen Ebenen der akademischen Arbeit umzusetzen. Mein Zweitgutachter Jörg Niewöhner steuerte gerade in der entscheidenden Phase wichtige, richtungsweisende Impulse bei. Am Institut für Europäische Ethnologie der Philosophischen Fakultät der Humboldt-Universität zu Berlin fand ich nicht nur einen Arbeitsplatz sondern auch ein inspirierendes, kollegiales Umfeld. Und dank der finanziellen und ideellen Förderung durch das Studienwerk der Heinrich-Böll-Stiftung e.V. und das Caroline von Humboldt-Programm konnte ich diese Arbeit am 26. Oktober 2017 schließlich erfolgreich verteidigen. Almut Sülzle begleitete meine Forschung von Anfang an mit stets den richtigen Fragen. Mit Stefan Heissenberger teilte ich Lust und Leid der teilnehmenden Fußballforschung in Form von Gedanken und Beobachtungen aber auch von Schmerzmitteln und Kühlpads. Die Supervisionsgruppe Sport und Geschlecht half mir, die vier großen „Fs“ - Forscherin, Feld, Feminismus und Fußball - zu entflechten und zeigte mir, dass gemeinsame Datenanalyse kreative Freiräume braucht, und dass sie großen Spaß bereiten kann. Meine Kolleg_innen standen mit Ratschläge, Diskussionsbereitschaft und moralischem Rückhalt bereit: Christoph Bareither, Robert Birnbauer, Daria Buteiko, Mandy de Wilde, Thomas Franssen, Urmila Goel, Janine Hauer, Dominik Kleinen, Lydia-Maria Ouart, Marie Therese Reichenbach, Svenja Reinke, Todd Sekuler, Andrea Vetter und Georg Weichert. Pia Mann, Johanna Kösters und Valerie Assmann bereicherten die Arbeit als ,wahre Kollaborateurinnen' immens. Ulf Heidel danke ich für sein wertvolles Lektorat. Marianne und Anselm Faust, Jakub Limanowski sowie Anna Hartmann verdan- 
ke ich hervorragende Korrekturen, vor allem aber den entscheidenden Rückhalt im Privaten. Gewidmet ist diese Arbeit meiner Freundin Jenny. Von ihr lernte ich, wie nah sich Ernst und Albernheit im Fußball sein können, und sie erinnerte mich immer wieder daran, dass Sexismus und Heteronormativität nicht lediglich analytische Kategorien, sondern schmerzhafte Alltagserfahrungen sind. Ich danke Jenny für eine bedingungslose Freundschaft und dafür, dass sie mich selbst dann mit aufrichtiger Begeisterung zum Fertigstellen dieser Arbeit motivierte, als sie selbst kaum noch Kraft hatte. 


\section{Literatur- und Quellenverzeichnis}

Abu-Lughod, Lila. 1990. The romance of resistance: Tracing transformations of power through Bedouin women. American Ethnologist 17, Nr. 1: 41-55.

---. 1991. Writing against culture. In: Recapturing anthropology: working in the present, hg. von Richard Gabriel Fox, 466-479. Santa Fe: School of American Research Press.

Adam, Jens. 2016. Ko-laborative Stadtforschung: Wie tragen zivilgesellschaftliche Initiativen zur Stadt(raum)gestaltung bei? Projektpräsentation. Gehalten auf: Institutionskolloquium am Institut für Europäische Ethnologie, 14. Juni, HumboldtUniversität Berlin.

Adam, Jens und Asta Vonderau. 2014. Formationen des Politischen. Überlegungen zu einer Anthropologie politischer Felder. In: Formationen des Politischen: Anthropologie politischer Felder, hg. von dies., 7-31. Bielefeld: Transcript.

Adams-Lehmann, Hope Bridges. 1899. Die Gesundheit im Haus: Eine ärztliche Anleitung für das Verhalten der Frau im täglichen Leben und bei Frauenkrankheiten. Stuttgart: Süddeutsches Verlags-Institut.

Adorno, Theodor W. 2003. Kulturkritik und Gesellschaft I und II. Frankfurt am Main: Suhrkamp.

Agricola, Sigurd. 1997. Vereinswesen in Deutschland: eine Expertise im Auftrag des Bundesministeriums für Familie, Senioren, Frauen und Jugend. Stuttgart: Kohlhammer.

Ahmed, Sara. 2004. The cultural politics of emotion. London u.a.: Routledge.

---. 2010. Feminist killjoys (and other willful subjects). The Scholar and Feminist Online 8, Nr. 3: 1-8.

Alkemeyer, Thomas. 2006. Rhythmen, Resonanzen und Missklänge. Über die Körperlichkeit der Produktion des Sozialen im Spiel. In: Body Turn. Perspektiven der Soziologie des Körpers und des Sports, hg. von Robert Gugutzer, 265-295. Bielefeld: Transcript.

---. 2011. „Frauenfußball braucht eine eigene Mythologie“. Uni-Info 11, Nr. 6: 6. URL: http://www.presse.uni-oldenburg.de/uni-info/2011/6/thema.html (zugegriffen: 4.6.2016)

Allahyari, Rebecca Anne. 2001. The felt politics of charity: serving "the ambassadors of God" and saving "the sinking classes". In: Passionate politics: emotions and social movements, hg. von Jeff Goodwin, James M. Jasper und Francesca Polletta, 195-211. Chicago: University of Chicago Press.

Alvarez, Sonia E. 1999. Advocating feminism: the Latin American feminist NGO „,boom“. International Feminist Journal of Politics 1, Nr. 2: 181-209.

American Anthropological Association. 2012. Principles of Professional Responsibility. $A A A$ ethics blog. $1 . \quad$ November. URL: http://ethics.americananthro.org/category/statement/ (zugegriffen: 18.2.2017).

Anderson, Ben. 2010. Preemption, precaution, preparedness: anticipatory action and future geographies. Progress in Human Geography 34, Nr. 6: 777-798.

Appadurai, Arjun. 2000. Grassroots globalization and the research imagination. Public culture 12, Nr. 1: 1-19. 
---. 2004. The capacity to aspire: culture and the terms of recognition. In: Culture and public action, hg. von Vijayendra Rao und Michael Walton, 59-84. Stanford: Stanford University Press.

Arantes, Lydia Maria, Hrsg. 2014. Ethnographien der Sinne: Wahrnehmung und Methode in empirisch-kulturwissenschaftlichen Forschungen. Bielefeld: Transcript.

Archetti, Eduardo und Noel Dyck. 2003. Introduction - Embodied identites: reshaping social life through sport and dance. In: Sport, dance, and embodied identities, hg. von Eduardo Archetti und Noel Dyck, 1-22. Oxford: Berg.

Assiter, Alison. 2000. Feminist epistemology and value. Feminist Theory 1, Nr. 3: 329-345.

Ausserer, Caroline. 2016. Historische UN-Resolution zum besseren Schutz von LSBTI-Personen verabschiedet. boell.de. 26. Juli. URL: http://www.boell.de/de/2016/07/26/sogi-historische-un-resolution-schutz-lsbtipersonen (zugegriffen: 19.8.2016).

Auswärtiges Amt. 2012. Die Internationale Sportförderung des Auswärtigen Amtes. Homepage. $\quad$ sport.diplo.de. $11 . \quad$ Februar. URL: http://www.sport.diplo.de/content/die-internationale-sportf\%C3\%B6rderung-desausw\% $\%$ C3\%A4rtigen-amtes (zugegriffen: 3.9.2016).

Bale, John. 1993. The spatial development of the modern stadium. International Review for the Sociology of Sport 28, Nr. 2-3: 121-133.

Bareither, Christoph. 2014. Vergnügen als Doing Emotion - Beispiel YouTube. In: Macher - Medien - Publika. Beiträge der europäischen Ethnologie zu Geschmack und Vergnügen, hg. von Kaspar Maase, Brigitte Frizzoni, Christoph Bareither und Mirjam Nast, 36-49. Würzburg: Königshausen \& Neumann.

---. 2016. Gewalt im Computerspiel: Facetten eines Vergnügens. Bielefeld: Transcript.

Baron, Christian. 2016. Proleten, Pöbel, Parasiten: Warum die Linken die Arbeiter verachten. Berlin: Das Neue Berlin.

Bausinger, Hermann. 2006. Sportkultur. Tübingen: Attempto.

Becker, Brigitte, Marion Hamm, Katharina Eisch-Angus, Ute Karl, Judith Kestler, Sebastian Kestler-Joosten, Ulrike A. Richter, Sabine Schneider, Almut Sülzle und Barbara Wittel-Fischer. 2013. Die reflexive Couch. Feldforschungssupervision in der Ethnografie. Zeitschrift für Volkskunde 106, Nr. II: 181-203.

Behn, Sabine und Viktoria Schwenzer. 2006. Anmerkungen zu Sexismus und Gender Mainstreaming im Kontext von Fußball und Fanarbeit. Sozial Extra 30, Nr. 3: $45-48$.

Bendix, Regina. 2006. Was über das Auge hinaus geht: Zur Rolle der Sinne in der ethnographischen Forschung. Schweizerisches Archiv für Volkskunde 102: 71-84.

Benford, Robert D. und David A. Snow. 2000. Framing processes and social movements: an overview and assessment. Annual Review of Sociology: 611-639.

Bennett, Roberte S., K. Gail Whitaker, Nina Jo Woolley Smith und Anne Sablove. 1987. Changing the rules of the game: reflections toward a feminist analysis of sport. Women's Studies International Forum 10, Nr. 4: 369-379.

Berg, Eberhard und Martin Fuchs, Hrsg. 1993. Kultur, soziale Praxis, Text: die Krise der ethnographischen Repräsentation. Frankfurt am Main: Suhrkamp.

Bergman, Solveig, Hana Hasková, Katerina Pulkrábková, Minna Rantalaiho, Celia Valiente und Zuzana Uhde. 2012. Remaking social citizenship in multicultural 
Europe: women's movements agency in child-care politics and policies. In: $R e$ making citizenship in multicultural Europe: women's movements, gender and diversity, hg. von Beatrice Halsaa, Sasha Roseneil und Sevil Sümer, 94-117. Basingstoke u.a.: Palgrave Macmillan.

Bernal, Victoria und Inderpal Grewal. 2014. The NGO form. Feminist struggles, states, and neoliberalism. In: Theorizing NGOs: states, feminisms, and neoliberalism, hg. von Victoria Bernal und Inderpal Grewal, 1-18. Durham: Duke University Press.

---. 2014a. Feminisms and the NGO Form. In: Theorizing NGOs: states, feminisms, and neoliberalism, hg. von Victoria Bernal und Inderpal Grewal, 301-310. Durham: Duke University Press.

---. Hrsg. 2014b. Theorizing NGOs: states, feminisms, and neoliberalism. Durham: Duke University Press.

Bernstein, Mary. 2005. Identity politics. Annual Review of Sociology 31, Nr. 1: 47-74.

Betz, Gregor J. 2016. Vergnügter Protest. Wiesbaden: VS Verlag für Sozialwissenschaften.

Binder, Beate. 2009. Streitfall Stadtmitte: der Berliner Schlossplatz. Alltag \& Kultur Bd. 13. Köln: Böhlau.

---. 2013. Erkundungen in Feldern politischer Praxis von Geschlecht und Sexualität. In: Geschlecht - Sexualität: Erkundungen in Feldern politischer Praxis, hg. von Beate Binder, 7-13. Berlin: Panama.

---. 2014. Troubling policies. In: Formationen des Politischen: Anthropologie politischer Felder, hg. von Asta Vonderau und Jens Adam, 363-386. Bielefeld: Transcript.

Binder, Beate und Sabine Hess. 2011. Intersektionalität aus der Perspektive der Europäischen Ethnologie. In: Intersektionalität Revisited. Empirische, theoretische und methodische Erkundungen, hg. von Sabine Hess, Nikola Langreiter und Elisabeth Timm, 15-52. Bielefeld: Transcript.

---. 2013. Eingreifen, kritisieren, verändern. Genealogien engagierter Forschung in Kulturanthropologie und Geschlechterforschung. In: Eingreifen, Kritisieren, Verändern!?: Interventionen ethnographisch und gendertheoretisch, hg. von Binder Beate, Friedrich von Bose, Kartin Ebell, Sabine Hess und Anika Keinz, 22-54. Münster: Westfälisches Dampfboot.

Birrell, Susan und Diana M. Richter. 1994. Is a diamond forever? Feminist transformations of sport. In: Women, sport, and culture, hg. von Susan Birrell und Cheryl L. Cole, 221-244. Champaign: Human Kinetics.

Birrell, Susan und Nancy Theberge. 1994. Feminist resistance and transformation in sport. In: Women and sport: interdisciplinary perspectives, hg. von D. Margaret Costa und Sharon Ruth Guthrie, 361-376. Champaign: Human Kinetics.

Bischoff, Susanne. 1986. Die Lust am Leben eigener Bewegungsutopien im Patriarchat. Autonomie von Mädchen und Frauen im Sport. In: Frauen Bewegung Sport, hg. von Sylvia Schenk, 130-138. Hamburg: VSA.

---. 1993. ... auf Bäume klettern ist politisch. In: ...auf Bäume klettern ist politisch. Texte aus der Feministischen Bewegungs- und Sportkultur, hg. von Susanne Bischoff, 18-35. Hamburg: Frühlings Erwachen.

---. 2003. Nicht nur auf Bäume klettern ist politisch - ein rückblickender Geburtstagsgruß. In: Geburtstagsbroschüre, hg. von Seitenwechsel - FrauenLesben Sport- 
verein Berlin e.V., Kassel. URL: https:/www.seitenwechselberlin.de/archiv/nicht-nur-auf-baeume/

Bister, Milena D. und Jörg Niewöhner. 2014. Prolog: Ko-laboratives ethnographisches Forschen im Schnittfeld von Psychiatrie und Anthropologie. In: Alltag in der Psychiatrie im Wandel Ethnographische Perspektiven auf Wissen, Technologie und Autonomie, 6-10. Berlin: Panama.

Bonz, Jochen. 2016. Zur Interpretation von Emotionen in der ethnografischen Fankulturforschung. Verständnismöglichkeiten und Beispiele aus einer Studie über Fußballbegeisterung. In: Emotionen im Spiel. Beiträge zu einer Ethnologie des Sports, hg. von Friederike Faust und Stefan Heissenberger, 17-25. Berlin: Panama.

Bonz, Jochen, Katharina Eisch-Angus, Marion Hamm und Almut Sülzle, Hrsg. 2017. Ethnografie und Deutung: Gruppensupervision als Methode reflexiven Forschens. Wiesbaden: VS Verlag für Sozialwissenschaften.

Bordo, Susan. 2013 [1993]. Unbearable weight: feminism, Western culture, and the body. Berkeley: University of California Press.

Bourdieu, Pierre. 1983. Ökonomisches Kapital, kulturelles Kapital, soziales Kapital. In: Soziale Ungleichheiten, hg. von Reinhard Kreckel, 183-198. Göttingen: Schwartz.

--- 1987 [1980]. Sozialer Sinn: Kritik der theoretischen Vernunft. Frankfurt am Main: Suhrkamp

---. 1992. Rede und Antwort. Frankfurt am Main: Suhrkamp.

---. 1992a. Die verborgenen Mechanismen der Macht. Hg. von Margareta Steinrücke. Hamburg: VSA-Verlag.

---. 1993. Narzißtische Reflexivität und wissenschaftliche Reflexivität. In: Kultur, soziale Praxis, Text: die Krise der ethnographischen Repräsentation, hg. von Eberhard Berg und Martin Fuchs, 365-373. Frankfurt am Main: Suhrkamp.

---. 1997 [1979]. Die feinen Unterschiede. Kritik der gesellschaftlichen Urteilskraft. Frankfurt am Main: Suhrkamp.

---. 2004. Für eine engagierte Wissenschaft. In: Gegenfeuer 2. Für eine europäische soziale Bewegung, hg. von Pierre Bourdieu, 34-42. Konstanz: UVK.

Bourdieu, Pierre und Loïc Wacquant. 1996 [1992]. Reflexive Anthropologie. Frankfurt am Main: Suhrkamp.

Brändle, Fabian und Christian Koller. 2002. Goal!!! Kultur- und Sozialgeschichte des modernen Fußballs. Zürich: Orell Füssli.

Braun, Sebastian und Sebastian Finke. 2010. Integrationsmotor Sportverein: Ergebnisse zum Modellprojekt ,spin-sport interkulturell“. Wiesbaden: VS Verlag für Sozialwissenschaften.

Braunmüller, Birgit, Bettina Rulofs und Ilse Hartmann-Tews. 2016. Geschlechterverhältnisse und sportliche Leistung in den Printmedien. Eine Bildanalyse der Olympischen Sommerspiele 2004 Athen - 2008 Peking - 2012 London. In: Sport als Bühne. Mediatisierung von Sport und Sportgroßveranstaltungen, hg. von Andreas Hebbel-Seeger, Thomas Horky und Hans-Jürgen Schulke, 82-109. Aachen: Meyer \& Meyer Verlag.

Brednich, Rolf Wilhelm, Hrsg. 2001 [1988]. Grundriss der Volkskunde: Einführung in die Forschungsfelder der europäischen Ethnologie. Berlin: Reimer.

Breidenstein, Georg, Stefan Hirschauer, Herbert Kalthoff und Boris Nieswand. 2015 [2013]. Ethnografie: die Praxis der Feldforschung. Konstanz: UVK. 
Bresemann, Patrick und Gabriel Duttler. 2016. Kritik an RasenBallsport Leipzig. In: Fanverhalten im Sport. Phänomene, Herausforderungen und Perspektiven, hg. von André Schneider, Julia Köhler und Frank Schumann, 137-158. Wiesbaden: VS Verlag für Sozialwissenschaften.

Brill, Dunja. 2009. Queer Theory und kritische Subkulturforschung - ein überfälliger Brückenschlag. Bulletin Texte 36: 104-125.

Broad, Kendal L. 2001. The gendered unapologetic. Queer resistance in women's sport. Sociology of Sport Journal 18: 181-204.

Bröckling, Ulrich und Robert Feustel. 2012 [2010]. Einleitung: das Politische denken. In: Das Politische denken: zeitgenössische Positionen, hg. von Ulrich Bröckling und Robert Feustel, 7-18. Bielefeld: Transcript.

Bromberger, Christian. 2006. Der ethnologische Blick auf Sport, Fußball und männliche Identität. In: Arena der Männlichkeit, hg. von Eva Kreisky und Georg Spitaler, 41-52. Frankfurt am Main: Campus.

---. 2010. Sport, football and masculine identity. In: Stadium worlds: football, space and the built environment, hg. von Sybille Frank und Silke Steets, 181-194. London u.a.: Routledge.

Bröskamp, Bernd. 2011. Migration, Integration, interkulturelle Kompetenz, Fremdheit und Diversität: zur Etablierung eines aktuellen Feldes der Sportforschung. Eine Sammelbesprechung. Sport \& Gesellschaft 1, Nr. 8: 85-94.

Brown, L. David, Alnoor Ebrahim und Srilatha Batliwala. 2012. Governing international advocacy NGOs. World Development 40, Nr. 6: 1098-1108.

Brown, Michael F. 1996. On resisting resistance. American Anthropologist 98, Nr. 4: 729-749.

Brown, Wendy. 1995. States of injury: power and freedom in late modernity. Princeton: Princeton University Press.

---. 2009. Introduction. In: Is critique secular? Blasphemy, injury, and free speech, hg. von Talal Asad, Wendy Brown, Judith Butler und Saba Mahmood, 7-20. Berkeley: University of California Press.

Brunn, Frank Martin. 2016. Die Tugend der Bildung im Sport. Sozialmagazin. Die Zeitschrift für Soziale Arbeit, Nr. 6: 60-70.

Bryson, Lois. 1994. Sport and the maintenance of masculine hegemony. In: Women, sport, and culture, hg. von Susan Birrell und Cheryl L. Cole, 47-64. Champaign: Human Kinetics.

Bundesministerium für wirtschaftliche Zusammenarbeit und Entwicklung. O.J. Sport für Entwicklung. bmz.de. URL: http:/www.bmz.de/de/themen/sport-fuerentwicklung/index.html (zugegriffen: 9.3.2016).

---. O.J.a. Sport als übergreifendes Thema für Entwicklung nutzen. bmz.de. URL: http://www.bmz.de/de/themen/sport-fuerentwicklung/deutsches_engagement/index.html (zugegriffen: 9.3.2016).

---. 2014. 100 Tage Bilanz: Sport für Entwicklung. Bonn: BMZ. URL: www.pressebox.de/attachment/655827/28+Anl+100+Tage+Bilanz.pdf (zugegriffen: 6.8.2015).

---. 2015. Die Rolle des Sports in der deutschen Entwicklungszusammenarbeit. Bonn: BMZ.

URL: http://www.bmz.de/de/mediathek/publikationen/reihen/infobroschueren_flyer/inf obroschueren/Materialie268_sport_ez.pdf (zugegriffen: 9.3.2016). 
Burdsey, Daniel. 2008. Contested conceptions of identity, community and multiculturalism in the staging of alternative sport events: a case study of the Amsterdam World Cup football tournament. Leisure Studies 27, Nr. 3: 259-277.

Buschmann, Rafael und Michael Wulzinger. 2016. Football Leaks. Die schmutzigen Geschäfte im Profifußball. München: Penguin.

Butler, Judith. 1997. Körper von Gewicht: Die diskursiven Grenzen des Geschlechts. Frankfurt am Main: Suhrkamp.

---. 2001. Was ist Kritik? Ein Essay über Foucaults Tugend. eipcp.net. URL: http:/eipcp.net/transversal/0806/butler/de (zugegriffen 16.2.2017).

---. 2006. Gender Trouble: Feminism and the subversion of identity. London u.a.: Routledge.

---. 2013. So what are the demands? And where do they go from here? Tidal: Journal of Occupy Theory 2: 8-11.

Cahn, Susan. 1994. Crushes, competition, and closets: the emergence of homophobia in women's physical education. In: Women, sport, and culture, hg. von Susan Birrell und Cheryl L. Cole, 327-339. Champaign: Human Kinetics.

Calhoun, Craig. 2008. Foreword. In: Engaging contradictions: theory, politics, and methods of activist scholarship, hg. von Charles R. Hale, xiii-xxvi. Berkeley: University of California Press.

Caudwell, Jayne. 1999. Women's football in the United Kingdom: theorizing gender and unpacking the butch lesbian Image. Journal of Sport \& Social Issues 23, Nr. 4: 390-402.

---, Hrsg. 2006. Sport, sexualities and queer/theory. London u.a.: Routledge.

---. 2007. Queering the Field? The complexities of sexuality within a lesbianidentified football team in England. Gender, Place \& Culture 14, Nr. 2: 183-196.

---. 2011. Gender, feminism and football studies. Soccer \& Society 12, Nr. 3: 330344.

---. 2011a. 'Does your boyfriend know you're here?' The spatiality of homophobia in men's football culture in the UK. Leisure Studies 30, Nr. 2: 123-138.

Chakkalakal, Silvy. 2014. Die Welt in Bildern: Erfahrung und Evidenz in Friedrich J. Bertuchs „Bilderbuch für Kinder“ (1790-1830). Göttingen: Wallstein.

Chawansky, Megan und Lyndsay M.C. Hayhurst. 2015. Girls, international development and the politics of sport: introduction. Sport in Society 18, Nr.8: 877-881.

Cheah, Pheng. 2006. Inhuman conditions: on cosmopolitanism and human rights. Cambridge: Harvard University Press.

Chhachhi, Amita und Renée Pittin. 1999. Multiple identities, multiple strategies: confronting state, capital and patriarchy. In: Labour worldwide in the era of globalization: alternative union models in the new world order, hg. von Peter Waterman, 64-79. Basingstoke u.a.: Palgrave Macmillan.

Coalter, F. 2010. The politics of sport-for-development: Limited focus programmes and broad gauge problems? International Review for the Sociology of Sport 45, Nr. 3: 295-314.

Coffey, Amanda. 1999. The ethnographic self: fieldwork and the representation of identity. Thousand Oaks u.a.: Sage Publications.

Cole, Cheryl L. und Susan Birrell. 1986. Resisting the canon: feminist cultural studies. Vortragsentwurf. Gehalten auf: American Society for the Sociology of Sport, Edmonton, AB. 
Cornwall, Andrea, Jasmine Gideon und Kalpana Wilson. 2008. Introduction: reclaiming feminism: gender and neoliberalism. IDS Bulletin 39, Nr. 6: 1-9.

Costa, Margaret und Sharon Guthrie. 1994. Feminist perspectives: intersections with women and sport. In: Women and sport: interdisciplinary perspectives, hg. von Margaret Costa und Sharon Ruth Guthrie, 235-252. Champaign: Human Kinetics.

Cox, Barbara und Shona Thompson. 2000. Multiple bodies: sportswomen, soccer and sexuality. International Review for the Sociology of Sport 35, Nr. 1: 5-20.

Darnell, Simon. 2011. Identity and learning in international volunteerism: 'Sport for Development and Peace' internships. Development in Practice 21, Nr. 7: 974986.

---. 2012. Sport for development and peace: a critical sociology. London: Bloomsbury.

Davies, James und Dimitrina Spencer, Hrsg. 2010. Emotions in the field: the psychology and anthropology of fieldwork experience. Stanford: Stanford University Press.

Dawin-Herne, G. A. 1926. Die Frau und der Sport. Start und Ziel: 34-35.

Day, Richard J. F. 2004. From hegemony to affinity: the political logic of the newest social movements. Cultural Studies 18, Nr. 5: 716-748.

Deem, Rosemary und Sarah Gilroy. 1998. Physical activity, life-long learning and empowerment - situating sport in women's leisure. Sport, Education and Society 3, Nr. 1: 89-104.

Degele, Nina. 2012. The future is female - or feminine? gwi-boell.de. 6. Januar. URL: http://www.gwi-boell.de/en/2012/01/06/future-female---or-feminine (zugegriffen: 24.9.2015).

---. 2013. Fussball verbindet - durch Ausgrenzung. Wiesbaden: VS Verlag für Sozialwissenschaften.

Degele, Nina und Caroline Janz. 2011. Hetero, weiß und männlich? Fußball ist viel mehr! Berlin: Friedrich-Ebert-Stiftung.

Desjarlais, Robert. 1997. Shelter blues. Sanity and selfhood among the homeless. Philadelphia: University of Pennsylvania Press.

Deutsche Akademie für Fußball-Kultur. 2019. Der Fußball ist tot. Homepage. URL: https://www.fussball-kultur.org/tag/der-fussball-ist-tot/ (zugegriffen: 20.2.2019).

Deutsche Olympische Akademie, Hrsg. 2013. Olympische Charta 2014. Melsungen: Bernecker Verlag.

URL: http://www.dosb.de/fileadmin/Bilder_allgemein/Veranstaltungen/Sotschi_2014/ Olympische_Charta_2014.pdf (zugegriffen: 6.3.2015).

Deutscher Bundestag. 2010. Internationale Sportförderung durch die Bundesregierung. Antwort der Bundesregierung auf die Kleine Anfrage der Abgeordneten Viola von Cramon-Taubadel, Winfried Hermann, Ingrid Hönlinger, Josef Philip Winkler und der Fraktion BÜNDNIS 90/DIE GRÜNEN. Drucksache 17/4352. 23. Dezember. URL: http://dip21.bundestag.de/dip21/btd/17/043/1704352.pdf (zugegriffen: 29.4.2016)

---. 2014. 13. Sportbericht der Bundesregierung. Unterrichtung durch die Bundesregierung. $\quad$ Drucksache $5.3523 . \quad$ November. URL: http://dip21.bundestag.de/dip21/btd/18/035/1803523.pdf (zugegriffen: 3.3.2016). 
Deutscher Fußball-Bund. 2000. Mitglieder-Statistik 2000. URL: http://www.dfb.de/fileadmin/_dfbdam/25667-mitgliederstatistik_2000.pdf (zugegriffen: 22.4. 2016).

---. $2015.2015 . \quad$ Mitglieder-Statistik URL: http://www.dfb.de/fileadmin/_dfbdam/66210-Mitglieder-Statistik_2015.pdf (zugegriffen: 22.4.2016).

Deutscher Olympischer Sportbund, Hrsg. 2012a. Integration durch Sport. Ein Programm stellt sich vor. URL: http://www.integration-durch-sport.de/fileadmin/fmdosb/arbeitsfelder/ids/images/2014/Flyer_Programm_DEUTSCH.pdf (zugegriffen: 23.3.2016).

---. 2012b. Bach: Sport muss politisch sein - aber neutral. dosb.de. 5. Februar. URL:

http://www.dosb.de/ru/leistungssport/spitzensport-

news/detail/news/bach_sport_muss_politisch_sein_aber_neutral (zugegriffen: 24.3. 2016).

DFC Kreuzberg. O.J. Selbstverständnis. Homepage. URL: https://www.dfckreuzberg.de (zugegriffen: 10.2.2016; Website inzwischen inaktiv).

Dietze, Gabriele. 2012. Intersektionalität im deutschen Strafraum: „Race“, Gender und Sexualität und die deutsche Nationalmannschaft. Feministische Studien 1, Nr. 12: 53-65.

Diketmüller, Rosa. 2002. Frauenfußball im Zeichen der Globalisierung - Chancen und Risiken. In: Global players - Kultur, Ökonomie und Politik des Fußballs, hg. von Michael Fanizadeh, Gerald Hödl und Wolfram Manzenreiter, 203-226. Frankfurt am Main: Brandes \& Apsel.

---. 2006. Frauenfußball - Ein Paradigmenwechsel. In: Arena der Männlichkeit. Über das Verhältnis von Fußball und Geschlecht, hg. von Eva Kreisky und Georg Spitaler, 347-365. Frankfurt am Main: Campus.

Discover Football. O.J. About us. Homepage. URL: http://www.discoverfootball.de/en/home/about-us/ (zugegriffen: 4.4.2015).

Discover Football. 2013. Frauen Macht Fußball. Ein Handbuch von DISCOVER FOOTBALL. Berlin: Fußball und Begegnung e.V. URL: http://www.discoverfootball.de/fileadmin/user_upload/Handbuch/DISCOVER_F OOTBALL_Handbuch_de.pdf.

Discover Football. 2013a. DISCOVER FOOTBALL the female perspective. Broschüre, Print-Version.

Donnelly, Peter. 2009. Sport and human rights. In: Sport and foreign policy in a globalizing world, hg. von Steven J. Jackson und Stephen Haigh, 33-46. London u.a.: Routledge.

Dreyfus, Hubert L., Paul Rabinow und Michel Foucault. 1994 [1982]. Michel Foucault: Jenseits von Strukturalismus und Hermeneutik. Weinheim: BeltzAthenäum.

Dunning, Eric. 1994. Sport as a male preserve: Notes on the social sources of masculine Identity and its transformations. In: Women, sport, and culture, hg. von Susan Birrell und Cheryl L. Cole, 163-178. Champaign: Human Kinetics.

Dworkin, Shari L. und Michael A. Messner. 2002. Just do...what? Sport, bodies, gender. In: Gender and sport: a reader, hg. von Sheila Scraton und Anne Flintoff, 17-29. London u.a.: Routledge.

Edelman, Marc. 2001. Social movements: changing paradigms and forms of politics. Annual Review of Anthropology: 285-317. 
---. 2005. When networks don't work: the rise and fall and rise of civil society initiatives in Central America. In: Social movements: an anthropological reader, hg. von June C.Nash, 29-45. Malden u.a.: Blackwell.

Edwards, Michael und David Hulme. 1996. NGO performance and accountability: introduction and overview. In: Beyond the magic bullet: NGO performance and accountability in the post-cold war world, hg. von dies, 3-16. West Hartford: Kumarian Press.

---. 1996a. Beyond the magic bullet? Lessons and conclusions. In: Beyond the magic bullet: NGO performance and accountability in the post-cold war world, hg. von dies., 219-227. West Hartford: Kumarian Press.

---, Hrsg. 1996b. Beyond the magic bullet: NGO performance and accountability in the post-cold war world. West Hartford: Kumarian Press.

Eisenberg, Christiane. 1999. „English Sports“ und deutsche Bürger: eine Gesellschaftsgeschichte 1800-1939. Paderborn: Schöningh.

---. 2014. Fußball als globales Phänomen. Historische Perspektiven. APuZ. Aus Politik und Zeitgeschichte 26: 7-15. URL: http://www.bpb.de/shop/zeitschriften/apuz/28247/sport-und-politik-gesellschaft (zugegriffen: 3.7.2016).

Elling, Agnes und Annelies Knoppers. 2005. Sport, gender and ethnicity: practises of symbolic inclusion/exclusion. Journal of Youth and Adolescence 34, Nr. 3: 257268.

Emerson, Robert M., Rachel I. Fretz und Linda L. Shaw. 1995. Writing ethnographic fieldnotes. Chicago: University of Chicago Press.

Eng, Heidi. 2006. "We are moving up like a hard-on!" Doing sex/uality in sport. NORA - Nordic Journal of Feminist and Gender Research 14, Nr. 1: 12-26.

---. 2008. Doing sexuality in sport. Journal of Homosexuality 54, Nr. 1-2: 103-123.

Enslin, Elizabeth. 1994. Beyond writing: feminist practice and the limitations of ethnography. Cultural Anthropology 9, Nr. 4: 537-568.

Eribon, Didier. 2016. Rückkehr nach Reims. Berlin: Suhrkamp

Escobar, Arturo. 1997. The making and unmaking of the third world through development. In: The post-development reader, hg. von Majid Rahnema und Victoria Bawtree, 85-93. London: Zed Books.

Esteva, Gustavo und Medhu Suri Prakash. 1997. From global thinking to local thinking. In: The post-development reader, hg. von Majid Rahnema und Victoria Bawtree, 277-289. London: Zed Books.

Ewing, Martha E., Lori A. Gano-Overway, Crystal F. Branta und Vern D. Seefeldt. 2002. The role of sports in youth development. In: Paradoxes of youth and sport, hg. von Margaret Gatz, Michael A. Messner und Sandra J. Ball-Rokeach, 31-47. Albany: State University of New York Press.

Eyerman, Ron. 2006. Performing opposition or, how social movements move. In: Social performance: symbolic action, cultural pragmatics, and ritual, hg. von Jeffrey C. Alexander, Bernhard Giesen und Jason L. Mast, 193-217. Cambridge, New York: Cambridge University Press.

Fabian, Johannes. 1993. Präsenz und Repräsentation. In: Kultur, soziale Praxis, Text: die Krise der ethnographischen Repräsentation, hg. von Eberhard Berg und Martin Fuchs, 335-564. Frankfurt am Main: Suhrkamp.

Färber, Christine und Jochen Geppert. 2004. Bericht der externen Organisationsberatung über das Pilotprojekt Gender Mainstreaming im Bezirksamt Friedrichshain- 
Kreuzberg von Oktober 2003 bis März 2004. Potsdam: Competence Consulting. URL: $\quad$ https://www.berlin.de/ba-friedrichshain-kreuzberg/politik-undverwaltung/aemter/schul-und-sportamt/sportfoerderung/ 12.11.2015).

Fassin, Didier. 2008. Beyond good and evil? Questioning the anthropological discomfort with morals. Anthropological Theory 8, Nr. 4: 333-344.

---. 2017. The endurance of critique. Anthropological Theory 17 Nr. 1: 4-29.

Faust, Friederike 2017. Taktiken der Anerkennung. Wie sich Fußballerinnen ein Mitspielrecht erkämpfen. In: Ballsport, Gender, Transkulturalität, hg. von Julia Haß und Stephanie Schütze, 57-82. Berlin: Panama.

---. 2017a. „Nichts macht mich so glücklich wie Fußball, obwohl ich da ständig unglücklich bin“. In: Ethnografie und Deutung: Gruppensupervision als Methode reflexiven Forschens, hg. von Jochen Bonz, Katharina Eisch-Angus, Marion Hamm und Almut Sülzle, 259-271. Wiesbaden: VS Verlag für Sozialwissenschaften.

Faust, Friederike und Corinna Assmann. 2014. Queering Football - Körperpraktiken im Frauenfußball zwischen Normierung und Destabilisierung der Geschlechterordnung. Body Politics. Zeitschrift für Körpergeschichte 2, Nr. 3: 145-177.

Faust, Friederike und Johanna Kösters. 2016. The joy of the killjoys. Pain and pleasure among women's football activists. In: Emotionen im Spiel. Beiträge zu einer Ethnologie des Sports, hg. von Friederike Faust und Stefan Heissenberger, 72 83. Berlin: Panama.

Faust, Friederike und Stefan Heissenberger. 2016. Eine Frage des Trainings. Der Forscher*innenkörper als Erkentnissubjekt. In: Körpertechnologien. Ethnographische und gendertheoretische Perspektiven, hg. von Katrin Amelang, Beate Binder, Sven Bergmann, Anna-Carolina Vogel und Nadine Wagener-Böck, 6882. Berlin: Panama.

---. 2016a. Emotionen im Spiel. Eine Einleitung. In: Emotionen im Spiel. Beiträge zu einer Ethnologie des Sports, hg. von dies., 7-16. Berlin: Panama.

---, Hrsg. 2016b. Emotionen im Spiel. Beiträge zu einer Ethnologie des Sports. Berlin: Panama.

Ferguson, James. 1990. The anti-politics machine: „development,“ depoliticization, and bureaucratic power in Lesotho. Minneapolis: University of Minnesota Press.

---. 2004. Power Topographies. In: A companion to the anthropology of politics, hg. von David Nugent, 383-399. Malden u.a.: Blackwell.

Ferguson, James und Akhil Gupta. 2002. Spatializing states: toward an ethnography of neoliberal governmentality. American Ethnologist 29, Nr. 4: 981-1002.

FIFA. 2014. Erklärung der FIFA zu Russland 2018. fifa.com. 25. Juli. URL: http://de.fifa.com/worldcup/news/y=2014/m=7/news=erklarung-der-fifa-zurussland-2018-2408085.html (zugegriffen: 30.3.2016).

Fisher, Caitlin und Sophia Davis. 2016. Gleiches Geld für gleiches Spiel? Ungleichheit auf dem Rasen. Prager Frühling. Magazin für Freiheit und Sozialismus 25: $160-167$.

Fisher, Julie. 1994. Is the iron law of oligarchy rusting away in the Third World? World Development 22, Nr. 2: 129-143.

Fisher, William F. 1997. Doing good? The politics and antipolitics of NGO practices. Annual Review of Anthropology: 439-464. 
Flam, Helena und Debra King. 2006. Introduction. In: Emotions and social movements, hg. von Helena Flam und Debra King, 1-18. 14. London u.a.: Routledge.

Fletcher, Kim und Peter St. Pierre. 2014. Sports in the psychological and social demobilization of child soldiers. In: Sports, peacebuilding and ethics, hg. von Linda M. Johnston, 85-102. New Brunswick, New Jersey: Transaction Publishers.

Flick, Uwe. 2016 [2002]. Computer in der qualitativen Sozialforschung. In: Qualitative Sozialforschung: eine Einführung, hg. von ders., 451-472. Reinbek: Rowohlt.

Fluehr-Lobban, Carolyn. 2008. Collaborative anthropology as twenty-first-century ethical anthropology. Collaborative Anthropologies 1: 175-182.

Forbes, Ann Armbrecht. 1999. The importance of being local: villagers, NGOs, and the world bank in the Arun valley, Nepal. Identities 6, Nr. 2-3: 319-344.

Foucault, Michel. 1978. Dispositive der Macht: über Sexualität, Wissen und Wahrheit. Berlin: Merve.

---. 1992 [1978]. Was ist Kritik? Berlin: Merve.

---. 1992a [1990]. Andere Räume. In: Aisthesis. Wahrnehmung heute oder Perspektiven einer anderen Ästhetik, hg. von Karlheinz Barck, 34-46. Leipzig: Reclam.

--- 1994 [1981]. Archäologie des Wissens. Frankfurt am Main: Suhrkamp.

---. 1996. Diskurs und Wahrheit: die Problematisierung der Parrhesia. BerkeleyVorlesungen 1983. Berlin: Merve.

---. 2003. Die Wahrheit und die juristischen Formen. Frankfurt am Main: Suhrkamp.

---. 2005. Polemik, Politik und Problematisierung. In: Schriften in vier Bänden = Dits et écrits Bd. 4: 1980-1988, hg. von Daniel Defert und François Ewald, 724-734. Frankfurt am Main: Suhrkamp.

---. 2014 [1987]. Der Wille zum Wissen. Frankfurt am Main: Suhrkamp.

Fraser, Nancy. 1997. Justice interruptus: critical reflections on the ,postsocialist“ condition. London u.a.: Routledge.

Freeman, Jo. o.J. [1972]. The tyranny of structurelessness. Onlineressource: URL: https://www.jofreeman.com/joreen/tyranny.htm (zugegriffen: 2.2.2019).

Friedrich, Sebastian. 2015. Problem und Diskurs. Das Potenzial des Problematisierungsbegriffs bei Michel Foucault für eine ideologiekritische Diskursanalyse. In: Work in progress. Work on progress. Beiträge kritischer Wissenschaft. Doktorand innen-Jahrbuch 2015 der Rosa-Luxemburg-Stiftung, hg. von Marcus Hawel, Lisa Doppler, Paul Fischer-Schröter und Martin Schröder, 29-42. Hamburg: VSA-Verlag.

Fürtjes, Oliver. 2012. Fußball und der Mythos vom Proletariersport. Forum Wohnen und Stadtentwicklung 6: 317-325.

Füssel, Marian und Tim Neu. 2010. Doing discourse. In: Diskursiver Wandel, hg. von Achim Landwehr, 213-235. Wiesbaden: VS Verlag für Sozialwissenschaften.

Generalsekretär der Vereinten Nationen. 2004. Universal language of sport brings people together, teaches teamwork, tolerance, secretary-general says at launch of international year. un.org. 11. Mai. URL: http:/www.un.org/press/en/2004/sgsm9579.doc.htm (zugegriffen: 24. März 2016).

Geukler-Palmert, Ingrid. 2014. Darmstadt-Strafe. Rosa Trikot für den Flop des Monats. bild.de. 9. Mai. URL: http://www.bild.de/sport/fussball/sv-darmstadt98/rosa-trikot-fuer-den-flop-des-monats-37537124.bild.html (zugegriffen: 19. September 2016). 
Gibbs, Graham S., Susanne Friese und Wilma Mangabeira, Hrsg. 2002. Forum Qualitative Sozialforschung / Forum: Qualitative Social Research 3, Nr. 2, Sonderheft: Using technology in the qualitative research process. URL: http://www.qualitative-research.net/ index.php/fqs/issue/view/22 (zugegriffen: 14.9.2016).

Gilroy, Sarah. 1989. The emBody-ment of power: gender and physical activity. Leisure Studies 8, Nr. 2: 163-171.

Giulianotti, Richard. 2004. Human rights, globalization and sentimental education: the case of sport. Sport in Society 7, Nr. 3: 355-369.

Gmünder, Stefan und Klaus Zeyringer. 2018. Das wunde Leder. Wie Kommerz und Korruption den Fußball kaputt machen. Belrin: Suhrkamp.

Goodwin, Jeff, James M. Jasper und Francesca Polletta. 2001. Introduction: why emotions matter. In: Passionate politics: emotions and social movements, hg. von Jeff Goodwin, James M. Jasper und Francesca Polletta, 1-27. Chicago: University of Chicago Press.

Göttlich, Andreas. 2008. König Fußballs neue Kleider: Die Integrationsvorstellungen deutscher Sportverbände. In: Mittendrin im Abseits: ethnische Gruppenbeziehungen im lokalen Kontext, hg. von Sighard Neckel und Hans-Georg Soeffner, 211234. Wiesbaden: VS Verlag für Sozialwissenschaften.

Gould, Deborah B. 2009. Moving politics: emotion and act up's fight against AIDS. Chicago: University of Chicago Press.

Graeber, David. 2016. Reflections on reflections. HAU: Journal of Ethnographic Theory 6, Nr. 2: 5-9.

Gräff, Friederike. 2012. Streit der Woche. Ist Sport politisch? taz.de. 5. August. URL: https://www.taz.de/!5094369/ (zugegriffen: 11.3.2016).

Griffin, Pat. 1998. Strong women, deep closets: lesbians and homophobia in sport. Champaign: Human Kinetics.

---. 2002. Changing the game: homophobia, sexism and lesbians in sport. In: Gender and sport: a reader, hg. von Sheila Scraton und Anne Flintoff, 193-208. London u.a.: Routledge.

Grimm, Carmen. 2016. Zwischen Crew-Love und Peer-Pressure. Synchronisierte Körper und Vergeschlechtlichungen in einem Mädchen*straßenfußballteam. In: Emotionen im Spiel. Beiträge zu einer Ethnologie des Sports, hg. von Friederike Faust und Stefan Heissenberger, 84-94. Berlin: Panama.

Grisard, Dominique. 2015. Pink. En/gendering a color. Solar Initiative. Current: Pink, Nr. 2: 26-29.

Gruber, Manuela. 2013. Die Zukunft is(s)t vegetarisch: der Wandel von einer fleischdominierten Esskultur zu einer vegetarischen Ernährungsweise. Hamburg: Diplomica.

Gugutzer, Robert. 2004. Soziologie des Körpers. Bielefeld: Transcript.

---. 2011. Körperpolitiken des Sports. Zur sportiven Verschränkung von Körper, Geschlecht und Macht. In: Die Sexualisierung des Sports in den Medien, hg. von Daniela Schaaf und Jörg-Uwe Nieland, 34-56. Köln: Harlem Verlag.

Güldenpfennig, Sven. 2000. Sport, Kritik und Eigensinn: der Sport der Gesellschaft. Sankt Augustin: Academia.

Gülfirat, Suzan. 2009. Football under cover: Fußball - Integration - Geschlechterrollen. boell.de. 3. November. URL: https://www.boell.de/de/2009/03/11/footballunder-cover-fussball-integration-geschlechterrollen (zugegriffen: 19.4.2017). 
Gunn, Simon und Rachel Bell. 2003. Middle classes: their rise and sprawl. London: Phoenix.

Günther, Susanne. 1996. Zwischen Scherz und Schmerz - Frotzelaktivitäten in Alltagsinteraktionen. In: Scherzkommunikation: Beiträge aus der empirischen Gesprächsforschung, hg. von Helga Kotthoff, 81-108. Opladen: Westdeutscher Verlag.

Gupta, Akhil und James Ferguson. 1997. Culture, power, place: an ethnography at the end of an era. In: Culture, power, place: explorations in critical anthropology, hg. von dies., 1-29. Durham: Duke University Press.

Haas, Felix. 2016. Die erbärmliche Aufregung über eine Frau als EMKommentatorin. stern.de. 17. Juni. URL: http://www.stern.de/sport/fussball/em2016/em-2016-im-zdf--claudia-neumann-und-die-erbaermliche-aufregung6906654.html (zugegriffen: 24.3.2017).

Habermas, Jürgen. 1962. Strukturwandel der Öffentlichkeit: Untersuchungen zu einer Kategorie der bürgerlichen Gesellschaft. Frankfurt am Main: Suhrkamp.

Hacking, Ian. 2007. Kinds of people: moving targets. Proceedings of the British Academy 151: 285-318.

Hagemann-White, Carol. 1993. Die Konstrukteure des Geschlechts auf frischer Tat ertappen? Methodische Konsequenzen einer theoretischen Einsicht. Feministische Studien 11, Nr. 2: 68-78.

Halberstam, Jack. 2012. Global female masculinities. Sexualities 15, Nr. 3-4: 336354.

Hall, Stuart. 2006 [2001]. Encoding/Decoding. In: Media and Cultural Studies: Keyworks, hg. von Meenakshi Gigi Durham und Douglas Kellner, 163-173. Malden u.a.: Blackwell.

Hamm, Marion. 2013. Engagierte Wissenschaft zwischen partizipativer Forschung und reflexiver Ethnographie. Methodische Überlegungen zur Forschung in sozialen Bewegungen. In: Eingreifen, Kritisieren, Verändern!?: Interventionen ethnographisch und gendertheoretisch, hg. von Binder Beate, Friedrich von Bose, Kartin Ebell, Sabine Hess und Anika Keinz, 55-72. Münster: Westfälisches Dampfboot.

Hansen, David M., Reed W. Larson und Jodi B. Dworkin. 2003. What adolescents learn in organized youth activities: a survey of self-reported developmental experiences. Journal of Research on Adolescence 1, Nr. 13: 25-55.

Haraway, Donna. 1995. Situiertes Wissen. Die Wissenschaftsfrage im Feminismus und das Privileg einer partialen Perspektive. In: Die Neuerfindung der Natur: Primaten, Cyborgs und Frauen, hg. von Carmen Hammer und Immanuel Stieß, 73-97. Frankfurt am Main: Campus.

Hargreaves, Jennifer. 1994. Sporting females: critical issues in the history and sociology of women's sports. London u.a.: Routledge.

---. 1999. The „Women's International Sports Movement“: local-global strategies and empowerment. Women's Studies International Forum 22, Nr. 5: 461-471.

---. 2000. Heroines of sport: the politics of difference and identity. London u.a.: Routledge.

Haring, Merten. 2010. Sportförderung in Deutschland Eine vergleichende Analyse der Bundesländer. Wiesbaden: VS Verlag für Sozialwissenschaften.

Harvey, David. 2005. A brief history of neoliberalism. Oxford: Oxford University Press. 
Harvey, Jean, John Horne, Parissa Safai, Simon Darnell und Sébastien CourchesneO'Neill. 2013. Sport and social movements: from the local to the global. London: Bloomsbury.

Haß, Julia. 2017. Und es gibt sie doch. Frauen, die Fußballspielen. Historische und aktuelle Dynamiken von Amateurfußball und Geschlecht in Rio de Janeiro. In: Ballsport, Gender, Transkulturalität, hg. von Julia Haß und Stephanie Schütze, 73-93. Berlin: Panama.

Haunss, Sebastian. 2004. Identität in Bewegung: Prozesse kollektiver Identität bei den Autonomen und in der Schwulenbewegung. Wiesbaden: VS Verlag für Sozialwissenschaften.

Hayhurst, Lyndsay. 2013. Girls as the 'new' agents of social change? Exploring the 'girl effect' through sport, gender and development programs in Uganda. Sociological Research Online 18, Nr. 2: o.S. URL: http://www.socresonline.org.uk/18/2/8.html (zugegriffen: 22.7.2015).

Heinemann, Klaus. 1996. Staatliche Sportpolitik und Autonomie des Sports. In: Sportpolitik. Sozialwissenschaftliche Analysen, hg. von Günther Lüschen und Alfred Rütten, 177-197. Stuttgart: Naglschmid.

Heinrich-Böll-Stiftung. 2016. Es wird eng - Handlungsspielräume für Zivilgesellschaft. Berlin: Heinrich-Böll-Stiftung. URL: https://www.boell.de/sites/default/files/fuer_demokratie.pdf?dimension1=ds_shri nking_de (zugegriffen: 11. März 2016).

Heissenberger, Stefan. 2010. Der entgrenzte Körper: Über Gewaltaspekte und dominante Männlichkeit in Fußballmannschaften. In: Gesundheit in Bewegung: Impulse aus Geschlechterperspektive, hg. von Ilse Hartmann-Tews, Britt Dahmen und Diana Emberger, 107-102. Sankt Augustin: Academia.

---. 2018. Schwuler* Fußball: Ethnografie einer Freizeitmannschaft. Bielefeld: Transcript.

Helms, Elissa. 2014. The Movementization of NGOs? Women's Organizing in Postwar Bosnia-Herzegovina. In: Theorizing NGOs: states, feminisms, and neoliberalism, hg. von Victoria Bernal und Inderpal Grewal, 21-49. Durham: Duke University Press.

Hemmings, Clare. 2012. Affective solidarity: feminist reflexivity and political transformation. Feminist Theory 13, Nr. 2: 147-161.

Hennies, Rainer und Daniel Meuren, Hrsg. 2009. Frauenfussball: der lange Weg zur Anerkennung. Göttingen: Werkstatt.

Herzog, Markwart. 2013. „Sympathien zu den balltretenden Amazonen“. Die Anfänge des Frauenfußballs im 1. FC Kaiserslautern. In: Frauenfussball in Deutschland: Anfänge - Verbote - Widerstände - Durchbruch, hg. von ders., 87-112. Stuttgart: Kohlhammer.

Hess, Sabine und Michi Knecht. 2008. Reflexive Medikalisierung im Feld moderner Reproduktionsmedizin: Zum aktiven Einsatz von Wissensressourcen in gendertheoretischer Perspektive. In: Wissen und Geschlecht. Beiträge der 11. Arbeitstagung der Kommission für Frauen- und Geschlechterforschung, hg. von Elisabeth Timm, Nikola Langreiter, Michaela Haibl, Klara Löffler und Susanne Blumesberger, 169-194. Wien: Institut für Europäische Ethnologie.

von der Heyde, Judith und Jochem Kotthaus. 2016. Editorial: Wettkampf. Sozialmagazin. Die Zeitschrift für Soziale Arbeit, Nr. 6: 3. 
Hickel, Jason. 2012. Liberalism and the politics of Occupy Wall Street. Anthropology of this century 4: 1-9. URL: http://eprints.lse.ac.uk/43389/ (zugegriffen 21.12.2016).

Hilhorst, Dorothea. 2003. The real world of NGOs: discourses, diversity, and development. London: Zed Books.

Hill Collins, Patricia. 1990. Black feminist thought: knowledge, consciousness, and the politics of empowerment. Boston: Unwin Hyman.

Hirschauer, Stefan. 2001. Das Vergessen des Geschlechts. Zur Praxeologie einer Kategorie sozialer Ordnung. Hg. von Bettina Heintz. Geschlechtersoziologie. Sonderheft 41/2001 der Kölner Zeitschrift für Soziologie und Sozialpsychologie, Nr. 41: 208-235.

Hirschauer, Stefan und Klaus Amann, Hrsg. 1997. Die Befremdung der eigenen Kultur: zur ethnographischen Herausforderung soziologischer Empirie. Frankfurt am Main: Suhrkamp.

Hirschman, Albert O. 1970. Exit, voice, and loyalty: responses to decline in firms, organizations, and states. Cambridge: Harvard University Press.

Hodžić, Saida. 2014. Feminist bastards. Towards a posthumanist critique of NGOization. In: Theorizing NGOs: states, feminisms, and neoliberalism, hg. von Victoria Bernal und Inderpal Grewal, 221-247. Durham: Duke University Press.

---. 2017. The twilight of cutting. African activism and life after NGOs. Oakland: university of California Press.

Hoffmann, Eduard und Jürgen Nendza. 2011 [2005]. Verlacht, verboten und gefeiert: zur Geschichte des Frauenfussballs in Deutschland. Weilerswist: Ralf Liebe.

Höhn, Sabine. 2013. The object of activism: documents and daily life in Namibian NGOs. PoLAR: Political and Legal Anthropology Review 36, Nr. 1: 99-117.

Holmes, Douglas und Georges Marcus. 2005. Cultures of expertise and the management of globalization: toward the re-functioning of ethnography. In: Global assemblages. Technology, politics, and ethics as anthropological problems, hg. von Aihwa Ong und Stephen Collier, 235-252. Malden u.a.: Blackwell.

Horeni, Michael. 2016. Der Zustand des Fußballs. Täuschen, tricksen - und aufschreien. faz.net. 3. Januar. URL: http://www.faz.net/aktuell/sport/fussball/bundesliga/von-rudi-voeller-bis-rogerschmidt-fussball-regeln-selten-beachtet-14092774.html (zugegriffen: 26.10.2016).

Humphreys, Michael und Tony Watson. 2009. Ethnographic practices: from 'writingup ethnographic research' to 'writing ethnography'. In: Organizational ethnography: studying the complexities of everyday life, hg. von Sierk Ybema, Dvora Yanow, Harry Wels und Frans Kamsteeg, 40-55. Thousand Oaks u.a.: Sage Publications.

Ibrahim, Joseph. 2015. Bourdieu and social movements: ideological struggles in the British anti-capitalist movement. Basingstoke u.a.: Palgrave Macmillan.

Illius, Bruno. 2003. Feldforschung. In: Ethnologie: Einführung und Überblick, hg. von Bettina Beer und Hans Fischer, 73-98. Berlin: Dietrich Reimer.

Ipsen, Detlev. 2002. Die Kultur der Orte. Ein Beitrag zur sozialen Strukturierung des städtischen Raums. In: Differenzierungen des Städtischen, hg. von Martina Löw, 233-245. Opladen: Leske und Budrich. 
Islam, Gazi. 2015. Practitioners as theorists: para-ethnography and the collaborative study of contemporary organizations. Organizational Research Methods 18, Nr. 2: 231-251.

Jackson, Steven J. und Stephen Haigh. 2009. Introduction. Between and beyond politics: sport and foreign policy in a globalizing world. In: Sport and foreign policy in a globalizing world, hg. von Steven J. Jackson und Stephen Haigh, 1-10. London u.a.: Routledge.

Jacobsen, Dirk. 2015. Die sportbezogene internationale Entwicklungszusammenarbeit von in Deutschland ansässigen Organisationen: ausgewählte Akteure, Inhalte, Strategien. Dissertation, Berlin: Freie Universität. URL: http://www.diss.fuber-

lin.de/diss/servlets/MCRFileNodeServlet/FUDISS_derivate_000000018448/Diss ertation_Jacobsen_Dirk_Sport_Entwicklung.pdf(zugegriffen: 7.6.2016).

Jakubowska, Honorata. 2014. Gender verification in sport as a surveillance practice: An inside and outside perception. Surveillance \& Society 11, Nr. 4.

Jasper, James M. 2011. Emotions and social movements: twenty years of theory and research. Annual Review of Sociology 37, Nr. 1: 285-303.

Jeggle, Utz. 2014. Verständigungsschwierigkeiten im Feld. In: Das Fremde im Eigenen: Beiträge zur Anthropologie des Alltags, hg. von Bernhard Tschofen, Reinhard Johler, Monique Scheer und Thomas Thiemeyer, 17-37. Tübingen: Tübinger Vereinigung für Volkskunde.

Josuttis, Konstantin. 2017. Die dunkle Seite des Balles. 34 Spieltage und ein Finale. Hildesheim: Arete.

Kaschuba, Wolfgang. 1995. Deutsche Bürgerlichkeit nach 1800 - Kultur als symbolische Praxis. In: Bürgertum im 19. Jahrhundert, hg. von Jürgen Kocka, 2:92-127. Göttingen: Vandenhoeck und Ruprecht.

---. 1997. Sportivität: Die Karriere eines neuen Leitwertes. Anmerkungen zur „,Versportlichung“" unserer Alltagskultur. In: Sportphilosophie, hg. von Volker Caysa, 229-256. Leipzig: Reclam.

---. 1999. Einführung in die Europäische Ethnologie. München: Beck.

---. 2010. Verlorene Bürgerlichkeiten? In: Alltag als Politik - Politik im Alltag. Dimensionen des Politischen in Vergangenheit und Gegenwart. Ein Lesebuch für Carola Lipp, hg. von Michaela Fenske, 81-96. Berlin: Lit.

---. 2014. What cities know about: urban spaces as civic labs. In: IBA_SUMMIT. knowledge I based I urbanism, hg. von Internationale Bauaustellung Heidelberg $\mathrm{GmbH}$, 34-40. URL: http://iba.heidelberg.de/files/iba_summit_dokumentation.pdf_ (zugegriffen: 15.2.2016).

---. 2016. Vom Wissen der Städte. Urbane Räume als Labore der Zivilgesellschaft. In: Urbane Aushandlungen. Die Stadt als Aktionsraum, hg. von Dominik Kleinen und Cornelia Kühn, 13-30. Berlin: Panama.

Katzenstein, Mary Fainsod. 1998. Faithful and fearless: moving feminist protest inside the church and military. Princeton: Princeton University Press.

Kauffman, L. A. 1990. The anti-politics of identity. Socialist Review 90, Nr. 1: 67-80.

Keller, Reiner. 2008 [2005]. Wissenssoziologische Diskursanalyse: Grundlegung eines Forschungsprogramms. Wiesbaden: VS Verlag für Sozialwissenschaften.

Kistner, Thomas. 2012. Fifa-Mafia: Die schmutzigen Geschäfte mit dem Weltfußball. München: Droemer Knaur. 
Klausner, Martina, Milena D. Bister, Jörg Niewöhner und Stefan Beck. 2015. Choreografien klinischer und städtischer Alltage. Ergebnisse einer ko-laborativen Ethnografie mit der Sozialpsychiatrie. Zeitschrift für Volkskunde 2, Nr. 111: 214235.

Klein, Gabriele und Michael Meuser. 2008b. Fußball, Politik, Vergemeinschaftung. Zur Einführung. In: Ernste Spiele: zur politischen Soziologie des Fussballs, hg. von dies., 7-16. Bielefeld: Transcript.

Klein, Marie-Luise. 2011. Migrantinnen im Sport - Zur sozialen Konstruktion einer „Problemgruppe“. In: Migration, Integration und Sport: Zivilgesellschaft vor Ort, hg. von Sebastian Braun und Tina Nobis, 125-135. Wiesbaden: VS Verlag für Sozialwissenschaften.

Kleinen, Dominik und Cornelia Kühn. 2016. Urbane Aushandlungen. Die Stadt als Aktionsraum. In: Urbane Aushandlungen. Die Stadt als Aktionsraum, hg. von dies., 7-12. Berlin: Panama.

Klöppel, Ulrike. 2010. Foucaults Konzept der Problematisierungsweise und die Analyse diskursiver Transformationen. In: Diskursiver Wandel, hg. von Achim Landwehr, 255-263. Wiesbaden: VS Verlag für Sozialwissenschaften.

Koch, Konrad. 1900. Die Erziehung zum Mute durch Turnen, Spiel und Sport: Die geistige Seite der Leibesübungen. Berlin: Gaertner.

Kothari, Rajni. 1997. The agony of the modern state. In: The post-development reader, hg. von Majid Rahnema und Victoria Bawtree, 143-151. London: Zed Books.

Kratzmüller, Bettina. 2005. „quae beneficia e medio statio Isthmiorum die sua ipse voce pronuntiavit" - Stadion und Politik in der Antike. In: Das Stadion: Geschichte, Architektur, Politik, Ökonomie, hg. von Matthias Marschik, Rudolf Müllner, Georg Spitaler und Michael Zinganel, 91-126. Wien: Turia + Kant.

Kreisky, Eva und Georg Spitaler, Hrsg. 2006. Arena der Männlichkeit: über das Verhältnis von Fußball und Geschlecht. Frankfurt am Main: Campus.

Krook, Mona Lena. 2006. Reforming representation: the diffusion of candidate gender quotas worldwide. Politics \& Gender 2, Nr. 3: 303-327.

Krüger, Michael. 2004. Adorno, der Sport und die Kritische Sporttheorie. Sportwissenschaft 34, Nr. 1: 21-32.

Krull, Patrick. 2007. "Die Zukunft des Fußballs ist weiblich". welt.de. 29. Oktober. URL: https://www.welt.de/sport/article1306397/Die-Zukunft-des-Fussballs-istweiblich.html (zugegriffen: 2.2.2019)

Kuby, Eva. 1986. Politische Frauenvereine und ihre Aktivitäten 1848 bis 1950. In: Schimpfende Weiber und patriotische Jungfrauen: Frauen im Vormärz und in der Revolution 1848/49, hg. von Carola Lipp, 248-269. Baden-Baden: Elster Verlag.

Kugelmann, Claudia. 1993. Sport für Frauen - Raum für Frauen - Weg für Frauen? Feministische Studien 2, Nr. 11: 140-144.

Kuhn, Gabriel. 2014. Die Linke und der Sport. Münster: Unrast.

Lackner, Theresa. 2012. Europameisterschaft. Fußballer gegen Boykott. süddeutsche.de. $15 . \quad$ Mai. URL: http://www.sueddeutsche.de/muenchen/ebersberg/europameisterschaft-fussballund-politik-trennen-1.1347990 (zugegriffen: 11.3.2016).

Laclau, Ernesto und Chantal Mouffe. 2001 [1985]. Hegemony and socialist strategy: towards a radical democratic politics. London: Verso.

Lang, Barbara. 1998. Mythos Kreuzberg: Ethnographie eines Stadtteils (1961 - 1995). Frankfurt am Main: Campus. 
Lang, Sabine. 1997. The NGOization of feminism. In: Transitions, environments, translations: feminisms in international politics, hg. von Joan Wallach Scott, Cora Kaplan und Debra Keates, 101-120. London u.a.: Routledge.

---. 2013. NGOs, civil society, and the public sphere. Cambridge: Cambridge University Press.

Langenohl, Andreas. 2009. Zweimal Reflexivität in der gegenwärtigen Sozialwissenschaft: Anmerkungen zu einer nicht geführten Debatte. Forum Qualitative Sozialforschung / Forum: Qualitative Social Research 10, Nr. 2: o.S. URL: http://www.qualitative-research.net/index.php/fqs/article/view/1207/2722 (zugegriffen: 5.4.2016).

Lash, Scott. 1994. Reflexivity and its doubles: structure, aesthetics, community. In: Reflexive modernization: politics, tradition and aesthetics in the modern social order, hg. von Ulrich Beck, Anthony Giddens und Scott Lash, 110-173. Stanford: Stanford University Press.

Lassiter, Luke Eric. 2004. Collaborative ethnography. ANTHRONOTES 25, Nr. 1: 120.

---. 2005. Collaborative ethnography and public anthropology. Current Anthropology 46, Nr. 1: 83-106.

Lau, Kimberly J. 2011. Body language. Sisters in shape, black women's fitness, and identity politics. Philadelphia: Temple University Press.

Leigh, Mary H. und Thérèse M. Bonin. 1977. The pioneering role of Madame Alice Milliat and the FSFI* in establishing international trade and field competition for women. Journal of Sport History 1, Nr. 4: 72-83.

Lengwiler, Martin und Jeannette Madarász. 2010. Das präventive Selbst. Eine Kulturgeschichte moderner Gesundheitspolitik. Bielefeld: Transcript.

Leutheusser-Schnarrenberger, Sabine. 1997. Beim FC Starnberg. In: Doppelpässe: Fußball \& Politik, hg. von Norbert Seitz, 242-243. Frankfurt am Main: Eichborn.

Leve, Lauren. 2014. Failed development and rural revolution in Nepal: rethinking subaltern consciousness and women's empowerment. In: Theorizing NGOs: states, feminisms, and neoliberalism, hg. von Victoria Bernal und Inderpal Grewal, 50-92. Durham: Duke University Press.

Lindner, Rolf. 1981. Die Angst des Forschers vor dem Feld. Überlegungen zur teilnehmenden Beobachtung als Interaktionsprozeß. Zeitschrift für Volkskunde 77, Nr. 1: 51-66.

---. 1983. Von sportsmen und einfachen Leuten. Zur Sozialgeschichte des Fußballsports. In: Der Satz „Der Ball ist rund“ hat eine gewisse philosophische Tiefe: Sport, Kultur, Zivilisation, hg. von Rolf Lindner, 22-36. Berlin: TransitBuchverlag.

---. 2001. Von der Feldforschung zur Feld-Forschung. In: Dazwischen: zur Spezifik der Empirien in der Volkskunde, hg. von Klara Löffler, 13-16. Wien: Selbstverlag des Instituts für Europäische Ethnologie.

---. 2003. Vom Wesen der Kulturanalyse. Zeitschrift für Volkskunde 99: 177-188.

Lindner, Rolf und Heinrich Th. Breuer. 1978. „Sind doch nicht alles Beckenbauers“: zur Sozialgeschichte des Fussballs im Ruhrgebiet. Frankfurt am Main: Syndikat.

Linne, Carina Sophia. 2011. Freigespielt: Frauenfussball im geteilten Deutschland. Berlin: Be.Bra Wissenschaftsverlag. 
Linska, Marion. 2012. Selbst-/Reflexion in der Kultur- \& Sozialanthropologie. Norderstedt: Books on Demand.

Lipp, Carola. 1986. Frauen und Öffentlichkeit. Möglichkeiten und Grenzen politischer Partizipation im Vormärz und der Revolution 1848. In: Schimpfende Weiber und patriotische Jungfrauen: Frauen im Vormärz und in der Revolution 1848/49, hg. von Carola Lipp, 270-307. Baden-Baden: Elster Verlag.

Lönnecker, Harald. 2013. „,... das macht man doch nicht!“ Frauenfußball an deutschen Hochschulen 1919-1935. In: Frauenfussball in Deutschland: Anfänge - Verbote - Widerstände - Durchbruch, hg. von Markwart Herzog, 201-222. Stuttgart: Kohlhammer.

Löw, Martina. 2001. Raumsoziologie. Frankfurt am Main: Suhrkamp.

Low, Setha M. und Sally Engle Merry. 2010. Engaged anthropology: diversity and dilemmas. An introduction to Supplement 2. Current Anthropology 51, Nr. 2: 203-226.

Marcus, George E. 2009. Introduction. Notes toward an ethnographic memoir of supervising graduate research through anthropology's decades of transformation. In: Fieldwork is not what it used to be: learning anthropology's method in a time of transition, hg. von James D. Faubion und George E. Marcus, 1-34. Ithaca: Cornell University Press.

Markovits, Andrei S. und Steven L. Hellerman. 2004. Die „Olympianisierung“ des Fußballs in den USA: Von der Marginalisierung in der amerikanischen Mainstream-Kultur zur Anerkennung als ein alle vier Jahre stattfindendes Ereignis. Sport und Gesellschaft-Sport and Society 1, Nr. 1: 7-29.

Markula, Pirkko, Hrsg. 2005. Feminist sport studies: sharing experiences of joy and pain. Albany: State University of New York Press.

Marschik, Matthias. 2004. „Kehren wir endlich zurück zum Sportlichen...“ Die neutrale Inszenierung von Sportereignissen. SWS-Rundschau 44, Nr. 2: 111-131.

---. 2004a. Sport als , leerer Signifikant' - Die Neutralisierung des Sportes als Bedingung seiner kulturellen Bedeutungen. Kurswechsel 10, Nr. 2: 35-43.

Marschik, Matthias, Rudolf Müllner, Georg Spitaler und Michael Zinganel. 2005. Einmarsch ins Stadion: Einleitung. In: Das Stadion: Geschichte, Architektur, Politik, Ökonomie, hg. von dies., 7-18. Wien: Turia + Kant.

Martischnig, Michael. 1982. Vereine als Träger von Volkskultur in der Gegenwart am Beispiel Mattersburg. Wien: Verlag der Österreichischen Akademie der Wissenschaften.

Marx Ferree, Myra und Carol McClurg Mueller. 2007. Feminism and the women's movement: a global perspective. In: The Blackwell companion to social movements, hg. von David A. Snow, Sarah A. Soule und Hanspeter Kriesi, 576-607. Malden u.a.: Blackwell.

McAdam, Doug. 1989. The biographical consequences of activism. American Sociological Review 54, Nr. 5: 744-760.

McDonald, Mary G. 2015. Imagining neoliberal feminisms? Thinking critically about the US diplomacy campaign, 'Empowering Women and Girls Through Sports'. Sport in Society 18, Nr. 8: 909-922.

McRobbie, Angela. 2009. The aftermath of feminism: gender, culture and social change. Thousand Oaks u.a.: Sage Publications.

Meier, Marianne. 2004. „Zarte Füsschen am harten Leder ...": Frauenfussball in der Schweiz 1970 - 1999. Frauenfeld: Huber. 
Mekata, Motoko. 2000. Building partnerships toward a common goal: experiences of the international campaign to ban landmines. In: The third force: the rise of transnational civil society, hg. von Ann Florini, Nihon Kokusai Kōryū Sentā und Carnegie Endowment for International Peace, 143-176. Tokyo: JCIE und CEIP.

Menasse, Eva. 2005. Vienna. Köln: Kiepenheuer und Witsch

Merkel, Udo, Hrsg. 2014. Power, politics and international events: socio-cultural analyses of festivals and spectacles. London u.a.: Routledge.

Merry, Sally Engle, Peggy Levitt, Mihaela Şerban Rosen und Diana H. Yoon. 2010. Law from below: women's human rights and social movements in New York City. Law \& Society Review 44, Nr. 1: 101-128.

Mertz, Elizabeth und Andria Timmer. 2010. Introduction. Getting it done: ethnographic perspectives on NGOs. PoLAR: Political and Legal Anthropology Review 33, Nr. 2: 171-177.

Messner, Michael A. 1994. Sports and male domination: the female athlete as contested ideological terrain. In: Women, sport, and culture, hg. von Susan Birrell und Cheryl L. Cole, 65-80. Champaign: Human Kinetics.

Meyer, Dorit. 2012. Bericht zur konzeptionellen Weiterentwicklung des Frauen- und Mädchensports im Bezirk Friedrichshain-Kreuzberg. URL: https://www.berlin.de/ba-friedrichshain-kreuzberg/politik-undverwaltung/beauftragte/gleichstellung/ (zugegriffen: 3.7.2016).

Michels, Harald. 2016. Fußball als Wettkampfsport in der Sozialen Arbeit. Sozialmagazin. Die Zeitschrift für Soziale Arbeit, Nr. 6: 42-50.

MINEPS V. 2013. Über die Konferenz. Mineps2013.de. URL: http://www.mineps2013.de/index.php?id=7 (zugegriffen: 14.2.2017).

---. 2013a. Berliner Erklärung [Declaration of Berlin]. 30. Mai. URL: http://www.mineps2013.de/fileadmin/Dokumente/pdf/130531_MINEPS\%20V_B erliner\%20Erk1\%C3\%A4rung\%20DE.pdf (zugegriffen: 14.2.2017).

Mittag, Jürgen. 2011. Sport und Protest. APuZ. Aus Politik und Zeitgeschichte 16-19: 9-14.

Mittag, Jürgen und Jörg-Uwe Nieland. 2017. Das Ende der Lizenz zum Machterhalt? Das System FIFA und die Grenzen von Opposition und Protest in internationalen Sportorganisationen. In: Verbände unter Druck: Protest, Opposition und Spaltung in Interessenorganisationen, hg. von Detlef Sack und Christoph Strünck, 197-216. Wiesbaden: VS Verlag für Sozialwissenschaften.

Mohanty, Chandra Talpade. 2003. "Under western eyes" revisited: feminist solidarity through anticapitalist struggles. Signs: Journal of Women in Culture and Society 28, Nr. 2: 499-535.

Mohr, Sebastian und Andrea Vetter. 2014. Körpererfahrung in der Feldforschung. In: Methoden der Kulturanthropologie, hg. von Christine Bischoff, Walter Leimgruber und Karoline Oehme-Jüngling, 101-116. Bern: UTB.

Mouffe, Chantal. 2014. Agonistik. Die Welt politisch denken. Frankfurt am Main: Suhrkamp.

Mosse, David. 2004. Is good policy unimplementable? Reflections on the ethnography of aid policy and practice. Development and Change 35: 639-671.

---. 2005. Cultivating development: an ethnography of aid policy and practice. Anthropology, culture, and society. London: Pluto Press. 
---. 2006. Anti-social anthropology? Objectivity, objection, and the ethnography of public policy and professional communities. Journal of the Royal Anthropological Institute 12, Nr. 4: 935-956.

Müller, Juliane. 2013. Migration, Geschlecht und Fussball zwischen Bolivien und Spanien. Netzwerke, Räume, Körper. Berlin: Reimer.

Müller, Marion. 2007. Das Geschlecht des Fußballs - Zur „Polarisierung der Geschlechtscharaktere“ im Fußball. Sport und Gesellschaft - Sport and Society 4, Nr. 2: 113-141.

---. 2016: Constructing Gender Incommensurability in Competitive Sports: Sex/Gender Testing and the New Regulations on Female Hyperandrogenism. Human Studies 39: 405-431.

---. 2009. Fußball als Paradoxon der Moderne: zur Bedeutung ethnischer, nationaler und geschlechtlicher Differenzen im Profifußball. Wiesbaden: VS Verlag für Sozialwissenschaften.

Musil, Robert. 1955. Tagebücher, Aphorismen, Essays und Reden. Hamburg: Rowohlt.

Najafi, Ayat und David Assmann. 2008. Football Under Cover. Drehbuch: Ayat Najafi, David Assmann, Marlene Assmann, Corinna Assmann, Valerie Assmann. Deutschland: Flying Moon Filmproduktion GmbH/Assmann Filmproduktion. Uraufführung (DE) am 10.02.2008, Berlin, 89'.

Nathaus, Klaus. 2009. Organisierte Geselligkeit: deutsche und britische Vereine im 19. und 20. Jahrhundert. Göttingen: Vandenhoeck \& Ruprecht.

Newman, Janet. 2012. Working the spaces of power: activism, neoliberalism and gendered labour. London: Bloomsbury.

---. 2013. Spaces of power: feminism, neoliberalism and gendered labor. Social Politics: International Studies in Gender, State \& Society 20, Nr. 2: 200-221.

---. 2014. Governing the present: activism, neoliberalism, and the problem of power and consent. Critical Policy Studies 8, Nr. 2: 133-147.

Newton, Esther. 1989. The mythic mannish lasbian: Radclyffe Hall and the new women. In: Hidden from History. Reclaiming the gay and lesbian past, hg. von Martin Dubermann, Martha Vicinus und George Chauncey Jr., 281-293. New York: New American Library.

Nieland, Jörg-Uwe. 2013. Weltmeisterschaften als Sprungbretter der medialen Wahrnehmung des Frauenfußballs? Die Berichterstattung in deutschen Printmedien im Zeitverlauf. In: Frauenfussball in Deutschland: Anfänge - Verbote - Widerstände - Durchbruch, hg. von Markwart Herzog, 241-261. Stuttgart: Kohlhammer.

Niewöhner, Jörg. 2014. Raum aus anthropologischer Perspektive. In: Theorien in der Raum- und Stadtforschung: Einführungen, hg. von Jürgen Ossenbrügge und Anne Vogelpohl, 14-23. Münster: Westfälisches Dampfboot.

---. 2016. Yhteistyöstävä antropologia: kuinka edistää refleksiivisyyttä kokeellisesti. In: Etnologinen tulkinta ja analyysi. Kohti avoimempaa tutkimusprosessia, hg. von Jukka Jouhki und Tytti Steel, 81-125. Tallinn: Ethnos. (Englische Version: Co-laborative anthropology. Crafting reflexivities experimentally. Zugänglich über academia.edu. URL: https://www.academia.edu/26429278/COLABORATIVE_ANTHROPOLOGY_CRAFTING_REFLEXIVITIES_EXPERIMENTALL Y) 
Ochsner, Andrea. 2012. Gender und kulturelle Identität in Bend it like Beckham. Frauenfußball als doppelte Entkolonialisierung. In: Spielen Frauen ein anderes Spiel?, hg. von Gabriele Sobiech und Andrea Ochsner, 155-170. Wiesbaden: VS Verlag für Sozialwissenschaften.

Okely, Judith. 2007. Fieldwork embodied. The Sociological Review 55: 65-79.

Ommert, Alexandra. 2016. Ladyfest-Aktivismus: queer-feministische Kämpfe um Freiräume und Kategorien. Bielefeld: Transcript.

Ortner, Sherry B. 1996. Making gender: the politics and erotics of culture. Boston: Beacon Press.

---. 2006. Anthropology and social theory: culture, power, and the acting subject. Durham: Duke University Press.

Palzkill, Birgit. 1995. Zwischen Turnschuh und Stöckelschuh: die Entwicklung lesbischer Identität im Sport. München: Verlag Frauenoffensive.

Parks, Thomas. 2008. The rise and fall of donor funding for advocacy NGOs: understanding the impact. Development in Practice 18, Nr. 2: 213-222.

Pelak, Cynthia F. 2005. Negotiating gender/race/class constraints in the new South Africa: a case study of women's soccer. International Review for the Sociology of Sport 40, Nr. 1: 53-70.

Petchesky, Rosalind P. 2003. Global prescriptions: gendering health and human rights. London: Zed Books.

Petermandl, Werner. 2005. Geht ihr aber ins Stadion ... Ein althistorischer Blick auf das Sportpublikum wie es war, wie es ist und wie es immer sein wird. In: Das Stadion: Geschichte, Architektur, Politik, Ökonomie, hg. von Matthias Marschik, Rudolf Müllner, Georg Spitaler und Michael Zinganel, 127-152. Wien: Turia + Kant.

Pfister, Gertrud, Hrsg. 1980. Frau und Sport. Frankfurt am Main: Fischer.

---. 1991. Zur Geschichte des Diskurses über den „weiblichen“ Körper (1880-1933). In: Bewegungs(t)räume. Frauen Körper Sport, hg. von Birgit Palzkill, Heidi Scheffel und Gabriele Sobiech, 7-14. München: Frauenoffensive.

---. 1999. Sport im Lebenszusammenhang von Frauen: ausgewählte Themen. Schorndorf: Hofmann.

---. 2003. Doing sport is doing gender - Sport and socialisation from a constructivist perspective. In: New approaches to exercise and sport psychology - theories, methods and applications. XIth European Congress of Sport Psychology, Copenhagen, 22-27 July 2003., hg. von Reinhard Stelter und Kirsten Kaya Roessler. Copenhagen: Institute of Exercise and Sport Sciences, University of Copenhagen.

---. 2003a. The challenges of women's football in east and west Germany: A comparative study. Soccer \& Society 4, Nr. 2-3: 128-148.

---. 2006. „Auf den Leib geschrieben“ - Körper, Sport und Geschlecht aus historischer Perspektive. In: Handbuch Sport und Geschlecht, hg. von Ilse HartmannTews, Bettina Rulofs und Dorothee Alfermann, 26-39. Schorndorf: Hofmann.

---. 2011. Die Darstellung von Frauen im Mediensport - Kontinuitäten und Veränderungen. In: Die Sexualisierung des Sports in den Medien, hg. von Daniela Schaaf und Jörg-Uwe Nieland, 57-80. Köln: Herbert von Harlem Verlag.

---. 2012. „Lasst sie Fußball spielen“ - Ärztinnen als Anwältinnen des Mädchenfußballs an der Wende vom 19. zum 20. Jahrhundert. In: Spielen Frauen ein anderes Spiel?, hg. von Gabriele Sobiech, 41-59. Wiesbaden: VS Verlag für Sozialwissenschaften. 
Pfister, Gertrud und Kari Fasting. 2004. Geschlechterkonstruktion auf dem Fußballplatz. Aussagen von Fußballspielerinnen zu Männlichkeits- und Weiblichkeitskonzepten. In: Die lokal-globale Fußballkultur - wissenschaftlich beobachtet, hg. von Dieter H. Jütting, 137-152. Münster: Waxmann.

Phillips, Anne. 2010. What's wrong with essentialism? Distinktion: Scandinavian Journal of Social Theory 11, Nr. 1: 47-60.

Phipps, Alison. 2016. Whose personal is more political? Experience in contemporary feminist politics. Feminist Theory 17, Nr. 3: 303-321.

Pilz, Gunter A. 2013. Sport, Fairplay und Gewalt. Beiträge zur Jugendarbeit und Prävention im Sport. Hildesheim: arete Verlag.

Pinter, Markus. 2005. Die Ehrentribüne als politische Bühne. In: Das Stadion: Geschichte, Architektur, Politik, Ökonomie, hg. von Matthias Marschik, Rudolf Müllner, Georg Spitaler und Michael Zinganel, 323-340. Wien: Turia + Kant.

Ploder, Andrea und Johanna Stadlbauer. 2013. Autoethnographie und Volkskunde? Zum Potenzial wissenschaftlicher Selbsterzählungen für die volkskundlichkulturanthropologische Forschungspraxis. Österreichische Zeitschrift für Volkskunde LXVII /116, Nr. 3+4: 373-404.

PoLAR, Hrsg. 2010. Symposium: ethnographic perspectives on NGOs. PoLAR: Political and Legal Anthropology Review 33, Nr. 2.

Polat, Yasmin. 2016. In Kreuzberg soll es den ersten Fußballplatz nur für Frauen geben. tagesspiegel.de, $18 . \quad$ Mai. URL: http://www.tagesspiegel.de/berlin/berliner-fussball-in-kreuzberg-soll-es-denersten-fussballplatz-nur-fuer-frauen-geben/13601204.html (zugegriffen: 14.1.2017).

Probyn, Elspeth. 2000. Sporting bodies: dynamics of shame and pride. Body \& Society 6, Nr. 1: 13-28.

Profé, Alice. 1980 [1928]. Frauensport aus ärztlicher Sicht. In: Frau und Sport, hg. von Gertrud Pfister, 113-115. Frankfurt am Main: Fischer.

---. 1980a [1928]. Soll auch die Frau Leibesübungen treiben? In: Frau und Sport, hg. von Gertrud Pfister, 124-125. Frankfurt am Main: Fischer.

Puig de la Bellacase, María. 2012. 'Nothing comes without its world': thinking with care. The Sociological Review 60, Nr. 2: 197-216.

Rabinow, Paul. 2004. Anthropologie der Vernunft: Studien zu Wissenschaft und Lebensführung. Frankfurt am Main: Suhrkamp.

Rabinow, Paul, George E. Marcus, James D. Faubion und Tobias Rees. 2008. Designs for an anthropology of the contemporary. Durham: Duke University Press.

Rahnema, Majid. 1997. Towards post-development: searching for signposts, a new language and new paradigms. In: The post-development reader, hg. von Majid Rahnema und Victoria Bawtree, 277-289. London: Zed Books.

Ramanath, Ramya. 2007. Limits to institutional isomorphism: examining internal processes in NGO-government interactions. Nonprofit and Voluntary Sector Quarterly 38, Nr. 1: 51-76.

Ramanath, Ramya und Alnoor Ebrahim. 2010. Strategies and tactics in NGOgovernment relations: insights from slum housing in Mumbai. Nonprofit Management and Leadership 21, Nr. 1: 21-42.

Raz, Florian. 2014. Die Mär vom unpolitischen Sport. tageswoche.ch. 24. Januar. URL: https:/www.tageswoche.ch/de/2014_04/sport/631316/\#hintergrund (zugegriffen 11.3.2016). 
Reckwitz, Andreas. 2003. Grundelemente einer Theorie sozialer Praktiken: eine sozialtheoretische Perspektive. Zeitschrift für Soziologie 32, Nr. 4: 282-301.

---. 2008. Praktiken und Diskurse: eine sozialtheoretische und methodologische Relation. In: Theoretische Empirie: zur Relevanz qualitativer Forschung, hg. von Herbert Kalthoff, Stefan Hirschauer und Gesa Lindemann, 188-209. Frankfurt am Main: Suhrkamp.

Revillard, Anne und Laure Bereni. 2016. From grassroots to institutions: women's movements studies in Europe. In: Social movement studies in Europe: the state of the art, hg. von Guya Accornero und Olivier Fillieule, 156-172. New York: Berghahn.

Riles, Annelise. 2007. The network inside out. Ann Arbor: University of Michigan Press.

Robertson, Jennifer Ellen. 2002. Reflexivity redux: a pithy polemic on ,ppositionality“. Anthropological Quarterly 75, Nr. 4: 785-792.

Robertson, Roland. 1995. Glocalization: time-space and homogeneity-heterogeneity. In: Global modernities, 25-44. Thousand Oaks u.a.: Sage Publications.

Rolshoven, Johanna. 2012. Zwischen den Dingen: der Raum. Das dynamische Raumverständnis der empirischen Kulturwissenschaft. Schweizerisches Archiv für Volkskunde 108: 156-169.

Rose, Lotte. 1992. Körper ohne Raum. Feministische Studien 1: 113-120.

Rose, Nikolas und Peter Miller. 1992. Political power beyond the state. The British Journal of Sociology 43, Nr. 2: 172-205.

Roth, Silke. 2006. Sisterhood and exclusionary solidarity in a labor women's organization. In: Emotions and social movements, hg. von Helena Flam und Debra King, 189-206. London u.a.: Routledge.

Rottenberg, Catherine. 2014. The rise of neoliberal feminism. Cultural Studies 28, Nr. 3: 418-437.

Roy, Arundhati. 2014. The NGO-ization of resistance. Massalijn.nl. 9. April. URL: http://massalijn.nl/new/the-ngo-ization-of-resistance/ (zugegriffen: 18.10.2016).

RPO. 2010. „Charme Offensive des DFB. Von Kickerinnen zu schillernden Schönheiten. “ $\quad$ rp-online.de. $10 . \quad$ Dezember. URL: https://rponline.de/sport/fussball/frauen/wm/von-kickerinnen-zu-schillerndenschoenheiten_aid-12517919 (zugegriffen: 4.10.2013).

Rundfunk Berlin-Brandenburg. 2015. Berliner Fußball-Verband wieder ohne Frau im Präsidium. rbb-online.de. 5. Juli. URL: https://www.rbbonline.de/sport/beitrag/2015/05/ruecktritt-der-einzigen-frau-im-praesidium-desberliner-fussball.html (zugegriffen: 11.11.2015).

Sabo, Donald. 1993. Psychological impacts of athletic participation on American women: Facts and fables. In: Sport in contemporary society: an anthology, hg. von D. Stanley Eitzen, 374-387. New York: St. Martin's Press.

Sandoval, Chela. 2000. Methodology of the oppressed. Minneapolis: University of Minnesota Press.

Sands, Robert R. 2002. Sport Ethnography. Champaign: Human Kinetics.

Sattler, Simone. 2014. Computergestützte qualitative Datenbearbeitung. In: Methoden der Kulturanthropologie, hg. von Christine Bischoff, Karoline Oehme-Jüngling und Walter Leimgruber, 476-487. Bern: Haupt.

Schaaf, Daniela. 2012. „Lieber Barbie als Lesbe?“ Dispositionen von Sportjournalisten und Sponsoren zum heteronormativen Körperideal im Frauenfußball. In: 
Spielen Frauen ein anderes Spiel?, hg. von Gabriele Sobiech, 139-154. Wiesbaden: VS Verlag für Sozialwissenschaften.

---. 2013. Vom „Mannweib“ zur „sexy Kickerin“. Veränderungen und Kontinuitäten im redaktionellen Auswahlprozess des Frauenfußballs. In: Frauenfussball in Deutschland: Anfänge - Verbote - Widerstände - Durchbruch, hg. von Markwart Herzog, 263-284. Stuttgart: Kohlhammer.

Schaaf, Daniela und Jörg-Uwe Nieland. 2011. Der Widerspenstigen Zähmung. Zur Sexualisierung des Frauenfußballs. Das Argument: Zeitschrift für Philosophie und Sozialwissenschaften 53, Nr. 1: 61-67.

---. 2011a. Anmerkungen zur Sexualisierung des Sports in den Medien. In: Die Sexualisierung des Sports in den Medien, hg. von Daniela Schaaf und Jörg-Uwe Nieland, 9-33. Köln: Harlem Verlag.

Scheer, Monique. 2011. Welchen Nutzen hat die Feldforschung für eine Geschichte religiöser Gefühle? Vokus 21, Nr. 1/2: 65-77.

---. 2012. Are emotions a kind of practice (and is that what makes them have a history)? A Bourdieuian approach to understanding emotion. History and theory 51, Nr. 2: 193-220.

Scheffer, Thomas. 2013. Die trans-sequentielle Analyse - und ihre formativen Objekte. In: Grenzobjekte: soziale Welten und ihre Übergänge, hg. von Reinhard Hörster, Stefan Köngeter, Burkhard Müller und Stephan Wolff, 87-114. Wiesbaden: VS Verlag für Sozialwissenschaften.

Schmidt, Robert. 2012. Soziologie der Praktiken: konzeptionelle Studien und empirische Analysen. Berlin: Suhrkamp.

Schmidt-Lauber, Brigitta. 2007. Das qualitative Interview oder: Die Kunst des RedenLassens. In: Methoden der Volkskunde: Positionen, Quellen, Arbeitsweisen der europäischen Ethnologie, hg. von Silke Göttsch-Elten und Albrecht Lehmann, 69-188. Berlin: Reimer.

Schokosport. O.J. Schokosport - Tanz, Bewegung und Selbstverteidigung für Frauen. Homepage. URL: https://www.schokosport.de (zugegriffen: 8.3.2016).

Scholl, Armin. 2013. Die Gegenöffentlichkeit sozialer Bewegungen: Zwischen strategischer Kommunikation und Verständigungsorientierung. In: Strategische Kommunikation, hg. von Ulrike Röttger, Volker Gehrau und Joachim Preusse, 201234. Wiesbaden: VS Verlag für Sozialwissenschaften.

Schönberger, Klaus. 1995. Arbeitersportbewegung in Dorf und Kleinstadt. Zur Arbeiterbewegungskultur im Oberamt Marbach 1900 - 1933. Tübingen: Tübinger Vereinigung für Volkskunde.

---. 2014. Protest! Von der Koordination zum Projekt? In: Protest, Empörung, Widerstand: zur Analyse von Auflehnungsbewegungen, hg. von Iuditha Balint, Hannah Dingeldein und Kathrin Lämmle, 19-30. Konstanz: UVK.

Schouten, Peer. 2009. Theory Talk \#34: James Ferguson on Modernity, Development, and Reading Foucault in Lesotho. Theory Talks. 22. November. URL: http://www.theory-talks.org/2009/11/theory-talk-34.html (zugegriffen: 7.6.2016).

Schrey, Martina. 2015. Das Klischee des „Altherren-Clubs“ stimmt noch immer. inforadio.de. 23. Februar. URL: http://www.inforadio.de/hintergrundmaterial/sport/216836.html (zugegriffen: 26.4.2017).

Schütze, Stefanie und Julia Haß. 2017. Fußball als Zugehörigkeitsraum. Amateurteams bolivianischer Migrantinnen in Sao Paolo. In: Ballsport, Gender, Transkulturalität, hg. von Julia Haß und Stephanie Schütze, 115-32. Berlin: Panama. 
Schuller, Mark und David Lewis. 2017. Engagements with a productively unstable category: anthropologists and non-governmental organizations. Current Anthropology 58, Nr. 5, 634-651.

Schultz, Jaime. 2014. Qualifying times: points of change in U.S. women's sport. Urbana: University of Illinois Press.

Schuster, Nina. 2008. Queere Räume? Strategien queerer Raumproduktion und ambivalente Politiken der Sichtbarkeit. In: Queer leben - queer labeln? (Wissenschafts)kritische Kopfmassagen, hg. von Judith Coffey, 128-144. Freiburg: Fwpf.

Schütte, Wilfried. 1996. „Die schäbige Geeje auf dem edlen Bratschenkasten“: Scherzbeziehungen und soziale Welten - ein Konzept zwischen Anthropologie und Konversationsanalyse. In: Scherzkommunikation: Beiträge aus der empirischen Gesprächsforschung, hg. von Helga Kotthoff, 193-221. Opladen: Westdeutscher Verlag.

Schütze, Fritz. 1983. Biographieforschung und narratives Interview. Neue Praxis 13, Nr. 3: 283-293.

Schwanhäußer, Anja. 2010. Kosmonauten des Underground: Ethnografie einer Berliner Szene. Interdisziplinäre Stadtforschung. Frankfurt am Main: Campus.

Scott, Joan Wallach. 1991. The evidence of experience. Critical inquiry 17, Nr. 4: 773-797.

---. 2012. The fantasy of feminist history. Durham: Duke University Press.

Scraton, Sheila, Caudwell, Jayne und Holland, Samantha. 2005. „Bent it like Patel“: centring „race“, ethnicity and gender in feminist analysis of women's football in England. International Review for the Sociology of Sport 40, Nr. 1: 71-88.

Scraton, Sheila und Anne Flintoff, Hrsg. 2002. Gender and sport: a reader. London u.a.: Routledge.

Seitenwechsel e.V. 2018. "Feministischer Sport: gestern - heute - morgen". 30 Jahre Seitenwechsel. URL: https://www.seitenwechselberlin.de/fileadmin/upload_maedchensport/bilder/pdfs/Programmflyer-web.pdf (zugegriffen: 2.5.2019).

---. O.J. Play like a girl. Mädchensport bei Seitenwechsel e.V. Homepage. URL: http://leyla.seitenwechsel-berlin.de/leyla-rennt-sport.htm (zugegriffen: 8.5.2019).

Selmer, Nicole. 2013. Der andere Fußball. Pferderennen mit Eseln? In: Frauenfussball in Deutschland: Anfänge - Verbote - Widerstände - Durchbruch, hg. von Markwart Herzog, 49-62. Stuttgart: Kohlhammer.

Sharma, Aradhana. 2008. Logics of empowerment: development, gender, and governance in neoliberal India. Minneapolis: University of Minnesota Press.

---. 2014. The state and women's empowerment in India. In: Theorizing NGOs: states, feminisms, and neoliberalism, hg. von Victoria Bernal und Inderpal Grewal, 93113. Durham: Duke University Press.

Sharpe, Erin K. 2008. Festivals and social change: intersections of pleasure and politics at a community music festival. Leisure Sciences 30, Nr. 3: 217-234.

Sherry, Emma. 2010. (Re)engaging marginalized groups through sport: the Homeless World Cup. International Review for the Sociology of Sport 45, Nr. 1: 59-71.

Siliman, Jael. 1999. Expanding civil society: shrinking political spaces - the case of women's non-governmental organizations. Social Politics 6, Nr. 1: 23-53.

Simpson, Meghan. 2009. Intersectionalities of gender and class in the wake of Kyrgizstan's march 2005 events. In: Gender dynamics and post-conflict reconstruction, 137-154. Frankfurt am Main: Peter Lang. 
Skeggs, Beverley. 1999. Matter out of place: visibility and sexualities in leisure spaces. Leisure Studies 18, Nr. 3: 213-232.

Sobiech, Gabriele. 2011. Die „Logik der Praxis“. Zur Herstellung einer kompetenten Mitgliedschaft im Frauenfußball. Spectrum 23, Nr. 2: 41-69.

Sobiech, Gabriele und Andrea Ochsner, Hrsg. 2012. Spielen Frauen ein anderes Spiel? Geschichte, Organisation, Repräsentationen und kulturelle Praxen im Frauenfußball. Wiesbaden: VS Verlag für Sozialwissenschaften.

Spaaij, Ramon. 2011. The glue that holds the community together? Sport and sustainability in rural Australia. In: The social impact of sport: cross-cultural perspectives, hg. von Ramon Spaaij, 24-38. London u.a.: Routledge.

Speed, Shannon. 2005. Dangerous discourses. PoLAR: Political and Legal Anthropology Review 28, Nr. 1: 29-51.

Sperling, Valerie, Myra Marx Ferree und Barbara Risman. 2001. Constructing global feminism: transnational advocacy networks and Russian women's activism. Signs 26, Nr. 4: 1155-1186.

Sperschneider, Eva, Annika Schauer und Marlene Schauer. 2012. DISCOVER FOOTBALL - Mit Fußball für Gleichberechtigung und Solidarität. Feministische Studien, Nr. 1: 110-118.

Spivak, Gayatri Chakravorty. 1996. Subaltern studies: deconstructing historiography. In: The Spivak reader: selected works of Gayatri Chakravorty Spivak, hg. von Donna Landry und Gerald M. MacLean, 203-236. London u.a.: Routledge.

Sterchele, Davide. 2015. De-sportizing physical activity: From sport-for-development to play-for-development. European Journal for Sport and Society 12, Nr. 1: 97120.

Sterchele, Davide und Chantal Saint-Blancat. 2013. Keeping it liminal. The Mondiali Antirazzisti (Anti-racist World Cup) as a multifocal interaction ritual. Leisure Studies: $182-196$.

Stevenson, Christopher L. 1975. Socialization effects of Participation in sport: a critical review of the research. Research Quarterly. American Alliance for Health, Physical Education and Recreation 46, Nr. 3: 287-301.

Stoetzler, Marcel und Nira Yuval-Davis. 2002. Standpoint theory, situated knowledge and the situated imagination. Feminist Theory 3, Nr. 3: 315-333.

Strüver, Anke und Claudia Wucherpfennig. 2012. Spielerisch-sportliche Raumaneignung. Zur Performativität von Körpern und Räumen im Spiegel aktueller Forschung. Feministische Studien, Nr. 1: 66-72.

Struzik, Justyna. 2019. unveröffentliches Manuskipt.

Sülzle, Almut. 2011. Fussball, Frauen, Männlichkeiten: eine ethnographische Studie im Fanblock. Frankfurt am Main: Campus.

---. 2014. Fußball und Männlichkeit. Vortrag gehalten auf: Workshop zu Sexismus und Homophobie im Fußball, 26. Juli, Berlin.

---. 2016. Emotionen pur? Feldforschungssupervision als Auswertungsinstrument in der ethnografischen Sportforschung. In: Emotionen im Spiel. Beiträge zu einer Ethnologie des Sports, hg. von Friederike Faust und Stefan Heissenberger, 2636. Berlin: Panama.

Tauschek, Markus, Hrsg. 2013. Kulturen des Wettbewerbs: Formationen kompetitiver Logiken. Münster: Waxmann.

Thayer, Millie. 2010. Making transnational feminism: rural women, NGO activists, and northern donors in Brazil. London u.a.: Routledge. 
---. 2010a. Translations and refusals: resignifying meanings as feminist political practice. Feminist Studies 36, Nr. 1: 200-230.

Theberge, Nancy. 1987. Sport and women's empowerment. Women's Studies International Forum 10, Nr. 4: 387-393.

---. 1994. Towards a feminist alternative to sport as a male preserve. In: Women, sport, and culture, hg. von Susan Birrell und Cheryl L. Cole, 181-192. Champaign: Human Kinetics.

---. 2003. "No fear comes": adolescent girls, ice hockey, and the embodiment of gender. Youth \& Society 34, Nr. 4: 497-516.

Theweleit, Klaus. 2004. Tor zur Welt: Fussball als Realitätsmodell. Köln: Kiepenheuer \& Witsch.

Thieler, Kerstin und Laura Schibbe, Hrsg. 2016. FrauenBewegung. Geschlechtergeschichte und Sport. Kassel: Ariadne. Forum für Frauen- und Geschlechtergeschichte. Stiftung Archiv der deutschen Frauenbewegung.

Thoma, Matthias. 2013. „Mädchen, ihr müsst einen Club gründen“. Frauenfußball in Frankfurt am Main. In: Frauenfussball in Deutschland: Anfänge - Verbote - Widerstände - Durchbruch, hg. von Markwart Herzog, 65-86. Stuttgart: Kohlhammer.

Tomkowiak, Ingrid. 2014. Die Farbe Rosa. In: Macher-Medien - Publika. Beiträge der Europäischen Ethnologie zu Geschmack und Vergnügen, hg. von Kaspar Maase, Brigitte Frizzoni, Christoph Bareither und Mirjam Nast, 177-192. Würzburg: Königshausen u. Neumann.

Tsing, Anna Lowenhaupt. 2005. Friction: an ethnography of global connection. Princeton: Princeton University Press.

Tuan, Yi-Fu. 2011 [1977]. Space and place: the perspective of experience. Minneapolis: University of Minnesota Press.

Tucholsky, Kurt. 1961. Das Mitglied. In: Gesammelte Werke Bd. 2, hg. von Mary Gerold-Tucholsky und Fritz J. Raddatz, 457-458. Berlin: Rowohlt.

UN Inter-Agency Task Force on Sport For Development and Peace. 2005. Sport as a tool for development and peace: towards achieving the United Nations Millennium Development Goals. URL: http://www.un.org/sport2005/resources/task_force.pdf (zugegriffen: 24.3.2016).

Valentine, Gill. 1989. The geography of women's fear. Area 21, Nr. 4: 385-390.

---. 1993. (Hetero)sexing space: lesbian perceptions and experiences of everyday spaces. Environment \& Planning D: Society \& Space 11: 395-413.

Vermeulen, Jeroen und Paul Verweel. 2011. Participation in sport: bonding and bridging as identity work. In: The social impact of sport: cross-cultural perspectives, hg. von Ramon Spaaij, 98-111. London u.a.: Routledge.

Verran, Helen. 2010 [2001]. Science and an African logic. Chicago: University of Chicago Press.

Vigh, Henrik. 2006. Navigating terrains of war: youth and soldiering in GuineaBissau. Oxford, New York: Berghahn.

---. 2009. Motion squared. A second look at the concept of social navigation. Anthropological Theory 9, Nr. 4: 419-438.

Wacquant, Loïc. 2003. Leben für den Ring. Boxen im amerikanischen Ghetto. Konstanz: UVK.

---. 2011. Habitus as topic and tool: reflections on becoming a prizefighter. Qualitative Research in Psychology 8, Nr. 1: 81-92. 
Wagner, Lorenz. 2017. Nach Ihrer Pfeiffe. Süddeutsche Zeitung Magazin 21: 8-15.

Walgenbach, Katharina, Gabriele Dietze, Lann Hornscheidt und Kerstin Palm, Hrsg. 2007. Gender als interdependente Kategorie: neue Perspektiven auf Intersektionalität, Diversität und Heterogenität. Opladen: Budrich.

Warneken, Bernd-Jürgen. 2006. Die Ethnographie popularer Kulturen: Eine Einführung. Wien: Böhlau.

Warneken, Bernd-Jürgen und Andreas Wittel. 1997. Die neue Angst vor dem Feld. Ethnographisches research up am Beispiel der Unternehmensforschung. Zeitschrift für Volkskunde 93, Nr. 1: 1-16.

Warner, Michael. 1993. Introduction. In: Fear of a queer planet: queer politics and social theory, hg. von Michael Warner, vii-xxxi. Minneapolis: University of Minnesota Press.

---. 2002. Publics and counterpublics (abbreviated version). Quarterly Journal of Speech 88, Nr. 4: 413-425.

Weber, Max. 2002 [1922]. Wirtschaft und Gesellschaft: Grundriss der verstehenden Soziologie. Tübingen: Mohr.

Weinreich, Jens. 2011. „Gott schütze uns vor Frauenfußball“. deutschlandfunk.de. 8. Mai. URL: https://www.deutschlandfunk.de/gott-schuetze-uns-vorfrauenfussball.1346.de.html?dram:article_id=196380 (zugegriffen: 5.2.2019).

Weiß, Anja. 2001. Rassismus als symbolisch vermittelte Dimension sozialer Ungleichheit. In: Klasse und Klassifikation, hg. von Anja Weiß, Cornela Koppetsch, Albert Scharenberg und Oliver Schmidtke, 79-108. Opladen: Westdeutscher Verlag.

---. 2013. Rassismus wider Willen ein anderer Blick auf eine Struktur sozialer Ungleichheit. Wiesbaden: VS Verlag für Sozialwissenschaften.

Wellgraf, Stefan. 2012. Hauptschüler: zur gesellschaftlichen Produktion von Verachtung. Bielefeld: Transcript.

Wenger, Etienne. 2008. Communities of practice: learning, meaning, and identity. Cambridge: Cambridge University Press.

Wenger-Trayner, Etienne und Beverly Wenger-Trayner. 2015. Learning in landscapes of practice: a framework. In: Learning in landscapes of practice: boundaries, identity, and knowledgeability in practice-based learning, hg. von Etienne Wenger-Trayner, Mark Fenton-O’Creevy, Steven Hutchinson, Chris Kubiak und Beverly Wenger-Trayner, 13-30. London u.a.: Routledge.

West, Candance und Don H. Zimmerman. 2009. Accounting for Doing Gender. Gender \& Society 23, Nr. 1: 112-122.

Whitson, David. 2002. The embodiment of gender: discipline, domination, and empowerment. In: Gender and sport: a reader, hg. von Sheila Scraton und Anne Flintoff, 227-240. London u.a.: Routledge.

de Wilde, Mandy. 2015. Brave new neighbourhood: affective citizenship in Dutch territorial governance. PhD thesis. Amsterdam: University of Amsterdam. URL: https://pure.uva.nl/ws/files/4502345/161461_GEDRUKT_Brave_new_neighbour hood Dissertation Mandy de Wilde DEF.p̄df (zugegriffen: 16.11.2016).

Woodward, Alison E. 2004. Building velvet triangles: gender and informal governance. In: Informal governance in the European Union, 76-93. Cheltenham: Edward Elgar Publishing.

Young, Iris Marion. 1980. Throwing like a girl: a phenomenology of feminine body comportment motility and spatiality. Human Studies 3, Nr. 1: 137-156. 
Young, Kevin. 1997. Women, sport and physicality: preliminary findings from a Canadian study. International Review for the Sociology of Sport 32, Nr. 3: 297305.

Ziai, Aram. 2006. Zwischen Global Governance und Post-Development. Entwicklungspolitik aus diskursanalytischer Perspektive. Münster: Westfälisches Dampfboot. 


\section{Friederike Faust Fußball und Feminismus}

\section{Eine Ethnografie geschlechterpolitischer Interventionen}

Fußball, ein Männersport? Was lange selbstverständlich war, wird zunehmend bezweifelt. Die Geschlechterverhältnisse sind in Bewegung geraten und Frauen fordern ihren Platz auf dem Rasen. Die ethnografische Studie begleitet eine feministische Frauenfußballorganisation bei ihren lokalen und internationalen Aktivitäten und zeigt die Chancen, aber auch Grenzen, die entstehen, wenn die vermeintlichen Gegensätze „Fußball“ und „Feminismus" aufeinandertreffen.

Wie wird unter den gegenwärtigen günstigen, aber spannungsreichen Umständen auf geschlechterpolitischen Wandel hingearbeitet? Mit vielen empirischen Beispielen veranschaulicht die Autorin, wie feministische Fußballerinnen innerhalb der komplexen Landschaft spätkapitalistischer Demokratien pragmatisch, aber von Idealen geleitet für Geschlechtergerechtigkeit kämpfen. Besonderes Augenmerk ist dabei auf die Spannungen zwischen Aktivismus und NGO sowie zwischen fußballerischer Leistung und feministischer Emanzi-pation gelegt, in denen die Akteurinnen beständig navigieren müssen. Die Studie schließt an Diskussionen um Sport, Geschlecht und Feminismus an und kommentiert zugleich politikethnologische Debatten um NGOs, Soziale Bewegungen und Formen des politischen Handelns.

Die Autorin: Dr. Friederike Faust, Wissenschaftliche Mitarbeiterin am Institut für Europäische Ethnologie der Humboldt-Universität zu Berlin

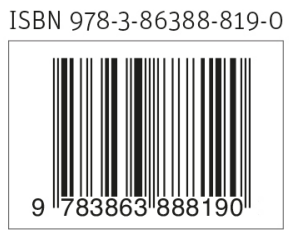

